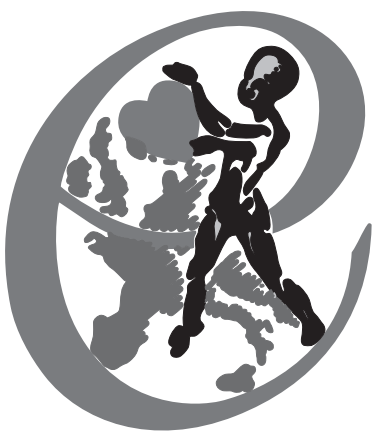

Volume 27 • Supplement S2 • Pages S1-S148

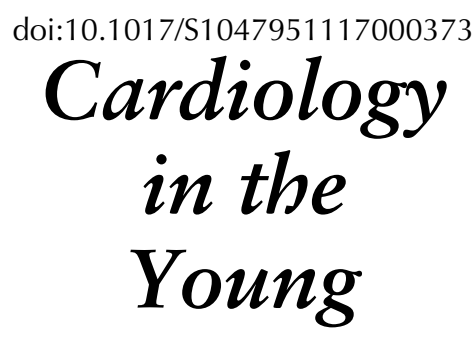

(C) 2017 Cambridge University Press

\title{
51st Annual Meeting of the Association for European Paediatric and Congenital Cardiology (AEPC)
}

MP1-1

Screening newborns for congenital heart disease using echocardiography at high altitude in China

Li J.(1), Qi H.(2), Xie S.(1), Li J.(1), Dai T.(2),

Chen H.(2)

Capital Institute of Pediatrics, Beijing, China(1); Women's and

children's hospital of Qinghai province, Qinghai, China(2)

Introduction: Screening newborns for complex congenital heart disease (CHD) relies widely on pulse oximetry and clinical examination. However, this screening method is not applicable to those living at high altitude, because of variedly decreased arterial saturations at different altitudes in different ethnics. Furthermore, altitude hypoxia induces pulmonary hypertension that is main morbidity in children with simple CHD with left to right shunt. We aimed to screen newborns for CHD using echocardiography at high altitude.

Methods: Echocardiography was performed in consecutive asymptomatic 645 newborns (aged 3 to 5 days; 286 girls and 359 boys; 158 Han, 419 Hui and 62 Tibetan) born in the Women's and Children's Hospital in Xi Ning (2 260 meter), Qinghai province between March 2015 and April 2016. Demographic data was obtained.

Results: Parents of the 645 newborns resided at altitudes from 1800 to 4080 meters (median 2260m). Among them, 600 were born at $\geq 37$ gestational weeks, and 45 born during 30 to 36 weeks. CHD was diagnosed in 171 (78 girls and 93 boys; 46 Han, 104 Hui and 14 Tibetan), making the prevalence of $26.5 \%$. Among them, 123 had secundum atrial septal defect, 14 had ventricular septal defect and 56 had patent ductus arteriosus. No correlation between the prevalence of CHD and ethnics $(p=0.27)$ or altitudes $(p=0.88)$. Additional 456 babies had patent foramen ovale. There was no complex CHD. One month follow up was made in 92 out of 645 babies. Among them, patent ductus arteriosus $(n=16)$ was closed in all. Secundum atrial septal defect $(n=23)$ and ventricular septal defect $(n=3)$ remained unchanged in size except for one spontaneous closure in each type of the CHD. Patent foramen ovale $(n=74)$ remained open except 3 .

Conclusions: The prevalence of simple CHD with left to right shunt CHD is substantial, without complex CHD. Screening newborns for CHD using echocardiography has important implications at high altitude. Follow-up remains challenging due to the poor socio-economical conditions, and must be reinforced in order to provide early repair and prevent from significant pulmonary hypertension.
MP1-2

Beyond aortic dilatation - Positive effect of angiotensin II receptor blockers on mitral valve prolapse in a retrospective analysis of pediatric patients with Marfan syndrome

Olfe J., Pesch J., Mueller G.C., Seggewies F., Stark V., Kozlik-

Feldmann R., Mir T.S.

Clinic for Pediatric Cardiology, University Heart Center,

Universitätsklinikum Hamburg Eppendorf, Hamburg, Germany

Objectives: In recent years, treatment with Angiotensin-IIreceptor-blockers (ARB) in patients with Marfan syndrome (MFS) has been well established. All studies, so far, focused on the the aortic root dilatation. No study has focused on the effect on the regularly appearing mitral valve prolapse (MVP) in MFS. The aim of our study was to analyse the treatment effect of ARB on the degree of MVP in pediatric patients with MFS.

Methods: Since 2008 we investigated 395 patients $(11.4 \pm 5.5 \mathrm{y})$ with suspected MFS. 139 patients were diagnosed with MFS. 61 patients showed MVP. We retrospectively analysed database data and 2D-echocardiograms. Because of the high error measuring the actual MVP in millimeter, mitral anular diameter (MAD) was used as a surrogate parameter for MVP in MFS (Pini R, 1989, Circulation). Patients in the treatment group received oral Valsartan, control patients had no treatment. Patients taking beta-blockers or having being operated on the aorta were excluded to clearly indicate group allocation.

Table 1. Demographic and mitral valve date before therapy initiation or at first visit $(\mathrm{t} 0)$ and at last follow up $(\mathrm{t} 1)$ in patients with MFS without treatment (control) or treated with an angiotensin receptor blocker (ARB).

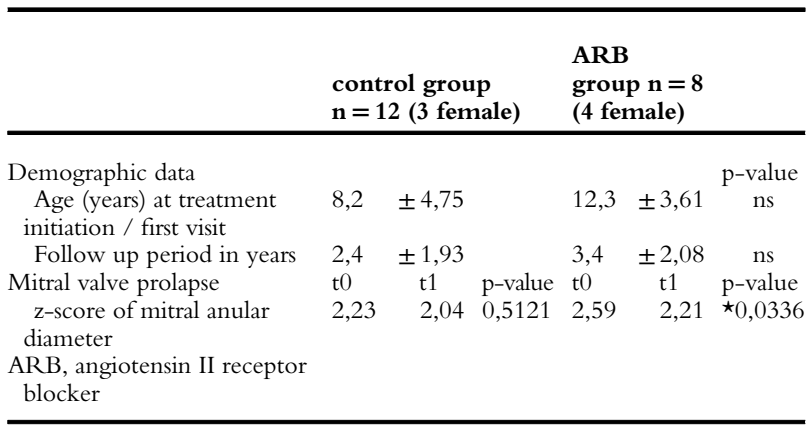




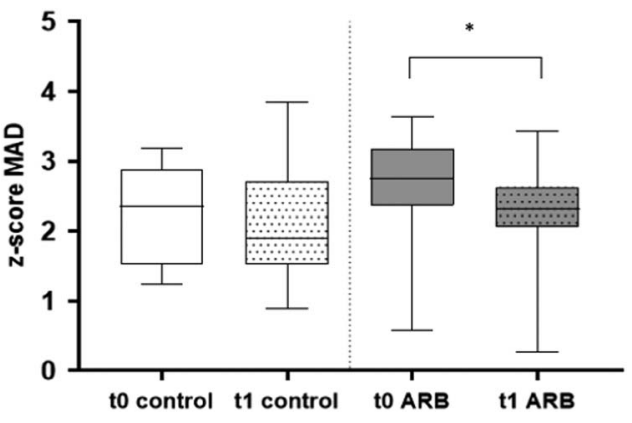

Results: Prevalence of MVP in pediatric Marfan patients was $43,8 \%$. In the control group the $z$-score of the MAD did not change significantly during the observation period. In patients treated with an ARB the $z$-score of the MAD was significantly reduced. Detailed data is shown in table 1 and graph 2.

Conclusion: Prevalence of MVP in children with MFS is 40 times higher than in a healthy population (Sattur S, 2010, Exp Clin Cardiol). In our study, treatment with an ARB in pediatric patients with MFS leads to a significant reduction of the MAD as a surrogate parameter for MVP. This first data suggests another positive effect of ARB treatment in MFS beyond the growth restrictive effect on the aorta. Future studies have to analyse greater numbers of patients, the long term effect and develop better imaging methods for the detailed analysis of MVP e.g. MRI.

\section{MP1-3}

Cardiopulmonary exercise test (CPET) and dobutamine stress cardiac magnetic resonance imaging (CMR) in young adults after neonatal arterial switch operation (ASO) in transposition of the great arteries (TGA)

Hövels-Gürich H. (1), Hamada S. (2), Kirschfink A. (2), Mühler E. (1), Kerst G. (1), Marx N. (2), Frick M. (2)

Department of Paediatric Cardiology, University Hospital Aachen, Germany (1); Department of Cardiology, University Hospital Aachen, Germany (2)

Objectives: Monocentric prospective study to evaluate objective exercise capacity, rate and extent of stress induced myocardial ischemia and abnormal pulmonary blood flow distribution (PBFD) at rest and under dobutamine stress.

Methods: 49 unselected patients (age 18-28y) underwent CPET (bicycle) and CMR (1.5 Tesla) at rest (coronary scan, cine, phase contrast flow) and under dobutamine (DSMR; cine, phase contrast flow), followed by angiography and late gadolinium enhancement (LGE).

Results: CPET: All patients reached maximal exercise effort (HRmax $174.0 \pm 17.3 / \mathrm{min})$. Peak oxygen uptake ( $\%$ of predicted peak VO2) (Wasserman): $85.8 \pm 10.4 \%$ (borderline normal); norm percentile (Dubowy, 2008): $13.2 \pm 12.2$ (z-value $=-1$ ). Ventilatory efficiency $\mathrm{VE} / \mathrm{VCO} 2$ at anaerobic threshold: $26.9 \pm 2.6$ (normal 23 - 30).

CMR: Coronary arteries: 1x known prox. LAD occlusion (collateralized via RCA; coronary type AB1), 1x proximal LCA-occlusion, LIMA-bypass). No other stenosis or kinking of the proximal coronary arteries. Coronary classification (Sauer): 38x A1 (normal type), 5x AB1 (RCX originates from RCA), 6x B1 (right single ostium, LCA retroaortal). 3 patients reclassified compared to operation report.
Ventricular function: LV: no regional wall motion abnormalities; $5 x$ EF $<55 \%$ (43\%-54\%). 1x non compaction cardiomyopathy. RV: all EF $>48 \%$. 1x RVOT-aneurysm.

Neo-aortic regurgitation: $2 \mathrm{x}$ moderate (reg. fraction $16 \%$ and $19 \%$ ). DSMR: 47/49 reached target heart rate; $1 \mathrm{x}$ termination at $120 /$ min due to complex ventricular ectopic beats, $1 \mathrm{x}$ termination at $160 /$ min due to trigger problems. 1 patient with known LAD occlusion had strong chest pain and hypokinesia in 1 segment under maximum stress.

PBFD at rest: 6/49 patients had prior intervention for RVOT/ PA-stenosis. 6/49 abnormal PBFD (>2:1). 4/49 relevant stenosis of the mean PA $(>2.5 \mathrm{~m} / \mathrm{s}) ;->$ cumulative rate of relevant RVOT/PA stenosis or abnormal PBFD:12/49 (25\%).

PBFD under DSMR: no worsening compared to PBFD at rest. On individual patient level, no worsening of abnormal PBFD.

LGE: no myocardial scar. 1/49 intramyocardial fibrosis.

Conclusion: CPET functional status was borderline normal. CMR found no new proximal coronary stenosis or kinking. 2/49 DSMR were pathologic. 12/49 patients (25\%) had relevant RVOT/PA stenosis (6 new, 6 prior known). PBFD did not worsen under dobutamine stress.

MP1-4

Safety And Short-Term Efficacy Of Transition From Bosentan To Macitentan In Patients With Pulmonary Arterial Hypertension: Preliminary Results

Aypar E., Alehan D.

Hacettepe University Hospital, Pediatric Cardiology Department, Ankara, Turkey

Introduction: Macitentan (Opsumit) is an orally active, potent, second-generation dual endothelin receptor antagonist (ERA) indicated for the treatment of pulmonary arterial hypertension (PAH). Macitentan's effectiveness was established in the pivotal SERAPHIN study in 2013. While peripheral edema, hepatotoxicity, and anemia appear to be class effects of the ERAs, clinical trials of macitentan indicate that its unique pharmacokinetics decrease the incidence of these side effects significantly compared to previous ERAs. We aimed to evaluate the safety and short-term efficacy of transition from bosentan to macitentan in patients with $\mathrm{PAH}$.

Methods: This is a single institution, 24 weeks prospective study. Patients on bosentan therapy were switched to oral macitentan $10 \mathrm{mg}$ once daily. Patients on combination treatment continued sildenafil. Patients were evaluated at baseline, 12 and 24 weeks. Endpoints included: change from baseline in 6-minute walk distance (6-MWD), WHO functional class (WHO FC), baseline oxygen saturation, oxygen saturation after 6-MWD test, systolic pulmonary artery pressure (sPAP) measured by echocardiography, brain natriuretic peptide (BNP) levels, incidences of adverse events (AE).

Results: 6 patients (mean age: 20.8 \pm 5.1 years (12-26), body weight: $55.2 \pm 16.1 \mathrm{kgs}(27-73), 4$ females, 2 males) completed 24 weeks of macitentan therapy. 4 had Eisenmenger syndrome, 2 had residual PAH after surgery. Median follow-up period: 13 years (7-23). All patients were in WHO FC II. 4/6 patients were on monotherapy (bosentan), 2/6 patients were on combination therapy (bosentan and sildenafil). Patients were on bosentan therapy for $7.7 \pm 2.7$ years (6-13). One patient with Down syndrome could not complete 6-MWD test. Macitentan significantly improved exercise capacity (6-MWD) from baseline (mean: $469 \mathrm{~m}$ ), at week 12 (mean: $510 \mathrm{~m},+41 \mathrm{~m}$ ), at 24 week (mean: $519 \mathrm{~m},+50 \mathrm{~m})(\mathrm{p}<0.05)$ and oxygen saturation after 6-MWD test from baseline (mean: \%81), at week 12 (mean: \%83), 
at 24 week (mean: \%87) $(\mathrm{p}<0.05)$ and decreased sPAP measured by echocardiography from baseline (mean: $100 \mathrm{mmHg}$ ), at week 12 (mean: $85 \mathrm{mmHg},-15 \mathrm{mmHg}$ ), at 24 week (mean: $85 \mathrm{mmHg}$, $-15 \mathrm{mmHg})(\mathrm{p}<0.05)$. There was no significant change in WHO FC, baseline oxygen saturation, BNP levels. None of the patients had AE (peripheral edema, anemia, hepatotoxicity).

Conclusions: In this short term preliminary study, transition from bosentan to macitentan significantly improved exercise capacity (6-MWD), oxygen saturation after 6-MWD test, hemodynamic parameters and well tolerated without any AE.

\section{MP1-5}

Out of hospital cardiac arrest and cardiopulmonary resuscitation in children with congenital heart defects Holmstrøm H. (2), Jortveit J. (1), Eskedal L. (1), Døhlen G. (2), Birkeland S. (2)

Sorlandet Hospital, Arendal, Norway (1); Oslo University Hospital, Oslo, Norway (2)

Background: Congenital heart defects are the most common birth defects and are an important cause of death in children. Some children with congenital heart defects are affected by cardiac arrest out of hospital, but little is known about the occurrence and consequences of such events. Early cardiopulmonary resuscitation may prevent death. In this nationwide cohort study, we describe the rate of out of hospital cardiac arrest and the rate and results of cardiopulmonary resuscitation in children 2-18 years old with congenital heart defects.

Methods: Data concerning all live births in Norway in 1994-2009 were retrieved from the Medical Birth Registry of Norway, the patient administrative systems at all hospitals in Norway, the Oslo University Hospital's Clinical Registry for Congenital Heart Defects and the Norwegian Cause of Death Registry. Survivors were followed through 2012, and supplementary information for the deceased children was retrieved from medical records at Norwegian hospitals. Cardiac arrests related to cardiac surgery were excluded.

Results: Among the 943871 live births in Norway 1994-2009, $11272(1.2 \%)$ children had a congenital heart defect. We identified nine $(0.1 \%)$ children $2-18$ years old who experienced out of hospital cardiac arrest. Early cardiopulmonary resuscitation was initiated in all patients. Return of spontaneous circulation occurred in four children, but two of them died of anoxic brain damage within a few days. Two children survived without serious sequelae.

Conclusions: Out of hospital cardiac arrest was infrequent among children 2-18 years old with congenital heart defects. Cardiopulmonary resuscitation was initiated in all, but only $22 \%$ survived.

\section{MP1-6 \\ Long-term follow-up after catheter ablation of accessory pathways in children \\ Backhoff D., Klehs S., Müller M.J., Schneider H.E., Paul T.,} Krause $U$

Department of Pediatric Cardiology and Intensive Care Medicine, Georg August University Medical Center, Göttingen, Germany

Background: Catheter ablation of the accessory pathway (AP) is treatment of choice for children with WPW syndrome. Data on long-term results $\geq 3$ years after $\mathrm{AP}$ ablation in pediatric patients is limited.

Patients and Methods: Between 10/2002 and 06/2015, a total of 268 patients $<18$ years of age had successful AP ablation. Median age at ablation was 11.6 (IQR 7.9-14.3) years, median follow-up was 5.6 (IQR 3.1-9.1) years, minimal follow-up was 1 year. Recurrence after ablation was defined as documentation of either pre-excitation on ECG or SVT attributable to AP.

Results: During follow-up, a total of 29/268 subjects experienced recurrence. 13/29 (45\%) patients had recurrence within the first month post ablation, and 23/29 (79\%) within the first year. After successful ablation, calculated freedom from SVT or ventricular pre-excitation was $91 \%$ after one year, $90 \%$ after three years, $90 \%$ after five years and $88 \%$ after eight years. In a Cox regression model of the whole study group, left-sided AP localization, low number of RF applications, short fluoroscopy and short procedure times were associated with long term success while presence of structural heart disease and multiple APs were associated with recurrence. Age, body weight and pre-excitation on ECG prior to ablation did not impact long-term success. No late complications attributable to AP ablation were observed.

Conclusions: RF ablation of AP in children was associated with early recurrence within the first post-ablation year in approximately $9 \%$ of the patients. Late recurrences after 3 years were rare.

\section{MP1-7}

Pediatric patients with KCNJ2 mutations: wide clinical spectrum in a special disorder

Zambrano M.D.(1), Cesar S.(1), Carro E.(1), Campuzano O.(2), Maya K.(3), Viadero M.T.(4), Alegria I.(4), Sanchez-de-Toledo J.(1), Brugada J.(1), Sarquella-Brugada G.(1)

Pediatric cardiology department, Hospital Sant Joan de Déu, Barcelona, Spain (1); IDIBGI-Cardiovascular genetic center, Universitat de Girona, Girona, Spain (2); Pediatric cardiology department, Instituto Hispalense de pediatría, Sevilla, Spain (3); Pediatric cardiology department, Hospital Marqués de Valdecilla, Santander, Spain (4)

Introduction: Andersen-Tawil syndrome (ATS) is a rare disorder characterized by periodic paralysis, cardiac arrhythmias and dimorphic features. ATS has been associated with mutations in the KCNJ2 with autosomal dominant inheritance pattern. The aim of the study is to analyze the electrocardiographic pattern of these patients.

Methods: Retrospective review of patients with KCNJ2 mutations in our unit. Clinical features, treatment, procedures, EKG stress test, 24h-Holter measurements and follow up are described. QT and QU intervals were measured in lead V2 with Bazzet formula, and electrocardiographic criteria for ATS.

Results: Family history, clinical features, genetics and treatment are shown in table 1.

\begin{tabular}{|c|c|c|c|c|c|c|}
\hline $\begin{array}{l}\text { Pat- } \\
\text { ient }\end{array}$ & $\begin{array}{l}\text { Age } \\
(y)\end{array}$ & Family History & $\begin{array}{l}\text { Dimor- } \\
\text { phic } \\
\text { features }\end{array}$ & Arrhythmia & Mutation & Treatment \\
\hline 1 & 8 & $\begin{array}{r}\text { Mother KCNJ2 } \\
\text { asymptomatic }\end{array}$ & Yes & $\begin{array}{l}\mathrm{PVC} \text { and } \\
\mathrm{PMVT}\end{array}$ & $\begin{array}{l}\mathrm{KCNJ} 2 \mathrm{p} .189 \\
\mathrm{R}>\mathrm{I}\end{array}$ & $\begin{array}{l}\text { Flecainide } \\
\text { Beta- } \\
\text { Blocker }\end{array}$ \\
\hline 2 & 6 & $\begin{array}{l}\text { Mother KCNJ2 + } \\
\text { ICD }\end{array}$ & Yes & No & $\begin{array}{l}\text { KCNJ2 } \\
\text { p.144G }>\text { A }\end{array}$ & No \\
\hline 3 & 11 & $\begin{array}{l}\text { Mother } \\
\text { KCNJ2 + asymptomatic }\end{array}$ & No & No & $\begin{array}{l}\mathrm{KCNJ} 2 \mathrm{p} .82 \\
\mathrm{R}>\mathrm{Q}\end{array}$ & No \\
\hline 4 & 15 & $\begin{array}{l}\text { Mother } \\
\text { KCNJ2 + asymptomatic }\end{array}$ & No & PVC-couplet & $\begin{array}{l}\text { KCNJ2 p. } 82 \\
\text { R }>\text { Q }\end{array}$ & $\begin{array}{l}\text { Beta- } \\
\text { blocker }\end{array}$ \\
\hline 5 & 5 & No & Yes & $\begin{array}{l}\text { PVC, PMVT, } \\
\text { TV } \\
\text { bidirectional }\end{array}$ & $\begin{array}{l}\text { KCNJ2 p.218 } \\
\text { R > W }\end{array}$ & $\begin{array}{l}\text { Flecainide } \\
\text { Beta- } \\
\text { blocker }\end{array}$ \\
\hline
\end{tabular}

All patients were females, mean age at diagnosis of 7.6 y (IQR 7; SD5.1). Four were index cases. Prominent U-waves in leads 
V2-V5 and in the inferior leads were detected in all patients. "U on P" sign was observed during tachycardia in all cases. Patients 1, 4 and 5 had the "tee on pee" sign and prolongation of the QT interval after premature ventricular contractions (PVC). Mean QT interval was $433 \mathrm{~ms}$ (IQR60, SD4), mean QU interval was $591 \mathrm{~ms}$ (IQR32.5, SD106). Two patients required sympathectomy due to refractory polymorphic ventricular tachycardia; the others are asymptomatic or well controlled with pharmacological therapies. The mean follow up time was 4 years, there were no malignant events and none need ICD.

Conclusions: Patients with KCNJ2 mutation have wide clinical and electrocardiographic manifestations, from asymptomatic to refractory PMVT. EKG features constantly seen were "U on P" during tachycardia, "tee on pee" sign and QT interval prolongation after PVC. Flecainide and beta-blocker therapy seems to be effective, but patients with refractory PMVT may need other drugs and sympathectomy to prevent malignant events.

\section{MP1-8 \\ First data of the RIKADA study: risk stratification of children and adolescents with primary cardiomyopathies}

Degener F. (1,2,3), Al-Wakeel-Marquard N.(1,3), Schmitt B.(1,3), Kelm M.(1), Kühne T.(1,2), Berger F.(1,2,3), Messroghli D. (1,3,4), Klaassen S. $(2,3,5)$

Deutsches Herzzentrum Berlin, Klinik für Angeborene Herzfehler/ Kinderkardiologie, Berlin, Germany (1); Charité Universitätsmedizin Berlin, Klinik für Pädiatrie mit Schwerpunkt Kardiologie, Berlin, Germany (2); DZHK (German Centre for Cardiovascular Research), partner site Berlin, Germany (3); Deutsches Herzzentrum Berlin, Klinik für Innere Medizin - Kardiologie, Berlin, Germany (4); Experimental and Clinical Research Center, a joint cooperation between the Charité Medical Faculty and the Max-Delbrück-Center for Molecular Medicine, Berlin, Germany (5)

Introduction: Primary cardiomyopathies (CMP) and their outcome in children and adolescents are heterogeneous. Some are diagnosed prenatally; others are first diagnosed with sudden cardiac death (SCD) or acute heart failure. The aim of the RIKADA study is to give a systematic characterization of these patients and first degree family members, and develop a risk stratification which allows detecting patients with high risk for heart failure or SCD.

Methods: We performed preliminary analyses of the first index patients enrolled into the RIKADA study between February 2014 and October 2016. The study design consists of physical examination, ECG, echocardiography, if applicable cardiopulmonary exercise testing, cardiovascular magnetic resonance (CMR) imaging, and blood analyses including molecular genetic testing. Follow-up examinations are performed every three years in index patients, every six years in siblings (until the age of 21 years).

Results: By October 2016, 48 patients with CMP (35\% dilated CMP, DCM; 29\% hypertrophic CMP, HCM; 19\% left ventricular noncompaction CMP, LVNC; 4\% arrhythmogenic right ventricular CMP, ARVC; $2 \%$ combined HCM/LVNC) were included. Median age (IQR) of index patients was 8.5 (13.6) years, 60\% male, BSA 1.07 (1.07) $\mathrm{m} 2.25 \%$ showed heart failure signs. Pro brain natriuretic peptide (proBNP) was 1496.0 (6359.3) $\mathrm{pg} / \mathrm{ml}$ (39/48; $\min 6.9$; $\max 782$ 47.0); maximum oxygen consumption $33.5(17.38) \mathrm{ml} / \mathrm{kg} / \mathrm{min}(21 / 48)$. CMR: LV-EF 57.5 (13)\% ( $\min 21 ; \max 88 ; 22 / 48)$, LV-EDV $95.35(35.7) \mathrm{ml} / \mathrm{m}^{2}$ $(22 / 48) .8 \%$ underwent heart transplantation, $4 \%$ died. Between
CMP groups there were significant differences in LV-EF $(p=0.0065)$ and LV-EDV $(p=0.0201)$. DCM patients had significantly higher proBNP levels $(\mathrm{p}<0.001)$ and $\mathrm{LV}$ volumes $(\mathrm{p}<0.001)$, and lower LV-EF $(\mathrm{p}<0.001)$ compared to the LVNC group. Frequencies of NYHA groups III (5/7) and IV (4/4)were higher in DCM and RCM patients. Furthermore, proBNP levels were higher in these groups (DCM: $3655 \mathrm{pg} / \mathrm{ml}$ (12287.9); RCM: 2384 pg/ml (6634).

Conclusions: Morphologic and functional data significantly varied between the CMP groups, especially between DCM and LVNC. Patients with DCM and RCM patients seem to be at higher risk for severe disease courses. Further follow-ups will enable a detailed risk stratification and may detect groups whuch benefit from an earlier intensive pharmacological heart failure treatment.

\author{
MP1-9 \\ Left ventricular apical pacing in children - feasibility and \\ long-term effect on ventricular function \\ Kovanda J., Janousek J., Lozek M., Kubus P. \\ Children's Heart Centre, 2nd Faculty of medicine and Motol University \\ hospital, Prague, Czech Republic
}

(Supported by MH CZ - DRO, University Hospital Motol, Prague, Czech Republic 00064203)

Aim: Left ventricular (LV) pacing has been reported to preserve LV function in chronically paced children with atrio-ventricular (AV) block. We sought to evaluate long-term results of LV apical pacing (LVAP) in patients with both structurally normal heart and congenital heart disease.

Patients and methods: 36 patients with complete spontaneous $(\mathrm{N}=22$, group $\mathrm{A})$ and surgical $\mathrm{AV}$ block $(\mathrm{N}=14$, group $\mathrm{B}$, systemic LV in all) received an epicardial VVIR $(\mathrm{N}=19)$ or DDDR $(\mathrm{N}=17)$ pacemaker at the median age of 1.69 (IQR 0.04-4.39) years. Bipolar ventricular pacing leads (Medtronic 4968) were placed at the LV apex using a subxiphoid approach $(\mathrm{N}=18)$ or sternotomy $(\mathrm{N}=18)$. After median follow-up of 2.9 (IQR 1.9-6.2) years echocardiography and exercise stress testing was performed. Data were compared to age-matched normal controls $(\mathrm{N}=25$, group $\mathrm{C})$.

Results: Pacemaker implantation was uneventful, there was no death and all patients were on LVAP at the end of follow-up. Probability (3 and 6 years after implantation) of absence of pacemaker-related surgical revision (elective generator replacement excluded) was 89.0 and $89.0 \%$, resp. Probability of freedom from battery depletion was $91.4 \%$ and $77.8 \%$, resp. Ventricular thresholds at given pulse duration did not change between discharge and last follow-up: mean 0.71 (SD 0.22) V and $0.84(0.37) \mathrm{V}, \mathrm{p}$ NS. There was no significant difference in LV end-diastolic volume index (LVESVi) and ejection fraction (LVEF) between groups A, B and C: LVESVi = mean 49 (SD 13), $50(16)$ and $50(10) \mathrm{mL} / \mathrm{m} 2 \mathrm{BSA}, \mathrm{LVEF}=\operatorname{median} 65,64$ and $65 \%$, $\mathrm{p}$ NS for both. Inter- and intra-LVsynchrony was preserved in groups A and B. Maximum oxygen uptake was, however, lower in group $\mathrm{A}=$ mean 33.5 (SD 5.8) and $\mathrm{B}=33.9(6.1) \mathrm{ml} / \mathrm{kg} / \mathrm{min}$ as compared to group $\mathrm{C}=40.8 \quad(6.6) \mathrm{ml} / \mathrm{kg} / \mathrm{min}, \mathrm{p}=0.009$. In group A (free from structural heart disease) LVEF and the Z-score of LV end diastolic diameter did not change significantly during pacing.

Conclusions: LVAP carries complete preservation of LV function and synchrony. Pacing-related complications are rare and probability of continued LVAP is excellent despite patient growth. 


\section{MP1-10}

Update on baseline data and first 1-year follow-up from the German multi-centre myocarditis registry in pediatric patients: "MYKKE"

Degener, F. (1,2,3), Opgen-Rhein B.(2), Schmidt F.(4), Weigelt A.(5), Wagner R.(6), Müller G.(7), Rentzsch A.(8), Racolta A.(9), Papakostas K.(10), Wiegand G.(11), Ruf B.(12), Hannes T. (13), Reineker K.(14), Kiski D.(15), Khalil M.(16), Fischer M.(17), Steinmetz M.(18), Fischer G.(19), Pickardt T.(20), Messroghli D. $(1,21,3)$, Schubert S. $(1,3)$ on behalf of the MYKKE consortium Deutsches Herzzentrum Berlin, Klinik für Angeborene Herzfehler/ Kinderkardiologie, Berlin, Germany (1); Charité Universitätsmedizin Berlin, Klinik für Pädiatrie mit Schwerpunkt Kardiologie, Berlin, Germany (2); DZHK (German Centre for Cardiovascular Research) partner site Berlin, Germany (3); Medizinische Hochschule Hannover, Kinderkardiologie, Hannover, Germany (4); Universitätsklinikum Erlangen, Kinderkardiologie, Erlangen, Germany (5); Herzzentrum Leipzig, Kinderkardiologie, Leipzig, Germany (6); Universitäres Herzzentrum Hamburg, Kinderkardiologie, Hamburg, Germany (7); Universitätsklinikum des Saarlandes, Kinderkardiologie, Homburg, Germany (8); Herz- und Diabeteszentrum NRW, Zentrum für angeborene Herzfehler, Bad Oeynhausen, Germany (9); Klinikum Links der Weser, Strukturelle und angeborene Herzfehler / Kinderkardiologie, Bremen, Germany (10); Universitätsklinikum Tübingen, Klinik für Kinder- und Jugendmedizin, Kinderkardiologie, Tübingen, Germany (11); Deutsches Herzzentrum München, Technische Universität München, Angeborene Herzfehler/Kinderkardiologie, München, Germany (12); Uniklinik Köln, Kinderkardiologie, Köln, Germany (13); Universitäts-Herzzentrum Freiburg Bad Krozingen, Klinik für angeborene Herzfehler und Pädiatrische Kardiologie, Freiburg, Germany (14); Universitätsklinikum Münster, Klinik für Kinder- und Jugendmedizin/Pädiatrische Kardiologie, Münster, Germany (15); Universitätsklinik Gießen, Abteilung für Kinderkardiologie, Giessen, Germany (16); LMU, Abteilung Kinderkardiologie und Pädiatrische Intensivmedizin, München, Germany (17); Universitätsmedizin Göttingen, Kinderherzklinik Göttingen, Klinik für Pädiatrische Kardiologie und Intensivmedizin, Göttingen, Germany (18); Universitätsklinikum Schleswig-Holstein, Klinik für angeborene Herzfehler und Kinderkardiologie, Kiel, Germany (19); Kompetenznetz für angeborene Herzfehler, Berlin, Germany (20); Deutsches Herzzentrum Berlin, Klinik für Innere Medizin - Kardiologie, Berlin, Germany (21)

Objectives: There are only few data on epidemiology, diagnosis and therapy of myocarditis in children and adolescents. Our first data revealed a high incidence of young children with severe heart failure, maybe due to immunological differences. With our new data we confirm the first baseline data on age-related clinical differences in this patient group and show follow up data.

Methods: After an initial 6-month pilot phase, MYKKE was opened in June 2014 as a prospective multi-centre registry for patients from pediatric heart centers, university hospitals and community hospitals in Germany. Inclusion criteria are age $<18$ years, hospitalization for suspected myocarditis and written consent.

Results: By October 31st 2016, 18 centers across Germany are actively participating and have enrolled 207 patients. Baseline data show clear trends for age: $0-<2$ years $(24 \%), 2-12$ years $(20 \%)$ and $13-18$ years $(56 \%)$; gender: male $67 \%$. Decrease of exercise capacity $(72 \%)$ was the leading symptom, followed by dyspnea (39\%), angina (38\%), arrhythmia (30\%), feeding intolerance $(22 \%)$ and syncope (13\%). Sudden cardiac death was only rare (3\%). 56\% had an infection less than 6 weeks ago, respectively $35 \%$ fever. Patients $0-<2$ years had again the highest incidence of reduction of ejection fraction (EF) below 30\% (55\%) compared with age groups
$2-12 y(39 \%)$ and $13-18 y(9 \%)$. Furthermore they and the 2-12 year group had the highest need for VAD therapy (20\%) according to $53 \%$ patients with decompensation $(2-12$ years: $41 \%$; $13-18$ years: $9 \%$ ). Most children died in the $2-12$ years group (10\%). The overall mortality was $3.9 \%$. By now we have the follow up data of 38 patients with a median time of 11 month after first visit. The median EF increased significantly from $47 \%$ at initial presentation to $60 \%$ at follow up $(\mathrm{p}<0.001)$.

Conclusions: With its ongoing enrollment, MYKKE is a successful multi-centre registry for myocarditis in children and adolescents. The data underline two age peaks with a severe clinical course and more adverse events in the youngest patients. MYKKE serves as a platform for deriving diagnostic criteria and will in future facilitate interventional studies.

\section{MP1-11}

Long-term follow up of bradi-arrhytmias after surgical correction of atrioventricular septal defects in a singlecenter registry of 551 children: Down syndrome is a risk factor or not?

Calvieri C., Di Mambro C., Silvetti M.S., Tamburri I., Zanoni S., Placidi S., Albanese S., Brancaccio G., Carotti A., Iorio F.S., Drago F. Bambino Gesù Children Hospital, Rome, Italy

Background: Atrioventricular septal defects (AVSD) represent nearly $7 \%$ of all congenital heart diseases and have been described often associated to Down Syndrome (DS). Our aim was to explore the incidence of atrioventricular block (AVB) and sinus node dysfunction (SND) onset after cardiac surgery, requiring pacemaker (PMK) implantation in a large single center cohort during a maximum follow-up of 34 years and to determine the impact of DS.

Methods: Demographic information, cardiac diagnosis, age at first surgical repair, surgical procedure, age at PMK implantation of 522 partial and complete AVSD who underwent intracardiac repair from 1982 to 2016 were reviewed from the system database of Our Institution.

Results: DS was present in 202 cases (38.7\% of overall population). Thirty-eight patients $(7.3 \%)$ underwent to permanent PMK implantation for early or late AVB or SND. AVB was present in 26 patients $(5 \%)$, of which $20(3.8 \%)$ with early onset and $6(1.2 \%)$ with late onset (median 5 years [IQ 4-9,5 years] after surgery), while 12 patients $(2,3 \%)$ had SND, of which 10 (1.9\%) with late onset (median 10,5 years [3,5-15,2 years IQ] after surgery).

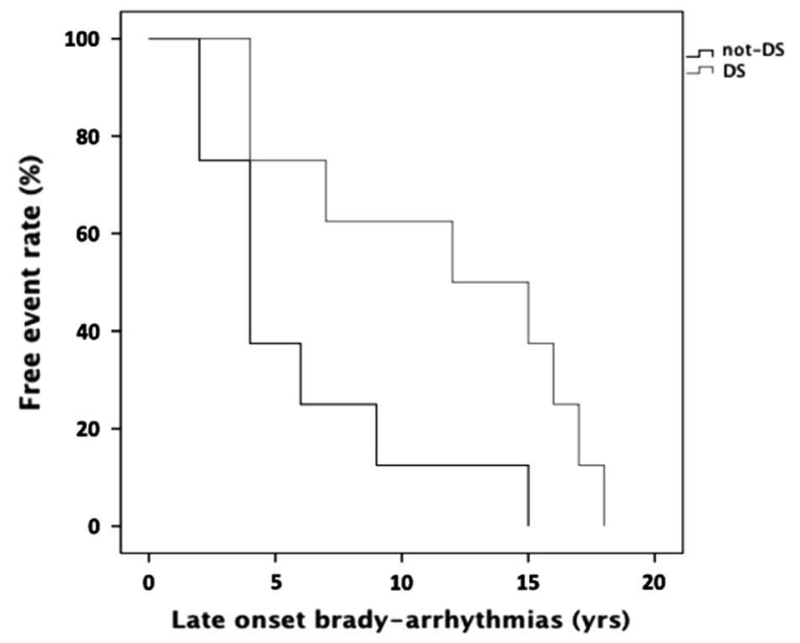


Children who undergone surgical repair for a partial AVSD had more frequently an early AVB than those with complete AVSD $(p=0.019)$, while no difference was found for late AVB onset $(p=0.68)$. Meanwhile, late SND onset was significantly more frequent in patients with complete AVSD than in partial AVSD $(p=0.017)$. At Kaplan Meier survival curve, the presence of DS was significantly associated with late onset of bradi-arrhythmic disorders (occurring at a medium of $5,7 \pm 4,4$ years in non-DS patients compared to $11,6 \pm 5,8$ years in DS patients). Finally, we have clearly noted that the incidence of AVB has gradually declined over the years, while the DNS has maintained the same percentage.

Conclusions: Our findings recommend a close follow up to early identify potential life-threating bradi-arrhytmias in this high risk patients. Furthermore, Down syndrome seems to not increase the risk for bradiarrhytmia disorders but to be related with a late timing onset of PMK implantation.

\section{MP1-12}

\section{Mechanisms underlying abnormal cardiorespiratory} response in Fontan patients

Domanski O. (1,2), Houeijeh A. (1), Guillaume M.P. (1), Gras P. (1), Recher M. (1), Bonnet M. (1), Matran R. (1,3,4), Godart F. (2,3) CHU Lille, Department of Echocardiography and Physiology, F-59000 Lille, France (1); CHU Lille, Department of Pediatric Cardiology and Congenital Heart Disease, F-59000 Lille, France (2); Univ. Lille, F-59000 Lille, France (3); Institut Pasteur de Lille, F-59000 Lille, France (4)

Background: Low peak oxygen consumption (peak VO2) is a strong predictor of mortality in adults with congenital heart disease. In univentricular hearts after Fontan procedure, peak VO2 is low at $59 \%$ of predicted value for the 50th percentile. But the mechanisms underlying this abnormal cardiorespiratory response to exercise are not well established.

Objectives: We aimed to determine which parameters can predict low peak VO2 $(<59 \%$ of predicted value) in Fontan patients.

Methods: Twenty one patients (mean age $23 \pm 8$ years) including 13 univentricular hearts, 5 hypoplastic left heart syndromes, 2 tricuspid atresia and 1 congenitally corrected transposition of the great arteries prospectively underwent cardiopulmonary exercise testing (CPX) with arterial blood gas measurements.

Results: Peak VO2 was low as expected $(24.4 \mathrm{ml} / \mathrm{kg} / \mathrm{min})$. All patients despite normal oxygen saturation, had shunt with $\mathrm{PaO} 2+\mathrm{PaCO} 2<120 \mathrm{mmHg}$ (mean $96 \pm 8 \mathrm{mmHg}$ ) at rest. Twelve patients had peak VO $2<59 \%$ of predicted value, which is considered $<50$ th percentile in Fontan population. In this group, resting oxygen saturation was lower $(94.3 \pm 1.8 \%$ vs $96.7 \pm 1.4 \%$, $\mathrm{p}=0.02)$, alveolar-arterial gradient was higher at rest $(52 \pm 9 \mathrm{mmHg}$ vs $44 \pm 5 \mathrm{mmHg}, \mathrm{p}=0.03)$ and at exercise $(58 \pm 10 \mathrm{mmHg}$ vs $50 \pm 6 \mathrm{mmHg}, \mathrm{p}=0.04)$. Secondly, these patients had lower exercise heart rate $(122 \pm 38 \mathrm{bpm}$ vs $169 \pm 25$ bpm, $p=0.002)$, lower chronotropic reserve $(49 \pm 31 \mathrm{bpm}$ vs $79 \pm 17 \mathrm{bpm}, \mathrm{p}=0.009)$ and had more often pacemakers (3 patients versus $0, p=0.05)$. Finally, in this group with altered peak VO2, we found more Fontan procedures (or modified Fontan) than extracardiac total cavopulmonary connections (5 patients or $42 \%$ vs 1 or $11 \%, p=0.03)$, patients were older ( $27.6 \pm 7$ years vs $16.8 \pm 6.4$ years, $\mathrm{p}=0.0009)$, and converted to extracardiac cavopulmonary connection later (at $21.1 \pm 13.9$ years old vs $3.8 \pm 2.4$ years old, $\mathrm{p}=0.002$ ).

Conclusion: Severe alteration of aerobic capacity evaluated by peak $\mathrm{VO} 2$ measurement in Fontan patients is correlated to the severity of cyanosis, the incapacity to rise heart rate at exercise and the type of surgery. Assessment of aerobic capacity with arterial blood gas measurements seems worthwhile to understand abnormal cardiorespiratory response in this population.

\author{
MP1-13 \\ Significant Diameter Heterogeneity Between Aortic Root \\ and Aortic Annulus in Bicuspid Aortic Valve \\ Fournier A.(1), Meloche-Dumas L.(1), Dallaire F.(2), Blais S.(2), \\ Dahdah N.(1) \\ CHU mère-enfant Sainte-Justine, Montréal, Canada (1); CHU \\ Sherbrooke, Sherbrooke, Canada (2)
}

Introduction: Bicuspid aortic valve (BAV) is associated with aortic dilatation and potential dissection. There are 3 major patterns of leaflets fusion in BAV: left and right coronary leaflets fusion (BAV-1), right and non-coronary leaflets fusion (BAV-2), and the rare three-leaflet fusion (BAV-3). We sought to determine the pattern of dilatation according to commonest types of BAV fusion, and whether this could modify patient management. The embryologically different true bicuspid valve is not included in this study.

Methods and Results: In this retrospective cross-sectional study, echocardiography reports were reviewed to evaluate the homogeneity of normalized diameters of the aortic valve, the aortic root and the ascending aorta according to fusion morphology. From 216 BAV patients followed at CHU Sainte-Justine between 2009 and 2014 (mean age at echocardiography 1 was $7.19 \pm 5.56$ years; $74.5 \%$ males), 155/216 (71.8\%) had BAV-1, and 61/216 (28.2\%) had BAV-2; $\mathrm{p}=\mathrm{NS}$ for age. Aortic valve stenosis was present in $66 / 216(30.6 \%)$ patients, with a lower prevalence 39/155 (25.2\%) in BAV-1 compared to $27 / 61$ (44.3\%) in BAV-2 $(\mathrm{p}=0.006)$. Aortic coarctation affected both groups similarly (26/155 (16.8\%) vs. 10/61 (16.4\%) respectively; $\mathrm{p}=\mathrm{NS})$. There was no statistically significant difference in the ascending aorta between the study groups (Table 1). However, BAV-1 had increased aortic root Z-score compared to BAV-2 $(\mathrm{p}=0.0007)$ despite a trend towards larger aortic annulus in BAV-2 $(\mathrm{p}=0.11)$. The difference was not significant between BAV-1 and BAV-2 in cases with valvular stenosis $(0.67 \pm 1.50$ vs. $0.17 \pm 1.73 ; p=0.48)$, but significant in the absence of stenosis $(0.79 \pm 1.57$ vs. $-0.25 \pm 0.98 ; p=0.00035)$ and in the absence of coarctation of the aorta $(0.88 \pm 1.53$ vs. $0.04 \pm 1.36 ; p=0.0011)$. Altogether, there was no significant differences in the ascending aorta dimension in either case (BAV-1 vs. BAV-2, or valvular stenosis vs. no stenosis, or coarctation of the aorta vs. no coarctation).

Conclusions: Although not necessarily dilated (mean $\mathrm{Z}<2.0$ ), type1 BAV fusion aortic roots are larger than in type-2. The observed difference suggests potential modifications in patient approach with this valvular disease according to the type of fusion.

\section{MP1-14 \\ Late Enlargement of Radiofrequency Lesions in Children: This is really truth? \\ Melo S., Pisani C., Miura N., Rochite C., Scanavacca M. Institute of Heart from São Paulo Brazil USP}

Introduction: Despite the current clinical use of radiofrequency (RF) catheter ablation in children, the effects of RF lesions in immature myocardium remain unknown. This study was specifically designed to investigate the late occurrence of RF lesions in the myocardium in development.

Methods And Results: Magnetic resonance imaging and coronary angiography were performed in 20 patients, who underwent RF 
catheter ablation due diagnosis of arrhythmia under the age of 18 years after signing consent forms. Fourteen patients were female with median age of 15(Q1:10 Q:16) years-old. AVRT ablation was performed in 5, AVNRT in 13, AT in 1 and Mahaim fiber in 1 patient. The MRI was perfomed at median time of 6 years (Q1:5 Q3:8,75) after the ablation. The mean weight, height and body mass index at the time of MRI were, respectively, $66 \pm 9 \mathrm{Kg}$, $168 \pm 7 \mathrm{~cm}$ and $23.4 \pm 2,4$. Mild ventricular fibrosis was found in 2 patients ( 1 after Mahaim fiber ablation and 1 after AVNRT). Also, mild atrial fibrosis was detected in 5 patients (AVRT (1), Mahaim fiber (1) and AVNRT (3) ablations). The fibrosis' site corresponded to the RF application sites. Angiotomographies performed in the same period of the MRI were normal. All patients were asymptomatic.

Conclusions: Clinically significant fibroses were not found in atrial or ventricular myocardium in this small series of patients who underwent RF ablation catheter under the age of 18 years. These findings may have implications when deciding for RF ablation of supraventricular tachycardias in children.

\section{MP1-15}

Normal values of the pediatric ECG/VCG with a focus on the ventricular gradient and QRST-angle

Kamphuis V.P. (1,2,3), Blom N.A. (1,3), Ten Harkel A.D.J. (1), Van Zwet E.W. (4), Man S. (5), Maan A.C. (6), Swenne C.A. (6) Department of Pediatric Cardiology, Leiden University Medical Center, Leiden, The Netherlands (1); Netherlands Heart Institute, Utrecht, The Netherlands (2); Department of Pediatric Cardiology, Academic Medical Center, Amsterdam, The Netherlands (3); Department of Medical Statistics, Leiden University Medical Center, Leiden, The Netherlands (4); Physiologic, Amsterdam, The Netherlands (5); Department of Cardiology, Leiden University Medical Center, Leiden, The Netherlands (6)

Introduction: The diagnostic performances of the electrocardiogram (ECG) and the vectorcardiogram (VCG) are relatively similar. However, additional spatial information (notably, the ventricular gradient, VG, and the spatial QRS-T angle, SA) can be derived from the VCG, not directly accessible in the 12-lead ECG and not explored in conventional VCG diagnostics. In an adult population, the VG and SA have been shown to provide extra diagnostic and prognostic value. However, normal values of VG and SA have not been published for children. Therefore, our study aims to assess normal values of the pediatric ECG and the vectorcardiographic VG and SA.

Methods: ECGs of children under 18 years old with a structural and functional normal heart, demonstrated by a normal echocardiogram, physical examination and ECG, were retrospectively selected from the pediatric cardiology outpatient clinic data. Also, ECGs of reportedly healthy first-year medical students aged 18-24 years were added. ECG/VCG analysis was done with the Matlabbased LEADS program. VCGs were synthesized by the Korsmatrix and VG (spatial QRST integral) and SA (planar angle between the spatial QRS and T axes) were calculated. Normal values (presented as 2 nd and 98th percentiles) were assessed by quantile regression with smoothing by splines.

Results: The study group consisted of 1124 subjects. Trends of heart rate, QRS duration, QT time and QTc agree with the literature. Graphs for normal values of the spatial QRS-T angle and ventricular gradient are shown in Figure 1. SA shows a dip at age 7 (Fig. 1a). VG shows an increase in magnitude over time (Fig 1b). Conclusions: Normal values of the pediatric ECG and VCG (VG and SA) were established. These normal values are important for future studies using VG and SA for risk stratification in structural heart disease in children.
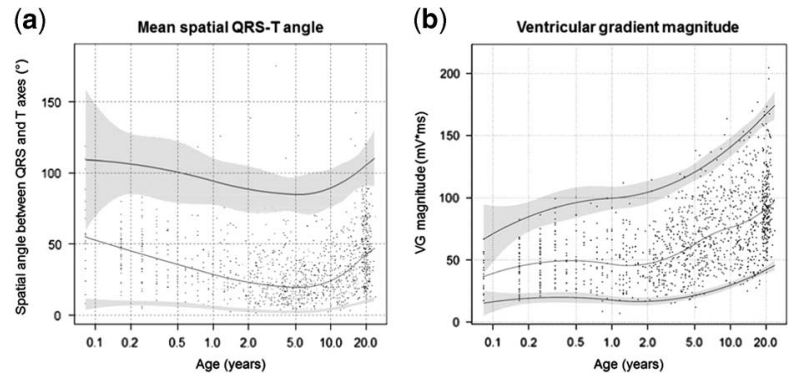

\begin{abstract}
MP1-16
Outcome of bioprosthetic valves in pulmonary position implanted for reconstruction of the right ventricular outflow tract in adults with congenital heart disease Poruban R. (1), Gebauer R. (1,2), Popelová J. (2), Timko F. (2), Slezáková D. (1), Skalský I. (2), Janoušek J. (1). Children's Heart Centre, 2nd Faculty of medicine and Motol University hospital, Prague, Czech Republic (1); Department of Cardiac Surgery, Na Homolce Hospital, Prague, Czech republic (2)[Supported by MH CZ DRO, University Hospital Motol, Prague, Czech Republic 00064203]
\end{abstract}

Objectives: Pulmonary valve replacement (PVR) is the most frequent surgical procedure in adult patients with congenital heart diseases (ACHD). However, none of used valve substitutes is ideal. The aim of the study was to determine the impact of PVR on clinical outcome and right ventricular function.

Methods: We retrospectively reviewed the outcome and performance of bioprosthetic valves implanted in the pulmonary position. Since 2005 to April 2015, 133 consecutive patients with a median age of 35 (range 18.4 to 70.4 ) years underwent valve implantation: Carpentier-Edwards Perimount Magna ${ }^{\mathrm{TM}}$ $(\mathrm{N}=106)$, St. Jude $\operatorname{Trifecta}^{\mathrm{TM}}(\mathrm{N}=10)$, St.Jude Medical Epic ${ }^{\mathrm{TM}}$ $(\mathrm{N}=11)$, St. Jude Toronto ${ }^{\mathrm{TM}}(\mathrm{N}=4)$ and other $(\mathrm{N}=2)$. Median follow-up was $4.3(0.1-11.0)$ years. Patients diagnoses included tetralogy of Fallot $(n=84)$, pulmonary stenosis $(n=33)$, complex diagnoses $(n=14)$ or other $(n=2)$. One hundred twenty nine patients had 182 previous surgical procedures. Eighty-two patients (61\%) had concomitant surgical procedures.

Results: There were no perioperative deaths, and $1(0.8 \%)$ late death. Probability of freedom from reoperation was $100 \%, 99 \%$, and $86.1 \%$ at 1,4 , and 8 years, respectively. Probability of freedom from valve dysfunction (pulmonary insufficiency more than moderate and or stenosis $>40 \mathrm{mmHg}$ ) was $98.3 \%, 90.2 \%$, and $56.9 \%$, respectively. There was a significant improvement $(p<0.001)$ in NYHA functional class. Echocardiography confirmed lasting reduction in pulmonary $(p<0.001)$ and tricuspid valve regurgitation $(p<0.001)$, decrease in the size of tricuspid valve annulus $(p<0.001)$ and improvement of right ventricular function $(p<0.001)$.

Conclusions: PVR using bioprosthetic valves has a low mortality and carries lasting improvement in functional status and right ventricular function in ACHD patients. Freedom from re-operation and valve dysfunction is acceptable. Further studies are needed to compare longterm performance of different valves types in the pulmonary position.

MP1-17

Right ventricular diastolic dysfunction in pediatric patients with ventricular pre-excitation

Khamnagadaev I.A., Termosesov S.A., Kokov L.S., Shkolnikova M. A., Garipov R.S., Volkova Y.Y., Ilich I.L.

Research and Clinical Institute of Pediatrics at the N.I. Pirogov Russian National Research Medical University, Moscow, Russian Federation 
Objectives: To evaluate the impact of ventricular pre-excitation on right ventricular (RV) diastolic function in children with WolffParkinson-Wight syndrome (WPW).

Methods: 44 pts (21 females) were included into the study. Main group - 21 asymptomatic WPW. Control group - 23 pts with absence of ventricular pre-excitation. In this pts atrioventricular nodal re-entrant tachycardia had been diagnosed. ECG, 24-hour Holter monitoring and echocardiography were performed before and 3 days after radiofrequency catheter ablation (RCA). Type of RCA: main group - RCA of accessories pathway (AP), control group - RCA of slow pathway of antrioventricular node. Invasive right ventricular pressure was recorded immediately before and 40 minutes after RCA. First negative derivate $(-\mathrm{dp} / \mathrm{dtmax})$ and time constant $(\tau)$ of RV pressure fall were calculated. Those data have been included into logistic regression model. Regression coefficients, odds ratio and probability of RV diastolic function disturbance depending on localization of AP were calculated.

Results: RV diastolic function was impaired in 10 (48\%) pts in main group before interventional treatment. There were no RV diastolic dysfunctions after RCA in all cases. Disturbance of RV diastolic function was not found in control group before and after RCA in all pts. The probability $(\mathrm{P})$ of RV diastolic function disturbance has been calculated using regression model. In pts with right-side AP P was 0.9, in parasaseptal - 0.7, in left-side - 0.1 $(\mathrm{p}<0.05)$. There was no significant difference in RV diastolic function in symptomatic and asymptomatic WPW.

Conclusion: Abnormal ventricular excitation via AP causes RV diastolic dysfunction in asymptomatic WPW. Probability of RV diastolic function disturbance in right-side AP higher than in paraseptal AP. Left-side AP associated with low risk of RV diastolic dysfunction.

\section{MP1-18}

Failing Right Ventricle in Hypoplastic Left Heart Syndrome: myofiber architecture reconstruction by Diffusion-Weighted Magnetic Resonance Imaging

Campanale C. M. (1), Scherrer B. (2), Afacan O. (2), Majeed A. (3), Singh J.(2), Warfield S.(2), Sanders S. P.(2).

Ospedale Pediatrico Bambino Gesu, Roma - Italy (1); Boston Children's Hospital, Boston - USA (2); Children's Hospital of the King's Daughter's, Atlanta - USA (3)

Introduction: Heart failure is a common cause of death in young adults with hypoplastic left heart syndrome (HLHS). DiffusionWeighted MR Imaging (DWMRI) studies described myofiber disarray in hypertrophic and dilated cardiomyopathies. Our hypothesis is that myofiber disarray is present in the systemic right ventricle (RV) of hypoplastic left heart syndrome (HLHS).

Methods: The explanted heart from a 14 yo with HLHS was fixed and imaged using a 3T Diffusion MR scanner. Diffusion Compartment Imaging (DCI) was computed and data displayed in glyph format for qualitative description of myofibers. Realtime Tractography was performed for 3D reconstruction. Histology sections were taken after imaging for comparison with diffusion data.

Results: The failing systemic RV in HLHS is markedly hypertrophied, dilated and bizarrely shaped (Fig A). The epicardial layer around most of the RV is formed by a homogeneous stratum of circumferential myofibers, while the endocardial layer is remarkably disarrayed (Fig B). Some smaller muscle bundles are organized with parallel fibers (Figure C1-C3) while larger bundles, which appear to be composites, show myofiber disarray, largely between component bundles (Fig D). The apical whorl is disrupted with chaotic arrangement of fibers (Fig E). In addition, we observed myocardial whorls or vortices and abrupt fiber tract interruptions (Figure F) within the wall.

Discussion: For the first time myofiber disarray in the RV of an HLHS has been demonstrated by DW-MRI. Myofibers and myocardial bundles arrayed at large angles result in inefficient contraction and ventricular dysfunction, as was present in this patient. These findings support our hypothesis that disordered myocardium contributes to heart failure in these patients. Further, myocardial whorls could be the substrate for arrhythmia, another frequent cause of death in young adults with congenital heart disease. Our findings support further study of myocardial architecture in congenital heart defects.
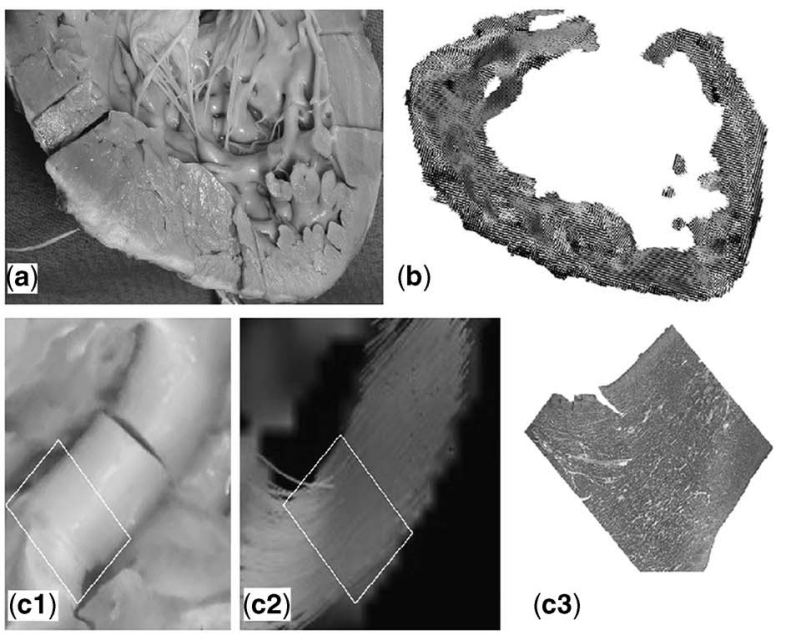

(c3)
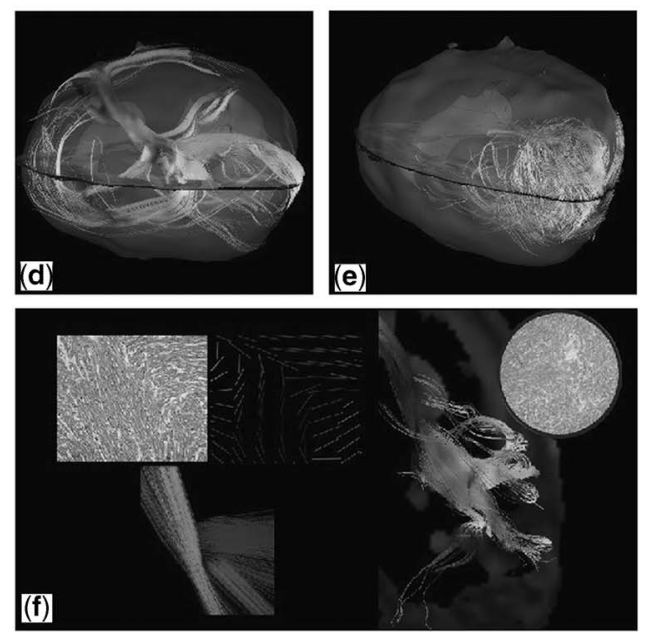

MP1-19

Takotsubo Cardiomyopathy in a woman with Pulmonary Atresia, Ventricular Septal Defect, and Major AortoPulmonary Collateral Arteries

Parrella C. (2), Oreto L.(1), Chinali M.(1), Valenti V. (1), D'Anna C. (1), Lumia F. (1), Iacomino M. (1), Leonardi B. (1), Del Mastro A.(1), Ricci R.(3), Drago F. (1)

Ospedale Pediatrico Bambino Gesù, Roma (1)

AOU Federico II, Napoli (2)

Ospedale Santo Spirito in Saxia, Roma (3)

Takotsubo cardiomyopathy (TC) is a transient reversible stressinduced cardiomyopathy. Although TC is frequently described in adults, it is rarely reported in Grown-Up Congenital Heart Disease $(\mathrm{GUCH})$ patients. 
We report the case of a 43 year-old woman who came to our emergency department complaining of acute chest pain and dyspnea after an angry debate.

At birth she was affected by pulmonary atresia, ventricular septal defect and major aorto-pulmonary collateral arteries. During childhood she underwent right Blalock-Taussig shunt, left Blalock Taussig shunt and left pulmonary artery reconstruction. When she was 35 years old, ventricular septal defect closure and unifocalization was performed; one year later, replacement of the ascending aorta and stenting of right pulmonary artery were performed.

Physical examination evidenced tachycardia, tachypnea, oxygen saturation $68 \%$ on oxygen $81 / \mathrm{min}$, blood pressure $140 / 80 \mathrm{mmHg}$. Electrocardiogram showed mild ST depression. Echocardiography revealed severe ventricular dysfunction, akinetic mid to apical left ventricular segments and normally contracting basal segments, left ventricular dilation and severely depressed ejection fraction (EF), $30 \%$. Troponin was still normal even 1 hour after the access to the hospital.

Three hours after symptoms onset, the patient was hemodynamically stable. Troponin I turned mildly positive, electrocardiogram showed negative $\mathrm{T}$ waves. Coronary angiography evidenced no coronary disease. According to Mayo Clinic criteria, TC was diagnosed.

After 4 days, echocardiography showed normal left ventricle volume and improved left ventricular systolic function (EF: 50\%), whereas apical hypokinesia persisted.

After 45 days, echocardiography documented recovered systolic function (EF:55\%) and no more apical dysfunction.

Conclusions: Few cases of TC in GUCH patients have been described. This is the first reported case of TC in a patient with pulmonary atresia, ventricular septal defect and major aortopulmonary collateral arteries.

Of note, all reported cases in GUCH patients involved young women, which is quite unusual in the setting of TC, since postmenopausal women are more likely to be prone to TC.

It should be considered that the number of GUCH patients is dramatically increasing and therefore we might expect to observe TC even more frequently and in older ages.

MP1-20

Clinical, neurohormonal and psychological characteristics predict on a long-term basis, adverse cardiac events in patients with congenital heart defects

Kourkoveli P., Apostolopoulou S., Kiaffas M., Moutson A., Rammos S. Onassis Cardiac Surgery Center, Department of Paediatric Cardiology and Congenital Heart Disease Athens, Greece

Introduction: Patients with congenital heart disease (CHD) face problems regarding their medical condition per se, like heart failure, but also their psychological status. Aim of this study is to identify the clinical, psychological and neurohormonal predictors of survival of patients with CHD, and define cut-off values.

Methods: Sixty consecutive clinical stable patients, mean age $28.9 \pm 11.4$ years old with various forms of CHD were included. Patients' neurohormonal, psychological status, and exercise capacity were assessed through plasma B-type brain natriuretic peptide (BNP) and interleukin 6 (IL-6), Beck depression inventory and Zung depression scale questionnaires and cardiopulmonary exercise test (CPX). Patients were followed for major cardiovascular events (MACE), including death or hospitalization for $5.1 \pm 1.1$ years.

Results: Most patients were symptomatic (48.3\% with NYHA II and $36.7 \%$ with NYHA III) and 17 (28.3\%) of them were cyanotic at rest. Mean plasma concentrations of BNP and IL-6 were
$106.6 \pm 98.6 \mathrm{pg} / \mathrm{ml}$ and $2.4 \pm 2.6 \mathrm{pg} / \mathrm{ml}$ respectively. 17 patients $(28.3 \%)$ were characterized as depressed. Patients with depression had higher plasma BNP levels $(\mathrm{p}=0.030)$, limited exercise capacity, as expressed with peak VO2 $(\mathrm{p}=0.019)$ and higher probability of experiencing a major adverse cardiac event (MACE) compared to non-depressed patients (95\% CI: 1.630 to 3.616, $\mathrm{p}<0.05) .22$ patients (36.6\%) experienced a MACE, among them 8 patients (13.3\%) died. BNP, IL-6, peak VO2, VE/VCO2 were proved to be strong predictors of survival; BNP value $>241 \mathrm{pg} / \mathrm{ml}$ predicted MACE with a sensitivity of $65.38 \%$ and a specificity of $73.53 \%$ (Area Under the ROC Curve, i.e. AUC $=0.693$, $\mathrm{p}<0.0001)$, IL-6 value $>1.54 \mathrm{pg} / \mathrm{ml}$ predicted MACE with a sensitivity of $61.53 \%$ and a specificity of $73.53 \%$ (AUC $=0.627$, $\mathrm{p}<0.0001), \mathrm{VE} / \mathrm{VCO} 2$ value $>38$ predicted MACE with a sensitivity of $73.08 \%$ and a specificity of $76.47 \%$ (AUC $=0.808$, $\mathrm{p}<0.0001$ ) and peak VO2 value $\leq 21.4 \mathrm{ml} / \mathrm{Kg} / \mathrm{min}$ predicted MACE with a sensitivity of $76.92 \%$ and a specificity of $70.59 \%$ $(\mathrm{AUC}=0.794, \mathrm{p}<0.0001)$ respectively.

Conclusions: Patients with CHD share common characteristics with patients with heart failure. BNP, IL-6 levels, exercise parameters and depression strongly predicted MACE and can be used with their cut off values for routine risk stratification in this population.

MP2-1

Do self- and proxy-reports of cognitive problems reflect intellectual functioning in children and adolescents with congenital heart defects?

Buratti S.(1,2), Ryberg C. (1,2), Broberg M. (2), Sunnegårdh J. (1) Department of Pediatric Cardiology, The Queen Silvia Children's Hospital, Sahlgrenska University Hospital, Gothenburg, Sweden (1) Department of Psychology, University of Gothenburg, Gothenburg, Sweden (2)

Aim: Children with congenital heart defects who suffer from cognitive impairments and school difficulties need to be identified as early as possible in order to set appropriate interventions in place that may enhance the school situation and quality of life for these children. Identifying children and adolescents at risk for cognitive difficulties requires specific screening tools. This study assessed such a tool - Pediatric Quality of Life Inventory Cardiac Module subscale: Cognitive Problems - to investigate whether proxyreported and self-reported cognitive problems were associated with measured intellectual functioning in children and adolescents with congenital heart defects.

Method: The sample consisted of 184 children/adolescents aged 3, 5,9 , and 15 years. The severity of the congenital heart defects diagnoses was categorized into three groups (mild, moderate, or severe) for all age groups. For all age groups, we collected proxyratings of cognitive problems and for the 5-, 9-, and 15-year-olds we also collected self-reported cognitive problems. Intellectual functioning was measured with the Wechsler scales. The control variables were socio-economic status and severity of diagnosis.

Results: A strong association was found between the parent's ratings of cognitive problems and the children's and adolescents' results on the Wechsler scales. This association was present for all ages, including the 3-year olds. As for the self-reports an association was only found between the 15 -year-olds self-report of cognitive problems and their results on the Wechsler scales.

Conclusions: To identify children with cognitive problems as early as at the age of three years, parent-rated Pediatrics Quality of Life subscale: Cognitive Problems can be used as a screening tool. For 15-year-olds, the self-report ratings can be used as a screening tool. We also suggest a cut off score of 80 for both the 15-year olds as well as the proxy reports. If the score falls below 80 the child should be formally evaluated using standardized test. 
MP2-2

Are Adults with Congenital Heart Defects adequately supplied in Medical Care?

Oberhoffer R. (1,2), Neidenbach R.(1), Pieper L. (3), Sanftenberg L. (4), Schelling J. (4), Lang N. (1), Trepakov A. (1), Nagdyman N.(1), Ewert P.(1), Kaemmerer H.(1)

Department of Paediatric Cardiology and Congenital Heart Defects, German Heart Centre Munich, Germany (1) Chair of Preventive pediatrics, Department of Sport and Health Sciences, Technical University of Munich, Germany (2), Chair of Behavioural Epidemiology, Technical University of Dresden, Germany (3), Institute for General Practice, University Hospital of the Ludwig-Maximilians-University Munich, Germany (4)

Objective: The number of Adults with Congenital Heart Disease (ACHD) in the western world is steadily rising. Nearly all ACHD have relevant residua and sequelae, requiring life-long follow-up. At least in Germany, the majority is NOT tied to a certified ACHD specialist or centre. For cardiac and noncardiac problems, ACHD consult general practitioners (GP), who are, however, frequently not qualified to manage congenital heart disease (CHD). This may put ACHD at risk and may adversely affect morbidity and mortality. Moreover, opportunities of initiating preventive care are mostly missed.

The current study provides an overview of primary health care of ACHD in Germany for the first time.

Methods and Results: A questionnaire-based survey of 116 ACHD (48.3\% female, mean age of $34.8 \pm 12.5$ years) and 126 GP's ( $35.7 \%$ female, mean age $54.6 \pm 9.1$ years) was analyzed.

Results of GP's: All ACHD consult GP's for noncardiac problems. $69 \%$ of the GP's also treat specific ACHD related cardiac problems. In up to $62 \%$ GP's consult (paediatric) cardiologists, but ACHD-specialists in only $25.4 \% .63 .5 \%$ of the GP's are not informed about the existence of ACHD facilities. GP's deal with all CHD types and severity grades, in 58\% even with moderate/ severe forms. Management tasks include heart failure, arrhythmias, endocarditis, pulmonary vascular disease and impeding comorbidities. GP's offer advice in social medical aspects and preventive measures, although specifically unskilled.

Results of ACHD: $41.1 \%$ of ACHD are uninformed about specific medical facilities, although suffering from significant side effects (arrhythmia, heart failure, pulmonary hypertension). ACHDsupport groups are unknown in $44 \% .70 \%$ of the ACHD surveyed need advice about exercise capacity, insurance, occupational skills and/or pregnancy. In 90\%, patients' needs concerning daily life questions and preventive measures are underprovided.

Conclusion: GP's have to guide ACHD with cardiac -/noncardiac problems to provide adequate diagnosis, treatment and preventive measures. According to study-results, ACHD and their caregivers are insufficiently informed about ACHD facilities. Experts and centres must become more visible and cooperate stronger with GPs to provide modern management in treatment and therapy.

\section{MP2-3}

4D-Flow-MRI in patients with Fontan circulation for evaluation of pulmonary artery blood distribution Lehner A. (1), Ulrich S.(1), Fernandez Rodriguez S.(1), Dalla-Pozza R.(1), Fischer M.(1), Haas N.(1), Kramer H.(2), Curta A.(2) Deparment of Pediatric Cardiology and Pediatric Intensive Care, University Hospital Munich, Campus Großhadern, Munich (1); Department of Radiology, University Hospital Munich, Campus Großhadern, Munich (2)

Objectives: 4D-flow-MRI is an arising tool to assess complex flow patterns in the heart and great vessels. We evaluated the feasibility of this imaging technique in patients after Fontan operation (FO) and attempted to visualize and quantify caval blood flow distribution and vorticity towards the pulmonary arteries (PAs).

Methods: 4D-flow-MRI scans were acquired in $10 \mathrm{FO}$ patients (age 7-21 years, mean 12,8 $\pm 4,2$ ) with extracardiac tunnel in 9 and 1 atriopulmonary connection. According to our MRI protocol, scans were performed at a velocity encoding (VENC) of $100 \mathrm{~cm} / \mathrm{s}$ with spatial resolution of $2.2 \times 1.8 \times 1.8 \mathrm{~mm}$ and 10 time-frames/ heart beat (HB). Flow pattern of caval inflow and distribution to the pulmonary arteries were visualized using time-resolved colour-coded path lines. All acquired data were processed offline using the manufacturer's software.

Results: In our patient group blood from the superior and inferior vena cava (SVC;IVC) drained predominantly to the RPA (53\% vs. $47 \%$ ). LPA blood supply / HB tended to be lower but with higher flow velocity compared to the right side $(12,68 \mathrm{ml} / \mathrm{HB}$ vs. $15,68 \mathrm{ml} / \mathrm{HB} ; 62,2 \mathrm{~cm} / \mathrm{s}$ vs. $49,7 \mathrm{~cm} / \mathrm{s})$ consistent with anatomically smaller or distorted LPAs. Colour-coded path lines revealed IVC blood predominantly draining to the RPA within our patient cohort in contrary to recently published data. However, within our group IVC and SVC (Glenn and Fontan tunnel) were rather placed orthogonal to each other than with a distinct offset to one or the other side as reported by others.

Conclusion: In this proof-of-concept study 4D-flow-MRI in Fontan patients was feasible with promising results. Further multicenter studies with larger patient numbers are warranted to correlate different surgical methods to the resulting PA blood flow distribution and its possible impact on late complications of the Fontan operation like protein-losing enteropathy.

\section{MP2-4}

Echocardiographic prevalence of cardiac malpositions, at birth and in children 5-15 years: Data from two large cross sectional observational studies

Saxena A., Ramakrishnan S., Roy A., Juneja R.

All India Institute of Medical Sciences, New Delhi, India

Introduction: Cardiac malposition refers to an abnormal intrathoracic position of the heart and is due to disturbance of cardiac looping. The heart may lie on right side, left side or in the midline. Abnormalities of atrial and visceral situs may be associated and if so, congenital heart disease (CHD) is almost always present. The prevalence of cardiac malposition varies in different series, perhaps due to undiagnosed cases of situs inversus with dextrocardia where CHD is unlikely. We present our data on prevalence of cardiac malposition based on two large cross sectional echocardiographic screening studies, at birth and in school children.

Methods: 20,307 newborns underwent screening echocardiography for CHD within 48 hours of birth in a general hospital setting in the city of New Delhi, India. The other cohort was of 14,724 school children, aged 5-15 years, residing in rural areas close to New Delhi, in whom echocardiography was performed as a part of screening for rheumatic heart disease. Both datasets were analyzed for presence of cardiac malposition and its association with structural heart disease.

Results: Of the 20,307 newborns $(53.5 \%$ males) screened, 5 had cardiac malpositions (prevalence $0.25 / 1000,95 \%$ CI $0.11-0.58 /$ 1000), all were males. Three had situs inversus with dextrocardia. One of these three had a severe CHD (complete atrioventricular septal defect with pulmonary atresia), other two had no CHD. Situs solitus with dextrocardia and situs solitus with mesocardia was present in one case each, both had cyanotic CHD (complete 
transposition and corrected transposition with ventricular septal defect, pulmonary stenosis, respectively). Of the 14,724 school children $(52.5 \%$ males) screened in 5-15 years age group (mean age $10.6 \pm 2.8$ years), two (both females) had cardiac malposition (prevalence 0.14/1000, 95\% CI 0.04-0.50/1000), both having situs inversus, dextrocardia. CHD was not seen in any of these.

Conclusions: Situs solitus dextrocardia is the commonest type of cardiac malposition, CHD is absent in majority. Survival in those with no CHD is expected to be comparable to that of control population. Patients with other types of cardiac malpositions are associated with significant CHD and their survival is likely to be reduced.

\section{MP2-5}

Improving continuity of care : Can a multidisciplinary approach and a pharmaceutical network improve discharge prescriptions for congenital heart disease patients?

Volpi E., Laws E., Baratta S., Tonazzini S., Alduini S., Zizevskikh M., Da Valle P., Donnini D., Baroni M., Assanta N., Biagini S. Fondazione G Monasterio Ospedale Del Cuore, paediatric and adult cardiac surgery and cardiology, Massa, Italy

Background: Continuity of care is essential at hospital discharge. Congenital heart disease is often treated in centres which are distant from the patients actual home and the handover of discharge prescriptions is problematic as a number of patients require galenic formulations for doses that are not available commercially with the risk that these may be prepared late. Mistakes can also be made by the discharge doctor and a control by an expert pharmacist can help to reduce errors.

Aim: To create a network between the specialized institutes pharmacy department and other health authorities pharmacies at a regional and national level to cover these needs. Control discharge prescriptions and improve patient and family knowledge to prevent errors.

Method: Multidisplinary team: pharmacists, cardiologists, nurses. Network development between national hospital pharmacies. Discharge prescriptions controlled by pharmacist. Direct contact of hospital pharmacies unless already included within the network. Clear instructions for preparation and use of galenic drugs. Educative written and oral session with the patient and family by the pharmacist and discharge nurse.

Results: The project began in July 2015 and from one involved hospital has developed into all local regional health authority hospital and territorial pharmacies plus a further 17 in 7 other regions totaling 36 . Of 401 patients $15.7 \%$ were resident outside the region and $11.7 \%$ of prescriptions were galenic. $65.1 \%$ of patients were aged under 18 years. The pharmacists compared the prescriptions made in the second half of 2015 (777 prescriptions for 198 patients) with the first half of 2016 (775 precriptions for 199 patients). An analysis of the first year of the teams activity showed that the overall number of prescription errors that may induce misinterpretation from the patient/family was considerably reduced from $49.4 \%$ to $5.2 \%$. The most frequent error was the lack of exact timing for administration (example twice a day) which dropped from 353 errors to 17.

Conclusion: The preliminary results are good with improved handover to primary and personal care and better patient satisfaction. The network will continue to be developed and electronic prescriptions will be introduced to further enforce correct discharge prescription.

\section{MP2-6}

Three Dimensional Printed Models For Surgical Planning Of Complex Congenital Heart Defects: An International Multicenter Study

Valverde I. (1,2,3,4), Gomez G.(1), Hussain T.(3,5), Suarez-Mejias C.(1), Velasco M.N.(3,4), Byrne N.(3), Ordoñez A.(2), GonzalezCalle A.(1), Anderson D.(3,4), Hazekamp M.G.(6), Roest A.A.W. (6), Rivas J.(1), Uribe S.(7), El-Rassi I.(8), Simpson J.(3,4), Miller O. (3,4), Ruiz E.(9), Zabala I.(9), Mendez A.(10), Gallego P.(1), Manso B.(1), Prada F.(11), Cantinotti M.(12), Ait-Ali M.(12), Merino C. (13), Parry A.(14), Poirier N.(10), Greil G.(5), Razavi R.(3,4), Gomez-Cia T.(1), Hosseinpour A.R.(1)

Paediatric Cardiology, Cardiothoracic Surgery and Technological Innovation Group. Hospital Virgen del Rocio, Seville, Spain (1); Cardiovascular Pathology Unit, Institute of Biomedicine of Seville, IBIS. Hospital Virgen de Rocio/CSIC/University of Seville, Seville, Spain. (2); Division of Imaging Sciences and Biomedical Engineering, King's College London, The Rayne Institute, St. Thomas' Hospital, London, United Kingdom. (3); Department of Paediatric Cardiology, Evelina London Children's Hospital, Guy's and St. Thomas' NHS Foundation Trust, London, UK (4); Children's Health CMC Dallas \& UT Southwestern, USA (5); Paediatric Cardiology and Cardiothoracic Surgery Departments, Leiden University Medical Center, Leiden, Netherlands (6); Biomedical Imaging Center, Pontificia Universidad Católica de Chile, Santiago, Chile (7); Paediatric Cardiology Unit, American University of Beirut AUB, Beirut, Lebanon (8); Paediatric Cardiology Unit, Hospital Regional Universitario Malaga, Malaga, Spain (9); Department of Paediatrics, Division of Paediatric Cardiology, Sainte-Justine University Hospital Center, Montreal, Quebec, Canada (10); Paediatric Cardiology Unit, Hospital Sant Joan de Deu, Barcelona, Spain (11); Fondazione G. Monasterio, CNR-Regione Toscana, Pisa, Italy (12); Paediatric Cardiology Unit, Hospital Reina Sofia, Córdoba, Spain (13); Paediatric Cardiology Unit, Bristol Children Hospital, Bristol, UK (14)

Introduction: Three-dimensional printed models (3D-models) provide an unrivalled spatial appreciation of patient-specific cardiovascular structures. However, the impact of this technology on surgical planning in complex congenital heart disease (CHD) is yet to be demonstrated.

Methods: A prospective, observational, case-crossover study involving 10 international centres and 40 patients with complex CHD (median age 3 years, range 1 month- 34 years) was conducted. Magnetic resonance imaging and computed tomography were used to acquire and segment the $3 \mathrm{D}$ cardiovascular anatomy. Models were fabricated by fused deposition modelling of polyurethane filament. We sought to evaluate (1) 3D-model dimension accuracy by comparison with medical images, (2) utility

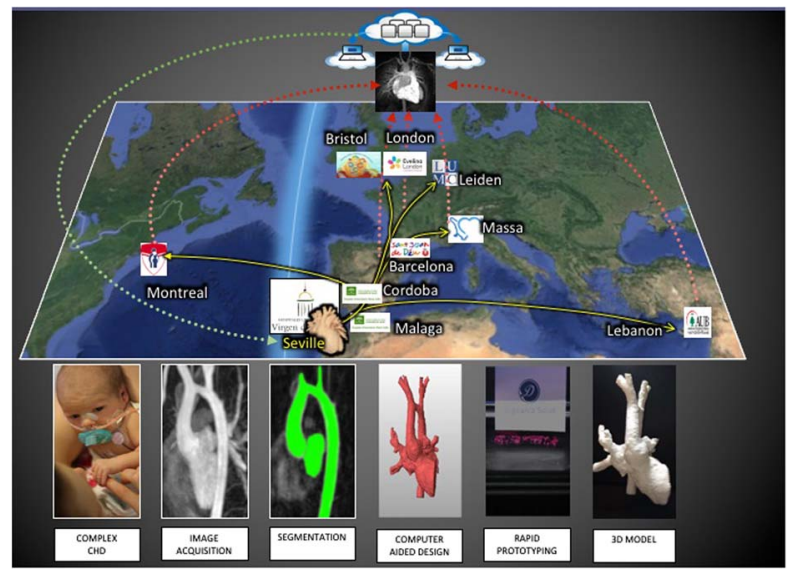


of the 3D models by evaluation of subjective satisfaction questionnaire and (3) incremental diagnostic value of 3D-models to plan surgery, comparing decisions made in two scenarios: During routine clinical practice and after inspection of a 3D-model.

Results: 3D models accurately replicate anatomy with a mean bias of $-0.27 \pm 0.73 \mathrm{~mm} .13$ surgeons and 30 paediatric cardiologists completed a satisfaction survey. $96 \%$ agree or strongly agree that 3D models provided better understanding of CHD morphology and reduced the chance of complications. 3D models changed the surgical decision in $45 \%$ of the cases. Consideration of a 3D-model refined the planned biventricular repair, achieving an improved surgical correction in $20 \%$ of cases. In $15 \%$ of cases initially considered for conservative management or univentricular palliation, inspection of the 3D-model enabled successful biventricular repair.

Conclusions: 3D models are accurate replicas of the cardiovascular anatomy and improve the understanding of complex CHD. This results in a change in the surgical decision making in $45 \%$ of the cases, allowing a better refinement of surgical strategy and surgical correction in cases that would conventionally be considered technically infeasible.

\section{MP2-7}

\section{Carotid Intima Media Thickness and Arterial Stiffness in} Smoking and Passive Smoking Children

Kilic Z., Bozdag O.(1), Karabel D.(1), Yildirim A.(1), Canik A.(2), Alatas O.(2), Musmul A.(3), Ucar B.(1)

Department of Pediatric Cardiology, Faculty of Medicine, Eskisehir Osmangazi University, Eskisehir, Turkey (1); Department of

Biochemistery, Faculty of Medicine, Eskisehir Osmangazi University, Eskisehir, Turkey (2); Department of Biostatistics, Faculty of Medicine, Eskisehir Osmangazi University, Eskisehir, Turkey (3)

Introduction and objectives: Cigarette smoking leads serious health problems furthermore passive smoking is also hazardous. Carotid intima media thickness (CIMT) and arterial stiffness are come into use to identify atherosclerosis risk and an early warning of myocardial infarction and stroke. In our study, Carotid intima media thickness and arterial stiffness in smoking and/or passive smoking group compare with non-smokers to clarify if this parameters are available to use in risk analysis of cardiovascular diseases.

Methods: We reached 2358 adolescents by questionnaires. 802 blood and urine samples from volunteers were collected for serum cholesterols, fasting glucose, insulin and urine cotinine. Participants divided into 3 groups according to questionnaires and cotinine results; smokers (n:103), passive smokers (n:104) and non-smokers (n:102). After carry out the exclusion criterias CIMT by high resolution B-mode ultrasound, osilimetric measurement of pulse wave velocity (PWV) and augmentation index by tonometers were evaluated in 309 participants (mean age: $15.5 \pm 1.09$ ) respectively.

Results: There's no statistically significant difference between groups by age, sex, body weight, height, BMI, systolic and diastolic blood pressures. CIMT and arterial stiffness did not differ among age and sex.(p>0.05). CIMT measurements obtained from both left and right carotis artery, all of the mean, minimum and maximum scale assesements showed CIMT thickens in passive smokers compare to non-smokers $(\mathrm{p}<0.05)$. There's statistically significant difference in both CIMT and arterial stiffness parameters between smokers and non-smokers $(p<0.001)$.

Conclusions: These results indicate, even in the early ages, smoking is a major risk factor for cardiovascular diseases and passive smoking is as vital as first hand smoking. CIMT measurements and arterial stiffness parameters can be use to estimate subclinical atherosclerosis in childhood.

\section{MP2-8}

Non-invasive assessment of arterial function in overweight and obese children and adolescents

Jakab A.E. (1), Hidvégi E.V.(2), Illyés M. (2), Cziráki A. (2),

Bereczki Cs. (1)

University of Szeged, Szeged, Hungary (1); University of Pécs, Pécs, Hungary (2)

Background: Overweight $(\mathrm{OW})$ and obesity $(\mathrm{O})$ are pandemic all over the World. Obesity may cause early atherosclerosis enhancing cardiovascular (CV) risk. Arterial function parameters (AFPs) predict the $\mathrm{CV}$ risk in adults. We may suppose the alteration of these parameters in OW and $\mathrm{O}$ children and adolescents, as well. Aims: To determine the frequency of $\mathrm{OW}$ and $\mathrm{O}$ in a large population of children and adolescents; to find differences in AFPs measured in patients and healthy subjects.

Patients and Methods: 6,824 (3,673 boys) healthy children and adolescents aged 3-18 years were examined. OW, O and systolic/ and-or diastolic hypertension were defined by the relevant guidelines. The AFPs (aortic pulse wave velocity [PWVao], aortic augmentation index [Aixao], aortic systolic blood pressure [SBPao]) were measured by a non-invasive, occlusive-oscillometric, invasively validated device. Four patient groups were created in both gender (OW, OW with increased systolic blood pressure $[\mathrm{OW}+\mathrm{ISBP}], \mathrm{O}, \mathrm{O}+\mathrm{ISBP})$. Results were compared to those measured in sex and age matched control groups.

Results: 518 (14.1\%) OW and 274 (7.5\%) O boys, 397 (12.6\%) OW and 174 (5.5\%) O girls, totally 915 (13.4\%) OW and 448 $(6.6 \%) \mathrm{O}$ children and adolescents were found. PWVao was increased in all patient groups, but significant differences were found only in groups of OW + ISBP and O + ISBP in both genders $(5.8 \mathrm{~m} / \mathrm{s}$ vs. $6.2 \mathrm{~m} / \mathrm{s}, 5.6 \mathrm{~m} / \mathrm{s}$ vs. $6.2 \mathrm{~m} / \mathrm{s}$ in boys; $5.7 \mathrm{~m} / \mathrm{s}$ vs. $6.2 \mathrm{~m} / \mathrm{s}, 5.7 \mathrm{~m} / \mathrm{s}$ vs. $6.1 \mathrm{~m} / \mathrm{s}$ in girls; $\mathrm{p}<0.001)$. No differences were found regarding Aixao. SBPao were increased in all patient's groups significantly (OW boys: $101.8-104.3 \mathrm{mmHg}$, OW girls: $101.0-103.4 \mathrm{mmHg}(\mathrm{p}<0.001)$, OW + ISBP boys: 102.6-121.6 mmHg, OW + ISBP girls: $100.2-120.0 \mathrm{mmHg}$ $(\mathrm{p}<0.0001)$, O boys: $100.6-103.2 \mathrm{mmHg}, \quad \mathrm{O}$ girls: 99.3$102.4 \mathrm{mmHg}(\mathrm{p}<0.001)$, O + ISBP boys: $101.3-120.3 \mathrm{mmHg}$, O + ISBP girls: $101-119.6 \mathrm{mmHg}(\mathrm{p}<0.0001)$.

Conclusions: Total prevalence of overweight and obesity was $20.0 \%$ in our population. PWVao was increased in all patient groups. SPBao was significantly increased in all patient groups. SPBao may serve as a surrogate marker in the procedure of $\mathrm{CV}$ risk stratification in OW and $\mathrm{O}$ children and adolescents. On the other hand, the increased SPBao indicates increased CV risk for these patients.

\section{MP2-9 \\ Clinical accuracy of annual cardiac computed tomography angiography to predict coronary event in adult and pediatric heart transplant recipients \\ Guillard O. (1,3), Rohnean A. (2), Tô N.T. (1), Paul J.F. (2), Houyel L. (1) \\ Hôpital Marie Lannelongue, Plessis-Robinson (France) (1); Institut Mutualiste Montsouris, Paris (France) (2); Hôpital Necker - Enfants malades, Paris (France) (3)}

Introduction: Cardiac allograft vasculopathy (CAV) is a major cause of late heart graft failure. Because the transplanted heart often remains denervated, the first symptom of myocardial ischemia can be sudden death, and routine periodic screening is thus 
recommended. Computed tomography coronary angiography (CTCA) has replaced conventional coronary angiography (CCA) for annual screening of heart transplant patients in our institution since September 2003, and has demonstrated a good sensibility and negative predictive value compared to CCA.

The aim of this study was to evaluate accuracy of CTCA annual screening to predict coronary events in heart transplant recipients. Method \& Results: From September 2003 to September 2016, 628 CTCA were performed annually in 110 patients including 47 children transplanted under 18. Age at first CTCA was $37.2+/$ - 17.8 years; mean follow-up duration was $12.4+/-6.3$ years. All patients had immunosuppressive tritherapy (anticalcineurins, mycophenolate mofetil, corticoids).

All CTCA were interpretable but 20 with uncertainties: poor visibility of distal coronary network in 14 , artifacts in 6 . Sixty-two patients had at least 1 pathological CTCA, requiring 41 CCA in 35. Coronary lesions were analyzed according to ISHLT classification. Both examinations were concordant in 27/41; Lesions were overestimated by CTCA in 11, underestimated in 3 . Twenty-one patients experienced at least 1 coronary event: necessity of percutaneous revascularization in 17 , sudden death in 3 (coronary origin not proved), myocardial infarction in 1 . No proved coronary event happened in children. Risk factors for coronary events were usual cardiovascular risk factors (body mass index $>2 \mathrm{SD}$, dyslipidemia, diabetes), ischemic cardiomyopathy as the cause for transplantation, and age $>18$ years at transplantation. Positive CTCA $<1$ year-interval with stenosis $>50 \%$ can predict the occurrence of a proved coronary event in heart transplant recipients with a sensitivity of $100 \%$, a specificity of $75 \%$, a negative predictive value of $100 \%$, and a positive predictive value of $45 \%$.

Conclusion: The sensitivity and negative predictive value of annual CTCA to predict proved coronary events after heart transplantation is excellent. This confirms the use of CCTA as a non-invasive and lower cost alternative to CCA for detection of CAV in heart transplant patients.

MP2-10

The efficacy of prenatal and postnatal screening for critical congenital heart defects

Narayen I.C.(1), Duineveld M.D.(2), Haak M.C.(1), Clur S.A.(2), Blom N.A. $(1,2)$

Leiden University Medical Center, Leiden, the Netherlands (1); Academic Medical Center of Amsterdam, Amsterdam, the Netherlands (2)

Introduction: The addition of pulse oximetry (PO) to routine postnatal care may lead to a higher detection rate of critical congenital heart defects (CCHD) before hospital discharge. However, there still is little information about the efficacy of PO screening for CCHD relative to the prenatal detection with fetal ultrasound screening. Aim of this study was to explore the efficacy of PO screening in relation to the prenatal detection rate of CCHD.

Methods: A literature search in PubMed was performed. Data were extracted and used to calculate prenatal and PO detection rates, standard error and the false positive rate. Data have been used for regression analysis weighted by study cohort size and weighted by standard error. Data have also been used to determine which CCHD are diagnosed most frequently by PO screening.

Results: 20 studies were included. A clear relationship between PO detection rate and prenatal detection rate was found. Correlation coefficients of $\mathrm{R}=0.903$ and $\mathrm{R}=0.857$ were determined with regression analyses weighted by study cohort size and standard error respectively. High prenatal detection rates were associated with lower PO detection rates, and vice versa (figure 1). A wide variety in false positive rate of PO screening was found, and there appears to be a relationship between prenatal detection rate and false positive rate.

Conclusion: Results of PO screening are strongly influenced by the prenatal detection rate of CCHD. Thus, prenatal detection rate should be taken into account when making the decision to implement PO screening in routine postnatal care.

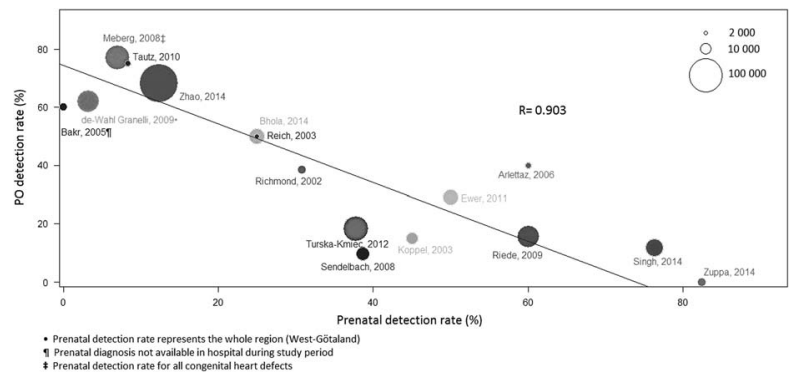

Figure 1.

Bubble chart of pulse oximetry (PO) and prenatal detection rates for individual studies with regression line weighted by study cohort size $(y=74.421-1.007 x)$.

\section{MP2-11}

Coronary artery variants/anomalies in patients with tetralogy of Fallot: should we look more carefully?A retrospective study of 226 Egyptian patients (A single centre experience)

Abd El-Naby A.(1),(2), Mahrous M.(1), Elsayed Ahmed A.(1), Ali N. (1), El Mozy W.(1), Kharabish A.(1), Romeih S.(1)

Aswan heart Centre, Aswan, Egypt(1)Cardiology department, Tanta University, Tanta, Egypt(2)

Background: Coronary artery anatomy has an important impact on the surgical management of patients with tetralogy of Fallot (TOF). The commonest reported anomaly is the left anterior descending artery (LAD) arising from the right coronary artery (RCA) and crossing right ventricle out flow tract (RVOT). Other variants/ anomalies are less reported and may be underestimated. Therefore, their clinical influence has not been investigated yet.

Aim: To study the origin and course of each coronary artery in Egyptian patients with TOF, and report the incidence of different coronary artery variants/anomalies.

Methods: A retrospective study to evaluate all coronary arteries, origin and course, by MSCT in TOF patients presented to Aswan Heart Centre during the period from 2013 to 2016.

Results: 226 TOF patients with median age 5 years (range between 9 months -40 years), male 124 (55\%).

Group I: Normal coronary arteries anatomy was reported in 155 patients (67\%). Group II: Coronary artery crossing the RVOT was reported in 23 patients (11\%) [Left main artery (LMA) arising from right coronary cusp (RCC) (5 patients), LAD arising from RCC (9 patients), large conal branch (8 patients), and RCA arising from left main artery (LMA)(1 patient)] Figure 1-I. Group III: Other coronary artery variants/anomalies were reported in 49 patients (22\%) [bridging of LAD (25 patients), ectasia (20 patients), LAD arising from RCC passing between aorta and pulmonary artery in a malignant course (1 patient), RCA arising from LMA passing between aorta and right atrium (2 patients), Left circumflex artery from RCA passing between aorta and left atrium (1 patient)] Figure 1-II. Conclusion: In patients with TOF; LAD crossing the RVOT is an important coronary anomaly; however, it is not the commonest one. In this study, other coronary variants/anomalies (especially bridging and ectasia) have been reported to be more frequent. 


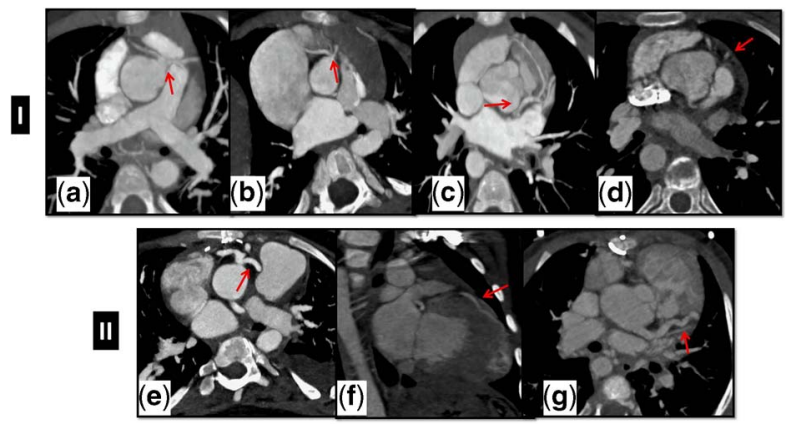

Figure 1.

I) shows a coronary artery crossing the RVOT, A) LMA arises from $R C C, B) L A D$ arises from $R C C, C) R C A$ arises from $L M A, D)$ large conal branch. II) Shows other coronary artery variants/anomalies, E) LAD arises from RCC and passes between aorta and pulmonary artery, F) bridging of $L A D, G)$ ectatic $L A D$. RVOT: right ventricular outflow tract, LMA: left main artery, LAD: left anterior descending artery, RCC: right coronary cusp, RCA: right coronary artery.

Those other variants/anomalies should be carefully evaluated and their clinical influence should be further investigated.

MP2-12

Cardiopulmonary exercise test among children with congenital heart diseases : a multicenter study Amedro P. (1,4), Gavotto A. (1,5), Guillaumont S. (1,2), Picot M.C. (3), Matecki S. $(4,5)$

Pediatric and Congenital Cardiology Department, University Hospital, Montpellier, France (1); Pediatric Cardiology and Rehabilitation Unit, Saint-Pierre Institute, Palavas-Les-Flots, France (2); Epidemiology and Clinical Research Department, University Hospital, Montpellier, France (3); Physiology and Experimental Biology of Heart and Muscles Laboratory - PHYMEDEXP, UMR CNRS 9214 - INSERM U1046, University of Montpellier, Montpellier, France (4); Pediatric Functional Exploration Laboratory, Physiology Department, University Hospital, Montpellier, France (5)

Introduction: Cardiopulmonary exercise test (CPET) is recommended in the follow-up of adults with congenital heart diseases (CHD). In children few centers have the expertise of this test. However, we recently showed that maximum oxygen uptake (VO2max) is correlated to health-related quality of life in children with CHD.

Purpose: We aimed to perform CPET among a large cohort of CHD children and to compare their VO2max to that of a control population. We also intended to identify variables that most impacted $\mathrm{VO} 2 \max$ in this population.

Methods: We included all children aged less than 18 who performed a complete CPET between 2010 and 2015 in 2 French pediatric CHD tertiary care centers. CHD group was defined upon Houyel's classification. Children with no chronic disease, no treatment and normal physical examination were included in the control group. Data were adjusted on age and gender. The impact of CPET and clinical variables on $\mathrm{VO} 2 \mathrm{max}$ was studied with multivariate analysis. Results: 798 children (496 CHD and 302 controls) were included in the study. VO2max was significantly lower in the CHD group, overall and for each sub-group $(\mathrm{p}<0.05)$. However, mean VO2max in the CHD group was good (93\% of predicted value). Children with single ventricles had the lowest value (72\%). VO2max significantly decreased with age in the CHD group. VO2max was impacted by left ventricle ejection fraction, right ventricle systolic pressure, left outflow tract obstacle or regurgitation, right outflow tract regurgitation and abnormal pulmonary function test. VE/VCO2 slope was more elevated in right heart diseases. Anaerobic threshold, oxygen uptake efficiency slope (OUES) and oxygen pulse correlated well to VO2max.

Conclusion: VO2max among children with CHD is not as altered as in adults but remained significantly lower than normal children. We recommend performing CPET in routine follow-up of theses patients. We should now focus on pediatric cardiac rehabilitation among CHD children.

MP2-13

Risk of significant pulmonary regurgitation after percutaneous balloon valvuloplasty for congenital pulmonary stenosis in long-term follow-up

Meyer-Szary J. (1), Sabiniewicz R. (1), Dorniak K. (2), Kwiatkowska J. (1) Department of Paediatric Cardiology and Congenital Heart Diseases, Medical University of Gdańsk, Poland (1), Department of Radiology, Medical University of Gdańsk, Poland (2)

Introduction: The percutaneous balloon pulmonary valvuloplasty (PBPV) has become the golden standard for treatment of congenital pulmonary stenosis (PS). Earlier studies have shown excellent outcome in regards of stenosis relief and neglectable risk of pulmonary regurgitation (PR) if proper balloon diameter was selected. Recently it has been shown, that in long term follow-up, this procedure carries a risk of functionally significant PR. This study aims to assess the degree of PR and its effect on right ventricular $(\mathrm{RV})$ dimension and function by MRI which is considered the reference method.

Methods: Fifty-six patients treated by PBPV underwent echocardiography according to the current guidelines protocol after a median of 13,6 $(5,7-24,2)$ years of observation. None qualified for late repeat PBPV for restenosis. On the basis of PR grade 21 patients (all cases of severe, random half of moderate) were enrolled in MRI study. Data is presented as mean \pm standard deviation or median (range) dependently on distribution.

Results: MRI was conducted 16,3 $(10,3-23,2)$ years after PBPV at $22,0(10,6-45,5)$ years of age, $52 \%$ were female. The RV end diastolic volume index (RVEDVI) was 119,0 $\pm 29,0 \mathrm{ml} / \mathrm{m} 2$ exceeding normal in $50 \%$ and exceeding $150 \mathrm{ml} / \mathrm{m} 2$ (a threshold value for PV replacement (PVR) in tetralogy of Fallot survivors) in 4 patients (20\% of the examined by MRI and 7\% of the study cohort). End systolic (RVESVI) was $53,6 \pm 17,3 \mathrm{ml} / \mathrm{m} 2$, exceeding normal in $45 \%$ and fulfilling PVR criteria in 1 ( $5 \%$ of MRI and $2 \%$ of the study cohort). RV ejection fraction was $54,8 \pm 6,1 \%$, ranging from $42 \%$ to $68 \%$ - within normal in all patients. PR fraction was $15 \%$ $(2 \%-47 \%)$ - mild in $60 \%$, moderate in $30 \%$, severe in $10 \%$ (2 patients, $4 \%$ of the study cohort). None of patients with moderate PR by echocardiography had severe abnormalities by MRI.

Conclusions: Survivors of PBPV for congenital PS require life-long follow-up. Despite mostly excellent outcomes, there is low but considerable risk of severe PR in the long-term.

MP2-14

Chest pain in the pediatric emergency department: can we improve management?

Ovaert C., Gallucci L., Mancini J., Bremond V., Bresson V., Pailhous S., Moreau E., Boutin A.

Timone Enfants, AP-HM, Marseille, France

Introduction: No precise recommendations exist as to the management of chest pain in children. Admissions in the pediatric 
emergency room with this symptom are increasing. The aim of our study was to analyze etiology and management of chest pain in our pediatric emergency department. The additional purpose was to elaborate an algorithm for future management.

Methods: Retrospective analysis of all children admitted with chest pain (main symptom), between 01-2014 and 05-2015, in our pediatric emergency department. Continuous variables were expressed as mean $+\mathrm{SD}$. Univariate analyses were performed using Chi2 test or Fisher exact test, to analyze relationships between qualitative variables. A $p$ value $<0.05$ was considered as significant.

Results: 224 children were included (0.5\% of total admissions). Sex ratio was $1.17(\mathrm{M} / \mathrm{F})$. Mean age was 10,5 + 3,35 years (range 3 to 18 years). In addition to detailed clinical history and examination, following tests had been performed: ECG (98.2\%), chest X-ray $(91,5 \%)$, general blood tests $(59.8 \%)$, troponin $(55,4 \%)$, myoglobin (42\%), CPK (19,2\%), BNP (14,3\%), echocardiography (20.1\%). The $\%$ of abnormal tests was as follow: ECG $6,4 \%$, chest X-ray $9,3 \%$, general blood tests $8.9 \%$, troponin $4.8 \%$, myoglobin $3.2 \%$, CPK 6,3\%, BNP 0\%, echocardiography 13,3\%. Final diagnosis was: musculo-parietal (59.8\%), psychogenic (15.2\%), digestive $(11.2 \%)$, pulmonary $(7.1 \%)$, cardiac $(3.1 \%, \mathrm{n}=7)$ and potentially cardiac $(3.6 \%, \mathrm{n}=8)$. The 7 confirmed cardiac etiologies consisted of 3 pericarditis, 3 worsening of pre-existing cardiac disease and 1 abnormal right coronary artery implantation. No significant difference in \% of ECG and enzyme levels performed was found between the different groups. Based on our findings we elaborated a new algorithm with retrospectively high sensitivity and reduced use of non-clinical tests. Additional test will be limited to those patients with warning personal or family history, abnormal cardiovascular examination or abnormal ECG.

Conclusion: Frequency of admissions, age distribution and etiologies are similar to those described in the literature. Cardiac causes remain very rare. Too many unnecessary tests are performed. A new algorithm has been elaborated in order to remain highly sensitive but to reduce unnecessary tests. Clinical and financial interest of this algorithm will need to be tested.

\section{MP2-15}

Stereological analysis of mitochondria in hypertrophic cardiomyopathy - A proposal for pathological diagnostic criteria of mitochondrial cardiomyopathy

Takeda A., Yamazawa H., Izumi G., Sasaki O., Abe J., Sasaki D. Department of Pediatrics, Hokkaido University Hospital, Sapporo, Japan

Introduction: Mitochondrial density in myocardium is highly consistent within each species according to the energy supply-demand balance. Thus, increased mitochondrial density may indicate excessive myocardial demand or adaptation to the reduced mitochondrial respiratory capacity. It is not well known whether mitochondrial density differ depending on the two status.

Methods: Eight patients with hypertrophic cardiomyopathy (HCM) were studied. Respiratory chain (RC) enzymatic assay and electron microscopy were performed using endomyocardial biopsy samples. Four patients were diagnosed as mitochondrial cardiomyopathy (MCM) with redued RC enzymatic activity. In each electron microscope images from endomyocardial biopsy samples, 18-25 sampling areas per each patient were studied. In each sampling area, mitochondrial volume density (Vvmit), myofibrillar volume density (Vvmyo), and mitochondrial surface area density (Svmit) were calculated using multipurpose test system for stereological analysis. Thus, mitochondrial volume density to myofibrillar and mitochondrial volume density ratio (Vvmit/ (Vvmyo + Vvmit)) and mitochondrial surface density to volume density ratio (Svmit/Vvmit) were calculated.
Results: In MCM group, all the patients showed reduced activity of complex I. Two patients also had reduced activity of complex IV. Vvmit, Svmit and Vvmit/(Vvmyo + Vvmit) were significantly higher than non-MCM group (0.57 vs $0.31,2.91$ vs $1.81,0.63$ vs 0.37 , respectively). Svmit/Vvmit was significantly higher in nonMCM group (5.21 vs 5.90).

Discussion: MCM group had significantly increased density and larger in size of mitochondria in myocardium. These data suggest that stereological quantification of mitochondria could be a pathological diagnostic criteria for mitochondrial cardiomyopathy. In addition, increased mitochondrial density in non-MCM group have a great impact on excessive energy consuming, which could be a prognostic factor.

Conclusions: Stereological analysis of mitochondria in hypertrophic cardiomyopathy could be a pathological diagnostic criteria of mitochondrial cardiomyopathy

\section{MP2-16 \\ Multi-parametric echocardiographic assessment for preoperative decision making in surgical treatment of atrioventricular septal defect \\ Schleiger, A., Buracionok, J., Miera, O., Schmitt K.B., Yigitbasi M., Berger F., Ovroutski $S$. \\ Department for Congenital Heart Disease and Pediatric Cardiology, Berlin, Germany}

Introduction: Surgical management of unbalanced atrioventricular septal defect (AVSD) remains challenging because of a high mortality rate and difficulties in decision making between biventricular repair (BVR) and univentricular palliation (UVP) in borderline cases. In this study we aimed to define echocardiographic criteria for imbalance and prove their predictability for mortality and reoperation rate.

Methods: From 1986 to 2016664 patients diagnosed with AVSD were identified in our institution. 522 patients received AVSD repair, $2.5 \%$ of these patients had unbalanced AVSD but were suitable for biventricular strategy. Of 253 patients pre- and postoperative echocardiographic data were available for retrospective analysis. For echocardiographic assessment modified atrioventricular valve index (mAVVI), ventricular cavity ratio (VCR), left ventricular inflow index (LVII) and right ventricle/ left ventricle inflow angle were measured. Left atrioventricular valve reduction index (LAVRI) was calculated to estimate left atrioventricular valve area after cleft closure. Correlation was proved between echocardiographic indices, surgical strategy, postoperative course, survival and reoperation rate.

Results: mAVVI was the only index to distinguish between balanced and unbalanced AVSD and predict surgical strategy (<0.19: UVP, >0.4:BVR; Jegatheeswaren et al.). Borderline cases $(\mathrm{n}=13)$ received biventricular repair and had no higher mortality or reoperation rate. Inflow angle positively correlated with ventilation time $(\mathrm{p}=0.01)$ and predicted early postoperative course. LAVRI $<0.5$, moderate mitral valve regurgitation and a mean gradient $>3 \mathrm{mmHg}$ at discharge were associated with reoperation. Conclusion: No echo index correlated with mortality. mAVVI seemed usable for preoperative decisions concerning interventricular balance. Correlation of right ventricle/left ventricle inflow angle with ventilation time may indicate the importance of ventricular septal defect size for postoperative course. LAVRI, residual mitral valve regurgitation or stenosis strongly correlated with reoperation rate. A multi-parametric echocardiographic assessment using indices for ventricular and atrioventricular valve size can facilitate decision making in borderline cases of unbalanced AVSD and surgical management of the atrioventricular valve. 
MP2-17

Early Detection Of Myocardial Dysfunction After Anthracycline Treatment in Asymptomatic Pediatric Cancer Patients: Comparison Of Left Ventricular Global Strain Measured With Triplane and 2D Speckle Tracking Cilsal E.(1), Oguz A.D. (2), Tunaoglu F.S.T. (2), Kula S. (2) Adana Numune Training and Education Hospital, Adana, Turkey (1); Gazi University Hospital, Ankara, Turkey (2)

Objective: Two-dimensional (2D) speckle tracking echocardiography (STE) are more useful to determine subclinical dysfunction than conventional methods as they enable global and regional evaluation of left ventricle in which patients exposed to anthracyclines. We firstly aimed to determine the left ventricular strain of pediatric cancer patients with normal ejection fraction treated with anthracycline using the 2D STE. We also compared global longitudinal strain values with using conventional 2D-STE and Triplane-STE in patients receiving anthracyclines.

Methods: This study included 23 cross-sectionally enrolled pediatric cancer patients receiving anthracycline chemotherapy (median age: 14 years, range 6-19) and 17 controls matched for age, gender and body surface area. Patients had received a median cumulative dose of $150 \mathrm{mg} / \mathrm{m}^{2}$ (range $60-360 \mathrm{mg} / \mathrm{m}^{2}$ ). In all children standard 2D, M-mode, Doppler and 2D-STE and triplane-STE imaging data were obtained.

Results: In patient group after anthracycline exposure some of the changes in cardiac parameters were demonstrated: 1. Patient group had a higher heart rate when compared with controls $(p=0.016)$. 2. Despite having a normal ejection fraction and shortening fraction values from "Pulsed" Doppler based measurements only pulmonary vein flow ratio showed a significant difference between two groups $(p=0.018)$. 3. Measurements were taken from the base of the interventricular septum; ETs values were significantly decreased and MPIs values were significantly increased in patients; measurements are taken from the base of the left ventricular free wall; $\mathrm{m} S$ velocities were showed statistically significant difference (respectively $p=0.022, p=0.042$ and $\mathrm{p}=0.001)$. 4. Following anthracycline exposure, pediatric cancer patients had a lower longitudinal and circumferential myocardial deformation of the left ventricle (respectively $p=0.003, p=0.01$ ). 5. Longitudinal strain values measured with Triplane method was significantly reduced in anthracycline group. Correlation between Longitudinal strain values measured with $2 \mathrm{D}$ and $3 \mathrm{P}$ STE were also demonstrated in anthracycline group (Table 1).

Conclusion: Systolic and diastolic functions are considered in asymptomatic patients with normal ejection fraction after chemotherapy. This study confirms the subclinical LV dysfunction in

Table 1. Global Longitudinal Peak Strain values of patient versus control group.

\begin{tabular}{|c|c|c|c|c|c|c|}
\hline $\begin{array}{l}\text { GLPS } \\
(\%)\end{array}$ & $\begin{array}{l}\text { Patient } \\
\text { group } \\
\text { 2D }\end{array}$ & Triplane & $\begin{array}{l}P \\
\text { value }\end{array}$ & $\begin{array}{l}\text { Control } \\
\text { group } \\
\text { 2D }\end{array}$ & $\begin{array}{l}\text { P value } \\
\text { Triplane }\end{array}$ & \\
\hline A4CH $\mathbb{S}$ & $-18,5 \pm 2,8$ & $-18,1 \pm 3,1$ & 0,655 & $-20,1 \pm 2,1$ & $-19,8 \pm 1,7$ & 0,659 \\
\hline $\mathrm{A} 3 \mathrm{CH} \mathbb{S}$ & $-18,6 \pm 3,0$ & $-17,9 \pm 3,1$ & 0,547 & $-21,3 \pm 2,5$ & $-21,6 \pm 2,7$ & 0,294 \\
\hline $\mathrm{A} 2 \mathrm{CH}$ & $-18,6 \pm 1,8$ & $-18,8 \pm 3,4^{\star}$ & 0,020 & $-19,3 \pm 2,3$ & $-19,4 \pm 2,4$ & 0,904 \\
\hline $\operatorname{Avg} \rrbracket$ & $-18,5 \pm 2,1$ & $-18,3 \pm 2,7 \star$ & 0,021 & $-20,3 \pm 1,4$ & $-21,4 \pm 1,7$ & 0,543 \\
\hline
\end{tabular}

$\mathrm{A} 3 \mathrm{CH}$, apical 3 chamber view; $\mathrm{A} 4 \mathrm{CH}$, apical 4 chamber view; $\mathrm{A} 2 \mathrm{CH}$, apical 2 chamber view, Avg, global average GLPS; GLPS, global longitudinal peak systolic strain

Data are expressed as mean \pm SD.

$\S \mathrm{P}<0.05$ between the patient and control group

$\star \mathrm{P}<0.05$ between $2 \mathrm{D}$ and Triplane approach patients after receiving anthracyclines with using Doppler and STE methods. We also exhibit the feasible and reproducible use of the triplane STE analysis for assessment of global LV function in the pediatric population.

\author{
MP2-18 \\ Novel Method for the Definition of Left Ventricular \\ Hypertrophy Improves Identification of Impaired Cardiac \\ Mechanics in Children with CKD: a 2D Echocardiography \\ Strain Study \\ Chinali M., Matteucci M.C.(1), Esposito C.(1), Doyon A.(2), \\ Franceschini A.(1), Del Pasqua A.(1), Iacomino M.(1) Schaefer F.(2), \\ Emma F.(1), Rineli G.(1), Drago F.(1) \\ Bambino Gesù Children Hospital, Rome, Italy (1); University of \\ Heidelberg, Heidelberg, Germay (2)
}

Background: We have recently suggested a simplified indexation approach to define left ventricular hypertrophy in children and adolescents of both genders (J Peds 2016). Objective of the present study was to verify whether our proposed indexation improves identification of abnormal cardiac phenotype in a population with high prevalence of $\mathrm{LVH}$.

Methods: Overall 238 children with available echocardiographic data, from the 4C multicenter European study on CKD were included. Presence of LV hypertrophy was defined using traditional partition values $(\mathrm{LVM}>38 \mathrm{~g} / \mathrm{m} 2.7)$ and by our recently suggested approach [LVM $>(45 \mathrm{~g} /(\mathrm{m} 2.16+0.09)]$. Differences in the two methods in the identification of children with impaired systolic function by traditional and 2D Strain indices of cardiac mechanical function were reported.

Results: Using the traditional partition value LV hypertrophy (tLVH) could be identified in 147 children representing $62 \%$ of the total population. In contrast, our proposed method identified LV hypertrophy (nLVH) in 142 children, representing 59\%\% of the whole population. Despite major accordance among methods (kappa score 0.88), tLVH was identified in 15 patients without $\mathrm{nLVH}$, while $\mathrm{nLVH}$ was present in only 10 patients without tLVH. Of note, children with tLVH, but not nLVH were significantly younger as compared to the rest of the population $(p<0.05)$ and did not show any reduction in traditional measures of chamber cardiac function (EF: 67.5 vs $68.4 p=n s$ ). In contrast patients with nLVH showed mildly lower indices of mechanical function by both midwall fractional shortening (14.8 vs $17.8 \%$; $\mathrm{p}<0.05)$ and on both the 2D strain radial $(26.5$ vs $32.4 \%)$ and the circumferential vector (21.2 vs $26.5 \%)$.

Conclusions: Traditional definition of LV hypertrophy mildly overestimates the prevalence of LVH in children with CKD younger children, in which no abnormalities in cardiac function can be found. Our proposed simplified approach for the definition of LV hypertrophy overcomes this issue, significantly improving risk stratification in CKD children.

\section{MP2-19 \\ Sudden death of patients with Kawasaki disease under school supervision in last 30 years \\ Ayusawa M., Kato M., Watanabe H., Komori A., Abe Y., Nakamura T., Jinbo S., Kamiyama $H$. \\ Department of Pediatrics and Child Health, Nihon University, Tokyo, Japan}

Objectives: Although students with Kawasaki disease (KD) are quite common in Japan, fatal events due to KD seem to be decreasing. 
It is necessary for school caregivers to be informed about the current situations of KD in school.

Method: All reports including ECG, death or health certificates, and autopsy reports of all sudden cardiac death (SCD) registered to the mutual aid system for accidents under school supervision in Japan during 30 years between 1984 and 2013 were reviewed.

Results: Fourteen cases (0.79\%) were enrolled from a total of 1768 sudden death. Graders were from 3rd in primary school to 12 th in high school. Twelve were male students. Eleven cases occurred among 1299 (0.85\%) SCD before 1999, and 3 cases did from among 469 SCD's (0.64\%) after 2000. In 14 victims, 13 cases occurred during exercise, and one case that received coronary artery bypass graft surgery dies suddenly while sitting on a chair. Reports of 11 cases proved that they had coronary artery lesion (CAL) and/or myocardial infarction; some of them were reveled by autopsies. However, in 3 cases were not reported that they have CAL, one is diagnosed as acute heart failure, and 2 other cases were reported as arrhythmia after autopsies. Actually those 3 cases were not restricted acting any sports. Eight cases were restricted competitive or strenuous sports strictly. Concerning to KD, there has been no report of survivors who was resuscitated from SCA. Conclusions: Before 1999, there was a possibility that the restriction of exercise was not observed strictly for students with CAL. Incidence of SCD due to CAL slightly decreased after 2000, however, students with past history of KD have possibility of outof hospital cardiac arrest induced by life-threatening arrhythmia.

\section{MP2-20 \\ Paediatric catheterization X-ray exposure reference doses: a single centre experience \\ Padrini M. (1), Baffoni L. (1), Castaldi B. (1), Bordin G. (1), Maschietto N. (1), Riccardi L. (2), Paiusco M. (2) Milanesi O. (1) (1) Department of Women's and Children's Health - University of Padova, (2) Department of Physics - University of Padova}

Background: There is a rising concern among the International Scientific Community over radiation exposure during cardiac catheterization in paediatric patients. To optimize radiation dose the states within the European Union are required to establish and use Diagnostic Reference Level (DRL). However at the time there is a lack of precise benchmarks for hemodynamic procedures. Aim Of The Study: A) To assess the radiation exposure levels in pediatric interventional cardiology and evaluate the main factors affecting it. B)To propose Local Diagnostic Reference Levels (DRLs) for diagnostic and interventional cardiac catheterization. C) To compare the data obtained before $(01 / 2014-11 / 2015)$ and after (12/2015-06/2016) the change of instrumentation protocols. Methods: We analyzed 97 interventional cardiology (IC) procedures, 41 diagnostic and 56 therapeutic, performed in pediatric patients (age range 6 days - 18 years) between January and June 2016. Fluoroscopic data were collected and analyzed digitally using the "Radiation Dose Monitor" software (Medsquare). Local DRLs were determined for each dosimetric parameter (DAP, Air Kerma and fluoroscopy time) and established on the third quartile value of the dosimetric parameters for diagnostic and interventional procedures.

Results: The median DAP value was $5,62 \mathrm{~Gy} \mathrm{~cm} 2$ for the entire sample. For diagnostic procedures was $6,48 \mathrm{~Gy} \mathrm{~cm} 2$ and 4,89 Gy $\mathrm{cm} 2$ for therapeutic procedures; no statistically significant difference was found between the two groups. The third quartile DAP value obtained in diagnostic and therapeutic procedures was respectively: 4,09 Gy cm2 and 12,14 Gy cm2 for " 5 to $15 \mathrm{Kg}$ " group and 20,17 Gy cm2 and 14,17 Gy cm 2 for " 15 to $30 \mathrm{Kg}$ " group. The change of protocols reduced the dose: the media DAP value decreased from $17,43 \mathrm{~Gy} \mathrm{~cm} 2$ to $11,87 \mathrm{~Gy} \mathrm{~cm} 2(\mathrm{p}<0.05)$.

Conclusion: This study represents the first Italian systematic and digital collection of dosimetric data in order to establish local DRL for diagnostic and therapeutic catheterization. In our centre there is a reduction of dose compared to data collected in the previous two years. It's important an ongoing review and updating of this data, due to the progression of the operator skills, develop new technologies and replacing equipment.

\section{MP3-1}

Chylothorax following paediatric cardiac surgery: a case control study of potential risk factors and the impact of a standardised management protocol

Day T. G. (1), Zannino D. (2), Golshevsky D. (1), d'Udekem Y. (3), Brizard C.(3), Cheung M.H. $(1,2,4)$

Department of Cardiology, Royal Children's Hospital, Parkville, Victoria, Australia (1); Heart Research Group, Murdoch Children's Research Institute, Parkville, Victoria, Australia (2); Department of Cardiac Surgery, Royal Children's Hospital, Parkville, Victoria, Australia (3); Department of Paediatrics, University of Melbourne, Parkville, Australia (4)

Objective: To investigate risk factors for the development of post-operative chylothorax following paediatric congenital heart surgery, and to investigate the impact of a management guideline on management strategies and patient outcome.

Methods: All patients with chylothorax following cardiac surgery at the Royal Children's Hospital, Melbourne, over a 48-month period beginning in January 2008 were identified. A control group (matched for age, date of operation, and sex) was identified. To investigate potential risk factors, univariable and multivariable logistic regression models were constructed with paired analysis. To examine the effect of a standardised management protocol, data before and after the implementation of the guideline were compared.

Results: 121 cases of chylothorax were identified, with 121 controls, matched for age at operation, date of operation, and sex. The chylothorax incidence was $5.23 \%$. Increasing surgical complexity (univariable OR 0.17 of RACHS-1 group 1 vs group 6 , $\mathrm{p}=0.02$ ), closed heart operations (OR 0.07 open vs closed, $\mathrm{p}<0.001$ ), and re-do chest incisions (OR 10.0 re-do vs virgin, $\mathrm{p}<0.001)$ were significantly associated with chylothorax. The impact of a standardised management protocol had no significant impact on either drain duration or management strategy.

Conclusions: We have replicated the previously reported association between surgical complexity and chylothorax risk, and have shown for the first time that re-do chest openings are also associated with a significantly increased risk. The implementation of a standardised management protocol in our institution did not result in a significant change in either chylothorax drainage duration, or management strategy.

\section{MP3-2 \\ Late management of truncus arteriosus: 20 years of humanitarian experience Gouton M.(1,2), Lucet V.(1), Bical O.(1), Leca F.(1) Mécénat-Chirurgie Cardiaque - Paris - France (1); Cardiologie Congénitale Montsouris - Paris - France (2)}

Background: the spontaneous evolution of the truncus arteriosus is poor because of the earliness of PAH. In developed countries, children are operated in the neonatal period with excellent long- 
term results. Late diagnosis and management remain rare, except in developing countries.

Aim: to describe the prognosis of children with truncus arteriosus diagnosed and tardily managed in developing countries.

Results: for 20 years, the humanitarian organization Mécénat-Chirurgie Cardiaque (France) has supported 41 children with truncus arteriosus from developing countries. The average age at management was 3 years old. Late diagnosis and management can be explained by the lack of adequate facilities in the developing countries. 8 children without shunt symptoms had to be recused, and 33 have been operated (32 repair surgery, 1 pulmonary banding). Surgery was decided if clinical and radiological symptoms of shunt were persistent, particularly if the cutaneous oxygen saturation was above $88 \%$, regardless of hemodynamic calculation of pulmonary vascular resistance, which was not carried out systematically. The postoperative course was marked by $\mathrm{PAH}$ crisis requiring $\mathrm{NO}$ ventilation and sildenafil in $30 \%$ of cases. The operative mortality has been $1 / 33$. The median stay in ICU was 5 days. The 32 children were able to return home without treatment and are followed up by the corresponding cardiologists, in relation with the French team of MCC. None of the children were lost to follow-up, with a mean follow-up of 3.4 years after surgery. At last follow-up, 1 child died six months after his surgery (infective endocarditis on the tube), 1 child had a massive truncal valve insufficiency, 5 had a significant stenosis of the RV-PA tube (3 symptomatic children are waiting for tube change), and 2 have already been reoperated 4 and 6 years after the complete repair to change the tube.

Conclusion: Late management of truncus arteriosus (even after two years of age) is possible with good long-term results and surgery remain possible without prior hemodynamic examination up to an advanced childhood if persist signs of left-to-right shunt, in particular a saturation above $88 \%$.

\section{MP3-3}

Bioimpedance spectroscopy measurements of phase angle and height for age are predictive of outcome following surgery for congenital heart disease

Magee A.G., Marino L.V., Meyer R., Johnson M., Newell C., Johnstone C., Magee A., Sykes K., Wootton S., Pappachan J.V.P. University Hospital Southampton, Southampton, UK.

Objective: Children with congenital heart disease (CHD) are often growth restricted (low weight- and/or height-for-age) and this increases mortality risk. The estimation of body composition using bioelectrical impedance spectroscopy (BIS), has been used in a variety of clinical settings, and BIS derived phase angle has been suggested as a surrogate marker of nutritional/prognostic status. We sought to describe the relationship between nutritional status, phase angle and post-operative outcomes.

Methods: 122 children with CHD following cardiac surgery (March 2015 to April 2016). Outcome variables included growth, mechanical ventilation, PICU length of stay (PICU LOS) and BIS $\mathrm{PA}$ at $50 \mathrm{~Hz}$. BIS measurements were taken before-surgery, postoperatively; day 0, day 2 and on discharge from hospital. Nutritional status was evaluated pre-operatively, and moderate malnutrition defined as height-for-age $\mathrm{z}$-score (HAZ) $\leq-2 ; 28.5 \%$ of infants and $20.6 \%$ of children met this criteria. Regression analysis was used to investigate the relationship between phase angle, HAZ and clinical outcomes.

Results: are presented as odds ratios (95\% confidence interval). A phase angle of $\leq 2.7$ was associated with increased duration of mechanical ventilation OR $4.1(1.3-12.4, p=0.01)$. PA of $\leq 2.7$ on day 2 was associated with an increased PICU LOS OR 7.8 $(2.7-22.45, p<0.001)$. HAZ $\leq-2$ was associated with increased mechanical ventilation OR $1.9(1.4-2.7, \mathrm{p}<0.001)$ and PICU LOS OR $1.8(1.1-2.7, \mathrm{p}=0.008)$. When the model was adjusted for age, risk factors and length of surgery, both a day 2 phased angle of $\leq 2.7$ and HAZ $\leq-2$ were associated with increased PICU LOS $(p=0.001$ and $p=0.04$ respectively). The model explained $81.7 \%$ of the variability in PICU LOS.

Conclusions: A pre-operative low HAZ is associated with poorer post-operative resilience, which when used with a phase angle measure of $\leq 2.7$ on day- 2 post-operatively may identify those with at increased risk of prolonged PICU-LOS.

\section{MP3-4 \\ Impact of higher central venous pressure on brain development and neurocognitive outcome in children before Fontan procedure at $\mathbf{2}$ to 3 years of age}

Knirsch W. (1,2), Mayer K. (1,2,3), Scheer I. (2,3), Tuura R. (2,3), Beck I. (2,4), Wetterling K. (5), Schranz D. (6), Hahn A. (7), Latal B. $(2,4)$, Reich B. (6)

Pediatric Cardiology, Pediatric Heart Center (1), Children's Research Center (2), Diagnostic Imaging, MR Center (3), Child Development Center (4), University Children's Hospital Zurich, Switzerland; Child Development Center, Frankfurt/Main, Germany (5); Pediatric Heart Center (6), Pediatric Neurology (7), University Hospital Giessen, Germany

Objectives: After bidirectional cavopulmonary anastomosis central venous pressure (CVP) increases and may influence further brain development in children with single ventricle until Fontan procedure. Therefore, we analysed hemodynamic impact on brain development before Fontan procedure.

Methods: In a prospective two-center study we analysed 32 children with single ventricle $(59.4 \%$ male, 25 with Giessen Hybrid approach, seven with classical Norwood approach) at a mean age of $27.4 \pm 4.0$ months (range 18.2 to 34.8 ). Children with genetic comorbidities were excluded. We evaluated hemodynamic values before Fontan procedure during routine cardiac catheterization and used semi-automated segmentation of cerebral MRI scans and neurodevelopmental assessment with Bayley-III Scales.

Results: Elevated CVP (measured in superior caval vein) and pulmonary artery pressure (PAP) correlated with smaller deep grey matter volume (CVP: $\mathrm{r}=-.40, \mathrm{p}=.03$; PAP: $\mathrm{r}=-.38, \mathrm{p}=.04$ ). Elevated pulmonary capillary wedge pressure (PCWP) was associated with both, smaller grey matter $(r=-.42, p=.03)$ and white matter $(r=-.42$, $\mathrm{p}=.03$ ) volumes. Increased CVP, PAP and PCWP were associated with greater cerebrospinal fluid (CSF) volumes (CVP: $r=.59$, $\mathrm{p}=0.001$; PAP: $\mathrm{r}=.43, \mathrm{p}=.02$; PCWP: $\mathrm{r}=.43, \mathrm{p}=.03)$. Larger CSF volumes correlated negatively with Bayley-III Scales: Cognitive Composite Scale $(r=-.45, p<0.001)$, and Language Composite Scale $(\mathrm{r}=-.46, \mathrm{p}<0.001)$, whereas total brain volumes did not.

Conclusions: Hemodynamics in children after establishing bidirectional cavopulmonary anastomosis may influence brain growth and cerebrospinal fluid volume. Of note, these MRI findings are correlated with adverse outcome. Further studies are needed to clarify the etiologic mechanisms responsible for this association.

\footnotetext{
MP3-5

Is optical coherence tomography superior in comparison to coronary angiography and biopsy regarding the detection of cardiac allograft vasculopathy? Urlich S.M. (1), Lehner A. (1), Schramm R. (2), Fischer M. (1), Dalla Pozza R. (1), Haas N.A. (1) Department of Pediatric Cardiology, Ludwig-Maximilians-University Munich, Germany (1); Department of Heart Surgery, LudwigMaximilians-University Munich, Germany (2)
} 
Objectives: Cardiac allograft vasculopathy (CAV) is a crucial problem concerning the long-term prognosis after heart transplantation, in adults and in children. Up to now, coronary angiography is considered to be the gold standard for diagnosis of CAV. However, because of the longitudinal hyperplasia of the intima without circumscribed stenosis especially the early stages of CAV are very difficult to detect using coronary angiography. In what extent biopsies should be used for detection of CAV is uncertain, yet. Intravascular imaging, for example optical coherence tomography (OCT), seems to be a good alternative for early diagnosis also of mild stages of CAV. In this study, we compared the results of OCT with coronary angiography and myocardial biopsy.

Methods: Retrospective analysis of all OCT examinations after pediatric heart transplantation in the department for pediatric cardiology and intensive care medicine at the LudwigMaximilians-University in Munich. Comparison of these results with simultaneously performed coronary angiography and myocardial biopsy of the right ventricle.

Results: Between June 2013 and August 2016 forty-seven patients underwent altogether sixty-nine OCT-examinations. In these examinations only 5 patients showed no sign of CAV according to the OCT-examination. Stanford I was detected in 23 OCTexaminations, Stanford II and III respectively in 16 examinations and Stanford IV in 9 OCT-examinations. In contrast only 21 of 69 coronary angiographies showed pathological results. Even regarding 25 cases with moderate to severe CAV with Stanford III and IV the coronary angiography was unremarkable in 32 percent. In nine patients, results of OCT-examination were compared with simultaneously performed myocardial biopsies. According to OCT two patients showed no CAV, three patients Stanford I, two patients Stanford II and two patients Stanford IV. In contrast myocardial biopsy was unremarkable in terms of CAV in seven cases. Two times myocardial biopsy showed a moderate, nonstenotic CAV. Interestingly in both cases OCT was unremarkable. Conclusion: For the early detection of CAV the OCT-examination is superior to the coronary angiography. However to detect also mild changes peripheral a combination with myocardial biopsy could be helpful. To evaluate the relevance of these mild CAV regarding the long-term survival further studies are necessary.

\section{MP3-6}

New stress-induced cardiomyopathy risk criteria in children and adolescents in high-achievement sports (Moscow)

Degtyareva E., Linde E., Jdanova O., Ovsyannikov D.

RUDN-University, Moscow, Russian Federation

Immunological imbalance with the hyperproduction of proinflammatory cytokines, predominantly TNF-a, may be of importance in the pathological transformation of athlete's heart and development of "stress cardiomyopathy" in children and adolescents in high-achievement sports in the event of inadequate overexertion.

Objective: to establish risk criteria of stress cardiomyopathy in young athletes based on comparison of the "quality" of adjustment to maximum physical activity reflecting the degree of myocardial damage.

Methods: A total of 100 young sportsmen of high and intermediate athletic qualification, athletes 10-19 years of age were examined. The clinical and functional state of cardiovascular system was assessed along with the sufficiency of energy supply during peak activity using a gas analyzer; the titers of anti-endothelial, anticardiomyocyte and anti-smooth muscle antibodies, antibodies to the myocardial conducting tissues, the level of proinflammatory cytokines (TNF-a, IL-6.8), troponin I, heart fractions of LDH, $\mathrm{CPK}$ and $\mathrm{CPK}-\mathrm{MB}$ were examined.

Results: In the beginner group, 5-fold increase in the level of TNF-a was observed in 3\% of sportsmen, 3 -fold increase - in $17 \%$, while normal value - in $46 \%$. In $67 \%$ of cases, the effective increase in the maximum physical performance $(\mathrm{PP})>6$ months of trainings and the improvement the oxygen transport system, the dynamics of immunological markers was characterized by a decrease in the early afterload level of proinflammatory cytokines.A significant negative correlation was obtained between the level of TNF-a at rest with athletes $(r=-0.7)$ and the training load in hours per week $(r=-0.65)$. A significant negative early afterload correlation was observed between the level of TNF-a and the maximum oxygen pulse ( $r=$ $-0.9)$, the titers of anti-endothelial $(\mathrm{r}=-0.67)$, anti-cardiomyocyte $(r=-0.76)$, anti-smooth muscle antibodies $(r=-0.89)$, antibodies to cardiac conducting tissue $(r=-0.94)$. Early afterload average values of the levels of proinflammatory cytokines and titers of anti-myocardial antibodies showed a statistically significant correlation with the training load in hours per week: $r=-0.79$ for TNF-a, $r=-0.94$ for IL-6, $r=-0.6$ for IL- 8 , which can serve as an objective criterion of the adequacy of the training process. The obtained data are the basis for changes in the intensity of training intensity and the objective criterion for cardiac protection.

\section{MP3-7 \\ Long-term follow up after single ventricle palliation: Comparison of different palliation methods \\ Sames-Dolzer E. (1), Gierlinger G.(1), Kreuzer M. (1), SchrempfJ. (1), Benedikt P. (1), Grohmann E. (1), Tulzer G. (1), Mair R. (1) \\ Children's Heart Center Linz, Austria}

Objectives: Patients with functional single ventricle physiology undergo different types of primary palliation at newborn age. This study analyzes patients characteristics and compares mortality and morbidity during long-term follow up between 4 palliation groups depending on primary surgical procedure.

Methods: Our single center retrospective study includes 459 consecutive patients who aimed at a Fontan circulation. Patients were operated between 1997 and 2014 and followed until the end of 2015 or death. 4 palliation groups were formed: Group A: no primary palliation needed due to balanced circulation; Group B: systemic to pulmonary artery shunt in case of severe pulmonary artery stenosis or atresia; Group C: pulmonary artery banding in case of pulmonary volume overload; Group D: Norwood procedure. 22,5\% of the nonNorwood patients (32 pts.) had dextrocardia or heterotaxy syndrome. Results: Group A: None of the 39 patients without stage I operation died during follow up. Group B: 71 patients received a mBTS (57 pts.), or other shunts (14 pts.) Total mortality rate was $12,68 \%$. Group C: 32 patients needed a PAB. 21,88\% of them died during long-term follow up. Group D: 317 patients underwent a Norwood procedure. The 3 stage associated mortality rates were $18,9 \%, 10,2 \%$ and $3,4 \%$, respectively.

In total 280 patients are followed after the Fontan procedure with a mortality rate of 2,5\% (7 pts.) during a median follow up of 4,21 years (range 2 months $-14,5$ years) after Fontan. 2 patients underwent heart transplantation and 271 patients are alive with Fontan physiology.

Conclusions: $69,06 \%$ of our single ventricle patients needed a Norwood procedure and this group showed the highest overall mortality rate of $28,71 \%$, followed by group C (PAB) $21,88 \%$ and Group B (shunt) 12,68\%. Patients without stage 1 procedure showed 100\% survival. The long-term results after the Fontan procedure are very good with more than 95\% survival without HTX independent of primary palliation. 
MP3-8

Predictors of re-intervention after aortic coarctation repair in childhood - a population based study

Ylinen M.(1,2)*, Tyni V.(1)*, Pihkala J.(1), Sairanen H.(1),

Sarkola T.(1)

Helsinki University Central Hospital/Children's Hospital and University of Helsinki, Helsinki, Finland (1); Kuopio University Hospital, Kuopio, Finland (2); * shared first author position.

Introduction: Recurrent coarctation of the aorta (reCoA) may occur regardless of primary treatment modality including surgery or balloon angioplasty/stent. Residual CoA and small transverse arch have been proposed as etiological factors. The aim of the present study was to evaluate the incidence of reCoA and to explore risk factors predicting the need for re-intervention.

Methods: 294 patients with isolated CoA were treated (surgery $\mathrm{n}=251$, angioplasty $\mathrm{n}=43$ ) in Helsinki Children's Hospital between years 2000 and 2012 with a follow-up until 2014 (median 8.0 years). Pre- and post-procedure baseline characteristics were retrospectively collected from clinical records.

Results: In all, 51/294(17.3\%) of the patients developed reCoA after the initial procedure, 40/251(15.9\%) after surgery and 11/43 $(25.6 \%)$ after angioplasty. The median time to second procedure was 3.4 and 16.8 months, respectively. In the surgery group, younger age, smaller weight and small pre-procedure transverse arch were associated with reCoA (Table). There were no need for re-interventions if aortic arch reconstruction was used as a primary technique $(\mathrm{n}=15)$. In the angioplasty group, bicuspid aortic valve was more common and post-procedure arm-leg blood pressure gradient (BPGr) was higher among reCoAs (Table), all but one reCoAs had post-procedure BPGr at least $10 \mathrm{mmHg}$. The best cut off post-procedure BPGr value was $7,6 \mathrm{mmHg}$ based on ROCanalysis, and predicted reCoA with a sensitivity and specificity of $60 \%$. The best cut off for pre-procedure transverse arch Z-score was -2.8 SD with a sensitivity of $38 \%$ and specificity of $79 \%$. In logistic regression, post-procedure BPGr and small transverse arch

\begin{tabular}{|c|c|c|c|c|}
\hline \multirow[b]{2}{*}{$\begin{array}{l}\text { Baseline } \\
\text { characteristics }\end{array}$} & \multicolumn{2}{|l|}{ Surgery } & \multicolumn{2}{|l|}{ Angioplasty } \\
\hline & $\begin{array}{l}\text { No } \\
\text { ReCoA } \\
\mathrm{n}=211\end{array}$ & $\begin{array}{l}\mathrm{ReCoA} \\
\mathrm{n}=40\end{array}$ & $\begin{array}{l}\text { No } \operatorname{ReCoA} \\
n=32\end{array}$ & $\begin{array}{l}\mathrm{ReCoA} \\
\mathrm{n}=11\end{array}$ \\
\hline $\begin{array}{l}\text { Age, surgery in days, } \\
\text { angioplasty in } \\
\text { years, median } \\
\text { (range) }\end{array}$ & $\begin{array}{l}23 \\
(1-6378)\end{array}$ & $\begin{array}{c}10 \\
(1-250)^{\star}\end{array}$ & $\begin{array}{l}3,1 \\
(0,7-14,3)\end{array}$ & $\begin{array}{c}5,7 \\
(0,9-15,5)\end{array}$ \\
\hline $\begin{array}{l}\text { Weight }(\mathrm{kg}) \text { at } \\
\text { primary } \\
\text { procedure, } \\
\text { median (range) }\end{array}$ & $\begin{array}{r}4,1 \\
(1,1- \\
98,5)\end{array}$ & $3,8^{\star}(2,3-7,2)$ & $14,2(6,8-72,5)$ & $\begin{array}{l}20,0 \\
\quad(9,1-63,9)\end{array}$ \\
\hline Sex (male, \%) & $146(69 \%)$ & $26(65 \%)$ & $22(69 \%)$ & $9(82 \%)$ \\
\hline $\begin{array}{l}\text { Bicuspid aortic } \\
\text { valve }(\%)\end{array}$ & $97(46 \%)$ & $19(48 \%)$ & $4(13 \%)$ & $6(55 \%)^{\star}$ \\
\hline $\begin{array}{l}\text { Pre-procedure } \\
\text { transverse arch } \\
\text { diameter, Z-score, } \\
\text { mean (SD) }\end{array}$ & $-1,5(2,0)$ & $-2,2(1,2)^{\star}$ & $-0,1(1,0)$ & $-0,8(1,4)$ \\
\hline $\begin{array}{l}\text { Pre-procedure } \\
\text { systolic arm-leg } \\
\text { gradient, mmHg, } \\
\text { mean (SD) }\end{array}$ & $35,1(18,6)$ & $34,6(18,8)$ & $27,3(19,1)$ & $31,7(20,6)$ \\
\hline $\begin{array}{l}\text { Post-procedure } \\
\text { systolic arm-leg } \\
\text { gradient, mmHg } \\
\text { mean (SD) }\end{array}$ & $3,7(11,3)$ & $5,3(10,0)$ & $9,6(12,9)$ & $27,6(15,1)^{\star}$ \\
\hline
\end{tabular}

$\star_{\mathrm{p}}<0,05$, Fisher's exact test / Mann-Whitney U-test / Independent sample T-test were independent predictors of reCoA when arch reconstructions were removed from the analyses.

Conclusions: The need for re-intervention after CoA repair in childhood is common and the associated risk factors differ between the primary treatment modalities. Both residual $\mathrm{CoA}$ and small aortic arch were independent predictors of development of reCoA.

MP3-9

Pulmonary arterial hypertension related to complete transposition of the great arteries: outcomes after arterial switch

Ma K., Li S., Hua Z., Yang K., Yan J., Qi L., Zhang S., Chen Q. Fuwai Hospital, Beijing, China

Introduction: Transposed great arteries (TGA) is the most common complex congenital heart defect leading to pulmonary arterial hypertension (PAH). Clinical scenario of patients with TGA-PAH after arterial switch operation (ASO) remains largely unknown. This study aimed to investigate mid- to long-term outcomes focusing on pulmonary vascular physiology.

Methods: Consecutive patients from 2010 to 2014 were retrospectively included. The inclusive criteria included 1). diagnosed as TGA-unrestrictive VSD, 2). age $>6$ months, 3). underwent ASO and 4). $\mathrm{mPAP}>25 \mathrm{mmHg} / \mathrm{PCWP}<15 \mathrm{mmHg}$. Patients co-present with collateral vessels, left ventricular outflow tract obstruction and cardiac positional abnormality were excluded.

Results: A total of 83 patients were included with an overall mortality of $13.3 \%(11 / 83)$. Fifty patients $\leq 1$ years old were divided into group 1 and the others ( 33 patients) $>1$ years old were in group 2. Eight deaths $(72.7 \%)$ were related to PAH and all of them occurred within 1 year after ASO. The figure displays the probability freedom from PAH related deaths, which was 93.6\%, $89.5 \%$, and $89.5 \%$ at 6 months, 1 year and 5 years, respectively. Only the mPAP immediately after ASO was identified as independent risk factor for mortality $(\mathrm{OR}=0.8, \mathrm{p}=0.020)$ in multivariate analysis. Among the 33 patients with abnormal mPAP at discharge, the $\mathrm{PAH}$ related deaths were less in those with regular postoperative oral drug treating PAH $(2 / 22$ vs. $6 / 11, \mathrm{p}=0.015)$. Conclusions: Patients with an age $<1$ year can undergo ASO with favorable outcomes and have better survival rate than those older than 1 year of age. Deaths were associated with mPAP immediately after ASO. Regular oral drug is indicated in patients with abnormal postoperative mPAP.

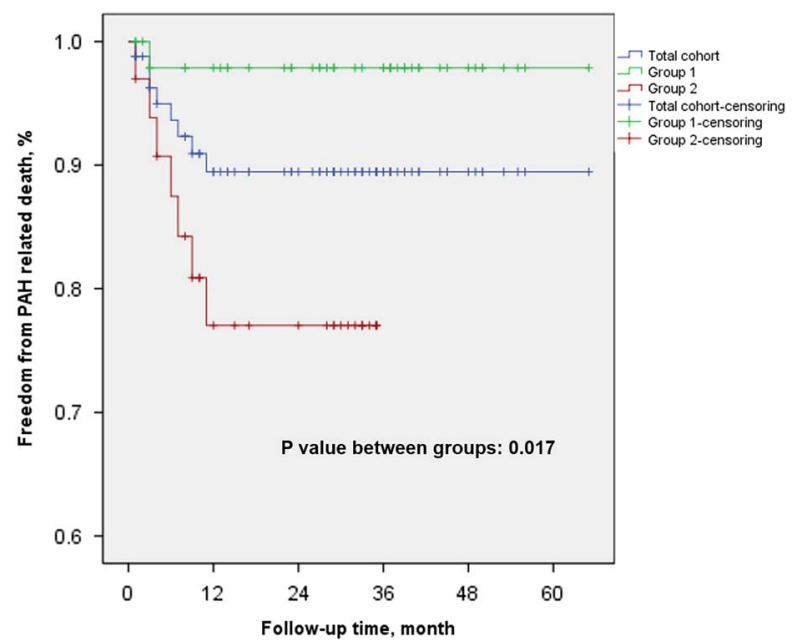


MP3-10

Aortic root growth in children with Marfan syndrome: evidence for gender differences

Muiño-Mosquera L., De Groote K., Vandekerckhove K., Panzer J., De Wilde H., De Wolf D., De Backer J.

Ghent University Hospital, Ghent, Belgium

Background: Aortic root (AoR) dilation and subsequent dissection are the most life threatening complications of Marfan syndrome (MFS). Known determinants of aortic dissection include the diameter and growth rate. Knowledge of risk factors favouring rapid AoR growth is important to tailor treatment.

Data regarding gender differences in children with MFS are scarce. Our aim was to study whether AoR growth in this group differs depending on the gender.

Methods: We retrospectively reviewed the echocardiography and clinical data of our cohort of MFS patients younger than 25yrs. MFS was diagnosed after familial screening in $50 \%$ or due to clinical features of MFS in the rest. All patients had an underlying FBN1 gene mutation. First digital echocardiography available was considered as baseline. Last echocardiography at follow-up was used to calculate AoR growth rate.

Results: Forty MFS patients were included in this study. Twentyseven $(69,2 \%)$ were males. Mean age and mean AoR diameter at baseline were $8,41 \pm 3,9 \mathrm{yrs}$ and $29,5 \pm 4,82 \mathrm{~mm}$ (z-score $3,29 \pm 1,95$ ) respectively.

During a mean follow up time of $4,63 \pm 2,95 \mathrm{yrs}$ no aortic dissection occurred. Eight patients (20\%) underwent elective AoR replacement at a mean age of $15 \pm 3,7 \mathrm{yrs}$. All these patients were males. One patient died of arrhythmia a few months after elective AoR replacement during orthopaedic surgery.

Mean AoR growth in this period was $1,1 \pm 0,68 \mathrm{~mm} / \mathrm{yr}$. There was a significant difference between males and females $(1,36 \pm$ $0,65$ versus $0,51 \pm 0,30 \mathrm{~mm} / \mathrm{yr} ; \mathrm{p}<0001)$ and between operated males and non-operated males $(2,02 \pm 0,53$ vs $1,09 \pm 0,49 \mathrm{~mm} / \mathrm{yr}$; $\mathrm{p}<0001)$. Operated males also had significant higher AoR $\mathrm{z}$-score at baseline in comparison to non-operated males $(5,35 \pm 1,43$ vs $2,68 \pm 1,47 ; \mathrm{p}<0001)$.

Conclusion: In this study males with MFS had higher AoR growth rate and higher incidence of elective AoR replacement than females with MFS. This supports the argument that there is a gender difference.

The group of males undergoing elective AoR replacement had significantly higher AoR z-score at baseline and more rapid AoR growth during follow-up. These results suggest that there is a group of MFS patients showing rapid AoR growth from a very young age. Further study is necessary to elucidate why this happens.

\section{MP3-11}

Primary Repair of Common Arterial Trunk: Is It Safe After Four Months of Age?

Hascoet S., Vergnat M., Ly M., Lebret E., Boet A., Roussin R., Horer $J$.

pôle des cardiopathies congénitales, Hôpital Marie Lannelongue, Le Plessis-Robinson, France

Introduction: Repair of common arterial trunk (CAT) is usually performed before 4 months of age. Late repair has been associated with unfavorable outcome. However, limited contemporary data support this finding. We assessed short-term outcome after CAT repair beyond 4 months of age, and investigated risk factors for poor outcome.

Methods: All patients undergoing CAT repair beyond ("late") 4 months of age (oversea referrals) from 1995 to 2015 were included.
Early Outcomes and potential risk factors for prolonged intensive care unit (ICU) stay ( $>7$ days) and treated post-operative pulmonary hypertension (PAH) (NO and/or other PAH-specific therapies) were assessed by means of logistic regression.

As a reference-value for CAT normal early outcome, results of concomitant regular age repair (before ("early") 4 months of age) were used.

Results: Patients "late" $(\mathrm{n}=34)$ presenting had at median age of 10 months (range 4m.-11y.). Preoperatively, median arterial oxygen saturation $(\mathrm{SaO} 2)$ was $90 \%$ (range $75-96 \%)$ and pulmonary vascular resistances (PVR) $(\mathrm{n}=10,29 \%) 8.9 \mathrm{WU}$ (range 3.8 19.2 WU). A valved conduit for right ventricular outflow tract reconstruction and complete closure of all septal defects were performed in all patients $n=34(100 \%)$.

When juxtaposed to "early" repair $(n=132)$ results, "late" repair patients did not demonstrate any difference in mortality and postoperative $\mathrm{PAH}$ rates, while ICU and ventilation time were significantly shorter in this group.

In multivariate analysis, age at surgery, preoperative PVR and catheterization were not predictor for mortality, postoperative $\mathrm{PAH}$, or prolonged ICU stay. $\mathrm{Sa} 02$ was a significant predictor for postoperative $\mathrm{PAH}$ and prolonged ICU stay, respectively $(\mathrm{OR}=$ $0.85,95 \%$ CI $0.72-0.99, \mathrm{p}=0.04)(\mathrm{OR}=0.84,95 \% \mathrm{CI} 0.73-0.97$, $\mathrm{p}=0.02)$.

Survival after hospital discharge was 100\% (median follow up time 2.2 years, $62 \%$ complete).

Conclusion: Early timing for CAT repair should not be revised given the naturally-selected character of the studied cohort.

For these patients, late repair is feasible without increased/prohibitive morbidity and mortality rates.

Preoperative $\mathrm{Sa} 02$, as main predictor for adverse outcome, is pivotal-decisive for operability assessment.

PVR can be reliably estimated by $\mathrm{SaO} 2$.

MP3-12

Impact of plasma protein deposition on Staphylococcus aureus adhesion to tissues used for RVOT reconstruction Veloso T.R. (1), Veloso R.P.L. (1), Claes J. (1), Liesenborghs L. (2), Van Kerckhoven S. (2), Jashari R. (3), Gewillig M. (1), Hoylaerts M.F. (2), Meyns B. (4), Heying R. (1)

(1) Cardiovascular Developmental Biology, Department of Cardiovascular Sciences, KU Leuven, Leuven, Belgium; (2) Center for Molecular and Vascular Biology, Department of Cardiovascular Sciences, KU Leuven, Leuven, Belgium; (3) European Homograft Bank, Saint Jean Clinique, Brussels, Belgium; (4) Division of Clinical Cardiac Surgery, Department of Cardiovascular Sciences, KU Leuven, Leuven, Belgium

Introduction: Congenital heart disease encompasses anatomical defects, such as malformations on the right ventricular outflow track (RVOT) that can be surgically corrected using a cryopreserved pulmonary homograft $(\mathrm{CPH})$ in first line and as an alternative xenografts such as bovine jugular vein (BJV) conduits which are also available stent-mounted for percutaneous catheter interventions. Although a good therapeutic alternative several clinical studies report an increased risk of stenosis, immunogenicity, thrombus formation or even infective endocarditis of BJV xenografts. In this study we investigate the role of plasma protein deposition on Staphylococcus aureus adhesion to several tissues used for RVOT reconstruction.

Methods: Similar tissue pieces prepared as for clinical use $(\mathrm{CPH}$, BJV conduit, pericardium patch and decellularized pericardium patch) were incubated for $2 \mathrm{~h}$ at $37 \mathrm{oC}$ with $30 \mu \mathrm{g} / \mathrm{ml}$ of fluorescently labelled Fibrinogen or $50 \mu \mathrm{g} / \mathrm{ml}$ of fluorescently labelled immunoglobulin (obtained from frozen pooled human plasma) 
resuspended in $200 \mathrm{~g} / \mathrm{L}$ of human albumin. Then, S. aureus 8325-4 (fluorescently labelled) adhesion to the same tissues was assessed under flow conditions (10 min at 1000s- 1 ) after $2 \mathrm{~h}$ of incubation at $37 \mathrm{oC}$ with PBS or frozen human pooled plasma using a micro-parallel flow chamber. Protein deposition and bacterial adhesion was assessed and quantified by fluorescence microscopy.

Results: Pericardium patch presented significant higher protein deposition $(\mathrm{P}<0.05)$ compared to $\mathrm{BJV}$ and $\mathrm{CPH}$ wall and leaflet. Although not significant, there is a trend to higher fibrinogen deposition on BJV tissue compared to $\mathrm{CPH}$. On the opposite, $\mathrm{CPH}$ presented higher $(\mathrm{P}<0.05)$ immunoglobulin deposition compared to BJV tissue. After incubation with frozen human pooled plasma $\mathrm{S}$. aureus adhesion under flow conditions increased $(\mathrm{P}<0.05)$ compared PBS.

Conclusions: Our results indicate that plasma protein deposition modulate $\mathrm{S}$. aureus adhesion to the tested tissues. Plasma protein adhesion might also play an important role after tissue implantation in vivo and may contribute to the onset of inflammation and infection as a clinical complication. In the future we will focus on the impact of plasma protein deposition on platelet adhesion and thrombus formation, endothelial cell and inflammatory cells adhesion and how these can modulate associated S. aureus adhesion and infection.

\section{MP3-13}

Is it really worthy an early complete repair in a Fallot situation?

Monteagudo M. (1), Aroca A. (1), Polo L. (1), Gonzalez-Rocafort A. (1), Sanchez R. (1), Bret M. (2), Rey J. (1), Sanabria P. (3), Pérez A. (3), Uceda A. (4), Labrandero C. (4)

Congenital Cardiac Surgery. Universitary Hospital La Paz, Madrid, Spain (1). Radiology. Universitary Hospital La Paz, Madrid, Spain (2).

Anaesthesia. Universitary Hospital La Paz, Madrid, Spain (3). Paediatric. Cardiology. Universitary Hospital La Paz, Madrid, Spain (4).

Objectives: The precise moment of surgery of a Fallot situation (FS) is still nowadays a controversy. We compare 2 groups of patients who underwent complete repair (CR) before/after 90 days (90d) of life. Differences in mortality, morbidity and need of redo were analysed.

Methods: A retrospective study of all patients under 18 years with CR of FS in our centre was held, between January 2007 and October 2016, dividing them in > / $\leq$ of 90d. SPSS 20.2 was used. Results: 130 CR, 27 (21\%) $\leq 90 \mathrm{~d}$ (10 newborn). No differences between genders. Median age in CR: 1,5 $(0,8-2,4)$ months versus 6 (5-9). Median weights: $3,9 \pm 1,2 \mathrm{~kg}$ versus $7,3 \pm 2,8$. Diagnoses in Table 1. Prenatal diagnosis: $37 \%$ in $\leq 90 \mathrm{~d}$ versus $11 \%>90 \mathrm{~d}$ $(\mathrm{p}=0002)$. Patent ductus: $40 \%$ in $\leq 90 \mathrm{~d}(63 \%$ prostaglandins dependence) versus $10 \%>90 \mathrm{~d}(\mathrm{p}<0001)$. Previous palliation: 21

Table 1. Diagnoses.

\begin{tabular}{lcl}
\hline & $\begin{array}{l}\mathrm{RC} \leq \mathbf{9 0} \text { days } \\
(\mathbf{n}=\mathbf{2 7})\end{array}$ & $\begin{array}{l}\mathrm{RC}>90 \text { days } \\
(\mathbf{n}=\mathbf{1 0 3})\end{array}$ \\
\hline TOF & $\mathbf{7 8 \%}$ & $\mathbf{7 9 \%}$ \\
TOF AVP & $\mathbf{0 \%}$ & $\mathbf{3 \%}$ \\
DORV TOF & $\mathbf{1 1 \%}$ & $\mathbf{5 \%}$ \\
VSD + PA & $\mathbf{7 \%}$ & $\mathbf{8 \%}$ \\
VSD + PS & $\mathbf{4 \%}$ & $\mathbf{2 \%}$ \\
DCRV + VSD & $\mathbf{0 \%}$ & $\mathbf{3 \%}$ \\
\hline
\end{tabular}

Table 2. Type of Surgery.

\begin{tabular}{lll}
\hline & $\begin{array}{l}\mathrm{RC} \leq 90 \text { days } \\
(\mathrm{n}=27)\end{array}$ & $\begin{array}{l}\mathrm{RC}>90 \text { days } \\
(\mathrm{n}=103)\end{array}$ \\
\hline Transannular & $90 \%$ & $78 \%$ \\
Infraannular & $7 \%$ & $9 \%$ \\
RA-PA & $4 \%$ & $11 \%$ \\
$\begin{array}{l}\text { Surgery } \\
\text { Conduit }\end{array}$ & $0 \%$ & $2 \%$ \\
\hline
\end{tabular}

Table 3. Complications.

\begin{tabular}{lccc}
\hline & $\mathbf{R C} \leq \mathbf{9 0} \mathbf{d}$ & $\mathbf{R C} \mathbf{> 9 0} \mathbf{d}$ & $\mathbf{p}$ value \\
\hline Delayed sternum closure & $30 \%$ & $7 \%$ & $\mathrm{p}=0,004$ \\
ECMO & $4 \%$ & $2 \%$ & $\mathrm{n} . \mathrm{s}$ \\
JET & $22 \%$ & $15 \%$ & $\mathrm{n} . \mathrm{s}$ \\
Pacemaker & $4 \%$ & $2 \%$ & $\mathrm{n} . \mathrm{s}$ \\
Pleural/peritoneal efussion & $22 \%$ & $22 \%$ & $\mathrm{n} . \mathrm{s}$ \\
Dialysis & $22 \%$ & $10 \%$ & $\mathrm{n} . \mathrm{s}$ \\
Inhospital infection & $22 \%$ & $10 \%$ & $\mathrm{n} . \mathrm{s}$ \\
Inhospital redo & $26 \%$ & $11 \%$ & $\mathrm{p}=0,041$ \\
\hline
\end{tabular}

(9 modified Blalock-Taussig, 12 hemicorrections). Previous palliation in $\leq 90 \mathrm{~d} 4 \%$, versus $19 \%$ in $>90 \mathrm{~d}$. There has been no palliation in the last 3 years. Type of surgery in Table 2. Cardiopulmonary bypass times (minutes): $122 \pm 24$ in $\leq 90 \mathrm{~d}$ versus $136 \pm 31$ in $>90 \mathrm{~d}$ $(\mathrm{p}=0,03)$ and aortic cross clamp time (minutes): $86 \pm 18$ in $\leq 90 \mathrm{~d}$ versus $99 \pm 22$ in $>90 \mathrm{~d}(\mathrm{p}=0005)$. Differences in intubation times (hours), 107(77-217) in $\leq 90 \mathrm{~d}$ versus $24(5-108)$ in $>90 \mathrm{~d}(\mathrm{p}=0003)$; intensive care unit (ICU) (days), 11(7-22) in $\leq 90 \mathrm{~d}$ versus $7(5-12)$ in $>90 \mathrm{~d}(\mathrm{p}<0001)$ and hospitalization (days), 19(14-38) in $\leq 90 \mathrm{~d}$ versus $12(8-20)$ in $>90 d(p<0001)$. Overall mortality: $2(1,5 \%) .1$ $(3,7 \%)$ in $\leq 90 \mathrm{~d}$ versus $1(1 \%)$ in $>90 \mathrm{~d}$, without statistical differences. No newborn mortality. Complications: $70 \%$ in $\leq 90 \mathrm{~d}$ versus $43 \%$ in $>90 \mathrm{~d}(\mathrm{p}=0,01)$. Type of complications, Table 3 .

Follow up (years): $3,9 \pm 2,5$ in $\leq 90 \mathrm{~d}$ versus $3,7 \pm 2,7$ in $>90 \mathrm{~d}$. No differences in late mortality: $1(3,8 \%)$ in $\leq 90 \mathrm{~d}$ versus $0 \%>90 \mathrm{~d}$, or need of redo during follow-up: $1(3,8 \%)$ in $\leq 90 \mathrm{~d}$ versus $8(7,8 \%)$ in $>90 \mathrm{~d}$. Conclusions: In our experience, CR in FS before and after $90 \mathrm{~d}$ (newborn included) has no differences regarding inhospital mortality; however it does in intubation times, ICU days and hospital stay, as well as complications. There are no differences in mortality or need of redo during follow-up. We will postpone elective CR until $>90$ d, keeping early CR for symptomatic patients.

MP3-14

Evaluation of the different therapeutic options in patients with congenitally corrected transposition of the great arteries

Dewals W.(1), Dessy H.(2), Demanet H.(2), De Wolf D. (3), François K. (3), Benatar A. (1)

(1) Universitair Ziekenhuis Brussel, Brussels, Belgium, (2) Hôpital

Universitaire des Enfants Reine Fabiola, Brussels, Belgium, (3)

Universitair Ziekenhuis Gent, Gent, Belgium.

Objective: The surgical approach for treatment of congenitally corrected transposition of the great arteries (ccTGA) remains challenging, though ideally one would favor 'anatomical' repair. 
We aimed to compare the outcome of different treatment strategies.

Subjects and methods: We retrospectively collected data from two Belgian surgical centers providing 47 patients $(23$ males). Type of operation, demographic and follow-up data was recorded.

Results: Median age at latest follow-up (FU) was 16 years (y) (0-26). Four patients were lost after a mean FU of $14 y$. One early death occurred related to prematurity. One patient died unexpectedly at the age of 2 months (m). Six patients with no associated defects did not undergo surgery. At a median FU age of $18 \mathrm{y}$ there was no failure of the systemic ventricle and no significant tricuspid regurgitation (TR).

Surgical patients were divided in two groups: univentricular repair (group I) versus biventricular repair (group II). The latter group was subdivided into group IIa in whom the morphologically left ventricle was restored in the systemic circulation and group IIb who underwent physiologic repair.

Group I comprised 13 patients (mean FU 123m). There was no surgical related mortality. One late mortality due to acute ventricular failure was reported.

Group IIa comprised 16 patients (4 atrial/arterial switch, 1 hemiMustard/bidirectional Glenn/Rastelli, 11 atrial switch/Rastelli) with mean FU of $127 \mathrm{~m}$. Group IIb comprised 7 patients (mean FU 220m). Postoperative mortality was $25 \%$ in group IIa versus $0 \%$ in group IIb. There was no late mortality in group IIa whilst 2 patients of group IIb required heart transplantation and one patient had TR 3/4. Re-intervention rate was similar in both groups (respectively $31 \%$ and $28 \%$ ). In each group, 2 patients had mechanical valves. Two patients of group IIa had an atrial flutter. 13 patients required pacemaker implantation $(5 / 7$ in group IIb and $6 / 16$ in group IIa). Overall in half of the patients, pacemaker implantation was intervention related.

Conclusion: At a median FU age of 16y, outcome was not superior in patients who underwent anatomic repair.

\section{MP3-15}

Atrial Septostomy in Children with Primary Pulmonary Hypertension

Degano Iglesias L. A., Yevenes Ruiz S., Manchola Linero A., Betrian Blasco P., Gran Ipiña F., Torrent Vernetta A., Sabaté Rotés A., Abella R., Moreno Galdo A., Albert Brotons D.C.

University Children 's Hospital Vall d'Hebron, Barcelona

Introduction: Atrial septostomy (AS) is one of the therapeutic strategies for the treatment of severe pulmonary hypertension $(\mathrm{PH})$ and right ventricular failure (VD) in children in whom medical therapy has failed. It has been developed as an alternative/ bridge treatment to lung transplantation. In the following study, we describe our experience with emphasis on the safety of the procedure and the improvement of symptoms.

Material and methods: Descriptive, retrospective study based on the review of the medical records of all children affected by HP with AS controlled in our center.

Results: AS was performed in 11 children (54.5\% males). The average age was 4 years ( 4 months to 11 years). The time between diagnosis of HP and AS was on average 20.4 months, $40 \%$ had syncope and worsening of clinical status, rest of cases had RV dysfunction despite the optimized pulmonary vasodilator therapy (VDP).

AS percutaneous was performed in 10 ( 8 using the Diabolo Fenestrated Stent Technique for mounting a Stent; 1 by Amplatzer atrial septal occluder with self-made fenestration, and 1 with exclusive balloon dilation) and 1 was surgical. All were guided by transesophageal echocardiography. AS size was selected based on SatHb (over 90\%). 2 patients had HP crises requiring treatment increased VDP.

There was no peri-procedure mortality. After the AS 5 children required mechanical ventilation and 1 ECMO. At follow-up, 2 children died due to progression of HP (other associated complications) within 6 months after the AS; 2 were transplanted, one at 4 months by progressive hypoxia and the other at 2 years. Of the remaining 7 after a median follow-up time of 32 months they are stable. Syncope disappeared and improvements in RV function have been shown using echocardiography.

Conclusion: AS in children with $\mathrm{HP}$ is a safe procedure with low morbidity and mortality that allows a high percentage of cases to improve their clinical situation and delay lung transplantation.

\author{
MP3-16 \\ Hybrid palliation for hypoplastic left heart syndrome and \\ variants: a single center 5-year experience \\ Manuri L.(1), Oreto L. (2), Saitta M. (1), Iannace E.(1), Iorio F.S.(1), \\ Guccione P. (2), Agati S. (1) \\ (1) Pediatric Cardiology Mediterranean Center-OPBG, Taormina, Italy, \\ (2) Bambino Gesù Children's Hospital, Rome, Italy
}

Background: Hybrid palliation is an alternative to Norwood stage I for the initial management of hypoplastic left heart syndrome (HLHS). We aimed to report our experience with hybrid strategy in HLHS over 5 years in a tertiary care Institution.

Methods and Results: Forty- eight consecutive patients with HLHS or variants underwent hybrid palliation at a mean age of 2.7 days and mean weight of $3.08 \mathrm{Kg}$. Balloon atrioseptostomy was required in 5 patients and surgical septectomy in 2 cases. In-hospital death was $11 \%$ (5 patients, including 2 preterms and 1 congenital atrioventricular block). Three high-risk patients were diverted to rescue Norwood operation between 37 and 60 days, with 2 early deaths. Interstage mortality was 11\% (5 patients, including 1 preterm and 2 intracranial hemorrhage). Fifteen patients required interventional procedures during interstage (mean age 3 months), such as atrial septal dilatation ( 3 cases) or stenting ( 7 cases), ductal stent dilatation (9 cases) or restenting ( 2 cases). Three patients underwent stage II operation in other Institutions. At a mean age of 6 months, 17 patients had comprehensive Norwood stage I-II, whereas 15 received biventricular repair. Survival after surgical stage II was $79 \%$ and 93\%, respectively. All 11 patients after comprehensive I-II and $4 / 13$ after biventricular repair required interventional procedures for pulmonary branches stenosis, either balloon dilatation (16 procedures) or stenting (7 procedures). Six patients underwent Fontan operation and 4 are currently awaiting. Overall survival is $59 \%$.

Conclusions: In our experience hybrid palliation has been the treatment of choice for HLHS and variants, unless contraindicated by the presence of aortic reverse coarctation or excessive ductal diameter.

Despite the tendency described by the Society of Thoracic Surgeons Congenital Heart Surgery 2015 Database, that institutions with higher hybrid use are expected to have low annual HLHS case volume (<5/year), our average case volume is almost double (9/year). Besides, our in-hospital mortality lies below that reported by STS database. Moreover, hybrid approach resulted in a successful bridge to biventricular repair in as much as 7 patients with hypoplastic mitral and aortic valves, who would have not been eligible for biventricular repair at birth. 
MP3-17

Influence of shear stress on Staphylococcus aureus adhesion to endothelial cells mediated by Fibronectinbinding protein $A$ and Clumping factor $A$ in an in vitro model of endovascular infections

Claes J. (1), Veloso T.R. (1), Mancini S.(2), Entenza J.M.(2), Moreillon P.(2), Hoylaerts M.F.(1), Heying R. (1)

KU Leuven, Leuven, Belgium (1); University of Lausanne, Lausanne, Switzerland (2)

Introduction: Staphylococcus aureus (S. aureus) is a pathogen most frequently associated with endovascular infections such as infective endocarditis. In order to cause endovascular infections, S. aureus needs mechanisms to adhere to endothelial cells lining the vessel wall and subendothelial matrix. Various staphylococcal surface proteins have been described to mediate adhesion to host cells and to extracellular matrix proteins, e.g. fibronectin (Fn)-binding protein A (FnBPA) and clumping factor A (ClfA). However, most of these interactions are studied in static conditions, and may not be representative for endovascular infections in the human body, where $\mathrm{S}$. aureus initial adhesion to the vessel wall occurs in flowing blood. Lactococcus lactis (L. lactis) are poorly pathogenic bacteria known to lack adhesion molecules for human matrix proteins. L. lactis expressing single staphylococcal surface proteins have been described before to study the contribution of single staphylococcal surface proteins in S. aureus infective endocarditis. In the present study we aimed to investigate how ClfA, FnBPA and FnBPA subdomains (A, A $+16, A B C, C D)$ contribute to bacterial adhesion to endothelial cells and subendothelial matrix in flow conditions.

Methods: We investigated adhesion of L. lactis expressing ClfA, FnBPA or FnBPA subdomains to Fn, Fg, intact and damaged endothelial cells and fibrin under shear stress. Fluorescently labelled L. lactis were perfused over a surface coated with Fn, Fg endothelial cells or fibrin in a micro-parallel flow chamber.

Results: We observed that the adhesion to Fn involved the CD domain and the A + 16 domain of FnBPA not only in static conditions, but also under shear stress. Adhesion to Fg was facilitated by ClfA and the A16 + domain. The adhesion to intact endothelial cells was mediated by $\mathrm{Fn}$ and when $\mathrm{Fg}$ was added to the bacterial perfusate, adhesion of ClfA and the A16+ domain increased.

Conclusion: Our data show that the adhesion of S. aureus to endothelial cells via FnBPA is primarily mediated by Fn under shear stress. Further studies will focus on concomitant infections with the specific L. lactis strains to allow to rank the importance of $\mathrm{Fn}$ and $\mathrm{Fg}$ mediated S. aureus adhesion in bacterial recruitment to the vessel wall.

\section{MP3-18}

Lower ministernotomy, a safe alternative for the repair of congenital heart diseases

Garcia-Vieites M.(1), Lozano-Balseiro M.(2), Rueda-Nuñez F.(2),

Portela-Torron F.(1), Cuenca-Castillo J.J.(1), Bautista-Hernandez V. (1).

(1) Complejo Hospitalario Universitario A Coruña. Cardiovascular

Surgery. A Coruña. Spain. (2) Complejo Hospitalario Universitario A

Coruña. Pediatric Cardiology. A Coruña. Spain.

Introduction: Lower ministernotomy represents a minimally invasive surgical technique which has been utilized for the repair of a wide variety of congenital heart defects with excellent surgical and cosmetic outcomes. The aim of our study is to report our results with lower ministernotomy for the repair of congenital heart diseases.
Methods: From January 2010 through November 2016, 156 consecutive congenital patients (122 pediatric $(78,2 \%)$ ) underwent lower ministernotomy approach at our center. We analyzed inhospital and follow-up outcomes.

Results: Median age at the time of surgery was 3,42 years (range from 3 months to 79 years). 122 patients $(78,2 \%)$ were pediatric and 92 female $(58,97 \%)$. Operative techniques were atrial septal defect closure $(n=93(59,61 \%)$, atrial septal defect closure with tricuspid annuloplasty $(n=6(3.84 \%))$, sinus venosus septal defect repair $(n=5(3,2 \%))$ ventricular septal defect repair $(n=37$ $(23,71 \%))$, and atrioventricular canal repair $(n=14(8,97 \%))$. There were no deaths or major in-hospital complications. Cardiopulmonary bypass and cross clamp times were 57,14 $\pm 19,57$ and $32,10 \pm 13,92$, respectively. 149 patients were extubated in the first eight hours $(95,51 \%) .3$ patients required conversion to full median sternotomy (1,92\%), 2 adults and one pediatric patient, both were successfully corrected and discharged home without any complications. There was no AV block in our study. For a medium follow up of 13 months (range from 1 month to 64 months) there were no deaths, reinterventions or reoperations and no significant significant residual defects were found.

Conclusions: At our center lower ministernotomy represents a safe alternative for the repair of congenital heart defects in pediatric and adult populations. Lower ministernotomy should become the technique of choice for these populations.

\begin{tabular}{lcc}
\hline & $\begin{array}{l}\text { Paediatric } \\
\text { population } \\
\text { N= 122 }\end{array}$ & $\begin{array}{l}\text { Adult } \\
\text { population } \\
\text { N=34 }\end{array}$ \\
\hline Age & $3,75 \pm 3,73$ & $45,20 \pm 14,32$ \\
Patology & $71(58,19 \%)$ & $22(64,7 \%)$ \\
$\quad$ OS ASD & 0 & $6(17,6 \%)$ \\
OS ASD + TR & $35(28,68 \%)$ & $2(5,8 \%)$ \\
VSD & $12(9,8 \%)$ & $2(5,8 \%)$ \\
AVC & $4(3,27 \%)$ & $1(3,1 \%)$ \\
SV ASD & $117(95,9 \%)$ & $32(94,11 \%)$ \\
Early extubation & $1(0,8 \%)$ & $2(5,8 \%)$ \\
Conversion to full & $5(4,1 \%)$ & $1(2,9 \%)$ \\
sternotomy & $2(1,6 \%)$ & 0 \\
Pleural effusion & 0 & 0 \\
Pericardial effusion & $1(0,8 \%)$ & $9(26,47 \%)$ \\
AV block & $2 \pm 1,19$ & $3 \pm 4,19$ \\
Postoperative AF & $3,25 \pm 1,51$ & $4,14 \pm 1,90$ \\
Media ICU stay (days) & & \\
Media postoperative in- & & \\
hospital stay & &
\end{tabular}

Pre and postoperative data divided into adult and paediatric population.

MP3-19

Glial Fibrillary Acid Protein - a potential biomarker for cerebral injury in neonates and infants undergoing surgery for congenital heart disease

Hansen J.H. (1), Kissner L. (1), Chitadze G. (2), Logoteta J. (1), Jung O. (1), Dütschke P. (3), Scheewe J. (4), Kramer H.-H. (1,5) Department of Congenital Heart Disease and Pediatric Cardiology, University Hospital of Schleswig-Holstein, Campus Kiel, Kiel, Germany (1); Institute of Immunology, University Hospital of Schleswig-Holstein, Campus Kiel, Kiel, Germany (2); Department of Anesthesiology and Intensive Care Medicine, University Hospital of Schleswig-Holstein, Campus Kiel, Kiel, Germany (3); Department of Cardiovascular Surgery, University Hospital of Schleswig-Holstein, Campus Kiel, Kiel, 
Germany (4); DZHK (German Center for Cardiovascular Research), partner site Hamburg/Kiel/Lübeck (5)

Objectives: Mild to moderate neurodevelopmental impairment is common in children with congenital heart disease requiring cardiac surgery as neonates or infants. Perioperative hypoxicischemic brain injury might contribute to adverse neurodevelopment. Monitoring techniques and biomarkers for perioperative brain injury are of special interest. We evaluated Glial Fibrillary Acid Protein (GFAP), an astrocyte cytoskeleton protein, as a biomarker for neuronal cell damage in neonates and infants ( $<1$ year) undergoing cardiac surgery. The relation between GFAP levels and perioperative cerebral tissue oxygenation was evaluated.

Methods: Serum levels of GFAP were measured before and 0, 12, 24 and 48 hours after surgery (Human GFAP ELISA, Abbexa ${ }^{\circledR}$ ). Cerebral tissue oxygenation $(\mathrm{ScO} 2)$ was derived by near infrared spectroscopy (INVOS, Covidien ${ }^{\circledR}$ ) for 12 hours before, during and for 48 hours after surgery. Cerebral oxygen extraction was estimated by calculating the difference between arterial and cerebral tissue oxygen saturation $(\Delta \mathrm{SaO} 2 \mathrm{ScO} 2)$. Normal GFAP levels were determined based on preoperative values. GFAP levels above the 90th percentile were defined as elevated.

Results: GFAP levels and $\mathrm{ScO} 2$ were obtained in 22 neonates and 30 infants. Preoperative GFAP was not different between groups. Among postoperative measurements, only median GFAP at 24 hours was higher in neonates $(2.48 \mu \mathrm{g} / \mathrm{l}$ [IQR: $1.43-5.26]$ vs. 1.48 [IQR: 0.51-3.45]). Elevated GFAP levels between 24 and 48 hours were found in $8(36 \%)$ neonates and $5(17 \%)$ infants $(\mathrm{p}=0.121)$.

In neonates, median age at surgery was higher in cases with elevated compared to normal postoperative GFAP (6 [4-7] vs. 3 [2-4] days, $p=0.006)$. In neonates with elevated GFAP, mean intraoperative $\mathrm{ScO} 2$ was lower compared to cases with normal GFAP $(68 \pm 8 \%$ vs. $79 \pm 7 \%, p=0.004)$. Early postoperative and mean $\Delta \mathrm{SaO} 2 \mathrm{ScO} 2$ of the first 12 postoperative hours were higher $(41 \pm 9 \%$ vs. $30 \pm 10 \%, \mathrm{p}=0.016$ and $34 \pm 9 \%$ vs. $25 \pm 8 \%$, $\mathrm{p}=0.032)$.

In infants, mean $\mathrm{ScO} 2$ of the entire postoperative course was lower in cases with elevated GFAP ( $61 \pm 9 \%$ vs. $69 \pm 8 \%, p=0.047)$.

Conclusion: GFAP might be a useful biomarker for subclinical brain injury in neonates and infants undergoing cardiac surgery. Neurodevelopmental outcomes and the association to GFAP levels and cerebral tissue oxygenation still need to be determined.

\section{MP3-20}

\section{Creation of the Fontan circulation in sheep: A survival} model

Van Puyvelde J., Rega F., Minami T., Claus P., Cools B., Gewillig M., Meyns B.

University Hospitals Leuven, Leuven, Belgium

Introduction: Patients with a single-ventricle survive thanks to the Fontan palliation. Nevertheless, there is a growing number of Fontan patients with progressive heart failure. To validate therapeutic options in these patients we developed a chronic Fontan large animal model.

Methods: A Fontan circulation was surgically created in 26 sheep $(63.9+/-6.2 \mathrm{~kg})$ without the use of cardiopulmonary bypass. The superior vena cava was anastomosed end-to-side to the pulmonary artery. The inferior vena cava was connected to the pulmonary artery by an ePTFE conduit and the inferior vena cava-right atrium junction was ligated (Fig. 1). In that way, all venous return, except coronary sinus blood flow, was drained passively to the pulmonary circulation. Heart rate, arterial blood pressure, central venous pressure and cardiac output $(\mathrm{CO})$ were recorded at the start of the surgery and after Fontan completion. Animals were followed for 21 weeks. A postoperative cardiac MRI was performed at 2 weeks in a small subgroup of animals $(n=3)$.

Results: Total cavopulmonary connection was successfully performed in all 26 animals. Eleven animals (42\%) died in the first 24 hours postoperatively. After creation of the Fontan circulation, central venous pressure increased significantly $(4.7 \pm 2.9$ vs. $14.9 \pm 2.5 \mathrm{~mm} \mathrm{Hg}, \mathrm{p}<0.001)$, mean arterial blood pressure decreased significantly $(66.4 \pm 14.9$ vs. $56.4 \pm 13.5 \mathrm{~mm} \mathrm{Hg}$, $\mathrm{p}<0.001)$ and cardiac output decreased significantly $(5.05 \pm 2$ vs. $1.7 \pm 1 \mathrm{~L} / \mathrm{min}, \mathrm{p}<0.001)$. Right ventricular output was significantly lower than total cardiac output $(0.34 \pm 0.24$ vs. $4.64 \pm 2.06 \mathrm{~L} / \mathrm{min}, \mathrm{p}<0.01)$ in the subgroup of animals that underwent a cardiac MRI. Four animals completed the 21 weeks follow-up period.

Conclusions: This study demonstrates that it is feasible to create a chronic animal model of the Fontan circulation. This animal model not only could facilitate future studies on the pathophysiology of the failing Fontan circulation but might also play a crucial role in the development and the study of advanced therapeutic approaches, like cavopulmonary assist devices, to treat patients with a failing Fontan circulation.

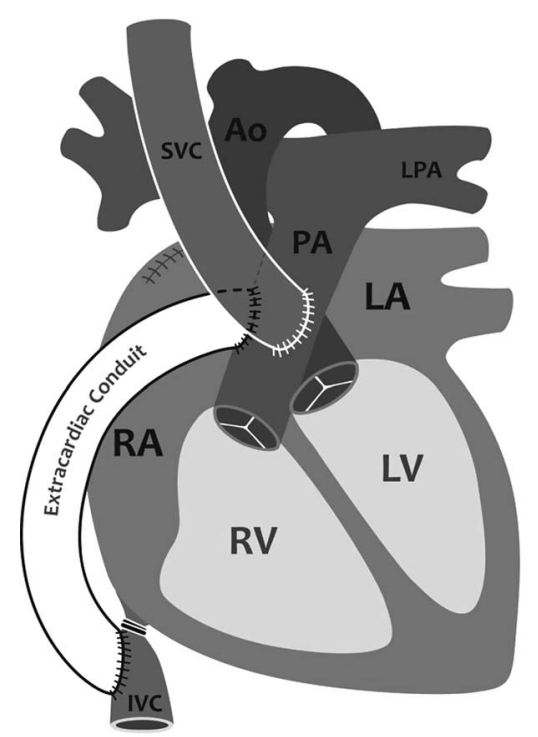

Figure 1.

Schematic illustration of the experimental technique. SVC: Superior vena cava, IVC: Inferior vena cava, Ao: Aorta, PA: Main pulmonary artery, LPA: Left pulmonary artery, LA: Left atrium, RA: Right atrium, $L V$ : Left ventricle, $R V$ : Right ventricle.

\section{MP4-1}

\section{Cracking of coronary stents}

Tanase D., Gendera K., Georgiev S., Genz T., Eicken A., Ewert P. German Heart Centre Munich, Germany

Because of their low-profile design, premounted stents can be delivered via smaller sheaths than manually crimped stents, allowing stent treatment in smaller patients. Off-label use of premounted coronary stents offers just a temporally benefit because they are only dilatable to small diameters, leading to a mismatch between maximal stent diameter and natural vessel growth. 
Therefore they are generally avoided in pediatric patients with only anecdotal reports in life threatening situations when surgery in not available. However, even if the total patient number is low, there are some children with implanted coronary stents needing further treatment options.

Methods and results: In a bench test a Coroflex Blue $4 \times 13 \mathrm{~mm}$ - and a Medtronic Integrity 4 x 9mm stent were inflated with ultra- high pressure balloons. Consecutive inflation of both stents with a Conquest $6 \mathrm{~mm}$ and $8 \mathrm{~mm}$ balloon with 40 atmospheres (rated burst pressure $40 \mathrm{~atm})$, cracked the stents.

The same inflation protocols were applied in a patient with early chylous effusions after total cavopulmonal anastomosis (TCPC). The patient's left pulmonary artery (LPA) had formerly been stented with a Medtronic Integrity Stent $4 \times 9 \mathrm{~mm}$, which could not be removed surgically during the TCPC operation. By cracking the coronary stent, the LPA stenosis was treated successfully.

Conclusion: Coronary stents are no longer a one way treating option in children with congenital heart disease. They may be cracked with recently available ultra-high pressure balloons opening new possibilities to treat stenosis even in the newborn period. Care needs to be taken to increase the balloon size diameters by small increments $(2 \mathrm{~mm})$ to avoid severe stent length shortening.

\section{MP4-2}

Percutaneous Closure of Arterial Duct with Amplatzer Duct Occluder II Additional Sizes in a high-volume tertiary referral centre

Santoro G., Mahmoud H.T., Gaio G., Giordano M., Palladino M.T., Iacono C., Di Nardo G., Esposito R., Argiento P., Fratta F., Morelli C., Ricci C., Russo M.G.

Paediatric Cardiology, A.O.R.N. "Ospedali dei Colli", 2nd University of Naples, Naples, ITALY

Introduction: Transcatheter closure of $\mathrm{AD}$ remains challenging in low body weight patients and those who have a persisting shunt following a previous attempt at interventional closure. Recent technical advances in device design may address these issues. This study aimed to report a large, single-center experience of percutaneous arterial duct (AD) closure using Amplatzer Duct Occluder II Additional Sizes device (ADO II-AS)(St. Jude Medical Corp, St. Paul, MN, USA).

Methods: From May 2011 to October 2016, 113 patients underwent attempted percutaneous closure of AD with ADO II-AS at our Institution. Mean age and weight were $4.8 \pm 8.1$ years (range $0-48$ ) and $21.4 \pm 20.6 \mathrm{~kg}$ (range 3-93), respectively. Fifteen patients $(11.5 \%$ ) were $\leq 6 \mathrm{~kg}$ (age $3.5 \pm 2.0$ months; weight $4.7 \pm 1.1 \mathrm{~kg}$ ). Arterial duct morphology was type A in $65(57.5 \%)$, type $\mathrm{B}$ in $1(1 \%)$, type $\mathrm{C}$ in $33(29.2 \%)$, type $\mathrm{D}$ in $7(6.1 \%)$ and type $\mathrm{E}$ in 6 patients $(5.3 \%)$, respectively. Arterial approach was used to negotiate and deploy the occluding device in 103 patients (91.2\%).

Results: $\mathrm{AD}$ diameter was $2.2 \pm 0.6$ (range 1.5-4.5) resulting in $\mathrm{QP} / \mathrm{QS}$ of $1.9 \pm 0.7$ (range 1-3.3). Mean pulmonary artery pressure and $\mathrm{PA} /$ aortic pressure ratio were $19.3 \pm 5.0 \mathrm{mmHg}$ (range $12-38$ ) and $0.34 \pm 0.14$ (range 0.14-0.95), respectively. Successful device deployment was achieved in 110 patients (97.3\%). Neither procedural morbidity nor mortality was recorded. Immediate, $24 \mathrm{~h}$ and mid-term (30 \pm 17 months) complete occlusion was recorded in $71 \%, 98.1 \%$, and $100 \%$ of patients, respectively.

Conclusion: In our experience, trans-catheter closure of AD of different sizes and morphologies using ADO II-AS is highly feasible, safe and effective also in challenging anatomic/clinical settings.
MP4-3

Antenatal referral for hospice care: A New approach to pregnancy birth and beyond

Barwick S., Wray M.T.

Leeds Teaching Hospitals NHS Trust, Leeds, U.K.

Paediatric and specifically neonatal palliative care has changed significantly over the past few years and is now integrated into the care of children and their families. This approach can begin before birth with palliative care for the family of a fetus with major abnormalities including Congenital Heart Disease (CHD).

When the diagnosis is made antenatally of severe CHD with a single ventricle circulation, several options for the antenatal and postnatal management are discussed. These include continuing the pregnancy and attempting to undertake palliative surgery, undergoing termination or opting for comfort care after delivery. Support from the hospice is discussed briefly at the time of diagnosis and at the follow up appointment, if the family wish, written information is given and a referral is made. It is stressed that referral to the hospice is primarily for support and does not commit the family to following the comfort care pathway. An initial visit to the hospice is arranged or a home visit if this better suits the family circumstances. A member of the hospice staff is the available to attend subsequent hospital appointments for support. They also provide regular counselling for parents, siblings and grandparents. In the Yorkshire and Humber Fetal Cardiology department 54 antenatal diagnoses were made of a single ventricle anatomy between October 2015 and September 2016. Of these 20 were on-going within our service (29 TOP, 3 IUD and 2 chose referral to another centre). 8 declined hospice referral but $60 \%$ of the ongoing pregnancies received antenatal and postnatal support.

If comfort care is chosen postnatally, redirection of care involving the hospice is seamless and if palliative surgery is chosen then respite care is offered.

\section{MP4-4 \\ Prophylactic endocardial lead extraction in pediatric patient}

Maltret A. (1), Horvilleur J. (2)

Centre de référence "Malformations cardiaques Congénitales Complexes$M 3 C$ ", Université Paris-V, Necker-Enfants Malades, AP-HP, Paris, FRANCE (1); Institut Cardiovasculaire Paris Sud, Hopital Privé Jacques Cartier, Massy, FRANCE (2)

Introduction: The rate of failing endocardial leads in the pediatric population is high (18\% to $30 \%)$, and abandoned endocardial lead has its own burden. In another hand, safety of leads extraction in pediatric patients with or without CHD have been reported. Because of those "pediatric" specificities, our institution decided to systematically consider prophylactic extraction of endocardial leads in pediatric patients at device replacement time, or during follow up if lead function trouble appeared. We report here the results of this management.

Patients and Methods: Between July 2011 and October 2016, lead extraction was considered for all patient with a transvenous pacemaker with either dysfunctional lead or growth related stretching of the lead on the chest X-rays. All extraction indications were Class IIb, none extraction was performed because active infection. In 5 years, 24 patients underwent 25 extraction procedures of 27 pacing leads. At implantation, mean patient age was 6 years old (range 2 to 9.5 years), mean weight was $23.6 \mathrm{kgs}$ (range 9.9 to 25) and mean height $123 \mathrm{~cm}$ (range 80 to 127). Mean duration of lead implantation was 7.9 years.

Results: Complete lead extraction was achieved in 16 procedures (64\%), for 5 patients the very distal tip of the lead remained 
included in the myocardium (20\%). So, clinical success reaches $84 \%$. Extraction procedure was aborted for 4 patients (16\%) because of intense fibrotic binding site along the vascular access or within the heart making the risk of those extraction overweighting the expected benefit.

Statistically significant risk factor of complete lead extraction failure were age of implanted lead over 8 years and vicious reserve loop (fig 1).

No major complication occurred.

Conclusion: Transvenous pediatric pacing systems are under great stress du to linear growing and active carriers. Reserve loop which are supposed to allow patient's growth can also jeopardized the extraction feasibility by hinder the stylet placement. Prophylactic lead extraction should be considered before or at the time of battery depletion, as the older the lead, the more hazardous the extraction procedure gets.

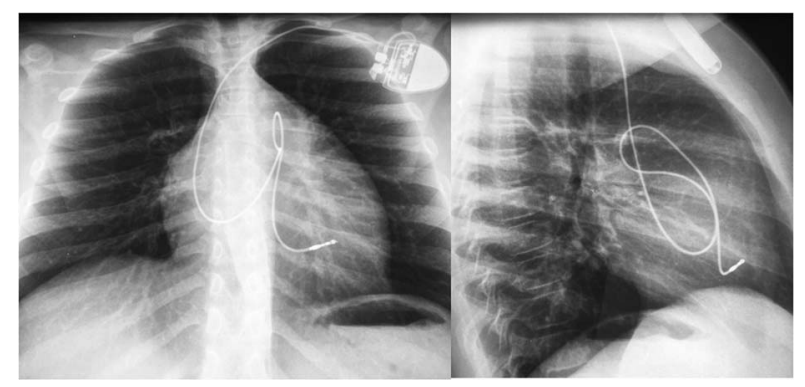

Figure 1.

exemple of vicious reserve loop.

\section{MP4-5}

Tissue Doppler Imaging in diagnosing fetal Long-QT syndrome: the development and validation of a novel diagnostic approach

Vink A.S. (1,2), Kuipers I.M.(2), De Bruin-Bon H.A.C.M.(1), Wilde A.A.M.(1), Blom N.A.(2,3), Clur S.A.B.(2)

Department of Cardiology, Heart Centre, Academic Medical Centre, Amsterdam, The Netherlands (1); Department of Pediatric Cardiology, Emma Children's Hospital, Academic Medical Centre, Amsterdam, The Netherlands (2); Department of Pediatric Cardiology, Willem-Alexander Children's Hospital, University Medical Centre Leiden, Leiden, The Netherlands (3)

Introduction: A definite diagnosis of Long-QT syndrome (LQTS) in a fetus is impossible without invasive DNA analysis, due to the unavailability of a fetal ECG. We aimed to develop and validate a novel diagnostic approach in children using color Tissue Doppler Imaging (cTDI) in order to provide a potential marker for the prenatal diagnosis of LQTS.

Methods: Twenty-four healthy children were age- and gendermatched to LQTS patients with a confirmed pathogenic mutation. A 12-lead ECG and myocardial contraction duration (CD) on cTDI recordings were cross-sectionally obtained. Validation of the $\mathrm{CD}$ was done by comparing the parameter to a previously defined cTDI measurement in adult patients. Inter-method and intraobserver variability was presented in intraclass-correlation coefficients (ICC). Receiver-operating characteristic (ROC) analysis was done and the optimal cut-off value for $\mathrm{CD}$ was determined. Feasibility was tested in a pilot study among fetuses.

Results: LQTS children had a longer CD compared to controls $(\mathrm{p}=0.008)$, while there was no statistical difference in heart rate $(p=0.145)$. CD had a high reproducibility $(\mathrm{ICC}=0.94)$, and reliably associates with the QT-interval $(\mathrm{ICC}=0.75)$ and the previously defined cTDI measure $(\mathrm{ICC}=0.86$ ). The area under the curve for CD was 0.71 , and an optimal cut-off value of $422 \mathrm{~ms}$ showed a $54 \%$ sensitivity and a $83 \%$ specificity in diagnosing LQTS. The measurement of the CD was feasible in fetuses. Conclusion: Myocardial CD assessed by cTDI was prolonged in LQTS children, and correlated reliably with the QT-interval. Measuring the CD in fetuses seems feasible, and has the potential for diagnosing fetal LQTS. Examples of the CD measurement in fetal twins are shown in the figure.

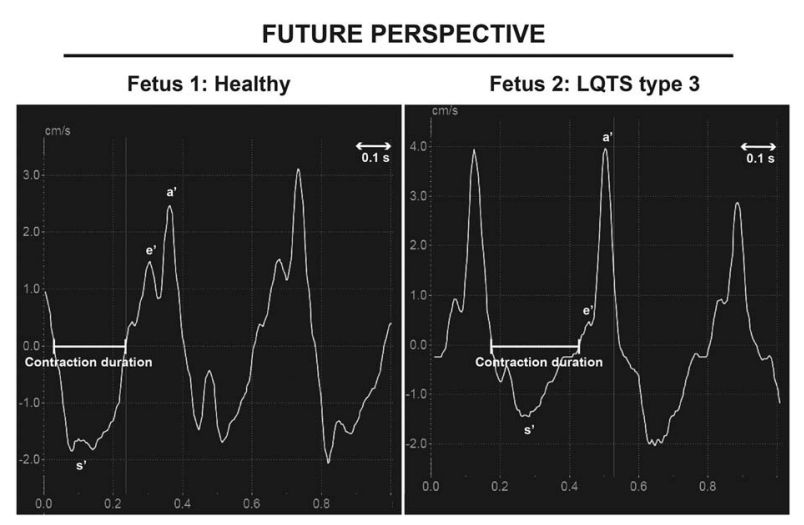

MP4-6

Radiation doses from paediatric interventional cardiology in France: a multicentre study

Baysson H., Dreuil S., De Zordo-Banliat F., Bernier M.O.

Institute for Radiology and Nuclear Safety (IRSN), Fontenay-auxRoses, France

Objectives: Children with congenital heart disease frequently undergo interventional cardiology procedures (ICP) for diagnostic or therapeutic purposes. Despite the clear clinical benefit to the patient, the complexity of these procedures may result in high cumulative radiation exposure. This issue is particularly relevant for children given their greater sensitivity to radiation and the longer life span during which radiation health effects can occur. In France, an epidemiological cohort study, named Coccinelle (French acronym for «Ladybird»), specifically designed to provide further knowledge on the potential cancer risk associated with paediatric ICP is currently carried out. In the framework of this study, some comprehensive information has been obtained on typical levels of radiation doses for ICP in France.

Methods: In this analysis, all children who have undergone at least one ICP before age 16 and over the period 2009-2013 are included from five major French paediatric cardiology hospitals. For each ICP, air-kerma area product (PKA) and fluoroscopy time (FT) are retrieved retrospectively when available. The median, first and third quartiles, minimum and maximum values of PKA and FT are calculated.

Results: Over 4,000 ICP have been analysed. The main ICP categories investigated were: Diagnostic, Patent Ductus Arteriosus (PDA) closure, Atrial Septal Defects (ASD) closure, Valvuloplasty and Angioplasty. Results will be presented for each ICP category, according to patients' age and weight. They will be discussed in light of available literature and published data.

Conclusion: The justification as well as the benefit-risk ratio of ICP is well established. However, the increasing use of ICP in paediatric population stresses the need of setting up reference levels and keeping doses to children as low as possible. 
MP4-7

The Amplatzer Duct Occluder II Additional Sizes device for transcatheter PDA closure in preterms infants:

Monocentric experience

Chalard A.(1), Lang M. (2), Cosserant B. (3), Rusé F. (3), Lusson J.R.

(1), Boeuf B.(2), Dauphin C. (1)

(1) Cardiologie, CHU Clermont-Ferrand, (2) Reanimation pédiatrique et néonatale, CHU Clermont-Ferrand, (3) Réanimation chirurgie cardiovasculaire, CHU Clermont-Ferrand, France

Introduction: Patent ductus arteriosus (PDA) in preterm infants continues to be a significant clinical problem contributing importantly to both morbidity and mortality. We reports a new technique for transcatheter PDA closure in extremely preterm infants using new commercially available technology.

Purpose: To present our initial experience with the Amplatzer Duct Occluder II Additional Sizes (ADOIIAS) device.

Methods: Hospital records and catheterization reports of all premature neonates ( $<32$ weeks gestation) who underwent transcatheter PDA closure between March 2015 and March 2016 were reviewed. After only venous femoral punction, the PDA was closed using a 4Fr delivery system from the pulmonary side. Echocardiography and fluoroscopy were performed for the guidance of ductal closure. Contrast angiography was not used in any patient.

Results: 7 premature infants born at gestational ages ranging between 25 and 30 weeks (median 27 weeks) underwent PDA closure. Median age was 18 days [ 10 - 32 days], and median weight was $1203 \mathrm{~g}$ [860 g - $1400 \mathrm{~g}$ ] ADOIIAS devices were successfully deployed in all cases. Complete closure was achieved in all patients. Median fluoroscopy time was $5,79 \mathrm{mn}(4,1-8,31)$. Median total dose-area product was $16.78 \mu \mathrm{Gy} / \mathrm{m}^{2}$ (4.7-39). We doesn't have major procedural complications. There were no instances of device migration, residual PDA, left pulmonary artery coarctation or aortic coarctation at mid term outcomes. All patients were alive at the time of this report.

Conclusion: We demonstrates that transcatheter PDA closure can be successfully performed in extremely preterm neonates using the new ADOIIAS device with a high success rate and low incidence of complications. This report also describes a novel transvenous approach using a combination of echocardiography and judicious use of fluoroscopy to avoid arterial access in this fragile patient.

\section{MP4-8}

Fetal versus postnatal echocardiogram accuracy in the diagnosis of interrupted aortic arch Larios G., Jaeggi E., Yoo S-J., Dutil N., Thakur V. The Hospital for Sick Children, Division of Cardiology. University of Toronto

Introduction: Accurate prenatal diagnosis of interrupted aortic arch (IAA) and type allows for comprehensive fetal and genetic counselling and postnatal management. Our study aims to evaluate the rate and accuracy of fetal (FE) versus postnatal (PNE) echocardiogram for IAA presence and type in patients with usual associated lesions (UAL), such as ventricular septal defects, or complex associated lesions (CAL, e.g. truncus arteriosus, TA).

Methods: Retrospective review of the FE and PNE in patients with suspected IAA was analysed, from 2004 to 2016. Patients were grouped as FE or PNE based on timing of initial suspicion of IAA. Final diagnosis of IAA and type was confirmed in the operating room, on axial images or autopsy. The presence and impact of UAL or CAL in the diagnosis was analysed.

Results: Of 96 subjects conforming the study group, 44 IAA were suspected on FE and 52 on PNE. Of the 44 subjects with FE, 11
(25\%) pregnancies were terminated, $4(9 \%)$ resulted in stillbirth and $7(16 \%)$ were misdiagnosed as IAA and were found to have coarctation of the aorta. Of the 52 found on PNE, 6 had a missed diagnosis on FE. All PNE patients had diagnosis confirmed. Of 73 confirmed IAA, there were 25 type A (34.2\%), 47 type B (64.4\%) and 1 type $\mathrm{C}(1.4 \%)$. FE detected 20/27 subjects and PNE 52/52 (Sensitivity $74.1 \%$ versus $100 \%, \mathrm{p}=0.0005)$. FE detected correctly the type of IAA in $11 / 20$ (55\%) and PNE in 48/52 (92.3\%), $\mathrm{p}=0.001$. A higher proportion of CAL was observed during FE, $18(41 \%)$ subjects versus $8(27 \%)$ patients in the only PNE group. The presence of CAL did not interfere with the performance of the tests $(\mathrm{p}=0.38)$.

Conclusions: Fetal diagnosis of IAA remains challenging with a lower detection rate and accuracy compared to PNE. Careful evaluation of 3 vessel tracheal and long axis views, especially when usual associated cardiac anomalies are found, is required to improve the accuracy of FE. Improved fetal diagnosis is important to allow women to have different options in pregnancy management and the option for genetic testing and counselling.

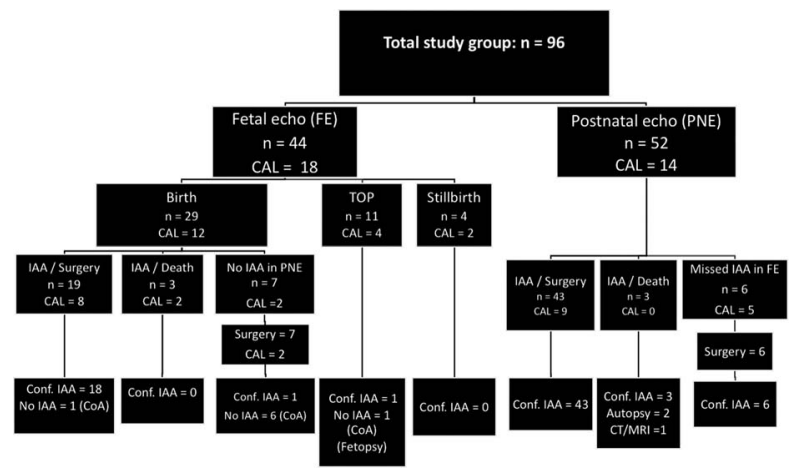

Figure 1.

Distribution of the subjects according to type of study and outcome. CAL: Complex associated lesions; CoA: Coartation of the aorta; Conf. IAA: Confirmed IAA (in the operating room, axial images or autopsy/fetopsy, or true positive); IAA: Interrupted aortic arch; FE: Fetal echocardiogram; PNE: Postnatal echocardiogram; NC: Not confirmed diagnosis; No IAA: Incorrectly diagnosed as IAA (false positive); TA: Truncus arteriosus; TOP: Termination of pregnancy; UAL: Usual associated lesions.

MP4-9

Cardiac Erosion after the Transcatheter Closure of Atrial Septal Defect Using Amplatzer Septal Occluder: Japanese Nationwide Experience

Akagi T. (1), Yazaki S. (2), Tomita H. (2), Hara H.(3), Takayama M. (3) Okayama University, Okayama, Japan (1), Japanese Society of Pediatric Interventional Cardiology (2) and Japanese Association of Cardiovascular Intervention and Therapeutics (3) registry

Introduction: Catheter closure of atrial septal defect (ASD) is an accepted highly successful alternative to surgical repair. In catheter closure of ASD using Amplatzer Septal Occluder, a cardiac erosion followed by a pericardial tamponade is an infrequent but potentially lethal complication and is difficult to speculate to occur especially before the device deployment. We retrospectively reviewed Japanese Interventional Pediatric Cardiology (JPIC) and Cardiovascular Intervention and Therapeutics (CVIT) database to clarify the incidence of cardiac erosion in Japan. These database includes all procedures performed, as well as all complications in Japan. 
Methods: Since August 2005 to December 2015, 7223 patients underwent transcatheter closure of ASD using Amplatzer Septal Occluder in Japan. Among this cohort, 13 patients (0.18\%) developed cardiac erosion. Erosion occurred from 1 day to 6 years after the procedure. Significant hemodynamic intolerance due to cardiac tamponade developed 2 patients. Three of 14 were developed erosion before the discharge hospital (within 3 days), rather remaining 10 patients developed after the discharge. Age at procedure ranged from 7 to 63 years, without specific high risk age. No procedure related death was observed in Japan. Individual information was indicated in table. In our data base, definite risk factor related cardiac erosion could not be clarified. Results:

\begin{tabular}{|c|c|c|c|c|}
\hline Subject & Site Reported Adverse Event Type & $\begin{array}{l}\text { Days from } \\
\text { Implant to } \\
\text { Event }\end{array}$ & $\begin{array}{c}\text { Age } \\
\text { (years) }\end{array}$ & $\begin{array}{c}\text { Device } \\
\text { Size }(\mathrm{mm})\end{array}$ \\
\hline 1 & Cardiac Erosion with tamponade & 136 & 13 & 20 \\
\hline 2 & Cardiac Erosion & 1 & 33 & 20 \\
\hline 3 & Cardiac Erosion & 164 & 11 & 20 \\
\hline 4 & Cardiac Erosion & 199 & 9 & 18 \\
\hline 5 & Cardiac Erosion & 161 & 38 & 30 \\
\hline 6 & Cardiac Erosion & 38 & 7 & 22 \\
\hline 7 & Cardiac Erosion with tamponade & 3 & 44 & 26 \\
\hline 8 & Cardiac Erosion & 17 & 11 & 28 \\
\hline 9 & Cardiac Erosion & 98 & 47 & 24 \\
\hline 10 & Cardiac Erosion & 83 & 28 & 28 \\
\hline 11 & Cardiac Erosion & 2382 & 12 & 24 \\
\hline 12 & Cardiac Erosion & 162 & 30 & 11 \\
\hline 13 & Cardiac Erosion & 1 & 63 & 19 \\
\hline
\end{tabular}

Conclusions: Compare to other information including United States, the incidence of cardiac erosion seems to be equal or slightly lower in Japan.

\section{MP4-10}

Assessment of linear insertion of atrioventricular valves in fetuses with postmortem cardiac MRI

Blondiaux E. (1), Autret G. (2), Dhombres F. (3), Gonzales M. (3), Clément O. (2), Jouannic J.M. (3), Houyel L. (4).

Service de Radiologie, Hôpital Trousseau - Hôpitaux Universitaires de l'Est Parisien (APHP), Université Pierre et Marie Curie, Paris VI, Paris, France (1); Plateforme Imageries du Vivant, Paris Cardiovascular Research Center, INSERM U970, Université Paris Descartes, Sorbonne Paris Cité, Paris, France (2); Service de médecine foetale, Hôpital Trousseau - Hôpitaux Universitaires de l'Est Parisien (APHP), Université Pierre et Marie Curie, Paris VI, Paris, France (3); Service de chirurgie des cardiopathies congénitales, Hôpital Marie-Lannelongue, CMR-M3C, Université Paris-Sud, Le Plessis-Robinson, France (4).

Background: Linear insertion of the atrioventricular valves (ILVAV) was described in 2002 by C. Fredouille et al. as an echocardiographic sign in the fetus associated with aneuploidy. These authors raised the hypothesis that LIAVV could represent a minor form of atrioventricular septal defect (AVSD). However, no anatomic or embryologic explanation has been provided yet to confirm this hypothesis. To elucidate the pathological significance of LIAVV, we compared postmortem high-spatial resolution cardiac magnetic resonance imaging (MRI) with foetopathological examination for the visualization of linear insertion of atrioventricular valves (LIAVV) in human fetal hearts. This work will be a preliminary step towards a larger scale study, with as a final aim to confirm or not the belonging of LIAVV to the spectrum of AVSD. Methods: Two normal hearts, 1 AVSD and 2 LIAVV (ranging from 17 to 34 weeks of gestation) were randomly selected among the fetal heart specimens of the anatomic collection of the French Reference Center for Complex CHD. Postmortem cardiac MRI was performed with a 4.7-T imager. Heart specimens were rinsed and placed in airtight bags filled with 1-2 $\mathrm{ml}$ of distilled water. 3D and $2 \mathrm{D}$ Turbo-RARE sequences in four-chamber, short axis and left ventricular long-axis planes were performed with a minimal

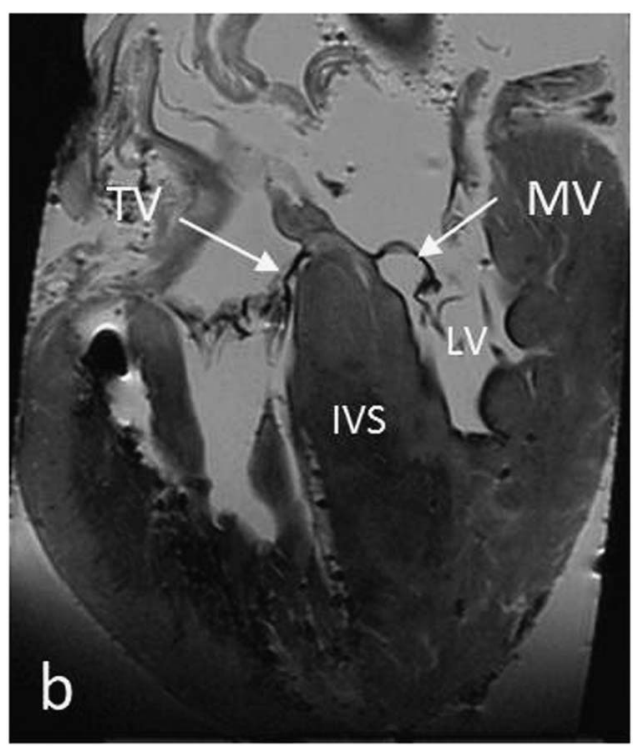

isotropic or in-plane resolution of $156 \mu \mathrm{m}$. MR images were compared to the foetopathological examination. Quantitative analysis included measurement of the distance between the insertion of the medial leaflets of the tricuspid and mitral valves and of the left ventricular inlet/outlet distance ratio.

Results: There was a perfect agreement between MR and foetopathological examination for identification of valves insertion, evaluation of mean mitral valve-tricuspid valve distance $(1.63 \mathrm{~mm}$ for normal hearts; $0.89 \mathrm{~mm}$ in hearts with LIAVV) and evaluation of inlet-outlet distance ratio $(>0.9$ in normal hearts, $<0.80$ in LIAVV, $<0.5$ in AVSD). Both hearts with LIAVV had a deficiency of the inlet ventricular septum closed by the insertions of a bi-leaflet mitral valve, explaining the lack of offsetting of the AV valves.

Conclusions: Postmortem cardiac MRI provides results similar to foetopathological examination for the assessment of LIAVV without needing specific preparation of the heart.

MP4-11

Outcomes of antenatal and postnatal diagnosis of TAPVC with unbalanced ventricles: 13 years' experience at a single cardiac surgical centre

Fernandes P., Mantagou L., Lim J.S.L.

Alder Hey Children's Hospital Foundation Trust, Liverpool, UK

Aim: To review the diagnosis, management and outcomes of patients with antenatal and postnatal diagnosis of total anomalous pulmonary venous connection (TAPVC) associated with unbalanced ventricles.

Methods: Retrospective review over 13 years from cardiac and fetal cardiac database at a single tertiary cardiac centre. 
Results: We reviewed 30 cases of TAPVC with associated complex congenital cardiac lesion between 2003 and October 2016. Of these, $19 / 30$ cases were associated with unbalanced ventricles. 16 were diagnosed antenatally with a complex congenital cardiac lesion (9 with antenatal suspicion of anomalous pulmonary veins) and 3 were diagnosed postnatally. See Table 1 for outcomes.

Table 1. Outcome of Antenatal and Postnatal diagnoses

\begin{tabular}{lcl}
\hline Outcome & Antenatal (16) & Postnatal (3) \\
\hline TOP & 3 & - \\
Born Alive & 13 & 3 \\
Compassionate Care & $5(1$ alive; 4 dead $)$ & 3 (1 alive; 2 dead $)$ \\
Active Management & $8(2$ alive; 6 dead $)$ & 0 \\
\hline
\end{tabular}

Amongst the live born antenatal patients who elected to proceed with surgery $(8 / 13)$, only $2 / 8$ are alive (currently aged 18 months and 7 years). The remaining patients who had undergone surgery $(6 / 8)$ - had only survived between 6 days to 44 weeks (median days of survival 22 days).

In the group who elected for compassionate care, 1 of 5 from the antenatal group is still alive at 6 months, and 1 of 3 from the postnatal groups is still alive at 7 years.

Table 2 . Isomeric status of live born

\begin{tabular}{lll}
\hline Isomerism & Antenatal (13) & Postnatal (3) \\
\hline Non-isomeric & 2 & 1 \\
Right & 9 & 2 \\
Unknown & 2 & 0 \\
\hline
\end{tabular}

Table 3. Types of TAPVC of live born

\begin{tabular}{lll} 
Types & Antenatal (13) & Postnatal (3) \\
\hline Supracardiac & 2 & 1 \\
Cardiac & 1 & 0 \\
Infracardiac & 7 & 1 \\
Mixed & 3 & 1 \\
\hline
\end{tabular}

We also reviewed the isomeric status and types of TAPVC, the breakdown are shown in tables $2 \& 3$.

Conclusions: With our data we have found that the outcome of TAPVC with unbalanced ventricles is poor, even with patients who are non-isomeric. The antenatal diagnosis of TAPVC with complex anatomy remains challenging, however should be suspected and evaluated further in the context of complex cardiac diagnosis.

\section{MP4-12}

Foetal intrapericardial Teratoma: relevance of the EXIT stategy

Quarti A., Iezzi F., Pozzi M.

Pediatric And Congenital Cardiac Surgery and Cardiology, Ospedali Riuniti, Ancona, Italy
Introduction: Teratoma is a neoplasm with derivatives from multiple germinal layers. Despite it has been described in many body sites, its presentation inside the pericardium is rare and often fatal.

The foetal ultrasound, and an accurate decision plan, are of great importance to increase the chance of survival for the newborn. The EXIT strategy can be used to assist the baby during the neoplasm removal.

Methods: A 31 year-old gravida 1, presented at our Institution with a 30 week foetus having a pericardial mass. An ultrasound revealed that the mass was heterogeneous and produced a complete right atrium collapse. A foetal magnetic resonance was deemed necessary. It showed the presence of cystic cavities, vessels and soft tissue, making the diagnosis of foetal Teratoma more likely.

A multidisciplinary team work plan was outlined. The mother was closely monitored and the foetal ultrasound was repeated once a day. In the first week the mass showed increase in its size and the pericardial effusion became significant. The team was concerned of potential cardiac tamponade physiology and a pericardiocentesis was considered to be potentially dangerous for the baby. In the end, it was decided to proceed to a caesarean section. It was judged that the use of an Ex Utero Intrapartum Treatment (EXIT) was the safer way to treat the baby.

Results: The mother was taken to the operating room and an additional surgical bed was prepared next to the mother for the newborn. A $2 \mathrm{~kg}$ baby was delivered via cesarean section and intubated prior to cutting the umbilical cord. As expected, the baby experienced a severe cardiac tamponade physiology. A sternotomy was rapidly performed. The tumor was easily identified attached to the aorta. It was carefully removed and the right atrium immediately reached a normal shape. The subsequent clinical course was uneventful. The histology revealed that the mass was effectively a Teratoma.

Conclusions: Since the tumor is benign in origin, the treatment of choice is surgical removal. Early ultrasound and MRI could optimize the timing of the removal. The EXIT strategy is helpful in order to minimize the surgical risk.

MP4-13

Transcatheter Pulmonary Valve implantation in native and postsurgical, non conduit right ventricular outflow tracts. A single center experience

Giugno L., Chessa M., Butera G., Piazza L., Conforti E., Pluchinotta F. R., Micheletti A., Negura D.G., Saracino A., Giusti G., Carminati M. Pediatric and Adult Congenital Heart Centre, IRCCS Policlinico San Donato, San Donato M.se MI, Italy.

Introduction: PPVI is a viable alternative to surgery in patients with RV outflow tract dysfunction. The extension of PPVI to patients without conduit has the potential to expand those patients eligible to benefit from nonsurgical restoration of RVOT function.

Methods: Between June 2010 and October 2016, 34 pts underwent percutaneous pulmonary valve implantation in native outflow tract in our centre. Primary underlying diagnosis was tetralogy of Fallot $(n=26)$, pulmonary valve stenosis $(n=3)$, pulmonary atresia with intact ventricular septum $(n=2)$, ventricular septal (VSD) defect with pulmonary valve stenosis $(n=2)$, transposition of great artery with VSD and pulmonary valve stenosis $(n=1)$. RV outflow tract remodelling included: transannular patch $(n=29)$, pulmonary valvotomy $(n=3)$, arterial switch $(n=1)$, percutaneous pulmonary valvuloplasty $(\mathrm{n}=1)$. The leading problem consisted of a pulmonary stenosis $(n=2)$ and pulmonary regurgitation $(\mathrm{n}=32)$.

Results: Technical success was 53\% (18/34 pts). The PPVI was not feasible in $47 \%$ of pts $(16 / 34)$ for coronary artery compression 
during sizing balloon of RVOT with not compliant balloon $(\mathrm{n}=2)$, diameter of RVOT $>$ of $30 \mathrm{~mm}$ at sizing balloon $(\mathrm{n}=14)$. Pre stenting was performed in all cases at the time of valve implantation with bare stent (Andrastent). The mean number of stents used for pre-stenting was $1.6 \pm 0,5$ (range 1 to 2 ). The Melody valve was implanted in 4/18 pts with Ensemble $22(n=3)$ and $20(\mathrm{n}=1)$. The Edwards Sapien valve was implanted in 14 pts. Valve size were $23 \mathrm{~mm}(\mathrm{n}=1), 26 \mathrm{~mm}(\mathrm{n}=7), 29 \mathrm{~mm}(\mathrm{n}=6)$. Instability of the valve occurred in $1 \mathrm{pt}$, which required insertion of two covered stents and second $26 \mathrm{~mm}$ Sapien valve with good results. No patient had clinically significant pulmonary regurgitation or stenosis after PPVI. Complications occurred in 3 patients $(n=2$ tricuspid valve injury that required surgical repair, $n=1$ mild hemoptysis which did not require treatment). At a mean follow-up of $12,4 \pm 9$ month (range 1 to 24 month), there were no episodes of endocarditis. There was preserved valve function during follow-up.

Conclusion: In our experience PPVI is a feasible procedure in about $50 \%$ of patients after surgical repair without the conduit with low rate of complications.

MP4-14

Absorb Bioresorbable stents in children: indications, initial experience and mid term results

Malekzadeh-Milani S., Sitefan F., Boudjemline Y.

Centre de Référence Malformations Cardiaques Congénitales Complexes

- M3C, Necker Hospital for Sick Children, Paris, France.

Background: Stents are used in various indications in the paediatric population. Bioresorbable stents are of great interest in this growing population because growth of the vessel is theoretically preserved. Little is known about the behaviour of these stents in children and in other vessels than coronaries. We report our experience with Absorb bioresorbable stents (Abbott).

Material and methods: All patients who received a resorbable stent between July 2015 and May 2016 were reviewed. Demographics, indication for cardiac catheterisation and for stenting, type of stent and medium term results are reported.

Results: Between July 2015 and May 2016, 16 children had Absorb stent implantation. Mean age was 6.3 years ( 3 days- 16.3 years). Mean weight was $21.8 \mathrm{~kg}(3-55 \mathrm{~kg})$. Mean BSA was $0.79(0.21-$ 1.64). Indications for stent implantation were: coronary artery stenosis in 6 patients (post arterial switch: $\mathrm{N}=3$, post coronary reimplantation: $\mathrm{N}=2$; post Kawasaki disease: $N=1$ ), pulmonary artery stenosis in 5 patients (post operative PA stenosis: $N=3,1$ LPA stenosis in unoperated neonatal Tetralogy of Fallot, 1 lobar stenosis in arterial tortuosity syndrome), PDA stent after perforation of pulmonary atresia in a neonate and stenosis of various arteries in 4 patients. Amongst these patients, there was 1 patient with diffuse inflammatory arteritis, 1 mid aortic syndrome, 1 renal artery stenosis and 1 neonatal aortic coarctation. Primary success was reported for all patients with good angiographic results and no effraction. 11 patients had a second elective catheterisation: 3 stents failure were noted: 2 stents fracture (one in the PA, one in the aortic neonatal coarctation) and one intrastent proliferation in the aorta and renal artery in a patient with inflammatory arteritis were observed. These patients had further stenting during the second procedure. Diameter of stents used were $2,5 \mathrm{~mm}(\mathrm{~N}=5), 3 \mathrm{~mm}$ $(\mathrm{N}=6)$ and $3,5 \mathrm{~mm}(\mathrm{~N}=13)$.

Conclusion: Resorbable stent is an alternative to conventional stent in small children and coronaries in children. Intermediate results are more than satisfactory. In this small series, 3 mid- term failures were noted. Careful follow-up and second catheterisation has to be considered if bioresorbable stent is implanted.

\section{MP4-15}

A New Covered Stent for Complex Coarctation

DeGiovanni J.V. (1), Morgan G.J. (2), Ciuffreda M. (3)

Queen Elizabeth Birmingham UK (1); Denver Children's USA (2);

Papa giovanni XX111 Bergamo Italy (3)

Introduction: Recent evidence is providing guidance on the need for covered stent use in lesions such as coarctation of the aorta and right ventricular outflow tract stenting. Currently the only FDA approved covered stent is the Cheatham-Platinum stent, but development in this area is urgently needed. Andratec have developed the Optimus covered stent as an alternative covered stent for similar clinical indications. This is a laser cut cobalt chromium stent with a double layer of PTFE sandwiching the stent struts. It's hybrid design offers a wider range of expansion with less shortening and potentially more predictable behaviour of the covering.

We describe the first human use of the covered Optimus stent in patients with coarctation of the aorta.

Methods and Results: We successfully implanted the Optimus covered stent in 7 consecutive cases in patients whose preprocedural anatomy looked challenging for currently available covered stents (Fig 1A). Six of the patients had native coarctation with one recoarctation following surgical repair. Median age was 46yrs (range 33-69yrs). There were no significant complications with reduction in the median invasive gradient from $23 \mathrm{mmHg}$ to $0 \mathrm{mmHg}$. The length of stents used ranged from $33 \mathrm{~mm}$ to $57 \mathrm{~mm}$ with a median shortening after expansion of $13 \%$. Post-procedural follow-up with magnetic resonance imaging or computed tomography has not shown evidence of fracture or migration or re-narrowing (Fig 1B). The median duration of follow-up is 8months.

Conclusions: Preliminary results show that the Optimus covered stent is safe and efficacious for use in patients with coarctation of challenging morphology. A systemic trial will be required to evaluate this stent for more widespread practice.

MP4-16

Ductus-stenting in complex congenital heart disease - early and long-term results in the Netherlands

Bökenkamp R.(1), van Beynum I.M. (2), Molenschot M.C. (3), Roofthooft M.T.R. (4), Dalinghaus M. (2), Krings G. (3), Berger R.M. F. (4), Blom N.A. $(1,5)$

Pediatric Cardiology LUMC, Leiden(1); Erasmus MC, Rotterdam(2); UMC Utrecht(3); UMC Groningen(4); AMC, Amsterdam(5) The

Netherlands

Introduction: Stenting of the ductus arteriosus has become an alternative for initial palliation in neonates with complex congenital heart disease.

Methods: To gain insight into the use and complications of ductal stenting, the patients from the 4 tertiary referring hospitals in the Netherlands were evaluated by means of a questionnaire.

Results: Between January 2010 and March 2015 ductal-stenting was performed in 57 patients. Median age at implantation was 22,4 (1- 84 days), median weight $3,3(1.9-4.2 \mathrm{~kg})$. Most patients $(\mathrm{n}=51)$ had a duct-dependent systemic circulation. Diagnoses were: HLHS and variants $n=28$, borderline $L V n=7$, IAA B with VSD $n=12$, TOF/PA/VSD $n=6$, HRHS $n=2$, others $n=2$. Implantations were performed percutaneously in 42 patients. In 15 patients the transthoracic route was used. In total 88 stents of various materials (self-expandable Nitinol-stents, bare metal stents, coronary-stents) were implanted. For the systemic circulation the median stent diameter was $8(6-10 \mathrm{~mm})$ and for the pulmonary circulation 3 (3-3.5 mm). Procedural complications without 
mortality occurred in $6(10.5 \%)$ patients. Stent dislocations in 4 and thrombotic complications in 3. During Follw-up 10 late stent stenoses needed treatment. Finally out of the 29 patients with a potentially biventricular circulation $26(89 \%)$ could be corrected $21(72 \%)$ were long-term survivors. From the 28 patients with a univentricular circulation 17 (40\%) are alive after staged-surgery. Conclusions: Ductal stenting in the Netherlands is mainly used in duct-dependent systemic circulation. There was no mortality during implantation. The long-term results of bilateral banding and ductus-stenting in potentially biventricular circulations are encouraging. Patient selection may have influenced the poor results of ductus-stenting in the univentricular circulation.

\section{MP4-17}

Transcatheter closure of Tubular (Type C) PDA's in Patients with Pulmonary Hypertension

Celebi A., Yucel I.K., Bulut M.O., Balli S., Oner T., Akgun G. Dr. Siyami Ersek Hospital for Cardiology and Cardiovascular Surgery, Department of Pediatric Cardiology, Istanbul, Turkey

Introduction: There are known groups of patients with patent ductus arteriosus (PDA) where transcatheter occlusion is technically more challenging. Among these are infants with large PDAs and older patients with tubular PDAs (type C). In this report, we will present our experience in transcatheter closure of Type $\mathrm{C}$ PDA's.

Method: Between 2005-2016, 872 patients underwent transcatheter PDA closure in our clinic. 40 of them had Type C PDA.

Results: The median age was 8 months (range 1 month to 6.5 years). The age groups were as follows: $<1$ year, $29 / 40$ patients (73\%); $1-2$ years, $9 / 40(22 \%)$ and $>2$ years $2 / 40$ (5\%). Median weight was $5.9 \mathrm{~kg}$ (range $3-15$ ) with $53 \%$ of patients weighing less than $6 \mathrm{~kg}$. The median measurements for minimal ductal diameter, length, and aortic ampulla were, $4.7 \mathrm{~mm}$ (range 3-11.7), $11 \mathrm{~mm}$ (4.3- 24), and $7.7 \mathrm{~mm}$ (4-14), respectively. Pulmonary hypertension was found in all. PDA was closed with Duct occluder I (DOI) in 31, vascular plug (VP) 2 in four, VP I in two, VP4 in one, DO II in one and DO II AS in one. Procedure was successful in $37 / 40(93 \%)$. Two patients sent to surgery after embolization of VP I and DOI. Significant aortic obstruction occurred before releasing the DOI device in another. Device displaced to descending aorta after 24 hours and repositioned with a bioptome in one. Occlusion rate was $100 \%$ after six month follow up. DOI slightly protrude to aortic lumen without significant obstruction in $13 / 31$ infants. In these peak velocity at descending aorta decreased at follow up.

Conclusion: Although technically challenging, transcatheter closure of large Type C PDA especially in infants with various devices is possible. It is important that the skirt of the device to be embedded into the duct for a more stable position. In a relatively small aorta, a larger aortic retention skirt diameter of DOI is one clinical concern; however, the somatic growth of aorta allows unimpeded aortic flows on late follow-up. In order to prevent aortic obstruction, plugs can be used to occlude the long Type C PDA especially in infants.

\section{MP4-18}

Feasibility of volumetric methods in the assessment of right ventricular (RV) function in fetuses

Duliban J. (1), Pietrzak M.(1,3), Grzyb A. (1,2), Dangel J. (1,3)

(1) Reference Center for Fetal Cardiology, Warsaw Hospice for Children

Foundation; (2) Cardiology Department, The Children's Memorial

Health Institute; (3) 2nd Department of Obstetric and Gynecology,

Medical University of Warsaw
Introduction: Estimation of right ventricle (RV) function with standard 2D echocardiography is challenging due to its complex geometry and irregular muscle fibers arrangement. In fetuses, different loading conditions and "systemic" role of the RV should be taken into account. The aim of our study was to assess the usefulness of new technique, 4D imaging of RV which is commonly used in adult and children echocardiography but it's role in fetal echo is unknown. According to our knowledge this is the first study using this method prenatally.

Methods: Epiq7 and volumetric 5-1 probe was used to store the fetal heart images in 3Dzoom mode. Volumes were analyzed using 4D RV Function TOMTEC Arena program. 59 examinations between 18 and 39 weeks of pregnancy were performed: 41 normal, 5 hypoplastic left heart syndrome (HLHS), 13 aortic stenosis (SA): 2 before and after fetal balloon aortic valvuloplasty (fBAV), 5 without BAV and 4 after BAV. Volumetric parameters: RVend-diastolic volume (EDV), end-systolic volume (ESV), stroke volume (SV) and ejection fraction (EF) were analyzed, as well as RV longitudinal freewall (RVLS freewall) and septal strain (RVLS septum). Tei index measured by pulsed Doppler was used for comparison with $4 \mathrm{D}$ measurement, as a reference method in evaluation of RV function in our department.

Results: Analysis was easier in pregnancies above 30 weeks. RV EDV increases during gestation in all studied groups and it was from $0,4 \mathrm{ml}$ in 18 weeks up to $8 \mathrm{ml}$ in 36 weeks. Significant negative correlation between RV Tei index and EF was revealed among HLHS and SA fetuses (rho $=-0.6364$, p-value $<0,01$ ), but not in normal fetuses (rho $=-0.0050, \mathrm{p}$-value $=0,98$ ) in which coefficient of variation was the lowest. EF in fetuses with HLHS was lower than in normal fetuses, significance can not be calculated due to small HLHS group. RVLS freewall correlates significantly with EF in all studied cases. It was significant improvement of RV image and function in two fetuses after fBAV.

Conclusions: Volumetric methods are feasible in assessing fetal RV. $\mathrm{EF}$ is a promising parameter in detecting impaired RV function. Role of right ventricle longitudinal freewall strain as an alternative measure of RV function require further investigation.

\section{MP4-19}

Outcomes of congenitally disconnected and non confluent branch pulmonary arteries - a single centre experience over 24 years

Durairaj S., Alias A., Stickley J., Barron D., Stumper O., Mehta C. Birmingham Children's Hospital, Birmingham, UK

Aim: To describe our experience of all children presenting with congenitally disconnected non-confluent branch pulmonary arteries. We studied the pattern of presentation, diagnostic modalities, intervention and outcomes.

Methods: Retrospective review of data of children with diagnosis of disconnected pulmonary arteries between 1989 and 2013. We included all children with diagnosis of non-confluent branch pulmonary arteries with or without alternative pulmonary blood flow including pulmonary atresia. We excluded acquired disconnection of pulmonary arteries, and anomalous origin of branch pulmonary artery from aorta.

Results: 35 patients were identified. $85 \%$ of our population presented during infancy (age range from 4 days to 17years) with slight female preponderance (females 20 vs males 15). The commonest mode of presentation was in association with congenital cardiac condition (cyanotic in 30, non-cyanotic in 4) except for one patient with isolated disconnection with coexisting lung hypoplasia. The most common congenital cardiac lesion was Tetralogy of Fallot. 
The disconnected branch pulmonary artery was on right-sided in $6(17 \%)$ vs left-sided in $22(83 \%)$. The supply to the disconnected segments was from ductus arteriosus in 22 patients, the aortic arch was right-sided in $10(28 \%)$ vs left-sided $20(56 \%)$ and unknown in $5(12 \%) .8$ patients had associated genetic syndrome.

Diagnosis was confirmed by cardiac catheterisation in 21 patients (60\%). Median age at first intervention was 10 months (1 to 254 months). Primary intervention was trans-catheter in 6(18\%) and surgery $28(82 \%)$. Average number of procedures after initial intervention was 5 (range 1-9). One patient had unsuccessful recruitment. At last follow up, 24 patients with full correction were doing clinically well and 1 had developed with pulmonary hypertension. 2 remains cyanotic and awaiting further intervention. Mortality in this series was three patients $(8 \%)$.

Conclusion: Congenitally disconnected branch pulmonary arteries is a rare abnormality. Most patients will need staged palliation approach before complete repair with good long term outcome.

\section{MP4-20}

Initial experience of percutaneous PDA closure in preterm and low birth weight infants

Malekzadeh-Milani S. (1), Patkai J. (2), Sitefane F. (1), Mirc M. (3), Bonnet D. (1), Boudjemline Y. (1)

(1) Centre de Référence Malformations Cardiaques Congénitales Complexes - M3C, Necker Hospital for Sick Children, George Pompidou European Hospital, Assistance Publique des Hopitaux de Paris, Paris, France, (2) Service de Réanimation et médecine néonatale de Port Royal, Paris, France, (3) SErvice de réanimation néonatale, CHI Montreuil, France

Background: Percutaneous PDA closure is a routine procedure with low complications rate. It is the gold standard treatment for children over $6 \mathrm{~kg}$. There are increasing reports of PDA closure in low weight babies. We report our initial experience in this specific population.

Material and methods: From March 2014 until October 2016, all preterm or low birth weight infants with percutaneous PDA closure were included. Demographic and procedural data and complications were reviewed.

Results: 23 patients were included. Mean gestational age was 30 weeks $(+/-4)$. Mean birth weight was $1.38 \mathrm{~kg}(+/-0.7)$. Mean age at the PDA closure was $50+/-29$ days. Mean weight was $1.9 \mathrm{~kg}(+/-0.75, \min 0.8-\max 2.9) .13$ patients were below $2000 \mathrm{~g}$. PDA was tubular in 18 patients. 7 patients with weight above $2,5 \mathrm{~kg}$ had arterial and venous accesses. 14 patients had venous access only and 2 patients had arterial sheathless access only. Devices used were ADO II AS in 20 patients, coil in 1 and Microvascular Plug Q5 in 2. Procedure was conducted with fluoroscopy and echocardiography. Mean procedural and fluoroscopy time were respectively $24+/-7$ and $4,3+/-2$ minutes $(3.6$ minutes in patients $<2000 \mathrm{~g})$. Mean radiation exposure was $8.9+/-11.5 \mathrm{mG}$ and $21.4 \mu \mathrm{Gm} 2(+/-26)$. Mean dye injection was $6.7 \mathrm{ml}(+/-5)(3 \mathrm{ml}$ in patients $<2000 \mathrm{~g})$. There was one perprocedural coil embolisation with successful retrieval and further closure with ADOII AS $4 \star 2$. There were no access complications, no post procedural blood transfusion, no renal failure and no death. One transient myocardial incompetence required dobutamine infusion for 48 hours. Follow up echocardiography confirmed absent LPA or aortic arch obstruction. We gradually modified our technique to adapt to very small babies below 1000G. We used shorter device over time. Radiation exposure was decreased by using low frame rate fluoroscopy and avoiding cine angiography. We had no complication.
Conclusion: Percutaneous PDA closure is a very good alternative to surgery in small infants. With an adapted technique, it can be performed with good early and mid term results. Transcatheter technique might become first line treatment of PDA closure in low weight patients.

\section{O1-1}

Right ventricular electrical activation in patients with repaired tetralogy of Fallot: insights from electro-anatomic mapping and MRI

Fournier E. (1), Jalal Z. (1,2), Sacher F. (1,2), Bordachar P. (2,3), Derval N. (2,3), Cochet H. (2), Teijeira Fernandez E. (2,3),

Haissaguerre M. (2,3), Iriart X. (1), Thambo JB. $(1,2)$

University Hospital of Bordeaux, Paediatric and congenital heart defects unit, Pessac, France (1); IHU Liryc, Electrophysiology and Heart Modeling Institute, fondation Bordeaux Université, F-33600 PessacBordeaux, France (2); University Hospital of Bordeaux, Electrophysiology and Ablation Unit, Pessac, France (3).

Background: Presence of prolonged QRS duration in patients with repaired tetralogy of Fallot (TOF) is considered as a risk factor for sudden death and associated with altered hemodynamics. It has been suggested that QRS duration mainly reflects abnormalities of the RV outflow tract (RVOT) rather than the $\mathrm{RV}$ body itself. We aimed to better understand the RV electrical activation pattern in these patients using activation mapping and MRI.

Methods: 61 adults (36 yo [25-48], median QRS duration $159 \mathrm{~ms}$ [140-171]) referred for either catheter ablation or pulmonary valve replacement late after TOF repair underwent an ECG, MRI with fibrosis analysis and an invasive RV activation mapping (Carto 3Biosense Webster). RV activation delay and RV activation time (RVAT) were studied and respectively defined as the delay between the reference surface ECG and the first RV electrogram (EGM) and, the duration between the first and the last RV EGM. RV electrical pattern was also analysed.

Results: RVAT was prolonged in all patients with median duration of $135 \mathrm{~ms}$ [120-156]). The delay between QRS onset and earliest

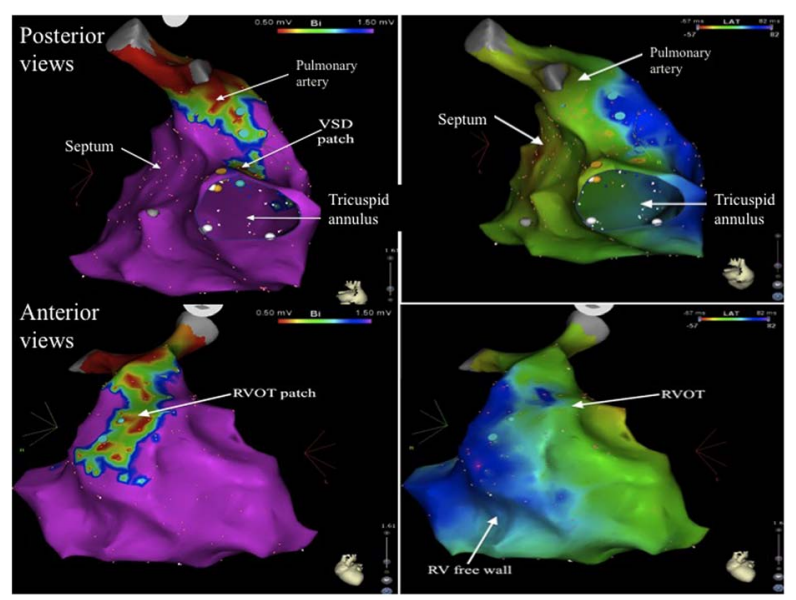

Figure 1.

Anterior and posterior views of $R V$ activation maps (right side) and voltage activation map (left side) in the same patient. RVOT, right ventricular outflow tract; VSD, ventricular septal defect; IVS, interventricular septum; TA, tricuspid annulus; $P A$, pulmonary annulus. 
RV activation was lengthened at $20 \mathrm{~ms}$ [7-34] reflecting the absence of RV purkinje activation and the left to RV activation. For most patients, the electrical activation pattern was similar, starting from the septum $(78.7 \%)$, following by the apex $(68.3 \%)$, the infundibulum (67.8\%) and the RV free wall was the latest activated $(77.6 \%)$. When the infundibulum was the latest region activated, the RVAT was longer (164 ms [120-156]) and the MRI RVOT scar surface area larger $\left(17.7 \mathrm{~cm}^{2}\right)$. RV dilation at MRI (RVEDV $\geq 150 \mathrm{ml} / \mathrm{m}^{2}$ ) was associated with RVAT prolongation reflecting the link between hemodynamic and electrophysiologic properties. QRS duration and fragmentation were weakly correlated with RVAT $(r=0.44, p=0.631)$.

Conclusion: RV activation is delayed in patients with repaired TOF and reflects an homogeneous activation pattern that is not only the consequence of an infundibular disease but also reflects a slow conduction in the RV free wall. This results could help to select eligible patients for resynchronization therapies.

\section{O1-2 \\ Long-term outcomes and prognosis of patients with isolated corrected Transposition of the Great Arteries \\ Chabchoub S., Bozio A., Bakloul M., Martin-Bonnet C., Perouse de Monclos T., Ducreux C., Veyrier M., Di Filippo $S$. \\ Cardiovascular Hospital Louis Pradel, Université Claude Bernard, Hospices Civils de Lyon, Lyon, France}

This study was to assess the long-term outcome of children and adults diagnosed with congenital corrected TGA (ccTGA) and no significant associated lesion.

Material and Methods: All patients with ccTGA were included in the retrospective single-center study. Clinical features, echocardiographic parameters and long term outcomes were collected. Pronostic factors for poor outcome were assessed.

Results: 55 patients were included (30 males), aged $16.7 \pm 18.4 \mathrm{y}$ at diagnosis (0 to $77 \mathrm{y}, 25 \%$ less than $1 \mathrm{y}$ of age): 13 (23.6\%) diagnosed antenatally, 25 fortuitously (45.5\%) and 17 had symptoms (30.9\%). Associated mild lesions were present in 23 cases (small VSD, mild pulmonary valve stenosis, small ASD, mild isthmus stenosis) and complete atrioventricular block at diagnosis was present in 7 . Follow-up was $17.9 \pm 12.7 \mathrm{y}$. NYHA class I, II, III and IV distribution at onset was respectively: $70 \%, 18 \%, 7 \%$ and $3.6 \%$, and changed to respectively: $31 \%, 38 \%, 24 \%$ and $6.7 \%$ at end-FU. Echocardiographic RV function was normal on $87 \%$ of cases, $13 \%$ had moderate and $0 \%$ had severe RV dysfunction: percent changed to respectively $73 \%, 18 \%$ and $9 \%$ at end-FU. Tricuspid regurgitation was grade $0,1,2$ or 3 in $33 \%, 49 \%, 9 \%$ and $9 \%$ of cases respectively at onset FU and evolved to $13 \%, 48 \%, 24 \%$ and $16 \%$ at end-FU respectively. HF occurred in 8 cases $(14.5 \%)$ at the age of $37.5 \pm 13.7 \mathrm{y}$ (7 to $61 \mathrm{y})$, AVB occurred in $20(36.4 \%)$ at age $31.4 \pm 19.4 \mathrm{y}$ ( 5 to $72 \mathrm{y}$ ) and arrhythmias in 16 (29\%), over FU. One patient died from uncontrolled $\mathrm{HF}$ at $54 \mathrm{y}$ of age. Eight patients had surgery ( 3 tricuspid plasties, 2 tricuspid replacements, 3 PA bandings and 1 heart transplantation). Nineteen pregnancies occurred (11 females) with no death. Echocardiographic RV function correlated with MRI measurements $(r=0.7, p=0.005)$. $\mathrm{RV}$ dysfunction was associated with TR grade $(\mathrm{p}=0.025)$. HF correlated with TR grade $(\mathrm{p}=0.015)$ and pacemaker implantation $(p=0.026)$. Freedom for HF was $50 \%$ at age of 36 years.

Conclusion: Isolated ccTGA shows significant worsening of clinical status, RV function and TR grade over twenty years follow-up of this young age population. These data should raise the issue of early LV conditioning for double switch option in isolated ccTGA.
O1-3

Cardiac morbidity and mortality in patients with RASopathy syndrome: an european multi centric study Calcagni G. (1), Limongelli G. (2), D'Ambrosio A. (1), Gesualdo F. (1), Digilio M.C. (1), Baban A. (1), Albanese S.B. (1), Versacci P. (3), De Luca E. (3), Ferrero G.B. (4), Agnoletti G. (4), Baldassarre G. (4), Banaudi E. (4), Marek J. (5), Kaski J.P. (5), Tuo G. (5), Russo M.G. (2), Pacileo G. (2), Milanesi O. (6), Messina D. (6), Marasini M, (7), Cairello F. (7), Formigari R. (8), Brighenti M. (8), Dallapiccola B. (1), Tartaglia M. (1), Marino B. (3)

Bambino Gesù Children's Hospital, Rome, Italy (1); Monaldi Hospital, II University of Naples, Naples, Italy (2); Sapienza University, Rome, Italy (3); University of Turin, Italy (4); Great Ormond Street Hospital for Children, London, UK (5); University of Padova, Padua, Italy (6); Giannina Gaslini Institute, Genoa, Italy (7); Sant'Orsola Malpighi Hospital, Bologna, Italy (8)

Introduction: A detailed characterization of cardiac morbidity and mortality in RASopathies is lacking.

Methods: A multi-centric, observational, retrospective cohort study was conducted in seven cardiac centers to systematically collect and analyze available data on cardiac involvement, morbidity and mortality. The clinical records of 371 patients with confirmed molecular diagnosis of RASopathy were reviewed. Mortality was described as crude mortality, cumulative survival and restricted estimated mean survival. Multivariable regression analysis was used to study the effect of each mutated gene on cardiac defects, number of interventions and risk of intervention.

Results: Cardiac defects were found in $80.3 \%$ of cases. More than half had pulmonary stenosis (PS), followed by hypertrophic cardiomyopathy (HCM) (27\%) and atrioventricular canal defect (AVC) (4.4\%). $R A F 1$ mutations were the best predictors of cardiac involvement in the study population. $R A F 1$ and $B R A F$ mutations were positively associated with HCM, while PTPN11 defects with AVC and, less robustly, with PS. Among patients with heart disease $(n=298)$, almost half underwent a percutaneous and/or surgical intervention. Mortality was relatively low, less than 3\%. Among patients with HCM, those with age $<2$ years and young adults were at higher risk for fatal events, which were related to their cardiac involvement in most of the cases, while those with biventricular obstruction and carrying PTPN11 mutations had higher risk of cardiac death. Overall, crude mortality was $0.29 / 100$ patients-year. Cumulative survival was $98.8 \%, 98.2 \%$, $97.7 \%, 90.2 \%$, at $1,5,10$, and 25 years, respectively. Restricted estimated mean survival at 25 years follow-up was 24.2 years.

Conclusions: in our cohort of patients with RASopathy, cardiac involvement was common, and required percutaneous or surgical intervention in almost half of cases. The risk of intervention was higher in individuals with Noonan syndrome and PS carrying PTPN11 mutations, and lower among patients with Costello syndrome and cardiofaciocutaneous syndrome. Mortality was relatively low in RASopathies. However, the association between HCM, a subset of PTPN11 mutations, with a peculiar age distribution (infants and young adults), and the coexistence of left and right side obstruction, may predict early mortality, including immediate postoperative events and life-threatening events as sudden death.

\section{O1-4}

Characteristics and outcomes of heart failure-related hospitalization in adults with congenital heart disease Ladouceur M. (1, 2), Karsenty C. (2). Pontnau F. (2), Ben Moussa N. (2), Malekzadeh-Milani S. (1), Boudjemline Y.(1), Legendre A. (1), Iserin L (2), Bonnet D.(1)

Necker, Paris France (1), Hôpital Européen Georges Pompidou, Paris France (2) 
Background: Heart failure (HF) is the main cause of death in adult patients with congenital heart disease (ACHD). We aimed to characterize HF-related hospitalization of ACHD and to determine HF risk factors and prognosis in this population.

Methods: We prospectively included 471 ACHD admitted in our unit during 24 months. Clinical and biological data and HF management were recorded. Major cardiovascular events were recorded for HF ACHD.

Results: HF was the main reason for hospitalization in $13 \%$ ( $n=76 / 583$ hospitalizations). HF ACHD were significantly older (median age $44 \pm 14$ vs. $37 \pm 15$ years old, $\mathrm{p}<0.01$ ), with more complex congenital heart disease $(\mathrm{p}=0.04)$ and had more frequent pulmonary arterial hypertension $(\mathrm{PAH})(29.1 \%, \mathrm{p}<0.01)$, past history of HF $(45.5 \%, p<0.01)$ and atrial arrhythmia $(61.8 \%$, $\mathrm{p}<0.01)$ than in patients without HF. Mean hospital stay of HF patients was longer $(12.2$ days vs. 6.9 days, $\mathrm{p}<0.01)$ and $25 \%$ of patients required intensive care. Twenty percent $(11 / 55 \mathrm{HF}-$ patients) died, $18 \%(n=10 / 55)$ were readmitted for $\mathrm{HF}$, and $11 \%$ $(6 / 55)$ had heart transplantation during the median follow-up of 18 months $95 \% \mathrm{CI}[14-20]$. In multivariate analysis, past history of $\mathrm{HF}(\mathrm{OR}=9,895 \% \mathrm{CI}[5,7-16,8], \mathrm{p}<0.01), \mathrm{PAH}(\mathrm{OR}=6,295 \%$ $\mathrm{CI}[3,5-10,7], \mathrm{p}<0.01)$ and atrial arrhythmia $(\mathrm{OR}=3,695 \% \mathrm{CI}$ $[2,2-5,9], \mathrm{p}<0.01)$ were the strongest risk factors of $\mathrm{HF}$ in ACHD. The risk of cardiovascular events was 19-fold higher after HF-related hospitalization.

Conclusions: HF is emerging as a leading cause of morbidity and mortality in ACHD population. Earlier diagnosis and more active management are required to improve outcomes of ACHD HF.

\section{O1-5}

NYHA classification in adults with congenital heart disease: Relation to objective measures of exercise and outcomes

Bredy C. (1,2), Ministeri M. (1,3), Kempny A. (1), Alonso-Gonzalez R. (1), Swan L. (1), Uebing A. (1), Diller G. (1,4), Gatzoulis M.A. (1), Dimopoulos K. (1)

Royal Brompton Hospital, London, UK (1); CHU Arnaud de Villeneuve, Montpellier, France (2); Centro Cuore Morgagni Pedara, Catania, Italy (3); Munster University, Munster, Germany (4)

Introduction: We aimed to validate NYHA functional classification and its relation to objective limitation based on cardiopulmonary exercise testing (CPET) in adult with congenital heart disease (ACHD) and to long-term outcome.

Methods: This is a retrospective study included all ACHD patients who underwent a CPET between 2005 and 2015 at the Royal Brompton. Effort-related dyspnoea was graded according to NYHA classification and divided in subgroups (2A, 2B, 3A and $3 \mathrm{~B})$. All diagnoses were graded according to the Bethesda Classification. Patients' outcomes, including hospitalization and allcause mortality were completed.

Results: 2781 patients (mean age $33.8 \pm 14.2$ years at CPET) representing the full range ACHD were included. There was a strong correlation between NYHA functional class, peak V02 and VE/VCO2 slope $(p<0.0001)$. NYHA was also correlated with ACHD severity according to the Bethesda classification $(\mathrm{p}<0.0001)$. Although a large number of NYHA class 1 patients did not achieve a "normal" CPET, NYHA classification was nevertheless a strong predictor of mortality with an 8.7-fold increased mortality risk in class 3 compared to class 1 (HR 8.68, 95CI: 5.26-14.35, p<0.0001). Furthermore, dividing ACHD patients in class 2 subgroups appeared to carry additional prognostic information, but not so for class 3 subgroups.
Conclusion: Despite known limitations, NYHA classification relates to objective measures of exercise and predict long term outcome in ACHD. Our data suggests potential merits from subdividing NYHA functional classification 2 into subgroups $\mathrm{A}$ and $\mathrm{B}$, but this needs validation in further studies. NHYA classification should be routinely recorded in the periodic assessment of ACHD.
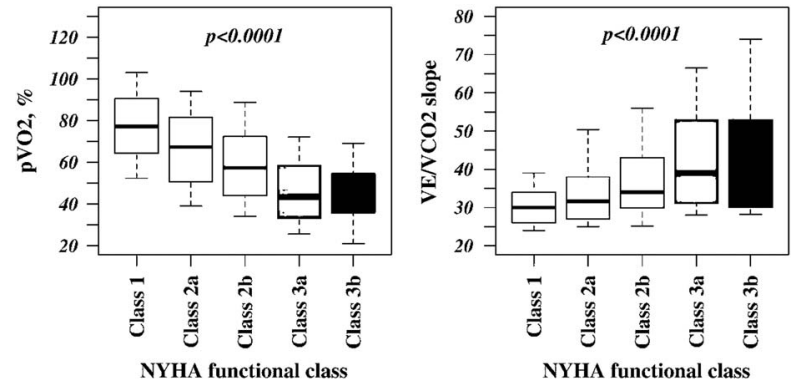

Figure 1.

Peak VO2 and VE/VCO2 according to detail NYHA functional class.

O1-6

Chronic Kidney Disease in Adolescents with Congenital Heart Disease

Bojan M.(1), Mirabile C.(1), Pieroni L. (2), Froissart M. (3), Bonnet

D. $(1,4)$

(1) CHU Necker-Enfants Malades, Paris, France, (2) CHU

Montpellier, France, (3) INSERM, Villejuif, France, (4) Université

Paris-Descartes, Paris, France

Background: Chronic kidney disease (CKD) is an important prognostic factor in young adults with congenital heart disease (CHD) [1]. Although it is likely that CKD is manifest early in CHD patients, the prevalence among adolescents is not known. Albuminuria is acknowledged to be the earliest marker of glomerular injury, and the most important predictor of decline in glomerular filtration rate (GFR) in children with CKD [2]. The new KDIGO 2012 guidelines highlight the importance of albuminuria for CKD screening [3]. The present study estimate the prevalence of CKD in CHD adolescents.

Methods: After information and written consent, CKD was assessed in patients aged 10 to 18 years with various CHD, attending the cardiologic outpatient clinic at our institution for follow-up after cardiac surgery in infancy. CKD stage was assessed according to the new KDIGO 2012 criteria [3]. CKD assessment used the bedside Schwartz equation [4] based on serum creatinine, and the Zappitelli equation based on cystatine C[5] to estimate GFR, and measurement of albuminuria. Because of the prevalence of microalbuminuria in healthy individuals, $(3-30 \mathrm{mg} / \mathrm{mmol}$ creatinine) [6], i.e. $6.2 \%$ in males and $13.4 \%$ in females, results are shown as estimates of the excess risk, calculated as the observed minus the expected number of patients with albuminuria.

Results: $20.34 \%$ of patients had albuminuria stage A2 (3-30 mg/ mmol creatinine) and $\mathrm{A} 3(>30 \mathrm{mg} / \mathrm{mmol}$ creatinine), allowing to estimate the excess risk of CKD associated with CHD at $11.20 \%$, 95\%CI [4.36\%-18.35\%]. Patients with Fontan palliation had the highest prevalence of albuminuria, $45.4 \%$. No patient had CKD according to the estimated GFR, but $10.92 \%$, 95\%CI [5.83\%$16.81 \%]$ had isolated mild decrease in GFR $(<90 \mathrm{ml} / \mathrm{min} / 1.73 \mathrm{~m} 2)$. Conclusions: Evidence of CKD has been identified in the present population based on albuminuria but not on GFR. These results call for routine, periodic screening for CKD, in particular for albuminuria, in all CHD patients to obtain prognostic information and identify patients who may benefit from early intervention. 


\section{References}

1. Dimopoulos K, Circulation 2008.

2. Wingen AM, Lancet 1997.

3. KDIGO 2012. Kidney international (Suppl).

4. Schwartz GJ, JASN 2009.

5. Zappitelli M, AJKD 2006.

6. Jones CA, AJKD 2002.

\section{O2-1}

Covered versus base stent for the treatment of aortic coartctation: systematic review of currently available clinical evidence

Butera G., Faccini A., Lunardini A., Piazza L., Chessa M., Fesslova V., Carminati $M$.

Policlinico San Donato IRCCS, San Donato Milanese, Italy

AIMS: To summarise data from studies where covered (CS) or bare (BS) stents were used to treat aortic coarctation.

Methods and Results: Electronic databases, journals and major international conference proceedings were systematically searched for pertinent clinical studies comparing the two methods of closure (percutaneous and surgical) published up to December 2015. Primary endpoints: occurrence of death and of major early complications. Major complications were defined as life-threatening events requiring immediate therapy; permanent functional or anatomic lesion; any aortic wall injury (dissection/ acute aneurysm); complication needing surgery.

Fifty-five original studies (total of 2046 patients: 434 CS, 1612 BS) were included. All studies were non-randomized. No death was encountered in CS subjects while it occurred in 4 subjects in the BS group $(0,4 \%$; $95 \%$ CI $0,22-0,58 \%)$. Quantitative synthesis of major complications after procedure showed a 3\% (95\% CI 2,2-3,8\%) rate in CS subjects while a $6.3 \%(95 \%$ CI 5,2-7,4\%) rate in BS subjects. Conclusions: The largest cohort to date of patients treated by using covered or bare stent shows that covered stent has a significantly lower rate of total early post-procedural complications.

\section{O2-2}

Results of transcatheter pulmonary valvulation in children $<30 \mathbf{~ k g}$

Sobrino Baladrón A., Rodriguez Ogando A., Ballesteros Saturnino F., Gil Jaurena J.M., Zunzunegui Martinez J.L.

H.G.U. Grgorio Marañón, Madrid (Spain).

Introduction: Although widely accepted, indications for percutaneous pulmonary valve replacement $(\mathrm{PPVr})$, are limited to prosthetic conduits in the right ventricle outflow tract (RVOT) in patients $\geq 30 \mathrm{~kg}$.

Aim: To evaluate the outcomes of Melody valve insertion in patients $<30 \mathrm{Kg}$.

Methods: We analysed procedural and outcomes data from 25 patients $<30 \mathrm{~kg}$ ( 9 patients $<20 \mathrm{~kg}$ ), who underwent Melody valve implantation for a native/patched RVOT $(\mathrm{N}=12)$, prosthetic conduit (12) and bioprosthesis $(\mathrm{N}=1)$.

Results: Median age and weight was 6.9 years $(1.5-13)$ and $21.2 \mathrm{~kg}$ (9-29.8). PPVr indication was regurgitation in 11, stenosis in 1 and mixed in 13. All procedures were successful. PPVr was performed through the femoral vein in 16 cases, jugular vein in 8 cases and transapical-perventricular in 1 case. Pre-stenting was performed in $95 \%$ of cases, $18 / 24$ in the same procedure. No significant regurgitation was recorded after the procedure, and the transpulmonary gradient was significantly reduced. Early minor complications occurred in 3 cases (10\%) The median hospital stay was 3 days (2-5). Median follow-up was 44 months (4-82). During follow-up, one patient underwent a new PPVr (valve-in-valve procedure); one patient required overdilation of the prosthesis; and one patient developed stent fracture. Follow-up with MRI demonstrated significant improvements in right ventricular volumes and function.

Conclusion: PPVr s highly feasible in children $<30 \mathrm{~kg}$, in both, native RVOT and prosthetic conduits, and mid-term follow-up, demonstrates good haemodynamic results and appears promising.

\section{O2-3}

Successful creation of an ovine pulmonary stenosis-regurgitation model simulating a Tetralogy of Fallot

Cools B., Claus P., Van Puyvelde J., Gewillig M., Rega F. UZ - KUL Leuven, Belgium

Introduction: Patients with surgically corrected tetralogy of Fallot (TF) often develop severe pulmonary regurgitation (PR) with chronic right ventricular volume overload, leading to adverse outcomes. We created an ovine survival model simulating the pathophysiology of TF to study the effects of right ventricular remodeling due to stenosis and regurgitation.

Methods: In lambs (weight $27.4 \pm 5.3 \mathrm{~kg}$ ), a pulmonary valve stenosis (PS) was created by placing a PTFE strip around the pulmonary artery through a right thoracotomy (Figure A). Four months later a bare metal stent was anchored across the pulmonary valve in the PTFE strip (Figure B) thereby relieving the stenosis and creating pulmonary valve insufficiency (Figure C). Melody valve implantations into this bare metal stent at different time intervals (5 months to 10 months) are ongoing (Figure D). Follow up by means of MRI was performed to assess remodeling and reversed remodeling of the RV.

Results: All animals survived the initial surgical phase $(\mathrm{n}=9)$. Two animals died during bare metal stent implantation (ventricular fibrillation $n=1$; PA rupture by balloon dilation $n=1$ ). MRI showed signs of RV hypertrophy prior to relief of stenosis compared to healthy controls. Total RV cardiac output (CO) was $2.2 \pm 0.7 \mathrm{~L} / \mathrm{min}$ after PS, $5.0 \pm 0.8 \mathrm{~L} / \mathrm{min}$ immediately after bare stent implantation and $3.5 \pm 0.1 \mathrm{~L} / \mathrm{min}$ SD after 5 months of PR. Animals had an important PR 5 months after bare metal stent implantation $(32 \pm 2.3 \%)$. The RV-LV EDV ratio was $1.6 \pm 0.3$ significantly higher compared to $0.9 \pm 0.0$ in healthy controls $(\mathrm{n}=3)(\mathrm{p} 0.06)$. (a)

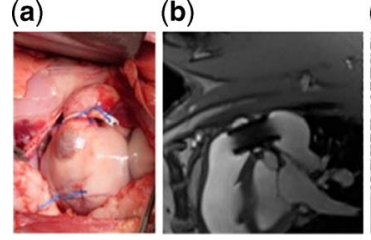

(c)

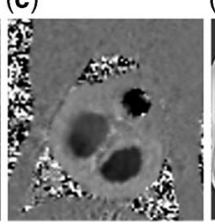

(d)

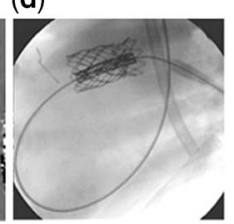

Conclusions: The creation of an ovine survival TF model with initial pulmonary valve stenosis and secondary regurgitation (mimicking the effect of surgical repair) is feasible. All hallmarks of the TF physiology (ventricular hypertrophy after stenosis - ventricular dilatation due to PR) were realized. This model forms good basis to study the timing of pulmonary valve replacement in TF.

Legend figure: A/ PTFE strip supra-annular and marker infundibular B/ MRI showing bare metal stent across the native pulmonary valve C/ MRI phase short axis showing massive PR D/ Deployment of Melody valve in bare stent. 


\section{O2-4}

The Melody ${ }^{\mathrm{TM}}$ valved stent: up to 10 years of experience

Cools B.(1), Budts W.(1), Boshoff D.(1), Heying R.(1), Vanagt $W$.

(2), Troost E.(1), Frerich S.(2), Gewillig M.(1)

UZ Leuven, Belgium (1)AZM Maastricht, The Netherlands (2)

Introduction: The Melody valve became commercially available in 2006. It has been shown to be a safe and effective intervention, but medium term results are still being gathered. The evaluation of the valve function and its risks from a prospective ongoing registry.

Methods: Single center experience; all data are collected from a dedicated prospective registry started in $2006.97 \%$ are pre-stented until stiff tube. The analyzed data: leaflet function (echocardiography), re-interventions, mortality, endocarditis (modified Duke's criteria) and stent fractures (chest X-ray).

Results: 159 valves were implanted 2006-2016 in 158 patients. The indication was stenosis $44 \%$, regurgitation $33 \%$ and mixed $23 \%$; male/female ratio was $2: 1$, the mean age at implant was $19.6 \mathrm{y}$ (3.5 - 81.6). Original conduit diameter had shrunken to mean $19 \mathrm{~mm}$ and was re-expanded to $22 \mathrm{~mm}$.

1 / Overall survival was $98 \%$ at 10 years, no procedural or valve related deaths.

2/ Graft survival was $92.1 \%$ at 10 years; re-intervention overall 11\%: explantation of valve in $4.4 \%$ (endocarditis $n=6$, subvalvular stenosis $\mathrm{n}=1$ ); repeat PPVI was $1.9 \%$ and balloon dilation for somatic growth $5 \%$.

3/ Endocarditis occurred in 9.4\% (15/159) with a freedom from endocarditis of $78 \%$ at $10 \mathrm{y}$. The maximal annual incidence of endocarditis was 3.3\% in 2015 with a steep decline after changes in behaviour. Mean age at endocarditis was $18.2 \mathrm{y}$ (8.1-45.6), male $87 \%$.

4/ Chest X-ray was performed in $87 \%$ of the patients showing a stent fracture in $8 \%$ median $2.9 \mathrm{y}(0.4-6.9)$ after implant. In no patients the fractures had hemodynamic significance.

5/ Valve function: a/ RVOT obstruction: mean RVOT PIG $65 \pm 17 \mathrm{mmHg}$ at implant drops to $23 \pm 11 \mathrm{mmHg}$ post implant and is $38 \pm 17 \mathrm{mmHg} 10 \mathrm{y}$ later. $\mathrm{B} /$ Indication regurgitation : median $4 / 4$ at implant drops to $0 / 4$ post and is $1-2 / 4$ at $10 \mathrm{y}$ follow-up.

Conclusions: Ten year follow-up of the Melody ${ }^{\mathrm{TM}}$ valved stent shows good long term preservation of the valve function. We observed no replacement for intrinsic valve dysfunction except endocarditis (3.8\%). The Melody valve is competitive with surgical conduits despite a higher incidence of SBE.

\section{O2-5}

\section{Unusual Techniques for Percutaneous Pulmonary Valve Replacement using the Melody valve}

Godart F., Houeijeh A., Guillaume M.P., Gras P., Domanski O

Paediatric Cardiology and Congenital Heart Disease, Lille, France

Percutaneous pulmonary valve replacement (PPVR) with the Melody valve (Medtronic) may be challenging in patients with unfavourable RVOT. Different techniques have been proposed to overcome this problem: jailing and/or Russian dolls techniques or the folded valve techniques.

From March 2015 to October 2016, 11 patients (5F/6M) with a mean age of $19 \pm 10$ years (11-45 years) underwent these procedures. Initial pathology included tetralogy of Fallot $(n=7)$, transposition of the great vessels $(n=2)$, pulmonary valve stenosis $(n=1)$, and aortic valve stenos $(n=1)$. These patients had undergone a median of 2 previous surgical repairs (1-5). The RVOT had been previously repaired with a transannular patch and 1 patient had homograft. The indications for pulmonary valve replacement were: significant pulmonary regurgitation $(n=7)$ and a mixed lesion $(n=4)$. All patients had before the procedure, MRI study and CT scan to delineate the exact morphology of the RVOT. Before implantation, balloon dilatation of the RVOT with control aortography to obviate any coronary artery compression was performed in all. Prestenting was realized in all with LD max stent (Ev3).

A $22-\mathrm{mm}$ Melody valve was implanted in 9 patients, a $20-\mathrm{mm}$ valve in 2 (9/11 under left ventricular pacing). The folding techniques were employed in 8 patients, the PA branch jailing in 7 , and the Russian dolls technique in 3 . The folding technique on both extremities of the stent $(n=1)$ and only on distal end $(n=7)$ was performed because of short pulmonary artery trunk with early PA bifurcation. These advanced techniques were combined in 6 patients. RVOT dilated up to $25-26 \mathrm{~mm}$ in diameter could be thus corrected by PPVR with a $22 \mathrm{~mm}$ Melody using these techniques. During follow-up (1 to 19 months), no patient had reintervention. No endocarditis was observed.

These advanced techniques for PPVR using the Melody valve can extend the classical indications. Native patched RVOT up to $25-26 \mathrm{~mm}$ in diameter can be repaired. However, these initial results are promising but more experience and longer follow-up are mandatory.

\section{O2-6 \\ Endocarditis risk following percutaneous pulmonary valve implantation is higher with Melody valve compared to Sapien valve}

Hascoet S., Mauri L., Claude C., Fournier E., Horer J., Brenot P., Riou J.Y., Petit J.

Pôle des cardiopathies congénitales, Hôpital Marie lannelongue, Le PlessisRobinson, France

Objectives: To compare the risk of infective endocarditis (IE) after percutaneous pulmonary valve implantation (PPVI) with the Sapien and Melody valves.

Background: The incidence of IE after PPVI is estimated at 3\% per year with the Melody valve. The Sapien valve is a more recently marketed valve used for PPVI.

Methods: We retrospectively included consecutive patients who underwent PPVI at a single center between 2008 and 2016. IE was diagnosed using modified DUKE criteria.

Results: PPVI was performed in 79 patients (Melody valve, 40.5\%; Sapien valve, 59.5\%). Median age was 24.9 y [18.1-34.6]. IE occurred in $8(10.1 \%)$ patients at a median of 1.8 years (min 1.0, $\max 5.6$ ) after surgery. Causative organisms were methicillin-sensitive Staphylococcus aureus $(n=3)$, Staphylococcus epidermidis $(n=1)$, Streptococcus mitis $(n=1)$, Aerococcus viridans $(n=1)$, Corynebacterium striatum $(n=1)$ and Haemophilus influenzae $(\mathrm{n}=1)$. All 8 cases occurred after Melody PPVI $(25.0 \%$ versus $0.0 \%, \mathrm{P}<0.0001)$. The incidence of IE was 5.7\% (95\%CI, 2.9\%-11.4\%) per person-year after Melody PPVI. The Kaplan-Meier cumulative incidence of IE with Melody PPVI was 24.0\% (95\%CI, 12.2-43.9\%) after 4 years and $30.1 \%$ (95\% CI $15.8-52.5 \%$ ) after 6 years, compared to $0.0 \%$ with Sapien PPVI after 4 years $(\mathrm{p}<0.04$ by Log-Rank test). There was a trend toward a higher incidence of IE in the first 20 patients with Melody PPVI (who received prophylactic antibiotics during the procedure only) and in patients who had percutaneous interventions, dental care, or non-cardiac surgery after PPVI.

Conclusion: IE after PPVI may be less common with the Sapien compared to the Melody valve. 
O3-1

Minimum acceptable oxygen delivery during cardiopulmonary bypass in neonates

Di Corte F., Gioia E., Mirabile C., Barbanti C., Pouard P., Bojan M. Anesthesiology and Critical Care Department, Necker-Enfants Malades Hospital, Assistance Publique Hopitaux de Paris, Paris, France

Introduction: The target of an optimal perfusion during cardiopulmunary bypass $(\mathrm{CPB})$ is mantaining the balance between oxygen consumption (VO2) and oxygen delivery (DO2). The level of the critical DO2, where the VO2 becomes supply dependent, has not been explored in neonates who are known to have higher metabolic rates than adults. The present study aims to identify the level of DO2 where the aerobic metabolism switches to anaerobic metabolism during normothermic neonatal CPB.

Methods: In a retrospective cohort of neonates, the DO2 was calculated from the CPB parameters recorded during aortic crossclamping. Several normothermic DO2 thresholds were chosen between $270 \mathrm{ml} \mathrm{min}^{-1} \mathrm{~m}^{-2}$ (critical DO2 in adult CPB) and $380 \mathrm{ml} \mathrm{min}^{-1} \mathrm{~m}^{-2}$ (average value of DO2 in the present population). Assuming that the $\mathrm{VO} 2$ varies with body temperatures, the DO2 thresholds were adjusted for temperatures recorded during CPB. The observed DO2-time integrals were calcultated below the temperature-adjusted threshold (Figure). Hyperlactatemia $(>3.3 \mathrm{mmol} / \mathrm{L})$ measured off-bypass was used to identify the imbalance between DO2 and VO2. The ability of the different DO2 thresholds to predict hyperlactatemia off-bypass was explored using the ROC methodology.

Results: Overall, 8356 time points were analysed in 75 patients. Both the observed DO2-time integrals below the 350 and $360 \mathrm{ml} \mathrm{min}^{-1} \mathrm{~m}^{-2}$ DO2 thresholds were discriminant for hyperlactatemia off-bypass, with a ROC areas of $0.633,95 \%$ CI $0.502-0.763$ and $0.666,95 \%$ CI $0.538-0.793$, respectively. When the DO2 was maintained below $360 \mathrm{ml} \mathrm{ml} \mathrm{min} \mathrm{m}^{-1} \mathrm{~m}^{-2}$ for more than $21 \mathrm{~min}$ or when DO2 was mantained below the $350 \mathrm{ml} \mathrm{min}^{-1} \mathrm{~m}^{-2}$ threshold for more than 17 minutes the proportion of patients with hyperlactatemia concentration exceeded $70 \%$.

Conclusions: When the DO2 level is mantained below $350-360 \mathrm{ml} \mathrm{min}^{-1} \mathrm{~m}^{-2}$ during normothermic cardiopulmonary bypass in neonates, there is a high risk to induce anaerobic metabolism, as assessed by hyperlactatemia off-bypass. Several limitations related to the study design do not allow the statement on whether this is the critical DO2 level in neonatal CPB. However, $350-360 \mathrm{ml} \mathrm{ml} \mathrm{min}{ }^{-1} \mathrm{~m}^{-2}$ is likely to represent the minumum acceptable DO2 required on normothermic neonatal bypass in order to maintain aerobic metabolism.

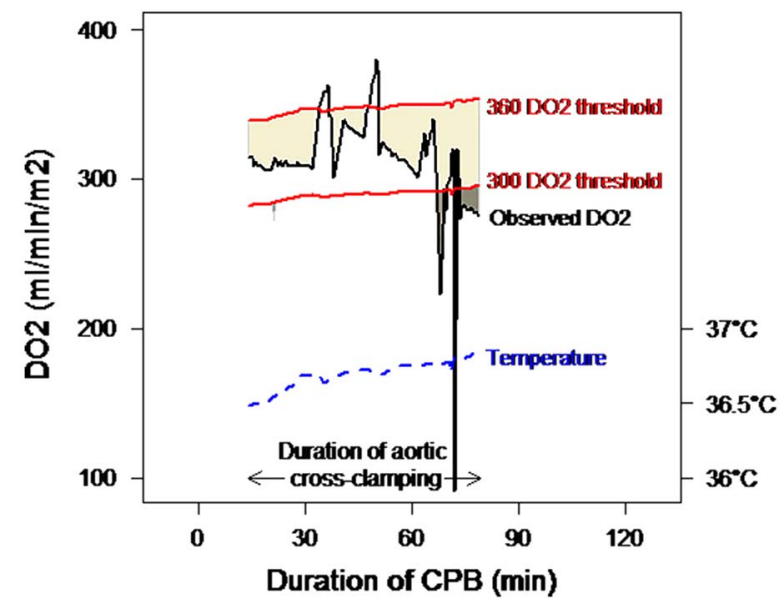

O3-2

Impact of Cardiopulmonary Bypass Time on Motor, Development in Children and Adolescents with Congenital Heart Disease

Häcker A.-L. (1,2), Reiner B. (1,2), Weberruss H. (1), Hager A. (2), Oberhoffer R. (1,2), Ewert P. (2), Müller J. (1,2)

Chair of Preventive Pediatrics, Technical University of Munich, Munich, Germany (1); Department of Pediatric Cardiology and Congenital Heart Disease, German Heart Centre Munich, Technical University of Munich, Munich, Germany (2)

Introduction: Heart surgeries requiring low-flow cardiopulmonary bypass increase the risk of long-term brain impairments and foster neurologic and fine motor deficits in children with congenital heart disease (CHD). This study analyzes the association between the accumulated cardiopulmonary bypass time $(\mathrm{CPB})$ and the motor development in children with CHD.

Patients and Methods: From July 2014 to July 2016 motor development of 504 children with various CHD (190 females, $13.1 \pm 3.1$ years old) was assessed by five tasks (FITNESSGRAM $\left.{ }^{\circledR}\right)$, converted to a motor score and compared to a recent healthy reference cohort. 161 (31.9\%) children with CHD exhibit impaired motor development defined by a score lower than one standard deviation compared to the reference.

Results: 276 of those children had $1.9 \pm 1.1$ cardiopulmonary bypass surgeries with an accumulated CPB time of $179 \pm 115$ minutes. In multivariable logistic regression, every increase by 10 minutes of $\mathrm{CPB}$ time resulted in a $6 \%(\mathrm{OR}=1.006,95 \%$ $\mathrm{CI}=1.002-1.010, \mathrm{p}=.002)$ increase in risk for impaired motor development. Dividing the accumulated CPB time into several shorter surgeries decreased the risk $(\mathrm{OR}=0.602,95 \% \mathrm{CI}=$ $0.376-0.964, p=.008)$. Moreover, compared to patients with left heart obstruction, patients with isolated shunt had a 1.9-fold $(p=.037)$, right heart obstruction 2.1-fold $(p=.024)$, and Fontan circulation 4.0-fold $(\mathrm{p}<.001)$ increased risk.

Conclusion: Longer accumulated CPB time increased the risk of an impairment in motor development, especially when long $\mathrm{CPB}$ time results from fewer surgeries. To minimize potential impairment, shortening of $\mathrm{CPB}$ time or usage of alternative procedures is recommended.

\section{O3-3}

New insight in edema formation after Fontan surgery; the contribution of osmotic pressure in plasma and interstitium

Indrebo M.M.(1,2), Berg A.(3,4), Guthe H.J.T.(3), Holmstrom H. (1,2), Wiik H.(4), Norgård G.(1)

Oslo University Hospital, Oslo, Norway (1); University of Oslo, Oslo, Norway (2); Haukeland University Hospital, Bergen, Norway (3); University of Bergen, Bergen, Norway (4)

Introduction: Despite improved result of Fontan surgery, fluid balance and edema formation are major challenges. Fontan circulation is dependent on elevated central venous pressure, however, it may lead to fluid accumulation in the tissues. While increases in hydrostatic pressure will favour transmicrovascular filtration, fluid accumulation is restricted by safety factors to limit edema formation. We hypothesize that chronically increased hydrostatic pressure leads to adaptive changes in plasma and interstitial colloid osmotic pressures. In this study we have measured the colloid osmotic pressure (COP) of plasma and interstitial fluid in children undergoing TCPC procedure.

Methods: This study had a prospective, descriptive design. 11 children (age 2,8 -4,9 year) undergoing TCPC surgery were 
included. Interstitial fluid and blood samples were obtained during the procedure. Interstitial fluid was harvested by nylon wicks when the child was in general anesthesia. Before surgery two wicks were implanted subcutaneously. One wick was removed before start of cardiopulmonary bypass (CPB), second wick was removed at the end of surgery. A third wick was implanted at the end of operation and removed after one hour. A fourth wick was placed and removed 24 hours later. Blood samples were taken at wick removal. Plasma and interstitial fluid were measured by a colloid osmometer.

Results: Before the procedure plasma COP ( $\mathrm{mmHg}$ ) was $20,0 \pm 0,8$ and interstitial COP $14,1 \pm 1,1$. During surgery with the use of $\mathrm{CPB}$ the colloid osmotic pressure gradient was reduced with a subsequent increase in osmotic pressure gradient 24 hours after start of surgery. The plasma COP was slightly reduced throughout the procedure with a normalization after surgery while interstitial COP slightly increased during the use of CPB with a significantly drop after surgery.

Conclusions: Fontan surgery with the use of cardiopulmonary bypass changes the colloid osmotic pressure gradient that may influence edema formation. The significant increase in gradient after surgery will favor transport of fluid into the capillaries and may be an important factor to counteract edema formation seen in these patients.

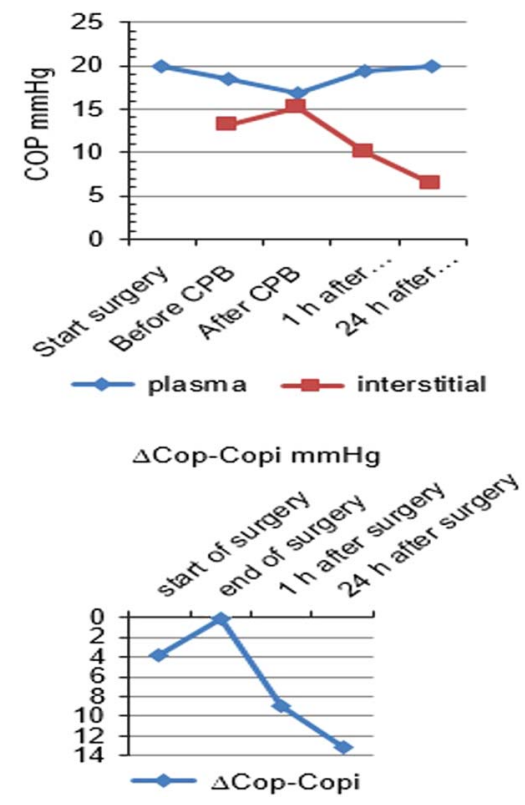

\section{O3-4}

Surgical Outcome Of Atrioventricular Valve Replacement For Children With A Functional Single Ventricle

Ide Y., Murata M., Ito H., Kanno K., Imai K., Ishido M., Fukuba R., Sakamoto K.

Mt. Fuji Shizuoka Children's Hospital, Shizuoka, Japan

Objectives: Atrioventricular valve (AVV) regurgitation remains a big issue in the treatment of children with a functional single ventricle (fSV). Some patients require AVV replacement (AVVR) during the early stage of life for uncontrolled regurgitation even after aggressive AVV plasty. We sought to clarify the current outcome of AVVR for children with fSV.

Methods: A retrospective review of $13 \mathrm{fSV}$ patients who underwent AVVR at a tertiary pediatric cardiac center from January 1999 to
March 2016. There were 8 (62\%) Heterotaxy and 2 (15\%) HLHS patients. Nine (69\%) had common AVV morphology. All AVVRs were performed by a single surgeon after at least one time (1-4) of AVV plasty and 9.6 months (7days-7.9years) after the final AVV plasty. AVVR was indicated for an AVV regurgitation more than moderate grade at Glenn stage (3), 2nd. interstage (5), Fontan stage (1) and post-Fontan stage (4). Current follow-up is obtained in all of the surviving patients.

Results: Median age and body weight at AVVR was 2.7 $(0.28-16.7)$ years and $8.7(3.5-33.5) \mathrm{kg}$, respectively. Replaced valves were bio-prosthesis in 3 and mechanical valve $(\mathrm{MeV})$ in 10 . Valve size ranged from 16 to $29 \mathrm{~mm}$. There were 6 mortalities (inhospital;4 late;2) due to heart failure (4), stuck valve (1), thrombosis (1) during $3.7+/-4.3$ years of follow up period. All 3 bioprosthesis were replaced to $\mathrm{MeV}$ due to progressive stenosis 2.2 (1.1-2.4) years after the first AVVR. Two MeV were stuck and replaced 5.0 and 0.80 years after implantation, respectively. Overall survival after the first AVVR was 61\% (1year) and 53\% (5 years). Freedom from re-intervention was 73\% (1year) and 22\% (5 years). Lower body weight at AVVR was involved in higher in-hospital mortality $(6.5 \mathrm{~kg}$ vs $14.7 \mathrm{~kg}, \mathrm{p}=0.0492)$.

Conclusions: The results of AVVR in fSV patients are disappointing with high mortality and morbidity. Bio-prosthesis degenerates rapidly and requires early re-AVVR, while $\mathrm{MeV}$ has high incidence of stuck valve and thrombosis. AVVR should be put off as late as possible if ventricular function is maintained properly by AVV plasty.

\section{O3-5 \\ Outcomes of Modified Konno Procedure in Obstructive Hypertrophic Cardiomyopathy in Children Laredo M., Khraiche D., Raisky O., Bonnet D., Vouhe P.R. Unité Médico-Chirurgicale de Cardiologie Congénitale et Pédiatrique, Centre de référence Malformations Cardiaques Congénitales Complexes- M3C, Hôpital Necker Enfants Malades, APHP, Université Paris Descartes, Sorbonne Paris-Cité, Paris, France.}

Objectives: Modified Konno procedure provides definitive relief of obstruction in children with complex congenital left ventricular outflow tract (LVOT) stenosis. The objective was to evaluate its results in children with obstructive hypertrophic cardiomyopathy (OHCM).

Methods: We retrospectively analyzed the records of the 68 consecutive patients who underwent the modified Konno ventriculoplasty for OHCM in our center between 1991 and 2015.

Results: The median age at surgery was 8 years $(1.2$ month -19 years). Familial history of HCM was present in 27 (40\%) children. Idiopathic HCM and RASopathy were the most frequent causes, with $47(70 \%)$ and $19(28 \%)$ patients, respectfully. $36(53 \%)$ patients were NYHA III or IV. $58(95 \%)$ patients were on $\beta$-blocker therapy. Mean septal thickness was $22.8 \pm 7.5 \mathrm{~mm}$ (median z-score: +15.9). Mean maximal LVOT gradient was $94 \pm 39 \mathrm{mmHg}$. An associated procedure was necessary in $25 \%$. Following procedure, in-hospital mortality rate was 5/68 (7\%) and was associated with age at procedure less that 1-year, low weight, Noonan syndrome and right ventricular outflow tract obstruction. Occurrence of post-operative complete heart block (CHB) was $13 \%$ and was not significantly associated with any baseline characteristic. Median follow-up time was 5.3 years. Freedom from death, heart transplantation, resuscitated sudden cardiac death and implantable cardioverter-defibrillator appropriate shock in hospital survivors was $70 \%$ at 20 -year follow-up and was associated to the presence of an associated lesion requiring a supplementary concomitant procedure $(\mathrm{HR}=8.78, \mathrm{p}=0.01)$ and to baseline 
septal thickness $(\mathrm{HR}=1.19, \mathrm{p}=0.01)$ in multivariate analysis. 20 -year cumulative incidence of reintervention was $24 \%$ and was associated to pre-procedure mitral valve dysplasia in multivariate analysis $(\mathrm{HR}=4.75, \mathrm{p}=0.049)$. All patients had long-standing complete relieve of LVOT obstruction, with a mean residual gradient of $11 \pm 6.6 \mathrm{mmHg}$. At last evaluation, functional status was dramatically improved, with $81 \%$ of patients being NYHA I. Yet $22 \%$ had signs of diastolic impairment, which was associated to baseline septal thickness $(\mathrm{HR}=1.12, \mathrm{p}=0.04)$.

Conclusions: The Modified Konno procedure allowed definitive obstruction relief in pediatric OHCM and dramatic improve of functional status, at the cost of a high rate of $\mathrm{CHB}$. Event-free survival after surgery in this pediatric population with severe hypertrophy was good.

\section{O3-6}

Recurrent Coarctation after Surgical Repair for Coarctation of the Aorta- A-15 Year Retrospective Study Hansson K. (1), Malm T. (2), Johansson Ramgren J. (2), Liuba P.(1) Pediatric Cardiology (1) and Cardiac Surgery (2), Skåne University Hospital, Lund, Sweden

Background: Coarctation of the aorta (CoA) accounts for 5-8\% of all congenital heart defects. Although the current treatment strategies have evolved during the past decades, some patients still develop recoarctation with subsequent need for reintervention. We sought to assess the prevalence of reinterventions among children born between 2001 and 2015 with earlier CoA repair at the Skåne University Hospital.

Method: We conducted a retrospective survey of all children born between January 2001 and June 2015 who were surgically treated for CoA at our center. The patients were identified from The Swedish Registry of Congenital Heart Disease and from the surgical database. Data on birth characteristics, age at diagnosis, age at surgical or transcatheter repair, type of repair, blood pressure gradient arm-leg after the primary repair, and the number, type and age at reinterventions due to recoarctation were gathered from the hospital's digital database.

Results: A total of 288 patients (median age at surgery: 15 days) were identified. Thirteen $\%(n=38)$ of patients needed one or more reinterventions (surgical: $n=3$; balloon dilatation: $n=35$ ). The majority of these patients $(n=33)$ underwent their first reintervention (surgical: $\mathrm{n}=3$; balloon dilatation: $\mathrm{n}=30$ ) during the first 2 years of life. No association was found between $>20 \mathrm{~mm}$ $\mathrm{Hg}$ arm-leg blood pressure at hospital discharge and the need for later reintervention $(\mathrm{p}=0.3)$.

Conclusion: Approximately 1 of 10 patients who undergo surgical repair for $\mathrm{CoA}$ require reintervention. In most cases, reinterventions after surgery are needed during the first 2 years of life. Arm-leg blood pressure gradient early after CoA repair does not predict risk for reintervention.

\section{O4-1}

Echocardiographic Nomograms for Upper Abdominal Aorta Pulsed-Doppler Parameters and Size in Healthy Caucasian Children

Cantinotti M. (1), Franchi E. (1), Scalese M. (1), Viacava C. (1), Assanta N. (1), Crocetti M. (1), Marotta M. (1), Kutty S. (2), Spadoni I. (1)

1Fondazione G. Monasterio CNR-Regione Toscana, Massa and Pisa, Italy 2 University of Nebraska Medical Center/Children's Hospital and Medical Center, Omaha, NE, USA

Background: Abdominal aorta pulsatility and blood flow patterns are important indicators for the diagnosis, as well as estimation of disease severity in congenital heart disease. Despite their routine use, reference values for these indexes are lacking.

Methods: We prospectively studied abdominal aorta pulsed-wave Doppler systolic peak velocity, deceleration time, systolic and diastolic duration, and two-dimensional vessel diameters in systole and diastole in healthy Caucasian Italian children. Heteroscedasticity was accounted for by White or Breusch-Pagan test. Age, weight, height, heart rate (HR) and body surface area (BSA) were used as independent variables in different analyses to predict the mean values of each measurement. Structured Z scores were then computed.

Results: In all, 853 subjects (age 0 days- 17 years; $45 \%$ females; BSA $0.12-2.12 \mathrm{~m} 2$ ) were studied. The Haycock formula was used when presenting data as predicted values (mean \pm 2 SDs) for a given BSA and within equations relating echocardiographic measurements to BSA. The predicted values and Z-score boundaries have been presented.

Conclusions: We report pediatric echocardiographic nomograms for multiple proximal abdominal aorta parameters including pulsed-wave Doppler peak systolic velocities, deceleration time, systolic-diastolic wave duration and two-dimensional vessel diameter variations. Our results demonstrate significant variations of these functional indexes with age that should be taken into account in clinical practice. At lower ages, steeper and shorter pulsed-wave Doppler peak velocity and limited pulsatility should be expected as physiologic findings.
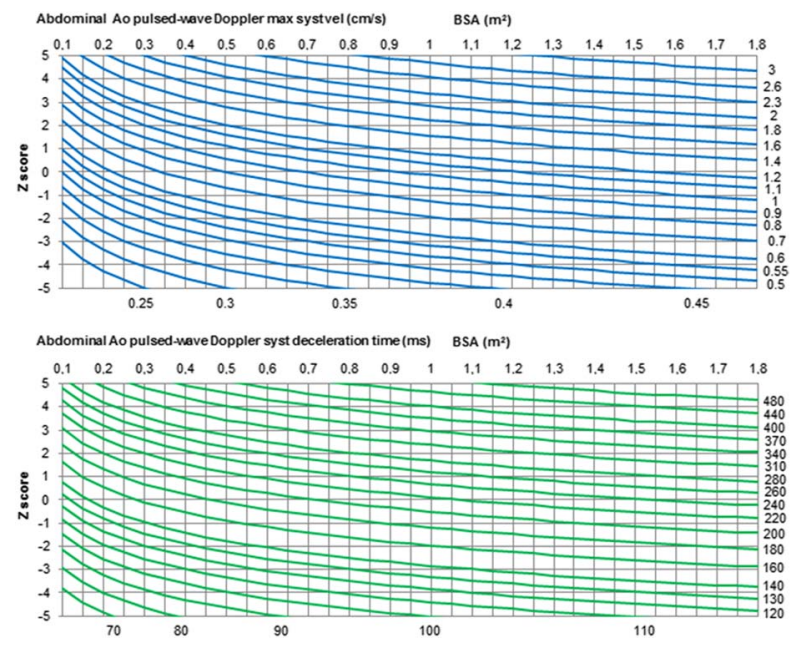

Figure 1.

$z$-score charts of abdominal aorta pulsed-wave Doppler peak systolic velocity and systolic deceleration time.

O4-2

Prognostic Potentialities of Lung Ultrasound Findings in Children Undergoing Cardiac Surgery for Congenital Heart Disease

Cantinotti M. (1), Franchi E. (1), Scalese M. (2), Giordano R. (1), Melo M. (1), Remoli E. (1), Clemente A. (1), Haxiademi D. (1), Moschetti R. (1), Ait Ali L. (2)

Fondazione Gabriele Monasterio CNR-Regione Toscana (1) Massa, Italy. Istituto di Fisiologia Clinica, Massa-Pisa Italy(2)

Background: Lung ultrasound (LUS) is gaining consensus for the diagnosis of pulmonary disease in acute setting. Despite pulmonary complications are very common in pediatric cardiac surgery. The use of LUS remain limited. Our aim was to test the prognostic potentialities of LUS in pediatric cardiac surgery. 
Methods: In 120 children (mean age: $2.8+/-4.6$, range: 0.09-20) undergoing pediatric cardiac surgery, LUS examinations have been performed at 12-48 hours, after surgery. For each hemithorax 3 major areas (anterior/lateral/posterior) have been evaluated separately. The presence and the degree of the following have been evaluated: 1) pleural effusion and atelectasis, classified with a score from 0 to 3 (none/trivial, small, moderate, and large) 2) pulmonary congestion assessed as percentage of B-lines (from 0 to $100 \%$ in each segment and globally). Primary end-point were: time to extubation (TE) and time of intensive care unit (ICU).

Results: B lines were present in all post-operative patients (Table 1). Right pleural effusion was diagnosed in $28 \%(20 \%$ mild, $6 \%$ moderate); and left pleural effusion in $42 \%$ (36\% mild, $5 \%$ moderate, $1 \%$ severe). Atelectasis in the right lung were found in $64 \%$ (47.4\% mild, $12 \%$ moderate, $7 \%$ severe), while left atelectasis in $75 \%$ (50\% mild, $18 \%$ moderate, $9 \%$ severe).

At univariate analysis significant correlation of B-lines with time to extubation and ICU time were noted. The correlations were significant in all scanning areas with only slightly differences among segments (Table-1).

Severe left atelectasis was positively associated with extubation time $(\mathrm{p}=0.05)$.

At multivariate analysis linear regression: the percentage of $\mathrm{B}$ lines in the anterior areas was identified as an independent variables for extubation time (95\% CI: 0,16-1,79, p=0 019)

Conclusions: LUS findings, particularly the degree of pulmonary congestion, may have a significant prognostic relevance in pediatric cardiac surgery. Further studies are required to validate and reinforce these data.

Table 1. B lines at 12-48 hours: incidence and correlation with outcome.

\begin{tabular}{lccc}
\hline B Lines & Percentage & Time ICU & $\begin{array}{c}\text { Time to } \\
\text { extubation }\end{array}$ \\
\hline Global score & $57 \%+/-21$ & $\begin{array}{c}\mathrm{r}=0,17, \\
\mathrm{p}=0,09\end{array}$ & $\begin{array}{c}\mathrm{r}=0.25, \\
\mathrm{p}=0.02\end{array}$ \\
$\begin{array}{c}\text { Posterior } \\
\text { segments }\end{array}$ & $55,7 \%+/-28$ & $\mathrm{r}=0,1$, \\
$\mathrm{p}=0,2$ & $\mathrm{r}=0,25$, \\
$\begin{array}{c}\text { Anterior } \\
\text { segments }\end{array}$ & $41 \%+/-19$ & $\mathrm{r}=0,0,32$, \\
$\mathrm{p}=0,01$ & $\mathrm{r}=0,2, \mathrm{p}=0.01$ \\
$\begin{array}{c}\text { Lateral } \\
\text { segments }\end{array}$ & $44 \%+/-19 \begin{array}{c}\mathrm{r}=0,2, \\
\mathrm{p}=0,03\end{array}$ & $\mathrm{r}=0.22$, \\
\hline
\end{tabular}

O4-3

\section{Giant aneurysms: a gender-specific complication of} Kawasaki disease?

Kuipers I.M. (2), Dietz S.M. (1), Kuipers I.M. (2), Tacke C.E.A. (1), Koole J.C.D. (1), Hutten B.A. (3), Kuijpers T.W. (1).

Department of Pediatric Hematology, immunology and infectious diseases, Emma Children's Hospital, Academic Medical Centre (AMC), Amsterdam, The Netherlands (1); Department of Pediatric Cardiology, Emma Children's Hospital, AMC, Amsterdam, The Netherlands (2); Department of Clinical Epidemiology, Biostatistics and Bioinformatics, AMC, Amsterdam, the Netherlands (3).

Background: Kawasaki disease (KD) is a pediatric vasculitis of unknown origin. Its main complication is the development of coronary artery aneurysms (CAA) with giant CAA at the end of the spectrum. Methods: In this cohort study, we evaluated the association between patient characteristics and the development of giant CAA based on z-scores. Multivariable, multinomial logistic regression analysis was used to identify variables associated with giant CAA.
Results: A total of $301 \mathrm{KD}$ patients, comprising of 216 patients without enlargement, 45 with small-sized, 19 with medium-sized, and 21 with giant CAA with all echocardiographies at our center were retrospectively included. Remarkably, $95 \%$ of patients with giant CAA were boys. In addition to 'no/late intravenous immunoglobulin (IVIG) treatment', 'male gender' (OR 15.56, 95\% CI 1.86-130.07), 'age <1 year' (OR 8.06, 95\% CI 2.56$25.35)$, and 'IVIG re-treatment (6.38, 95\% CI 1.86-21.88)' were significantly associated with an increased risk of giant CAA, with patients without enlargement as reference. Compared to patients medium-sized CAA, 'IVIG re-treatment' was significantly associated with giant CAA. The majority of giant CAA continued to increase in size during the first 40 days.

Conclusions: We identified risk factors associated with an increased risk of giant CAA. The difference in variables between the giant CAA group and the other CAA subgroups suggests a separation between patients with the treatment-resistant giant CAA and the other IVIG-responsive patients, in which gender may be factored as a most relevant genetic trait. The increase in size during the first 2 months indicates the need for repeated echocardiography.

\section{O4-4}

Regression and complications of z-score based giant aneurysms in a Dutch cohort of Kawasaki disease patients Kuipers I.M. (2), Dietz S.M. (1), Kuipers I.M. (2), Koole J.C.D. (1), Breur J.M.P.J. (3), Fejzic Z. (4), Frerich S. (5), Dalinghaus M. (6), Roest A.A.W. (7), Hutten B.A. (3), Kuijpers T.W. (1)

Department of pediatric hematology, immunology and infectious diseases, Emma Children's hospital, Academic Medical Centre (AMC), Amsterdam, The Netherlands (1); Department of pediatric cardiology, Emma Children's hospital, AMC, Amsterdam, The Netherlands (2); Department of pediatric cardiology, Wilhelmina Children's hospital, University Medical Centre Utrecht, Utrecht, The Netherlands (3); Department of pediatric cardiology, Amalia Children's hospital, Radboud University Medical Centre, Nijmegen, The Netherlands (4); Department of pediatric cardiology, Academic hospital Maastricht, Maastricht, The Netherlands (5); Department of pediatric cardiology, Sophia Children's hospital, Erasmus Medical Centre, Rotterdam, The Netherlands (6); Department of pediatric cardiology, Willem-Alexander Children's hospital, Leids University Medical Centre, Leiden, The Netherlands (7); Department of Clinical Epidemiology, Biostatistics and Bioinformatics, AMC, Amsterdam, the Netherlands (8)

Introduction: Kawasaki disease (KD) is a pediatric vasculitis. Its main complication is the development of coronary artery aneurysms (CAA), with giant CAA at the end of the spectrum. We evaluated regression and event-free rates in a non-Asian cohort of patients with giant CAA using the current $z$-scores adjusted for bodysurface area instead of absolute diameters.

Methods: KD patients with giant CAA (z-score $\geq 10$ ) visiting our outpatient clinic between January 1999 and September 2015 were included. Patient characteristics and clinical details were extracted from medical records. Regression was defined as all coronary arteries having a z-score of $\leq 3$. A major adverse event was defined as cardiac death, myocardial infarction, cardiogenic shock or any coronary intervention. Regression-free and event-free rates were calculated using the Kaplan-Meier method.

Results: We included 52 patients with giant CAA of which 45 had been monitored since the acute phase. The 1-year, 2-year and 5year regression-free rates were $0.86,0.78$ and 0.65 , respectively. The 5-year, 10-year and 15-year event-free rates were $0.79,0.75$ and 0.65 , respectively. Four children whose CAA would not have been classified as 'giant' based on absolute diameters instead of z-scores, had experienced an event during follow-up. 
Conclusions: We found a high percentage of children in whom the lumen of giant CAA completely normalized. Four children not classified as 'giant' based on absolute diameters with z-scores of $\geq 10$, experienced a cardiac event. Hence, the use of $z$-scores seems to be justified.

\section{O4-5 \\ How long does it take for coronary artery aneurysms to resolve after Kawasaki disease? \\ Hillary C., Jaber D., Khader Y., Tulloh R.M.R. \\ Department of Congenital Heart Disease, Bristol Royal Hospital for Children, Bristol, UK.}

Introduction: Kawasaki disease (KD) is the commonest acquired heart disease in the Western world, with incidence 9.2/100,000 under 5 years in the UK. 25\% will have coronary artery aneurysms $(\mathrm{CAA}+)$ despite intravenous immunoglobulin therapy. At the current time, the time course for outcome of smaller aneurysms is not clear in the Western Europe population.

Methods: We retrospectively studied all children with a new diagnosis of KD from 1.1.00 to 31.7.16 in our cardiac network (population 5.8 million). Demographics (including height and weight) and echocardiographic data were recorded, along with the coronary size at diagnosis and at each follow-up. $Z$ scores were recorded or calculated and those with $z \geq 2.5$ were classified $\mathrm{CAA}+$. Data is presented as median (range), $\mathrm{p}<0.05$ was significant (Kruskal-Wallis).

Results: 443 patients (281 boys 63.6\%) with diagnosis of KD, presented at 42 months (18 days - 15 years). 420/426, where treatment was known, received immunoglobulin along with high dose aspirin. $92(20.7 \%)$ had CA involvement. For CAA + vs CAA- there were more boys $(67 \%$ vs $62 \%, p=0.04)$, and they were younger (453 vs 1440 dys, $\mathrm{p}=0.00021$ ). 81 had involvement of the left main CA and 59 of the right CA. 28 had bright coronaries but with $z<2.5$ all resolving in 124 (30-404) days. All but $1(2.9 \%)$ of 37 with $z$ between 2.5 and $<5$ resolved in $284(20-$ 5534) days. Of those 17 with $z$ between 5 and <10, 29\% did not resolve but the rest did in 456 (127-2508) days. Of those 10 with giant aneurysms of $z \geq 10,80 \%$ did not resolve and the rest took up to 4831 days to do so. Those who were over 1 year with $\mathrm{z}$ score $>10$ never resolved, whereas some of the younger children grew fast, helping to normalise the CAA + .

Conclusions: We present a large population based series of KD and show, for the first time in Europe, the time course of CAA + resolution at different $z$ scores. Such CAA + are more likely to be severe in younger boys, but even small aneurysms may persist in the long term.

\section{O4-6 \\ Echocardiographic predictors of survival in paediatric pulmonary hypertension}

Lammers A.E. (1), Diller G.P. (2), Haworth S. G. (3), Marek J.(3), Moledina S.(3)

University Hospital Münster, Paediatric Cardiology, Münster, Germany (1); Universitätsklinikum Münster, ACHD Clinic, Münster, Germany (2), Paediatric Cardiology, Great Ormond Street Hospital for Children, London, UK (3)

Introduction: Transthoracic echocardiography (TTE) remains the most important non- invasive tool for clinical-follow up of patients with pulmonary hypertension $(\mathrm{PH})$. Data on which echocardiographic parameters are predictive of survival in children are sparse.
Methods: Fourty-one children (age $8.0 \pm 5$,6 years, 44\% male, 58\% WHO class III) with confirmed PH and structurally normal hearts were included (66\% with idiopathic PH). All underwent a standardized, protocolized TTE at a tertiary centre for paediatric $\mathrm{PH}$. A combined end-point of death and transplantation was used.

Results: During a median follow-up of 4.4 years [IQR 2.0-9.2 years], 16 patients met the combined endpoint (including 13 deaths). On univariate Cox proportional survival analysis, the following echocardiographic parameters were significantly related to outcome: left ventricular systolic and diastolic eccentricity indices (HRs 1.06; $\mathrm{p}=0.02$ and 1.16; $\mathrm{p}=0.004$, respectively), right ventricular systolic and diastolic diameters (HRs 1.06; $\mathrm{p}=0.02$ and $1.75 ; \mathrm{p}=0.01)$, pulmonary valve dimension $\mathrm{z}$-score (HRs 1.26; $\mathrm{p}=0.02)$, right atrial area $\mathrm{z}$ - score (HR 1.21, $\mathrm{p}=0.002)$ as well as E- and A-wave tissue Doppler velocities on the left ventricular side (HRs 1.25; $\mathrm{p}=0.04$ and $0.80 ; \mathrm{p}=0.04$ ). On multivariable analysis left ventricular diastolic eccentricity index (HR 1.26, $p=0.03)$ and right atrial area $z$-score (HR 1.16, $\mathrm{p}=0.02$ ) remained predictive of outcome (joint c-statistic 0.73 , $\mathrm{p}=0.003)$. In addition, mixed venous oxygen saturation on cardiac catheterization (HR $0.95, p=0.04)$, oxygen saturation in room air (HR 0.85, $\mathrm{p}=0.01)$ and heart rate at rest $(\mathrm{HR} 0.97$, $\mathrm{p}=0.045)$ were predictive of outcome.

Conclusions: Larger right atrial size, as well as the degree of interseptal shift towards the left ventricle (eccentricity index) appear to be particularly associated with adverse outcome in paediatric $\mathrm{PH}$ patients. These parameters were superior to RV function in the current study. These findings underline the importance of interventricular interaction and left-ventricular compromise in patients with PH. Standardized TTE protocols focusing on outcome prediction require further attention and should be integrated into clinical practice. Ideally, these results should be confirmed in larger multi-centre trials.

\section{O5-1 \\ Long-term outcome of patients with congenital heart disease undergoing cardiac resynchronization therapy Kubuš P. (1), Rubáčková Popelová J. (2), Kovanda J. (1), Sedláček K. (3), Janoušek J. (1) \\ Children's Heart Centre, 2nd Faculty of Medicine, Charles University in Prague and Motol University Hospital, Prague, Czech Republic (1); Department of Cardiac Surgery, Na Homolce Hospital, Prague, Czech Republic (2); Cardiology Department, Institute for Clinical and Experimental Medicine, Prague, Czech Republic (3)}

Objectives: Cardiac resynchronization therapy (CRT) is rarely used in patients with congenital heart disease (CHD) and follow-up in available studies is short. We sought to evaluate long-term impact of CRT in patients with CHD.

Methods: Thirty consecutive patients with structural CHD $(\mathrm{N}=28)$ or congenital atrioventricular block $(\mathrm{N}=2)$ aged median 12.9 (IQR 6.5-18.2) years at CRT-P implantation were followed for median 9.0 (IQR 4.5-11.4) years on CRT. CRT was performed for systemic left ventricular $(\mathrm{LV}, \mathrm{N}=12)$ and right $(\mathrm{RV})$ or single $(\mathrm{SV})$ ventricular $(\mathrm{N}=18)$ failure and was associated with additional cardiac surgery in 13 patients. CRT response was defined as an increase in systemic ventricular ejection fraction or fractional area of change (FAC) by $>10$ points and improved or unchanged NYHA class at the end of follow-up. Actuarial survival probability was calculated for 5 and 10 years after CRT implantation.

Results: Freedom from cardiovascular death or heart failure hospitalization was 92.0 and $82.3 \%$, respectively. No patient underwent heart transplant. Surgical revision of the pacing system 
was performed in 3 patients and pacing system extraction due to infection in 1 patient. CRT therapy was terminated in other 5 patients due lead dysfunction (exit block). Freedom from CRT complications leading to surgical system revision (elective generator replacement excluded) or therapy termination was 81.4 and $70.1 \%$ at 5 and 10 years, respectively. Overall probability of an uneventful therapy continuation was 74.6 and $56.3 \%$, respectively. Upgrade to CRT-D was performed in 1 patient. Ejection fraction or FAC changed from median 29.5\% (IQR 22.3-35.0) before CRT to median 40.3\% (IQR 32.0-54.0; $<<0.001$ ) at the end of follow-up. NYHA class improved from median 2.0 to 1.5 $(\mathrm{p}=0.002)$. CRT response tended to be more frequent in systemic LV (9/12 patients) than RV or SV (6/18 patients, $\mathrm{p}=0.060)$.

Conclusions: Long-term CRT in patients with CHD is associated with acceptable survival and sustained improvement of systemic ventricular function in $50 \%$ of patients. Probability of device complications necessitating surgical revision or therapy termination is however high.

\section{O5-2}

\section{Use of implantable loop recorders in children with syncope}

Polyakova E., Trofimova T., Shkolnikova $M$.

Children's Center of cardiac arrhythmias, Moscow, Russian Federation

Prior studies have demonstrated a high value of the implantable loop recorders (ILR) in the diagnostic work-up in adults with unexplained syncope $(\mathrm{UnS})$ and palpitations. Evidence of indications and benefits of ILR in the pediatric population is still insufficient. Patients' and family history, physical examination are unable to determine the mechanism of syncope in $15-20 \%$ of children with recurrent UnS to avoid risks associated with arrhythmogenic syncope. ILR allow prolonged monitoring of heart rhythm for periods from a few days to several years, making it possible to detect inherited arrhythmias in children with UnS.

Methods: ILRs were implanted in 273 children (49\% boys) aged 2.5 to $17(12.2 \pm 4.4)$ with recurrent UnS. Incidence of syncope varied from once a week to once a year. Personal and family history, physical examination including ECG, stress test, holter monitoring, tilt-table and other tests were unable to identify the cause of syncope.

Results: For 242 pts ILR monitoring was completed due to the symptom-rhythm correlation or detection of arrhythmia (50\% clinically positive cases) or due to the end of 36-mnth follow-up. Arrhythmogenic syncope were diagnosed in 29\% (71 from 242). $40 \%$ reveal confirmed positive cases were caused by arrhythmias. Among arrhythmic events $94 \%$ are related to bradyarrhythmias, 46 caused by severe asystole and 13 caused by AV block. Ventricular fibrilation was diagnosed only in 3 pts with long QT syndrome. 60 pts had asystole lasting 3 to $30 \mathrm{~s}, 35$ pacemakers and 3 cardioverter defibrillators were implanted. The frequency of complications was $1.8 \%$.

Conclusion: The efficiency of ILR monitoring in children with $\mathrm{UnS}$ is $50 \%$. Arrhythmogenic syncope were diagnosed in $29 \%$ cases (mostly bradicardia). So, in children ILR technology is an efficient and secure method for diagnosing the cause of UnS.

\section{O5-3}

Evaluation of implantable cardioverter defibrillator therapy in preventing sudden cardiac death in paediatric patients in the Netherlands

Nanhekhan D.F.F. (1), Kammeraad J.A.E. (2), Blom N.A. (1), Van de Heuvel F.(3), Blank C. (4), Reimer A. (5), Clur S.A. (6), Rammeloo L.A.J. (7), Ten Harkel A.D.J. (1)
Leiden University Medical Center, Leiden, The Netherlands (1); Erasmus MC-Sophia, Rotterdam, The Neterlands (2); University Medical Center Groningen, Groningen, The Netherlands (3); University Medical Center Utrecht, Utrecht, The Netherlands (4); University Medical Center Nijmegen, Nijmegen, The Netherlands (5); Amsterdam Medical Center, Amsterdam, The Netherlands (6); Free University Hospital, Amsterdam, The Netherlands (7)

Background: Implantable Cardioverter Defibrillator (ICD) therapy has shown to be effective in the prevention of sudden cardiac death (SCD) for more than two decades. Lead integrity problems, inappropriate shocks and infections are main concerns of ICD therapy particularly in children.

We aimed to analyse our experience with ICD treatment in children.

Methods: A multicentre retrospective review of all patients below 18 years at implant was performed. Primary outcomes were mortality, re-intervention, appropriate and inappropriate shock and the occurrence of complications.

Results: Between 1990 and 2016, 170 (102 male) children in the Netherlands underwent ICD implantation. The median age at implantation was 13 years (9-16). In 93 patients the ICD was implanted as primary prevention. Underlying cardiac diseases were primary electrical disease (48\%), congenital heart disease (6.5\%) and cardiomyopathy (40\%). A transvenous ICD system (TV-ICD) was implanted in $65 \%(\mathrm{n}=110), 24 \%$ had a non-transvenous system (NTV-ICD) and $11 \%$ had a subcutaneous ICD (S-ICD). The follow-up time was 5 (2-9) years. 11 patients died (6.5\%) due to cardiac disease, 9 had a heart transplant (5\%) and 10 had the ICD removed (6\%). 71 patients experienced complications (42\%) including infection $(\mathrm{N}=17)$, lead dysfunction $(\mathrm{N}=68)$ and pleural effusion $(\mathrm{N}=5)$. In patients $<12$ years the occurrence of complications was significantly higher $(56 \%)$ compared to patients $\geq 12$ years $(32 \%)(p=0.002)$. More patients with NTV ICD systems experienced complications (67\%), compared to TV systems $(35 \%)$ and S-ICDs $(28 \%)(\mathrm{p}=0.001) .53$ patients $(32 \%)$ experienced appropriate shocks; 26 patients $(16 \%)$ experienced inappropriate shocks. The number of patients with inappropriate shocks was significantly less compared to earlier reports. The main cause of inappropriate shocks were lead fracture $(\mathrm{N}=5)$, $\mathrm{T}$-wave over sensing $(\mathrm{N}=5)$ and supraventricular tachycardia $(\mathrm{N}=11)$. Less patients with a cardiomyopathy had inappropriate shocks (6\%) compared to patients with congenital heart disease $(27 \%)$ and primary electric disease $(23 \%)(\mathrm{p}=0.006)$.

Conclusion: In the paediatric ICD patients complications are more likely to happen in patients $<12$ years and those with a NTV system. Compared to earlier reports there were significantly less inappropriate shocks. Patients with a cardiomyopathy have less inappropriate shocks.

\section{O5-4}

s-ICD implantation in the young: low BMI as a predictor of surgical complications

Pazzano V., Silvetti M.S., Verticelli L., Battipaglia I., Grifoni G., Saputo F.A., Remoli R., Drago F.

Paediatric Cardiology and Arrhythmology Unit, Medical-Surgical Department of Paediatric Cardiology, Bambino Gesù Children Hospital, Rome, Italy

Introduction: The use of Implantable Cardiac Defibrillators (ICDs) to prevent sudden cardiac death from malignant ventricular arrhythmias is increasing in children. An entirely subcutaneous system (s-ICD) is an option that offers many advantages. However, larger size of the device can cause complications in small patients. 
Methods: This is an observational study, from a single-centre, on s-ICD implantation in children and in young patients with Congenital Heart Disease (CHD). Data were collected prospectively in an Italian Registry, and analysed retrospectively. Values are reported as median (range).

Results: Between 2013 and 2016, 17 patients were considered for s-ICD implantation. Five (29\%) did not pass ECG screening criteria (broad QRS, high T wave), twelve underwent implantation. Nine (75\%) were females, age was 15y (10-29) with 8 (67\%) $<18$ y; weight was $55 \mathrm{~kg}$ (38-82), BMI 21.4 (18.2-27.9). Six patients $(50 \%)$ had a cardiomyopathy ( 3 ARVC, $3 \mathrm{HCM}$ ), five $(41.6 \%)$ a surgically repaired CHD, and one (8.3\%) LQTS. One was implanted in secondary prevention. The first six patients received a s-ICD 1010SQ-RX device, in the following six an Emblem s-ICD was implanted. In the first six cases, three incisions (axillary, xifoid and superior sternal) were performed, in the following six, superior incision was avoided. Shock zone was set at 210-220 bpm, with a conditional zone at $180 \mathrm{bpm}$.

During 12 (1-32) month follow-up, four patients (41.6\%) experienced device-related surgical complications: three upper sternal wound dehiscence (one effectively treated, two with system explantation for infection) and one ICD pocket infection requiring explantation. All patients needing explantation had a BMI $<20$, which showed $100 \%$ sensitivity, $89 \%$ specificity in predicting this outcome. All wound dehiscences occurred after procedures performed with three incisions $(3 / 6)$. One patient had an appropriate shock, and one had inappropriate shock due to T wave oversensing.

Conclusions: Paediatric patients seem at high risk of S-ICD pocket complications, with a BMI $<20$ representing an important risk factor. A lower number of incisions seems to reduce the incidence of surgical complications. Of note, a relevant number of candidates do not fulfil ECG screening criteria.

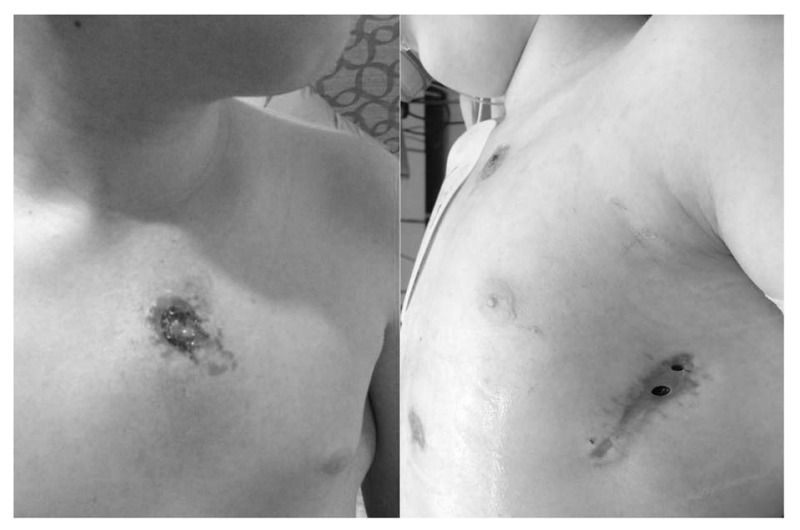

O5-5

Infants and Small Childrens with the Extracardiac Implantable Cardioverter-Defibrillator - One Decade of Experience

Müller M.J., Backhoff D., Willberg J., Schneider H.E., Paul T., Krause $U$.

Department of Pediatric Cardiology and Intensive Care Medicine, University Medical Center, Georg August University, Goettingen, Germany

Objective: Implantable cardioverter-defibrillators (ICD) are lifesaving in patients with high risk for sudden cardiac death (SCD) from ventricular tachycardia/fibrillation (VT/VF). Special requirements need to be met to provide adequate ICD therapy in infants and young children because of small body size, faster heart rates and a higher level of activity. Programming as well as Implantation technique have to be tailored according to the demands of the small patients. We report a decade of experience with a completely extracardiac (EC) ICD implanted in infants and young children.

Methods: A retrospective data analysis enrolling all pediatric patients who had an EC-ICD implanted at our institution between July 2004 and December 2015 was performed. Demographic parameters, indications for ICD implantation, ICD programming as well as adequate and inadequate ICD therapies were analyzed.

Results: Data from a total of 37 patients (13 females) could be analysed. Underlying diseases included cardiac channelopathies in 25 patients, various forms of cardiomyopathies in 10 patients and congenital heart defects in 2 patients, respectively. ICD implantation was for primary prevention of SCD in 11 (29.7\%) patients and for secondary prevention in the remaining 26 (70.3\%). Single chamber systems were implanted in 27 (73\%) and dual chamber systems in $10(27 \%)$. At implantation, mean age was 5.4 years (0.2-11.6) years, mean body weight was $20(4.6-42) \mathrm{kg}$. In all patients VF detection zones were set to $240-260 \mathrm{~ms}$, whereas VT detection was inactivated in all patients. Initial detection was at 24-30 cycles whereas redetection was $9-12$ beats. During mean follow-up of 4.9 (0.7-11.4) years, appropriate shocks were documented in 11 patients (primary prevention $n=1$, secondary prevention $n=10 ; n . s$.$) . Inappropriate shocks due to T$ wave oversensing $(\mathrm{n}=1)$, rapidly conducted atrial tachycardia $(\mathrm{n}=2)$ and lead dislocation $(\mathrm{n}=1)$ were observed in 4 patients $(10.8 \%) .22$ surgical revisions were performed in 13 patients (35.1\%) due to malfunction of the ICD. At the time of data assessment all 37 patients were alive.

Conclusions: EC-ICD was effective and safe for avoiding SCD in infants and young children. Inadequate discharges were in a range as previously reported. Surgical revisions were frequent.

\section{O5-6 \\ Efficacy of a modified ICD insertion technique in children in protecting against sudden cardiac death \\ Dave H., Balmer C., Podonyi A., Schmiady M., Weber R., Schweiger M., Huebler $M$. \\ Children's Heart Centre and Children's Research Centre, University Children's Hospital, Zurich, Switzerland}

Introduction: A variety of aetiopathologies subject children to a high risk of ventricular arrhythmias. Implantable cardioverter defibrillators are life-saving. However, the enormous size of the ICD compared to the small size of children means that innovatiave techniques are needed to adapt this technology to children.

Methods: We analyzed 28 children subjected to an epicardial ICD procedure from 2004 to 2014 . The median age and weight of the cohort was $12(2.9-20)$ years and $42(13.5-97) \mathrm{kg}$. The indications included hypertrophic cardiomyopathy (14), restrictive or dilatative cardiomyopathy (6), electric cardiomyopathy (CPVT, long QT, Brugada syndrome)(10) and other causes (4). In 7 patients ICD was implanted in conjunction with an intra-cardiac procedure (most often resection of hypertrophic obstructive LVOT). The indication was primary prophylaxis in 18 and secondary prophylaxis in 10 patients. A left axillary thoracotomy used to place electrodes onto the left atrial appendage and left ventricle was the preferred approach. A shock electrode was placed across the rib cage so as to ensure that maximum ventricular muscle mass was exposed to the shock vector between the ICD 
placed in the epigastrium and the shock electrode. In majority of patients, the ICD was placed in a pocket created between the parietal pericardium and the diaphragm (through an epigastric incision).

Results: 1 patient (listed for transplantation for dilatative cardiomyopathy) died. Kaplan Meier survival was $90.9 \pm 8.7 \%$ at 12 years follow-up. The atrial and ventricular pacing thresholds were a median of $0.5(0.3-1.75)$ and $1(0.3-4.5) \mathrm{V}$ respectively. The respective $\mathrm{P}$ and $\mathrm{R}$ waves were 5(1.7-16.5) and 12.7(5.5-30) $\mathrm{mV}$ respectively. The pleural coil resistance was 61.5(43-107) Ohms. A test induction of ventricular fibrillation (inducible in 25 ICD systems) was recognized and successfully converted with a median of 20(10-30)Joule energy. 8 shocks in 4 patients successfully terminated ventricular arrhythmias during follow-up. Inappropriate shocks happened in 2 patients (1 due to broken ventricular electrode). Freedom from complete ICD system replacement was $87.1 \pm 6 \%$ and freedom from electrode/coil revision was $61.4 \pm 9.3 \%$ at 10 years (Figure).

Conclusions: Modern ICD technology can be adapted to the paediatric demands. Our epicardial/pleural modified approach leads to excellent short and midterm results with regards to procedural success, efficacy as well as overall patient outcome.

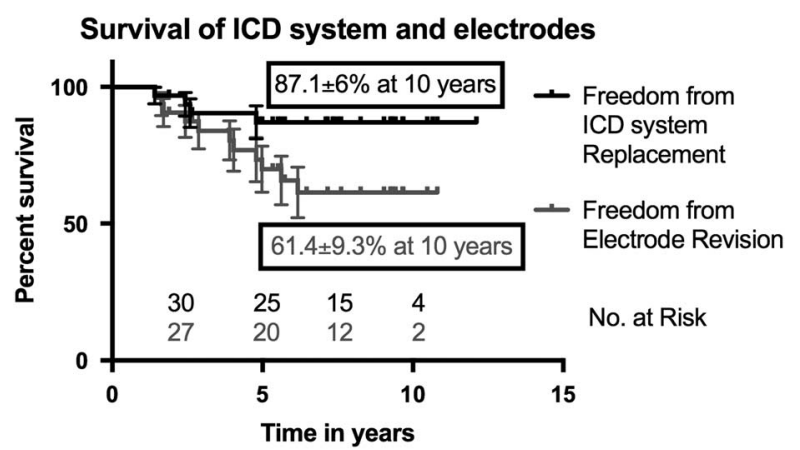

O6-1

Towards Non-Invasive Assessment of Central Venous Pressure Variations using Real Time and Quantitative Liver Stiffness Estimation

Villemain O. (1), Sitefane F. (1), Pernot M. (2), Malekzadeh-Milani S. (1), Tanter M. (2), Bonnet D. (1), Boudjemline Y. (1)

M3C Necker-Enfants Malades, Paris, France (1); Institut Langevin U979, Paris, France (2)

Objectives: The main purpose of this study was the real-time evaluation of the variation impact of central venous pressure $(\mathrm{CVP})$ on liver stiffness (LS) by shear wave elastography (SWE) in a cohort of children with heart disease.

Background: SWE has been showed and used as a non-invasive, quantitative and reproducible approach to assess LS. LS has been reported to be associated with fibrosis but there is also a potential dependence of LS with the CVP.

Methods: 103 children (6.8 \pm 5.5 years) referred to our institution for diagnostic or interventional right heart catheterization (RHC) were prospectively enrolled. CVP and LS were measured simultaneously at baseline and after $15 \mathrm{ml} / \mathrm{kg}$ of volume loading. Inferior vena cava (IVC) diameter and pulsed-Doppler profile of hepatic veins were also evaluated. Plasma level of NT-pro-BNP was assayed during the RHC.

Results: At baseline RHC, mean CVP was $7.4 \pm 2.9 \mathrm{~mm} \mathrm{Hg}$ [range 3-16] and mean LS was $9.0 \pm 5.8 \mathrm{kPa}$ [4-46.1].
After volume loading, mean CVP increased significantly to $10 \pm 3.3 \mathrm{~mm} \mathrm{Hg}[3-18] \quad\left(\mathrm{p}<10^{-4}\right)$ and mean LS increased significantly to $14.4 \pm 9.1 \mathrm{kPa}[4.3-72]\left(\mathrm{p}<10^{-4}\right)$.

LS significantly correlated with CVP $[\mathrm{r}=0.89, \mathrm{p}<10-4$; $\mathrm{CVP}=(\ln (\mathrm{LS})-1) / 0.145]$. Optimal cut-off value of LS for detection of $\mathrm{CVP}>10 \mathrm{mmHg}$ was $10.8 \mathrm{kPa}(\mathrm{Se}=89.3 \%, \mathrm{Sp}=86.0 \%)$, with an area under the curve of 0.946 (95\% CI 0.920 to 0.971 ; $\mathrm{p}=0.01)$.

Beyond this correlation, LS is sufficient to provide an indirect and reliable measurement of quantitative CVP variations.

IVC diameter, pulsed-Doppler profile of hepatic veins and NTpro-BNP were less robust than LS to estimate CVP.

Conclusions: Here, we show that LS measurement using SWE is a reliable surrogate of quantitative estimation of the CVP. It can also be used to measure CVP changes in real time. LS could potentially be a useful non-invasive tool for evaluation and follow-up of acute and chronic right heart failure.

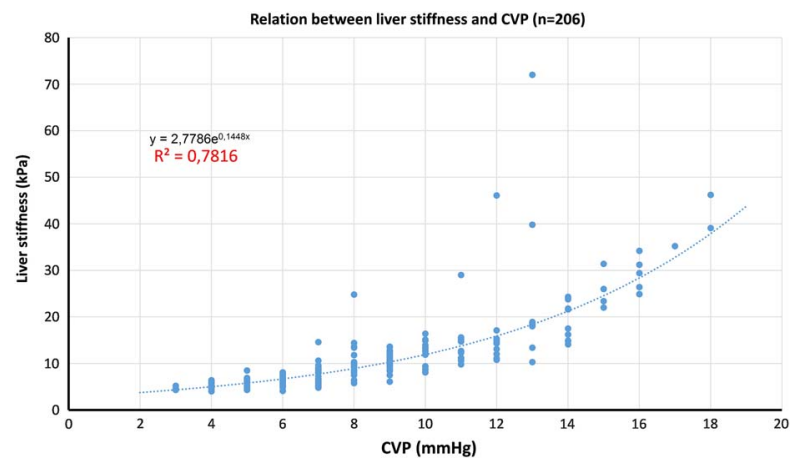

O6-2

Reduced heart rate reserve in Fontan patients: chronotropic incompetence or chronotropic protection? Eyskens B., Cools B., Claessen G., La Gerche A., Van De Bruaene A., Heying R., Roggen M., Bogaert J., Claus P., Budts W., Heidbuchel H., Gewillig M.

University Hospital Leuven, Belgium

Background: Patients with a Fontan circulation have limited exercise tolerance and impaired heart rate (HR) response to exercise. It remains to be determined whether this impaired chronotropic response reflects pathology of the sinoatrial node or is a consequence of altered cardiac hemodynamics. We aimed to evaluate the adequacy of heart rate and acceleration throughout exercise relative to metabolic demand and cardiac output.

Methods: 10 Fontan patients with normal sinus rhythm (all TCPC, age $19.6 \pm 4.0$ years, age at TCPC $7.2 \pm 4.6$ years; left ventricle in 8 ) and 20 healthy controls underwent supine bicycle exercise to near maximal exertion during cardiac magnetic resonance imaging with simultaneous invasive pressure recording via a pulmonary and radial artery catheter. Cardiac index $(\mathrm{CI})$, stroke volume (SVi) and HR were assessed and exercise-induced increase $(\Delta)$ in CI, SVi and HR relative to the exercise level and increase in oxygen consumption (VO2) were calculated.

Results: Maximal exercise capacity and HR reserve was lower in Fontan patients compared to controls; HR and HR reserve when expressed in \% of maximum was lower suggesting chronotropic incompetence (fig A). In contrast, at every exercise level, HR and increases in $\mathrm{HR}$ relative to workload and $\mathrm{VO}_{2}$ in absolute values were higher than in controls (fig $\mathrm{B}$ ). The change in CI relative to 
the change in $\mathrm{VO}_{2}\left(\Delta \mathrm{CI} / \Delta \mathrm{VO}_{2}\right)$ was similar between groups; Fontan patients had a higher increased $\Delta \mathrm{HR} / \Delta \mathrm{VO}_{2}$ and reduced $\Delta \mathrm{SVi} / \Delta \mathrm{VO}_{2}$. The lower exercise capacity and $\mathrm{HR}$ reserve in Fontan patients was associated with failure of the systemic ventricle to maintain end-diastolic volume and SVi during exercise, suggesting insufficient preload reserve.

Conclusions: At every exercise intensity chronotropic responsiveness is preserved in Fontan patients, indicating normal sino-atrial function. However, exercise capacity and maximum $\mathrm{HR}$ are attenuated, likely as a consequence of reduced ventricular filling Bainbridge reflex (1915). "Chronotropic incompetence" is therefore not a correct term to describe the diminished heart rate reserve in Fontan patients. Increases in HR at maximal exercise would most likely further reduce SVi because insufficient preload reserve, which might be harmful: the term "chronotropic protection" appears more appropriate !
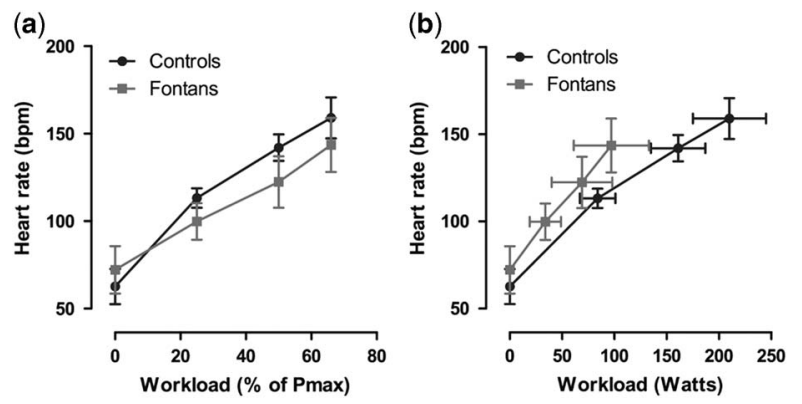

O6-3

\section{Carotid intima-media thickness in children with congenital heart disease}

Reiner B. (1,2), Oberhoffer R. (1,2), Ewert P. (1), Müller J. (1,2) Department of Pediatric Cardiology and Congenital Heart Disease, German Heart Centre Munich, Technical University Munich, Munich, Germany (1); Institute of Preventive Pediatrics, Technical University Munich, Munich, Germany (2)

Introduction: Carotid intima-media thickness (cIMT) is a noninvasive marker for cardiovascular risk. Adults with congenital heart disease (CHD) are known at higher risk for developing further cardiovascular diseases. Thus, the aim of this study is to get known more about risk factors in young CHD patients and to identify high risk diagnostic subgroups.

Methods: For this cross-sectional study 319 children (116 girls) aged 6-18 years (12.3 years \pm 3.3 ) were recruited between May 2015 and October 2016 in our institution. Far wall cIMT was assessed non-invasively via ultrasound, while central blood pressure (systolic $=\mathrm{cSBP}$ and diastolic $=\mathrm{cDBP}$ ) and pulse wave velocity (PWV) were evaluated using a Mobil-O-Graph ${ }^{\circledR}$. Further, anthropometrical data and medication were recorded. According to their heart defect the children were divided into five diagnostic subgroups: aortic stenosis/aortic coarctation $(n=90)$, atrial septal defect/ventricular septal defect/atrioventricular septal defect (shunts; $\mathrm{n}=83$ ), tetralogy of Fallot/pulmonic stenosis (ToF/PS; $\mathrm{n}=58)$, univentricular heart $(\mathrm{n}=55)$, transposition of the great arteries (TGA-switch; $\mathrm{n}=33$ ). Data were compared by using oneway ANOVA with Bonferroni's post-hoc tests. Two-sided p-values $<0.050$ were considered statistically significant.

Results: Mean cIMT in children with CHD was $0.462 \pm 0.041$ on average. Children with TGA-switch showed the thickest cIMT values with $0.486 \pm 0.044$. Therefore, it was significantly higher as it was in ToF/PS-patients $(0.451 \pm 0.036 ; \mathrm{p}=.001)$ or shunts
$(0.452 \pm 0.032 ; \mathrm{p}<.001)$. Additionally, cIMT values showed a significant correlation to height $(p=.027), \operatorname{PWV}(p=.029)$ and cSBP $(\mathrm{p}<.001)$, but not with $\mathrm{cDBP}(\mathrm{p}=.318)$. Still after correction for sex, age and height children with TGA showed sign. higher cIMT values $(B=.025 ; \mathrm{p}=.001)$ and patients with ToF/PS $(\mathrm{B}=-.013 ; \mathrm{p}=.025)$ or shunts $(\mathrm{B}=-.014 ; \mathrm{p}=.009)$ sign. lower cIMT values than children with other heart defects.

Conclusion: Children with TGA-switch presented the highest cIMT values and should be closely monitored. The relation of cSBP and CDBP on cIMT was already shown in first studies of healthy adults and could be confirmed in this young patient group. To compare patients data to norm values an own investigation of healthy children is planned, while variations in ultrasound protocols make a generalized application of norm values impossible.

O6-4

Cardiopulmonary exercise test in children with congenital heart diseases: correlation between ventilatory parameters and maximum oxygen uptake

Gavotto A. (1,2), Matecki S. (2, 5), Guillaumont S. (3), Picot M.C. (4), Amedro P. $(1,5)$

Pediatric and Congenital Cardiology Department, University Hospital, Montpellier, France (1); Pediatric Functional Exploration Laboratory, Physiology Department, University Hospital, Montpellier, France (2); Pediatric Cardiology and Rehabilitation Unit, Saint-Pierre Institute, Palavas-Les-Flots, France (3); Epidemiology and Clinical Research Department, University Hospital, Montpellier, France (4); Physiology and Experimental Biology of Heart and Muscles Laboratory PHYMEDEXP, UMR CNRS 9214-INSERM U1046, University of Montpellier, Montpellier, France (5)

Introduction: Ventilatory parameters measured by cardiopulmonary exercise test (CPET) are correlated to maximum oxygen uptake (VO2max) and morbidity in adults with chronic heart failure. Such parameters as oxygen uptake efficiency slope (OUES) and $\mathrm{VE} / \mathrm{VCO} 2$ slope are interesting in severe cardiac conditions when a maximal CPET is not possible. Very few data is available in children with congenital heart diseases (CHD). We aimed to identify the correlation between OUES, VE/VCO2 slope and VO2max in a large cohort of CHD and control children.

Methods: We included all children who performed a CPET between 2010 and 2015 in 2 French pediatric CHD tertiary care centers. The CHD group was defined upon Houyel's classification. Children with no chronic disease, no treatment and normal physical examination were included in the control group. Data were adjusted on age and gender for comparisons between both groups. Correlations were measured with Pearson coefficients.

Results: 798 children (496 CHD and 302 controls) were included. OUES was strongly influenced by gender and age $(\mathrm{p}<0001)$ and slightly correlated to $\mathrm{VO} 2 \max (\mathrm{r}=0,25 ; \mathrm{p}<0001)$. Values of OUES divided by the weight (OUES $/ \mathrm{kg}$ ) were close to VO2max (mean 48,6 $\pm 8,8$ for females and 39,4 $\pm 7,3$ for males). OUES $/ \mathrm{kg}$ was strongly correlated to VO2max in both groups $(r=0,83$; $\mathrm{p}<0$ 001). VE/VCO2 slope was influenced by age but not gender and did not correlate to VO2max in both groups. However VE/ $\mathrm{VCO} 2$ slope was more elevated in single ventricles (35.5 vs 29.6; $\mathrm{p}<0.001$ ), right heart diseases (33.4 vs 30.2; p <0.001), pulmonary restrictive syndrome ( 33.1 vs $30.3 ; \mathrm{p}<0.001)$.

Conclusion: OUES is strongly influenced by anthropometric variables. The "OUES/weight" value is more relevant in pediatric population and correlates extremely well to VO2max. We recommend using it for non maximal CPET. In pediatric CHD clinical trials, VE/VCO2 slope could also be used as an endpoint in right heart diseases and single ventricles. 


\section{O6-5 \\ What is the best wall thickness measure for indicating risk of sudden death in childhood hypertrophic cardiomyopathy?}

Östman-Smith I. (1), Sjöberg G. (2), Larsson P. (3), Rydberg A. (4), Fernlund E. (5)

Queen Silvia Chuildren's Hospital, Gotenburg, Sweden (1); Astrid

Lindgren Children's Hospital, Stockholm, Sweden (2); Academic

Hospital, Uppsala, Sweden (3); University Hospital, Umeå, Sweden (4), University Hospitals, Linköping and Lund, Sweden (5)

Background: In hypertrophic cardiomyopathy risk for sudden death is influenced by degree of cardiac hypertrophy but there is no agreement about which measure of hypertrophy is most useful in the growing child. A Z-score of $>6$ has been a proposed cut-off but not validated to be useful, other measures proposed have been a max wall thickness $>2 \mathrm{~cm}$, or septal thickness $>190 \%$ of the 95th percentile value for age (SEPPER).

Patients and Methods: Patients with a diagnosis of HCM before age 19 years attending all five regional centres of paediatric cardiology in Sweden have been studied. There were 28 patients with sudden death or re-suscitated cardiac arrest, and 99 patients with at least two years follow-up without heart-failure death (mean follow-up 11.1yrs). The wall thickness measure at last follow-up were quantified as maximall wall thickness, Z-score for maximal wall thickness (Detroit Z-score), SEPPER, or septum-to-cavity ratio, and frequency distribution histograms were used to find optimal cut-off denoting high risk, and subsequently relative risk, sensitivity and specificity was determined.

Results: Maximal wall thickness $>2 \mathrm{~cm}$ had a relative risk of 5.1 [95\%CI 2.1-12.6], a sensitivity of 83 [64-94)\% and a specificity of 62 [52-72]; Z-score $>6$ a relative risk of 5.7 [3.0-10.8], sensitivity $56[35-76] \%$, specificity 92 [84-96]\%, whereas a lower cut-off Z-score $>4.5$ gave a relative risk of 9.9 [3.1-31.4], sensitivity of 88 [69-97]\% and specificity of $69[59-79] \%$. SEPPER > 190 gave a relative risk of 8.0 [3.3-19.5], sensitivity of $82[63-94] \%$ and specificity $77[67-85]$, and septum-to-cavity ratio $>0.45$ a relative risk of 6.3 [2.5-15.7], sensitivity of $79[58-93] \%$ and specificity of $73[63-82] \%$. Most subjects with cardiac arrest had shown progressive disease with median increase in Z-score of +0.48 over time, but the majority of survivors had reduced their $Z$-scores in response to therapy, median -0.29 , $(\mathrm{p}=0.021)$.

Conclusion: The measures relating wall thickness to body size (Detroit Z-score) or age (SEPPER) discriminated best between a high and low risk, but a $Z$-score $>6$ is too high a cut-off to give a reasonable sensitivity; a cut-off $>4.5$ gives the highest sensitivity but at the cost of a lower specificity.

\section{O6-6 \\ Hepatic changes in the Fontan circulation: which modalities are useful for follow-up screening? \\ Francois K., Ackerman T., De Backer J., Demulier L., Geerts A., Van Vlierberghe H., Bové T. \\ Cardiac Surgery, Cardiology and Hepatology of the University Hospital Ghent, Gent, Belgium}

Objective: Fontan completion induces liver congestion, a precursor for liver fibrosis, cirrhosis and ultimately hepatocellular carcinoma. Timely detection of liver dysfunction is essential to identify patients at risk. We sought to determine which serum markers and liver imaging modalities could be most useful for follow-up screening.
Methods: In a prospective set-up, 28 Fontan patients, aged $24,2 \pm 5,1$ years, underwent echocardiography, blood analysis and liver imaging. Median interval since Fontan completion was 19,5 years (IQR 14,6-21,1 y). Serum analysis included trombocyte count (TRC), albumin, liver enzymes, $\alpha$-foetoprotein, $\alpha 1$-antitrypsin, and proBNP. Fibrosis indices APRI, FIB-4 and Forns-index, validated for liver fibrosis in hepatitis C, were calculated. Liver morphology and Dopplers of hepatic vein, portal vein, hepatic artery and superior mesenteric artery (SMA) were performed, and resistance indices (RI) calculated. Liver stiffness (LS) was measured with elastography. Correlations between the serum markers and imaging results, and relation to time indices (age, Fontan interval) were established.

Results: Lab analysis showed abnormal values for YGT, bilirubin, ALT, and TRC (in 74\%, 33\%, 33\% and 29\% of patients). APRI and FIB-4 were unindicative for liver fibrosis. Forns-index indicated moderate fibrosis in $24 \%$ of patients, only Forns index correlated significantly with Fontan interval $(p=0,05)$. Ultrasound liver morphology was normal in $61 \%$ of patients, in $22 \%$ and $17 \%$ lobulated contour and nodular hyperplasia was found. Doppler velocities and indices fell within the normal range. Lower AMS RI showed tendency to correlate with longer interval $(p=0,09)$. LS (mean $10,4 \pm 3,7 \mathrm{kPa}$ ) was abnormally increased in $96 \%$ of patients, higher LS was significantly related to longer interval $(p=0,02)$, lower AMS RI $(p=0,02)$, larger spleen $(p=0,02)$, and lower albumin $(p=0,01)$.

Conclusion: Adult Fontan patients show moderate signs of liver dysfunction. The usefulness of post-Fontan screening with classical fibrosis indices remains unclear. The morphologic changes we found sustain the use of ultrasound liver and spleen imaging in regular follow-up of Fontan patients, while liver Doppler indices seem to result largely normal. Liver stiffness, partially due to liver congestion, seems to overestimate the grade of fibrosis, but increases with time and higher mesenteric vascular resistance, and needs validation through sequential measurements.

\section{O7-1}

Invasive assessment of pulmonary endothelial function in Fontan patients

Latus H., Lederle A., Khalil M., Kerst G., Schranz D., Apitz C. Pediatric Heart Centre Giessen, Germany

Background: Pulmonary endothelial dysfunction due to a loss of pulsatile pulmonary blood flow is thought to be a major factor in the development of increased pulmonary vascular resistance (PVR) in Fontan patients causing significant morbidity and mortality. We therefore aimed to assess pulmonary endothelial function by vasodilator response to acetylcholine administered in segmental pulmonary arteries in children and adolescents with Fontan hemodynamics.

Methods: Twenty-four Fontan patients (mean age $12.0 \pm 7.8$ years, 10 females) underwent invasive cardiac catheterization including intra-arterial Doppler- and pressure measurements in a segmental pulmonary artery. Pulmonary flow reserve (PFR) was quantified as the change of average peak velocity (APV) in response to acetylcholine (Ach) infusion and local PVR was assessed by local pressure divided by velocity.

Results: APV significantly increased from $16.8 \pm 7.5$ to $20.3 \pm 6.9 \mathrm{~cm} / \mathrm{s}(\mathrm{p}=0.01)$ resulting in a mean PFR of $1.35 \pm 0.70$. Local PVR dropped from $0.72 \pm 0.32$ to $0.57 \pm 0.20 \mathrm{mmHg} / \mathrm{cm} / \mathrm{s}$ $(\mathrm{n}=18 ; \mathrm{p}=0.004)$. A significant relationship was found between baseline local PVR and PFR $(r=0.73, p=0.0006)$ as well as between local PVR and the percent decrease in local PVR 
$(r=0.52, p=0.02)$. No relationship was found between PFR as well as local PVR with global hemodynamics such as cardiac putput and global PVR.

Conclusions: This study is the first assessing local pulmonary endothelial function in Fontan patients. Given the difficulties in the assessment of global PVR in these patients, measurement of local pulmonary blood flow velocity and PVR may be helpful in the evaluation of adverse pulmonary vascular remodelling. Future research is required to study whether impaired local pulmonary endothelial function may be predictive for Fontan failure.

O7-2

Patient-Specific Computer Heart Model in Children with Dilated Cardiomyopathy as a Useful Tool to Guide Beta-Blocker Therapy in Pediatric Heart Failure: Preliminary Findings from the MD-Paedigree Study Chinali M.(1), Itu L.M. (2), Rinelli G. (1), Heimann T. (3), Neumann D. (3), Secinaro A. (1), Zaccagnini D. (4), Lisignoli V. (1), Ecabert O. (3), Drago F. (1)

Bambino Gesù Children Hospital, Rome, Italy (1); Transilvania University, Brasov, Romania (2); Siemens Healthcare GmbH, Erlangen, Germany (3); Lynkeus srl, Rome, Italy (4)

Background: Betablockers (BB) are an essential part of standard therapy in adult congestive heart failure (HF). However, no specific recommendation on the use of $\mathrm{BB}$ in children is present in consensus statements, suggesting that further studies are urgently needed. Our aim here was to predict the effect on cardiac function of heart rate-change caused by BB therapy in children with HF, using patient-specific mechanical heart models.

Methods: 60 children with DCM-heart failure underwent comprehensive imaging evaluation including 3DEchocardiography and Cardiac Magnetic Resonance. Clinical and imaging data were used to build patient-specific heart models. The model includes anatomical features, dynamic and biomechanical features, as well as electrophysiological characteristics. Integrating these patientspecific heart features, the model builds a whole-body-circulation model and is offerse a prediction on the effect of changes in mean heart and/or cuff pressure on cardiac geometry, function and contractile efficiency, by providing expected changes in LV volumes, ejection fraction and generating pressure-volume LV curves.

Results: Study included 7 boys and 5 girls (age $11 \pm 5$ years, range 1-17) in which BB therapy was either initiated or changed from baseline. Mean left ventricular ejection fraction at baseline was $40.3 \pm 5.6 \%$ and mean heart ranged from 77 to $125 \mathrm{bmm}$. At study baseline beta-blocker therapy was already present in 10 patients. In all cases beta-blocker of choice was carvedilol at a dose range of $6.25 \mathrm{mg}$ to $37 \mathrm{mg}$ per day. At follow-up beta-blocker therapy was present in all cases, except one in which therapy was withdrawn due to low compliance. Dose increase was reported in 8 patients, reduction in 3 and withdrawn in one. Mean dose change between baseline and follow-up was $+15.8 \mathrm{mg} /$ day. Mean change in heart rate ranged between -35 to $+31 \mathrm{bpm}$. Mean left ventricular ejection fraction change ranged from -11 to $+13 \%$. The computational model was able to predict well the ejection fraction at follow-up, with a correlation of 0.87 and a mean absolute difference of $4.58 \%$.

Conclusions: Our preliminary findings supports the hypothesis that our patient-specific heart model might be of clinical aim to define 'optimal' mean heart rate target, at which BB therapy for each patient should be targeted.
O7-3

Transcatheter Potts Shunt creation in children for suprasystemic pulmonary hypertension: initial and midterm results

Malekzadeh Milani S, Sitefane F., Sizarov A., Khraiche D., Mirabile C., Lenoir M., Levy M., Bonnet D., Boudjemline Y.

Centre de Référence Malformations Cardiaques Congénitales Complexes - M3C, Hôpital Necker Enfants Malades, Paris, France

Background: Reversed Potts shunt improves right ventricular function and physical exercise tolerance in patients with suprasystemic pulmonary arterial hypertension (PAH). Surgical shunt creation in such patients carries high mortality and morbidity risks. Proximity of left pulmonary artery (LPA) and descending aorta (DAo) permits creation of such a connection percutaneously potentially reducing procedure-related risks. Feasibility, safety and hemodynamic efficacy of the transcatheter Potts shunt in children was not reported.

Methods: Transcatheter Potts shunt creation using radiofrequencyguided vessel walls perforation followed by covered balloonexpandable stent placement connecting the DAo and LPA lumens. Procedural details, and clinical and echocardiographic follow-up data were prospectively collected.

Results: Stent-secured Potts shunt was successfully created in 6 children (mean age $11.0 \pm 4.2$ years) with drug-refractory suprasystemic PAH and deteriorating right ventricular function. Shunt creation produced nearly complete systolic pressure equilibration with right ventricular function improvement within days in all patients. After shunt creation, 2 patients with severe biventricular dysfunction and pericardial effusion pre-procedurally, demonstrated acute function worsening of the unloaded left ventricle resulting in cardiac arrest and died of irreversible brain at 3 and 10 days despite complete cardiac function recovery. Stent embolization occurred in one patient with successful placement of the second stent. Four patients survived the procedure without complications and all but one showed significantly improved clinical status at mean follow-up of $6 \pm 3$-months.

Conclusions: Transcatheter Potts shunt is feasible in children and results in drastic improvement of the clinical status in surviving patients. Careful selection of patients is important to ensure favorable outcome of the procedure.

\section{O7-4}

Myocardial collagen turnover and myocardial fibrosis are associated with systemic right ventricle dysfunction and wall stress in transposition of the great arteries palliated by atrial switch

Ladouceur M. (1, 2), Baron S.(2), Nivet-Antoine V. (1), Maruani G. (2), Soulat G. (2), Ferrera H. (2), Blanchard A. (2), Boutouyrie P. (2), Iserin L. (2), Paul JL.(2), Mousseaux E. (2)

Necker, Paris France (1)Hôpital Européen Georges Pompidou, Paris France (2)

Background: Heart failure is a serious event in patients with transposition of the great arteries (D-TGA) after atrial redirection surgery (Mustard-Senning operation). Mechanisms of the late failure of the systemic right ventricle (sRV) are poorly characterized. We aimed to determine the role of myocardial fibrosis in systolic and diastolic sRV dysfunctions.

Methods: Determinants of systolic and diastolic functions of sRV were prospectively studied in 49 patients (median age 32 years, male: female ratio $=3: 4$ ) with atrially switch D-TGA compared to 26 healthy subjects similar for age and sex. Diastolic and systolic functions of $\mathrm{sRV}$ were assessed using echocardiography and 
cardiac magnetic resonance imaging (CMR). Fibrosis extend within sRV myocardium was evaluated using gadolinium-enhanced magnetic resonance and serum turnover collagen biomarkers.

Results: Late gadolinium enhancement (LGE) was diagnosed in 35\% of patients with D-TGA, and collagen degradation biomarkers measured by MMP1/TIMP1 ratio were significantly increased in D-TGA compared to healthy subjects $(1.0 \times 10-2$ vs. $2.5 \times 10-2$, $\mathrm{p}=0.04)$. MMP1/TIMP1 as well as LGE lesions were significantly associated with $s R V$ wall stress $(r=0.77, p<0.01$. Figure $1 \mathrm{~A})$ and early sRV filling velocity/ early myocardial velocity $(r=0.53$, $p=0.02$. Figure $1 B)$. $s R V E F$ was strongly correlated with $s R V$ wall stress $(r=-0.74, p<0.01)$ and fibrosis markers (pro-MMP1/TIMP1, $\mathrm{r}=-0.81, \mathrm{p}<0.01$ Figure 1C, and LGE, $\mathrm{p}<0.01)$.
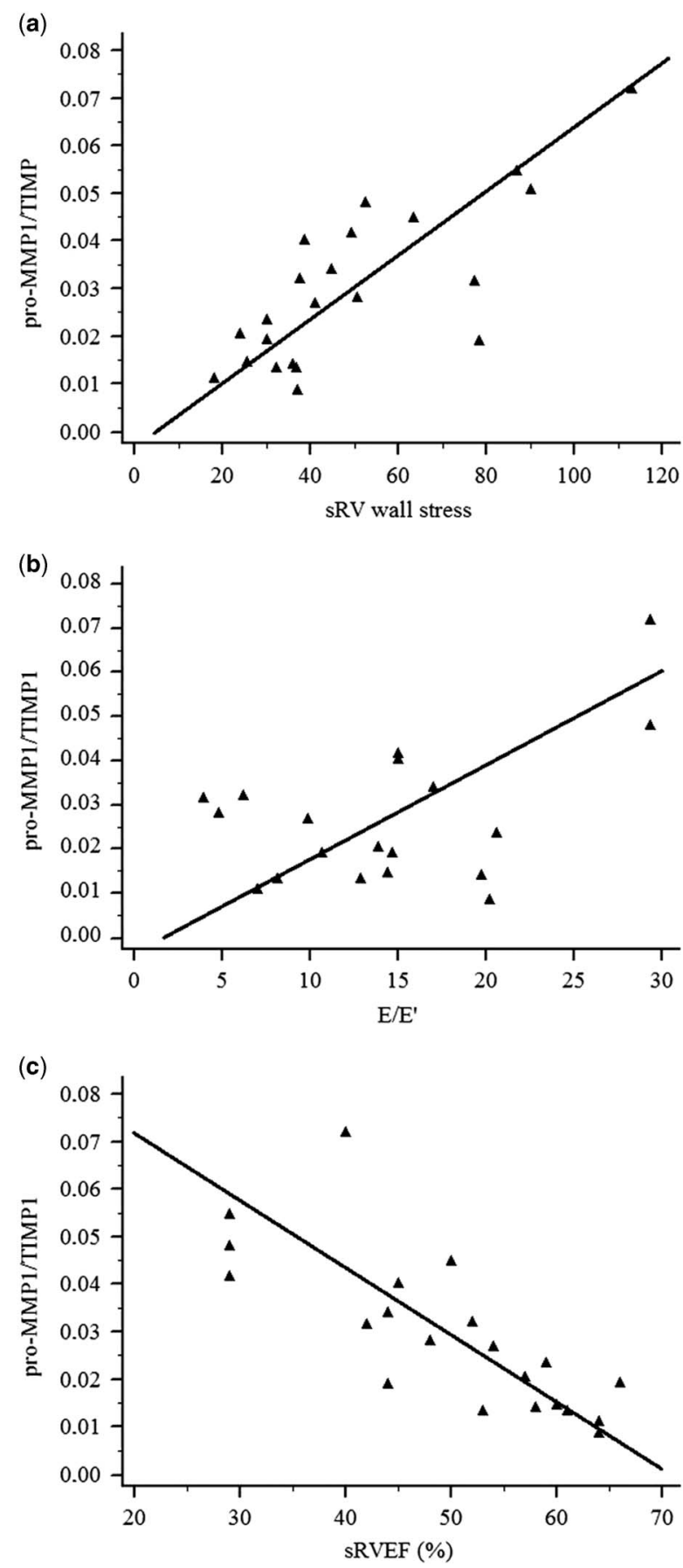

Conclusions: Impairment of diastolic and systolic functions of $\mathrm{sRV}$ is related to myocardial matrix remodeling, which is the consequence of longstanding RV pressure overload. Research in medical therapies that reduce fibrosis is warranted in this setting.

\section{O7-5}

The PPAR $\gamma$ Agonist Pioglitazone Reverses Angioobliterative Pulmonary Vascular Disease and Prevents Right Heart Failure through distinct Epigenetic and Metabolic Mechanisms

Legchenko E. (1), Küffner R. (2), Ferndandez-Gonzalez A. (3), Kourembanas S. (3), Jonigk D. (4), Hansmann G. (1)

Department of Pediatric Cardiology and Critical Care, and Pulmonary Vascular Research Center (PVRC), Hannover Medical School, Germany (1); Institute of Bioinformatics and

Systems Biology, German Research Center for Environmental Health, Helmholtz Center Munich, Germany (2); Division of Newborn Medicine, Department of Medicine, Boston Children's Hospital and Harvard Medical School, Boston, MA, USA (3); Institute of Pathology, Hannover Medical School, Member of the German Center for Lung Research (DZL), Hannover, Germany (4)

Background: So far, no intervention could fully reverse pulmonary arterial hypertension $(\mathrm{PAH})$ or even prevent pressure overload heart failure, in the well-established SuHx rat model that closely resembles human disease. We hypothesized that the PPAR $\gamma$ agonist pioglitazone (Pio) reverses angio-obliterative $\mathrm{PAH}$ and prevents heart failure in RV pressure overloaded rats through distinct epigenetic and metabolic mechanisms.

Methods: Male SD rats were injected either with no agent, vehicle (DMSO), or the VEGFR2 inhibitor SU5416 (4 groups): control normoxia $(\mathrm{ConNx})$; control/hypoxia (ConHx, 1x s.c. DMSO, 3wks hypoxia, 6wks room air); SU5416/hypoxia (SuHx, SU5416 $20 \mathrm{mg} / \mathrm{kg} /$ dose s.c. $\mathrm{x} 1$, 3wks Hx, 6wks Nx); SU5416/hypoxia treated with Pio (SuHx + Pio, SU5416 s.c. x1, 3wks Hx, 6wks $\mathrm{Nx}$, including $5 \mathrm{wks}$ of Pio treatment $20 \mathrm{mg} / \mathrm{kg} /$ day p.o.). Hemodynamics, RV/LV mass and volumes were assessed by closed-chest cardiac catheterization, MRI, ECHO, Fulton's index $(\mathrm{RV} / \mathrm{LV}+\mathrm{S})$. RNA expression studies (mRNASeq, single and miRNA array qPCR) were performed on rat $\mathrm{RV}$ and $\mathrm{LV}(\mathrm{N}=3$ / group), and on laser-capture microdissected explanted heart and lung tissue of IPAH HLTx patients and healthy donors $(\mathrm{N}=7-10)$. Pio-regulated mitochondrial function (fatty acid oxidation, ATP production) was assessed in rat neonatal ventricular cardiomyocytes.

Results: SuHx rats developed severe $\mathrm{PAH}$ and overt RV failure vs. ConNx and ConHx that was fully reversed and prevented by Pio administration ( $\mathrm{SuHx}+\mathrm{Pio})$, respectively: RVSP (91.1 vs. 28.8 vs. 32.2 vs. 34.2; $\mathrm{N}=5-9, \mathrm{p}<0.0001)$, RVEDP, RVEDV, RVH, and RVEF (77.8 vs. 74.9 vs. 48.0 vs. $75.4 ; \mathrm{N}=3-5, \mathrm{p}<0.001)$. RNASeq revealed 160 genes with differential expression in $\mathrm{SuHx}$ RVs (FDR 5\%), including Ctgf and Acss3. qPCR arrays identified several miRs that were altered in SuHx RVs and regulated by Pio, including miR-197 (up with SuHx, down with Pio; predicted to regulate Acss3), miR-146b (up with SuHx, down with Pio), and miR-133 (down with SuHx, up with Pio). Altered miR expression was confirmed in human plexiform lesions vs. small pulmonary arteries of HLTx patients.

Conclusions: To the best of our knowledge, PPAR $\gamma$ activation by pioglitazone is the first intervention that fully reverses angioobliterative PVD and prevents heart failure in a robust animal model, and as such is an attractive treatment option for clinical PAH. 
O7-6

Chronic home inotropic support in infants and children with heart failure improves survival and quality of life and may lead to long-term improvement or transplantation Apostolopoulou S.C., Vagenakis G., Tsoutsinos A., Laskari C.V., Rammos $S$.

Onassis Cardiac Surgery Centre, Athens, Greece

Introduction: Severe chronic heart failure (CHF) especially in infants and children is a serious condition with rapid progression and very high morbidity and mortality especially in countries with absence or low rate of heart transplantation. This population usually needs continuous in hospital or ICU care and inotropic support.

Methods: This study reviews our experience with chronic home intravenous inotropic support and/or monthly levosimendan infusion in patients with end-stage CHF unable to be weaned off inotropes and exit the hospital.

Results: The study population consisted of 20 critically ill patients aged $8.7 \pm 7.8(0.2-26.1)$ years with severe CHF (7 myocarditis, 6 dilated cardiomyopathy, 2 restrictive cardiomyopathy, 5 repaired congenital heart disease) needing continuous inotropic support in hospital and mostly treated in the ICU. A total of 17 patients received continuous infusion prepared daily by the caretakers with $5 \mathrm{mcg} / \mathrm{kg} / \mathrm{min}$ dobutamine and $0.5 \mathrm{mcg} / \mathrm{kg} / \mathrm{min}$ milrinone at home through a permanent central catheter for $1.0 \pm 0.8(0.2-3.0)$ years. Additionally, 14 of the patients on home intravenous inotropes and the remaining 3 patients of this population received monthly levosimendan infusions for $1.1 \pm 1.0$ (0.1-3.0) years. During $1.9 \pm 1.2(0.2-4.2)$ years of follow-up, 3 patients died of worsening CHF after 0.3-2.1 years of home intravenous inotropic support. All remaining patients remained in stable condition at home with close follow-up and good quality of life with only 2 line infections treated with intravenous antibiotics and 3 catheter reinsertions due to inadvertent removal. With stabilization, 5 myocarditis patients improved and discontinued inotropic support after 0.3-2.3 years, 1 cardiomyopathy had a left ventricular assist device and 2 underwent heart transplantation in another country. Conclusions: Chronic home intravenous inotropic support with or without levosimendan in patients with end-stage CHF is feasible for long periods with good results and few side effects even in small infants and children allowing stabilization, discharge from hospital, good quality of life. It may significantly prolong life and provide precious time for heart transplantation or myocardial remodeling, improvement and discontinuation of inotropes even after long periods of support. The last 2 statements gain great importance in countries with absence or low rate of heart transplantation especially in the paediatric population.

\section{O8-1 \\ Motor development and their Association to Health- related Quality of life in Children and Adolescents with Congenital Heart Disease \\ Remmele J. (1,2), Reiner B. (1,2), Weberruss H. (1), Oberhofer R. (1,2), Ewert P. (2), Müller J. $(1,2)$ \\ Chair of Preventive Pediatrics, Technical University Munich, Munich, Germany (1); Department of Pediatric Cardiology and Congenital Heart Disease, German Heart Center Munich, Munich, Germany (2)}

Objective: Reduced physical activity and health-related quality of life (HrQoL) is often reported in children and adolescents with congenital heart disease (CHD) compared to healthy counterparts. However motor development (MD) in patients with CHD is poorly explored, therefore this study investigated motor competence and its association with HrQoL.
Patients and Methods: In total 576 consecutive children and adolescents with CHD (225 girls, 12.9 \pm 3.1 years) participated in the FITNESSGRAM ${ }^{\circledR}$ for assessing muscular strength and flexibility by five tasks converted to a motor score and compared to a recent healthy reference cohort. Moreover they filled in the KINDL $®$ questionnaire. Results: MD was significant reduced (z-score: $-0.62 \pm 0.83$; $\mathrm{p}<.001)$ compared to controls and $175(30.4 \%)$ children and adolescents with CHD exhibit impaired MD defined by a z-score lower than one standard deviation compared to the reference. This hold true for shoulder flexibility (z-score: $-0.64 \pm 1.42$; $\mathrm{p}<.001$ ), lower limb flexibility ( $\mathrm{z}$-score: $-0.75 \pm 1.59 ; \mathrm{p}<.001$ ), trunk flexibility (z-score: $-1.34 \pm 1.46 ; \mathrm{p}<.001)$ and curl-ups (z-score: $-0.30 \pm 1.21 ; \mathrm{p}<.001)$. Except in push-ups there was no significant reduction. In multivariable regression corrected for age and gender, a higher MD was associated with higher HrQoL $(\mathrm{B}=1.155$, beta $=0.096, \mathrm{p}=.030)$.

Conclusion: MD in children and adolescents with CHD is reduced and the reduction in MD is also associated with worse HrQoL. It may therefore be indicated to pay more attention to MD in children and adolescents with CHD.

\section{O8-2}

Survival and neurocognitive outcome after cardiac ECLS in children - a single centre study

Logeswaran T. (1), Kehl A. (1), Valeske K. (2), Mueller M.(3), Bauer J. (1), Akintuerk H.(2), Schranz D.(1), Hahn A. (4), Thul J.(1)

Paediatric heart Centre, Giessen, Germany (1); Department of paediatric heart surgery, Giessen, Germany (2); Department of Anaesthesia, Giessen, Germany (3), Department of Neuropaediatrics, Giessen, Germany (4)

Background: The use of extracorporal membrane oxygenation in infants and children with cardiac disease is increasing; survival and neurological morbidity varies. The aim of this study was to examine neurodevelopmental long-term outcome and healthrelated quality of life (HRQoL) in school children who underwent cardiac surgery and required ECLS in infancy / early childhood.

Methods: Between 2000 and 2013, 91 children (median age of ECLS: three months; range: 0-203 months) required postoperative ECLS. After successfully weaning, 13 patients died during hospital stay; 56 children (62\%) were discharged home, respectively. Neurocognitive Follow-Up was performed in survivors between 5-18 years: 23 of 30 patients $(77 \%)$ were reexamined. Median age at follow up was eight years and three months. Re-Evaluation included a detailed neurological examination, assessment of the intelligence coefficient (IQ) by means of the Culture Fair Test and assessment of selective attention. Parental questionnaire were used to measure health related quality of life (HRQoL). Results were compared with those of 23 age- and sex-matched healthy comparison individuals.

Results: Median IQ was 97 in the study group (range: 45-124). IQ was poorer in patients after ECMO than in the comparison group $(p<.001)$. Neurological examination revealed handicap in three patients; however, fine motoric assessment showed significant lower scores on all motor domains in 12 patients (52\%). These results were significant poorer compared to the healthy comparison group $(\mathrm{p}<.001)$. In the attention test the group after ECMO showed no significant difference. Quality of life was described as significant poorer to that of typically developing peers.

Conclusion: ECLS is an important therapeutic issue in children with low cardiac output and survival is improving. Children after ECLS have higher risk for cognitive and motor impairments. Consequently, long-term neurodevelopmental follow up are necessary to provide early educational and therapeutic support. 


\section{O8-3}

Postmortem genetic analysis in paediatric patients. Familial implications

Sarquella-Brugada G.(1), Campuzano O.(2), Cesar S.(1), Carro E.(1), Sanchez-Molero O.(2), Iglesias A.(2), Fernandez A.(2), Castellà J.(3), Medallo J.(3), Brugada J.(1), Brugada R.(2)

Arrhythmia Unit, Hospital Sant Joan de Déu, Univesitat de Barcelona, Barcelona, Spain(1); IDIBGI-Centre de Genètica Cardiovascular, Universitat de Girona, Girona, Spain(2); Insitut de Medicina legal $i$ Forense de Catalunya, Barcelona, Spain(3)

Introduction: Despite exhaustive autopsy analysis, sudden death in young population remains without explanation in 50\% of the cases. Channelopathies and cardiomyopathies are a common cause of sudden death in the Young. This study tries to find cause of sudden death in children using molecular autopsy and to analyze familial implications of these findings.

Methods: We have performed 55 gene-panel DNA analysis from patients died of sudden death with negative autopsies. We have done familial segregation up to 3 generations and have analysed familial findings.

Results: We have analysed DNA from 75 unexplained sudden death individuals. In 30 patients (40\%) we have identified 79 genetic variants (27 in ion cannel genes, 52 in structural genes). In $33,3 \%$ of the cases we could perform familial analysis. Genetic variant carriers were clinical studying using standard protocols.

In 7 cases we have detected early signs of potentially lethal disease and therapeutic and preventive measures have been taken accordingly.

Conclusions: Our study remarks the importance of molecular autopsy as part of the forensic protocol in case of negative autopsy in Young population. Around $40 \%$ of all sudden deaths in paediatric population present a genetic variant that could justify the cause of death. Molecular autopsy is a useful tool with a high impact in morbi-mortality of relatives that might be at risk.

\section{O8-4 \\ Hypertrophic Cardiomyopathy in children: Genotypic- phenotypic correlation in a large Dutch cohort Vos J.S.(1), Bökenkamp R.(1), Barge-Schaapveld, D. Q.C.M. (2) Blank A.C.(4), Frohn-Mulder I.M.E.(5), Delhaas T.(6), Wolterbeek R.(3), Kuipers I.M.(7), Rammeloo L.(8), Reimer A.(9), Blom N.A.(1,7) Pediatric Cardiology (1) Clinical Genetics(2) Medical Statistics (3) LUMC, Leiden; Pediatric Cardiology UMC Utrecht(4); Erasmus MC, Rotterdam(5); MUMC + , Maastricht(6); AMC, Amsterdam(7); VUMC, Amsterdam(8); UMC St. Radboud, Nijmegen(9); The Netherlands}

Introduction: Hypertrophic cardiomyopathy (HCM) is a genetically determined myocardial disease characterized by left ventricular hypertrophy of various morphologies, with a wide array of clinical manifestations ranging from no complaints to sudden cardiac death (SCD). Although risk factors for cardiac events such as myocardial thickness have been described earlier, the consequences and/or benefits of genetic testing are still unclear.

Aim: To investigate the genotype-phenotype correlation in a Dutch cohort of unrelated children with HCM.

Methods: Multicenter retrospective study in 7 centers in the Netherlands on children (0-18 years) presenting between June 1978 and October 2016 with left ventricular wall thickness of $\geq 2$ SD above the population mean on echocardiography. Data were gathered till the latest visit before the patient turned. The genetic testing has changed over the years from DNA analysis by direct sequencing of all coding intron-exon boundaries of known HCM causing genes to next-generation-sequencing of a larger panel of HCM genes in the cases after 2012. Statistical analyses were performed by Kaplan-Meier curves and Poisson regression.

Results: Until now 78 patients $(69 \%$ male) were included with a mean follow time of 11,5 years. In 45 patients results from genetic tests were available, of which 31 patients $(65 \%)$ had one or more mutations and $14(30 \%)$ had no mutations. Mutations were located in the MYBPC3 (35\%), MYH7 (19\%), DSP (2\%), PRKAG2 (2\%), POPDC2 (2\%), MYPN (2\%) and TNNI3 (2\%) gene. VT/VFevents occurred earlier $(p=0.034)$ and more often $(p=0.0042)$ in genotype-positive patients. SCD occurred in four patients in the genotype-positive group in the absence of any echocardiographic differences with the genotype-negative group.

Conclusions: We showed that the cardiac event rate is higher in genotype-positive children with hypertrophic cardiomyopathy

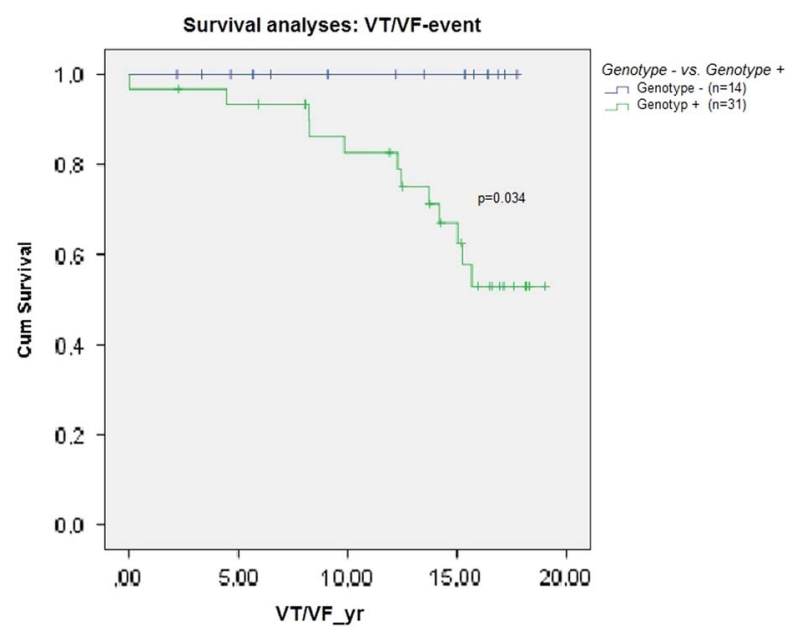

O8-5

(Cost)effectiveness of pulse oximetry screening after homebirths and early discharge: a prospective study of 20.000 newborns

Narayen I.C.(1), Blom N.A.(1), Onland W.(2), Clur S.A.(2), Rammeloo L.A.(3), van den Dungen F.A.(3), van Kaam A.H.L.C. (2), te Pas A.B.(1), POLAR study group (4)

Leiden University Medical Center, Leiden, the Netherlands (1); Academic Medical Center of Amsterdam, Amsterdam, the Netherlands (2); VU Medical Center, Amsterdam, the Netherlands (3); different affiliations in the Netherlands (4)

Introduction: Pulse oximetry screening (POS) for critical congenital heart defects (CCHD) is increasingly implemented worldwide. However, it is unknown whether POS is (cost)effective in settings with homebirths and very early discharge. We assessed this in the Netherlands, where there is a high homebirth rate and early discharge after delivery in hospital.

Methods: POS was performed in hospitals and by community midwives at home or at the policlinic. Measurements were taken $\geq$ one hour after birth and on day two or three, fitting the visit scheme of community midwives. Primary outcome is the accuracy, the sensitivity and specificity. Secondary outcomes are false positive rate and costs per timely diagnosis.

Results: Inclusion ends January $1^{\text {st }}$ 2017. Complete final results will be presented at the meeting. From July 2015 to November 201622.825 newborns were included; 19.737 had a measurement in the first hours after birth, 14.175 newborns had a measurement at day 2 or 3. Out of 7 antenatally undiagnosed CCHDs, 4 were detected 
with POS (sensitivity 57\%). Accuracy for CCHD screening was $98.97 \%$ with a specificity of $98.98 \%$. The false positive rate was $1.0 \%$ (232 cases) with serious non-CCHD illness diagnosed in 82 infants, including non-critical CHD, infections, pulmonary hypertension and wet lungs (figure 1). No pathology was found in 66 newborns and data are not complete for 84 false positive screenings. 47 cardiac ultrasounds were made.

Conclusions: POS screening detected critical illnesses in newborns.

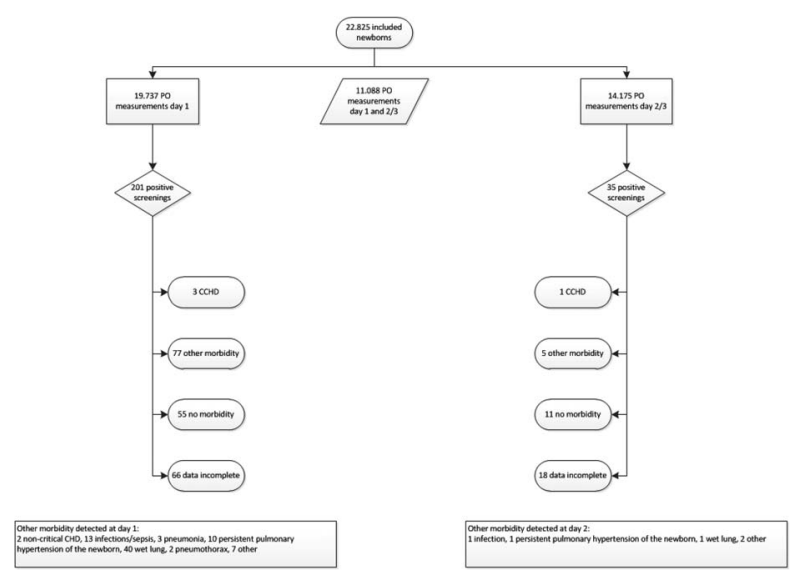

O8-6

Effectiveness of the methylprednisolone-prednisolone combination therapy in 1 st line treatment of high risk Kawasaki disease

Yoshikane Y. (1), Miyamoto T. (2), Hirose S. (2), Ogawa A. (1) Department of Pediatrics, Fukuoka university Chikushi hospital, Fukuoka, Japan (1); Department of Pediatrics, Faculty of Medicine, Fukuoka University, Fukuoka, Japan (2)

Introduction (or Basis or Objectives): IVIG is utilized as the primary treatment for Kawasaki disease (KD). However, 10-20\% of patients do not respond to IVIG. There are three scoring systems for the prediction of non-response to IVIG in Japan. Steroid combination therapy for initial treatment is recommended for high risk cases in the 2012 guidelines from the Japanese society of pediatric cardiology and cardiac surgery. In our hospital we started methylprednisolone combination therapy for high risk patients, however they often needed additional treatment due to a relapse of their condition. On this basis of the fact, we changed the protocol to methylprednisoloneprednisolone (IVMP-PSL) combination therapy. Objective is to evaluate efficacy of IVMP-PSL combination therapy.

Methods: We defined those who have high scores in 1 or 2 out of the three scoring systems as a moderate high risk patient. From January 2007 to August 2016, a total of 68 hospitalized moderate high risk patients were enrolled. The patients were separated into three groups. Group I $(\mathrm{n}=31)$ : who were administered only IVIG, Group M ( $\mathrm{n}=10)$ : who were administered methylprednisolone and IVIG, and Group P $(\mathrm{n}=27)$ : who were administered methylprednisolone, IVIG and prednisolone, which was tapered every 2 or 3 days, and discontinued within 10 to 14 days after the onset of fever. The necessity of additional therapies and clinical course were compared.

Results: The necessity of additional therapies in group $\mathrm{M}$ was higher than that in group P (40\% $4 / 10$ vs. $26 \% 7 / 27$, not significant). The number of patients who still had a residual coronary lesion over 2 months in group $\mathrm{M}+$ group $\mathrm{P}$ was significantly lower than that in group I $(1 / 37,3 \%$ vs $6 / 31,19 \%, \mathrm{p}<0.05)$.
Conclusions: There is a possibility that methylprednisoloneprednisolone combination therapy is a beneficial strategy for the moderate high risk patients. Steroid combination therapy can reduce residual coronary lesions.

\section{O9-1}

Prophylactic Catheter Ablation of the Arrhythmogenic Substrates for Total Cavo-Pulmonary Connection Candidates

Toyohara K.(1), Nishimura T. (1), Shoda M. (2), Park I.S. (1)

Department of Pediatric Cardiology, Tokyo Women's Medical university, Tokyo, Japan (1), Department of Cardiology, Tokyo Women's Medical university, Tokyo, Japan (2)

Background: The substrates of supraventricular tachycardia (SVT) are often associated with candidates for total cavo-pulmonary connection (TCPC) procedure. Since SVT can be a serious complication and is mostly difficult to be cured by catheter ablation (CA) because of access limitation to the heart after TCPC. Therefore, pre-operative electrophysiological evaluation seems important to prospect the risk of SVT. The purpose of this study is to delineate the incidence of arrhythmogenic substrates in TCPC candidates and evaluate the result of prophylactic CA before TCPC.

Patients: From January 2010 to December 2015, we performed electrophysiological study (EPS) upon 29 consecutive patients (isomerism in 22, Ebstein anomaly in 3, ccTGA 2, dTGA 1 and tricuspid atresia in 1) before TCPC. Median age was 3 years and median weight was $15 \mathrm{~kg}$.

Results: In 26 patients (89\%) except 3 with Ebstein anomaly, distinct His bundle electrograms were recorded at the unusual sites. Coronary sinus did not exist in 25 patients. The type of SVT was arioventricular (AV) reentrant tachycardia (AVRT) with accessory pathway in 2 patients, AVRT involving twin AV nodes in 14, uncommon AV nodal reentrant tachycardia (AVNRT) in 2, common atrial flutter (AFL) in 5 and incisional atrial reentrant tachycardia in 5 and ventricular tachycardia in 1 . We achieved successful CA in 27 patients (93\%). We could not complete the isthmus block line for AFL in two failed cases after replacement of artificial common AV valve and valvoplasty of tricuspid valve. In 14 patients with AVRT involving twin AV nodes, we performed CA of unilateral AVN which showed recessive anterograde conduction. Iatrogenic ventricular dyssynchrony after CA was never observed in all 14 cases of our series. We could eliminate uncommon AVNRT by modification of one posterior AV node in two patients. One clinically significant complication of a stuck artificial common AV valve occurred after successful CA for AFL. The mean follow-up period was 3 years. In all patients after CA no recurrence of SVT was observed. Twenty eight patients underwent TCPC procedure successfully and safely.

Conclusion: Prophylactic CA of arrhythmogenic substrates in TCPC candidates may be an effective therapeutic option.

\section{O9-2}

Interest of coronarography in posteroseptal accessory pathways ablation in children

Alazard M. (1), Lacotte J. (1), Piechand J.F. (1), Maltret A. (1, 2) Institut Cardiovasculaire Paris Sud, Hopital Privé Jacques Cartier, Massy, FRANCE (1); Centre de référence "Malformations cardiaques Congénitales Complexes-M3C ", Université Paris-V, Necker-Enfants Malades, AP-HP, Paris, FRANCE (2)

Introduction: Radiofrequency catheter ablation has become a routine management of pediatric patients with accessory pathways. 
Nevertheless ablation of posteroseptal accessory pathways are challenging, especially in children, because of the proximity of the target site to coronary arteries. We report the interest of coronarography prior to catheter ablation in posteroseptal accessory pathways in children.

Method: Between July 2009 and December 2015, we registered 441 paediatrics electrophysiologicals ablations, 237 (54\%) concerned accessory pathways, among which we issued 55 (23\%) posteroseptal accessory pathways. We retrospectively reviewed those 55 posteroseptal accessory pathways ablation procedures $(20$ with decremental conduction and 35 typicals) concerning 50 paediatrics patients.

Results: The median patient age was 10.5 years old (range $3.5-18$ ) and median body weight was $39.3 \mathrm{~kg}$ (12-92). 71\% were symptomatic mostly with palpitations and $15 \%$ presented with heart failure due to tachycardia induced cardiomyopathy, all associated with decremental accessory pathways and permanent jonctionnal reciprocating tachycardia (PJRT). Radiofrequency ablation was performed using a $4 \mathrm{~mm}$ irrigated electrode catheter (Thermocool, Biosense Webster, Inc). 20 patients underwent a coronarography coupled to the 3D navigation system (Carto Univu). Among them, $30 \%$ had a coronary artery in close proximity to the ablation site which modified the course of treatment: 1 patient was switched for cryotherapy, 3 patients received limited RF energy (15W), the others 2 had coronary artery injury (figure 1 ). In the patient treated with cryotherapy the ablation was unsuccessful, among the 3 patients with limited RF, one had an early recurrence.

Conclusion: Children are more at risk of coronary artery injury because of their relatively small hearts with a more pronounced proximity of ablation sites to coronary artery, which are narrower vessels with less cooling than in adult. Ablation of posterior septal pathways specifically in children carries a risk of coronary artery injury, which is probably underestimated. Coronary angiography should be strongly considered before catheter ablation for posteroseptal accessory pathways in children. The use of merged 3D images and coronarography, the limitation of RF energy or the switch to cryotherapy are possible ways to limit the risk of coronary artery injury.

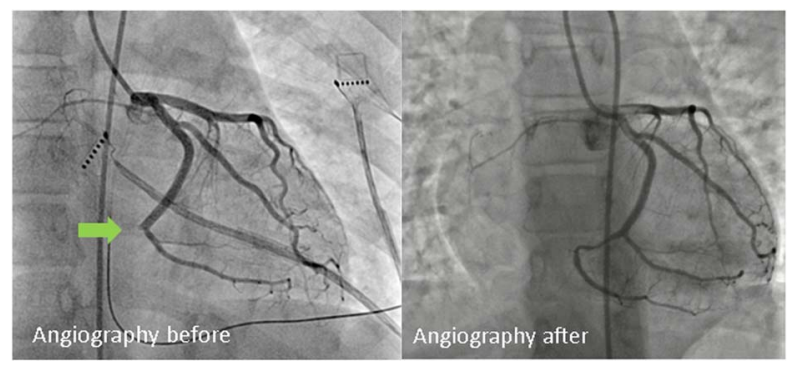

Figure 1.

Coronary injury secondary to RF ablation.

\section{O9-3}

Risk factors for Sudden Cardiac Death in Childhood Hypertrophic Cardiomyopathy: A systematic review and meta-analysis

Norrish G.(1), Cantarutti N. (1), Pissaridou E. (2), Ridout D. (2), Limongelli G. (3), Elliott P. (4) and Kaski J.P. (1)

Centre of inherited cardiovascular disease, Great Ormond Street Hospital, London, UK (1); UCL cardiovascular institute of science, London UK (1); Population, Policy and Practice Programme, UCL Great Ormond Street Institute of Child University College London, London, UK (2); Monaldi Hospital, Naples, Italy (3); Barts Heart Centre, University College London, London, UK (4)
Objective: To perform a systematic literature review and metaanalysis of clinical risk factors for sudden cardiac death in childhood hypertrophic cardiomyopathy.

Methods: Medline and Pubmed databases were searched for original articles published in English from 1963 through to December 2015 which included patients under 18 years with a primary or secondary end-point of either sudden cardiac death (SCD) or equivalent (aborted cardiac arrest or appropriate ICD discharge) or cardiovascular death (CVD).

Results: Twenty eight studies (3544 patients) met inclusion criteria. We identified four conventional major risk factors which were evaluated in at least 4 studies and found to be statistically associated with an increased risk of death in at least 2 studies: previous adverse cardiac event (pooled hazard ratio 5.4 (95\% CI 3.67-7.95), $\mathrm{p}<0.001, \mathrm{I} 2=0 \%$ ); non-sustained ventricular tachycardia (pooled hazard ratio 2.13 (95\% CI 1.21-3.74), $p=0.009, \quad \mathrm{I} 2=19 \%)$, unexplained syncope (pooled hazard ratio 1.89 (95\% CI .69-5.16), $\mathrm{p}=0.22, \quad \mathrm{I} 2=46 \%)$ and extreme left ventricular hypertrophy (pooled hazard ratio 1.80 (95\% CI 0.75-4.32), $\mathrm{p}=0.19, \mathrm{I} 2=21 \%$ ). Additional 'minor' risk factors included a family history of sudden cardiac death, gender, age, symptoms, ECG changes, abnormal blood pressure response to exercise, left atrial diameter and left ventricular outflow tract obstruction.

Conclusions: A lack of well-designed, large population based studies in childhood hypertrophic cardiomyopathy means the evidence-base for individual risk factors is not robust. We have identified four clinical parameters which are likely to be associated with increased risk of SCD, SCD-equivalent event or CVD. Multi-centre prospective studies are needed to further determine their relevance in predicting SCD in childhood HCM and to identify novel risk markers.

\section{O9-4}

Pediatric (aborted) sudden cardiac death: focus on cardiac etiologies and family history

Bakker A.M. (1), Buysse C.M.P. (1), Verhagen J.M.A . (1), Van Zellem H.L. (1), Rammeloo L.A.J. (2), Blom M.T. (3), Blom N.A. (3), Helbing W.A. (1), Tan H.L. (3), Kammeraad J.A.E. (1) 1.) Erasmus Medical Center, Rotterdam, the Netherlands 2.) VU Medical Center, Amsterdam, the Netherlands 3.) Academic Medical Center, Amsterdam, the Netherlands

Introduction: Sudden cardiac death (SCD) accounts for approximately $10 \%$ of total pediatric mortality. Studies on the etiology of (aborted) SCD < age of 40 years have shown a significant contribution of inheritable cardiac conditions (ICC). This knowledge stresses the importance of a thorough family history.

Aim: To identify the distribution of cardiac etiologies and the cardiac family history in pediatric (aborted) SCD victims.

Methods: (Aborted) SCD victims $<19$ years were identified from two prospectively developed resuscitation databases, including three tertiary referral centers, between 2002 till 2016. (Aborted) SCD was defined as an out-of-hospital unexpected and abrupt loss of consciousness with loss of vital signs, resulting in death or, if successfully resuscitated, survival to hospital discharge, with a (suspected) cardiac etiology. Medical records were retrospectively reviewed on etiology of (aborted) SCD and family history.

Results: 138 (aborted) SCD victims were identified. The median age was 1.9 years; $64 \%$ were male. $30 \%(n=41$ of 138$)$ was diagnosed with a (potential) ICC $(n=20$ primary electrical diseases, $n=21$ cardiomyopathies). $19 \%(n=26$ of 138$)$ was diagnosed with a primary non-ICC. In $51 \%(n=71$ of 138$)$ the etiology remained unspecified.

$24 \%(n=10$ of 41$)$ of patients with a (potential) ICC had a positive family history for (potential) ICC. In unspecified cases, the family 
history was reported in $45 \%$ ( $n=32$ of 71 ), of whom $16 \%$ ( $n=5$ of 32) was positive for (potential) ICC.

Conclusion: Up to one third of pediatric (aborted) SCD was due to (potential) ICC. A significant proportion of these children had a positive cardiac family history at time of (aborted) SCD. In the majority of unspecified (aborted) SCD cases, no cardiac family history was reported. Societal and medical awareness of the importance of a positive cardiac family history might improve the identification of children at risk for SCD, as well as provide unsolved etiologies in (aborted) SCD victims.

Family history

Positive Negative Unknown

Cardiac etiology, n (\%)

(Potential) ICC (primary

electrical disease,

cardiomyopathy)

Primary non-ICC

Unspecified

Total

$10(24.4) \quad 26(63.4) \quad 5(12.2)$

$3(11.5) \quad 13(50.0) \quad 10(38.5)$

$5(7.0) \quad 27(38.0) \quad 39(54.9)$

$18(13.0) \quad 66(47.8) \quad 54(39.1)$

\section{O9-5}

Cardiovascular profile in pediatric Myotonic Dystrophy type 1: are our patients at risk?

Cesar S. (1), Marzo L. (1), Carro E. (1), Nascimento A. (2), Ortez C. (2), Brugada J.(1), Sarquella-Brugada G.(1)

Pediatric cardiology department (1), Hospital Sant Joan de Déu, Barcelona, Spain, Pediatric Neurology department (2), Hospital Sant Joan de Déu, Barcelona, Spain

Introduction: Myotonic dystrophy-1(DM1) is a multisystem disease associated with cardiac conduction and structural abnormalities. Our objective is to describe cardiovascular evaluation in our paediatric population, both echocardiography and EKG measurements, and to correlate these features with CTG expansion size.

Methods: This retrospective study included 27 paediatric DM1 patients. We reviewed clinical features, transthoracic echocardiography images, EKG and cardiac 24h-Holter. We analysed (1) echocardiographic measurements with LVEF, LVED-LVES diameters, systolic and diastolic echo analysis (2) EKG features: rhythm, PR interval, QRS interval, QT and QTc. (3) cardiac 24h-Holter monitoring features:HR variability, mean SDNN, maximum RR, and qualitative findings, (4) and the CTG expansion size.

Results: No significant differences by gender (m55.56\%; f44.44\%); age was 5-19yo (m14.1). Only two symptomatic patients explaining dizziness, incomplete right branch block was detected in these cases. No sudden death was detected and no pacemaker or ICD were implanted. EKG and 24h-Holter findings:mean HR in the basal 12 leads EKG was $82 \mathrm{bpm}(40-130)$. The most frequent feature was an incomplete RBBB (26\%), and complete RBBB $(11 \%)$. Five patients $(18.5 \%$, older patients)had abnormal 24h-Holter:2 had second degree AV block Mobitz I,1 had first degree AV-block, 1 had asymptomatic monomorphic PVC, and the last one had severe sinusal bradicardia during the deep sleeping (min HR of 23bpm). Mean SDNN was obtained in $22 / 27$ patients. Analysis by age group showed a decreasing mean SDNN with age, so younger patients had a larger value SDNN than the older (group < 10yo mSDNN 170.7ms \pm 39 , and group $>15$ yo SDNN of $147.3 \mathrm{~ms} \pm 37$ ), with a significant variation between three age groups 5-10,10-15,15-20yo. No patients with structural heart disease. LVES and LVED diameters were normal,11\% had slightly reduced LVEF (52-55\%)with no relation with conduction disorders. No significant abnormalities in diastolic echo analysis. We didn't find significant relation between neurological condition, EKG/echo features and the CTG expansion (but only had an exact size in 6/27 patients,123-1667 CTG repeats).

Conclusions: (1) Pediatric patients showed mild conduction abnormalities that could be the precursors for high degree heart block, ventricular arrhythmias and sudden death in the DM1-adult population. (2) In our DM1-pediatric series, SDNN analysis showed that the lower values of SDNN the older DM1 patients. So, it is an abnormal SDNN behaviour that it could have the basis on para/sympathetic system, cardiac autonomic modulation and deterioration in baroreceptor function. (3) Echocardiographic structural abnormalities were described in DM1 population, but it had been detected in our paediatric series. (4) We cannot relate CTG repeats with the heart condition.

\section{O9-6}

Resting ECG and Signal-Averaged ECG can Detect Depolarization Abnormalities in Pediatric Catecholaminergic Polymorphic Ventricular Tachycardia Tofighi T. (1), (2), Hamilton R.M. (2)

Faculty of Medicine, University of Toronto, Toronto, CA (1); The

Hospital for Sick Children, Toronto, CA (2)

Introduction: Catecholaminergic Polymorphic Ventricular Tachycardia (CPVT) is characterized by ventricular arrhythmias (VA) in response to physical activity or emotional stress, resulting in presentations of cardiac syncope or sudden cardiac arrest (SCA) with an untreated mortality of $50 \%$. Despite limitations in nonambulatory patients, diagnosis currently relies on the presence of VA during exercise stress testing, although a negative result does not exclude a diagnosis of CPVT. We sought to assess the diagnostic utility of resting ECGs and Signal-averaged ECGs (SAECGs) in children subsequently diagnosed with CPVT.

Methods: We conducted a retrospective analysis of baseline ECG and SAECG data in 33 patients diagnosed with CPVT between March 1997- March 2016. A series of 192 waveform measurements across 12 leads were used for ECG comparisons, and for SAECG we extracted: 1) unfiltered (QRSDu); 2) filtered (QRSDf) QRS durations; 3) duration of high frequency (HFLA) signals; 4) terminal root-mean-square (RMS) voltage. A 1:2 age and gender match was used to compare ECG parameters from initial and most recent ECGs in 29 CPVT patients with a cohort of 88 normal school children using a 2 -sample t-test assuming equal variance. Data from this cohort of 150 control patients was used to derive BSA and gender corrected Z-score from regression equations.

Results: Baseline ECG data in 29 CPVT patients as compared to controls revealed statistically significant bradycardia $(p=0.016)$ and QRS prolongation $(\mathrm{p}=0.005)$. Raw QT was prolonged $(\mathrm{p}=<0.001)$ but rate-corrected QT was not. Baseline SAECGs were performed in 17 (52\%) of patients. For SAECG, the QRSDu was abnormal in 9 patients with an abnormal Z-score $>2$ (53\%). Abnormalities of QRSDf, HFLA and RMS were less common. QRSDu was not normally distributed, with patients distributed throughout $Z$ values of -1.0 to 4.5 (Figure 1).

Conclusion: ECG and SAECG are abnormal in a significant proportion of children with CPVT. SAECG abnormalities, particularly prolongation of unfiltered QRS duration, may be helpful in identifying CPVT, particularly in non-ambulatory patients who cannot formally complete exercise testing. Recent studies of patch clamp recordings in CPVT iPSC cardiomyocytes identify a decrease in action potential upslope $\mathrm{V}_{\max }$ that may explain QRS prolongation. 


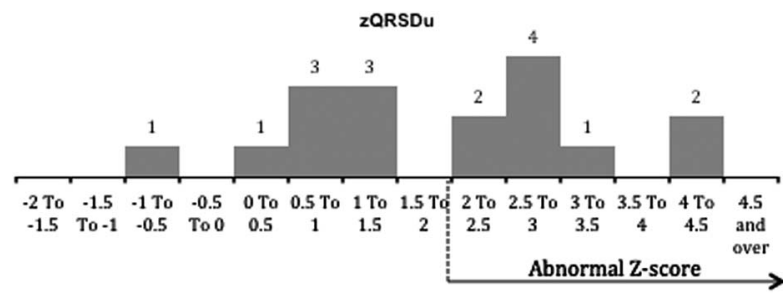

O10-1

Utility of 3D printing techniques for Interventional planning

Velasco Forte M.N. (1, 2), Byrne N. (1), Valverde I. (1, 2, 3), Pusphparajah K. (1, 2), Gomez-Ciriza G. (3), Rosenthal E. (2), Qureshi S. (2), Tandon A. (4), Razavi R. (1, 2 ), Krasemann T. (1), Hussain T. (1, 4).

King's College London, UK (1); Department of Congenital Heart Disease, Evelina London Children's Hospital, Guy's and St Thomas NHS Foundation Trust, UK (2); Cardiovascular Pathology Unit, Institute of Biomedicine of Seville, IBIS, Virgen del Rocio University Hospital/CSIC/University of Seville, Spain (3); 6. Dept. of Pediatrics, UT Southwestern Medical Center, 1935 Medical District Drive, Dallas

Background: The spatial relationships of anatomical structures in patients with complex congenital heart disease (CHD) can represent a challenge for interventional planning. In these cases, cardiac magnetic resonance (CMR) and multi-detector computed tomography (MDCT) are diagnostic tools that provide information which can be reproduced in a $3 \mathrm{D}$ printed model to assess the feasibility of interventional treatment.

Methods: Interventional planning was reproduced in cases in whom cardiac catheterisation was controvertible using a patientspecific 3D printed heart model. Segmentation was performed from previous imaging acquired dataset using Mimics software v.18. Models were fabricated by polyjet technology or fused deposition modelling. Cardiac catheterisation was performed in the model before proceeding in the patient.

Results: Fifteen patients were referred for cardiac 3D printing $(60 \%$ females; mean age:41 (1.5 - 65), 87\% adults). Four patients had partial anomalous pulmonary venous drainage, three had transposition of the great arteries (TGA), four had coronary artery fistula, one had an aortic aneurysm post aortic valve replacement, one had tetralogy of Fallot (TOF) and one had multiple ventricular septal defects (VSD). $67 \%$ of the patients underwent cardiac catheterisation, 1 case with coronary fistula and one TGA (13\%) were considered unsuitable after reviewing the model and 20\% are awaiting further management.

LPA stenosis motivated printing for $2 / 3$ of the patients with TGA and the TOF case. 3D models provided interventionalists with the opportunity to select stent length and size beforehand for all these cases. The remaining TGA had a previous atrial switch operation (Senning), developing a baffle leak that was considered unsuitable for stent closure as per the proximity of the defect to the mitral valve. All 3 coronary fistulas have successfully been closed. Occlusion of the aortic aneurysm was achieved using an AVP II device and a large VSD was closed with an amplatzer device in the case with multiple VSDs. All patients who underwent catheterisation were used had a successful result.

Conclusions: 3D models provide the opportunity to select materials and plan access routes before cardiac catheter intervention. This is beneficial where spatial complexity obstructs expert led treatment and interventional planning, providing the interventional cardiologist with confidence to address conditions that might otherwise be resolved with surgery. (a)

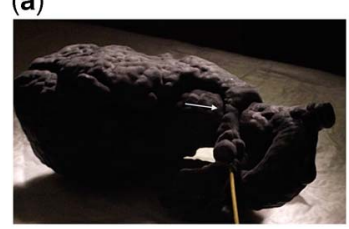

(b)

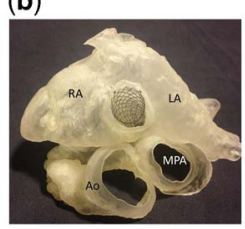

(c)

(d)

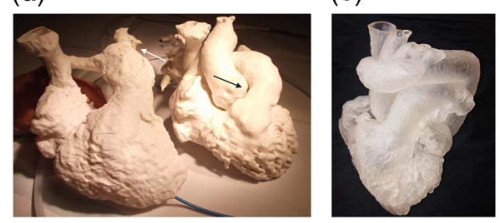

(f)

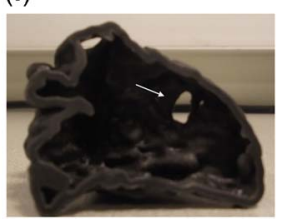

O10-2

Long-term results after implantation of an unrestrictive

Babystent in the infant population

Zartner P., Hraska V., Asfour B., Schneider M.

Deutsches Kinderherzzentrum Sankt Augustin, Germany

Rationale: All conventional stents suited for the implantation in newborns or infants have a maximum dilatable diameter below the expected growth of nearly all vessels in those patients. Either their use has to be avoided or later surgical removal is implicated.

Method: The Babystent (BS) is a premounted, balloon expandable metal stent of $6 \mathrm{~mm}$ diameter and $20 \mathrm{~mm}$ length. Due to its special design the stent struts will open up along the full length of the stent from a diameter of $10 \mathrm{~mm}$ on, so that there is no limiting upper diameter.

Population: Between 2010 and 2014, seventeen BS were implanted in 14 patients (mean age 4 months [ $1-14$ months]. All but one stent were placed into the aortic isthmus for coarctation. One stent was used to enlarge the superior vena cava in a 6 months old after Glenn anastomosis. Redilations were performed in all patients between one and 7 times. One BS was falsely explanted by a surgeon not realising the nature of the BS. Summarized follow-up time is 47.2 patient years ranging from 2 to 6 years.

Results: Sixteen BS are still in place in 13 patients. Circumferential integrity is lost in 5 patients with redilations of minimal $10 \mathrm{~mm}$. Mean BS diameter is $8.8 \mathrm{~mm}[7-12 \mathrm{~mm}]$. None of the patients has received a different stent in the position where the BS was implanted, no related re-operation was necessary. Instent tissue proliferation was found early in $5 \mathrm{BS}$, but was considered mild. A small dissection occurred in one patient after redilation.

Conclusion: The BS shows good results in treating coarctations. Despite intimal proliferation occurred quite frequently it was found early after implantation and did not grow later on. Multiple BS implantations in one patient are an option in hypoplasic vessels. The loss of the circumferential integrity of the BS does not necessarily lead to collapse of the stented area. As the BS comes premounted and does not tolerate curvy delivery routes, the treatment of coarctations seems to be the optimal location for BS placement.

O10-3

The role of interventional cardiology in the follow-up of TGA patients operated with Mustard technique Alvarez-Fuente M., Sánchez-Pérez I., Abelleira-Pardeiro C., Herraiz I., Bermudez-Cañete R., Villagrá-Albert S., Garrido-Lestache M.E., Fernandez-Pineda L., Tamariz R., del Cerro M.J.

Hospital Ramón y Cajal. Madrid. Spain. 
Background: TGA patients operated with Mustard technique present stenosis and dehiscence of the atrial baffles in their evolution. These residual lesions can be adequately treated percutaneously. Material and methods: Retrospective descriptive analysis of our experience in percutaneous treatment of baffle lesions after Mustard surgery.

Results: Between September 2006 and June 2016, 55 interventions were performed in 40 patients with Mustard surgery. Patients mean age was 26.9 years (SD 6.01), 60\% (40) were males and mean weight was $66 \mathrm{Kg}$ (SD 12.4). Indications for catheterization were: systemic baffle stenosis in $61.8 \%$ (34), deshiscence in $16,4 \%$ (9) and both lesions in 16,4\% (9). Of all the stenosis, 11 patients (25.6\%) presented a complete baffle obstruction (8 SVC baffle, 2 IVC baffle and 1 in both baffles). Nine (81.8\%) complete obstructions were treated successfully; perforation of the obstruction was performed in 8 patients with the stiff part of a coronary wire and in 3 with radiofrequency. After recanalization a sequential dilation was performed with increasing size balloons and a stent was implanted. Of the 18 dehiscence $13(72.2 \%)$ were treated succesfully, 7 with covered CP stent, 5 with ASO and one with ADO. Fourteen patients $(30.4 \%)$ carried an intravascular pacemaker at catheterization, in 9 of them a stent was implanted in the SVC baffle jailing the pacemaker cable ( $3 / 9$ patients the cable dysfunctioned). Median fluroscopy time was 32.5 minutes (IQR 23.93-51.90) and radiation dose 177 Gycm2 (IQR 127.0-260.0), being the maximum 109 minutes and 923 Gycm2 in a patient with complete obstruction.

Complications were observed in 9 interventions: 2 cases of atrial flutter after setnt implantation in SVC, pacemaker cable dysfunction in 3 patients, 1 stent migration and other minor vascular complications. No patients deceased.

Conclusions: Percutaneos treatment of Mustard surgery lesions is feasible and secure, when they are performed in units with experience in treatment and follow-up of these patients.

\author{
O10-4 \\ Pulmonary Artery Growth after Arterial Duct Stenting in \\ Completely Duct-dependent Pulmonary Circulation \\ Santoro G., Capozzi G., Mahmoud H.T., Gaio G., Giordano M., \\ Palladino M.T., Iacono C., Carrozza M., Esposito R., Ricci C., \\ Morelli C., Russo M.G. \\ Paediatric Cardiology, A.O.R.N. "Ospedali dei Colli", 2nd University \\ of Naples, Naples, ITALY
}

Introduction: Significant and balanced pulmonary artery (PA) growth following arterial duct (AD) stenting has already been reported in literature. However, no data are so far available about the role of this percutaneous approach in promoting PA growth in the case of congenital heart disease (CHD) with completely ductdepended pulmonary blood flow (CDD-PBF). Aim of this study was to evaluate the effect of AD stenting in this patho-physiologic setting.

Methods: PA growth was evaluated as Nakata Index and McGoon Ratio as well as individual PA z-score changes in 49 patients submitted to neonatal AD stenting according to their patho-physiology [CDD-PBF $(n=15)$ vs. multiple PBF sources $(n=34)$ ].

Results: Control angiography was performed 7.2 \pm 6.4 months (range 1-8, median 6) after AD stenting. In the whole population, significant and balanced PA growth was recorded (Nakata Index $+122 \pm 117 \%$; LPA z-score $+84 \pm 52 \%$; RPA z-score $+92 \pm 53 \%$ vs. pre-procedure, $\mathrm{p}<0.0001$ for all comparisons). Percentage increase of global and branch vessel size was not significantly different in patients with CDD-PBF as compared to those with multiple PBF sources (Nakata Index $89 \pm 90$ vs. $144 \pm 124 \%$; LPA z-score $63 \pm 40$ vs. $89 \pm 58 \%$; RPA z-score $74 \pm 35$ vs. $100 \pm 57 \%$, $\mathrm{p}=\mathrm{NS}$ for all comparisons) as was final absolute PA size (Nakata Index $237 \pm 90$ vs. $289 \pm 80 \mathrm{~mm} 2 / \mathrm{m} 2, \mathrm{p}=\mathrm{NS}$ ).

Conclusions: Percutaneous AD stenting was associated with significant and balanced PA growth in CHD with completely ductdependent pulmonary circulation over a short-term follow-up. Thus, it may be considered as a valuable alternative to surgical palliation in this subset of patients.

\section{O10-5}

Percutaneous Access to atrial mass in following TCPC surgery (Modified Fontan) for single ventricle physiology DeGiovanni J. V. (1,2), Mehta C. (1), Noonan P. (3), Clift P. (2), Grech V. (4), Spadoni I. (5)

Birmingham Children's (1); Queen Elizabeth Birmingham (2); Yorkhill Children's Glasgow (3): Mater Dei Hospital Malta (4); Heart Hospital G Pasquanucci Massa, Italy (5)

Introduction: The final palliation for single ventricle physiology these days utilises the surgical technique of Total Cavo Pulmonary Connection (TCPC) using a ptfe conduit between the inferior caval vein and the pulmonary arteries. Entry into the LA mass through the conduit is not easy, sometimes impossible and not without risk. We describe a technique to enter the atria mass (AM) from the right internal jugular vein, usually using a transeptal needle.

Method: The procedure is carried out under general anaesthesia and with TEE guidance.

Angiography is carried out simultaneously from the superior caval vein and the left atrium. This helps with the puncture site, direction of needle and distance between the PA and AM.

Results: We carried out this procedure in 6 patients, 5 children and 1 adult. The ages of the children ranged from 4 to 11 years and the adult was 30 years old. In 4 of the cases, the procedure was mainly carried out to create a stent fenestration, 2 within weeks of the TCPC and 2 much later for protein losing enteropathy (PLE). In 2 patients, concomitant IVC/conduit anastomosis stenting was carried out one with a $\mathrm{CP}$ stent and one with an Optimus stent because of documented stenosis. One older child required access to the AM for electrophysiology and radio frequency ablation and the adult patient required a permanent transvenous atrial pacing lead.

The procedures were all successful. In one patient, there was early closure of the stent fenestration and this was initially managed with i.v. TPA and once flow was reinstated, the stent was dilated with a ballon and has remained open an with no recurrence of the PLE. Conclusion: Access to the AM in TCPC Fontan is increasing, in part as this population is rising, they are living longer and hence will develop problems with age. Although we have used this technique safely and showed proof of concept for fenestration, permanent transvenous pacing and for arrhythmia therapy, other indications will arise in future.

O10-6

Transcatheter embolisation of the hepatic vascular anomalies - treatment of neonates with severe CHF secondary to hepatic AV shunt

Brzezinska-Rajszys G., Zubrzycka M., Kolesnik A., Rewers B., Markiewicz-Kijewska M., Kalicinski P., Kosciesza A.

The Children's Memorial Health Institute, Warsaw, Poland

Introduction: High-flow hepatic vascular anomalies with arteriovenous (AV) shunting manifest in neonates with congestive 
heart failure (CHF). Early transcatheter embolization is accepted lifesaving treatment of these patients.

The aim of the study was to present one center experience with transcatheter embolisation of the hepatic vascular anomalies in neonates with severe CHF secondary to hepatic AV shunt.

Material and methods: Seven neonates (age 3-67 days, med.19) with severe CHF related to significant hepatic AV shunt were treated percutaneously. Decision was taken in a multidisciplinary team. The main aim of percutaneous embolization was to improve cardiac status of patients. The diagnosis of hepatic vascular anomalies (multifocal hemangioma /hemangioendothelioma -3 pts, diffuse hemangioma /hemangioendothelioma $-1 \mathrm{pt}$, giant diffuse hemangioma $-1 \mathrm{pt}$, arteriovenous malformation $-2 \mathrm{pts}$ ) were confirmed by ultrasonography, computed tomography and arteriography in all cases. Embolization of vascular pathology with transarterial approach using simple, detachable and micro coils was technically successful with no complication.

Results: In 5 pts embolisation diminished signs of $\mathrm{CHF}$ and allowed for planning the later treatment (additional embolizations, surgical resection of localized tumours, banding of hepatic artery). In $1 \mathrm{pt}$ with diffuse hemangioma total hepatectomy with liver transplantation was successfully performed in age 23 days. One patient with giant diffuse hemangioma died due to multiorgan failure and sepsis at age 33 days. In last clinical and ultrasound/CT follow-up (21 - 156 mths, med. 94,65) six patients are in good general condition, with no or not significant hepatic AV shunt. Four of them had only percutaneous treatment.

Conclusions: 1. Treatment of neonates with severe CHF secondary to hepatic arteriovenous shunting is challenging and must be based on detailed diagnosis and multidisciplinary approach

2. Embolisation of liver hemangiomas or vascular malformation in neonates with severe CHF can be considered as a method of treatment or bridge to another surgical or percutaneous procedures.

3. With individualized strategy and use of various treatment options (percutaneous, surgical including transplantation) excelent results can be obtained in this most difficult and demanding patients.

\section{O11-1}

Natural History of Bicuspid Aortic Valve Disease Over First Two Decades of Life

Hagler D., Niaz T., Poterucha J.T., Olson T.M., Johnson J.N.

Mayo Clinic, Rochester Minnesota USA

Introduction: The natural history of bicuspid aortic valve (BAV) has not been elucidated in pediatric population. Our aim was to study the age wise changes in various echocardiographic parameters in patients with BAV over first two decades of life.

Methods: The Mayo Clinic echocardiography database was retrospectively analyzed to identify pediatric and young adult subjects ( $\leq 22$ years) diagnosed with BAV from 1990-2015. We analyzed all echocardiograms performed prior to any intervention on aortic valve or aorta and recorded measurements formatted as z-scores. Results: We identified a total of 1010 patients with BAV, out of which 619 had isolated BAV. The distribution of morphology of cusp fusion in isolated BAV was right-left (RL) in 419 (67.7\%), right-non coronary fusion (RN) in 197 (31.8\%) and left-non coronary fusion (LN) in $3(0.5 \%)$ subjects. There was an abnormal dilation of mid-ascending aorta (defined as z-score $>2$ ) during pediatric and young adult age. Subjects with RN fusion had significantly higher dilation than patients with RL fusion $(p<0.001)$. There was also a period of peak growth of aorta around 8 years of age. There was no significant sinus of Valsalva dilation. There was a higher mean aortic valve systolic gradient at birth due to congenital aortic stenosis but no significant progression was seen over time. However, aortic regurgitation showed age wise progression with higher grades in patients with RN cusp fusion $(\mathrm{p}<0.001)$. Conclusions: The natural history of BAV shows interesting patterns of disease progression in terms of growth of aorta and aortic valve abnormality over time based on valve morphology.
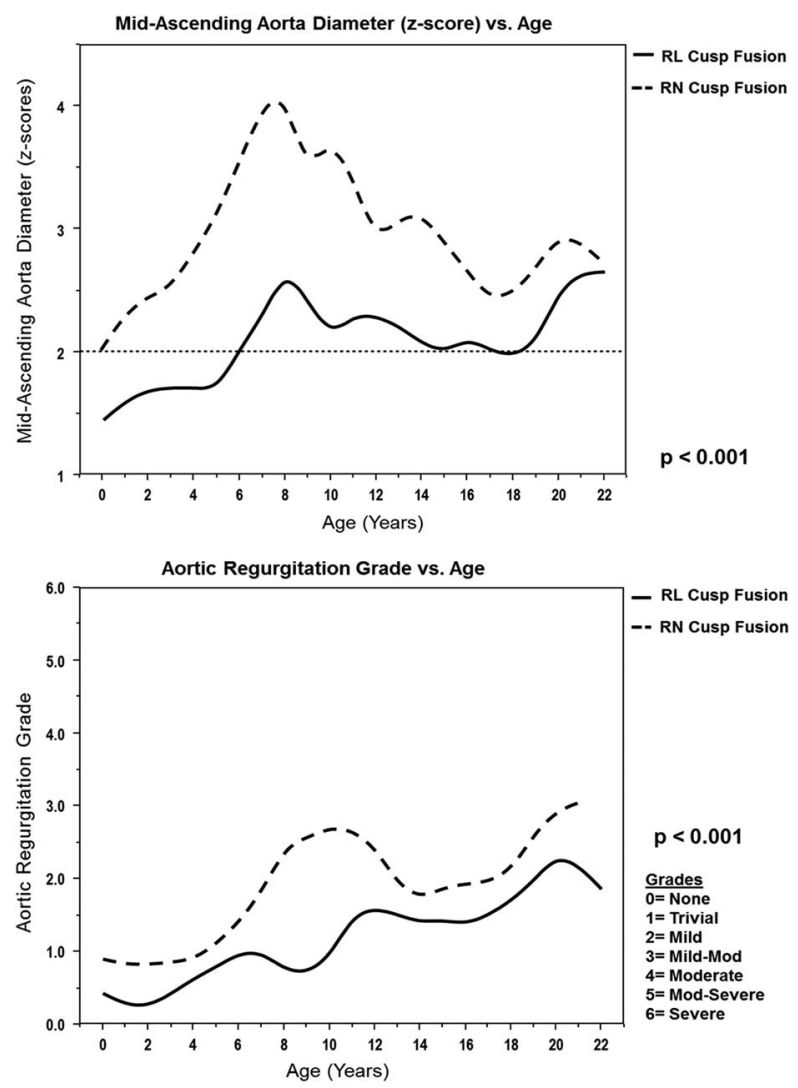

Figure 1.

Age wise progression of aortic diameter and aortic regurgitation grades in patients with BAV. Age in years is plotted along $x$ axis. Mid-Ascending aortic dimensions as $z$-scores and aortic regurgitation as grades are plotted along $y$ axis. Solid line represents $R L$ and dashed line represents $R N$ cusp fusion. P-values represent the difference between means of $R L$ and $R N$ cusp morphology in each category.

O11-2

Updating Pediatric Norms for Peak VO2 in a Clinical Setting

Nederend M.(1,2), Ten Harkel A. D. J. (2), Allison T. G.(1)

Mayo Clinic, Rochester, USA (1); Leiden University Medical Center, Leiden, the Netherlands (2)

Introduction: Predicting peak $\mathrm{VO}_{2}$ in a pediatric population presents with multiple challenges, including evolving sociocultural norms. Currently recommended norms for predicted peak $\mathrm{VO}_{2}$ based on sex and weight in children and adolescents date from 1984 (Cooper et al.). Our aim was to determine the applicability of those norms in current practice.

Methods: We reviewed the Mayo Rochester Integrated Stress Center database for the most recent cardiopulmonary exercise test performed by patients $\leq 18$ years from January 2011 to July 2016 . 
Patients with significant structural heart disease, use of negative chronotropic drugs, AICD implantation, sympathetic denervation, or obesity (CDC guidelines) were excluded. T-tests were used to determine differences in anthropometric variables, peak $\mathrm{VO}_{2}$, and percent predicted peak $\mathrm{VO}_{2}\left(\mathrm{PPVO}_{2}\right)$ between boys and girls. Sensitivity analysis was performed by excluding patients with submaximal effort $(\mathrm{n}=135$, peak respiratory exchange ratio $(\mathrm{RER})<1.05)$ on the test to determine if differences in $\mathrm{PPVO}_{2}$ could be due to differential test effort.

Results: 360 children (163 girls) with a mean age of 13 , range 6-18, for both boys and girls were analyzed. Mean height was $162 \pm 18.2$ for boys and $158.3 \pm 13.9 \mathrm{~cm}$ for girls $(p=0.033)$. BMI was comparable for boys and girls, $19.7 \pm 3.3$ and $19.9 \pm 3.1 \mathrm{~kg} / \mathrm{m}^{2}$, respectively $(\mathrm{p}=0.69)$. Average peak $\mathrm{VO}_{2}$ was $43.2 \pm 9.4 \mathrm{~mL} / \mathrm{kg} /$ $\mathrm{min}$ in boys compared to $35.6 \pm 7.9$ in girls $(\mathrm{p}<0.0001)$. $\mathrm{PPVO}_{2}$ was higher in girls (103.7 per cent [95\% CI: 99.9-107.5] compared to 93.5 per cent [95\% CI: $90.8-96.1] ; \mathrm{p}<0.0001)$. Sensitivity corrected $\mathrm{PPVO}_{2}$ increased for both boys $(96.8$ per cent $[95 \% \mathrm{CI}$ : 93.6-100.0]; $\mathrm{p}=0.063)$ and girls (109 per cent [95\% CI: $104.8-$ 113.8]; $\mathrm{p}<0.0001)$. Boy-girl differences remained statistically significant $(\mathrm{p}<0.0001)$.

Conclusion: Based on sensitivity analysis, girls achieved higher peak $\mathrm{VO}_{2}$ than predicted by the Cooper equations. These results may reflect different trends in physical activity in the United States in boys compared to girls over the 32 years since these prediction equations were published. For more accurate exercise test interpretation, we suggest adjusting predicted peak $\mathrm{VO}_{2}$ upwards by $10 \%$ for girls in current practice.

\section{1-3}

Contribution of semi quantitative MRI perfusion imaging in adolescents with suspicion of myocardial ischemia after the arterial switch operation

Atallah V., Kara M., Meot M., Raimondi F., Khraiche D., Bonnet D. Necker Enfants Malades Hospital, Paris France

Background: Late coronary artery events are extremely rare in adults after the arterial switch (ASO) for transposition of the great arteries (TGA).

Methods: Over a period of 3 years, we evaluated semi quantitative myocardial perfusion using MRI in 11 adolescents (8 males, 3 females; mean age 15.4 years) presenting with chest pain at exercise $(n=3)$ and/or with positive stress test during systematic follow-up $(n=8)$. We excluded patients who had known coronary artery anomalies and one patient in whom a previously unknown left coronary stenosis was identified during MRI. Coronary anatomy was analyzed using 3D heart imaging. Semiquantitative evaluation of myocardial perfusion was performed by the analysis of First-Pass perfusion images at rest and during dipyridamole infusion.

Results: Nine patients had normal coronary distribution and two single coronary artery. None of these patients had qualitative nor semi quantitative perfusion defect. All patients with normal coronary artery anatomy had an anterior reimplantation of left main stem immediately behind the pulmonary artery trunk but without stenosis. Only one patient with single coronary artery had anterior reimplantation of the coronary ostium.

Conclusions: Qualitative and semi quantitative perfusion was normal in adolescents with suspicion of myocardial ischemia during follow-up after the ASO for TGA. Anterior reimplantation of the left main stem was observed with a high frequency but did not translate into functional anomalies at MRI. These reassuring results do not exclude that regular follow-up of this population at risk for late cardiac events should be maintained.
O11-4

Aortic Elasticity and Carotis Intima Media Thickness in Children with Mitral Valve Prolapse

Gunay E.E., Ibıs Z., Cevik S.B., Akalin F.

Marmara University Faculty of Medicine, Department of Pediatric

Cardiology, Istanbul, Turkey

Introduction: Mitral valve prolapse (MVP) occurs due to proteoglycan accumulation, abnormal collagen composition, elastin fiber fragmentation which may also affect connective tissue and arterial wall. We investigated aortic elasticity, carotis intima-media thickness (CIMT) and their relation to joint hypermobility in patients with MVP.

Methods: The study included $41 \mathrm{MVP}$ patients (mean age $=13.4$ \pm 3.9 ; median: 14 , range:5-20 years, male/female $=15 / 26)$ and 43 controls (mean age $=12.8 \pm 3.4$; median: 13 , range: $6-19$, male/ female $=15 / 28)$. Blood pressures, hypermobility scores were obtained; Philips IE33 Ecocardiography machine equipped with $5 \mathrm{MHz}$ and L-11 Mhz linear transducers were used for measuring LV diameters and functions, valve morphology, systolic and diastolic diameters of aortic annulus, sinus valsalva (SV), sinotubuler junction (STJ), ascending, descending and abdominal aorta (AscAo, DescAo, AbdoAo) and CIMT. Z-scores, aortic strain, aortic distensibility (DI), aortic stiffness index (SI) were calculated. Results: In patients with MVP, Z-scores of aorta at level of anulus, SV, STJ were higher than controls $(p=0.023, p=0.011$, $\mathrm{p}=0.005$ respectively). Echocardiographic features; mitral anulus/ $\mathrm{m}^{2}, \mathrm{LVds} / \mathrm{m}^{2}, \mathrm{LVdd} / \mathrm{m}^{2}$ and mitral E were greater in MVP group $(p=0.001, p=0.004, p=0.022, p=0.008$, respectively). MVP group had higher Strain $(\%)$ and DI $(p=0.020,0.012$; respectively) and lower SI in ascAo $(p=0.019)$. Healthy children had higher CIMT than MVP patients $(p=0.020)$. Leaflet thickness (LT) correlated to CIMT $(p=0.033, r: 0.343)$ and SI $(p=0.039$ $\mathrm{r}: 0.356)$; but inversely correlated to strain and DI of SV ( $\mathrm{p}=0.015$, r:-0.398; $p=0.054$, r:-0.333 respectively). In both MVP and control groups, CIMT correlated to SI of SV $(p=0.001, r=0.54$; $\mathrm{p}=0.007, \mathrm{r}: 0.47$ respectively) and inversely correlated to strain and DI of SV $(p=0.005, r=-0.47 ; p=0.001, r=-0.49)$. Patients with LT greater than $5 \mathrm{~mm}$ had higher CIMT $(\mathrm{p}=0.035)$. Hypermobility score were slightly higher in MVP patients $(p=0.056)$ and correlated to DI and strain of DescAo $(p=0.002, r=-0.50 ; p=0.020$, $\mathrm{r}=-0.43)$ and DI AbdoAo $(\mathrm{p}=0.020, \mathrm{r}=-0.39)$.

Conclusions: Patients with MVP had larger aortic root and more distensible AscAo which may be related to the structure of aortic wall. Hypermobility inversely correlated to distensibility of DescAo and AbdoAo probably due to conduit-like behavior of aorta. The increase in CIMT and SI of ascAo with the increase in LT may warn about future risks for atherosclerosis.

\section{O11-5}

\section{Longitudinal assessment of global and segmental right ventricular deformation in children with hypoplastic left heart syndrome through their univentricular palliation} Kramer B., Charakida M., Bellsham-Revell H., Tibby SM., Simpson JM. Evelina Children's hospital, London, UK

Introduction: The univentricular palliation treatment in patients with hypoplastic left heart syndrome (HLHS) commonly consists of three stages; Norwood 1, superior cavopulmonary connection (SCPC) and total cavopulmonary connection (TCPC). Following each stage the loading conditions of the right ventricle (RV) change and these may impact on RV deformation. In this study, we evaluated longitudinal changes in myocardial deformation in patients with HLHS. Previous work from our group has shown that RV strain correlates with RV ejection fraction. 
Methods: Patients with HLHS who have successfully completed the univentricular palliation at Evelina Children's hospital between 2007-2016 were recruited for this study. RV systolic myocardial function was assessed at pre and post Norwood 1, pre and post SCPC and pre and post TCPC stage. Speckle tracking analysis was performed on apical four chamber views to measure global longitudinal strain (GLS) and segmental deformation. Differences in RV function according to LV morphology (mitral and aortic atresia (MA/AA) vs mitral stenosis and aortic atresia (MS/AA)) were also explored. All measurements were analysed by one observer using Q-lab version 10.5 (Philips Medical Systems, Andover, USA).

Results: Fifty-one patients with HLHS were identified (20 MA/ AA and $31 \mathrm{MS} / \mathrm{AA})$. Norwood 1, SPCP and TCPC operations were performed at $0.13 \pm 0.09,5.39 \pm 1.45$ and $40.16 \pm 7.87$ months respectively. None of the patients had significant tricuspid regurgitation. A trend towards highest values of GLS was noted prior to Norwood 1 (Table 1). No difference was observed between MA/AA, MS/AA groups with respect to GLS, whereas lower mid-septal strain was noted in the MS/AA group (p:0.013). Conclusions: In this study we demonstrated for the first time that patients with HLHS with MA/AA, MS/AA followed through Fontan palliation have similar GLS through all three stages of surgical palliation. A trend towards higher GLS in pre Norwood stage was noted which is consistent with $\mathrm{RV}$ volume loading prior to initial surgical palliation. The lower mid-septal strain in MS/AA group, possibly reflects their diseased fibrotic myocardium of the LV. These findings indicate that speckle tracking assessment tracks with physiological changes and can be used to monitor RV function in patients with HLHS.

Table 1. Speckle tracking echocardiographic parameters at each stage.

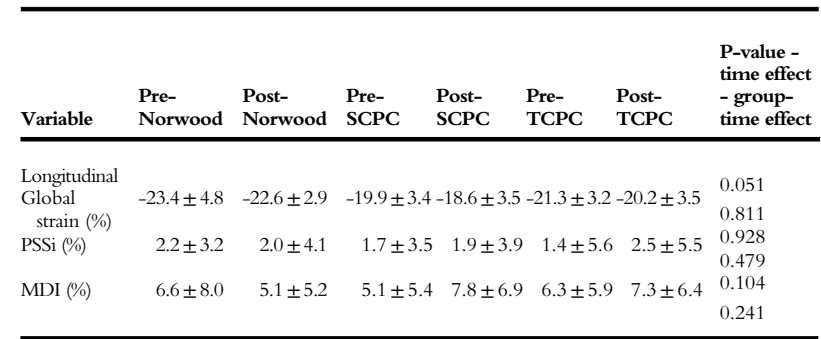

Mean \pm standard deviation

$\mathrm{SR}$, strain rate; PSSi, post systolic strain index; MDI, mechanical dyssynchrony index

\section{O11-6}

Association between obesity and abnormal postprandial hemodynamics in adolescents

Hauser J.A., Muthurangu V., Taylor A.M., Jones A.

UCL Centre for Cardiovascular Imaging, London, UK

Introduction: Early cardiovascular risk often remains undetected in the young as conventional means of risk stratification commonly rely on characterizing resting physiology. Using a novel dynamic MRI protocol, we hypothesized that ingestion of a high-carbohydrate, high-fat meal can unmask latent hemodynamic dyregulation in order to identify a pro-atherosclerotic state.

Methods: Eighty healthy teenagers (34 females; median age 16, IQR: 14 to 18 years), 34 of which were either overweight $(\mathrm{BMI}>25)$ or obese $(\mathrm{BMI}>30 ; \mathrm{n}=19)$ underwent the following protocol: After 12 hours of fasting, resting blood pressure (BP) was obtained and, using rapid MRI sequences, blood flow (BF) was measured in segments of the aorta (ascending and supra-iliac) as well as the renal, celiac and superior mesenteric arteries (CA and SMA). Following a high calorie meal $(1600 \mathrm{kcal})$, these measurements were repeated every 5 to 10 minutes for 1 hour. Interactions between hemodynamic measures and time, BMI Z score for age (zBFA) and sex were assessed in a multilevel regression model.

Results: In the fasting state, there was a significant association between zBFA and systolic BP $(2 \mathrm{mmHg}$ per zBFA increment, $\mathrm{p}<0.05)$, iliac and renal $\mathrm{BF}(0.09$ and $0.08 \mathrm{~L} / \mathrm{min}$ per zBFA increment, $\mathrm{p}<0.005$ each), and an inverse correlation of cardiac index $(\mathrm{CI})$ with zBFA $(0.18 \mathrm{~L} / \mathrm{min} / \mathrm{m} 2$ decrease per zBFA increment, $\mathrm{p}<0.001)$. By contrast, fasting perfusion of the SMA, the CA was not correlated with body weight. Following meal ingestion, there was a significant increase in CI, renal, celiac, mesenteric and iliac BF. The increase in BF to the SMA was significantly lower in overweight subjects than in the normal weight $(0.13 \mathrm{~L} /$ min decrease per zBFA increment after 1 hour, $p<0.05)$. By contrast, the remaining changes in $\mathrm{BF}$ were comparable across all weight groups.

Conclusions: For the first time we could demonstrate that postprandial mesenteric perfusion is impaired in overweight adolescents. Our findings suggest a mismatch between the postprandial metabolic demand of the small gut and the cardiovascular response in this population. Further research is necessary to better understand how this dysregulation influences atherosclerotic risk in the young.

O12-1

A Gene, MMP12, which encodes a Metalloprotease is Responsible for nearly $6 \%$ of Patients with Congenital Heart Defects and Heterotaxy

Veyrier M.(1), Guimier A.(2), Bajolle F.(3), El Malti R.(4), Oufadem M.(2), Di Filippo S.(1), Bole-Feysot C.(2), Masson C.(2), Schoen P. (5), Deleuze J.F.(6), Nitschké P.(2), Lyonnet S.(2), de Pontual L.(2), Bonnet D.(2), Amiel J.(2), Bouvagnet P.(4), Gordon C.T.(2)

Service de Cardiologie Pédiatrique, Hôpital Louis Pradel, Bron, France (1); Laboratory of Embryology and Genetics of Congenital Malformations, INSERM U1163, Institut Imagine, Paris, France (2); Unité MédicoChirurgicale de Cardiologie Congénitale et Pédiatrique (M3C), Hôpital Necker-Enfants Malades, Assistance Publique-Hôpitaux de Paris, Paris, France (3); Laboratoire Cardiogénétique, Hospices Civils de Lyon, Bron, France (4); German Heart Centre Munich, Munich, Germany (5); Centre National de Génotypage, Evry, France (6)

Introduction: Heterotaxy results from a failure to establish normal left-right asymmetry early in embryonic development. As a consequence, the normal positioning of thoracic and abdominal organs is disturbed with eventually complex cardiovascular malformations. Several genes were already associated with heterotaxy but the vast majority of heterotaxy cases remain elusive.

Method: Whole exome sequencing was performed in a family with a pair of dizygotic twins suffering from congenital heart defects and heterotaxy. In a second step, a cohort of 264 index cases with either heterotaxy (extracardiac and/or cardiac laterality defects such as dextrocardia or transposition of the great arteries; $n=154$ ) or non-heterotaxy Congenital Heart Defects (CHDs) such as isolated tetralogy of Fallot or common arterial trunk $(n=110)$ was sequenced after HaloPlex target enrichment of the MMP21 gene. Results: Patient 1, a girl, had interrupted IVC with azygous continuation, partial APVR, unbalanced AVC, cleft anterior mitral valve leaflet, hypoplastic LV, dextrocardia, common mesentery and polysplenia. Patient 2, a boy, had left SVC draining to 
coronary sinus, secundum ASD, abnormal atrioventricular connection, subarterial VSD, right aortic arch with mirror image branching, and PDA. Patient 1 died shortly after birth from haemodynamic failure. Whole exome sequencing of this affected dizygotic twins led to the identification of rare, compound heterozygous mutations in MMP21 (NM_147191.1): c.677T > C, p. Ile226Thr (paternally inherited) and c.1203G > A, p.Trp401* (maternally inherited). The cohort of 264 index cases included 25 syndromic cases in which heterotaxy or CHDs were associated with one or more other anomalies (for example, anal atresia, vertebral anomalies or cleft palate) and comprised 117 familial and 147 sporadic cases, with 34 index cases born to consanguineous parents. We identified nine cases with rare, biallelic MMP21 variations predicted to affect protein function; all variations were confirmed by Sanger sequencing and segregated with heterotaxy or other laterality defects and complex CHDs

Conclusion: Overall, the penetrance of laterality defects in our series was very high $(>90 \%)$ in comparison to that observed for Primary Ciliary Dyskinesia $(\sim 50 \%)$. On the basis of the above findings, MMP21 mutations account for $5.9 \%$ of non-syndromic heterotaxy cases.

\section{O12-2}

Exome Sequencing Reveals Overrepresentation of Rare Variants in ErbB Pathway Genes in Patients with Left Ventricular Outflow Tract Obstruction Defects

Helle E. (1,2,3), Ojala T. (3), Wernick R. (4), Ashley E. (1,2), Priest J. $(1,5)$

Stanford Center for Inherited Cardiovascular Disease, Stanford University School of Medicine, Stanford, CA, USA (1), Division of Cardiovascular Medicine, Stanford University School of Medicine, Stanford, CA, USA (2), Children's Hospital, University of Helsinki, Helsinki, Finland (3), Department of Genome Sciences, University of Washington, WA, USA (4), Division of Pediatric Cardiology, Stanford University School of Medicine, Stanford, CA, USA (5)

Introduction: Left ventricular outflow tract obstruction defects (LVOTO), such as bicuspid aortic valve, congenital aortic stenosis, coarctation of the aorta, and hypoplastic left heart, are thought to share a similar genetic basis. These defects are associated with significant heritability, yet few genes have been associated with LVOTO in humans and explain only a minority of cases.

The ErbB family receptor tyrosine kinases serve multiple functions during embryonic development, and signaling defects in these pathways are shown to lead to congenital cardiac malformations in mice. We determined the frequency of very rare variants in ErbB family genes ERBB2, ERBB3, and ERBB4 in a Finnish LVOTO patient population.

Methods: The exomes of 50 Finnish patients with LVOTO and their family members (total $n=112$ ) were sequenced at University of Washington, Center for Mendelian Genomics. Variant calling was performed with the Realtime Genomics Package (RTG version 3.4) in reference to the UCSC reference sequence (hg19). Synonymous single nucleotide polymorphisms (SNP) and variants with minor allele frequency over 0.01 in the Exome Aggregation Consortium (ExAC) database were excluded.

Results: A total of 11 probands had rare missense variants in the ErbB family receptor tyrosine kinase genes. One individual had two missense variants in the ERBB3 gene and one individual had a missense variant both in ERBB2 and ERBB3. We identified a very rare ERBB2 variant p.Arg599Cys that was present in all affected members in two unrelated families with multiple LVOTO patients in three generations. This ERBB2 variant is found only in one ExAC individual (MAF of 0.0007), and in none of the ethnically matched Finnish Sequencing Initiative Suomi population $(N=10,474)$. The variant is located in a highly conserved area of the ERBB2 gene. Both the CADD score (14.39) and the PolyPhen2 score (0.985) predict this variant to be pathogenic.

Functional work in endothelial cell models is currently being carried out to investigate the disease causing potential of these variants.

Conclusion: Very rare ErbB family receptor tyrosine kinase variants were overrepresented in this LVOTO cohort. A fifth of LVOTO probands had at least one potentially pathogenic variant in ERBB2, ERBB3, or ERBB4.

\section{O12-3}

Analysis of Pediatric Heart Failure Patients Identifies Novel Genetic Variants in Cardiac Disease Genes

Kühnisch J. (1), Herbst C. (1,2), Al-Wakeel N. (2), Degener F. (2), Messroghli D. (2), Berger F. (2,3), Klaassen S. (1,3), Experimental and Clinical Research Center (ECRC), Max-Delbrück-Centrum for Molecular Medicine (MDC), Charité - Medical Faculty Berlin, Berlin, Germany (1); Department of Congenital Heart Disease and Pediatric Cardiology, German Heart Institute Berlin, Berlin, Germany (2); Department of Pediatric Cardiology, Charité - University Medicine Berlin, Berlin, Germany (3)

Pediatric heart failure is a serious, life-threatening condition that may result from genetically defined cardiomyopathy. While the mechanisms leading to cardiomyopathy in adults are well-established, the underlying genetic mechanisms, early pathological events and additional disease promoting factors are poorly characterized in children.

To address the genetic defects leading to pediatric heart failure we analyzed a cohort of more than 100 patients ( $<18$ years) with hypertrophic cardiomyopathy (HCM), dilated cardiomyopathy (DCM), left ventricular noncompaction cardiomyopathy (LVNC), arrhythmogenic right ventricular cardiomyopathy (ARVC), and restrictive cardiomyopathy (RCM). Index patients and their first degree relatives were screened with next generation sequencing (NGS) for mutations in 174 target genes (Illumina TruSight Cardio Sequencing Panel). The detected genetic variants were bioinformatically filtered with a minor allele frequency (MAF) of $<0.001$, evaluated with in silico pathogenicity prediction tools and validated on the ExAC reference data base.

Detected variants were found in genes encoding for sarcomere proteins such as cardiac muscle alpha-actin 1 (ACTC1), alpha 2 actinin (ACTN2), lamin A/C (LMNA), myosin binding protein C 3 (MYBPC3), and myosin heavy chain 7 (MYH7). Most frequently, we found variants in MYH7 (>8 patients), MYBPC3 (>8 patients) and desmoplakin (DSP) ( $>5$ patients), which is consistent with observations in adult heart failure patients. Some patients carried known pathogenic variants such as the MYBPC3 splice site mutation c.927-2A $>$ G or the MYH7 mutation p.A428D. In addition, we observed novel variants that lead to a premature stop codon and that are pathogenic according to the American College of Medical Genetics and Genomics (ACMG) guidelines. We further identified novel pathogenic missense mutations in i) alpha 2 actinin (ACTN2) in a 6 year old patient with LVNC, ii) LIM domain-binding protein 3 (LDB3) in a 19 year old patient with HCM and iii) tafazzin (TAZ) in a 1 year old newborn with DCM. Interestingly, in about $30-40 \%$ of the analyzed index patients we did not identify pathogenic or likely pathogenic genetic variants. Our accumulated data suggest that in-depth clinical phenotyping and genetic analysis of pediatric index patients, affected as well as unaffected family members is essential to assess genetic variants in a pediatric heart failure cohort. 
012-4

Characterisation of Paediatric Hypertrophic Cardiomyopathy Patients with RASopathy Mutations Bolton C. (1,2), Norrish G. (1,2), Kaski J.P. $(1,2)$ Centre for Inherited Cardiovascular Diseases, Great Ormond Street Hospital, London (1); University College London Institute of Cardiovascular Science, London (2)

Aim: To compare clinical characteristics of patients with hypertrophic cardiomyopathy (HCM) and

RASopathy or sarcomeric gene mutations.

Methods: Retrospective data was collected for paediatric patients seen between 2014-2016, with a diagnosis of HCM (left ventricle (LV) wall thickness $\geq 2$ standard deviations above the predicted mean (z-score +2$)$, not explained by abnormal loading conditions) and a 'pathogenic' RASopathy or sarcomeric mutation.

Results: 29 patients had RASopathy mutations and 35 patients had sarcomeric. Patients with RASopathy mutations presented younger than sarcomeric patients (median age 4 months vs 9 years). A family history of HCM was uncommon in RASopathy patients $(10 \%$ vs $66 \%, \mathrm{P}<0.001)$. Compound mutations were not identified in RASopathy patients but were found in 5 sarcomeric patients.

\begin{tabular}{lcll}
\hline $\begin{array}{l}\text { RASopathy } \\
\text { Gene Mutation }\end{array}$ & Frequency & $\begin{array}{l}\text { Sarcomeric } \\
\text { Gene Mutation }\end{array}$ & Frequency \\
\hline PTPN11 & 12 & MYH7 & 17 \\
RAF1 & 9 & MYBPC3 & 15 \\
RIT1 & 4 & TNNT13 & 3 \\
HRAS & 2 & TNNT2 & 3 \\
BRAF & 1 & TPMI & 2 \\
SHOC2 & 1 & & 5 \\
Compound & 0 & Compound & 5 \\
\hline
\end{tabular}

LV hypertrophy distribution was more variable in RASopathy patients (59\% asymmetric septal, concentric 38\%), whereas $91 \%$ of sarcomeric patients had asymmetric morphology. Maximal wall thickness was lower in RASopathy patients (mean $\mathrm{Z}$ score 3.8 vs 4.6, $\mathrm{P}=0.054)$. However, $\mathrm{LV}$ outflow tract obstruction (LVOTO $>30 \mathrm{mmHg}$ gradient) and systolic anterior motion of mitral valve were more common in patients with RASopathy mutations $(48 \%$ vs $15 \% \mathrm{P}=0.005 ; 76 \%$ vs $49 \% \mathrm{P}=0.009$ respectively). Structural heart abnormalities were more frequent in RASopathy patients (valvulopathy $52 \%$ vs $14 \%, \mathrm{P}=0.002$; pulmonary stenosis $28 \%$ vs $0 \%, \mathrm{P}<0.001)$.

The majority (86\%) of RASopathy patients were asymptomatic in NYHA class 1 . Symptoms reported included: chest pain (17\%), palpitations (14\%), exertional breathless (48\%) and unexplained syncope $(7 \%)$. No deaths occurred during follow up (median follow up: 4.2 years). Ten sarcomeric patients (29\% vs $3 \%)$ underwent ICD implantation- $30 \%$ for secondary prevention. RASopathy patients were more likely to undergo myomectomy $(17 \%$ vs $6 \%, \mathrm{P}=0.234)$.

Compared to other RASopathy patients, RAF1 + mutations were associated with higher ventricular septal thickness and smaller LV diameters (mean $\mathrm{z}$ score 11.4 vs $5.5, \mathrm{P}=0.009$; and -2.2 vs -0.5 , $\mathrm{P}=0.002$ respectively).

Conclusion: HCM patients with RASopathy mutations were clinically distinct, presenting earlier in childhood with no family history. They had a more variable pattern of LV hypertrophy and a higher prevalence of LVOTO requiring myomectomy. Further studies are needed to characterise this population.
O12-5

Genetic characterization of childhood-onset cardiomyopathies in Finland

Vasilescu C. (1), Ojala T.H. (2), Brilhante V. (1), Ojanen S. (1), Palin E. (1), Alastalo T.P. (3), Koskenvuo J. (3), Hiippala A. (2), Jokinen E. (2), Jahnukainen T. (4), Lohi J. (5), Pihkala J. (2), Tyni T.A. (1,6), Carroll C.J. (1), Suomalainen A. $(1,7,8)$

Research Programs Unit, Molecular Neurology, University of Helsinki, Helsinki, Finland (1), Department of Pediatric Cardiology, Helsinki University Central Hospital and University of Helsinki, Helsinki, Finland (2), Blueprint Genetics, Helsinki, Finland (3), Department of Pediatric Nephrology and Transplantation, Helsinki University Central Hospital and University of Helsinki, Helsinki, Finland (4), Department of Pathology, Helsinki University Central Hospital and University of Helsinki, Finland (5), Department of Pediatric Neurology, Helsinki University Central Hospital and University of Helsinki, Helsinki, Finland (6), Department of Neurology, Helsinki University Central Hospital and Clinical Neurosciences, University of Helsinki, Helsinki, Finland (7), Neuroscience Center, University of Helsinki, Helsinki, Finland (8)

Background: Childhood-onset cardiomyopathies are severe heart disorders, mainly of genetic origin and typically monogenic, with dominant, recessive, maternal, or de novo occurrence. The majority of patients with these disorders are without molecular diagnosis, which is an essential step for detailed understanding of the disease, for prognostic evaluation, and treatment optimization. Methods: We collected a cohort of severe childhood-onset cardiomyopathies (KidCMP), mostly of Finnish ancestry and representative for the whole country. The 73 patients manifested with hypertrophic (HCM), dilated (DCM), restrictive, left-ventricular non-compaction, and histiocytoid cardiomyopathies, with a median age-of-onset of 0.33 years. We applied next-generation sequencing to identify the disease-causing variants: whole-exome sequencing and two targeted sequencing panels.

Results: We identified the genetic cause for the disease in $31 \%$ of the patients. Recessive, de novo, and dominantly inherited mutations formed each a third of diagnoses. Mitochondrial PPA2 was found to underlie infantile DCM with sudden death. NEK8 recessive variants were found to cause HCM with liver cirrhosis. We underscore that neonatal DCM can be caused by X-linked TAZ variants with good cardiac prognosis. Moreover, we report that recessive JPH2 variants cause early-onset cardiomyopathy, previously described in autosomal dominant adult disease. Of the 15 heterozygous diseasecausing variants identified in MYH7, ACTC1, TNNC1, TNNI3, JPH2, CALM1, CACNA1C, BAG3, TBX20, PTPN11, and RAF1, eleven were novel and ten de novo. Altogether, we found a highly heterogeneous genetic background, affecting multiple cellular pathways, with a high prevalence of de novo variants.

Conclusions: Even in a genetic isolate such as Finland, we did not find recessive founder variants, but show that de novo variants were a common cause of early-onset cardiomyopathies. We describe PPA2 as a gene for infantile DCM, and NEK8 underlying HCM with liver involvement. The disease-causing variants were typically familyspecific, emphasizing the importance of next-generation sequencing methods in routine diagnosis of these patients.

012-6

A novel compound heterozygous loss-of-function mutation in KIF20A is associated with a rare, lethal cardiomyopathy in two siblings

Louw J.J.(1,2), Verdood C. (2), Nunes Bastos R. (3), Chen X. (4), Corveleyn A. (2), Jia Y.(2), Breckpot J.(2), Gewillig M.(1), Peeters H. (2), Santoro M.(4), Barr F.(3), Devriendt K.(2)

Department of Congenital and Pediatric Cardiology, University Hospitals Leuven, Belgium(1); Centre of Human Genetics, University Hospitals Leuven, Katholieke Universiteit Leuven, Belgium (2); Department of 
Biochemistry, University of Oxford, Oxford, England, United Kingdom

(3); Laboratory of Endothelial Molecular Biology, Department of Oncology, Vesalius Research Center, VIB, Katholieke Universiteit

Leuven, Leuven, Belgium(4)

Introduction: We present a small family with three siblings of which two are affected by an undescribed and lethal cardiomyopathy, i.e. restrictive cardiomyopathy of the right ventricle. The parents are non-consanguineous of Caucasian descent. An autosomal recessive hypothesis is most likely, as this is a very rare and unique phenotype, occurring in a male and female sibling.

Methods: Exome sequencing analysis was done in unaffected and affected siblings. Genomewide SNP typing and parametric linkage analysis was done in a recessive model. Genes were identified in the linkage regions with variants in the patients, inherited from both parents, and for which the unaffected sibling is heterozygous or reference. One candidate gene was identified using functional data and genotype phenotype correlations. Results were confirmed by Sanger sequencing.

Results: We identified two compound heterozygous variants in the KIF20A as the most likely cause. Further functional studies were performed showing increased multiploidy segregation patterns and demonstrating delayed cell growth with lowered transcription and protein steady-state levels. Translational blocking of KIF20A in a zebrafish model resulted in a cardiomyopathy phenotype.

Conclusions: Reaching a genetic diagnosis in rare disorders remains a challenge. We illustrate that even in a small family with only two affected individuals, the identification of the underlying mutation is feasible, using a combination of the sophisticated genetic tools, functional studies and a zebrafish model.

\section{O12-7}

Using a zebrafish model to quantify a cardiomyopathy phenotype in a causal novel gene

Louw J.J.(1,2), Verdood C. (2), Nunes Bastos R. (3), Chen X. (4), Corveleyn A. (2), Jia Y.(2), Breckpot J.(2), Gewillig M.(1), Peeters H. (2), Santoro M.(4), Barr F.(3), Devriendt K.(2)

Department of Congenital and Pediatric Cardiology, University Hospitals Leuven, Belgium (1); Centre of Human Genetics, University Hospitals Leuven, Katholieke Universiteit Leuven, Belgium (2); Department of Biochemistry, University of Oxford, Oxford, England, United Kingdom (3); Laboratory of Endothelial Molecular Biology, Department of Oncology, Vesalius Research Center, VIB, Katholieke Universiteit Leuven, Leuven, Belgium (4)

Introduction: Cardiomyopathies are primary myocardial disorders with a structurally and functionally abnormal heart muscle. Reaching an etiological diagnosis is important for prognosis and counselling on recurrence risks. Gene identification through WES still represents a major challenge, especially since the genetics is heterogeneous. However, proving that a certain gene is a novel cause may require lengthy functional studies, including animal models. We studied a small family with two siblings presenting with a novel, lethal CMP. It was characterized by fetal-onset restrictive CMP predominantly affecting the right ventricle and leading to irreversible heart failure and early death. This phenotype is unique and has not been reported in literature.

Methods: Exome sequencing analysis was done in unaffected and affected siblings. Genomewide SNP typing and parametric linkage analysis was done in a recessive model. Embryos of wildtype, $\mathrm{Tg}$ (kdrl: EGFP)s843 and double transgenic Tg (gata1:DsRed2; kdrl: EGFP) zebrafish lines were used. The translational inhibition and splice site morpholino oligomer (MO) for zebrafish kif20a was injected into 1 to 2 cell stage embryos. Phenotypes were studied at
24, 30, 48, 72, 96120 and 144 hours post fertilization (hpf). The efficiency of $\mathrm{MO}$ on inhibiting kif20a expression was checked by Western blot assay.

Results: We identified two compound heterozygous functional variants in the KIF20A gene as the most likely cause in the family. In the zebrafish model, from day 2 onward a progressive cardiac phenotype was seen in $90 \%$ of the morphants with pooling of red blood cells proximal to the atrium, relative bradycardia, decreased cardiac function and cardiac oedema. Western blot showed a $74 \%$ protein reduction in kif20a ATG MO. To quantify cardiac function, confocal imaging of live embryos was done at 48, 72, 96 and $120 \mathrm{hpf}$. The atrium and ventricle was imaged separately and fractional shortening and heartrate is calculated. Histology is currently being performed.

Conclusions: Several mutations in known and novel genes causing $\mathrm{CHD}$ and CMP are being identified as pathogenic since the advent of Next Generation Sequencing. Proving the causality is crucial. We show that a zebrafish model can be used reliably to quantify the phenotype and cardiac function in a novel gene causing cardiomyopathy.

O13-1

Pulmonary vein flow impedance: an early predictor of cardiac dysfunction in intrauterine growth restriction Bravo-Valenzuela N.J.M., Zielinsky P., Peralta J.Z., Nicoloso L.H., Picolli A., Sulis N.M., Ritter C.C., Van der Sand L.F.

Instituto of Cardiology / University Foundation of Cardiology, Porto Alegre - Brazil

Objective: To test the hypothesis that increased pulmonary vein impedance is an early parameter of cardiac dysfunction in fetuses with IUGR due to placental insufficiency. We previously showed that pulmonary vein pulsatility index (PVPI) is higher in IUGR fetuses than in controls. In this study we evaluated the use of pulmonary vein Doppler in the assessment of the early-onset of cardiac dysfunction in fetuses with IUGR.

Methods: Twenty-seven (27) fetuses with IUGR (group 1), twenty-eight (28) fetuses with appropriate growth for gestational age (AGA) from hypertensive mothers (group 2) and twenty-eight (28) fetuses with AGA from healthy mothers (group 3 - control) were studied. Pulsatility indices (PIs) of pulmonary veins and ductus venosus were calculated by Doppler echocardiography [maximal velocity (systolic or diastolic peak) - pre-systolic peak/ mean velocity]. Obstetric ultrasound was used to assess fetal biometry and Doppler to assess the PIs of uterine, umbilical and middle cerebral arteries. Statistical analysis was conducted using ANOVA, post-hoc Tukey and Pearson's tests. P $<0.05$ was considered to be statistically significant.

Results: Mean gestational age was $31 \pm 3$ weeks in group 1 (IUGR), $30 \pm 3$ weeks in group 2 and $28 \pm 3$ weeks in the control group. Mean PVPI was significantly higher in group $1(1.32 \pm 0.4)$ as compared to group $2(1,1 \pm 0,3)(\mathrm{P}=0.01)$ and controls $(0,75 \pm 0.12) \quad(\mathrm{P}<0.001)$. In the IUGR group, a comparison between PVPI showed no significant correlation with DVPI $(\mathrm{r}=0.05 ; \mathrm{p}=0.79)$ or with cerebro-placental ratio (CPR) $(\mathrm{r}=0.01, \mathrm{p}=0.97)$. In group 2 , there was a positive correlation between PVPI and DVPI $(r=0.5 ; \mathrm{p}=0.04)$, but not between PVPI and CPR.

Conclusions: PVPI is higher in fetuses with IUGR due to placental insufficiency than in AGA fetuses from healthy mothers (controls) or from hypertensive mothers. Higher PVPI in IUGR reflects decreased LV compliance and altered LA dynamics. As LV dysfunction precedes RV, our results suggest that PVPI could be an early echocardiographic parameter in the assessment of fetal diastolic function in IUGR due to placental insufficiency. 
013-2

Isovolumic relaxation time is prolonged in fetal long QT syndrome

Clur S.A.(1), Vink A.S.(1), Robles de Medina P.(1), Blom N.A.(1), Wilde A.A.(1), Rydberg A.(2), Donofrio M.T.(3), Benson D.W.(4), Cuneo B. $(5,6,7)$.

Academic Medical Center, Amsterdam, The Netherlands (1); Department of Clinical Sciences, Pediatrics, Umeå University, Sweden (2); Children's National Medical Center, Washington, USA (3); Medical College of Wisconsin, Milwaukee, USA (4); University of Colorado School of Medicine, Denver, USA (5); Colorado Institute for Maternal and Fetal Health, Denver, USA (6); Division of Cardiology, Department of Pediatrics, Children's Hospital Colorado, Denver, USA (7).

Objective: Long QT syndrome (LQTS) is an inherited channelopathy responsible for unexplained fetal loss and sudden infant death. Prenatal diagnosis is challenging as fetal electrocardiograms are not available.

We hypothesized that the left ventricular isovolumic relaxation time (LVIRT), the mechanical correlate of repolarization, is prolonged in LQTS fetuses.

Methods: This was a retrospective multicenter case series. From fetal pulsed wave Doppler waveforms of mitral inflow and aortic outflow during sinus rhythm, we averaged 3 consecutive measurements of cycle length, LVIRT, ejection time (LVET) and isovolumetric contraction time (LVICT) in LQTS fetuses and controls. We normalized LVIRT, LVET and LVICT for cycle length as we anticipated a lower heart rate in the LQTS fetuses. We compared the measurements between the control and LQTS fetuses, and analyzed trends over time using a linear mixedeffects model.

Results: Eighty measurements from 29 LQTS fetuses (9-KCNQ1 (1 homozygous), 7-KCNH2, 7-SCN5A, 1-CALM 2 and 5-nongenotyped) were compared with 601 measurements from 466 controls. The LQTS fetal heart rates were significantly lower than the controls from 15 weeks of gestation (Fig. A). There were no significant differences between the N-LVET or N-LVICT between the two groups. The N-LVIRT was significantly longer in the LQTS fetuses after the $25^{\text {th }}$ week of gestation (Fig. B), $\mathrm{p}<0001$ at 26-30 and 32-36 weeks. The best cutoff point for the diagnosis of LQTS was a N-LVIRT of $11.21 \mathrm{msec}(95 \% \mathrm{CI}$ $11.02-12.32)$ at $32-36$ weeks with a $100 \%$ sensitivity $(95 \% \mathrm{CI} 1-1)$ and $79 \%$ specificity $(95 \% \mathrm{CI} 0.68-0.89)$ [AUC of 0.92 (95\%CI 0.84-0.96)].

Conclusion: The N-LVIRT is prolonged in LQTS fetuses from the $25^{\text {th }}$ week of gestation. We speculate that this prolongation reflects developmental changes in calcium transport and may show genotype specific effects. Together with the findings of sinus bradycardia, an increased fetal N-LVIRT duration may improve prenatal detection of LQTS. Further studies are needed to determine if the fetal LVIRT duration correlates with LQTS mutation type or perinatal ventricular arrhythmia risk.

(a)

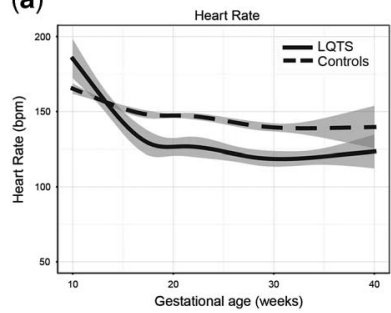

(b)

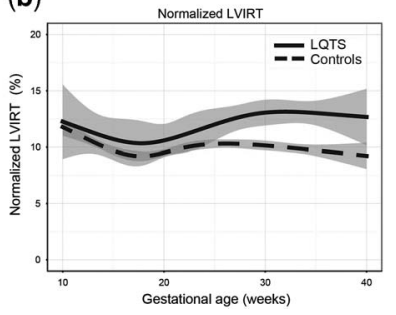

O13-3

Diagnosis, Management and Mid Term Outcome of Congenitally Corrected Transposition of the Great Arteries in the Fetus: a single centre experience

Tuo G., Paladini D., Cheli M., DeTonetti G., Santoro F., Marasini $M$.

Giannina Gaslini Institute, Genoa, Italy

Objectives: To describe the morphologic features and outcome of congenitally corrected transposition of the great arteries (ccTGA) as diagnosed during fetal life.

Background: Since now few case series have been published regarding the natural and surgical history of prenatally diagnosed ccTGA.

Methods: We included patients with a prenatal diagnosis of ccTGA between January 2005 and December 2015.

Results: 18 fetuses were diagnosed with ccTGA. Mean gestational age at diagnosis and at last fetal echocardiogram were respectively 23 weeks (17-33 weeks) and 35,5 weeks $( \pm 1,5) .17$ fetuses were referred for suspicion of cardiac malformation, one for dextrocardia. Only 2/18 fetuses presented an isolated form of ccTGA. Associated cardiac defects included an abnormal cardiac position in $6 / 18$, a ventricular septal defect (VSD) in $13 / 18$, pulmonary atresia in $6 / 18$ and pulmonary stenosis in $5 / 18$, tricuspid dysplasia in $5 / 18$, a right aortic arch in $2 / 18$. Two fetuses developed a complete atrioventricular (AV) heart block in the third trimester. None of them presented associated extracardiac abnormalities. Karyotype was tested prenatally in 4 fetuses and resulted normal. Five (28\%) pregnancies were interrupted whereas one fetus was lost at follow up. Mean gestational age at birth was 39 weeks, mean birth-weight $3200 \mathrm{~g}$ and mean oxygen saturation 95\%. 9/12 patients required one or more surgical procedures. Among them, 2 completed the Fontan circulation, 4 received the anatomical repair (2 the Senning and Rastelli procedure and 2 the double switch operation). One received a bidirectional cavopulmonary connection. Both fetuses with complete AV block required a permanent pacemaker implantation at birth. At a mean follow up of 5,1 years $( \pm 3,3) 2 / 12$ patients were lost at follow up whereas the others are alive and clinically well.

Conclusions: Isolated cases of ccTGA as well as cases of ccTGA with extracardiac or chromosomal anomalies were rare prenatally. The mid term outcome was reasonably good for all liveborn infants even if most of them needed one or more cardiac treatment. Prenatal counselling remains challenging for the fetal cardiologist because fetuses with an apparently favourable condition may then develop major changes particularly regarding to tricuspid valve and cardiac rhythm with significant modification of prognosis.

013-4

Anatomical and functional changes in foetal left ventricular myocardium

Castaldi B. (1), Cattapan I. (1), Fedrigo M. (2), Bordin G. (1), Padrini

M. (1), Susin F. (3), Angelini A. (2), Milanesi O. (1)

(1) Department of Women's and Children's Health - University of

Padova (2) Department of Thoracic adn Cardiovascular Sciences -

University of Padova (3) Department of Hydraulic Engineering -

University of Padova

Background: Left ventricular myocardium is a strongly anisotropic tissue in adults. Cardiomyocytes are arranged in a complex threedimensional network which forms a counterclockwise helix in the endocardial layer and a clockwise helix in the epicardial layer. This peculiar organization is fundamental to myocardial functionality 
because left ventricular contraction is not only longitudinally and radially oriented, but also characterized by ventricular twisting. Foetal physiological myocardial development dynamics has not been completely explained yet and the few studies about this topic focused mainly on the histology of mammalian hearts and not human ones.

Aim of the study: Speckle Tracking Echocardiography allows to study separately endocardial and epicardial functionality in vivo. Combining this data with morphometrical observation on histological sections of fetal human heart, we compared the development of the different myocardial layers with advancing gestational age.

Materials and methods: To study fetal myocardial function, we enrolled 72 foetuses and 39 premature babies without cardiac pathologies. We studied longitudinal endocardial and epicardial strain in these patients.

For the histological section, we studied 20 foetal hearts without cardiac pathologies from autoptical investigation. We determined every layer's thickness and cardiac fibres orientation in comparison with gestational age.

Results: Endocardial values of deformation are higher than epicardial ones. Epi/endocardial values ratio increase with gestational age (see figure). The epicardial layer, in fact, becomes more influencing. Histological data confirm our observations. All the layers increase their thickness during gestation but epicardium increases fourfold while trabecular and endocardial layers maintain the same value throughout pregnancy. As a consequence, also in this case we found an increasing epi/endo ratio.

Conclusions: Left ventricular myocardium maturation is a process that begins early during fetal life. It starts from the differentiation of a subendocardial layer. The development of the epicardial is slower and is completed late in the foetal life. Probably this process is led by the increasing pre- and after load in the third trimester of gestation. The creation of different phantom models of myocardial arrangements in order to assess the difference in pressure-volume curves between different layer arrangements at different gestational weeks is an on-going process.
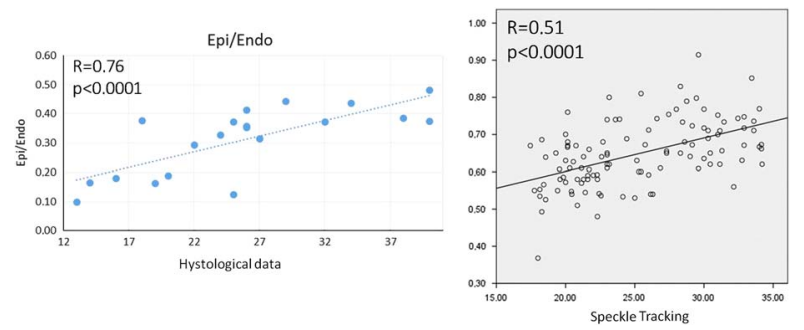

013-5

Persistent pulmonary hypertension in newborns with TGA - can it be predicted prenatally?

Grzyb A. (1,2), Kolesnik A. (1,2,3), Brzezinska-Rajszys G. (2), Brodzikowska A. (2), Zubrzycka M. (2), Rewers B. (2), Bokiniec R.

(5), Dangel J. (1,4) (1) Reference Center for Fetal Cardiology, Warsaw Hospice for Children Foundation, (2) Cardiology Department, The Children's Memorial Health Institute, (3) Descriptive Anatomy Department, Medical University of Warsaw, (4) 2nd Department of Obstetrics and Gynecology, Medical Univerrsity of Warsaw, (5) Neonatology and Neonatal Intensive Care Department, Medical University of Warsaw

Objectives: Transposition of great arteries (TGA) is one of the most common cyanotic congenital heart defects. Despite prenatal diagnosis, in cases with restriction at the level of foramen ovale and/or ductus arteriosus, it can be fatal in the newborn. When additionally complicated with persistent pulmonary hypertension of the newborn (PPHN), it poses even a greater danger, as standard therapy (PGE1 and balloon atrial septostomy (BAS) may fail to provide adequate intracardiac mixing and blood oxygenation.

The aim of this study was to search for predictive factors of pulmonary hypertension in the newborns with TGA, based on prenatal echocardiographic examinations.

Methods: We evaluated echocardiographic exams of fetuses with TGA diagnosed between 2011-2016 in the referral center for fetal cardiology. Follow-up data were collected from target pediatric cardiology departments.

Results: Out of 60 patients diagnosed with TGA, 16 showed signs of PPHN, requiring treatment with iNO (14), Epoprostenol (1) or both (1). 15/16 underwent BAS within the 1st day of life. In 14/16 fetuses signs of foramen ovale restriction and limited interatrial blood mixing were observed prenatally, usually before 35th week of pregnancy. Additionally, in 6 of them, restrictive or bidirectional flow in DA was observed, which implied increased pulmonary blood flow. In 2 remaining fetuses restriction of flow at FO level occurred after 38th week of pregnancy.

From 16 newborns with restrictive FO and PPHN 13 survived and underwent arterial switch operation successfully. From the remaining group, 3 newborns died before any attempt of intervention - in all of them there were signs of restrictive FO and in 1 - restrictive DA prenatally.

Conclusions: FO and DA flow in fetuses with TGA should be assessed repeatedly, especially just prior to delivery, as it can change during pregnancy. Restrictive flow across FO (especially longlasting), together with increased pulmonary flow in fetuses seem to be predictive of PPHN in the newborn with TGA. Such knowledge is of great importance for neonatologists and interventional cardiologists in order to provide the optimal and intensive treatment for the newborn. In all cases of TGA with PPHN prenatal diagnosis enabled to provide the optimal treatment and saved newborns' life.

\section{ON-1}

Educational involvement of nursing staff in a medical charity mission

Laciaková L., Blaškovanová N., Tláskal T.

Children's Heart Centre, Motol University Hospital, Prague, Czech Republic

Introduction: In September 2015 and March 2016 a combined team of nurses and doctors from our institution took part in the humanitarian project of the Ministry of Interior of the Czech Republic called MEDEVAC; in Phnom Penh, Cambodia. Together with other members of the team, we were asked to perform nursing supervision at the Department of Cardiac Surgery of the Calmette Hospital, where a series of paediatric surgical open heart procedures were performed. The main task was to educate local staff and possibly eliminate nursing mistakes which could potentially lead to patient's harm or to prolong the stay in the postoperative unit.

Methods: The main method used was education and supervision in oral form in English followed by demonstration. The education occurred mainly in the early postoperative period and was related to proper application of intravenous drugs, precise measurement of physiological functions, regular toilet of a respiratory tract and serving nutrition to patients. We preferred to use local material 
resources but displayed also materials imported from the Czech Republic. During our stay, we were able to organize meetings which were attended by both the principal and the head nurse. As part of these meetings, we tried to find solutions for these and other deficiencies in compliance with the personnel and local material supply.

Results: The local nursing staff showed great interest in deepening and acquiring new information and was able to utilise newly acquired knowledge almost immediately. Despite the rush associated with medical emergencies and the language barrier the local and visiting teams were able to achieve the main task as stated in the introduction.

Conclusion: The presentation highlights the importance of nursing staff participation in charity missions to perform education and supervision of the local staff, as well as to achieve best possible medical results.

ON-2

A crossover study of endotracheal suctioning methods in high risk cardiac infants

Tume L.N.(1), Baines P.(1), Guerrero R.(1), Hurley M.(2), Johnson

R.(1), Kalantre A.(1), Ramaraj R.(1), Ritson P.(1), Scott E.(1), Walsh

L.(1), Arnold P.(1)

Alder Hey Children's NHS, Liverpool, UK (1) and University of

Central Lancashire (2)

Background: Endotracheal suctioning is a risky procedure in in single ventricle infants. We examined the effect of endotracheal suction in the first 48 hours after first stage palliative surgery and to compare open (OS) and closed suctioning (CS) techniques.

Methods: A randomised crossover study combined with a prospective observational study in the first 48 hours postoperatively.

Results: 24 infants were recruited: 7 infants after Norwood-Sano, 11 modified Blalock-Taussig shunt (MBTS) and 6 after Pulmonary Artery Banding (PAB). The median age was 15 days (IQR 5-53) median weight (3.3 Kg IQR 2.8-3.6). All infants were sedated and muscle-relaxed for study measurements. There were statistically significant changes in heart rate $(p=0.002)$, all blood pressures (SBP $\mathrm{p}=0.022)$; (DBP $\mathrm{p}=0.009)$; (MBP $p=0.007)$, arterial saturation $(p=0.040)$ with OS considerably larger, but these were not clinically significant. We observed a reduction in flow velocity (on echocardiography) from $2.1 \mathrm{~m} / \mathrm{sec}$ to $1.67 \mathrm{~m} / \mathrm{sec}$ during suctioning, but recovery to baseline values was rapid. 211 suction episodes were recorded by bedside nurses during their routine suction. $62 \%$ of these suction episodes were classed as 'unplanned and 38\% 'planned'. Reasons for the unplanned suctions were most commonly (48\%) for acute desaturation (69\% having Spo2 $\leq 70 \%)$. Unit guidelines require an additional IV bolus of opiate (fentanyl) and muscle-relaxant are always administered pre-suction, yet this did not always occur. A serious adverse event (SAE) occurred in 9\% (19/211) of suction episodes. In the suction episodes where a SAE occurred $42 \%$ [8/ 19] did not have a bolus of sedation nor relaxant pre- suction and $42 \%$ [8/19] had both drugs pre-suction. 74\% [14/19] of adverse events occurred with open suctioning and $68 \%$ occurred on the night shift [13/19].

Conclusions: Under controlled suctioning conditions, there was statistically significant but no clinically significant changes in physiological parameters between open and closed suction and no adverse events. However, data collected by bedsides nurses demonstrated significant haemodynamic instability and adverse events, with less events occurring with CS. This suggests that CS is safer in less experienced cardiac PICU staff.
ON-3

Living With Fontan circulation- alive, active and happy? Adolescents and young adults experiences of their overall life situation

Berghammer M. (1,2), Rydberg A. (3), Ekman I. (1, 4), Hanseus K. (5) Karlsson J. (6)

University of Gothenburg, Centre for Person-Centred Care, www. GPCC.gu.se (1); Institute of Health science at University West, Trollhättan, Sweden (2); Department of Clinical Sciences at University of Umeå, Pediatrics, Umeå, Sweden (3); Institute of Health and Care Sciences, Sahlgrenska Academy at University of Gothenburg, Gothenburg, Sweden (4); Department of Clinical Sciences, Pediatrics, Lund University Hospital, SE-Lund, Sweden (5); Centre for Health Care Sciences, Örebro University Hospital, Örebro, Sweden (6)

Background: Adolescents and adults living with Fontan circulation belong to the first generation of patients with this condition surviving into adulthood. To what extent the heart defect affects their overall life situation therefore needs to be investigated deeper.

Aim: To explore the overall life situation of young persons living with Fontan circulation and to evaluate the impact on quality of life (QoL), health and sense of coherence.

Method: The patient sample $(\mathrm{n}=48)$ all undergone their surgical palliation before 1995. Two self-report questionnaires were used for evaluating the overall life situation including QoL, by the study-specific Essence of Existence questionnaire along with the Sense of Coherence (SOC)-scale.

Results: Of the initial patient population from $1995(\mathrm{n}=48)$, 34 was reached and 20 replied. The majority $(n=18)$ were satisfied/very satisfied with their QoL and considered their general health as good/very good $(n=16)$. The total mean SOC score was 61.3, showing significant correlations between SOC and QoL $(0.52, \mathrm{n}=20, \mathrm{p} 0.018)$, between SOC and self-esteem, $(\mathrm{r}=.71$, $\mathrm{n}=20, \mathrm{p} 0.001)$ and a positive association between SOC and better health perception (good/very good health) $(r=0.40$, $\mathrm{n}=20, \mathrm{p} .078)$. A majority $(\mathrm{n}=16)$ reported symptoms, both heart defect related and more general symptoms.

Conclusion: Present study shows that adolescents and adults are mainly satisfied with their life situation, their QoL and experience their health as good/very good, even when experience symptoms and restrictions in daily life. The result from the present study provides a deepened description of how life can be experienced when living with Fontan circulation.

Key words: Univentricular heart, Fontan circulation, Quality of life, questionnaire, sense of coherence (SOC).

\section{P-1}

Can psychostimulant drug therapy change the electrophysiological properties of low-risk manifest accessory pathways?: A case report

Karadeniz C.

Katip Celebi University, Department of Pediatric Cardiology, Izmir, Turkey

Introduction: Attention-deficit hyperactivity disorder (ADHD) is the most common psychiatric disorder in paediatric population. Despite their safety profile some concerns about the potential cardiovascular adverse effects such as arrhythmias and sudden cardiac death (SCD) still continue. The effects of psychostimulant drugs on the accessory pathway conduction properties with asymptomatic WPW pattern during the treatment of ADHD have not been evaluated before.

Case report: A 8-year-old boy diagnosed with ADHD was admitted to our paediatric outpatient clinic for cardiac evaluation before the 
initiation of psychostimulant drug therapy. His medical and familial history were unremarkable in terms of tachycardia and any cardiac event. On admission, cardiac and other system physical examinations were normal. Electrocardiographic (ECG) examination showed a WPW pattern as short PR and delta waves. Echocardiographic examination revealed normal cardiac structure and functions. An electrophysiological study (EPS) was performed for assessing the electrophysiological properties of the accessory pathway (AP) before starting the psychostimulant therapy. The pathway's effective refractive period (APERP) from the high right atrium was $290 \mathrm{~ms}$ and shortest preexcited RR interval (SPERRI) was $310 \mathrm{~ms}$ during atrial fibrillation. There was no any inducible supraventricular tachycardia observed during programmed or burst atrial pacing. With this electrophysiological properties the AP was accepted as low-risk. On the sixth month of psychostimulant therapy patient underwent second EP study for evaluating the effect of methylphenidate on the AP's electrophysiological properties. The APERP was $250 \mathrm{~ms}$ and SPERRI interval was $210 \mathrm{~ms}$ during rapid atrial pacing. Due to the high risk properties of AP and patient will continue to use psychostimulant drug therapy we decided to perform AP's ablation. During delta mapping there appeared to be an accessory pathway potential in right posteroseptal region. Ablation was performed with 6-mm tip Cryocatheter by using 3D electroanatomic mapping (The EnSite NavX system, St. Jude Medical, St Paul, MN, USA) guidance (Figure). There was no evidence of preexcitation after cryoablation. After the ablation therapy patient was discharged without any complication.

Conclusions: Patients with WPW pattern who will use psychostimulant drug therapy should be evaluated by electrophysiological study even if they are asymptomatic in terms of any arrhythmic event

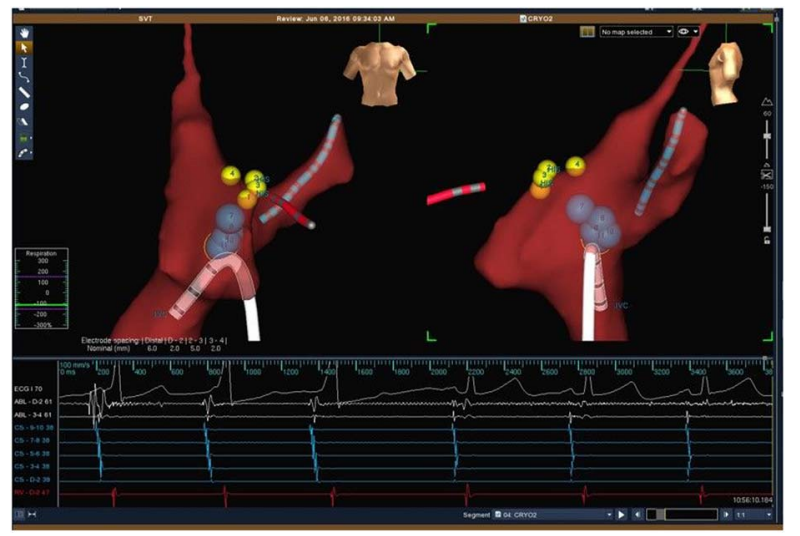

Figure.

$3 D$ electroanatomic mapping and ablation of $A P$.

\section{P-2}

Does obesity influence ventricular repolarization in children?

Yıldız N.Y. (1), Uçar T. (1), Berberoğlu M. (2), Şıklar Z. (2), Ramoğlu M. (1), Köse S.K. (3), Tutar E. (1), Atalay S. (1)

Ankara University School of Medicine, Department of Pediatric Cardiology, Ankara, Turkey (1); Ankara University School of Medicine, Department of Pediatric Endocrinology, Ankara, Turkey (2); Ankara University School of Medicine, Department of Biostatistics, Ankara, Turkey (3)

Introduction: In last decades it's reported that ventricular repolarization changes lead to sudden cardiac death in obese individuals. In this study; our aim is to investigate the relationship between ventricular repolarization changes, echocardiographic parameters with antropometric measures and metabolic syndrome laboratory parameters in obese children.

Methods: The study involved 163 subjects with a mean age ( \pm standart deviation) of $12.3 \pm 2.7$ years. A total of 81 individuals were obese (RBMI > 120) and 82 were healty non obese children (RBMI: 80-110). Anthropometric measurements of participants were performed and evaluated according to normograms. Obese patients were subdivided into two groups; metabolic syndrome (MS, n:25) and non metabolic sydrome obese (NMSO, n:56 ). Fasting plasma glucose, fasting insulin and lipid profile were performed. QT/QTc interval, QT/QTc dispersions on 12 lead ECG were measured and left ventricular systolic measurements were performed by echocardiography.

Results: Body weight, body mass index, relative body mass index and waist/hip circumferance ratio, systolic and diastolic blood pressures were found statistically higher in obese children than control group. Systolic and diyastolic blood pressure difference was significant only between MS and control groups. QT ve QTc dispersions were significantly higher in obese group regarding to healty controls. Difference between QT ve QTc dispersions in MS group compared with NMSO $(\mathrm{p}<0.001)$ and control group $(\mathrm{p}<0.001)$ and in NMSO group compared with control group $(\mathrm{p}<0.05)$ was statistically significant. QT ve QTc dispersions were influenced mostly by waist/hip circumferance ratio. Left ventricular mass index was significantly higher and EF was lower in obese group than control group. Positive correlation between LVMI and QT and QTc dispersions was detected. Relation between EF and QT ve QTc dispersions was not significant. Conclusions: QT/ QTc interval prolongation and increase in QT/ QTc dispersions on ECG may be found at early ages and subclinical left ventricular systolic dysfunction may also be detected on echocardiography in obese children therefore further investigations are necassary for evaluation of probable rhythm disturbances.

\section{P-3}

Post pediatric cardiac surgery arrhythmia - can we predict and prevent their occurrence in children after cardiac surgery?

Harpanahalli R.R, Maiya S.S, Pujar S.V

Narayana Institute of Cardiac Sciences, Bangalore, India

Introduction: Arrhythmias in immediate post pediatric cardiac surgery are not uncommon. Accurate incidence and their risk factors have been inadequately studied. This study was carried out to determine incidence of life threatening arrhythmias causing hemodynamic disturbances and determinants thereof in pediatric post cardiac surgery patients.

Methods: A prospective cohort study at a tertiary care hospital of India over 10 months. Base line data of all patients noted pre operatively. Patients were observed post operatively for arrhythmias causing hemodynamic disturbances. Ethical clearance was taken from institute ethics committee. Laboratory and clinical parameters were recorded on day of occurrence of arrhythmia or on first postoperative day in the children who had no arrhythmias. These parameters included those required to determine the variables listed in Table 1. Statistical analysis was done using SPSS 20 software. Mann Whitney test and Chi square test for comparison of proportions were used to determine significance of association.

Results: 2271 children undergoing cardiac surgery over a period of 10 months were included. The number of children who had life threatening arrhythmias requiring immediate treatment was 147 $(6.9 \%)$ (Group 1) and those without arrhythmias were 2124 (Group 2). The distribution of types of arrhythmias in group 1 is depicted in Figure 1. 
Table 1. $\star$ depicts the significance of association of various parameters between group $1 \&$ group 2 during immediate post cardiac surgery period.

\begin{tabular}{|c|c|c|}
\hline $\begin{array}{l}\text { Variable (Group } 1 \\
\mathrm{n}=147 \& \text { group } 2 \\
\mathrm{n}=2124)^{\star \star}\end{array}$ & p Value & Clinical implication \\
\hline $\begin{array}{l}\text { Significant association } \\
\text { present }\end{array}$ & & $\begin{array}{l}\text { These factors may predict arrhythmias. } \\
\text { Protective strategies may be as: } \\
\text { - Less invasive ventilation } \\
\text { - Intense monitoring, early correction } \\
\text { of biochemical factors } \\
\text { - Strict glycemic control } \\
\text { - Optimize inotropic support }\end{array}$ \\
\hline $\begin{array}{l}\text { Cardiopulmonary by-pass } \\
\text { time in minutes }\end{array}$ & $\underline{0.00}$ & \\
\hline $\begin{array}{l}\text { Cross clamping time in } \\
\text { minutes }\end{array}$ & $\underline{0.00}$ & \\
\hline $\begin{array}{l}\text { Post op ventilation duration } \\
\text { in hours }\end{array}$ & 0.00 & \\
\hline $\begin{array}{l}\text { Low Serum Ionized calcium } \\
\text { on day of arrhythmia } \\
(\mathrm{mmol} / \mathrm{L})\end{array}$ & 0.004 & \\
\hline $\begin{array}{l}\text { Hyperglycemia on day of } \\
\text { arrhythmia }(\mathrm{mg} / \mathrm{dL})\end{array}$ & 0.004 & \\
\hline $\begin{array}{l}\text { Low Partial pressure Oxygen } \\
\text { in arterial blood }\end{array}$ & 0.025 & \\
\hline $\begin{array}{l}\text { Adrenaline infusion } \\
\text { administered }\end{array}$ & $\underline{0.000}$ & \\
\hline $\begin{array}{l}\text { Milirione infusion } \\
\text { administered }\end{array}$ & $\underline{0.000}$ & \\
\hline $\begin{array}{l}\text { Isoprenaline infusion } \\
\text { administered }\end{array}$ & $\underline{0.000}$ & \\
\hline $\begin{array}{l}\text { More than three inotropes } \\
\text { administered }\end{array}$ & $\underline{0.003}$ & \\
\hline \multicolumn{3}{|c|}{ No Significant association present } \\
\hline Age in months & 0.102 & $\begin{array}{l}\text { Comparable age and gender } \\
\text { composition in both groups }\end{array}$ \\
\hline Gender & 0.448 & \\
\hline $\begin{array}{l}\text { Raised Pre-operative serum } \\
\text { Creatinine }\end{array}$ & 0.887 & \\
\hline $\begin{array}{l}\text { Raised Post-operative serum } \\
\text { Creatinine on day of } \\
\text { arrhythmia }\end{array}$ & 0.083 & $\begin{array}{l}\star \star \text { Age, bypass time, cross clamping time and } \\
\text { post-operative ventilation time are not } \\
\text { normally distributed. Hence non } \\
\text { parametric tests (Mann Whitney Test } \\
\text { was used) }\end{array}$ \\
\hline Pre-operative leuckocytosis & 0.053 & \\
\hline $\begin{array}{l}\text { Post-operative leuckocytosis } \\
\text { on day of arrhythmia }\end{array}$ & 0.735 & \\
\hline $\begin{array}{l}\text { Acidosis present on arterial } \\
\text { blood gas analysis }\end{array}$ & 0.929 & \\
\hline $\begin{array}{l}\text { Low Serum bicarbonate levels } \\
\text { on day of arrhythmia }\end{array}$ & 0.924 & \\
\hline $\begin{array}{l}\text { Low Serum Potassium on day } \\
\text { of arrhythmia }\end{array}$ & 0.47 & \\
\hline $\begin{array}{l}\text { Noradrenaline infusion } \\
\text { administered }\end{array}$ & 0.184 & \\
\hline
\end{tabular}

$\star$ Limited data and statistical calculations presented due to paucity of space

m AV disassociation
$=$ JET
$=$ Sinus node dysfunction
SVT
$=$ VT
$=$ Atrial flutter
$=$ Atrial fibrillation
$=$ Ventricular fibrillation
$=$ Complete heart block

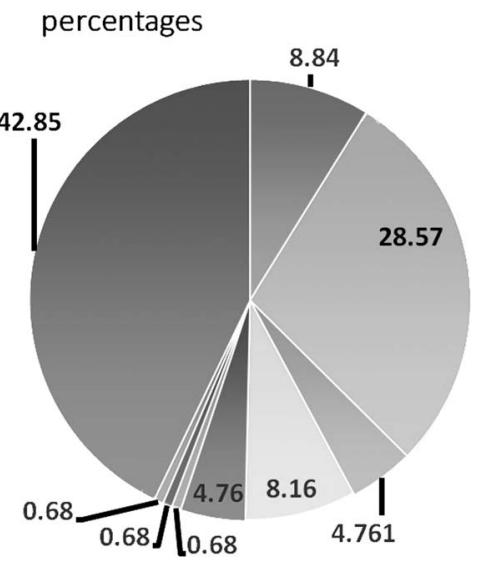

Conclusions: The incidence of arrhythmias are likely to reduce by being more intense in monitoring blood biochemistry and gases with strict glycemic control and being less invasive in interventions like cardiopulmonary bypass time, cross clamping time, ventilation duration and inotropic usage.

\section{P-4}

Miniaturized subcutaneous Holter: finally a paediatric tool Sarquella-Brugada G., Moll I., Cesar S., Carro E., Brugada J.

Pediatric Arrhythmia Unit, Hospital Sant Joan de Déu, Barcelona, Spain

Introduction: Paediatric health professionals are constantly inventing trying to adapt adult technology to our small patients. Miniaturized subcutaneous holter monitor with remote monitoring is a useful tool extensively used in adult population and, due to its small size, potentially useful in paediatrics.

The objective of our work is to analyse utility, implanting techniques and tracings quality of the subcutaneous holter monitor in paediatric population.

Methods: Retrospective analysis of 70 paediatric patients implanted with a miniaturized subcutaneous holter monitor in our institution.

Results: The indication for implant was syncope, palpitations, monitoring and titration of treatment in patients with high risk of malignant arrhythmias.

Implant technique was performed under mild anesthesia, modifying the technique recommended by the company, being at a mid-axillary subpectoral position and closure with surgical glue.

Event detection rate was high $(23 \%$ of our patients at first 6 months), quality of the electrograms was good (easy identification of $\mathrm{P}$ waves, supraventricular and ventricular extrasystoles, supraventricular tachycardias and asystole).

Our experience with remote monitoring has been acceptable in almost all patients. In $72 \%$ of them, different types of alerts have been received, finally being described as respiratory arrhythmia.

Concerning complications, two explants have been necessary (local infection) and one abrupt explant has been observed after a high intensity thoracic impact.

Conclusions: Miniaturized subcutaneous holter monitor is an excellent tool for paediatric population, taking into account the limitation of the false alerts in a so common paediatric arrhythmia such respiratory arrhythmia.

\section{P-5}

Flecainide test in paediatric population: safety, effectiveness and risk factors for a positive test

Sarquella-Brugada G.(1), Cesar S.(1), Carro E.(1), Reiter D.(1), Moll I.(1), Torner S.(1), Esquirol M.(1), Campuzano O.(2), Brugada J.(1) Pediatric Arrhythmia Unit, Hospital Sant Joan de Déu, Universitat de Barcelona, Barcelona, Spain (1); Centre de Genètica CardiovascularIDIBGI, Universitat de Girona, Girona, Spain (2)

Introduction: Flecainide test is a pharmacological test used in order to unmask Brugada-pattern EKG in patients with a suspected Brugada Syndrome (BrS). The aim of this study is to review data about Flecainide test and to predict the conditions for a positive test in our pediatric Arrhythmia Unit.

Methods: We retrospectively reviewed all our standardized Flecainide test $(2 \mathrm{mg} / \mathrm{kg}$ in $10 \mathrm{~min})$ performed from 2010 to 2016 in pediatric patients. Clinical data (demographic data, familial and personal clinical history, symptoms), EKG changes (basal and with fever), SCN5A mutation and data concerning Flecainide test are described and analysed. 
Results: We reviewed 81 pediatric patients, by gender (f54.3\%, $\mathrm{m} 45.6 \%$ ), median age 10.7y (SD4.2, IQR6). Familial clinical history of $\mathrm{BrS}$ was present in 65 patients $(80.2 \%)$. Of these patients, $29.6 \%$ had first-degree relatives with BrS.

We observed 67/81 negative Flecainide test (82.7\%), 11/81 positive $(13.5 \%)$ and $3 / 81$ uncertain $(3.7 \%)$. Four patients required monitoring during overnight hospitalization. One of the 11 positive Flecainide test had an abnormal EP study.

Syncope was the main reason to perform Flecainide test (22.2\%), but we detected other symptoms (palpitations, dizziness, febrile seizures/ unspecified convulsions and recovered sudden death). No symptoms in $58 \%$ of patients, so the test was performed for other reasons.

Statistical analysis by familial history, basal and febrile EKG, syncope and febrile seizures and SCN5A mutation was performed. We detected $8 / 11$ positive Flecainide test had familial history for BrS (72.7\%) and 9/11 had abnormal basal EKG defined by incomplete RBBB or 2-3 BrS pattern. Flecainide test was positive in $2 / 3$ cases of abnormal febrile EKG (66.6\%), and uncertain in the other one. Syncope was the main symptom in the positive Flecainide test patients $(3 / 11,17.6 \%)$; the other patients had no related symptoms. Febrile seizures were detected in 5/81 patients; one of them had a positive Flecainide test and 1 uncertain.

Within the 11 positive Flecainide test, 3 of them had SCN5A mutation $(27.2 \%)$. No SCN5A mutation was detected in patients with febrile seizures, and 1 was detected in patient with syncope. Conclusions: (1) We did not detected severe complications related with Flecainide test. (2) About 15\% of our patients had a positive test, and the symptom most related with that was syncope. (3) We detected the following risk factors for a positive Flecainide test: a) SCN5A mutation b) familial history for $\mathrm{BrS}$ (c) having an abnormal basal EKG.

\section{P-6 \\ QT correction during exercise testing: performance of new QT formulae computed from normal cohort at baseline Benatar A., Dewals W., Decraene T., Feenstra A. \\ Department of Paediatric Cardiology, Universitair Ziekenhuis (VUB), Brussels, Belgium}

Aim: We studied the performance of new QT correction formulae computed from electrocardiograms (ECG) from a normal cohort of children preceding exercise testing. Formulae were applied to all exercise stages and compared to QTc Bazett.

Methods: 74 healthy children underwent graded exercise tests (bicycle or treadmill), heart rate reached $>85 \%$ expected maximum for age. Digital 12 lead ECGs recorded and stored at baseline, maximum exercise, one, two, four, and six minutes post exercise (all erect posture). For all stages, ECGs retrieved, QT and RR intervals measured on screen (lead II or V5). From baseline ECG, the QT/RR and natural $\log \mathrm{RR} /$ natural $\log \mathrm{QT}$ were plotted. New formulae obtained from linear regression analysis. Regression analysis QT/RR computed QTc $=\mathrm{QT}+$ constant $\alpha \mathrm{x}$ (1-RR), whilst regression analysis natural $\log \mathrm{QT} /$ natural $\log \mathrm{RR}$ computed $\mathrm{QTc}=\mathrm{QT} / \mathrm{RR} \beta$. New formulae were applied for all stages procuring QTc values. QTc Bazett $(\mathrm{QTc}=\mathrm{QT} / \mathrm{RR} 1 / 3)$ was calculated for comparison. Descriptive and analytical statistics were calculated, significance level set $\mathrm{p}=<0,05$.

Results: Median age 10.4 years, mean 10.7 (range 5.05-15.9 years) Gender: 40 males and 34 females. $\alpha=0.19, \beta=0.4 \log$ QTc rest: median $409 \mathrm{~ms}+17$, peak $380 \mathrm{~ms}+22$, 1' $385 \mathrm{~ms}+23,2$ ' $403 \mathrm{~ms}+$ 25, 4' $416 \mathrm{~ms}+14,6$ ' $420 \mathrm{~ms}+14$; Linear QTc rest: median $412 \mathrm{~ms}+15$, peak $374 \mathrm{~ms}+14,1^{\prime} 381 \mathrm{~ms}+17,2$ ' $400 \mathrm{~ms}+16,4$ ' $414 \mathrm{~ms}+12$, 6' $419 \mathrm{~ms}+12$. QTc Bazett rest: Median $424 \mathrm{~ms}+20$, peak $426 \mathrm{~ms}+24,1^{\prime} 423 \mathrm{~ms}+27,2^{\prime} 435+27,4{ }^{\prime} 442 \mathrm{~ms}+16 ; 6$ ' $442 \mathrm{~ms}+17$. Median heart rate beats/minute rest $92+14$, peak $185+11,1^{\prime} 149+20,2^{\prime} 121+20,4^{\prime} 106+16,6^{\prime} 102+14$.
Conclusion: both new formulae exhibit significant QTc decline at peak exercise $\mathrm{p}<0.01$, returns to normal by 4 minutes. In contrast, Bazett QTc Increases at peak exercise, then dips and surges significantly $\mathrm{P}<0.01$. These new formulae appear promising in the evaluation of congenital long QT syndrome and drug QTc monitoring.

\section{P-7}

Feasibility and outcome of near zero fluoroscopy ablation procedures in children

De Wilde H., De Pooter J., Timmers L., Van Heuverswyn F., De WolfD. Ghent University Hospital, Ghent, Belgium

Introduction: Recent developments in mapping techniques allow to completely discard fluoroscopy in a great number of ablation procedures. We studied the feasibility and outcome of "near zero fluoroscopy ablation" in our pediatric population.

Methods: Data were retrospectively collected from twenty-four consecutive pediatric near zero fluoroscopy ablation procedures, performed from January to September 2016. Demographics, procedural parameters and outcome from these procedures were compared with previously collected data. Continuous data were analyzed using the 2-tailed Student's t test or the Mann-Whitney U test. Pearson Chi-square test was used for the categorical parameters.

Results: During a 10-month period twenty-four symptomatic children with a previously suspected or documented reentry tachycardia or pre-excitation underwent a radiofrequency (RF) ablation under general anesthesia. Fourteen patients (58\%) had RF applications in the slow pathway region because of slow/fast atrioventricular nodal reentry tachycardia (AVNRT). In 8 patients $(33 \%)$ there was overt conduction over an accessory pathway (AP), the remaining two had concealed conduction (3 right sided, 6 left sided and one mid-septal AP). The mean age at the time of the procedure was 11.4 years, the mean body weight 39.2 kilograms. The mean procedural time was 118 minutes.

In twenty children (83\%) all imaging was performed only with the use of a non-fluoroscopic 3-dimensional (3D) mapping system (Ensite Nav X). In four children fluoroscopy was used during the transseptal puncture for access to the left AV groove.

The acute success rate was $92 \%$ and there were no procedural complications except for self-limiting mild groin discomfort in one patient (4.2\%). One patient had recurrence of pre-excitation during follow-up. Conclusion: This report underlines the feasibility and impact of "near zero fluoroscopy ablation" during pediatric RF-ablation procedures for AVRT or AVNRT. As fluoroscopy was only used in four procedures, 20 children were cured without any exposure to radiation and its associated risks. Not surprisingly, a significant reduction in mean DAP was observed, without compromising procedural time, patient safety or outcome.

Performing near zero fluoroscopy ablation procedures in children under guidance of a non-fluoroscopic 3-D mapping system should therefore be considered as a standard of care in pediatric ablation procedures.

\section{P-8}

Management of electrical storm in a 14-year-old girl with Anderson-Tawil syndrome

Schmidt K., Follmann P., Pohler J., Fritsche K., Mitschke T., Zimmer M., Kriebel T.

Department of Paediatrics, Westpfalz-Klinikum, Kaiserslautern, Germany

Introduction: One prominent feature of Anderson-Tawil syndrome (ATS) is a propensity to considerable ventricular arrhythmia such as isolated premature ventricular contractions (PVC), bidirectional 
or polymorphic ventricular tachycardia (VT). However, the risk of Torsade de Pointes tachycardia or sudden cardiac arrest seems to be low in ATS. To the best of our knowledge, this is the first report of management of electrical storm in a patient with ATS.

Case Presentation: In a 14-year-old girl with clinical features and a positive genetic testing for ATS an ICD was implanted in 2007 due to a syncope and recurrent bidirectional VT. Despite the treatment with Flecainide and Metoprolol arrhythmia burden increased over the years up to $70 \%$ in the Holter monitor. Although VT was clinically well tolerated, ventricular function became compromised in the last 6 months with a shortening fraction (SF) of 20-25\%. Five days prior to hospital admission for a planned change of antiarrhythmic medication she suffered from a syncope due to self-limited VT. Some hours later the patient developed an electrical storm with recurrend hemodynamic instable VT. Despite sedation, intubation, and infusion of Esmolol and magnesium 11 internal and external (after deactivating the ICD to save battery life-time) defibrillations had to be performed. Left ventricular function was reduced to a SF of $15-20 \%$. After the injection and subsequent continuous infusion of Lidocaine rhythm stabilized and sinusrhythm in alteration with ventricular bigeminus was noted. In the next five days Lidocain infusion could be stopped and oral antiarrhythmic medication was changed from Metoprolol to Propranolol $(5 \mathrm{mg} / \mathrm{kg} / \mathrm{d})$ and from Felcainide to Imipramin $(4 \mathrm{mg} / \mathrm{kg} / \mathrm{d})$ resulting in a reduction of arrhythmia burden to $20 \%$ with mainly PVC/ventricular bigeminus in the Holter monitor. In the next 3 months arrhythmia burden remained stable and ventricular function returned to normal with a SF of $30-35 \%$.

Conclusion: Electrical storm is a rare but life-threatening complication of Anderson-Tawil syndrome which can acutely be treated by Lidocain infusion in our patient. As described once before Imipramin may play an important role in the chronic treatment of this channelopathy.

\section{P-9}

Rate Control by Transoesophageal Atrial Qverdrive Pacing for Refractory Supraventricular Tachycardia Paech C. (1), Janousek J. (2), Wagner F. (1), Gebauer R.A. (1) Department for Pediatric Cardiology, University of Leipzig - Heart Center, Leipzig, Germany (1), Children's Heart Centre, 2nd Medical Faculty of Medicine, Charles University in Prague and Motol University Hospital, Prague, Czech Republic. (2)

Introduction: Tachyarrhythmia-induced cardiomyopathy (TIC) is a rare yet life-threatening phenomenon in children. TIC has been defined as myocardial dysfunction that is entirely or partially reversible after control of the responsible tachyarrhythmia and is typically caused by an incessant supraventricular tachycardia. In cases of unsuccessful termination of the tachycardia cardiogenic shock may occur. Several authors favour the use of mechanical circulatory support in such cases. In view of these partly severe cases the authors would like to present the option of transoesophageal overdrive pacing (TOP) as a tool for initial heart rate control and hemodynamic stabilization of patients with TIC refractory to conventional strategies of tachycardia termination.

Methods: Chart review for patients receiving TOP during the last 5 years in two centers was performed.

Results: A case series of successful TOP including technique and outcomes is depicted.

Conclusion: Rate control through TOP is a safe and effective option to restore acceptable hemodynamics in infants with refractory supraventricular tachycardia and severe impairment of ventricular function. It presents the possibility of immediate heart rate control and offers time for myocardial recovery and safe implementation of antiarrhythmic drug therapy.

\section{P-10}

Surface electrocardiographic features and prevalence of arrhythmias in paediatric Friedreich's ataxia

Montañes M. E., Toledano M., Field E., Walsh H., Maguire K., Cervi

E., Kaski J.P.

Great Ormond Street hospital, London, UK

Introduction: Friedreich's ataxia (FA) is a progressive neurodegenerative disease inherited as an autosomal recessive trait. The diagnosis is usually made on the basis of neurological symptoms with progressive ataxia. Cardiac involvement, in the form of concentric non obstructive hypertrophic cardiomyopathy (HCM), is very common (up to $90 \%$ ). Heart failure in the $3 \mathrm{rd}$ and 4 th decades is the most common cause of death. However, data on the prevalence of arrhythmias in this population are scarce.

Methods: Clinical and electrocardiographic data from a cohort of 26 consecutive paediatric patients with FA evaluated between 2007 and 2016 were reviewed. Fourteen patients were female $(54 \%)$ and mean age at diagnosis was $10 \pm 3$ years. Patients were followed up for a median of 3 years (interquartile range 2 to 6 years).

Results: 24 (92\%) patients had echocardiographic features of HCM. Twenty five (96\%) showed abnormalities on their baseline ECGs, with only one patient, without cardiac involvement, having a normal ECG. The most common abnormalities were: inferolateral repolarisation abnormalities $[\mathrm{n}=24(92 \%)]$; voltage criteria for left ventricular hypertrophy $[\mathrm{n}=19(73 \%)]$ and pathological $\mathrm{Q}$ waves $[\mathrm{n}=4(15 \%)]$. Three patients $(11 \%)$ showed relatively small QRS voltages throughout and five (19\%) had an abnormal QRS axis (4 with right axis deviation and 1 with left axis deviation). None had evidence of preexcitation or conduction disease.

20 patients $(77 \%)$ underwent at least one 24 hour Holter monitor during follow up, including 8 patients (40\%) who were symptomatic (chest pain, $n=4$; palpitations, $n=3$; syncope, $n=1$ ). Four patients $(20 \%)$ were found to have tachyarrhythmias at follow-up: $2(10 \%)$ had isolated short runs of nonsustained supraventricular tachycardia (SVT); 1 had an 8 beat run of non-sustained ventricular tachycardia; and 1 had an episode of paroxysmal atrial fibrillation (AF). There were no sudden arrhythmic deaths.

Conclusions: ECG abnormalities are very common in FA, and 20\% had non-sustained arrhythmias at follow-up. These results suggest that 24 hour Holter monitoring should be performed routinely during follow up to detect underlying arrhythmias in FA.

\section{P-11}

The Effect of Metilpheniydate, Risperidone and Combination Therapy on ECG in Children With Attention-Deficit Hyperactivity Disorder

Karpuz D.(1), Hallioglu O.(1), Toros F.(2), Tasdelen B.(3)

Mersin University, Faculty of Medicine, Department of Pediatric Cardiology, Mersin, Turkey(1). Mersin University, Faculty of Medicine, Department of Child and Adolescent Psychiatry, Mersin, Turkey (2).

Mersin University, Faculty of Medicine, Department of Biostatistics and Medical Informatics, Mersin, Turkey (3).

Introduction: Attention-deficit hyperactivity disorder (ADHD) is a common neurodevelopmental disease in children for which the most commonly preferred medication is with metilphenydate and/or risperidone. However, concerns regarding the adverse effects of both drugs have increased recently. The aim of this study 
is to investigate ventricular repolarization on electrocardiogram (ECG) in a pediatric population receiving methylphenidate $(\mathrm{MPH})$, risperidone (RIS) and combined therapy.

Methods: A total of 215 patients between 6-12 years with ADHD/ conduct disorder receiving methylphenidate, risperidone and combined therapy for minimum 3 months and an untreated ADHD group $(n=76)$ were consecutively included in the study. Seventy healthy children in the same age group were enrolled as a control group. In the study, twelve lead ECG parameters including mean QT, corrected QT (QTc), T-peak to T-end (TpTe) intervals, $\mathrm{Tp} T$ e dispersion and $\mathrm{TpTe} / \mathrm{QT}$ ratio were compared. Results: Mean systolic and diastolic blood pressure values were found to be significantly higher in the MPH group compared to the untreated ADHD, combined therapy group and the healthy control group $(\mathrm{p}<0.05)$. QT interval, and QTc, TpTe interval, $\mathrm{TpTe}$ dispersion and $\mathrm{TpTe} / \mathrm{QTc}$ ratio values for groups receiving RIS, MPH and combined therapy were found to be significantly higher than both the control and untreated ADHD groups. Moreover, in the combined therapy group TpTe and TpTe/QTc values were higher than the single drug administration groups $(\mathrm{p}<0.05)$. TpTe and TpTe/QT ratio was significantly higher in the RIS group compared to that of the MPH group. $(\mathrm{p}<0.05)$. Conclusion: In conclusion, $\mathrm{TpTe}$ and $\mathrm{TpTe} / \mathrm{QT}$ ratio was found to be higher in the combination therapy group compared to the monotherapy group. It was also higher in the RIS group compared to the MPH group. These results suggested that combined therapy of these drugs had a more prominent impact on the $\mathrm{T}$ wave and RIS could be strongly associated with it.

\section{P-12}

Filling the gap in the detection and management of dysrhythmia - the life-vest

Fernandez Rodriguez S., Haas N.A., Lehner A., Birnbaum J., Fischer M., Schulze-Neick I.

Klinikum der Universität München - Campus Großhadern, Departement of pediatric cardiology and intensive care, Munich, Germany

Objectives: Palpitations and syncopes are frequent possible symptoms of dysrhythmias in pediatric patients and in patients with congenital heart diseases. The precise diagnosis can be influenced by a number of factors: non-compliance, rare occurence and short duration of the dysrhythmia. With the standard holter ECG there is only the option of 24-48-hour monitoring of the patient. Event monitors however are invasive and expensive procedures. To date there is no possibility to make a 30-day monitoring with a 3-channel ECG.

Methods: We used the 3-channel cordless ECG from Nuubo ${ }^{\circledR}$ in 15 consecutive patients and assessed the feasibility and accuracy. In addition questionaire regarding patient comfort was used. Diagnostic investigation were indicated because of palpitations, syncopes, previous history of dysrhythmia with new symptomes and for therapy monitoring. The average period of monitoring was 9 days (5-21 days). The median age was 22 years (1-61 years).

Results: The long-term ECG monitoring showed an excellent signal quality in all patients; $84 \%$ available in analysable form. No patient prematurely stopped the long-term ECG. Even patients with massive scoliosis, dextrocardia and other complicating circumstances such as patient compliance reached a good signal quality. In 4 patients dysrhythmia were detected which required a therapy adjustment. These periods of dysrhythmia were of short duration and did not happen every day so that it maight have stayed undetected with a standard holter ECG. 3 patients showed no changes in their known dysrhythmia and 8 patients showed normal ECG in rare episodes of palpitations or syncope. All the patients would choose to wear the Nubbo system again.
Conclusion: The cordless ECG from Nuubo ${ }^{\circledR}$ is a helpful diagnostic tool for patients with suspected rhythm disorders covering a wide age and size range. The monitored period was adapted to individual patients needs. The cordless ECG showed impressive signal quality and precise detection of arrhythmias.

\section{P-13}

The role of endomyocardial biopsy in establishing the etiology of progressive ventricular arrhythmias in children Vasichkina E.S., Mitrofanova L.B., Tatarsky R.B, Pervunina T.M, Lebedev D.S

Federal Almazov North-West Medical Research Centre, SaintPetersburg, Russia

Objective: This study aimed to assess the results of endomyocardial biopsy (EMB) to establish the etiology of progressive ventricular arrhythmias in children.

Methods: We studied 16 consecutive patients with progressive ventricular arrhythmias who underwent EMB.

The inclusion criteria were: age less than 18 year old, progressive ventricular arrhythmias, structurally normal heart or changes in a structure of the heart initially diagnosed as Arrhythmia induced cardiomyopathy (AIC).

Before EMB patients underwent a complete history, physical examination, laboratory studies (including thyroid function, CK, CK-MB, LDG, Troponin I, proBNP), echocardiography, ECG, Treadmill test and Holter monitoring. Cardiac MRI and genetic test were performed according to indications and physician's decision. In overall 74 biopsies were performed in 16 children. A histological analysis followed the Dallas Criteria, immunohistochemistry, polymerase chain reaction (PCR) used for interpretation of EMB. Samples were obtained from $>1$ region of the right ventricle.

Results: The mean age of patient population was $13,6+2,94$ year (from 7 to 17 year old). 56,25\% (9/16) patients had sustained or nonsustained VT, 43,75\% (7/16) - frequent ventricular ectopy (PVCs). Patients had palpitation $(43,7 \%)$, syncope $(31,2 \%)$, weakness and reduction of physical tolerance $(31,2 \%)$. Mild dilatation of at least one of the heart chambers (left/right ventricles, left atrium) was reported in $50 \%(8 / 16)$ patients at baseline.

In $2(10,5 \%)$ patients arrhythmogenic right ventricular dysplasia (ARVD) was diagnosed by EMB, MRI and genetic test (PKP2). Myocarditis was diagnosed based on histological and immunohistological analyses in 50\% $(8 / 16)$ of patients. PCR was positive for viral genome in four of them (2- Parvovirus B19, 1 - Enterovirus, 1 - Ebstein-Barr virus). Five of these patients had active myocarditis.

Conclusion: EMB is a valuable method for the diagnosis of the etiology of progressive ventricular arrhythmias in children. Half of pediatric patients with progressive ventricular arrhythmias had an unsuspected myocarditis according to the results of EMB.

\section{P-14}

Extension of right-sided $\mathrm{T}$ wave inversion to Lead V4: an ECG marker of atrial septal defect in children

Saito-Benz M. (1), Gnanapragasam J. (2)

University of Otago, Wellington, New Zealand (1), University Hospital Southampton, Southampton, UK (2)

Introduction: Atrial septal defect can be difficult to diagnose in childhood, for even relatively large lesions can remain asymptomatic in children. Moreover, fixed splitting of the second heart sound, which typically distinguishes it from an innocent murmur clinically, can be absent in young children. This study aims to improve the diagnostic value of ECG in children with ASDs. We 
hypothesise that, with the effect of volume overload and ventricular strain, ASDs may be associated with an extension of rightsided T-wave inversion to praecordial lead V4 in ECG.

Methods: All patients under the age of 18 years who underwent device or surgical closure of isolated secundum ASD at University Hospital Southampton between January 2008 and August 2012 were identified retrospectively using existing local database, and their ECGs prior to transcatheter/surgical intervention were reviewed. Children with multiple congenital cardiac abnormalities were excluded from the study. In order to avoid duplication, only one ECG was selected from each patient in any one year.

Results: A total of 84 patients and 177 ECGs were identified using the study methods described above. Of all ECGs reviewed, T-wave inversion was observed in V1 in 92\%, V2 in 54\%, V3 in 41\%, V4 in $33 \%, \mathrm{~V} 5$ in $4 \%$ and $\mathrm{V} 6$ in $0.6 \%$. The extension of $\mathrm{T}$-wave inversion to lead V4 was more commonly observed in children who required surgical closure for their ASDs rather than transcatheter device closure $(50 \%$ vs $22.4 \%, \mathrm{p}<0.0005)$. T-wave inversion in lead V4 was more commonly observed in younger age groups.

Conclusions: T-wave inversion in right precordial leads is seen in healthy children whereas T-wave inversion in the left precordial leads is considered abnormal in all age groups. This study suggests that extension of right-sided T-wave inversion to lead V4 is seen in up to a third of children with ASDs requiring either surgical or transcatheter intervention. Along with RSR' pattern in lead V1 and evidence of right ventricular enlargement in ECG, the extension of right-sided T-wave inversion to lead V4 should raise the suspicion of ASD in children with a clinically innocent flow murmur.

\section{P-15}

\section{Efficiency and safety of CARTO ablation of atrial} tachycardias in preschool children

Svintsova L., Dzhaffarova O., Plotnikova I., Krivolapov S., Batalov R., Popov S.

Tomsk National Research Medical Center the Russian Academy of Sciences "Cardiology Research Institute", Tomsk, Russia

Importance: Prolonged X-ray radiation during RFA is a risk of remote complications especially in children of yearly age. Navigation mapping in such category of patients is reasonable to reduce fluoroscopy time.

Aim: To assess the efficiency and safety of navigation mapping in RFA of atrial tachycardias in children.

Results: 30 RFA with CARTO system were performed to 23 children with atrial ectopic tachycardias during $2006-2016$ years. The patients' age was 5,0 years (IQR: 3,58-6.0), ranging from 7 months to 7 years old. 7 patients were less than 15 kilos during CARTO. The minimal age of the child who was performed CARTO was 7 months, the minimal weight $-7,4 \mathrm{~kg}$.

As minimal size of the diagnostic catheter used in this system is $7 \mathrm{Fr}$ (NaviStarTM), it is necessary to make a preliminary assessment of the diameter of femoral vessels by echocardiography.

Ectopic focus in most patients was in left atrium $(n=20)$. In two cases the approach to left atrium was performed by transseptal puncture, in one case - through patent foreman ovale.

The overall efficiency of CARTO ablation considering recurrences and retreatments was $91,3 \%$. Vessel injuries and other complications, associated with a procedure, were absent. Due to CARTO ablation decrease of fluoroscopy time in 2,3 times in comparison with standard fluoroscopy mapping ( $\mathrm{p}<0,001)$ was marked.

Conclusion: RFA with navigation mapping is effective and safe in children weighing more than 7 kilos and allow reducing time of $\mathrm{X}$-ray radiation to minimize complications associated with radiation exposure.

\section{P-16}

Signal-Averaged Electrocardiogram (SAECG) in children with ventricular arrhythmias

Turska-Kmiec A., Miszczak-Knecht M., Kosciesza A., Ziolkowska L., Brzezinska-Rajszys G., Zuk M., Kowalczyk-Domagala M.,

Sobielarska D., Czarnowska E.

Children's Memorial Health Institute, Warsaw, Poland

SAECG is a noninvasive technique for identification of low amplitude signals (LAS) at the end of the QRS complex, called ventricular "late potentials"(VLP) representing regions of abnormal myocardium with slow conduction. Abnormal SAECG poses the potential of identifying patients (pts) at increased risk of malignant ventricular arrhythmias (VA) and sudden cardiac death. There have been limited studies using SAECG in children, mainly in the postoperative congenital heart disease and in arrhythmogenic right ventricular cardiomyopathy (ARVC). Little is known about SAECG in the children with "idiopathic" VA.

The aim of the study was to evaluate SAECG in children with VA. Material and methods: Retrospective review of SAECG data from 187 pts with VA (ventricular ectopic beats > 2000/24hour, 70pts had ventricular tachycardia -VT, 53pts - VA with left bundle branch morphology-VA-LB), normal QTc, normal echocardiogram (initial diagnosis "idiopathic VA"). Radionuclide LVEF (left ventricular ejection fraction) was performed in all pts (decreased EF-20pts), qualitative 99mTcMIBI SPECT (rest/exercise) in $80 \mathrm{pts}=43 \%(\mathrm{PD}=$ perfusion defects- $60 \mathrm{pts})$. Mean age at VA diagnosis $8,8 \mathrm{yrs}$, at SAECG $=14,5 \mathrm{yrs}$. Magnetic resonance imaging $(\mathrm{MR}$ ) had $108 \mathrm{pts}=58 \%$ (small focuses of lipogenesis $-23 /$ $108 \mathrm{pts})$, endomyocardial biopsy (EMB) 36pts (19\%) $(\mathrm{MYO}=$ myocarditis-24, ARVC-6pts). No deaths were observed Statistical analysis: $\mathrm{p}<0.05$ considered to be statistically significant.

Results: VLP were found in 15/187 pts (8\%) without significant correlation with malignant VA (7/70pts with VT), symptoms (7/43pt), VA-LB (10/123pts), VA during exercise test (2/18pts), PD (9/60pts), MYO (5/24), abnormal MR (2/23pts). Longer filtered QRS had pts with age at VA diagnosis $>10 \mathrm{yrs}$ (96vs84ms, $\mathrm{p}=0000$ ), symptoms (96vs91ms, $p=0,01)$, sinus bradycardia (97vs $91 \mathrm{~ms}$, $\mathrm{p}=0$ 017), polymorphic VA (103vs91ms, $\mathrm{p}=0$ 014), PD (96vs88ms, $p=0011)$, decreased LVEF (97vs $91 \mathrm{~ms}, \mathrm{p}=0,04), \mathrm{VA}>50 \%$ of all beats in Holter ECG (104vs91ms, p=0,05), VA-LB superior axis (101ms, $p=0015)$. Lower RMS-40 values had pts $>10 y$ ys at VA diagnosis (55vs71microV, $p=0000)$, VA-RB superior axis (32microV, $\mathrm{p}=0035)$. Longer LAS had pts with age at VA diagnosis $>10 y r s$ (25vs22ms, $p=0012$ ), syncope (28vs23ms, $p=0024)$.

Conclusions: 1. Ventricular late potentials were found only in $8 \%$ of children with VA and normal echocardiogram but did not significantly correlate with malignant ventricular tachycardia and abnormalities in SPECT, EMB and MR. Discrete significant abnormalities in mean values of selected SAECG parameters were found in patients older than 10yrs age at VA diagnosis and especially regarding to mean QRSf duration.

\section{P-17 \\ Effects of Vagus Nerve Stimulation on Heart Rate Variability in Children with Epilepsy \\ Cetin I.I. (1), Serdaroglu A. (2), Hirfanoglu T. (2), Kurt G. (3), Capraz I. (4), Bilir E. (4), Arhan E. (2), Aydin K. (2) Ankara Children's Hematology Oncology Education and Research Hospital, Department of Pediatric Cardiology, Ankara, Turkey (1); Gazi University School of Medicine, Department of Pediatric Neurology, Ankara, Turkey (2); Gazi University School of Medicine, Department of Neurosurgery, Ankara, Turkey (3); Gazi University School of Medicine, Department of Neurology, Ankara, Turkey (4)}


Objectives: Vagus nerve stimulation (VNS) is a non-pharmacologic antiepileptic therapy for patients with refractory epilepsy who are not candidates for resective surgery or who have had resective surgery with unsatisfactory results. Although some studies have shown that VNS treatment is safe, well tolerated and effective in seizure reduction, there are only a few reports concerning effects of VNS on cardiac autonomy. The aim of this study was to investigate the effects of VNS on heart rate variability (HRV) in patients with refractory epilepsy.

Methods: Twenty patients (mean age $11.7 \pm 4.2$ years) with refractory epilepsy were evaluated in terms of interictal heart rate variability with 24-hour ECG recordings before, after 6 and 12 months of VNS treatment for each patient. We analyzed heart rate, pulse interval, frequency-dependent parameters including total power, low-frequency (LF), high-frequency (HF), LF/HF ratio, timedependent parameters including SDNN, SDNN index, SDANN, RMMSD, PNN50 and triangular index. HRV at both daytime and night were separately investigated for diurnal rhythm. The results were compared to age and gender matched healthy controls.

Results: The minimum and mean HR were increased, pulse interval was narrowed in patients before treatment compared to control group $(\mathrm{p}<0.05)$. HRV was depressed in patients before treatment in all frequency and time-dependent parameters compared to control group $(\mathrm{p}<0.05)$ (Table 1$)$. It was observed that, cardiac autonomic system is under the effect of sympathetic system especially at daytime, when increased LF/HF ratio was taken in to account, in patients before treatment $(\mathrm{p}<0.05)$. However, these differences between pre and post VNS implantation were seen especially at 6 months of treatment $(p<0.05)$ and there were no significant additional changes at 12 months of treatment. Even so, these parameters were still significantly different at 12 months of treatment than control group $(\mathrm{p}<0.05)$. There was also significant seizure reduction (almost 50\%) after VNS treatment $(p<0.05)$.

Conclusions: A significant improvement was observed in all HRV parameters after VNS therapy. Interestingly, maximum improvement was seen at 6 months of treatment and then no further improvement was observed. It can be explained by tolerability or adaptation of the autonomic system to the treatment.

\section{P-18}

The Etiology and Clinical Characteristics of Patients with Pulmonary Hypertension without Congenital Heart

Disease

Gümüştaş M., Dönmez Y.N., Alehan D.

Hacettepe University, Pediatric Cardiology, Ankara, Turkey

Pulmonary hypertension $(\mathrm{PH})$ is a rare progressive disorder in children that is associated with significant morbidity and mortality. Most common causes of $\mathrm{PH}$ in children include; congenital heart diseases, idiopathic pulmonary arterial hypertension, and rarely; connective tissue disorders and thromboembolism. We aimed, in our study, to evaluate the etiology and clinical features of study population that are not associated with congenital heart diseases other than the small atrial septal defects not causing serious $\mathrm{PH}$.

Methods and Results: From 2011 to 2016, 180 pediatric PH patients were evaluated for the clinical features and etiologic distrubition of PH. 37 patients with idiopathic pulmonary hypertension (IPAH) and $\mathrm{PAH}$ associated with other diseases were enrolled in our study. The baseline characteristics were detailed in Table 1.20 patients with IPAH, and 17 patients with PAH associated with other diseases were studied. Median age for IPAH was lower than PAH associated with other disease (4,7 to 7,2 years). Patients with $\mathrm{PAH}$ associated with other diseases included chronic renal failure $(\mathrm{n}=2)$, pulmonary disease $(n=7)$, portopulmonary hypertension $(n=1)$ veno-oclusive disease $(n=1)$ and systemic juvenile idiopathic arthritis $(n=1)$.
Syndromes associated with PAH were Down syndromes $(n=2)$, Allagille syndrome $(n=1)$, Dursun syndrome (congenital neutropenia, $n=1$ ) and Cleidocranial Dysostosis $(n=1)$. Six patients associated with pulmonary disease, three patients had interstitial lung disease, one patient had bronchiolitis obliterans, one patient had bronchopulmonary dysplasia and other one had obstructive sleep apnea syndrome. In IPAH patients, two patients had hematologic comorbidities (thalassemia carrier and hypofibrinogenemia).

discussion: Pulmonary hypertension is a progressive disease with a poor diagnosis. Due to lack of specific symptoms and signs especially in the early stage of the disease, the diagnosis is usually delayed. A systemic approach considering the coexistent disorders is required for the diagnosis. Hence, systemic classification is essential in determining the high-risk groups who should be screened.

\begin{tabular}{|c|c|c|}
\hline & IPAH $(n=20)$ & $\begin{array}{l}\text { PAH associated with } \\
\text { other diseases } \\
(n=17)\end{array}$ \\
\hline Age at diagnosis (years) & $4,7(0,5-14)$ & $7,2(0,1-17,7)$ \\
\hline Gender; female & $13(65 \%)$ & $8(47 \%)$ \\
\hline \multicolumn{3}{|l|}{ Comorbities, (n) } \\
\hline Secundum ASD & 9 & 3 \\
\hline Syndrome / Obesite / Renal & $-1-1-$ & $5 / 1 / 2$ \\
\hline $\begin{array}{l}\text { Hematologic / Pulmonary / } \\
\text { GIS }\end{array}$ & $2 /-/-$ & $1 / 6 / 2$ \\
\hline \multicolumn{3}{|l|}{ Hemodynamics at diagnosis } \\
\hline Catheterization & $\mathrm{n}=18$ & $\mathrm{n}=12$ \\
\hline $\begin{array}{l}\text { PVRi (WU.m2) / PVR/ } \\
\text { SVR }\end{array}$ & $15,4 / 61,1$ & $13,8 / 46,6$ \\
\hline Mean PAP $(\mathrm{mmHg})$ & $60 \pm 16,6$ & $42 \pm 16$ \\
\hline Echocardiographic $^{1}(\mathrm{n}) /$ PAP & $\mathrm{n}=2 / 87,5 \pm 3,5$ & $\mathrm{n}=5 / 51 \pm 19,8$ \\
\hline \multicolumn{3}{|l|}{ Pulmonary Therapy, $\mathrm{n}$} \\
\hline Anti congestive therapy & - & 5 \\
\hline Monotherapy $\star$ & 10 & 4 \\
\hline Combination & $6^{\circ}, 2^{\mathrm{x}}$ & $6^{\circ}, 1^{\mathrm{x}}$ \\
\hline Triple Therapy & $2^{\beta}$ & $1^{\beta}$ \\
\hline
\end{tabular}

$\mathrm{IPAH}=$ Idiopathic pulmonary artery hypertension, $\mathrm{PAH}=$ pulmonary artery hypertension, $\mathrm{ASD}=$ atrial septal defect, $\mathrm{PVR} i=$ Pulmonary Vascular Resistance index, PVR/SVR = ratio of pulmonary vascular resistance to systemic vascular resistance, $\mathrm{PAP}=$ pulmonary arterial pressure, $\mathrm{WU}=\mathrm{W}$ oods units, ${ }^{1}$ Measurement of the peak tricuspid regurgitant velocity, ${ }^{\star} E R A s$ (Endothelin receptors antagonist); ${ }^{\circ}$ ERAs and PDE-5 inh (phosphodiestere- 5 inhibitors);

${ }^{\mathrm{x}}$ ERAs and PGI2 (Prostacyclin analogue); ${ }^{\beta}$ ERAs, PDE-5 and PGI2

\section{P-19}

Predictive genotype testing in pediatric healthy phenotypes with family history of ARVC-5

Laser K.T.(1), Kececioglu D.(1), Milting H.(2)

Herz- E Diabeteszentrum NRW, Universitätsklinikum der

Ruhr-Universität Bochum, Klinik für angeborene Herzfehler, Bad

Oeynhausen, Germany (1); Herz- E Diabeteszentrum

NRW, Universitätsklinikum der Ruhr-Universität Bochum, Erich und

Hanna Klessmann-Stiftung für Kardiovaskuläre Forschung und

Entwicklung, Bad Oeynhausen, Germany (2)

Objectives: Autosomal dominant TMEM43-p.S358L mutation encoding for the ER-protein LUMA is a highly malignant fully penetrant missense mutation with a deleterious clinical phenotype causing ARVC5 and unpredictable sudden cardiac death especially in males. Syncopes were reported even in underaged males. In order to understand the disease course and the impact of imaging methods for the identification of the disease onset a close follow up of underaged carriers is of major relevance.

Methods: Three underaged mutation carriers were predictively tested for the Newfoundland mutation TMEM43 p.S358L (12 and 15ys male, 18 ys female) but are currently without clinical 
phenotype. We repeatedly examined these carriers by ECG, holter-ECG, echocardiography including deformation imaging, tissue Doppler, 3D-echo and cardiac MRI including volumetric data and vitality scan for 6 years follow up.

Results: Predictive testing of the underaged in combination with the family history had psychosocial impact for the kids. Of note, at present there were no significant early changes in morphologic, functional or electric alterations in all 3 adolescents. Task force criteria for ARVC were not fulfilled yet. Patients could be reassured concerning normal physical activities apart from competitive sports. Neither Beta blockade has been established nor ICD's have been implanted.

Conclusions: The advantage of predictive testing in these upcoming patient group is the possibility of early detection of disease in order to redefine the role of different established new imaging technologies as well as the reassurance of actual healthiness. On the other hand these adolescents have to cope with the problem of upcoming cardiac disease and the timing of medical as well as ICD-therapy has to be redefined. We recommend predictive testing of ARVC5 not before the age of ten years.

\section{P-20}

Incidence of Aspirin-Related Hepatotoxicity in Pediatric Cases with Acute Rheumatic Fever

Olgun H. (1), Bulgan M. (2), Ceviz N. (1), Yolcu C. (1), Sahin I.O. (1), Laloglu F. (1)

Atatürk University Faculty of Medicine Division of Pediatric

Cardiology, Erzurum, Turkey (1); Atatürk University Faculty of

Medicine Department of Pediatrics, Erzurum, Turkey (2)

Objective: This study aims to investigate the incidence of hepatotoxicity in pediatric patients who were diagnosed with acute rheumatic fever (ARF) and on aspirin treatment.

Methods: Between January 2008 and August 2015, a total of 173 pediatric patients who were diagnosed with ARF were retrospectively analyzed. Patients who had more than one ARF episodes were assessed separately for each aspirin treatment during the attack. Therefore, 281 ARF attacks in 258 patients were included in this study. Patients with ARF were divided into two main groups, including those who received aspirin and did not. Aspirin group was also divided into two subgroups including those who developed hepatotoxicity and did not. These patients were also classified according to whether they were symptomatic or not. The patients in the hepatotoxic group were divided to subgroups according to the treatment modification. Aspirin-related side effects were examined based on the anti-inflammatory doses and body weight. Incidence of hepatotoxicity was investigated in these groups.

Results: Of a total of 195 episodes, 83(42.6\%) were hepatotoxic. Of these, 18.5 were symptomatic, and 24.1 were asymptomatic hepatotoxicities. Gastrointestinal symptoms such as nausea and vomiting were present in the patients with symptomatic hepatotoxicity episodes and tinnitus was seen in five episodes. Hepatotoxicity symptoms occurred $14.7 \pm 10.6$ days after the initiation of aspirin treatment. Aspirin dose reduced during 24 hepatotoxicity episodes, while treatment discontinued and naproxen sodium was initiated during 21 episodes, and ibuprofen was initiated during an episode. Following treatment modification, elevated liver enzymes returned to normal values within $16.1 \pm 11.1$ days. Between two groups of patients with reduced dose of aspirin or who were switched to anti-inflammatory drugs, there was no statistically significant difference in the time to return to normal values of liver enzymes. Also, there were no significant differences in the incidence of hepatotoxicity in the patients receiving different anti-inflammatory aspirin doses.
Conclusion: Hepatotoxicity is common in the ARF patients receiving aspirin. Although these patients have no symptoms, they should be evaluated for liver enzymes on a regular basis. Reduction of the dose of aspirin or treatment modification with a new anti-inflammatory drug may improve hepatotoxicity in this patient population.

\section{P-21}

Two Patients with the Heterozygous R189H Mutation in ACTA2 and Complex Congenital Heart Defects expands the Cardiac Phenotype of Multisystemic Smooth Muscle Dysfunction Syndrome

Logeswaran T. (1), Friedburg C. (2), Akintuerk H. (3), Biskup S. (4), Graef M. (2), Neubauer B. (5), Schranz D.(1), Bouvagnet P.(6), Lorenz B.(2), Hahn A.(5)

Pediatric heart centre, Giessen, Germany (1); Department of opthalmology, Giessen, Germany (2); Department of pediatric heart surgery, Giessen, Germany (3); Center for Genomics and Transcriptomics, Tuebingen, Germany (4); Department of Neuropediatrics, Giessen, Germany (5); Laboratoire Cardiogénétique, Lyon, France (6)

De novo heterozygous mutations changing R179 to histidine, leucine or cysteine in the ACTA2 gene are associated with Multisystemic Smooth Muscle Dysfunction Syndrome (MSMDS).

(a)

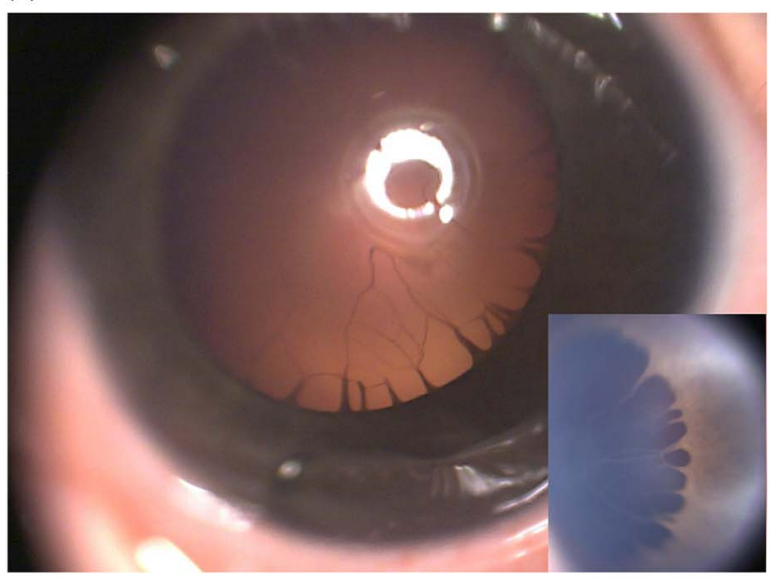

(b)

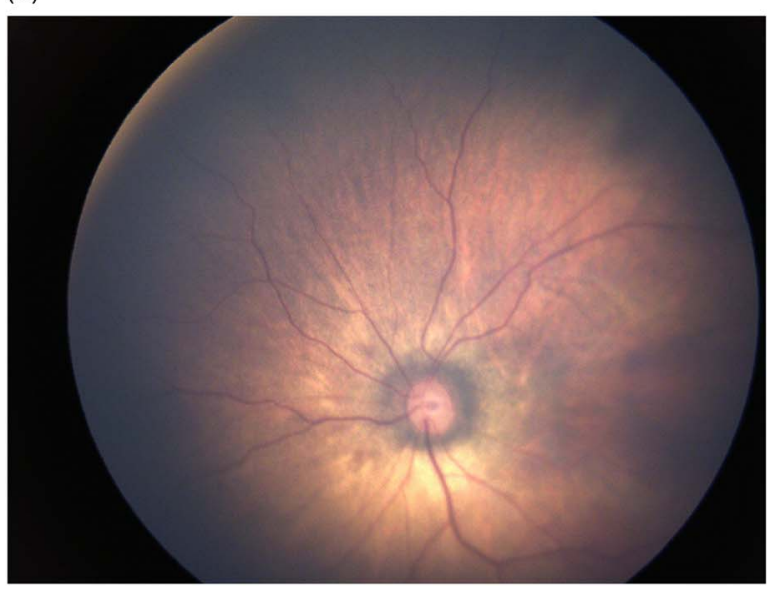

Figure 1.

Digital images of the iris of patient 1 showing a persistent extensive pupillary membrane with multiple wisks. The iris stroma appears hypoplastic, the pupil being only partially dilated. 
Characteristic hallmarks of this condition, caused only by these specific ACTA2 mutations, are congenital mydriasis (mid-dilated, non-reactive pupils, figure 1), a large persistent ductus arteriosus (PDA), aortic aneurysms evolving during childhood, and cerebrovascular anomalies. We describe two patients, a 3-day-old newborn and a 26-year-old woman, with this unique mutation in association with a huge PDA and an aorto-pulmonary window. In addition, one showed a coarctation of the aortic arch and the other a complete interruption of the aortic arch type A; thereby expanding the spectrum of cardiac congenital heart defect of this syndrome. Each patient displayed a huge PDA and an extracardiovascular phenotype consistent with MSMDS. These observations exemplify that a functional alpha 2 smooth muscle actin is necessary for proper cardiovascular organ development, and demonstrate that a very exceptional congenital heart defect (aortopulmonary window) can be caused by a mutation in a gene encoding a contractile protein of vascular smooth muscle cells.

\section{P-22}

Identify genetic hypomethylation and upregulation of toll-like receptors in Kawasaki disease

Huang Y.H., Li S.C., Kuo H.C.

Department of Pediatrics and Kawasaki Disease Center, Kaohsiung Chang Gung Memorial Hospital and Chang Gung University College of Medicine, Kaohsiung, Taiwan

Introduction: Kawasaki disease (KD) is an acute febrile systemic vasculitis of childhood characterized by profound elevated levels of proinflammatory and inflammatory cytokine. Toll like receptors (TLRs) act as the sensor arm of the innate immune system and induce proinflammatory cytokine expressions. The aim of this study is to survey TLR1-10 in different stages of KD and controls. Methods: A total of $18 \mathrm{KD}$ patients including before intravenous immunoglobulin (IVIG), at least 3 weeks after IVIG treatment, 18 health and 18 febrile controls were enrolled for survey. GeneChip ${ }^{\circledR}$ Human Transcriptome Array 2.0 was used with 6 cases pooling methods and Infinium HumanMethylation450 BeadChip Kit to evaluate methylation patterns of $\mathrm{CpG}$ markers on TLRs. Results: KD patients showed significantly differential expression of mRNA levels of TLRs compared to health and febrile controls, while only TLR 3 and 7 were not different between KD patients and controls. After IVIG treatment, the mRNA levels of TLRs except TLR3 were significantly down-regulated in the KD patients. On the contrary, the methylation status of $\mathrm{CpG}$ sites of TLR1, 2, 4, 6, 8, 9 demonstrates reverse tendency between two stage of KD samples and controls.

Conclusion: TLRs especially TLR 1, 2, 4, 6, 8, 9 may involve in the immunopathogenesis of KD. These results provide first evidences by TLRs that a bacterial-like inflammatory response play important role in $\mathrm{KD}$.

\section{P-23}

Solving syndromic congenital heart defects using WES in a Trio or Index-only approach

Louw J.J.(1,2), Corveleyn A. (2), Jia Y.(2), Breckpot J.(2), Gewillig M. (1), Devriendt K.(2)

Department of Congenital and Pediatric Cardiology, University Hospitals Leuven, Belgium (1); Centre of Human Genetics, University Hospitals Leuven, Katholieke Universiteit Leuven, Belgium (2)

Introduction: Congenital heart defects (CHD) are a major cause of infant morbidity and mortality. Reaching an etiological diagnosis is important for prognosis and counselling on recurrence risks.
Gene identification for CHD still represents a major challenge, especially since the genetics of CHD is heterogeneous. Syndromic CHD is defined as CHD with the presence of dysmorphism, additional major malformations and/or ID. Our aim is to evaluate the use of NGS in the detection of causal mutations of syndromic CHD in sporadic cases.

Methods: Exome sequencing using a trio approach (parents and child) was compared to an index-only analysis, filtering was done against in-house exomes. Data analysis was done using commercial and in-house developed software (Genomics Core/UZ Leuven). According to Ensembl only exonic, exonic/splicing and splicing variants were included. Synonymous variants were excluded. Variants occurring with a frequency of $<1 \%$ in the 1000 genomes project or with an unknown frequency were included. Splicing site changes occurring at less than 5 positions were considered as possible candidates.

Results: Initially, exome sequencing was done in 9 trio's. In 4 a pathogenic mutation was detected in respectively MEIS2 (c.998_1000del:p.Arg333del), DYRK1A (c.C1282T:p.R428X), EFTUD2 (c.671delG:p.G224fs) and SALL1 (c.1998_1999del: p.666_667del). We analyzed exomes of 4 additional index-only cases. As WES data of parents were not available, inherited variants could not be filtered out, and we experienced seriously limitations in the filtering process. Only one case was diagnosed after identifying a causal nonsense mutation in ANKRD11, (c.7189C > T, p.Gln2397^ causing KBG syndrome).

Conclusions: Trio analysis has a higher yield than index only analysis in syndromic CHD, due to an increased efficiency in filtering. A gene-first approach also leads to broadening the clinical spectrum of known syndromic genes. For example, MEIS2 has been previously suggested as a candidate gene for cleft palate, CHD and ID. In our patient with a MEIS2 deletion a more severe phenotype was seen than previously described in literature. This is most probably due to dominant-negative mechanisms. In addition, as others have demonstrated before, WES is an excellent means to identify new genes important in the development of cardiopathies.

\section{P-24}

Novel genetic mutation in BAG3 and TNNT2 in a Swedish family with a history of dilated cardiomyopathy and sudden cardiac death

Fernlund E. (1,5), Wålinder Österberg A. (1), Kuchinskaya E. (2), Gustafsson M. (3), Jansson K. (4), Gunnarsson C. $(2,6)$

Division of Pediatrics, Department of Clinical Experimental Medicine, Linköping University, Linköping (1), Department of Clinical Genetics, and Department of Clinical Experimental Medicine, Linköping University, Linköping (2), Department of Cardiology, Department of Medical and Health Sciences, Linköping University, Linköping (3), Department of Clinical Physiology, Department of Medical and Health Sciences, Linköping University, Linköping (4), Pediatric Heart Center, Lund University, Lund (5), Centre for Rare Diseases in South East Region of Sweden, Department of Medical and Health Sciences, Linköping University, Linköping (6), all Sweden.

Introduction: Familial dilated Cardiomyopathy (FDCM) is a rare cause of Dilated Cardiomyopathy (DCM), especially in childhood. Our aim was to describe the clinical course and the genetic mutations in a family with a history of sudden cardiac death and dilated cardiomyopathy, in which the proband was a four-month old infant presenting with respiratory problems due to DCM. In the family there was a strong family history of DCM.

Methods: DNA was analyzed initially from the deceased girl using next generation sequencing including 50 genes involved in cardiomyopathy. A cascade family screening was performed in the 
3-generation family after identification of the TNNT2 and the BAG3 mutation in the proband. Mutation carriers underwent clinical examination including biochemistry panel, cardiac ultrasound, Holter ECG and exercise stress test.

Results: The index patient presented with advanced DCM. After a severe clinical course, the baby had external left ventricular assist as a bridge to heart transplantation. 1.5 months post-transplant the baby suffered sudden cardiac death (SCD) though maximal treatment in the pediatric intensive care unit. The patient was shown to have two heterozygous genetic variants in TNNT2 c.518G > A (p.Arg173Gln) and BAG3 c.785C > T (p.Ala262Val). Three of five screened individuals (two females) appeared to be mutation carriers in the family. The mutation carriers of TNNT2 mutation presented with DCM, two patients had mild or moderate symptoms of heart failure and reported palpitations but no syncope or presyncopal attacks prior to the genetic diagnosis. The mutation carriers of TNNT2 and BAG3 mutation had more advanced DCM. In the second generation of the family there was two additional cases of SCD due to DCM, diagnosed by autopsy, but no genetic analysis was possible in these cases.

Conclusion: Our findings suggest that the mutations in TNNT2 and BAG3 are associated with a high propensity to life-threatening cardiomyopathy presenting from childhood and young adulthood.

\section{P-25}

\section{Type B Aortic Dissection in childhood and MYBPC3 mutation: a Case Report}

Aries E, El Louali F., Aldebert P., Pico H., Bal L., Chabrol B., Ovaert C.

Timone enfants, AP-HM, Marseille, France

Introduction: Non-traumatic aortic dissection is a rare disease in children. Management is standardized in adults. In children the diagnosis, etiology and treatment is less clear. We report an uncommon clinical observation with review of the literature.

Clinical Observation: This young boy presented at the age of 11 years with an ischemic stroke. Hypertrophic cardiomyopathy with non-compacted left ventricle but preserved systolic function was discovered. Genetic work-up revealed mutation of MYBPC3. Anti-coagulant therapy was introduced. At the age of 13 years he underwent surgery for significant scoliosis and pectus carinatus. Three months later he presented with thoracic pain concomitant with mild fever and inflammatory signs. The history was compatible with dry pericarditis and anti-inflammatory therapy was introduced. The pain improved but reappeared a few weeks later. A hyperechogenic retrocardiac mass was seen on echocardiography. CT scan revealed type B aortic dissection with 2 active entry points into the false lumen. There was no visceral impact. After medical-surgical multidisciplinary discussion, conservative approach was decided with medical control of arterial hypertension, discontinuation of anticoagulant therapy but introduction of anti-platelet therapy. Progressive thrombosis of the false lumen and closure of the 2 entry points was seen during the fooling 3 months, confirmed by CT Scan.

Discussion: The average age of patients with type $\mathrm{B}$ aortic dissection is 65 years. Acute mortality is high, approaching $40 \%$. The incidence among those under 21 years is extremely low (about $0.37 \%$ of cases), and exceptional before 15 years. It is most often associated with connective tissue diseases such as Marfan syndrome. Spontaneous aortic dissection together with MYBPC3 mutation and non-compaction cardiomyopathy has so far not been described.

\section{P-26}

Repeated syncopes caused by sinus nodal arrest requiring pacemaker implantation in patient with late-onset familial form of congenital central hypoventilation syndrome Klaskova E. (1), Drabek J. (2), Hobzova M. (3), Prochazka M. (4) Department of Paediatrics, University Hospital Olomouc and Faculty of Medicine and Dentistry, Palacky University Olomouc, Czech Republic (1); Institute of Molecular and Translational Medicine, University Hospital Olomouc and Faculty of Medicine and Dentistry, Palacky University Olomouc, Czech Republic (2); Department of Respiratory Medicine, University Hospital Olomouc and Faculty of Medicine and Dentistry, Palacky University Olomouc, Czech Republic (3); Department of Medical Genetics, University Hospital Olomouc and Faculty of Medicine and Dentistry, Palacky University Olomouc, Czech Republic (4)

Introduction: Congenital central hypoventilation syndrome (CCHS) is a rare genetic disorder resulting from mutations in the PHOX2B gene characterized by hypoventilation secondary to missing responses to both hypercapnia and hypoxia, that is thought to be caused by neural crest migration abnormalities. Baseline bradycardia, transient asystole or vasovagal syncope have been described in these patients. We present a rare case of girl with late-onset familial CCHS who experienced four syncopes caused by sinus nodal arrest with pauses of up to 20 requiring pacemaker implantation.

Patients and Methods: A 4-year-old girl, previously being hospitalised 5 times for respiratory failure from 6 weeks of age. She experienced two severe cyanotic attacks related to viral pneumonia at the age of 6 weeks and 4 months. At the age of one year, she was diagnosed with severe pulmonary hypertension resulting in acute right ventricle failure. Primary pulmonary, cardiac, neuromuscular and metabolic disease was excluded. Overnight videopolysomnography revealed non-apnoeic oxygen desaturation. Only then, CCHS became the highly probable cause, but this time the parents refused tracheostomy and mechanical ventilatory support. After further upper respiratory tract infection at the age of two years, she remained dependent on a ventilator while asleep.

At the age of three years and nine months, she experienced four syncopes caused by sinus nodal arrest with pauses of up to 20 s. Since permanent epicardial pacemaker implantation was performed, no syncopes occurred during a further follow-up.

Afterwards, she failed extubation and conversion to overnight noninvasive ventilatory support after pneumonia. Tracheostomy was done, and she was discharged home using a portable positive pressure ventilator during sleep. Her father was retrospectively found out to suffer from severe headache and excessive daytime sleepiness.

Results: Molecular genetic evaluation of PHOX2B gene was performed in both girl and her father, and casual polyalanine repeat expansion mutation c.741_755dup15 in exon 3 was found in heterozygous form.

Conclusions: Patients with CCHS demonstrate affected autonomic function including heart rate and blood pressure control probably being caused by absence of normal maturation of carotic body and visceral sensory ganglia as a part of dysregulation of autonomic nervous system.

\section{P-27}

First Evidence of Maternally Inherited Mosaicism in TGFBR1 in Loeys Dietz Syndrome. Literature Review of TGBR1\&2 Mosaicism

Baban A.(1), Magliozzi M.(2), Adorisio R.(1), Secinaro A.(3), Dietz H.(4), Vricella L.(4), Drago F.(1), Novelli A.(2), Amodeo A.(5) 1 Pediatric Cardiology and Cardiac Arrhythmia/Syncope Unit, Department of Pediatric Cardiology and Cardiac Surgery, Bambino Gesù Children's Hospital and Research Institute, Rome, Italy., 2 Laboratory of 
Medical Genetics, Bambino Gesù Children's Hospital and Research Institute, Rome, Italy., 3 Department of Imaging, Bambino Gesù Children's Hospital and Research Institute, IRCCS, Rome, Italy. 4 Howard Hughes Medical Institute and Institute of Genetic Medicine, Johns Hopkins University School of Medicine, Baltimore, Maryland, USA; Division of Pediatric Cardiology, Department of Pediatrics, Johns Hopkins University School of Medicine, Baltimore, Maryland, USA; Department of Medicine, Johns Hopkins University School of Medicine, Baltimore, Maryland, USA. 5 Mechanical Assistance Device Unit, Department of Pediatric Cardiology, Bambino Gesù Children's Hospital and Research Institute, Rome, Italy.

The index patient is the only male child of Caucasian nonconsanguineous parents, both having normal echocardiographic findings at baseline visit. The mother was operated for recurrent hemorrhage originating from the right buccal artery. She also recently presented with diminished right visual acuity for spontaneous vitreous detachment. On physical examination, the mother disclosed no signs of cranio-facial abnormalities.

Aortic dilatation of the proband was confirmed after birth and follow-up was instituted and carried out at another institution, where the diagnosis of oculo-cutaneous albinism was also made. PE disclosed macrosomia and bifid uvula. The suspicion of the coexistence of two distinct genetic defects was raised: oculocutaneous albinism and Loeys Dietz Syndrome (LDS). NGS approach identified a previously repoted, heterozygous, missense mutation c.1460G > A (p.Arg487Gln) of TGFBR1 (data confirmed by Sanger). Parents was tested for segregation study which identified a maternally inherited mutation in mosaicism. Specifically, the rate of mosaicism was $18 \%$ in peripheral blood cells and buccal cell swab DNA and around 10\% in DNA extracted from the hair root cells. The child was operated for aortic root replacement that was complicated by severe haemorhage and complete AV block that needed pacemaker positioning. He was discharged and is doing well at 5 months follow up.

Maternal head to pelvis MRI showed vascular asymmetry at head and neck regions mainly prevalent right vertebral and left carotid arteries without significant signs of arterial tortuosity. The volume rendering reconstruction of the gadolinium enhanced MR angiography showed mild tortuosity at the arch and mild ectatic proximal abdominal aorta and peculiarly the left ventricle vertical long axis cine image in diastolic phase revealed multiple myocardial clefts at the inferior basal segment and anomalous distribution of mitral papillary muscle without signs of regurgitation. The aorta showed a uniform dilatation of the abdominal aorta. Peculiarly the left ventricle showed myocardial inferobasal cleft. Literature review of mosaicism in TGFBR1 and TGFBR2 mutations are included.

This is the first report of somatic mosaicism of a TGFBR 1 mutation of a mother of a child with classic LDS.

\section{P-28}

\section{Congenital heart defect in patient with Noonan Syndrome} and RIT1 mutation

Calcagni G. (1), Digilio M.C. (1), Baban A. (1), Lepri F. (1), Pantaleoni F. (1), Dentici M.L.(1), Drago F. (1), Marino B. (2), Dallapiccola B. (1), Tartaglia M.(1)

Bambino Gesù Children's Hospital, Rome, Italy (1); Sapienza University, Rome, Italy (2)

Background: Noonan syndrome (NS) is a genetically heterogeneous, autosomal dominant disorder due to mutations in genes, the majority coding proteins with an established role in the RAS/ MAPK signalling pathway. RIT1 mutations have recently been identified as a new cause of NS. The aim of the study is to evaluate the prevalence and anatomic types of congenital heart defect (CHD) in NS patients heterozygous for mutations in this gene.

Methods: From January 2015 to January 2016, 9 patients with NS-causing RIT1 mutations have been diagnosed. All patients had 2D-color Doppler echocardiographic and ECG evaluation. CHD was diagnosed in all of them.

Results: Pulmonary valve stenosis (PVS) was the most recurrent defect accounting for six cases. Hypertrophic cardiomyopathy (HCM) and atrial septal defect ostium secundum type were observed in four and three cases, respectively, while partial atrioventricular canal defect (pAVCD) and ventricular septal defect (VSD) was documented in one individual each. All patient with PVS had also supravalvular pulmonary stenosis and dysplastic pulmonary valve. HCM was associated with PVS and muscular VSD in one patient, with PVS in an additional one. One patient with HCM with biventricular obstruction had cardiac transplantation at the second year of age. Lymphatic anomalies were present in 3 patients, including recurrent chylothorax in two cases, lymphedema of lower limbs in one.

Conclusions: 1) the prevalence of CHD in patients with RIT1 mutations is relatively high and needs further studies to be underlined; 2) a great variety of anatomic abnormalities in CHD is detected ranging from the classic PVS and HCM to the uncommon pAVCD; 3) HCM can be severe; 4) complications due to lymphatic anomalies should be taken into account in these patients especially at the postoperative stage due to higher risk of chylothorax.

\section{P-29}

Notch signaling is abnormally activated in cardiac progenitor cells of tetralogy of Fallot patients

Malashicheva A., Kozyrev I., Ignatieva E., Pervunina T., Grehov E., Gordeev M., Kostareva A.

Federal Almazov Medical Research Centre, Saint-Petersburg Russia

Tetralogy of Fallot (TOF) is a congenital heart defect that is characterized by four anatomical abnormalities: ventricular septal defect, biventricular connection of the aorta, subpulmonary stenosis, and right-ventricular hypertrophy. TOF is the most common cyanotic heart defect and the most common cause of blue baby syndrome. The cellular and genetic mechanisms of this defect are so far unclear. Recently fine-tuned sequential activation of Notch genes have been shown to be responsible for the proper heart chamber development. In addition mutations in several genes of the Notch pathway have been shown to be associated with TOF. The aim of this study was to analyze the activity of Notch pathway in the cardiac stem cells (CSC) derived from myocardial tissue of TOF patients.

We isolated CPC from 8 TOF patients (saturation 70-80\%, EF 60$80 \%$ ) and from 4 patients with ventricular septal defects (saturation $100 \%$, EF $70-80 \%)$. We analyzed the activity of Notch pathway by estimating the expression of Notch genes and receptors as well as the main Notch target gene HEY1 by qPCR. To test the differentiation capacity of CPC we differentiated CPC to cardiogenic, adipogenic and osteogenic lineages. To verify that the activation of Notch signaling influences the differentiation potential of $\mathrm{CPC}$ we infected the CPC with lentiviruses bearing activated Notch intracellular domain (NICD) and also estimated differentiation capacity of CPC. The CPC derived from TOF patients had significantly higher NOTCH1 and HEY1 gene expression. This elevated Notch activity corresponded to higher differentiation rate to cardiac lineage estimated by Nkx2.5, MEF2C and Troponin T expression by qPCR as well as alpha-actinin and Troponin I levels 
by ICH staining of differentiated cells. Activation of Notch by lentiviral NICD expression also caused elevation of cardiogenic markers in the CPC confirming the role of Notch signaling in determining cardiogenic potential of CPC.

Our data on CPC derived from TOF patients suggest direct involvement of Notch pathway dysregulation in the pathology of TOF and further confirms that fine-tuned Notch signaling is one of the key factors responsible for the appropriate heart development.

\section{P-30 \\ Clinical course of myocarditis complicated by atrioventricular block in young children \\ Vasichkina E.S., Boldina N.M., Pervunina T.M., Lebedev D.S. \\ Federal Almazov North-West Medical Research Centre, Saint- \\ Petersburg, Russia}

Objective: To study clinical and prognostic characteristics of clinical course of myocarditis complicated by high degree atrioventricular block (AVB) in young children during three years follow-up.

Methods: We enrolled 7 patients under the age of 1,5 years, mean age was 9,3 \pm 4,4 months (from 3 to 16 months), who had myocarditis complicated with AVB: complete AVB - 4 patients, seconddegree -3 . The duration of the AV conduction abnormalities before the admission in our clinic amounted at mean 3,9 $\pm 3,6$ months (from 2 weeks to 11 months).

We analyzed their clinical presentations, biochemistry and serology studies, chest X-rays, electrocardiography, echocardiography. The diagnosis of the myocarditis was made in the presence of association between first appear AVB and viral infection and in combination with: elevated markers of myocardial damage and/or cardiomegaly by chest radiography and/or increased left ventricular end diastolic and systolic dimensions with/without decreased ejection fraction. The etiologic factor of the myocarditis was determined in 3 patients: Varicella zoster, Mycoplasma pneumonia, Parvovirus B19.

The analysis of the dynamics of the patient condition was made by the comparison first and last visit in our clinic.

Results: Mean follow-up was 20,2 $\pm 12,6$ months (from 1,5 to 35 months).

During a follow-up period after therapy, cardiac enzymes returned to normal. All except one patient improved their symptoms and signs of heart failure. All patients showed positive dynamics of echocardiographic parameters with the left ventricular ejection fraction improved.

However, all our patients had persistent atrioventricular block, and three of them received permanent pacemaker implantation.

Conclusions: The outcome of conduction abnormalities complicated with myocarditis in young children is variable, but in our study atrioventricular block was irreversible.

\section{P-31}

Losartan attenuates apoptosis and fibrosis in adriamycininduced cardiomyopathy rat model

Hong Y.M., Kim K.C.

Departments of Pediatrics (1); Thoracic and Cardiovascular Surgery(2), Ewha Womans University School of Medicine, Seoul, Korea

Purpose: The precise mechanism for the pathogenesis in adriamycin (ADR)-induced cardiomyopathy has not been elucidated. Different hypotheses include the production of free radical species, an interaction with nucleic acid or nuclear components and disruption of a cardiac-specific program of gene expression. Programmed cell death or apoptosis has been proposed to be involved in cardiac dysfunction under some experimental and clinical conditions. Angiotensin (AT) II plays an important role in cardiac fibrogenesis by acting as a potent growth factor and cytokine for vascular smooth muscle cells, cardiac myocytes and cardiac fibroblasts, via activation of the AT type I receptor. Losartan is a selective AT1 receptor antagonist. It improves left ventricle function, prevents geometric remodelling, and prolongs survival in several heart diseases, such as hypertension, heart failure, ischemic heart disease, and diabetes mellitus. The purpose of this study was to investigate the changes of apoptosis and remodelling in adriamycin induced cardiomyopathy rat model after losartan treatment. Methods: Male Sprague-Dawley rats were separated into three groups: the control group (C group), A group (ADR $5 \mathrm{mg}$ /week for 3 weeks, intraperitoneal injection), L (losartan group) was treated with ADR ( $5 \mathrm{mg} /$ week for 3weeks) and losartan $(10 \mathrm{mg} /$ $\mathrm{kg} /$ day for 3 weeks). The rats were sacrified at week 3 . Changes of caspase-3, B cell leukemia/lymphoma (Bcl)-2, Bax, tumor necrosis factor (TNF)- $\alpha$, matrix metalloproteinase (MMP)-3, collagen 1 and collagen 3 proteins were estimated by westernblot analysis in the heart tissues after losartan treatment. Left ventricle was stained by Masson's Trichrome for evaluation of collagen contents.

Results: Left ventricular hypertrophy was significantly noted in the A group and it was significantly decreased in L group compared with A group. The protein expressions of caspase-3, Bax, MMP-3, TNF- $\alpha$, collagen 1 were significantly higher in the A group compared with the $\mathrm{C}$ group. They were significanly decreased after losartan treatment.

Protein expression of $\mathrm{Bcl}-2$ was decreased in $\mathrm{L}$ group, but it was not significantly different.

Collagen contents in LV were significantly increased in A group compared with $\mathrm{C}$ group and decreased in $\mathrm{L}$ group compared with A group.

Conclusion: Losartan decreased apoptosis, inflammation and fibrosis in LV tissues.

\section{P-32}

Combination of single nucleotide polymorphism of hemostatic system genes in patients with single ventricle Lugacheva Yu.(1), Kulagina I.(1), Kovalev I.(2), Krivoshchekov E.(1), Yanulevich O.(1), Plotnikova I.(1), Suslova T.(1)

Tomsk National Research Medical Center the Russian Academy of Sciences "Cardiology Research Institute"Tomsk, Russia(1), Research and clinical Institute for pediatrics at the Pirogov Russian National Research Medical University, Moscow, Russia(2)

Aim: To study the combination of single nucleotide polymorphism (SNP) of hemostatic system genes associated with thrombosis appearance in patients with single ventricle (SV).

Methods and materials: Molecular genetic study with diagnostics of SNP of hemostatic system genes was performed. The average age was $3,3(0,6 ; 5,0)$ years. Out of 102 examined children thrombosis was recorded in 13 patients with SV during follow up. Signs of thrombosis were not discovered in 89 patients during defect surgical correction. Study material was whole blood. DNA samples were tested for single nucleotide polymorphism in hemostatic genes: F2:20 210 G > A (Factor II), F5:1691 G > A (Factor V, Leiden Mutation), FGB: -455 G > A (Factor I), ITGA2:807 C > T (platelets collagen receptor GP Ia-IIa), ITGB3:1565T > C (platelets fibrinogenic receptor GP IIb-IIIa), PAI-1:-675 5G > 4G (plasminogen activator inhibitor I). Genotype was detected by polymerase chain reaction method using market reagent kit (DNA-Technology, Russia).

Results: Carriership of one polymorphism was discovered in 9 $(10,1 \%)$ examined children without thrombosis, and children with 
thrombosis had more than one polymorphism. The combination of two polymorphisms was disclosed in $16(18,0 \%)$ children with SV without thrombosis, and in $6(46,2 \%)$ children with thrombosis. Three polymorphisms were discovered in 35 (39,3\%) children without thrombosis and in $3(23,1 \%)$ patients with thrombosis. Polymorphism of four genes was detected in $20(22,5 \%)$ children with SV without thrombosis, and in $3(23,1 \%)$ children with thrombosis. Five polymorphisms were discovered in 7 (7,9\%) children without thrombosis, in $1(7,7 \%)$ patient with thrombosis. Six polymorphisms were marked in $1(1,0 \%)$ child without thrombosis, children with thrombosis had no that combination. Depending on a carriership of polymorphism combination statistically significant differences $(\chi 2=0,34, p=0,56)$ were not disclosed in comparative analysis of children with and without thrombosis.

Conclusion: As a result of molecular genetic analysis risk of thrombosis appearance depending on combination of single nucleotide polymorphism of hemostatic system genes in patients with single ventricle was not detected.

\section{P-33 \\ Markers of endothelial dysfunction in children with heart diseases}

Plotnikova I., Tupikina A., Svintsova L., Dzhaffarova O., Yanulevich O., Krivoshchekov E., Gusakova A.

Tomsk National Research Medical Center the Russian Academy of Sciences "Cardiology Research Institute"

Aim: To assess markers of endothelial dysfunction in children with dilated cardiomyopathy (DCMP) and single ventricle (SV) in a year after total cavopulmonary connection (TCPC).

Method and materials: 17 children with SV in a year after TCPC with extracardiac conduit and 11 patients with DCMP were examined. All patients had NYHA II. The average age was 8 years \pm 4.5 months. Biochemical markers of endothelial dysfunction were disclosed: the level of endothelin-1 and metabolites of nitric oxide (nitrate and nitrite). The normal range was endothelin-1 - 16,9 pg/ml, nitrogen nitrite (NO2) - 1,2 $\mathrm{mcmol} / \mathrm{l}$, nitrogen nitrate (NO3) $-12,3 \mathrm{mcmol} / \mathrm{l}$.

Results: The indices of median endothelin-1 exceeded regulatory values and were $57,7 \mathrm{pg} / \mathrm{ml}$ (IQR: 7,2-172,80) in patients with DCMP. Similar data were disclosed in patients with SV $-51,2 \mathrm{pg} /$ $\mathrm{ml}$ (IQR: 6,2-165,5). Increased endothelin-1 was discovered in 6 patients $(63,6 \%)$ with DCMP which were individually evaluated. It may be connected with pathogenetic mechanisms preceding DCMP in the form of inflammation in myocardium and damage of vessel endothelium by cytokines. The analyzed marker was increased in $56,3 \%$ (9 persons) of patients with SV that can be conditioned by prolonged hypoxia and be an early marker of pulmonary hypertension with low pressure in a late postoperative period. Values of median nitrite and nitrogen nitrate were increased in both groups. Significant differences were not disclosed, $\mathrm{p}=0,96$ and $\mathrm{p}=0,98$, correspondingly. Individual analysis showed that the level of nitrogen nitrate was increased in all patients with SV and DCMP. According to the same analysis of nitrite level only 3 patients with SV and 3 with DCMP had the normal value of the studied index.

Conclusion: The most examined children with NYHA II regardless of aetiopathogenesis had increased endothelin-1 level. That is one of the markers of endothelium dysfunction. The increase of metabolite of nitric oxide can be treated in two ways: first, it can be a compensatory mechanism in patients with HF fulfilling protection function, causing generalized vasodilation and reducing pulmonary hypertension, and, second, a result of progressive inflammation.

To support this hypothesis it is necessary to continue the prospective study.

\section{P-34}

Detection of cardiomyopathy in children with Marfan Syndrome with 2D strain echocardiography

Ratsimandresy M., Dulac Y., Hadeed K.

Children's hospital, Cardiopediatrie Toulouse France (1)

Introduction: Studies have found cardiomyopathy in adult patients with marfan syndrome (MS) whereas conventional echocardiographic parameters showed normal systolic function. The aim of our study was to evaluate the validity of the 2D strain echocardiography or "speckle tracking imaging" (STI) for the study of myocardial function in children with MS, and to assess the interest for cardiomyopathy detection in this population.

Methods and Results: Echocardiographic parameters of left ventricular systolic function were compared between the two populations (39 MS and 41 healthy children) in standard 2D, STI and cardiac MRI. The values of global strain longitudinal peak (SLG) of MS were compared with values of ejection fraction of the left ventricle (LVEF) assessed by cardiac MRI (gold standard). Relations between the two types of mutations (PTC and inframe) and altered strain were studied. Impaired SLG was significant for 7 segments. This segmental impairment predominated the basal level with apex-to-base gradient. Patients with more altered SLG appeared to have a greater dilatation of the ascending aorta.

Conclusion: This is the largest pediatric cohort who compare STI and MRI in children with SM. The interest for primary or secondary cardiomyopathy detection remains to be validated and consolidated with larger studies and could justify not only to focus on the aorta.

\section{P-35}

\section{Acquired von Willebrand syndrome in infants with} aortopulmonary shunt

Wiegand G.(1), Icheva V.(1), Budde U.(2), Hofbeck M.(1) Department of Pediatric Cardiology, University Children's Hospital, Tuebingen, Germany (1), MEDILYS Laborgesellschaft mbH, Hamburg, Germany (2)

Introduction: The acquired von Willebrand syndrome (aVWS) was first described in 1968 by Simone and colleagues in patients with autoimmune diseases. The aVWS is very rare in children, most frequently being described in connection with congenital heart defects including aortic stenosis, persistent ductus arteriosus, ventricular septal defect and pulmonary hypertension. The aVWS often results in increased bleeding tendency such as mucosal-, gastrointestinal- or surgical bleeding. Until now, there are no reports describing aVWS in infants with aortopulmonary shunts. Methods and Results: Between 07/15-07/16 we evaluated 11 infants younger than 3 months with univentricular hearts and aortopulmonary shunt $(9 \mathrm{x}$ Blalock-Taussig-Shunt, $1 \mathrm{x}$ central aortopulmonlary shunt, 1x Sano-Shunt (from the systemic right ventricle to the pulmonary artery)) and tested for avWD. The shunt operation was performed between day 5.-180. (median 8d), the blood samples were collected between days 18-260 after surgery (median 32d). In all these 11 patients we identified avWD with a reduction/loss of the largest VWF multimers. In 10 patients the collagen binding capacity was reduced, in 3 patients the VWF: Ag was slightly elevated, while in 8 infants it was in the normal range.

Conclusions: Despite the limited number of patients, we can presume that nearly $100 \%$ of the patients with aortopulmonary shunt present aVWS. Its pathogenesis is explained by the increased activation of the VWF under the influence of the turbulent flow within the shunt. The activated VWF is bound to its specific 
receptors located on the platelets and on the activated endothelial cells, and undergoes an ADAMTS 13 mediated proteolysis, which leads to the loss of large multimeres. First results show that the VWF swiftly normalizes shortly after suppression of the shunt dependent lung perfusion and switching to a cavopulmonary (Glenn) connection.

So far none of our patients demonstrated an increased bleeding tendency in everyday life. However, we must consider this anomaly as a potential cause of increased blood loss during cardiac catheterizations and operations. Knowledge of the existence of an avWD is therefore necessary for introduction of the replacement therapy with FVIII/VWF products.

\section{P-36}

Endomyocardial biopsy for monitoring pediatric heart transplant patients: 11 years' experience at a Turkish Heart Center

Erdogan I. (1), Varan B.(1), Surucu M.(2), Tokel K.(1), Sezgin A.(3) Department of Pediatric Cardiology Faculty of Medicine, University of

Başkent, Ankara-Turkey (1); Ankara Children's Hematology Oncology Education and Research Hospital, Ankara (2); Department of

Cardiovascular Surgery, Faculty of Medicine, Başkent University, Ankara-Turkey (3)

Introduction: Heart transplantation (HTX) has become an established therapy for patients with end-stage heart failure. Endomyocardial biopsy (EMB) still represents the gold standard for routine surveillance of rejection. We aim to report our experience regarding the use of $\mathrm{EMB}$ in monitoring heart transplant recipients.

Methods: We retrospectively evaluated all patients who underwent orthotopic HTX between 2005 and 2016 at our hospital. Mean age of the patients was 11.9 years ( 11 months-19 years). The route of the procedure, histopathological results and complications were recorded and evaluated with echocardiographic examination.

Results: Orthotopic HTX was performed in 38 children in the last 11 years. A total of $252 \mathrm{EMB}$ procedures were performed. Twenty of the biopsies were performed through the right or left internal jugular vein, others were performed through the right or left femoral vein.

Among the 252 biopsies, 196 (77\%) showed no evidence of cellular and humoral rejection. The remainder showed, mild (grade $1 \mathrm{R}$ ), moderate (grade $2 \mathrm{R}$ ) and severe (grade $3 \mathrm{R}$ ) rejection in $27(10.7 \%), 9(3.5 \%)$ and $2(0.8 \%)$ EMB, respectively, according to the ISHLT guidelines. Ten episodes $(3.9 \%)$ of acute humoral and $3(1.2 \%)$ episodes of suspected humoral rejection were detected. Only in 2 cases $(0.8 \%)$ the harvested EMB specimens contained no myocardial tissue.

Five patients who had biopsy-detected rejection had left ventricular ejection fraction (EF) between $0.45-0.60$ and 4 patients with rejection had EF below 0.45 . A patient with grade $1 \mathrm{R}$ cellular rejection had a normal EF but pericardial effusion. One had a normal biopsy result but systolic and diastolic dysfunction with echocardiography.

The rate of serious acute complications was $0.8 \%$ ( 2 of $252 \mathrm{EMB}$ ) in our study (one myocardial perforation and pericardial effusion with spontaneous resolution and one hematoma in the groin). Ther were no long-term complications.

Conclusion: The histopathological examination of right ventricular EMB still represents the gold standard of care for cardiac allograft rejection monitoring. EMB is an invasive, but safe and dedicated diagnostic procedure. However, the usefulness of recent noninvasive diagnostic approaches for detecting rejection remains to be further analyzed.
P-37

Prognostic Values Of Heart Rate And Oxygen Saturation In Patients With Pulmonary Arterial Hypertension Before And After Six Minute Walk Test

Kula S., Atasayan V., Tunaoğlu F.S., Oğuz D., Canbeyli F.

Gazi University Faculty of Medicine, Department of Pediatric

Cardiology, Ankara, Turkey

Objective: This study aims to determine the prognostic values of heart rate (HR) and transcutaneous oxygen saturation (Sat) which were measured before six minute walk test (6MWT), at the end of $6 \mathrm{MWT}$ and 5 minutes after $6 \mathrm{MWT}$ in children who are undergoing pulmonary arterial hypertension (PAH) specific treatment. Methods: This is a prospective review of 29 children who were aged between 7 and 17 years, diagnosed with PAH and underwent $6 \mathrm{MWT}$. Transcutaneous oxygen saturation and heart rate were measured before $6 \mathrm{MWT}$, at the end of $6 \mathrm{MWT}$ and 5 minutes after the completion of 6MWT (Sat0, Sat1, Sat2; HR0, HR1, HR2 respectively). HR increase was defined as $\mathrm{HR} 1$ minus $\mathrm{HR} 0$ and decrease in oxygen saturation was defined as Sat0 minus Sat1.

Results: The mean age of the children was $129 \pm 45$ months and the mean follow-up time was $58 \pm 40$ months. The mean proBNP concentrations did not change significantly before and after the administration of PAH specific treatment $(946.4 \pm 1754.8 \mathrm{pg} /$ $\mathrm{ml}$ vs $917.6 \pm 1920 \mathrm{pg} / \mathrm{ml} \mathrm{p}=0.11)$. However, the mean $6 \mathrm{MWT}$ distance increased significantly after the administration of PAH specific treatment $(400.2 \pm 107.8 \mathrm{~m}$ vs $436.2 \pm 119.2 \mathrm{~m}$, $\mathrm{p}=0.012)$. The 6MWT distance was significantly longer and HR1 was significantly higher in survivors $(453.3 \pm 96.5 \mathrm{~m}$ vs $250 \pm 135.2 \mathrm{~m}, \mathrm{p}=0.025$ and $122.8 \pm 18.4 / \mathrm{min}$ vs $94.3 \pm 19.1 /$ min, $p=0.034)$. HR 1 increased significantly but Sat 1 and Sat2 decreased significantly after PAH specific treatment $(p=0.017$, $p=0.03$ and $p=0.017$ respectively). While there was no significant correlation between World Health Organization functional classification (WHO-FC) and Sat1 before treatment, WHO-FC and Sat1 correlated negatively after treatment $(r=-0.435, p=0.021)$. Although WHO-FC and pro-BNP did not correlate before treatment, they correlated positively after treatment $(p=0.0001)$. There was no association between survival and heart rate increase whereas survival was positively associated with the decrease in oxygen saturation. If the decrease in oxygen saturation was $14.6 \%$, sensitivity increased to $100 \%$ and specifity was $58.3 \%$

Conclusions: PAH is a chronic disease which continues to progress despite the administration of specific therapy. The measurement of oxygen saturation with pulse oximeter during $6 \mathrm{MWT}$ might have prognostic importance in the follow up of children undergoing PAH specific treatment.

\section{P-38}

Correlation between Basic Pulmonary Hemodynamics and Pulmonary Vascular Resistance in Children with Pulmonary Hypertension associated with Congenital Heart Disease

Limsuwan A., Patipanvat S., Kladkaew W., Momsila P., Srichiangsa $T$.

Faculty of Medicine Ramathibodi Hospital, Mahidol University, Bangkok, Thailand

Background: Derived calculated pulmonary vascular resistance (PVR) has been routinely utilized as a parameter to evaluate children with pulmonary arterial hypertension associated with congenital heart disease (aPAH/CHD). Debatable whether the $\mathrm{PVR}$ is the ideal parameter to reflect the severity of aPAH/CHD 
due to the derived calculated value depends on oxygen consumption which is difficult and complicated to obtain the accurate value. To avoid using the oxygen consumption, the ratio between the PVR and systemic vascular resistance or Rp/Rs has been used instead of PVR. The objective of this study is to define whether the basic pulmonary hemodynamic parameters could be used as parameters to correlate with PVR and Rp/Rs.

Methods: Patients with aPAH/CHD underwent cardiac catheterization. Derived calculated PVR was calculated from transpulmonary pressure gradient divided by the pulmonary blood flow. The PVR was tested and showed to be directly correlated with the $\mathrm{Rp} / \mathrm{Rs}$. The pulmonary hemodynamics especially the diastolic pulmonary pressure (dPAP), diastolic pressure gradient (DPG: difference between the dPAP and pulmonary capillary wedge pressure) and the pulmonary pulse pressure (difference between the systolic pulmonary pressure and $\mathrm{dPAP}$ ) was compared and correlated with the PVR. The sensitivity and specificity of the pulmonary hemodynamic threshold value predict the PVR were determined.

Results: The cardiac catheterization data of 50 children (mean age 5.97 years, range 6 months to 18 years, 25 male) was retrospectively reviewed. The dPAP, DPG and PPP were correlated with the PVR, $r=0.83,0.84$ and 0.67 respectively. The $\mathrm{dPAP}>$ $24.5 \mathrm{mmHg}$ had $93.3 \%$ sensitivity and $81.8 \%$ specificity to predict PVR $>6$ WU. $\mathrm{m}^{2}$ with area under the curve of 0.92 . The ratio of the DPG $>14 \mathrm{mmHg}$ had $80 \%$ sensitivity and $78.8 \%$ specificity to predict PVR $>6$ WU.m ${ }^{2}$ with area under the curve of 0.898 .

Conclusion: The basic pulmonary hemodynamics particularly the dPAP and DPG could be used to assess the severity of aPAH/ CHD in children with reasonable correlation with the PVR. The $\mathrm{dPAP}$ has a better prediction of the PVR in comparison with the DPG and PPP.

\section{P-39}

Protein loosing enteropathy and cardiac disease in children and young adults

Perouse de Monclos T., Martin-Bonnet C., Bakloul M., Ducreux C., Veyrier M., Walton C., Ninet J., Henaine R., Metton O., Mitchell J., Di Filippo $S$.

Cardiovascular Hospital Louis Pradel, University Claude Bernard Lyon1, Hospices Civils de Lyon, Lyon France

The objective of this study was to assess the severity and the outcome of PLE in patients with congenital heart disease (CHD). Material and methods: Single-center retrospective chart-analysis of patients with CHD and diagnosed with PLE during follow-up. Cardiac disease past history, demographics, clinical data, therapeutic management and outcomes were assessed.

Results: 15 patients were included in the study (8 males). Underlying cardiac disease was: tricuspid atresia (4), double inlet ventricle (2), complex CHD (2), TGA with VSD and pulmonary stenosis (1), VSD and pulmonary hypertension (1), AVSD (1), pulmonary valve stenosis (1), TGA (1), total abnormal pulmonary venous return (1) and restrictive cardiomyopathy (1). Cardiac surgery was performed in 14 patients at the mean age of 5.4years (median 5y). Postoperative course was uncomplicated in 4 cases and 9 (60\%) presented prolonged pleural and/or pericardial effusion after surgery, 2 of them had a phrenic paralysis. Long-term cardiac events included arrhythmias in 3 cases, edemas in 2.

PLE was diagnosed at mean age of $8.5 y$ years (mean $7.5 y$ ). Mean time from surgery to PLE onset was 3.75years (median 0.8y). PLE was mild in 6 patients $(40 \%)$, moderate in $4(27 \%)$ and severe in $5(33 \%)$. No prognosis factors for severity of PLE was found.
All patients had myocardial function within normal range. Treatment for PLE included: diuretics and albumin supplementation in all cases, steroids in 4 , fenestration of Fontan in 3. Four cases (27\%) had poor outcome: of them, 2 patients died at 1.4 and $2.5 \mathrm{y}$ after onset of PLE because of uncontrolled compromised hemodynamics, and 2 were transplanted (1 PLE resolved within 6 months after transplantation and 1 died of uncontrolled graft failure). Eleven non-transplanted survivors (73\%) clinical status ranged in: NYHA I (5cases), NYHA II (4), or NYHA III (2). No PLE resolved in non-transplanted survivors.

Conclusion: PLE is a severe complication of CHD with high rates of mortality or transplantation. No significant predictive prognosis factor was found. No case resolved over time but disease was controlled in $73 \%$ of the cases.

\section{P-40}

Novel, atrial flow controlling (AFR) device to support balloon atrioseptostomy (BAS) in patients with pulmonary arterial hypertension (PAH)

Schulze-Neick I. (1), Lehner A.(1), Neurohr C.(1), Grünig E. (2), Heineking B. (1), Dalla-Pozza P. (1), Haas N.A. (1)

Paediatric and Congenital Cardiology and Intensive Care Medicine, Ludwig-Maximilian's University Munich (1), und Zentrum für Pulmonale Hypertonie, Thoraxklinik, Heidelberg (2), Germany

Introduction: Ballon Atrioseptostomy (BAS) remains unsatisfying in both the short and long term due to both uncertainties of the form and size of the created atrial septal defect (cASD) and the frequent later closure of it. Ad-hoc makeshift devices to address these problems have been reported, but present a risk per se, and do underline the need for a systematic and less risky solution.

Methods: A device very similar to conventional ASD-occluding devices, but with a central hole in it was designed and industrially produced in different sizes of outer diameter and of the inner hole, to provide both a controlled shunt when implanted into the cASD after BAS, and lasting patency of this atrial communication.

Results: In communication with the local Ethics Committee, two patients, (a) 39 year old male with very decompensated, nontransplantible clinical state despite multitherapy, with pulmonary arterial hypertension (PAH) and right heart (RV) failure, (b) 8 year old girl with recurrent syncope despite triple, both oral and inhaled $\mathrm{PAH}$-therapy, received a BAS according to the established PAH algorithm, and an AFR device to technically stabilize the BAScreated atrial septal defect.

The AFR implantation was fast, uncomplicated, remained in stable position and remained entirely patent at most recent checkup (>8 months post implantation). There was no need to repeat the BAS. Anticoagulation was provided with aspirin for 3 months. Both patients showed improved RV function. Further, patient (a) had marked clinical improvement with successful lung transplantation 2 months later; in patient (b) syncope did not occur again. Conclusions: The AFR device functioned as intended by stabilizing the BAS result, providing patency and delivering a controlled RLShunt over the observation time. AFR-implantation required only small-to-moderate sized cASD. Re-BAS did not occur, and anticoagulation required only mild agents. Taken together, this lowered the BAS risks and increased the BAS benefits, thus improving BAS risk/benefit ratio. As a result, BAS in its clinical value is upgraded. This changed risk-benefit profile might allow and justify an earlier use in the treatment algorithm of these diseases. These preliminary encouraging results need to be confirmed by a larger study. 


\section{P-41}

Gradual deterioration of vitamin $\mathrm{D}$ status and bone mass density in children and adolescents with Fontan circulation Diab S. G., Godang K., Myhre A.G., Sparr I., Almaas R., Døhlen G., Bollerslev J., Möller T.

Oslo University Hospital, Norway

Introduction: Patients with a Fontan circulation are at risk of malnutrition and growth deficit. Our group has shown high prevalence of vitamin D deficiency and low bone mass density in adolescents with Fontan circulation. The aim was to investigate vitamin D status and bone mass density in children with Fontan circulation at different ages. Methods: Patients were recruited from our national Fontan clinic and divided in two cohorts aged 5 to 9 years and 10 to 15 years respectively. We prospectively measured serum levels of 25-OHvitamin $\mathrm{D}$ and total body bone mass density by narrow fan-beam dual energy x-ray absorptiometry (DXA) scan. The DXA results were compared to age and gender matched reference data and expressed as Z-scores. Vitamin D and DXA results of the two cohorts were compared to the previous cohort of 17 patients aged 16 to 18 years. Current recommendations for patients with chronic illness suggest an optimal serum level of 75-125 nmol/L.

Results: We included 15 patients aged 5 to 9 years and 19 patients aged 10 to 15 years. Vitamin D levels in the two cohorts were $77.5 \pm 31.0 \mathrm{nmol} / \mathrm{L}$ (mean $\pm \mathrm{SD}$ ) and $61.6 \pm 30.2 \mathrm{nmol} / \mathrm{L}$ respectively, while the level in the adolescent cohort was $34.6 \pm 18.3 \mathrm{nmol} /$ L. The DXA scans for the two youngest cohorts showed z-scores of $0.7 \pm 0.9$ and $-0.7 \pm 1.4$, while the oldest cohort had a $z$-score of $-0.8 \pm 1.1$. Statistically significant differences are shown in figure 1 .

Conclusion: Both vitamin D status and bone mass density show agerelated differences in Fontan patients and a significant decrease through childhood and adolescence. Whether this is due to life style factors (i.e. sun exposure, dietary intake of vitamin D and physical activity) or due to a pathophysiological effect of the Fontan circulation is unknown. Future studies should investigate the pathogenesis of both vitamin D deficiency and impaired bone mass density, as well as determine the risk of bone related complications.

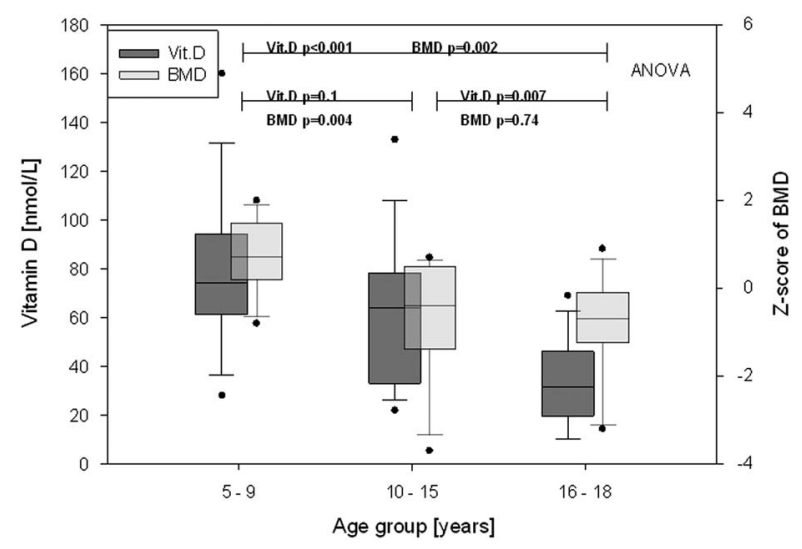

Figure 1.

Vitamin D serum level and bone mass density by age group.

P-42

Vitamin D status and bone mass density in adolescents with Fontan circulation

Diab S. G., Godang K., Myhre A.G., Sparr I., Almaas R., Døhlen G., Bollerslev J., Möller T.

Oslo University Hospital, Norway

Introduction: Although patients with a Fontan circulation are at risk of malnutrition and growth delay, the implications on vitamin $\mathrm{D}$ metabolism and bone health have barely been investigated.

Methods: We prospectively measured serum levels of 25-OH-vitamin D in a nationwide cohort of Fontan patients aged 16 to 18 years old. We also measured bone mass density by narrow fan-beam dual energy x-ray absorptiometry (DXA) scan. The DXA results were compared to age and gender matched reference data and expressed as Z-scores.

Results: Seventeen consecutive patients were recruited from our pre-transition national Fontan clinic. All 17 patients had vitamin D levels $<75.0 \mathrm{nmol} / \mathrm{L}$, which has been suggested as a lower limit in patients with chronic illness. Thirteen patients $(76 \%)$ had vitamin D insufficiency with levels $<50.0 \mathrm{nmol} / \mathrm{L}, 9(53 \%)$ patients had deficiency with values $<37.5 \mathrm{nmol} / \mathrm{L}$ and two patients $(12 \%)$ had severe vitamin D deficiency with non-measurable values $<12.5 \mathrm{nmol} / \mathrm{L}$. The DXA showed abnormally low z-scores of $-1.7 \pm 0.9($ mean $\pm \mathrm{SD})(\mathrm{p}<0.001)$ for columna and $-0.8 \pm 1.1$ $(p=0.009)$ for total body measurement respectively. There was no correlation between DXA result and vitamin D level.

Conclusion: Adolescent Fontan patients have a high prevalence of vitamin $\mathrm{D}$ deficiency and low bone mass density, however, without these two being correlated. Whether the vitamin D deficiency is due to limitations in sun exposure and dietary intake of vitamin $\mathrm{D}$, or if vitamin $\mathrm{D}$ deficiency and insufficient bone mass density are differently related to the Fontan circulation is unknown. Future studies should investigate pathogenesis of both vitamin D deficiency and low bone mass density, as well as determine fracture implications and identify interventions.

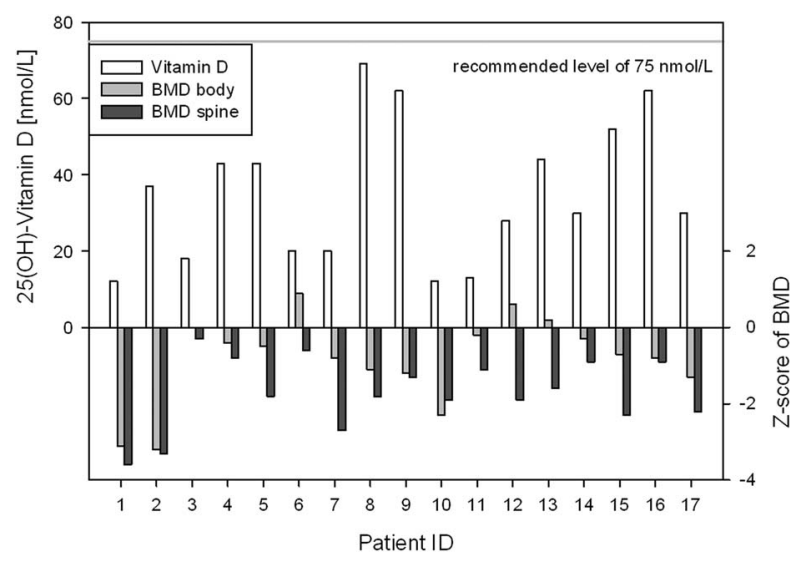

Figure 1.

Vitamin $D$ levels in $n m o l / L$ and bone mass density by $Z$-score for each of the patients.

P-43

Ibuprofen for the closure of Patent Ductus Arteriosus in preterm babies - Network experience from two Neonatal Intensive Care Units

Siddhi P.(1), Rasaiah V.(1), Storey I. (2)

Birmingham Women's Hospital, Birmingham, United Kingdom (1); Birmingham Heartlands Hospital, Birmingham, United Kingdom (2)

Background: The use of ibuprofen in the treatment of Patent Ductus Arteriosus (PDA) in preterm babies is well established. Ibuprofen has been associated with oxygen dependency beyond 28 days of life (1). The purpose of this study was to assess the effect of ibuprofen on the closure of PDA and analyse the incidence of severe chronic lung disease measured by the need for home oxygen. 
Method: This was a retrospective analysis of the data collected over a period of 3 financial years from two Neonatal Intensive Care Units in the Southern West Midlands Maternity and Newborn Network. Results: There were a total of 93 preterm neonates identified to have had Patent Ductus Arteriosus (PDA). $86 \%(\mathrm{n}=80)$ were ventilated at the time of treatment. Ibuprofen was commenced on an average on the eleventh day of life. Of those who received a single course of ibuprofen $(n=38), 71 \%(n=27)$ were found to have no PDA at discharge. Of those who received a second course of ibuprofen $(\mathrm{n}=16), 38 \%(\mathrm{n}=6)$ had no PDA at discharge. $23 \%$ $(n=21)$ underwent surgical ligation. 2 of the 3 who had an incomplete course of ibuprofen had spontaneous closure of PDA. There were 10 deaths. $46 \%(n=43)$ were discharged on home oxygen. Conclusion: Ibuprofen was shown to be effective in closing the duct in preterm babies. However the second course of Ibuprofen seemed less effective. Oxygen dependency in neonates at discharge was considerable. Though ibuprofen has known to have less immediate side effects compared to indomethacin, the longterm effects are yet to be established. We look forward to the randomised controlled study like OSCAR (Outcome of Selective early Closure of Patent Ductus ARteriosus in Preterm Babies) to help address the dilemma of managing PDA in preterm babies.

Reference

1. B Thébaud, T Lacaze-Mazmonteil, Patent ductus arteriosus in premature infants: A never-closing act. Pediatric Child Health. 2010 May-Jun; 15(5): 267-70.

PMCID: PMC2912622

\section{P-44}

Prenatal diagnosis of fetal aortic coarctation.

Meysonnier C. (1), Massoud M. (1), Joly H. (2), Bonnet C. (2), Veyrier M. (2), Di Filippo S. (2), Pangaud N. (2)

Centre Pluridisciplinaire de Diagnostic Prénatal, Hôpital Femme Mère

Enfant, Lyon-Bron, France (1), Service de cardiologie pédiatrique,

Hospices Civils de Lyon, Lyon-Bron, France (2)

Objectives: Prenatal diagnosis of fetal aortic coarctation (AoCo) is mainly suspected when cardiac ventricular or great vessels disproportion is encountered on prenatal ultrasound. Prenatal counseling remains challenging because of the lack of specificity of these criteria. The aim of this study was to evaluate the detection rate of fetal AoCo according to these criteria and to determine echocardiographic features associated with this diagnosis.

Methods: In this retrospective study, we reviewed the chart of 85 fetus referred for cardiac ventricular or great vessels disproportion between January 2010 and December 2015. 63 fetus were excluded because of hypoplastic left ventricle, complexe heart disease, interruption of the aortic arch. 37 fetus were included for suspicion of isolated AoCo and noted their postnatal follow-up. We compared the subject with and without AoCo. We analysed the right/left ventricular (RV/LV) diameters ratio, main pulmonary artery/ ascending aorta (MPA/AAo) diameters ratio, ductus arteriosus/aortic isthmus (DA/AOI) diameters ratio, presence of reverse flow in the aorta, persistent left superior vena cava, ventricular septal defect, bicuspid aortic valve. The postnatal follow up included the ultrasound data, timing of the surgery and the surgical approach.

Results: The AoCo was confirmed postnatally in 19 neonates $(51,4 \%)$, all requiring surgical treatment in the neonatal period 7 days after birth on average ( 2 days- 18 days). Significant statistical differences $(p=0005)$ were found when the diagnosis was suspected during the second trimester ultrasound scan $(p=0.005)$, when there was a reverse flow in the aorta $(21,1 \%$ vs $0 \% \mathrm{p}=0039)$ and the $\mathrm{RV} / \mathrm{LF}$ diameters ratio was significantly higher in case of coarctation at the third trimestre $(1,82$ vs $1,30 \mathrm{p}<0001)$. There was no significant difference for the $\mathrm{RV} / \mathrm{LF}$ diameters ratio at the second trimester $(p=0183)$, the MPA/AAo diameters ratio $(p=0132)$, the DA/AOI diameters ratio $(p=0079)$, the ventricular septal defect, the persistent left superior vena cava, and the prenatal diagnosis of bicuspid aortic valve.

Conclusion: Cardiac ventricular or great vessels disproportion is not a specific sign for the prenatal diagnostic of aortic coarctation. Further studies are needed to build prediction models integratting different ultrasound signs in order to improve the antenatal detection of this anomaly.

\section{P-45}

Echocardiographic evaluation of cardiac functions in newborns of mildly preeclamptic pregnant women within postnatal 24-48 hours

Mutlu K.(1), Karadas U.(2), Yozgat, Y.(3), Mese T.(1), Demirol M. (1), Coban S.(1), Karadeniz C.(1) Ozdemir R.(1) Orbatu D.(2), Karaarslan U.(1), Tavli V(1)

izmir Dr.Behcet Uz Children's Hospital, izmir, Turkey, Pediatric cardiology(1); MH Aegean Maternity, Teaching and Training Hospital, izmir, Turkey(2); Bezmialem Vakif University, Istanbul, Turkey (3)

Objective: The aim of this study is to detect the preeclampsiarelated cardiac dysfunction within postnatal 24-48 hours in newborns born from preeclamptic mothers.

Material and Method: Forty newborns born from mildly preeclamptic mothers consisted the study group and 40 healthy newborns matched with the study group in terms of gestational age, weight and gender consisted the control group. Cardiac functions of the study and the control group were evaluated using conventional echocardiography (shortening fraction, SF; mitral and tricuspid E/A with pulsed wave; peak systolic gradient of aortic and pulmonary valves) and myocardial performance index (MPI) within the first 24-48 hours of life and results were compared.

Results: Median age of the pregnant women in study group was 26 years $(\min 21, \max 35)$ and it was 25 years $(\min 20-\max 35)$ in control group. A statistically significant difference was not detected between groups with regard to weight, type of delivery, week of delivery and gender $(p>0005)$. A statistically significant difference was not detected between conventional echocardiography measurements of study and control groups. A significant difference was detected when left and right ventricle PW Doppler MPI measurements of study and control groups were compared (left ventricle MPI 0,37 $\pm 0,09$ and $0,26 \pm 0,11, \mathrm{p}<0.001$; right ventricle MPI $0,29 \pm 0,08$ and $0,26 \pm 0,07 \mathrm{p}<0035$ ).

Conclusion: According to the results of the study, elongation in right and left ventricle MPI was detected to be more significant compared to systolic and diastolic functions for determining preeclampsiarelated cardiac injury in newborns of preeclamptic mothers within 24-48 hours of their life. Ventricle functions of the newborns of preeclamptic mothers should also be evaluated with MPI measurement beside conventional echocardiography measurements.

\section{P-46 \\ Conotruncal Anomalies in The Fetus:Echocardiographic and Clinical Evaluation and Prognosis of 72 Patients Babaoglu K., Deveci M., Dogan Y., Tugral O., Yucesoy G. Kocaeli University School of Medicine Kocaeli, Turkey}

Objective: Majority of the conotruncal anomalies (CTA) are accurately diagnosed by fetal echocardiography. In this study, we 
aimed to evaluate the results of prenatally diagnosed patients with CTA.

Methods: The medical and fetal echocardiographic records of pregnant women admitted to our pediatric cardiology department between January 2011 and January 2016 were reviewed.

Results: A total of 4690 fetal echocardiographic investigations revealed $72(1.5 \%)$ fetuses with CTA. The gestation weeks at the time of diagnosis ranged between 14 to 36 weeks with a mean of $25.1 \pm 6.8$ weeks. Twenty-eight $(38 \%)$ of the fetuses with CTA had tetralogy of Fallot (TOF) which included absent pulmonary valve in $3(4.5 \%)$, pulmonary atresia in $2(3 \%)$ and complete atrioventricular septal defect in $3(4.5 \%)$. Twenty-three $(32 \%)$ of the patients had double outlet right ventricle, $10(13.8 \%)$ had transposition of great arteries, 3 (4\%) had truncus arteriosus and 8 (11\%) had posterior malalignment type ventricular septal defect and aortic arch anomalies. Postnatally, transthoracic echocardiography was performed in 51 fetuses and results were consonant with prenatal diagnosis, except 2 cases. $62 \%$ of the newborn babies were male. Chromosome analysis and karyotyping revealed trisomy 18 in 1, and Di George syndrome in 2 of the babies. Extracardiac anomalies accompanied in $23(32 \%)$ fetuses. Complete AV block was detected in a baby with TOF and complete atrioventricular septal defect. Seven fetuses died prenatally (3 termination of pregnancy, 4 intrauterine death) and 20 infants died postnatally. Total rate of mortality was $37.5 \%$.

Conclusion: Prenatal diagnosis of cases with conotruncal anomalies is established accurately with increasing experience by fetal echocardiography. However, posterior malalignment type ventricular septal defects and aortic arch anomalies are not easy to detect correctly in fetal period. As cases with CTA have associated extracardiac and chromosomal anomalies, they generally do not have good prognosis.

\section{P-47 \\ Sonographer led fetal echocardiography - an effective strategy for low risk cases \\ Forster J., Bainbridge S., Barwick S., Brown E.M., Hares D.L., Thomson J.D.R. \\ Fetal Cardiology, Leeds General Infirmary, Leeds, UK}

Introduction: Detailed fetal echocardiography is performed for familial, maternal and fetal indications. Pregnancies in which there is a relatively low risk of fetal cardiac abnormality include those in which there is a first degree relative with congenital heart disease (CHD), exposure to known cardiac teratogens or the existence of a variety of maternal illnesses. Higher risk pregnancies include the mothers in whom there is a suspicion of abnormality during an obstetric scan, raised nuchal thickness, monochorionic twins or suspected arrhythmias.

In our centre, after triage, low risk referrals are assessed and scanned in a dedicated clinic solely be an experienced cardiac sonographer Method: This audit was performed to assess the safety and effectiveness of the service described. Between October 2013 and September 2016 fetal echocardiography referrals were triaged by the fetal cardiac team and classed as low or higher risk according to agreed local and national guidelines. Indications for referral, an assessment of scan quality and presence of normality (or otherwise) were prospectively recorded. A pre-written information sheet was given to mothers whose fetus had a normal heart following assessment by the sonographer. When an abnormality was demonstrated, a fetal cardiologist and nurse specialist were available in the hospital for counselling.

Results: Over a 3 year period, 500 ladies were scanned in this sonographer led clinic. Of the 500 pregnancies, 431 were referred for family history of CHD. 7 of the 500 fetuses were found to have CHD and were then reviewed by a fetal cardiologist immediately following the scan. In addition, 2 unexpected intrauterine deaths were detected. There were no known incidences of false negative or false positive diagnosis of CHD made.

Conclusion: The sonographer led low risk fetal echocardiography clinic with an experienced sonographer, after appropriate referral triage, detected pathology in $1.4 \%$ of the cases. All of these parents received a Consultant consultation and counselling at the time of their appointment. The clinic has provided reassurance for the remaining parents where the fetal echo was normal. This service development has proved both cost-effective and time efficient, enabling additional resources to be targeted to higher risk cases.

\section{P-48 \\ Prenatal restriction of foramen ovale: important information for obstetrician, neonatologist, pediatric cardiologist or cardiac surgeon?}

Pluzanska J. (1,2), Moszura T. (2), Respondek-Liberska (1) Department of Prenatal Cardiology, Polish Mother's Memorial Hospital Lodz, Poland (1); Department of Cardiology, Polish Mother's Memorial Hospital Lodz, Poland (2)

Neonate with an isolated ductal dependent congenital heart defect is usually a candidate for cardiac surgery or intervention, regardless of the prenatal diagnosis. However, in some cases, fetal echocardiography results provide important information and the priority of these selected cases, should be known to pediatric cardiac surgeons, before the neonate's clinical deterioration.

Material and methods: Based on our computer database in our unit in the years 2012-2014 we selected fetuses who had an isolated congenital heart defect and restriction of the foramen ovale defined as its diameter of $4 \mathrm{~mm}$ or less, with left - right shunt across foramen ovale, $\mathrm{V} \max >70 \mathrm{~cm} / \mathrm{sec}$ along with a typical harsh sound during fetal ausculation during echocardiography and reversal flow in pulmonary veins.

Total of 18 cases were selected $(0,76 \%)$. One case resulted in termination of the pregnancy and in one case (in twin pregnancy) in premature delivery, giving a total of 16 cases after prenatal diagnosis in our center and delivery with postnatal management in our institution.

Results: There were 10 non-survivors and 6 survivors, In the group of non-survivors there were 5 cases of HLHS and in the group of survivors 4.

The only significant difference between survivors and nonsurvivors pertained to the fraction of newborns operated on up to 11 days, which was significantly higher among the survivors (5/ 6 vs. $2 / 8, p=0.031)$. Our data from this research has crucial value which might help pediatric cardiologists and pediatric cardiac surgeons choose the candidates for early cardiac surgery and information from prenatal echocardiography about restriction of the foramen ovale should be taken into consideration as valuable information for the whole team of specialists, suggesting priority for cardiac surgery.

Conclusions: 1) In the event of prenatal restriction of the foramen ovale early surgery by day 10 had a statistically better outcome in terms of survival compared to cases that underwent surgery at a later period at our Institute.

2) Information from prenatal echocardiography regarding restriction of the foramen ovale should be taken into consideration as valuable information suggesting priority for early cardiac surgery. 


\section{P-49}

French monocentric experience with antenatal diagnosis of Hypoplastic Left Heart Syndrome

Joly H.(1), Blondel de Joigny C.(1), Veyrier M.(1), Pangaud N.(1), Bertail-Galoin C.(1), Massardier J.(2), Walton C.(1), Di Filippo S.(1) (1) Cardiovascular Hospital Louis Pradel, Université Claude Bernard, Hospices Civils de Lyon, Lyon, France, (2) Obstetrics and Gynecology, Hopital Femme Mère Enfant, Lyon, France

The objective of this study was to assess the outcomes of fetus and decision making after prenatal diagnosis of hypoplastic left heart syndrome (HLHS).

Material and Methods: This study is a single-center retrospective analysis of all fetus diagnosed with HLHS syndrome from 2010 to 2016. Antenatal parameters included: mother age and gestational age of fetus at diagnosis, genetic testing, and prenatal outcomes. Postnatal parameters included: gestational age, birth weight, apgar score, echocardiographic measurements, and decision-making.

Results: Overall 70 foetus were included in the study, diagnosed at $26.4 \pm 5.2$ weeks of gestation, with: typical HLHS (54), unbalanced DORV (2) or AVSD (1), borderline LV (4), severe aortic coarctation and LV hypoplasia (3), double inlet ventricle (3) and complex CHD (3). Four cases had a chromosome anomaly. Thirty-seven terminations of pregnancy $(53 \%)$ at $25.2 \pm .3 .1 \mathrm{WG}$ and $25(36 \%)$ live births occurred; the remaining cases included: sudden in utero death (1), ongoing gestation (1), and lost of FU $(6=9 \%)$. Gestational age at birth was $38.8 \pm 1.9 \mathrm{wg}$ (34 to $42 \mathrm{wg}$ ), birth weight was $3180 \pm 600 \mathrm{~g}$ (1900 to $4300 \mathrm{~g}$ ); apgar score was $\leq 3$ in $16 \%$ and $>7$ in $80 \%$ of the cases. Postnatal echocardiographic analysis was concordant with prenatal assessment: HLHS (15), borderline LV (7), DORV (1), DIV (1). One patient died early after birth from uncontrolled cardiogenic shock and hypoxemia. Among the 24 remaining patients, 8 were un-operated and died (parents decision for compassionate care), and 16 underwent first-stage surgery (i.e. 23\% of overall 70 fetus) at the age of $10.8 \pm 6.2$ days ( 5 to $25 \mathrm{~d}$ ) from whom 7 had secondstage surgery at the age of $4.1 \pm 2.6$ months ( 1 to $7 \mathrm{mos}$ ).

Conclusion: Despite experience and improvement of surgical techniques in HLHS, decision making in a French tertiary-care center Pediatric cardiology department still prefer TOB or compassionate care to active surgical management.

\section{P-50}

Recurrence of congenital heart disease in offsprings of mothers with congenital heart disease screened by fetal echocardiography

Fesslova V. (1), Inversetti A.(2), Sigismondi C.(2), Brankovic J. (1) Center of Fetal Cardiology, Policlinico San Donato IRCCS, Milano, Italy (1); Dpt. Obstetrics/Gynecology, Ospedale San Raffaele, Milano, Italy (2)

Introduction: Pregnant women with congenital heart disease (CHD) are at risk of recurrence Preconceptional counselling is very delicate in this population and the data relative to the recurrence rates are of extreme use in this field.

Objective of the study: to analyze the recurrence of congenital heart disease (CHD) in offsprings of mothers affected with CHD.

Material and methods: Retrospective-prospective study of 310 pregnancies of 271 mothers with CHD studied by fetal echocardiography in our Center between Jan. 1995 and Dec.2015. Thirty nine women were followed-up during 2-3 pregnancies. Twenty seven women, all operated, had cyanotic CHD, in
34 pregnancies and 244 had acyanotic CHD, operated in 149, in 276 pregnancies. Thirty six had multiple familial risk (2-5 relatives) Results: Twenty three probands had CHD (total recurrence rate $23 / 310=7.4 \%$ pregnancies), $1 / 34$ pregnancies with cyanotic CHD (2.9\%), 22/244 pregnancies with acyanotic CHD (9\%).

When mother alone was affected, the recurrence was 21/ $235=8.9 \%$; when mother and another relative were affected the recurrence rate was $2 / 36=5.5 \%$. The specific recurrence was higher in VSD $(7 / 71=9.8 \%)$, ASD II (considered at age $>1 \mathrm{yr}$, diam. $>8 \mathrm{~mm}-7 / 88=7.9 \%)$, aortic stenosis $(3 / 34=8.8 \%)$, ductus arteriosus $(1 / 12=7.7 \%)$ and in AVSD (2/4cases). Concordant lesions occurred in 9 cases, partially concordant in 7 , discordant in 6 cases.

Conclusions: Our data confirm a relevant recurrence of CHD in affected mothers, despite the numerical limits of our population. This fact has to be taken in account in prenatal counselling.

\section{P-51}

The Short Axis Echocardiographic Scan. An Easy Implementation to Improve the Detection Rate of Perimembranous Ventricular Septal Defects in the Fetus Cavalli C. (1), Dall'Asta A. (2), Galli L. (2), Volpe N. (2), Weiss A. (1), Kiener A. (2), Kaihura C. (2), Frusca T. (2), Ghi T. (2) (1) Pediatric Hospital "P. Barilla"; (2) Unit of Obstetrics and Gynecology. Azienda Ospedaliero-Universitaria. Parma. Italy.

Background: The anatomic position of perimembranous ventricular septal defects (VSD) is definitely close to tricuspidal apparatus and their detection rate by fetal echocardiography (FE) is weak by the long axis and the 5-chamber views. Conversely to FE, neonatal echocardiography includes the basal short-axis view as part of a standard examination. We tried to assess if the introduction of the short axis view in FE may improve the prenatal detection rate of perimembranous VSDs.

Setting: Tertiary level Center for prenatal diagnosis in which all examinations are performed by a Gynecologist skilled in FE and a Pediatric Cardiologist together. This is a single Center prospective study.

Methods: From 2014 to present all the fetuses in whom an isolated subaortic VSD was suspected on the 5- chamber view of the fetal heart were assessed afterward by the short axis scan. The transducer was then rotated 90 degrees towards the left shoulder of the fetus to obtain the view of the right outflow tract surrounding the cross section of the aortic root. When present, a VSD is detectable both by 2D and Color Flow Doppler as an interruption of the aortic profile and as a reddish sequence respectively, just below the tricuspid valve. An echocardiography was performed for final diagnosis in all fetuses within 2 weeks after birth.

Results: Overall an isolated subaortic VSD was suspected by the long axis view in 18 fetuses at a mean gestational age of $22.1 \pm 2.7$ weeks. In $12 / 18$ fetuses $(66.7 \%)$ the VSDs were confirmed by the basal short axis and at postnatal follow-up the diagnosis was confirmed 10/12 (83.3\%). The remainder $2 / 12$ $(16.7 \%)$ were false positive cases. Eventually, neither of the 6 cases in which the VSD was not confirmed by the basal short axis view revealed a VSD at postnatal analysis.

Conclusion: The short axis view of the fetal heart seems useful in confirming the presence of a perimembranous VSD suspected by the classical 5-chamber and long axis views. The introduction of this scan could increase the detection rate of perimembranous VSDs which require a more specific counselling than other types of VSD 


\section{P-52}

The placenta on fetal MRI in congenital heart disease-can we learn something new?

Mlczoch E. (1), Gruber G. (2), Dekan S. (3), Michel-Behnke I. (1), Worda C. (4), Salzer-Muhar U. (1), Weber M. (2), Prayer D. (2), Kasprian G. (2)

Paediatric Heart Center Vienna, Vienna, Austria (1); Department of Neuroradiology, Vienna, Austria (2); Department of Pathology, Vienna, Austria (3); Department of Fetomaternal Medicine, Vienna, Austria (4)

Objective: Fetal MRI has shown to be a valuable tool to evaluate the placenta. Little is known about the placenta in the preseence of congenital heart disease (CHD). This fetal magentic resonance imaging study aimed to identify abnormal placental signals and pathologies throughout gestation in fetuses with CHD.

Methods: The 1.5 tesla magnetic resonance examinations of 199 fetuses with CHD were retrospectively investigated. The placenta was evaluated on T1, T2, echoplanar and diffusion weighted images. (GW 18-35).

Results: Fetuses with CHD show non age related premature maturation signs on $\mathrm{T} 1$ weighted images in $17 \%$, T2 weighted images in $18 \%$ and echoplanar sequences $12 \%$. Oedemtous placenta signals were noted in $6 \%$. Bleeding, Hematoma and Infarction was present in $8 \%$ on $\mathrm{T} 1,6 \%$ on $\mathrm{T} 2$ and $6 \%$ on echoplanar sequences. Fetuses with a right heart disease had significantly more placental inhomogenities than healthy fetuses.

Conclusion: This is the first study to show placental abnormalities on fetal MRI in fetuses with CHD.

The findings need to be investigated further.

\section{P-53}

Correlation between Basic Echocardiogram and Cardiac Magnetic Resonance of the Right Ventricle in Repaired Tetralogy of Fallot

Trisauvapak T., Limsuwan A., Khowsathit P.

Faculty of Medicine Ramathibodi Hospital Mahidol University, Bangkok, Thailand

Introduction: Cardiac magnetic resonance (CMR) has become a standard tool to evaluate the right ventricle $(\mathrm{RV})$. In repaired tetralogy of Fallot (rTOF), the right ventricular volume load is considered to be one of the key criterions for the pulmonary valve replacement. The objective of this study is to define whether the conventional echocardiographic measurement could be use as a parameter to define the right ventricular dilatation in comparison with the CMR measurement.

Methods: Patients with rTOF underwent CMR and echocardiogram. From the 4-chamber view and parasternal view in long axis, the right atrium (RA), RV and left ventricle (LV) dimensions were measured during diastole and indexed by body surface area. The RV echocardiographic measurement was compared and correlated with the RV volume index obtained from CMR. The sensitivity and specificity of the echocardigraphic threshold value predict the RV volume were determined.

Results: A total of 91 patients (mean age 14, range 12-18, 64 male) were recruited. The echocardiographic measurement of RV end diastolic diameter (RVEDD) index and RV/LV dimension ratio and the RA dimension were correlated with the RV end diastolic volume index (RVEDVi) obtained by CMR. The RVEDD index $>1.55 \mathrm{~cm} / \mathrm{m}^{2}$ had $81 \%$ sensitivity and $60 \%$ specificity to predict RVEDVi $>150 \mathrm{~mL} / \mathrm{m}^{2}$ with area under the curve of 0.79 . The ratio of the $\mathrm{RV} / \mathrm{LV}$ dimension $>0.5 \mathrm{~cm} / \mathrm{m} 2$ had $86 \%$ sensitivity and $60 \%$ specificity to predict RVEDVi $>150 \mathrm{~mL} / \mathrm{m}^{2}$ with area under the curve of 0.79 . While the RA dimension
$>10 \mathrm{~cm} / \mathrm{m} 2$ had $65 \%$ sensitivity and $69 \%$ specificity to predict RVEDVi $>150 \mathrm{~mL} / \mathrm{m}^{2}$ with area under the curve of 0.73 .

Conclusion: The basic echocardiogram parameter of the RV dimension could be used to assess the right ventricle volume load in rTOF with reasonable CMR correlation. The RVEDDi has a better prediction of the RV volume in comparison with the RV/ $\mathrm{LV}$ and RA dimension.

\section{P-54}

Cyanosis in a patient with corrected pulmonary atresia due to pulmonary arterial hypertension and nocturnal apnea effectively treated by non-invasive CPAP

Dirks S., Ovroutski S., Berger F.

Deutsches Herzzentrum Berlin, Germany

Objectives: Case report of a 41 year old male, with pulmonary atresia and ventricular septal defect, presenting with chronic cyanosis after pulmonary valve replacement and closure of the VSD at the age of 23 years and later interventional pulmonary valve replacement.

Methods: His physical capacity is declining due to dyspnea, equivalent to NYHA Class III. Resting $\mathrm{SpO} 2$ is $85 \%$. He is overweight (BMI 28.5). Echocardiography shows mildly decreased biventricular systolic function and right ventricular systolic pressure of $70-80 \mathrm{mmHg}$ and paradox ventricular septal movement. Mean pulmonary valve gradient is $23 \mathrm{mmHg}$. No right to left shunt. Bodyplethysmography shows low vital capacity ( $24.2 \%$ of normal) and low total lung capacity $(21.4 \%$ of normal). CPET shows a peak oxygen consumption of $8.6 \mathrm{ml} / \mathrm{min} / \mathrm{kg}(25 \%$ of normal), low breathing reserve of $10 \%$ and a drop in SpO2 to 74\%. Computertomography and MRI showed no pulmonary thrombosis, but RV hypertrophy, dilated proximal but rarefied distal pulonary arteries and right sided diaphragmatic paresis. Polysomnography revealed episodes of hypopnea $10.6 / \mathrm{h}$ and obstructive apnea $0.6 / \mathrm{h}$, snoring $101 \mathrm{~min}(24.2 \%)$. SpO2 during sleep averaged $81 \%$ (minimum 68\%). Resting CO2 was $49 \mathrm{mmHg}$; with increase at sleep to average $62 \mathrm{mmHg}$ (maximum $73 \mathrm{mmHg}$ ). A polysomnography using a non-invasive CPAP mask showed significant reduction of obstructive apnea to $0.1 / \mathrm{h}, \mathrm{CO} 2$ was much less with a mean of $56 \mathrm{mmHg}$ (maximum $60 \mathrm{mmHg}$ ) and $\mathrm{SpO} 2$ level was higher (mean 85.9\%).

Results: A cyanotic patient with no remaining right to left shunt and secondary pulmonary arterial hypertension and relevant restrictive lung disease due to diaphragmatic paresis and multiple thoracotomies shows moderate to severe respiratory and mild circulatory restriction, a drop of $\mathrm{SpO} 2$ at exercise. Hypopnea and obstructive apnea during sleep cause relevant worsening of $\mathrm{CO} 2$ and $\mathrm{SpO} 2$. All of these symptoms are improved by use of a CPAP-mask.

Conclusion: Sleep-apnea may aggravate the symptoms of chronic lung disease and pulmonary arterial hypertension, this should be recognized by polysomnography. Symptoms and long term prognosis may effectively be treated using non-invasive CPAP.

\section{P-55}

Are adults with congenital heart disease informed about their risk for infective endocarditis and treated in accordance to current guidelines?

Helm P.C. (1,2), Diller G.-P. (3), Kaemmerer H. $(4,5)$, Bauer U.M. M. (1,2), Asfour B. (6), Tutarel O. (4,5)

(1) National Register for Congenital Heart Defects, Augustenburger Platz 1, 13353 Berlin, Germany, (2) DZHK (German Centre for Cardiovascular Research), (3) Muenster University Hospital, Center for Adults with Congenital Heart Defects (EMAH-Center), AlbertSchweitzer-Str. 33, 48149 Muenster, Germany, (4) German Heart 
Centre Munich, Department of Paediatric Cardiology and Congenital Heart Defects, Technical University of Munich, Germany, (5) DZHK (German Centre for Cardiovascular Research), Partner Site Munich, Germany, (6) Asklepios Klinik Sankt Augustin GmbH, German pediatric heart center, Sankt Augustin, Germany

Background: Infective endocarditis (IE) is associated with significant morbidity and mortality. Whether and to what extent the guideline changes in the last decade have been implemented into clinical practice is unknown. Especially the knowledge level of patients has not been studied.

Purpose: To study the current knowledge level of ACHD patients regarding IE and antibiotic prophylaxis of IE.

Methods: Patients recruited via the German National Register for Congenital Heart Defects to an online survey about IE. 1458 individuals participated and 1211 patients with detailed clinical data available (mean age $30.5 \pm 11.8$ years, female $=44.1 \%$ ) were included into the study.

Results: Group A (IE prophylaxis recommended): 343 patients (mean age $31.8 \pm 11.2$ years, female $=53.1 \%$ ); Group B (IE prophylaxis is not required): 714 patients (mean age $29.1 \pm 10.8$ years, female $=53.9 \%$ ); Group C (a conclusive statement regarding IE prophylaxis not possible): 154 patients (mean age $33.9 \pm 15.7$ years, female $=57.1 \%$ ). A regular treatment in a tertiary medical environment took place in $76.1 \%$ (group A), 66.1\% (group B) and $40.9 \%$ (group C). Within the last five years, $67.3 \%$ (group A), $52.8 \%$ (group B) and $26.6 \%$ (group C) were informed by a physician about IE. In 47.8\% (group A), 28\% (group B) and 16.9\% (group C) the discussion was initiated by a physician. Knowledge regarding IE was present in $82.5 \%$ (group A), $73.2 \%$ (group B), and $62.3 \%$ (group C), while in $70.6 \%$ (group A), $37.7 \%$ (group B) and $16.9 \%$ (group C) an increased risk of IE was present according to their own opinion. Knowledge of antibiotic prophylaxis was present in $82.2 \%$ (group A), $63.7 \%$ (group B), and $42.2 \%$ (group C).

Conclusions: This study reveals important knowledge gaps regarding IE/antibiotic prophylaxis in ACHD. Even 20-30\% of ACHD with a strict recommendation for using antibiotic prophylaxis, did not possess knowledge about IE and misjudged their risk for IE. A discussion about IE and antibiotic prophylaxis should take place with every ACHD patient during regular clinical contacts to close this knowledge gap.

\section{P-56}

Superior Vena Cava - Right Atrium Junction Flow Pattern Post Transcatheter Patent Foramen Ovale Closure

Yalonetsky S., Tal R., Aharonson D., Gross G., Lorber A.

Adult Congenital Heart Service, Rambam Health Care Campus, Haifa, Israel

Introduction: Percutaneous transcatheter closure of patent foramen ovale (PFO) has been widely used in recent years, mostly for

(a)

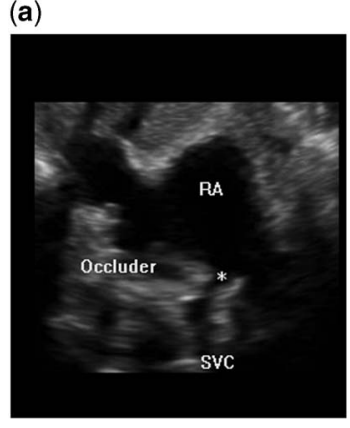

(b)

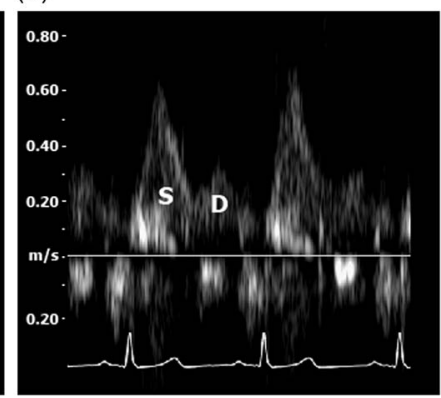

secondary prevention of cryptogenic stroke. A report of superior vena cava (SVC) obstruction by an atrial septal occluder device has raised our concern regarding blood flow disturbance at the SVCright atrial (RA) junction following percutaneous PFO closure. The aim of this study was to examine RA- SVC junction flow pattern in 21 patients ( 4 male, aged $52.7 \pm 9$ years) who underwent PFO device closure 4-120 months previously, in comparison to 21 age and sex matched controls ( 4 male, aged $51 \pm 8.5$ years) with structurally normal hearts.

Methods: All patients underwent transthoracic echocardiographic assessment of the SVC-RA junction in the subcostal view with the patient in recumbent position. SVC flow velocity was recorded by pulse wave Doppler with the sample volume placed at the entrance of the SVC to the right atrium (RA) as marked by asterix on the Figure, panel A. Five well-defined cardiac cycles recorded during quiet respiration were analyzed for each subject. The systolic and diastolic waveforms ( $\mathrm{S}$ and $\mathrm{D}$ respectively - Figure, panel B) were analyzed for flow velocity and waveform duration. The comparison between two groups was made using paired t-test. P value less then 0.05 was considered significant.

Results: Mean \pm SD values of the obtained haemodynamic parameters are summarized in the table:

\begin{tabular}{lccl}
\hline Parameters & $\begin{array}{l}\text { Post PFO } \\
\text { closure }\end{array}$ & $\begin{array}{l}\text { Control } \\
\text { group }\end{array}$ & $\begin{array}{l}\text { P } \\
\text { value }\end{array}$ \\
\hline $\begin{array}{l}\text { Systolic velocity }(\mathrm{cm} / \mathrm{sec}) \\
\text { Systolic wave duration } \\
(\mathrm{msec})\end{array}$ & $60 \pm 11$ & $64 \pm 17$ & 0.27 \\
$\begin{array}{l}\text { Diastolic velocity (cm/ } \\
\text { sec) }\end{array}$ & $30 \pm 8$ & $422 \pm 67$ & 0.4 \\
$\begin{array}{c}\text { Diastolic wave duration } \\
\text { (msec) }\end{array}$ & $320 \pm 75$ & $35 \pm 9$ & 0.1 \\
\hline
\end{tabular}

Conclusions: There was no significant difference in the haemodynamic parameters between patients who underwent transcatheter PFO closure, and age and sex matched controls. Therefore it seems that transcatheter PFO closure doesn't affect the normal blood flow at the SVC-RA junction. Further investigation using the higher number of patient in comparison with the age matched controls may be warranted.

\section{P-57}

Primary surgical repair of congenital heart defects beyond the age of 60

Chikada M., Ono H., Miyairi T., Nishimaki H., Kitanaka Y., Ohno M., Chiba K., Ro D., Nagata T., Endo H., Sakurai Y., Suzuki H., Kita $S$.

The department of Cardiovascular Surgery, St. Marianna University Hospital. Kawasaki, Japan

Objective: Our country has the longest average life span in the world. The number of newly found out patients with congenital heart defects is increasing by the progress of echocardiography and computed tomography. In this study, we tried to examine the recent primary surgical repair of congenital heart defects beyond the age of 60 .

Methods: Eleven patients with congenital heart defects beyond the age of 60 underwent primary surgical repair for the last decade in our hospital. The median age was 64 years (60 to 78). The operative procedures were as follows: atrial septal defect closure and tricuspid annuloplasty 7 cases, atrial septal defect closure and 
right partial anomalous pulmonary venous return repair one case, left partial anomalous pulmonary venous return repair one case, partial atrioventricular defect repair one case and rapture of valsalve aneurysm one case. The median follow up period was 3.5 years (1 to 10). The change of NYHA classification, BNP level and the number of given drugs related to congenital heart defects were investigated.

Results: There was no surgical death and no hospital death. And there was no late death during follow up period. NYHA classifications before operation were NYHA1 2 cases, NYHA2 7 cases and NYHA3 2 cases. After operation, NYHA classifications improved significantly. And NYHA classifications at follow up were NYHA1 10 cases and NYHA2 one case. Median BNP levels $(\mathrm{ng} / \mathrm{ml})$ before operation was 67 . And the median BNP levels were 117 after operation and 84 at follow up. The median numbers of given drugs related to the congenital heart defects was 1.5 (0-3) before operation, 3 (1-5) after operation and $3(0-4)$ at follow up.

Conclusions: The result of primary surgical repair of congenital heart defects beyond the age of 60 was satisfactory. NYHA classifications were improved by the surgery. But BNP level and the number of given drugs increased after operation.

\section{P-58 \\ Sex and Age Features in Adults with Congenital Heart Disease: Experience from a Tertiary Centre in Ukraine Lebid I., Khanenova V., Stogova O., Rudenko N., Yemets I. Ukrainian Children's Cardiac Center, Kyiv, Ukraine}

Objectives: To determine age peculiarity and risk factors depend on sex in adults with congenital heart disease ACHD presented at our institution.

Methods: Between 01.04.2011 and 31.12.2015, there were 2044 consecutive ACHD (11 patient's groups by nomenclature - more than $40 \mathrm{pts}$ in a group). All patients divided for patients who had a surgical/percutaneous intervention initially and never operated and two groups: Gr1 - ACHD after interventions in adulthood, Gr2 - adults, without any procedures elder 18 years.

Results: Of 2044 ACHD, 1059 (52\%) were male and 985 (48\%) women, median age $26.23 \pm 0.24$ (18-88 years). There was domination patients after surgical/percutaneous intervention $(\mathrm{n}=1295 ; 63.4 \%)$, compare without any intervention $(\mathrm{n}=749$; $36.6 \%)$. Gr2 ( $\mathrm{n}=1625 ; 79.5 \%)$ were prevailing compared Gr.1 $(\mathrm{n}=419 ; 20.5 \%)$. Overall mortality was $0.34 \%(\mathrm{n}=7), 5$ males $(0.47 \%)$ and 2 women $(0.20 \%)$. The majority ACHD $(n=1837)$ were younger than 40 years $(90 \%)$. Female ACHD had more often the risk factors: arrhythmia history $(\mathrm{n}=236$ women, $\mathrm{n}=201$ male, $\mathrm{p}<0.05)$, higher degree of heart failure, by NYHA $>1(\mathrm{n}=453$, $\mathrm{n}=285$ men, $\mathrm{p}<0.05$ ), which require more often of medications $(n=660, n=466, p<0.05)$. Men smoked more often $(n=169$, $\mathrm{n}=29, \mathrm{p}<0.05)$. Obesity, infective endocarditis had no significant differences depend on sex. Woman had prevalence in ASD $(n=286), \operatorname{VSD}(n=169)$, PDA $(n=106)$, men $(n=145$, $\mathrm{n}=129, \mathrm{n}=51$, respectively, $\mathrm{p}<0.05)$. Men had prevalence in congenital aortic valve malformations $(n=318)$ and CoAo $(\mathrm{n}=106)$, women $(\mathrm{n}=107, \mathrm{n}=59$, respectively, $\mathrm{p}<0.05)$. In Gr.1 women more often required cardiac surgery procedures at ASD $(n=90 ; 47 \%)$, and PAPVC $(n=14 ; 52 \%)$, males $(n=30$; $33 \%, \mathrm{n}=8 ; 25 \%$ respectively, $\mathrm{p}<0.05)$. The men in the same group in $\operatorname{ToF}(n=15 ; 32 \%)$, women $(n=3,9 \%)$.

Conclusions: The age peculiarities for ACHD were prevalence patients younger than 40 years old, with no significant gender differences. Woman had significantly more often arrhythmia history, higher degree of heart failure, more medications, prevalence in ASD, VSD, PDA, and more often required surgical/ percutaneous intervention in ASD and PAPVC. Men smoked more often, had significantly prevalence in congenital aortic valve malformations and CoAo, more often required procedures in ToF.

\section{P-59 \\ Frequency of associated congenital heart defects in Down syndrome \\ Hyder S.N., Mukhtar N., Aziz Z. \\ Children hospital EThe Institute of Child health, Lahore. Pakistan}

Patients with Down syndrome are prone to have congenital heart defects. Study was done to find the frequency of congenital heart defects in children with Down's syndrome in Children Hospital Lahore.

Material \& Methods: The descriptive study had directed by the Department of Cardiology in The Children's Hospital and the Institute of Child Health, Lahore, in year 2015. Fifty-eight phenotypically Down syndrome children coming to the cardiology department for echocardiography from birth to 13 years were included in this study. The 2 -dimension echocardiography had been done after detailed history and physical examination.

Results: Congenital heart defects were found in 29 out of 58 patients $(50 \%)$. Among the affected patients, 16 (55.2\%) were males and 13 (44.8\%) females with ratio of 1.2:1. Acyanotic lesions were common (79.31\%) than cyanotic lesion (20.69\%). Among the isolated lesions ventricular septal defect, patent ductus arteriosus and complete atrioventricular defects were the commonest defects $(20.69 \%)$ each, followed by pulmonary atresia $(6.89 \%)$, tetralogies of Fallot, transposition of great arteries and double outlet right ventricle (3.45\%) each. Among the mixed lesions ventricular septal defect with atrial septal defect was most common (6.89\%), followed by Coarctation of aorta patent ductus arteriosus, univentricle with atrial septal defect, and double outlet right ventricle and pulmonary atresia (3.45\%) each.

Conclusion: Heart defects are found in 50\% children with Down syndrome. The commonest in acyanotic lesion are ventricular septal defect, in cyanotic cardiac lesion pulmonary atresia and in case of mix lesion ventricular septal defect with atrial septal defect were found.

Key Words: Down syndrome, Congenital heart disease, Transposition of great arteries, Pulmonary atresia, Tetralogy of Fallot's, Ventricular septal defect.

\section{P-60}

\section{Aortic adult congenital heart disease: younger but worse} patients?

Monteagudo M., Polo L., Gonzalez-Rocafort A., Sanchez R., Rey J., Aroca $A$.

Universitary Hospital La Paz, Madrid, Spain

Introduction: Aortic root pathology is the second more frequent heart disorder in adults with congenital heart disease. Aortic valve abnormalities occur with a wide spectrum of severity and thus require different surgical techniques. These patients have ongoing morbidity and reduced long-term survival compared to patients with acquired aortic valve disease although they are normally younger patients. However most of them have had one or more surgical or percutaneous procedures which make them a group with higher comorbidities and mortality. The aim of this study was to evaluate the outcome after aortic surgery in adults with congenital heart disease. 
Methods: We analyze a total of fifty-four patients at our institution that underwent surgery for congenital aortic disease during a period of 8 years (2008-2016). All past medical history, physical examination, previous surgeries, clinical data, as well as cardiac assessment was obtained. Immediate postoperative management, complications, morbidity and mortality was researched. Patients were followed up after discharged from our institution.

Results: Age ranged from 19 to 70 years with a mean age of 38 . There were 22 women and 32 men. 37 of the 54 patients (68\%) have had one or more previous cardiac surgeries. The mean Euroscore was $10,25 \%$. Regarding risk factors, there were 3 patients with Down syndrome, 2 with Turner syndrome, 1 CATCH-22 syndrome and 3 endocarditis. The overall 30-day mortality was $5,5 \%(n=3)$. Data from surgical techniques includes 2 Konno surgeries, 2 biological aortic prostheses, 25 mechanical prostheses, 3 valve plasty, 3 posterior annular enlargement, 8 Bentall-Bono surgeries, 3 David-Standford and 8 supracoronary aortic replacement and mechanical prostheses. $33 \%$ of the patients had some type of complications. Leading causes of these were reintervention because of bleeding (4), atrial fibrillation (4), pneumonia (3) and 3 patients required a pacemaker due to complete heart block.

Conclusions: Adult patients with congenital heart disease continue to be affected by an increased morbidity and mortality when compared to general population with acquired heart disease. However, the data suggests that thus this greater risk, we can perform surgery in these patients with adequate outcomes, acceptable mortality and survival benefits.

\section{P-61}

\section{Cardiovascular risk in adolescents and adults with} congenital heart disease

Wacker-Gussmann A.(1,2), Thiel R.M.(1), Trappe K.(1), Oberhoffer R.(1,2), Ewert P. (1), Hager A.(1)

Department of Pediatric Cardiology and Congenital Heart Disease, Deutsches Herzzentrum München, Munich, Germany (1); Faculty of Sport and Health Sciences, Institute of Preventive Pediatrics, Munich, Germany (2)

Introduction: There is an increasing number of patients with congenital heart disease reaching older age. They are probable at risk for aquired cardiovascular diseases similar to the general population. We investigated, whether diagnosis of congenital cardiac disease, exposure to cardiovascular risk factors or oxygen saturation at rest influenced the early manifestation of atherosclerosis.

Methods: Patients with congenital heart disease were included in our prospective study. The early manifestation of atherosclerosis was quantified by an increase in pulsewave velocity, augmentation index or central blood pressure; aerobic capacity by cardiopulmonary exercise testing.

Results: In total, 188 patients (89 females, 99 males) were analyzed. Median age was 33 years (range 12-74 years). Patients with congenital heart disease presented only little exposure to cardiovascular risk factors. Hyperuricemia was found in $21 \%$, hypertension in $19 \%$, hypercholesterolemia in $18 \%$, hypertrigyceridemia in $17 \%$, obesity in $17 \%$, smoking in $4 \%$ and diabetes in $3 \%$. None of these risk factors was found in $46 \%$.

Patients with adiposity and hypertriglyceridemia had a significantly reduced peak oxygen uptake. Less exposure to cardiovascular risk factors resulted in a better aerobic capacity. On the other hand peak oxygen uptake was correlated to vascular stiffness measured as pulse wave velocity $(\mathrm{r}=-0.225, \mathrm{p}=0.005)$ and augmentation index $(\mathrm{r}=-0.371, \mathrm{p}<0.001)$.
Only patients with coarctation had best results for their aerobic capacity, despite they are particular at risk for atherosclerosis due to arterial hypertension and elevated central blood pressure.

Conclusions: Patients with congenital heart disease have low cardiovascular risk factors, maybe as a result of an intentional healthy lifestyle.

\section{P-62 \\ Brachial arterio-venous fistula to augment pulmonary artery flow in patients with complex congenital cyanotic heart disease \\ Warriner D., Oliver J., English K., Cullington D. \\ Leeds Teaching Hospitals NHS Trust, Leeds, UK}

Introduction: There are a variety of options to augment pulmonary blood flow in adults with complex, cyanotic, congenital heart disease. One method to augment pulmonary blood flow in is by creation of a peripheral arterio-venous fistula. Theoretically, this is the simplest, lowest risk surgical option to augment pulmonary flow for patients with a pre-existing cavo-pulmonary shunt and who do not have pulmonary hypertension.

Methods: We describe a case series of four patients who regularly attend our congenital unit who have cyanotic congenital heart disease, a pre-existing cavopulmonary shunt and undergone creation of an upper limb arterio-venous fistula (brachio-basilic or brachio-cephalic) within the last 6 years, to augment pulmonary flow. All patients had a prior history of worsening breathlessness, fatigue and cyanosis before their artero-venous fistula. We have summarised the published literature pertaining to arterio-venous fistula creation in relation to augmenting pulmonary blood flow (Table 1).

Table 1. The published literature for arterio-venous fistula in augmenting pulmonary blood flow in complex congenital heart disease.

\begin{tabular}{|c|c|c|c|c|}
\hline Author & Year & $\mathbf{n}=$ & Follow-up & Outcome \\
\hline Mitchell & 1989 & 5 & 2.5 years & Symptoms \\
\hline Magee & 1996 & 11 & 7.4 years & $\begin{array}{l}\text { Haemoglobin } / \mathrm{O}_{2} \\
\text { Saturations }\end{array}$ \\
\hline Hickey & 2010 & 21 & 11 years & Symptoms \\
\hline Quinonez & 2011 & 11 & 2.8 years & $\begin{array}{l}\text { Haemoglobin } / \mathrm{O}_{2} \\
\text { Saturations }\end{array}$ \\
\hline Quarti & 2011 & 6 & 1.3 years & $\begin{array}{c}\text { Haemoglobin/ } \\
\text { Haematocrtit }\end{array}$ \\
\hline Chanana & 2015 & 23 & $\mathrm{~N} / \mathrm{A}$ & $\mathrm{O}_{2}$ Sats \\
\hline
\end{tabular}

Results: Three patients had a sustained symptomatic improvement at mean follow-up of 28 months (range 6 to 72 months). In the patients who had an improvement in symptoms, there was a reduction in haemoglobin of $-17 \mathrm{~g} / \mathrm{L}$ (range $6-27$ ), a mean increase in oxygen saturation of $+11 \%$ (range $4-20$ ) and improvement in NYHA class of 1 (range 1-2). Complications were reported in two patients. One developed ischaemia of the hand due to arterial steal. Another developed decompensated heart failure due to the increase in volume loading of the ventricle. Conclusions: Peripheral AV fistula creation remains a valuable therapeutic intervention for some patients who have complex, cyanotic congenital heart disease, have a pre-existing cavo-pulmonary shunt, have progressive symptoms of breathlessness or worsening cyanosis but who have otherwise limited treatment 
options. Due to its simplicity, the option of creating an arteriovenous fistula is attractive, but it is not without adverse consequences too.

P-63

Atypical presentations of right to left shunting in two individuals with anomalous venous connections Bradley-Watson J. (1), Myerson S. (2), Kelion A. (2), Orchard E. (2) Royal Berkshire Hospital, Reading, UK (1); Department of Cardiology, John Radcliffe Hospital, Oxford, UK (2)

Introduction: We present two cases of patients with clinically significant right-to-left shunting via unusual anomalous venous connections not identified by transthoracic echocardiography preoperatively.

Case 1: A 68-year-old woman with a history of two previous intracerebral abscesses and multiple cortical infarcts was admitted for aortic valve surgery. During an on table TOE, there was opacification of the left atrium following injection into a left antecubital fossa vein. Post-operative cardiac CT demonstrated her left SVC draining into the left atrium with the left upper pulmonary vein draining into her left SVC. The right upper pulmonary vein drained anomalously via the right SVC into the right atrium. Given the risk of further embolic phenomenon, percutaneous occlusion of the Left SVC was performed above the connection to the Left Upper Pulmonary Vein.

Case 2: A 12-year old boy underwent repair of unobstructed supracardiac total anomalous pulmonary venous drainage as a neonate following diagnosis by transthoracic echocardiography. He remained cyanosed throughout childhood and cardiac MRI

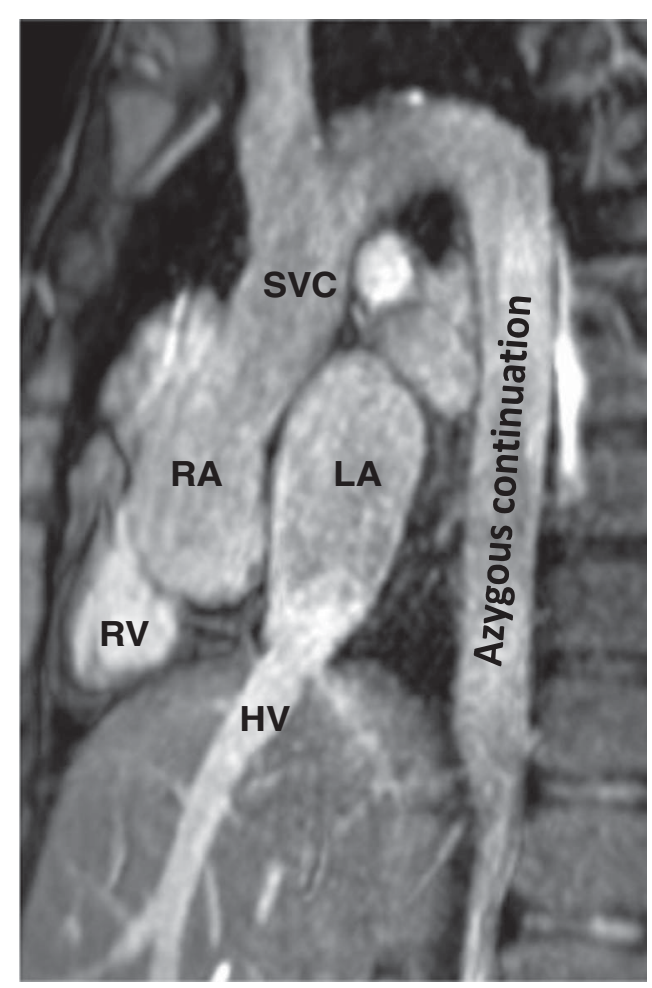

Figure 1.

Cardiac MR image in the sagittal plane showing an azygous continuation and a cyanotic shunt created by hepatic venous drainage directly into the left atrium. ultimately showed an interrupted inferior vena cava with azygous continuation to the SVC but with drainage of the hepatic veins directly to the left atrium, creating the cyanotic shunt.

Conclusions: These cases demonstrate the importance of a comprehensive work-up, including cross-sectional imaging, to fully characterise pulmonary and systemic venous drainage in patients with signs of right-to-left shunting.

\section{P-64}

Prevalence of arterial hypertension in children and adolescents after repair for coarctation: impact of the concomitant presence of complex congenital heart disease Giordano U., Chinali M., Franceschini A., Cafiero G., Turchetta A., Iorio F.S., Giannico S.

Department of Pediatric Cardiology and Cardiac Surgery - Bambino Gesu' Children's Hospital - Rome - Italy

Objective: This study was designed to evaluate the difference in prevalence of arterial hypertension among patients depending on the existence of associated congenital lesions.

Methods: We identified 235 children that were divided into two groups: those with isolated coarctation of the aorta and those with coarctation associated with complex congenital heart disease who had undergone biventricular correction and the data were retrospectively analyzed.

Results: There were 148 with isolated coarctation of the aorta and 87 with complex coarctation of the aorta. Patients were defined as hypertensive if they required antihypertensive treatment and/or when blood pressure was above 95th percentile. Patients with isolated coarctation of the aorta were significantly older than patients with complex coarctation of the aorta $(p<0.001)$ with a markedly higher prevalence of arterial hypertension ( $44 \%$ vs $24 \%$ ) in the isolated coarctation of the aorta group. The difference in the prevalence of hypertension remained significant in an analysis of covariance correcting for differences in age among groups $(p<0.001)$ demonstrating that the risk of developing hypertension for patients with complex coarctation of the aorta was reduced by more than a half as compared to isolated coarctation of the aorta (odds ratio 0.48).

Conclusions: We conclude that in patients after surgery for coarctation of the aorta, the association with complex congenital heart disease results in a significant reduced prevalence of late hypertension. Low systemic flow and pressure before surgery in patients with complex coarctation of the aorta might be associated with a lower rate of arterial hypertension

\section{P-65}

Evaluating the Long Term Effects of the Fontan Procedure on the Hepatic System

Sardar Z. M., Jenkins P.

University of Manchester, UK.

The Fontan procedure is a palliative procedure performed in univentricular patients which is associated with impaired pulmonary function and liver fibrosis. Currently no national guidelines exist for monitoring liver fibrosis in these patients therefore liver function tests (LFTs) and liver ultrasound (US) scans are used. However these tests are largely targeted at viral mediated fibrosis which has a different mechanism of injury to Fontan associated fibrosis. Acoustic radiation force impulse (ARFI) imaging is an elastography technique that may have an important role in assessing liver stiffness in these patients. We aimed to assess and compare the efficacy of LFTs, liver US and ARFI imaging in testing for 
liver fibrosis in Fontan patients. We also aimed to assess any relation between cardiopulmonary exercise test (CPET) variables and ARFI scores.

Data was collected retrospectively from the North West Regional ACHD Centre. Fifteen Fontan patients were identified with LFTs, liver US and ARFI results of which 12 also had CPET results. The sensitivity of LFTs and liver US was calculated and confidence intervals applied. Spearman's correlation coefficient was applied to the ARFI and CPET data.

The sensitivity of LFTs and liver US was found to be 6.6\% (95\% CI $0.3-34 \%)$ and $86 \%(95 \%$ CI $58-97 \%)$ respectively. ARFI identified liver fibrosis in all patients with $33 \%$ at F2, 33\% at F3 and $33 \%$ at $\mathrm{F} 4$. The $\%$ peak VO2 (62.6 vs $46, \mathrm{p}=0.2)$ and $\%$ predicted O2 (101.1 vs 88.9, $\mathrm{p}=0.6$ ) was found to be slightly lower in F2 patients with a higher VE/VO2 (32.5 and 42.0, $\mathrm{p}=0.2)$.

LFTs alone are not a suitable screening test for liver fibrosis in Fontan patients. Liver US provides greater information but is limited to identifying structural changes and cannot accurately quantify the degree of fibrosis. ARFI is a sensitive which requires further research in larger study samples to determine a role in routine hepatic monitoring of Fontan patients. This study has identified a weak association between impaired CPET variables and greater ARFI scores which requires further investigation in order to assess the prospect of another screening test for liver fibrosis.

\section{P-66}

\section{Decreased cardiovascular function late after coarctation} repair

Nederend I. (1,2,3), ten Harkel A.D.J.(1), Blom N.A.(1), de Geus, J. C. $(2,3)$

LUMC Medical centre, Leiden, the Netherlands (1); VU University, Amsterdam, the Netherlands (2); EMGO Institute for health and care research, Amsterdam, the Netherlands (3)

Introduction: Survival after repair of a coarctation of the aorta (CoA) is good but in adulthood late residua including hypertension, restenosis and heart failure are not uncommon. This study aims to evaluate cardiac function, heart rate variability, exercise capacity and physical activity in children late after CoA repair.

Methods: 32 patients (19 boys) after CoA repair and a healthy agematched control group underwent detailed echocardiography, maximal exercise testing and $24 \mathrm{~h}$ holter monitoring. Physical activity including physical education at school, regular biking/ walking behavior and sports participation was mapped using a questionnaire.

Results: Age of the patients and controls ranged from 8-18y (mean $13.3 \pm 2.9$ ). Patients were operated at a mean age of 1 year (range 16 days -5.8 years) and time after intervention was on average $12.3 y$. Left ventricular longitudinal strain and ejection fraction measured by $3 \mathrm{D}$ echocardiography were lower in patients compared to controls $(11.4 \pm 2.7$ vs. $14.6 \pm 3.3 \% \mathrm{p}=0.002$ and $45.9 \pm 6.8$ vs $52.0 \pm 8.2 \% ; p=0.006)$. Analysis of heart rate variability did not show any differences except for $24 \mathrm{~h}$ standard deviation of inter-beat-intervals (corrected for prevailing heart rate); which was lower in patients $(169.6 \pm 40.4)$ vs controls $(193.1 \pm 39.1 \mathrm{p}=0.004)$. Maximal oxygen uptake was lower in patients compared to controls (VO2peak $=40.8 \pm 9.6$ vs $44.6 \pm 7.2 \mathrm{~mL} / \mathrm{kg} / \mathrm{min} \mathrm{p}=0.031)$ as was the anaerobic threshold $(56.8 \pm 10.2$ vs $61.1 \pm 10.2 \%$ of VO2peak $p=0.040)$. Maximal heart rate was not different $(p=0.779)$. Diastolic blood pressure was higher at maximal exercise in patients $(68.4 \pm 14.7 \mathrm{mmHg})$ compared to controls $(58.8 \pm 15.9 \mathrm{mmHg}, \mathrm{p}=0.005)$. Patients were on average physically more active compared to controls but this was not statistically significant $(21.6 \pm 19.8$ vs $13.1 \pm 14.0$ METs/week $\mathrm{p}=0.058$ ). No relationships were found between cardiac function, heart rate variability and exercise capacity.

Conclusions: This study shows that 12 years after correction of a $\mathrm{CoA}$, cardiac function and exercise capacity are already decreased in childhood. However, autonomic function as measured by heart rate variability was not different. Decreased exercise capacity could not be explained by a lower weekly physical activity in patients.

\section{P-67}

Two Siblings With Primary Pulmonary Hypertension And Cleidocranial Dysostosis:Report Of A New

\section{Association}

Aypar E., Ertugrul I., Alehan D., Utine E., Kumbasar U., Demircin $M$.

Hacettepe University Hospital, Ankara, Turkey

Introduction: Cleidocranial dysostozis (CCD) (MIM 119600) is an autosomal dominant skeletal dysplasia characterized by abnormal clavicles, open fontanelles, short stature, dental anomalies and skeletal changes. We report two siblings with CCD and primary pulmonary hypertension $(\mathrm{PH})$. In literature, $\mathrm{PH}$ or other cardiovascular system abnormalities have not been reported previously in association with CCD. Two siblings, a 17 year-old girl and a 14 year-old boy were admitted with dyspnea. In both patients, physical examination showed narrow sloping shoulders that can be apposed at midline due to bilateral absence of clavicles, and variety of skeletal changes associated with CCD (Figure). Microarray analysis of the boy was normal.

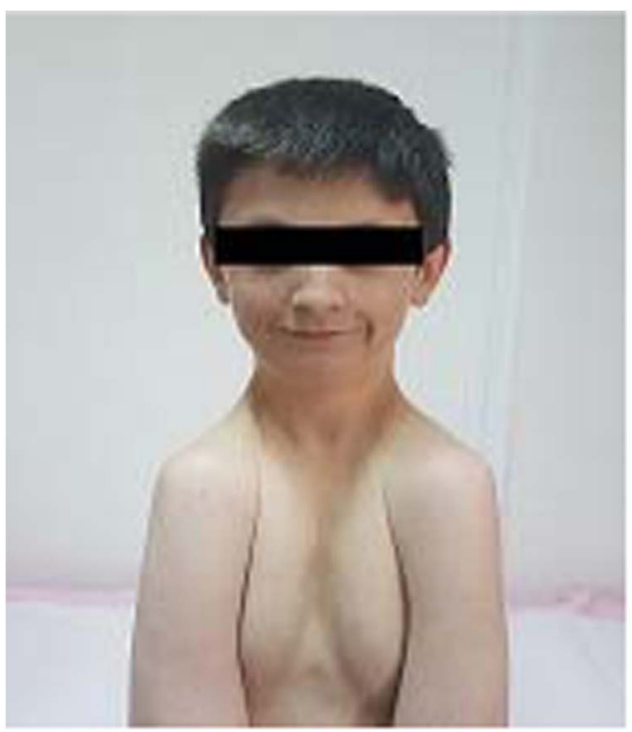

In both patients, echocardiography showed severe $\mathrm{PH}$, right heart failure, massive pericardial effusion (PE). Computed tomographic examination of the lungs were normal. Catheterization confirmed severe $\mathrm{PH}$ and exluded congenital heart disease. In the first patient, systolic pulmonary artery pressure (PAP): $90 \mathrm{mmHg}$, diastolic PAP:28 mmHg, mean PAP:51 mmHg, PCWP:11 mmHg, Qp/ Qs:1, Rp/Rs:66\%. In the second patient systolic PAP:76mmHg, diastolic PAP:37 mmHg, mean PAP:52 mmHg, PCWP:11 $\mathrm{mmHg}, \mathrm{Qp} / \mathrm{Qs}: 1, \mathrm{Rp} / \mathrm{Rs}: 57 \%$. Diagnostic examinations ruled out other causes of $\mathrm{PH}$. Despite specific $\mathrm{PH}$ therapy (bosentan $2 \mathrm{x}$ 
$125 \mathrm{mg}$, PO, inhaled iloprost) and thoracoscopic pericardial window surgery due to chronic massive PE, the first patient was lost during the first year. The second patient was started on bosentan $2 \times 125 \mathrm{mg}$ PO and sildenafil $3 \times 20 \mathrm{mg}$, PO and had pericardial window surgery. On $4^{\text {th }}$ year of follow-up, last echocardiography showed systolic PAP: $85 \mathrm{mmHg}$, mean PAP:50 mmHg. 6-min walk test: $275 \mathrm{~m}$, resting oxgen saturation:95\%. Potts shunt surgery was planned.

Conclusions: In literature, $\mathrm{PH}$ or other cardiovascular system abnormalities have not been reported previously in association with CCD. This is the first report of two siblings with primary $\mathrm{PH}$ and CCD.

\section{P-68}

Regression of Multiple Inoperable Cardiac Rhabdomyomas in a Neonate after Everolimus Treatment

Dogan M.T. (1), Ucar T. (1), Tunc G. (2). Erdeve O. (2), Tutar E. (1), Atasay B. (2), Arsan S. (2). Atalay S. (1)

Department of Pediatric Cardiology, Ankara University Medical School, Ankara, Turkey. (1); Department of Neonatology, Ankara University Medical School, Ankara, Turkey. (2)

Introduction: Rhabdomyomas are the most cardiac tumors diagnosed in neonates and infants. We presented a critically sick neonate with multiple inoperable cardiac rhabdomyomas that infiltrated most of the RVOT region intramural and caused obstructions.

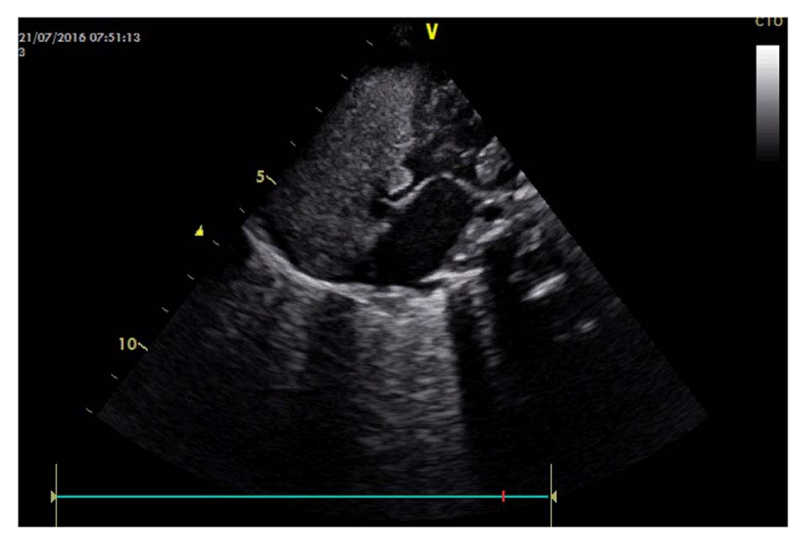

Case Report: The term male newborn was born at 39 weeks of gestation and 3550 gr. He was resuscitated after birth and then he was placed on mechanically ventilation. The postnatal echocardiography $(\mathrm{ECHO})$ revealed multiple rhabdomyomas in the left ventricle, right atrium, and right ventricle with evidence of right ventricle outflow-tract (RVOT) obstruction. The mass located in the RVOT was very large and infiltrated most of the RVOT region intramurally. He was not tolerate weaning from the mechanical ventilation. Postnatal cranial MRI revealed multiple hamartomas along the periventricular zone. Postnatal Renal Ultrasound revealed multiple angiomyolipomas that originate both kidneys. Everolimus (mammalian target of rapamycin (mTOR) inhibitor) therapy was started since the patient was not tolerate extubation and rhabdomyomas were inoperable and causes RVOT obstructions. The patient was tolerated the extubation 8 days following initiation of the medical therapy. Echocardiography at 2 weeks following initiation of everolimus therapy showed regression of the RVOT tumor. Tumor regression continued and at 2 months of age significant reduction in the rhabdomyomas was observed. The RVOT tumor was hardly visible without significant gradient across the RVOT. At the time of writing the child was continued everolimus therapy.

Conclusions: Our patient had a very large mass that infiltrated most of the RVOT region intramurally and caused obstructions. Our patient is a critically sick neonate, resuscitated after birth secondary to multiple inoperable cardiac rhabdomyomas causing right ventricular outflow-tract obstruction, who was successfully treated with everolimus (mammalian target of rapamycin (mTOR) inhibitor). Symptomatic cardiac rhabdomyomas might be managed with everolimus treatment. However further studies needed for effective dose of everolimus and its side effects in children.

\section{P-70}

Evaluation of the myocardial deformation and function by speckle trecking echocardiography and tissue Doppler imaging in infants and preschool children with iron deficiency anemia

Pehlivan B. (1), Cetin I.I.(2), Ozbek N.(3), Gursu H.A.(2), Azak E. (2), Kibar A.E.(2), Orgun A.(2), Surucu M.(2), Pamuk U.(2) Ankara Children's Hematology, Oncology Education and Research Hospital, Pediatrics, Ankara/Turkey (1); Ankara Children's Hematology, Oncology Education and Research Hospital, Pediatric Cardiology, Ankara/Turkey (2); Ankara Children's Hematology, Oncology Education and Research Hospital, Pediatric Hematology, Ankara/Turkey (3)

Objectives: The aim of the study was to assess the myocardial deformation and function by speckle tracking echocardiography (STE) and tissue Doppler imaging (TDI) in infants and preschool children with iron deficiency anemia.

Methods: Fourty patients (mean age 2,5 years, 19 female) diagnosed iron deficiency anemia and twenty healty children (mean age 3,6 years, 10 female) were studied prospectively. The conventional echocardiography, STE and TDI were performed in patients and control group. The left ventricular longitudinal global strain (LVLGS) and strain rate (LVLGSR), left ventricular circumferential global strain (LVCGS) and strain rate (LVCGSR), and right ventricular longitudinal global strain (RVLGS) and strain rate (RVLGSR) were examined by STE. The myocardial velocities $\left(\mathrm{S}_{\mathrm{m}}, \mathrm{E}_{\mathrm{m}}\right.$ and $\left.\mathrm{A}_{\mathrm{m}}\right)$ and time intervals [isovolumic contraction time (ICT), isovolumic relaxation time (IRT) and ejection time (ET)] at interventricular septum (IVS), left ventricular posterior wall, right ventricular lateral wall were examined by TDI.

Results: $\mathrm{S}_{\mathrm{m}}(6,0$ vs. $6,8 \mathrm{~cm} / \mathrm{s})$ and $\mathrm{E}_{\mathrm{m}}(11.0 \mathrm{vs} 13,2 \mathrm{~cm} / \mathrm{s})$ at IVS, $\mathrm{S}_{\mathrm{m}}$ $(5,6$ vs. $7,8 \mathrm{~cm} / \mathrm{s})$ and $\mathrm{E}_{\mathrm{m}}(13,8$ vs. $15.8 \mathrm{~cm} / \mathrm{s})$ at $\mathrm{LV}, \mathrm{E}_{\mathrm{m}}(14,8$ vs. $16,8 \mathrm{~cm} / \mathrm{s})$ at RV, ET (200,2 vs. $235,1 \mathrm{~ms})$ at IVS, ET (198,2 vs. $240,9 \mathrm{~ms})$ at LV, and ET (195,9 vs. $233,8 \mathrm{~ms})$ at RV were statistically significantly lower in patients than controls $(\mathrm{p}<0.05)$. MPI $(0,59$ vs. 0,45$)$ at IVS, IRT $(63,9$ vs. 56,1$)$, and MPI $(0,63$ vs. 0,47$)$ at LV, ICT $(60,9$ vs. 53,4$)$, and MPI $(0,6$ vs. 0,46$)$ at RV were statistically significantly increased in patients than controls. LVLGS (-22,7 vs. $-25,4 \%$ ), LVLGSR (0.43 vs. $\left.0.94 \mathrm{~s}^{-1}\right)$, RVLGSR (0.75 vs. $\left.0.99 \mathrm{~s}^{-1}\right)$, LVCGSR ( 0.76 vs. $\left.1 \mathrm{~s}^{-1}\right)$ were significantly decreased in patients than controls $(\mathrm{p}<0.05$ for LVLGS and LVCGSR, $\mathrm{p}=0.001$ for LVLGSR and RVLGSR).

Conclusions: Iron deficiency anemia results in both systolic and diastolic myocardial dysfunction even in children with normal conventional echocardiographic findings. The STE and TDI are usefull methods to evaluate myocardial function in these patients. 
Table 1 The tissue Doppler findings.

\begin{tabular}{lccc}
\hline & Patients & Controls & P \\
\hline IVSs & $6,03 \pm 0,81$ & $6,88 \pm 1,33$ & $\mathbf{0 , 0 1 9}$ \\
IVSe & $11,03 \pm 1,55$ & $13,20 \pm 1,62$ & $\mathbf{0 , 0 0 0}$ \\
IVSa & $5,84 \pm 1,46$ & $6,76 \pm 2,02$ & 0,080 \\
IVS ICT & $59,63 \pm 1,94$ & $56,50 \pm 8,92$ & 0,418 \\
IVS IRT & $57,33 \pm 9,95$ & $52,60 \pm 8,83$ & 0,073 \\
IVS ET & $200,20 \pm 33,87$ & $235,15 \pm 23,21$ & $\mathbf{0 , 0 0 0}$ \\
IVS MPI & $0,59 \pm 0,95$ & $0,45 \pm 0,05$ & $\mathbf{0 , 0 0 0}$ \\
LVs & $5,61 \pm 1,02$ & $7,81 \pm 1,08$ & $\mathbf{0 , 0 0 0}$ \\
LVe & $13,88 \pm 2,3$ & $15,83 \pm 2,36$ & $\mathbf{0 , 0 0 7}$ \\
LVa & $6,20 \pm 1,49$ & $6,91 \pm 1,41$ & 0,480 \\
LV ICT & $60,48 \pm 14,12$ & $57,90 \pm 9,62$ & 0,654 \\
LV IRT & $63,90 \pm 11,12$ & $56,10 \pm 9,33$ & $\mathbf{0 , 0 0 6}$ \\
LV ET & $198,28 \pm 29,7$ & $240,90 \pm 25,44$ & $\mathbf{0 , 0 0 0}$ \\
LV MPI & $0,63 \pm 0,11$ & $0,47 \pm 0,67$ & $\mathbf{0 , 0 0 0}$ \\
RVs & $9,22 \pm 1,9$ & $10,02 \pm 2,48$ & 0,252 \\
RVe & $14,8 \pm 3,13$ & $16,82 \pm 2,42$ & $\mathbf{0 , 0 0 6}$ \\
RVa & $8,65 \pm 2,62$ & $7,92 \pm 2,73$ & 0,367 \\
RV ICT & $60,98 \pm 12,59$ & $53,40 \pm 9,04$ & 0,032 \\
RV IRT & $59,3 \pm 10,29$ & $55,95 \pm 6,86$ & 0,299 \\
RV ET & $195,93 \pm 30,62$ & $233,80 \pm 24,98$ & $\mathbf{0 , 0 0 0}$ \\
RVMPI & $0,61+0,1$ & $0,46+-0,06$ & $\mathbf{0 , 0 0 0}$ \\
\hline
\end{tabular}

Table 2. The speckle tracking echocardiographic findings.

\begin{tabular}{lrrr}
\hline & \multicolumn{1}{c}{ Patients } & \multicolumn{1}{c}{ Controls } & P \\
\hline LVLGS & $22,73 \pm 4,46$ & $25,45 \pm 3,92$ & $\mathbf{0 , 0 4 5}$ \\
LVLGSR & $0,43 \pm 0,38$ & $0,94 \pm 0,35$ & $\mathbf{0 , 0 0 0}$ \\
RVLGS & $31,20 \pm 5,45$ & $26,75 \pm 4,85$ & 0,005 \\
RVLGSR & $0,75 \pm 0,48$ & $0,99 \pm 0,32$ & $\mathbf{0 , 0 0 0}$ \\
LVCGS & $28,50 \pm 7,42$ & $28,90 \pm 5,29$ & 0,660 \\
LVCGSR & $0,76 \pm 0,43$ & $1,04 \pm 0,35$ & $\mathbf{0 , 0 1 4}$ \\
\hline
\end{tabular}

\section{P-71}

Evaluation of the aortic leaflet morphology, aortic dimentions, elastic properties of the ascending aorta and left ventricular diastolic functions in children with wellfunctioning bicuspid aortic valve

Ekici F.(1), Uslu D.(1), Ŏğuz B.(2), Kardelen F.(1)

Akdeniz University Medical School, Department of Pediatric Cardiology (1); Biostatistic(2) Antalya, Turkey

Objective: To determine the relationship between aortic leaflet morphology and elastic properties and diastolic function in children with well functioning bicuspid aortic valve (BAV).

Methos: Aortic leaflet morphology, aortic dimension, elastic properties and diastolic functions were evaluated in 55 children with well-functioning BAV (mean ages; 9,06 years). Patients had a history of prior intervention, associated syndrome, congenital heart defects, moderate /severe aortic regurgitation or stenosis were excluded.

Patient were classified as two groups according to the aortic leaflet morphology; Group 1 consisted of patients had fusion between the right and noncoronary cusps (R-N), Group 2 consisted of patients had fusion between the right and left coronary cusp (R-L). Diastolic functions of the left ventricle were evaluated by conventional pulse wave Doppler and Tissue Doppler echocardiography. Aortic root (AR) and proxymal ascending aorta (AA) dimensions were expressed by z scores.

Dispensibility Index (DI) Strain and Strain Index (SI) of the ascending aorta were calculated according to previously reported formules.

Results: There was 25 patients in Grup 1 and 30 patients in Group 2. AA dilatation $(z$ score $>2$ or $=2)$ was common and occured in 26 of 55 cases (48\%). 21 patient had a mild AA dilatation ( $\mathrm{z}$ score $>2$ and $<4$ ) and 5 cases had a moderate AA dilatation $(z$ score $>4)$. AR dilatation ( $z$ score $>$ or $=2$ ) was seen only 3 cases and more common in group 2 than patient in group $1(\mathrm{p}<0,05)$. Patient in group 1 had more distensible and less stiff AA than patient in group 2. (DI: $11+4,4$ versus $8,0+3,4, \mathrm{p}=0007$, SI: $2,6+1,6$ versus $3,6+1,9, p=0,01)$.

Patient with dilated AA had lower DI and strain values than patients with normal sized AA. (Strain; 15,8 +8 versus 20,9+7,7) $(\mathrm{p}<0,05$ and $\mathrm{p}<0,05)$.

There were no differences in the myocardial velocities with regard to aortic leaflet morphologies. Elastic properties of AA were not related to myocardial diastolic functions in children with BAV

Conclusion: The aortic leaflet morphologies affect the elastic properties of AA, but not related to myocardial diastolic function in children with BAV.

\section{P-72}

Multimodality assessment of left ventricular mass in patients with congenital heart disease: What are the differences?

Michel M., Shabanah W., Körperich H., Kelter-Klöpping A., Laser K.T. Heart and Diabetes Center North Rhine-Westphalia, Bad Oeynhausen, Germany

Aims: Non-invasive assessment of left ventricular (LV) mass is important in the evaluation of patients with congenital heart disease. This study was designed to test the accuracy and reproducibility of real-time 3-dimensional echocardiography (RT3DE) in measuring LV mass as compared with the current gold standard of cardiac magnetic resonance imaging (MRI).

Methods: Subjects consecutively underwent RT3DE using a commercially available Toshiba Artida (SSH-880.CV) and MRI on a Philips 3.0T TX Achieva (R3.2) MRI scanner. RT3DE and MRI LV mass assessment was performed with two different analytical 4D-LV-analysis software packages each (RT3DE: Tomtec/ Toshiba, MRI: Philips IntelliSpace Portal, Release v7.0.1/ homemade HDZ MR-Tools software package).

Results: 20 patients (mean age $\pm \mathrm{SD}=17.4 \pm 15.0 \mathrm{yrs}$, range $=0.1$ to $72.9 \mathrm{yrs})$ and 20 controls $(13.0 \pm 3.3 \mathrm{yrs}, 7.1$ to $19.4 \mathrm{yrs})$ were included. Mean RT3DE derived LV mass was $98.2 \pm 38.3 \mathrm{~g}$ (Tomtec) and $94.7 \pm 38.4 \mathrm{~g}$ (Toshiba), respectively. Mean MRI derived LV mass was $89.1 \pm 38.0 \mathrm{~g}$ (Philips) and $90.7 \pm 39.9 \mathrm{~g}$ (HDZ), respectively. MRI derived LV mass was not significantly different (MRI Philips vs. MRI HDZ $\mathrm{p}=0.23$, Student's t-test). RT3DE derived LV mass was significantly overestimated compared with MRI derived LV mass (RT3DE Tomtec vs. MRI Philips $p=0.000$, RT3DE Tomtec vs. MRI HDZ $p=0.000$, RT3DE Toshiba vs. MRI Philips $\mathrm{p}=0.001$, RT3DE Toshiba vs. MRI HDZ $p=0.007$ ), and RT3DE derived LV mass (Toshiba) was significantly overestimated compared with RT3DE derived LV mass (Tomtec) $(p=0.009)$. LV mass assessment applying RT3DE and MRI was accurate and reproducible (intra-class correlation coefficients $>0.96$ ), Bland-Altman analysis demonstrated most narrow limits of agreement for intraoberserver and interobserver variability for MRI HDZ (MRI HDZ intraobserver: mean difference was $1.8 \%$, 2SD limits of agreement (LOA) 
$12.5 \%$ to $-8.8 \%$; MRI HDZ interobserver: mean difference $9.7 \%$, 2SD LOA $28.9 \%$ to $-9.4 \%$ ) and for RT3DE Tomtec (RT3DE Tomtec intraobserver: mean difference $-0.8 \%, 2$ SD LOA $10.1 \%$ to $-11.7 \%$; RT3DE Tomtec interobserver: mean difference 5.6\%, 2SD LOA18.1\% to $-6.9 \%$ ).

Conclusion: Although sophisticated RT3DE analysis software was used with satisfactory results, it cannot completely compensate for minor contrast which complicates border detection and leads to overestimation of LV mass. Old established hardware should be further improved in order to generate more competitive data compared to MRI.

\section{P-73}

Can cardiac recovery in chronic myocarditis be influenced by training during LVAD support?

Racolta A. (1), Milting H.(2), Lauenroth V.(3), Horst J.P.(1),

Klusmeier E.(1), Vcasna R.(1), Kantzis M.(1), Sandica E.(1), Kececioglu D.(1), Laser K.T.(1)

Herz-und Diabeteszentrum NRW, Center for Congenital Heart Defects, Bad Oeynhausen, Germany (1); Herz-und Diabeteszentrum NRW, Klessmann-Institut(EHKI), Bad Oeynhausen, Germany(2); Herz-und Diabeteszentrum NRW, VAD Koordination, Bad Oeynhausen, Germany(3)

Introduction: (or Basis or Objectives): 6 months old patient with massively impaired left ventricular function: EF 35\% [ejection fraction] LVEDD [left ventricular enddiastolic diameter] $44 \mathrm{~mm}$ $-2 \mathrm{SD}$, VTI [velocity time integral] aortal $<\mathrm{Pc}_{\mathrm{c}} 3$, Speckle-tracking [ST] longitudinal strain [GLS] 4Ch -6,5\%, 2Ch -6,4\%). Biopsy revealed histological diagnosis of dilative cardiomyopathy, DD chronical myocarditis. Genetic testing was performed for known cardiomyopathy encoding genes. LVAD- Berlin Heart $(\mathrm{BH}$, left ventricular assist device) implantation 2 days after admission. A weaning plan including cardiac muscle training was carried out over 14 weeks.

\begin{tabular}{llll}
\hline $\begin{array}{l}\text { Measurements } \\
\text { phase D }\end{array}$ & $\begin{array}{l}\text { Pump } \\
\text { on }\end{array}$ & $\begin{array}{l}\text { Pump off } \\
\mathbf{1 5} \text { min }\end{array}$ & $\begin{array}{l}\text { Pump off } \\
\mathbf{3 0 ~ m i n}\end{array}$ \\
\hline Heart Rate/min & 100 & 118 & 118 \\
VTI pulmonal (cm) & 15 & 16 & 16 \\
VTI aortal (cm) & 16 & 20 & 20 \\
LVEDV (ml/m2) & 79 & & 76 \\
LV-SV (ml) & 18 & & 15 \\
EF \% & 44 & & 50 \\
GLS4CH \% & -11 & -10 & -12 \\
GLS2CH \% & -10 & -10 & -15 \\
\hline
\end{tabular}

Methods: Follow up using clinical and echocardiographic data (ST, VTI and 3D-echocardiographic enddiastolic -[LVEDV], endsystolic-[LV-ESV], stroke-volumes [LV-SV] and EF). Cardiac muscle training for 2 hours/day with reduction of the $\mathrm{BH}$ to $55 /$ min for 3 weeks (phase A), extension to 4 hours/day for 2 weeks (phase B) and complete reduction to $55 / \mathrm{min}$ for 9 weeks (phase C). In the phase D: pump stop 30 minutes once per week under additional heparin bolus.

Results: The first reduction of pump resulted in LV dilatation and increasing mitral incompetence. The training (phase A) was carried on and revealed an improvement of ST from -8\% (GLS $2 \mathrm{C}$ ) and $-10 \%$ (GLS 4C) to $-13 \%$ and respectively $-11 \%$. The VTI's were initially between Pc. 3-10 and increased to Pc 10. Phase B showed at the beginning an increase of the LVEDV from $55 \mathrm{ml} / \mathrm{m}^{2}$ to $57 \mathrm{ml} / \mathrm{m}^{2}$. Afterwards it remained constant with $59 \mathrm{ml} / \mathrm{m}^{2}$ as well as VTI (10.Pc) and EF (35\%). During pump stop, we assessed an increase of the VTI's and EF and (last test in the attached Table). Final hemodynamic testing in the cath. lab: BH50/min: CI (cardiac index) $4,1 \mathrm{ml} / \mathrm{m}^{2} / \mathrm{min}$, CVS (central venous saturation) 60\%, LVEDP (left ventricular enddiastolic pressure) $8 \mathrm{mmHg}$, Off $\mathrm{BH} 30$ minutes: CI $3,6 \mathrm{ml} / \mathrm{m}^{2} / \mathrm{min}$, CVS $58 \%$, LVEDP $10 \mathrm{mmHg}$. The explantation of the device was successful.

Conclusions: Training of impaired cardiac muscle under LVAD in children with not acute myocarditis may be a possibility to prevent the direct way as bridge to transplant in selected patients. New echocardiographic tools may help assess improvement of function with impact on prognosis.

\section{P-74}

Pediatric Friedreich's Ataxia: new echocardiographic data for the follow-up

Cesar S. (1), Ganc G. (2), O’Callaghan M. (2), Sarquella-

Brugada G. (1)

Pediatric Cardiology department, Hospital Sant Joan de Déu, Barcelona, Spain, Pediatric Neurology department, Hospital Sant Joan de Déu, Barcelona, Spain

Introduction: The main cause of mortality in Friedreich's Ataxia is cardiac disease. Strict follow-up with clinical and echocardiographic data is essential in this group of patients. Latest data in some published adult series showed the role of relative wall thickness (RWT) and its relation with an early detection of concentric remodeling despite of a normal left ventricular mass index (LVMI). We present a retrospective description of echocardiographic measurements and their relation with demographic and genetic data.

Methods: We analysed retrospectively a cohort of 11 pediatric patients diagnosed with Fridreich's Ataxia. Demographic, genetics (GAA triplets), clinical and echocardiographic data were described. Echocardiographic data included left ventricle diameters and RWT (M-mode), systolic function (M-mode and Simpson), and

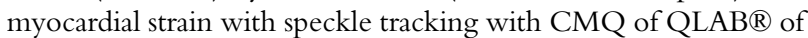
Philips (circumferential and longitudinal analysis). Qualitative and quantitative variables were analysed, establishing a significance level when $p<0.05$. The adjustment of the linear regression was with the R2 criteria. Statistical analysis performed with StatCrunchTM.

Results: A total of 11 patient were analysed: 8-19yo, mean age $14.3 \pm 3.55,72 \%$ females. Nine of them had symmetric hypertrophic cardiomyopathy, increased LVMI, and preserved left ventricle ejection fraction (LVEF,\%) and 2 had normal values of LVMI and borderline values for interventricular and posterior wall thickness. RWT value was $\geq 0.42$ (mean $0.61 \pm 0.12$ ), with no difference by gender $(p<0.05)$, so despite of a normal LVMI all of them showed a concentric remodeling pattern. We detected a correlation between intervertricular septum thickness and GAA expansion, not affected by gender or age, with a significant positive correlation $(\mathrm{P}=0.02 ; \mathrm{R} 2=0.37)$, but that observation was not significant for RWT values. Myocardial strain was analysed and we obtained decreased longitudinal peak-systolic values despite of a preserved LVEF.

Conclusions: (1)Concentric remodeling was observed in all our pediatric patients because of compatible RWT values, despite of normal LVMI. (2)GAA expansion showed a positive correlation with the interventricular septum thickness, but no with RWT. (3) Global longitudinal myocardial strain showed decreased values despite of a preserved ejection fraction. 


\section{P-75}

Evaluation of diffuse myocardial fibrosis by T1 mapping in children and adolescents with primary cardiomyopathies results from the RIKADA study

Al-Wakeel-Marquard N. (1,2), Degener F. (1,2,3), Kelm M. (1), Schmitt B. (1,2), Kühne T. (1,2,3), Klaassen S. $(2,3,4)$, Berger F. $(1,2,3)$, Messroghli D. $(2,5)$

Deutsches Herzzentrum Berlin, Klinik für Angeborene Herzfehler Kinderkardiologie, Berlin, Germany (1); DZHK (German Centre for Cardiovascular Research), partner site Berlin, Berlin, Germany (2); Charité - Universitätsmedizin Berlin, Klinik für Pädiatrie mit Schwerpunkt Kardiologie, Berlin, Germany (3); Experimental and Clinical Research Center, a joint cooperation between the Charité Medical Faculty and the Max-Delbrück-Center for Molecular Medicine, Berlin, Germany (4); Deutsches Herzzentrum Berlin, Klinik für Innere Medizin - Kardiologie, Berlin, Germany (5).

Introduction: Diffuse myocardial fibrosis is a common finding and associated with adverse outcome in adult cardiomyopathy (CM), yet data on its role in pediatric patients are sparse. Cardiovascular magnetic resonance (CMR) T1 mapping is a non-invasive tool to quantify diffuse myocardial fibrosis as reflected by extracellular volume (ECV). This study aimed to assess markers of diffuse myocardial fibrosis and their correlation with ventricular function in children and adolescents with primary CM.

Methods: In total, 13 prospectively enrolled patients (mean age $11.6 \pm 5.0$ years) with primary $\mathrm{CM}$ (dilated $\mathrm{CM}, \mathrm{n}=4$; hypertrophic $\mathrm{CM}, \mathrm{n}=4$; left ventricular non-compaction $\mathrm{CM}, \mathrm{n}=5$ ) were compared to 16 healthy controls (mean age $25.1 \pm 2.7$ years). T1 maps were generated with Modified Look-Locker Inversion recovery (MOLLI) T1 mapping in a midventricular short axis plane before and 15 minutes after bolus application of Gd-DOTA. ECV was calculated from T1 values of the left ventricular (LV) circumference, T1 from LV blood pool, and hematocrit. In the patient group, T1 and ECV were compared to volumetric CMR data.

Results: Median (interquartile range) native T1 in patients and controls was 1028 (44) ms and 995 (40) ms, respectively $(\mathrm{p}=0.009)$. No significant difference in ECV between patient and control group was found $(27[7] \%$ and $26[5] \%$, respectively; $\mathrm{p}=0.5$ ). In patients, native $\mathrm{T} 1$ correlated significantly with LVend-diastolic volume (EDV) indexed to body surface area $(r=0.8$, $\mathrm{p}=0.003$ ). Correlations between ECV and parameters of ventricular function were not observed.

Conclusions: Our results demonstrate a tendency towards increased diffuse myocardial fibrosis associated with LV-EDV in young patients with CM. However, the overall non-significant elevation of ECV may indicate an only minor role in the course of pediatric $\mathrm{CM}$. Further longitudinal studies are necessary to investigate the diagnostic and prognostic value of non-invasive ECV measurements in children and adolescents with primary CM.

\section{P-76}

Long term course of bicuspid aortic valve in patients with and without associated cardiac malformations

Lamy J., Ecarnot F., Tchaoussoff C., Hascoet S., Bernard Y. University Hospital Jean Minjoz, Besancon, France

Introduction: Evolution of bicuspid aortic valve (BAV) in patients with associated cardiac malformations remains largely unknown. We compared the course of isolated BAV with that of BAV associated with other cardiac malformations in a large cohort of patients. Methods: Single-centre, retrospective cohort study. All patients in whom BAV was diagnosed in a large regional referral university hospital between 01/1989 and 12/2015, and who underwent at least 2 echocardiographic examinations in our centre were eligible. Two groups were defined, namely "isolated BAV", and "associated BAV" (e.g. BAV associated with coarctation, other simple or complex cardiac malformations).

Results: 280 patients were included, $61.8 \%$ with isolated BAV and $38.2 \%$ with associated BAV, who were mainly children. Mean duration of follow-up (FU) was 9 years (range 0-39 years). Patients with associated BAV were younger and had less complications at diagnosis ( $\mathrm{p}<0.0001$ for both).

About half the population developed a new complication of BAV during $F U$, with no difference between groups $(p=0845)$, but patients with associated BAV had less worsening of their initial complication ( $\mathrm{p}<0.033)$. At the end of FU, $51.1 \%$ of the whole population had an aortic dilatation. More than half of the patients underwent cardiac surgery related to BAV in $50 \%$ of cases. Patients with associated BAV underwent more cardiac surgery $(p<0.0001)$, mainly for associated cardiac malformations and at a younger age $(p<0.0001)$.

Younger age at diagnosis (odds ratio, $\mathrm{OR}=0.63 ; 95 \% \mathrm{CI}=$ [0.5-0.9] per quartile) was significantly associated with the risk of complications during $\mathrm{FU}$, and the presence of a complication at diagnosis $(\mathrm{OR}=18,15 ; 95 \% \mathrm{CI}=[4.2-78.9])$ was associated with the occurrence of cardiac surgery related to BAV during FU. Associated BAV $(\mathrm{OR}=0.01 ; 95 \% \mathrm{CI}=[0.003-0.7])$ had a lower risk of surgery related to BAV. Cumulative incidence of moderate to severe aortic stenosis or regurgitation was $23.9 \%$ (95\% CI = [19-29.9]) and $45.1 \%(95 \% \mathrm{CI}=[37.9-53.1])$ respectively at 20 and 40 years of age, with no difference between both groups. Conclusion: In our study, associated BAV was not predictive of a higher risk of complications during FU.

P-77

Left atrial pediatric reference volumes using Real-time 3DEchocardiography

Linden K. (1), Goldschmidt F.(1), Winkler C.(1), Laser K.T.(2), DallaPozza R.(3), Herberg U.(1)

Universitätsklinikum Bonn, Bonn, Germany (1); Herz- und

Diabeteszentrum NRW, Bad Oeynhausen, Germany (2); Ludwig-

Maximilian Universität München, München, Germany (3)

Objectives: Left atrium (LA) size and function play a critical role in left ventricular filling. The assessment of LA size, reservoir and conduit function have been shown to be of prognostic value regarding various heart diseases. Real-time 3D echocardiography (RT3DE) allows the evaluation of left atrial volume changes throughout the cardiac cycle without geometric assumptions. However, pediatric reference values are missing. Aim of our study was to promote the understanding of the dynamic LA function and to create normal values for the pediatric population by RT3DE.

Methods: In a multicenter prospective study, in 218 healthy children and adolescents aged 1.5-256 months, RT3DE of the LA was performed (ie 33, Philips; Vivid 7, GE). Data were analyzed using 4D LV Function by TomTec, assessing maximal and minimal LA volume (Vmax, Vmin). The volume and time period prior to the active contraction of the LA (VpreA) was determined by adapting the visual rebound of the mitral valve as well as the $\mathrm{p}$-wave in ECG. Additionally, the cyclic volume change [CVC $(\mathrm{ml})=\mathrm{Vmax}-\mathrm{Vmin}], \quad$ active $-\quad[\mathrm{AE}(\%)=(\mathrm{VpreA}-\mathrm{Vmin}) / \mathrm{CVC})]$ and passive emptying $[\mathrm{PE}(\%)=(\mathrm{Vmax}-\mathrm{VpreA}) / \mathrm{CVC})]$, atrial active emptying fraction $[\mathrm{AEF}(\%)=(\mathrm{VpreA}-\mathrm{Vmin}) / \mathrm{VpreA})$, atrial passive emptying fraction $[\mathrm{PEF}(\%)=(\mathrm{Vmax}-\mathrm{VpreA}) / \mathrm{Vmax}]$ and the duration of atrial systole and diastole, as well as the active and 
passive share in atrial systole were calculated (Fig 1a). Inter- and intraobserver variability was assessed for all parameters. Percentiles were created using the LMS method.

Results: Figure 1b exemplarily shows percentiles for VpreA. Throughout measurements Vmax and VpreA increased with ageprogression and decreasing heart rate. AE and AEF increased with age, whilst PE and PEF declined. Furthermore, length of atrial systole increased with age progression, concomitant with shortening of atrial diastole duration. (a)

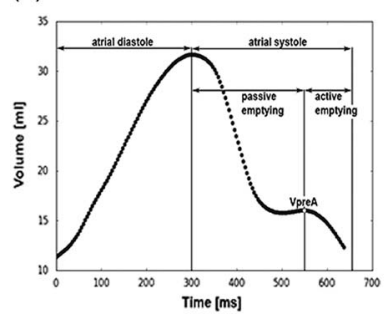

(b)

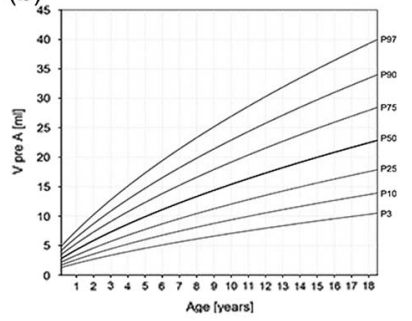

Conclusion: This study is the first to provide comprehensive reference data for LA volume and function on a large pediatric population using RT3DE. RT3DE is a promising non-invasive method to assess LA function in daily clinical use.

This study was funded by Fördergemeinschaft Deutsche Kinderherzzentren.

\section{P-78}

A case study: Description of the LV growth process in children with aortic stenosis based on 4D-Realtime Echocardiography

Wolf A., Winkler C., Herberg U., Breuer J., Linden K.

University of Bonn, Germany

Introduction: For the examination of left ventricular (LV) volume 4D-Realtime (4DRT) Echocardiography has been established as a safe and accurate method. In current literature, a serial long-term follow up study about the growth of the LV of children with congenital heart disease assessed by 4DRT Echo is missing. Furthermore, the current scientific approach evaluates fixed parameters such as EDV and ESV, but neglects temporal changes as well as the spatial structure of the LV. In our case study, we describe the LV growth of patients with aortic stenosis based on

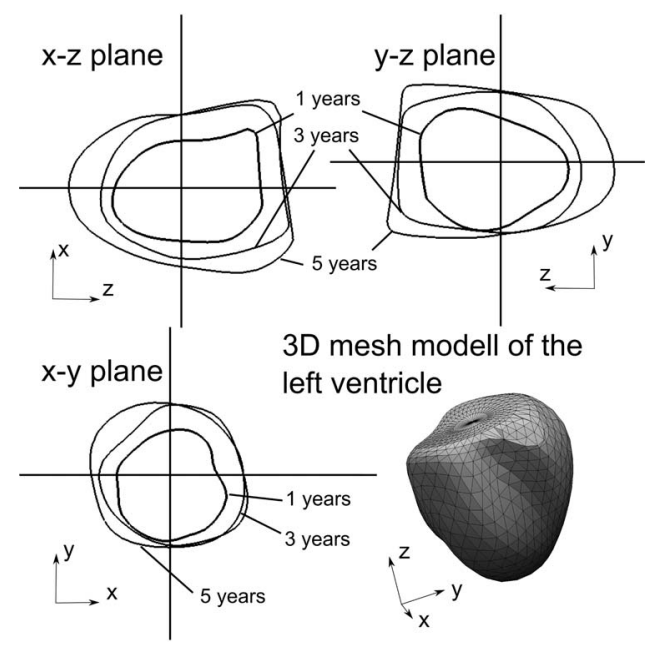

serial measurements. This case report illustrates a new approach for the assessment of data obtained by 4DRT, which will help to define the anatomy and evaluate the heart function.

Methods: 4DRT datasets of one patient with aortic stenosis were serially obtained using ie33 (Philips; X7-2 matrix transducer), starting from the first postnatal preinterventional echo until 7 years. Data were analysed using ImageArena 4.6 (TomTec) and the including tool 4D LV-Analysis 3.1. Surface models of the dynamic left ventricular volume changes were exported and used for a mathematical analysis. To visualize and assess the changes of the 3D structure of the surface models, the open source software ParaView was chosen.

Results: To illustrate serial changes of LV function and size, we compared the surface models for the enddiastolic volume of one patient for three ages (1,3 and 5 years) by plotting the contours as multiview orthographic projections. The figure shows $2 \mathrm{D}$ plots of different section planes and the 3D surface model for the first measurement. The contours reveal how the LV size changed over the time. Furthermore, we can detect regions of interest which show a very rapid or slow change. If compared to reference data, we can confer the diseased left ventricle with given reference mesh models.

Conclusions: In this study, we illustrated the complex changes of ventricular geometry in children with critical aortic stenosis during follow up. The derived knowledge is crucial to examine and understand the pathological growth process. This may result in a prospective estimation of longitudinal left ventricular growth and function and therefore therapeutic decision making.

\section{P-79}

Long-term fate of coronary arteries in Kawasaki disease Kaldararova M. (1), Kantorova A. (1), Vrsanska V. (1), Mikulas J. (2), Masura J. (1)

National Institute of Cardiovascular Diseases - Children's Cardiac Centre, Bratislava, Slovakia (1); National Institute of Cardiovascular Disease - Dpt. of Diagnostic and Interventional Radiology, Bratislava, Slovakia (2)

Introduction: Late or inadequate acute Kawasaki disease treatment may lead to coronary artery (CA) dilatation. Giant CA aneurysms are recognized to be irreversible and may cause life-threatening complications. Aim of the study was to analyse long-term morphological and functional outcome of patients with CA affection after Kawasaki disease.

Patients and methods: Performed was a retrospective analysis of 26 patients $(20 \mathrm{M} / 6 \mathrm{~F})$ followed at our institution between 1996-2016. Median age at diagnosis was 2.8 years (3 months 11.6 years); median follow-up 8 years ( 5 months -20 years).

Defined were baseline and late morphological CA characteristics (by echocardiography and CT-/coronarography), presence of myocardial ischaemia and/or ventricular dysfunction. According to baseline findings were patients divided in groups: 1 . with mild CA dilatation $(<5 \mathrm{~mm}$, without aneurysms), 2. small CA aneurysms (5-8 mm); 3. giant CA aneurysms ( $>8 \mathrm{~mm})$.

Results: Acute phase CA affection was as follows: both CA dilated in $13(50 \%)$, only left CA dilatation in $12(46.2 \%)$ and only right CA dilatation in 1 patient $(7.8 \%)$.

Group 1: 16 patients (61.6\%); in all patients mild CA dilatation normalized within 3 months.

Group 2: 5 patients (19.2\%); in all patients small CA aneurysms were persistent during follow-up. In $2(20 \%)$ late calcifications at aneurysm-site were found, though without significant stenosis; further 3 patients were without progression. No myocardial ischaemia or ventricular dysfunction were present. 
Group 3: 5 patients (19.2\%); giant aneurysms of both CA were present, though right CA was more severely affected (multiple aneurysms/stenosis). Despite anticoagulation therapy late complications (at median 6 years after acute phase) were present in 4 patients (80\%): in 1 progressive CA dilatation; in 3 complete right CA occlusion with collateral circulation, while in 1 of them later additional left anterior descending CA thrombosis occurred. In the latter 3 patients also myocardial ischaemia and/or ventricular dysfunction developed. One late death (20\%) occurred due to severe ventricular dysfunction.

Conclusions: After Kawasaki disease giant CA aneurysms have extremely high-risk potential for occlusion at any time during follow-up; this can lead to myocardial ischaemia, ventricular dysfunction or even death. Close clinical monitoring and repeated CA morphological and functional evaluation is therefor mandatory.

\section{P-80 \\ Complete correction of truncus arteriosus communis with or without conduit: 10 years experience \\ Kantorova A., Kaldararova M., Valentik P., Kovac M., Nosal M. National Institute of Cardiovascular Diseases, Children's Cardiac Centre, Bratislava, Slovakia}

Introduction: Truncus arteriosus communis (TAC) is corrected either by conduit implantation or by direct anastomosis between right ventricle (RV) and pulmonary artery (PA). The aim of our single-centre whole-country study was to evaluate long-term results of our patients after complete correction of TAC.

Methods: Between 2002-2015, 33 patients underwent twoventricle repair at our center. At the time of surgery median age was 16 days (5-447); median weight $3.1 \mathrm{~kg}$ (2.4-9.7). Thirty patients were followed up for median 6.7 years $(1-14$ years).

Postoperative clinical and echocardiographic data within last 10 years was analysed retrospectively comparing patients of Group I (conduit) $(\mathrm{n}=7)$ versus Group II (non-conduit) $(\mathrm{n}=26)$.

Results: Occurrence of moderate-to-severe proximal stenosis of RV-PA anastomosis/ conduit was not significantly different between the groups: $28.6 \%$ vs $19.2 \%(p=0.59)$. Proximal stenosis of RV-PA anastomosis did not require re-intervention in our patients; conduit stenosis required catheter dilation in one patient and conduit replacement in 2 patients.

Distal (pulmonary branch) stenosis was observed more frequently $(n=9)$, but without statistically significant difference between the groups: $42.8 \%$ vs $23.1 \%(p=0.29)$. All the patients underwent catheter balloon dilation ( 3 patients in Group I and 6 in Group II), followed by reoperation in 3 patients.

In our patients severe pulmonary regurgitation was statistically less frequent in Group I than in Group II: $14.3 \%$ vs $69.2 \%(p=0.008)$. Due to severe RV dilation 3 patients (Group II) required late conduit implantation.

Difference in freedom from any re-intervention between Group I and Group II was statistically not significant at 5 th year $65.4 \%$ vs $77.8 \%(p=0.20)$; was significant at 10 th year $42.8 \%$ vs $63.1 \%$ $(\mathrm{p}=0.01)$.

Medians of time of the first re-intervention were: 2.5 (Group I) vs 5.5 months (Group II) $(p=0.67)$ after complete correction.

Conclusion: Time to first re-intervention within couple of months after surgery, due to pulmonary branch stenosis, was comparable in both groups.

From long-term (10-year) perspective the conduit means higher risk of repeated interventions for the patients (due to conduit stenosis). On the other hand severe pulmonary regurgitation in $\mathrm{RV}$-AP direct anastomosis will later expectedly lead to additional re-interventions due to RV dilation.

\section{P-81}

Right Ventricular Myocardial Systolic Activation in children with Pulmonary Hypertension using Tissue Doppler Imaging

Mohamed W.G., Elsayed M.H., Elshahid G.S., Roushdy A.M.

Ain shams university, Cairo, Egypt

Background: Tissue Doppler imaging (TDI) has provided an objective means to quantify global and regional ventricular functions with improved accuracy and greater reproducibility than conventional echocardiography. This study was conducted to assess right ventricular (RV) myocardial systolic activation by TDI in children with pulmonary arterial hypertension (PAH).

Methods: Forty pediatric patients with $\mathrm{PAH}$ and 20 healthy controls, underwent standard echocardiogram and TDI. In the apical 4 chamber view the following regional parameters were evaluated in three different myocardial segments (RV basal lateral, basal septal and LV basal lateral): systolic (Sm), early- and late-diastolic (Em and $\mathrm{Am}$ ) peak velocities. RV myocardial systolic activation delay was defined as the difference in time to peak TDI systolic velocities between the RV basal lateral wall and basal septal. In addition, RV end-diastolic and end-systolic areas were measured to calculate RV fractional area change (FAC).

Results: Compared with the control group, pediatric patients with $\mathrm{PAH}$ showed reduced RV FAC; $37.6 \pm 14 \%$ versus $48 \pm 5 \%$ $(\mathrm{P}<0.0024)$. The patients group showed lower myocardial peak velocities and a significant activation delay compared with controls $(\mathrm{P}<0.0001)$. There was a significant negative correlation between RV myocardial systolic activation delay and RV FAC. RV myocardial systolic activation delay was even present in a subset of patients with normal FAC.

Conclusions: In PAH, RV myocardial systolic activation was markedly delayed and showed significant negative correlation with the RV FAC. RV myocardial systolic activation delay could offer a unique approach to predict early RV dysfunction before the decline in RV FAC is evident.

\section{P-82}

Effects of enzyme replacement therapy on cardiac disease in children with mucopolysaccharidosis type II

Lazea C. (1,2), Al-Khzouz C. (1,3), Bucerzan S. (1,3), Nascu I. (3), Asavoaie C. (2), Grigorescu-Sido P. (3)

University of Medicine and Pharmacy "Iuliu Hatieganu", Department Pediatrics I, Cluj-Napoca, Romania (1); Emergency Children Hospital, Clinic Pediatrics I, Cluj-Napoca, Romania (2); Emergency Children

Hospital, Department of Genetic Diseases, Cluj-Napoca, Romania (3);

Introduction: The prevalence and severity of cardiovascular disease in patients with mucopolysaccharidosis (MPS) type II is high and progressive, consisting in severe cardiac valve disease and ventricular hypertrophy. Enzyme replacement therapy in MPS type II may improve the organ impairment. The aim of the study was to characterize the cardiac results of enzyme replacement therapy in children with MPS type II.

Methods: The treatment of these patients consisted in weekly administration of recombinant form of human iduronate 2-sulfatase in dose of $0.5 \mathrm{mg} / \mathrm{kg}$, iv. We assessed the function of the mitral and aortic valves, left ventricular chamber dimensions, septal and posterior wall thicknesses and ventricular function in 15 patients with MPS type II every 6 months after starting of treatment.

Results: At diagnosis, all patients presented echocardiographic alterations. Mitral valve thickening with variable grades of regurgitation was diagnosed in all patients; Aortic regurgitation was 
present in 9 patients and aortic stenosis in 2 patients. Left ventricular hypertrophy was diagnosed in 7 patients. Mild pulmonary hypertension was present in 4 patients. The mean age of the patients at starting therapy was 6.1 years. Duration of treatment was 12 months in 3 patients, 2 years in 7 patients, 3 years in 2 patients, 4 years in 2 patients and 5 years in one patient. The treatment results on valvular heart disease were: stable disease in 8 patients, mild improvement in 2 patients and aggravation in 5 patients. Ventricular hypertrophy remained unmodified in 6 patients and worsened in one patient.

Conclusions: Enzyme replacement therapy had little effect on cardiac disease in children with MPS type II.

\section{P-83}

Assessment of Pulmonary hypertension in infants with Bronchopulmonary dysplasia. A review of literature and developing screening protocol

Raychaudhuri H., Ali I.

Barts and London NHS Trust, Dartford, UK

Introduction: Pulmonary hypertension $(\mathrm{PH})$ is a known complication of bronchopulmonary dysplasia (BPD) with a prevalence range of $8-25 \%$. Infants with $\mathrm{BPD}$-associated $\mathrm{PH}$ have reported mortality rates ranging from $14-38 \%$. BPD with concomitant $\mathrm{PH}$ has been reported to increase the risk of long-term pulmonary morbidity, as well as cognitive, behavioural and social problems. Given the short-term and possible long-term complications of $\mathrm{PH}$, it may be important to identify such infants so that appropriate medical treatment and follow-up plans may be implemented. Increasingly it has been recognised in literature that evolving pulmonary venous anomalies in infants with BPD has further contributed to development of $\mathrm{PH}$. Paediatric cardiologists are faced with increasing cases of this patient cohort. To the best of our knowledge, there is lack of a unified European guideline for

Flowchart for Management of Pulmonary hypertension (PH) in Broncho-Pulmonary Dysplasia (BPD)

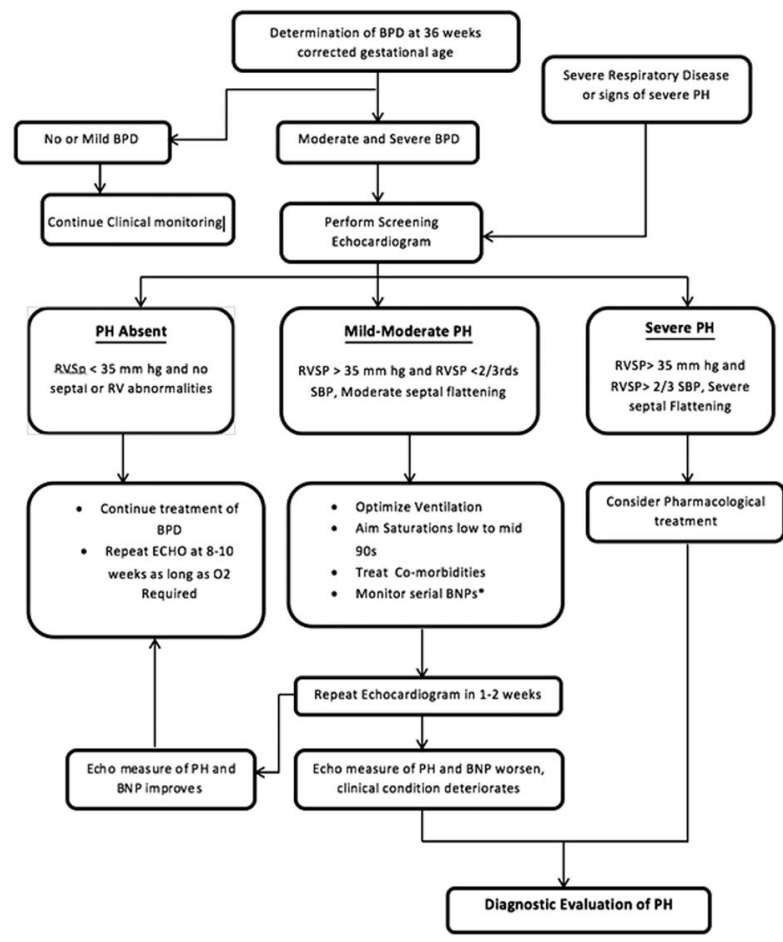

regular assessment of this patient group. This study aims at reviewing available evidence and developing a protocol which will help in echocardiographic screening of preterm infants with BPD at risk of developing PH.

Methods: We conducted a review of the literature regarding use of echocardiographic techniques for evaluation of $\mathrm{PH}$ in infants with bronchopulmonary dysplasia. Results from 21 different papers were assessed and analysed for this study.

Results: We identified seven parameters which can be used for detection of $\mathrm{PH}$ non-invasively using echocardiography from fifteen studies. On the basis of the available evidence, we came up with a screening algorithm which will help screen infants with BPD who are at risk of developing PH.

Conclusions: Paediatric $\mathrm{PH}$ has its own unique characteristics and its assessment cannot be extrapolated from adult studies. The clinical utility of echocardiography in predicting the presence and severity of pulmonary hypertension in patients with BPD has been well founded but larger studies are required to further evaluate these parameters for determining the severity of pulmonary hypertension at early stages of the disease.

\section{P-84}

Impact of personalized cardiac rehabilitation programs in postoperated congenital heart disease children

Fuertes Moure A., García-Cuenllas Álvarez L., Rodríguez-López

Domingo A., Centeno Jiménez M., Álvarez García-Rovés R., Maroto

Álvaro E., Medrano López C., Castillo Martín J.I.

Universitary Hospital Gregorio Marañón. Madrid. Spain.

Introduction and Objectives: Most patients with congenital heart disease (CHD) do not perform regular physical exercise. Consensus reports have stated that exercise should be encouraged and regularly performed in these patients. Postoperated children with CHD frequently associate disorders that impair their exercise capacity (ExC). We developed a personalized cardiac rehabilitation program (CRP) conceived to counteract their deconditioning.

Our aim was to characterize the effect of a personalized CRP through cardio pulmonary exercise test.

Material and Methods: 8 postoperated CHD patients were recruited during 2015 (ages 7-17 years): 2 heart transplants, 3 postoperated Tetralogy of Fallot, 3 Fontan procedures.

CRP started with clinical review, baseline echocardiography, submaximal ergometry and post-exercise echocardiography. These tests allowed a personalized CPR design: raining intensity was set at the transition zone between 1 st and 2 nd threshold. CPR consisted in $1 \mathrm{hr}$ supervised exercise 2-3 times/week, 3 months, including respiratory training, column table, and aerobic exercise. After CRP, tests were repeated to quantify its effects on ExC.

Results: Submaximal exercise tests in treadmill (limited by symptoms, including fatige) were performed (Bruce protocol). Ergometric tests showed effort time improvement $(+29 \%)$, increased METs $(+7.7 \%)$ and a decrease in baseline HR, without any other significant changes.

Restrictive spirometric pattern was predominant. CPET revealed a tendency to increase $\mathrm{VO} 2$ in $+7.4 \%(+2.2 \mathrm{ml} / \mathrm{kg} / \mathrm{min})$, oxygen pulse in $+2 \%$ and a tendency to decrease $\mathrm{O} 2$ and $\mathrm{CO} 2$ equivalents. $2 / 8$ patients had conflicting data, with decreased ExC. No adverse effects were found.

Conclusions: Despite our small smaple, a 3-month CRP could improve relevant ExC parameters. Exercise was safe and and improvement of fitness after a physical exercise training programme can be obtained. Routine use of formal CRPs might be a useful tool to reduce morbidity and improve quality of life in CHD. 


\section{P-85}

CPET in children with congenital heart disease: comparison between postoperated Tetralogy of Fallot and Fontan procedure

García-Cuenllas Álvarez L., Fuertes Moure A., Álvarez García-Rovés R., Centeno Jiménez M., Maroto Álvaro E., Medrano López C., Castillo Martín J.I.

Universitary Hospital Gregorio Marañón. Madrid. Spain.

Introduction: Postoperated children with complex congenital heart disease (CHD) have a decreased functional capacity (FxC) associated with death and hospitalization. Cardio pulmonary exercise test (CPET) assesses their FxC and shows specific patterns according to different physiological adaptations depending on the type of CHD.

Objective: The aim of this investigation was to define and compare the exercise performance of postoperated Tetralogy of Fallot children (PTF) against subjects that had undergone a Fontan procedure $(\mathrm{FP})$ through $\mathrm{CPET}$.

Methods: Retrospective observational study of $25 \mathrm{PTF}$ (mean age $12 \pm 3.2$ years, weight $43.2 \pm 14.55 \mathrm{~kg}$, height $146.7 \pm 14.4 \mathrm{~cm}$, $58 \%$ male) and 63 Fontan (mean age $11.8 \pm 3.4$ years, weight $41.9 \pm 17 \mathrm{~kg}$, height $146.5 \pm 17.5 \mathrm{~cm}, 56 \%$ male). Inclusion criteria admited pulmonary atresia with VSD in the PTF group and both right or left systemic ventricles in the FT group. Patients with pacemakers, sinus node dysfunction or junctional rhythm were excluded. Ramp treadmill ergometry (Bruce protocol) was performed with expired gas in all cases.

Results: Submaximal exercise tests limited by symptoms were performed, clinically and electrically negative in all cases, with no significant arrhythmias. $38 \%$ of FP reached $85 \%$ MPHR vs $32 \%$ in PTF. From the FP, $62 \%$ showed a normal spirometric pattern and $28.6 \%$ restrictive vs $56 \%$ with normal pattern and $20 \%$ restrictive in PTF group. CPET results were:

We found no significant differences between groups.

From a cardiovascular stadpoint $\mathrm{VO}_{2}$ was decreased in both groups, revealing impaired ExC. with normal $\mathrm{O}_{2}$ pulse (appropriate stroke volume).

\begin{tabular}{|c|c|c|}
\hline & $\begin{array}{l}\text { FONTAN } \\
\text { procedure } N=63\end{array}$ & $\begin{array}{l}\text { Postoperated T. of } \\
\text { FALLOT } N=25\end{array}$ \\
\hline Effort time (min) & $10.22 \pm 2.4$ & $10.3 \pm 2.7$ \\
\hline METs & $8.8 \pm 2.4$ & $9.3 \pm 3$ \\
\hline $\begin{array}{l}\text { Resting HR } \\
\text { (bpm) }\end{array}$ & $95 \pm 17$ & $89 \pm 13.8$ \\
\hline Max HR & $164 \pm 26$ & $169.7 \pm 17.3$ \\
\hline HRR & $69 \pm 27$ & $80.7 \pm 22.3$ \\
\hline $\begin{array}{l}\text { Max predicted } \\
\operatorname{HR}(\%)\end{array}$ & $77 \pm 13.5$ & $79.6 \pm 9.3$ \\
\hline $\begin{array}{l}\text { Resting SBP/ } \\
\text { DBP (mmHg) }\end{array}$ & $102 \pm 14 / 59 \pm 9$ & $97.4 \pm 10 / 56.6 \pm 8.4$ \\
\hline $\begin{array}{l}\mathrm{Max} \mathrm{SBP} / \mathrm{DBP} \\
(\mathrm{mmHg})\end{array}$ & $127 \pm 19 / 69.5 \pm 9$ & $125.4 \pm 10.3 / 71.2 \pm 7.5$ \\
\hline Double product & $20950 \pm 3372.2$ & $20938 \pm 2817$ \\
\hline $\mathrm{Max}_{\mathrm{VO}}(\%)$ & $76 \pm 19.8$ & $79.5 \pm 10.2$ \\
\hline $\mathrm{O}_{2}$ pulse $(\%)$ & $102.16 \pm 33$ & $101 \pm 18.5$ \\
\hline $\begin{array}{l}\mathrm{VE} / \mathrm{VCO}_{2} \text { slope } \\
\left({ }^{\circ}\right)\end{array}$ & $38 \pm 6.8$ & $33.4 \pm 8.3$ \\
\hline $\mathrm{O}_{2}$ equivalents & $36.9 \pm 6.2$ & $32.7 \pm 7.3$ \\
\hline $\mathrm{CO}_{2}$ equivalents & $39.3 \pm 7.5$ & $34.9 \pm 6.8$ \\
\hline $\begin{array}{l}\text { Breathing } \\
\text { Reserve }\end{array}$ & $39 \pm 12.3$ & $42 \pm 14.6$ \\
\hline OUES & $1.47 \pm 0.55$ & $1.57 \pm 0.51$ \\
\hline
\end{tabular}

FP group showed ventilatory inefficiency with higher Vslope and $\mathrm{CO}_{2}$ equivalents (V/Q disturbance), whilst in the PTF group those parameters were limit.

Conclusions: Functional capacity is decreased in both PTF and FP patients. Ventilatory efficiency variables revealed V/Q imbalance between FP subjects (according to their condition). This disturbance is only appreciated in PF when significant RV dysfunction develops.

\section{P-86}

Very-long-term observation after pulmonary valvuloplasty shows higher than expected risk of pulmonary insufficiency Meyer-Szary J., Sabiniewicz R., Kwiatkowska J.

Department of Paediatric Cardiology and Congenital Heart Diseases, Medical University of Gdansk, Poland

Introduction: It is generally accepted that percutaneous balloon pulmonary valvuloplasty (PBPV) has excellent short- and longterm outcomes in relieving valvular pulmonary stenosis.

Methods: Between 1992 and 2005, 84 patients were treated by PBPV and their data was retrospectively revived. There were two early and one late mortality. Four patients were converted to surgery due to supravalvular component of stenosis. Of the remaining 77 eligible patients, 21 were lost to follow-up and 56 responded to study invitation and underwent detailed echocardiography according to current guideline protocol.

Results: The average long-term observation period was 13,8 years (range 6-24), the average age at the final examination was 23,2 years (range 6-69) and $41 \%$ were male. On the day of the procedure the patients were 9,4 years old (range 0-60). The average pressure gradient by Doppler was $79 \mathrm{mmHg}$ (range 40-182), valvular annulus average diameter $1,5 \mathrm{~cm}$ (range $0,65-2,41$ ) and balloon-to-annulus ratio (BAR) was 1,25 (+/-0,18). There were four patients undergoing the procedure twice for lack of technical success at first attempt. After successful procedure the average gradient was $39 \mathrm{mmHg}$ and there was further drop to $12 \mathrm{mmHg}$ (range 4-42) at the final examination (both differences statistically significant). Only one patient was exceeding $40 \mathrm{mmHg}$ and none had indications for late repeat PBPV. Right ventricular dilatation was found in $4-51 \%$ of cases dependently on the echocardiographic parameter considered and $44 \%$ had dilated pulmonary artery. TAPSE was diminished in $7 \%$ of the patients, all having comorbidities. Using current quantification criteria $39 \%$ of patients had insignificant pulmonary insufficiency, $46 \%$ moderate and $14 \%$ severe. Mean vena contracta was $0,5 \mathrm{~cm}$ and maximum $1,6 \mathrm{~cm}$. There was no correlation of the degree of insufficiency and BAR as in vast majority of cases it was close to 1,3 . No significant arrhythmias were noted, $4 \%$ of patients were NYHA II, none had clinical signs of overt heart failure.

Conclusions: This very-long-term observational study confirms excellent stenosis relieve outcome but points out significant risk of pulmonary insufficiency. Despite good clinical status the above findings call for vigilant follow-up and in selected subset of patients an MRI study, because some patients may become candidates for pulmonary valve replacement.

\section{P-87}

3D derived Sphericity index for early detection of myocardial remodeling, a multicentic study in iron loaded asymptomatic beta thalassemia major patients

AbdelMassih A. F. (1), Agha H.M. (1), AbdelRahman M. Y. (1), Milanesi O.(4), Castaldi B.(4), Geranio G.(5), Frizziero L.(5), Putti M. C.(5), Kharabish A. (3), Esmail R. (1), El-Kamah G. (7), Hamdy M.(2), El-Baz H. (6), El-Behary N. (3), Hanna C. (8), El-Tagy H. (11), Mishriky M. A. (10), Mamdouh M. (10), Khalifa H. (10), Ayad N. (9), Livio A. (5) 
Pediatric Cardiology Unit, Pediatrics' Department, Specialized Children Hospital, Faculty of Medicine, Cairo University, Cairo, Egypt (1); Pediatric Hematology Unit, Pediatrics' Department, Specialized Children Hospital, Faculty of Medicine, Cairo University, Cairo, Egypt (2); Cardiac MRI unit, Radiology Department, Faculty of Medicine, Cairo University, Cairo, Egypt (3); Pediatric Cardiology unit, Department of Woman and Child health, Faculty of Medicine, Padova University, Padova, Italy (4); Pediatric Hematology unit, Department of Woman and Child health, Faculty of Medicine, Padova University, Padova, Italy (5); Clinical Pathology Department, Faculty of Medicine, Cairo University, Cairo, Egypt (6); Pediatric Hematology Department, National Research Center, Cairo, Egypt (7); Computer Science and software development department, American University of Cairo, Cairo, Egypt (8); Pediatric Residency Program, Faculty of Medicine, Cairo University, Cairo, Egypt (9); Cardiology Residency Program, Faculty of Medicine, Cairo University, Cairo, Egypt (10); Orthopedics' Residency Program, Faculty of Medicine, Cairo University, Cairo, Egypt (11)

Introduction: The ventricular remodeling that occurs with left ventricular (LV) dysfunction is characterized by a LV change from an ellipical to a spherical shape and is associated with a decreased exercise tolerance, and poor outcome. There are limited data on the occurrence and importance of LV remodeling in children with LV dysfunction. Studying LV sphericity index in beta thalassemia major patients with iron myocardial load might help in the validation of this index as powerful predictor of early cardiomyopathic changes in asymptomatic patients. This may help as well to extend its usage as potent screening tool in patients liable to develop LV dysfunction including other iron load disorders or anthracycline induced myocardial insult.

Objectives: To evaluate the effectiveness of the usage of "Sphericity Index" in measuring the degree of cardiac remodeling in iron loaded $\beta$-thalassemia major children.

Methods: Twenty-seven thalassemic patients (with a mean age of $11 \pm 3$ years) and 25 healthy control subjects matched for age and sex have been included in the study. Patients have been recruited from Pediatric Hematology Clinics in both Cairo University, Egypt $(\mathrm{n}=14)$ and Padova University, Italy $(\mathrm{n}=13)$. 3D Speckle tracking Echocardiography was performed to all patients and control subjects in addition to myocardial relaxometry $(\mathrm{T} 2 \star)$ by Cardiac MRI.

Results: Compared to normal subjects patients didn't exhibit statistical difference in LV EF; patients vs. Controls; $74.3 \pm 3.5 \%$ vs. $75.4 \pm 3.9 \%$, $\mathrm{p}=0.30$, while patients had a statistically significant reduction of left ventricular global longitudinal strain; $-16.7 \pm 0.8$ vs. $-18.7 \pm 1.2$; patients vs. controls, $\mathrm{p}<0.05$. Sphericity index was significantly higher in patients compared to controls; $36 \pm 6 \%$ vs. $27 \pm 3 \%$; $p<0.05$. The only parameter that showed statistical significant correlation with myocardial iron load as measured by $\mathrm{T} 2{ }^{\star}$ was the sphericity index with a correlation coefficient of $0.9 ; \mathrm{p}<0.05$.

Conclusions: 3D derived sphericity index can detect early iron induced myocardial remodeling in asymptomatic beta thalassemia major patients with preserved ejection fraction. Extending the study to larger cohorts may confirm its ability to replace the relatively expensive cardiac MRI derived myocardial relaxometry in iron load disorders.

\author{
P-88 \\ Body Surface Area Estimation In Girls With Turner \\ Syndrome: Implications For Interpretation Of Aortic \\ Sized Index \& Aortic Root Z scores \\ Fletcher, A. (1), McVey L., (1) Guaragna-Filo G., (2) Hunter L., (3) \\ Santoro R.I., (4) Mason A., (1) Wong S.C. (1) \\ Developmental Endocrinology Research Group, Royal Hospital for \\ Children, Glasgow, UK (1); Paediatric Endocrinology Unit, Faculty of
}

Medical Sciences, State University of Campinas, Brazil (2); Department of Paediatric Cardiology, Royal Hospital for Children, Glasgow, UK (3); Paediatric Cardiology Unit, Clinical Hospital of State University of Campinas, Brazil (4)

Objective: To assess the impact body surface area (BSA) formula selection has on aortic size index (ASI) and aortic root Z-score risk stratification in patients with Turner's syndrome (TS) monitored for aortic root dilatation.

Methods: We retrospectively accessed the aortic sinuses measurements, age, weight and height of a cohort of females with TS in Brazil presenting for routine monitoring of aortic root size. We utilised 6 commonly employed BSA formula (Dubios, Mosteller, Haycock, Boyd, Gehan and George and Furqan) to calculate ASI and aortic root $\mathrm{Z}$-scores. We compared the results of all formulae to Dubios using Bland-Altman plots. We further examined the percentage whose risk category changed employing cut off values of ASI $>2 \mathrm{~cm} / \mathrm{m} 2$ or Z-score $>2$ as "high risk", and ASI $>2.5 \mathrm{~cm} /$ $\mathrm{m} 2$ as "very high risk".

Results: Bland-Altman plots demonstrate a good general agreement between Dubios and 5 other BSA formulae (largest ASI mean error $-0.091 \mathrm{~cm} / \mathrm{m} 2$, largest aortic root Z-score mean error -0.25) however, at higher BSA values we note increasingly disparate ASI and Z-scores with a correlating impact on risk stratification. As an example the Boyd formula stratified $17 \%$ fewer as "high risk ASI" and 33\% fewer as "very high risk ASI" and 50\% fewer as "high risk Z-score", with potentially important implications on future management.

Conclusions: We recognise for the first time that BSA formula selection has an important impact on ASI- and Z-score-based risk stratification when monitoring TS patients for aortic dilatation, with potential implications on future follow up and management. Further work is required form a consensus on an appropriate BSA formula for stratifying aortic root dilatation in TS.

\section{P-89 \\ Incidentally detected small fistuluous flows in pulmonary artery by color Doppler; echocardiographic findings and follow-up results \\ Atik S. U., Saltik I. L., Eroglu A. G. \\ Istanbul University Cerrahpasa Medical Faculty, Istanbul, TURKEY}

Abnormal small fistulous flows in pulmonary artery can be detected on routine TTE examination in asymptomatic patients. Although they are strongly thought to be coronary fistulas, it is still controversial to manage these asymptomatic patients. Here in, we described the clinical characteristics and follow-up results of small fistulous flows in pulmonary artery which was incidentally detected on routine TTE examination in 82 patients.

Materials and methods: The study population consisted of 82 patients with abnormal small fistulous flows in pulmonary artery who had been studied at our institution between 1992 and 2016. Results: Most of the patients (\% 65.5) were male. The mean age at diagnosis was $4.9 \pm 4.7$ years (range 16 days to 26 years). The patients were followed during a median of 23 months up to 12 years. The echocardiography indication was cardiac murmur in 39 $(47.5 \%)$, routine cardiac control in $24(29 \%)$, nonspecific chest pain in $11(13.5 \%)$, and the rest for other reasons. Coronary arteries were evaluated as normal except 3 patients with mild left coronary artery dilatation. Additional cardiac anomalies were ventricular septal defect in 7 , atrial septal defect in 4 , patent ductus arteriosus in 4 , mitral valve prolapse in 3 , mitral and/or aortic insufficiency in 7. These fistulous flows were detected in three different regions in parasternal aorta short axis view. In $59(72 \%)$ patients, fistulous 
flow located in anterior aspect of main pulmonary artery (between pulmonary valve and bifurcation), in 17 (20.7\%) patients in aortic side of pulmonary artery and in 7 (7.3\%) patients in right pulmonary artery. As a further and invasive investigation, cardiac catheterization was performed in only 3 of 82 patients for different reasons. Echocardiographic diagnose of coronary artery to pulmonary artery fistula was confirmed with selective coronary angiography in these three patients. Spontaneous closure was detected in only one patient, the others remained almost unchanged during the follow-up.

Conclusion: We believe that although they are thought to be coronary fistulas, most asymptomatic pediatric patients with small fistulous flows in pulmonary artery may be managed conservatively and require no further investigation.

\section{P-90}

\section{Body Habitus and Hypermobility in Children with Mitral} Valve Prolapse

Ibis Z., Gunay E., Saylan Cevik B., Akalin F.

Marmara University Faculty of Medicine, Department of Pediatric Cardiology, Istanbul, Turkey

Introduction: Mitral valve prolapse (MVP) is often an isolated finding, however it also occurs in heritable disorders of connective tissue disorder. An increased prevalance of MVP in patients with hypermobility syndrome was demonstrated before. In this study we investigated the anthropometric properties, asthenic body habitus and hypermobility in patients with isolated MVP.

Methods: The study group consisted of 41 patients with MVP and 43 controls. MVP was diagnosed as superior displacement of mitral leaflets of more than $2 \mathrm{~mm}$ above mitral annulus during systole. None of them had associated systemic, cardiac or rheumatologic disease. Anthropometric measurements were obtained during physical examination. Height, weight, armspan, lower segment, anteroposterior and transverse chest diameter, chest circumference, abdominal circumference, hip circumference, sitting height, length of femur, cruris, arm and forearm were measured, Armspan/height ratio, armspan height difference, upper segment/ lower segment ratio, chest/height ratio, waist/height ratio, hip/ height ratio, anteroposterior chest/height ratio were calculated. Beighton Score System was used for assessment of joint hypermobility and Brighton criteria for benign joint hypermobility syndrome (BJHS).

Results: The study group included 15 boys and 26 girls between 5 to 20 years of age (mean $=13.4 \pm 3.9$; median $=14$ years); control group included 15 boys and 28 girls between 6 to 19 years of age (mean $=12.8 \pm 3.4$; median:13years). There was no significant difference in terms of age, weight, height, BSA between groups. Number of girls were higher in both groups. Armspan/height ratio and hip/height ratio were significantly higher in MVP group $(p=0.034$ and $p=0.024$ respectively). Mean hypermobility score was $1.92 \pm 2,09$ (median 2; range $0-8$ ), in MVP group and $1,18 \pm 1,84$ (median 0 ; range $0-8$ ) in control group $(\mathrm{p}=0.06$ ). BJHS was diagnosed in 5 MVP patients and 3 healthy children. Hypermobility score negatively correlated to age in MVP group $(\mathrm{p}=0,05 ; \mathrm{r}-0,42)$. Arthralgia was also common in MVP group $(\mathrm{p}=0.003)$.

Conclusion: Body habitus was more asthenic in children with isolated MVP than the normal population and joint elasticity was higher despite low incidence of real BJHS. Joint elasticity becomes less evident by aging in patients with MVP. These findings suggests common pathogenetic mechanisms affecting connective tissue both in skeletal and cardiac structures.

\section{P-91}

Evaluation of the ventricular functions, ascending aorta and aortic root widths in the asymptomatic patients with isolated bicuspid aortic valve

Yildirim A.(1), Altun Usta S.(1), Karaagac Turkmen A.(1), Sahin M. (1), Cftci O.(1), Sungur M.(1)

(1); Kartal Kosuyolu Heart and Training Hospital, Department of Paediatric Cardiology Istanbul, Turkey (2); Kartal Kosuyolu Heart and Training Hospital, Paediatrist, Istanbul, Turkey

Objective: Aortic root and/or ascending aorta dilatation is frequently encountered in patients with bicuspid aortic valve (BAV). The aim of this study is to evaluate the ventricular functions, ascending aorta and aortic root widths in asymptomatic patients with isolated BAV to define their prognosis.

Method: 3 to17 years old (mean 9,5 $\pm 3,9$ ) 49 patients (41 males, 8 females) with BAV with/out stenosis or insufficiency and 3 to 15 years old (mean $8,8 \pm 2,9) 20$ healthy controls (15 males, 5 females) were enrolled in this study. Their aortic root, ascending and descending aorta widths, aorta and sinus valsalva elasticities were measured. Aortic valve morphology, valve regurgitation (AR) jet length and directions were recorded. Tissue Doppler Imaging (TDI) was performed to obtain views from interventricular septum (IVS), mitral and tricuspid free wall in apical four chamber position. Blood natriuretic peptide (BNP) levels were measured.

Results: No difference was found between the age, height, weight, systolic and diastolic blood pressures of two groups. The most common valve morphologies in these patients were: right to left cusp fusion (40.8\%), right non-cusp fusion (36.7\%), bicuspid $(18.3 \%)$ and left non-coronary cusp fusion $(4.08 \%)$. The most common two directions of AR jets were central (27\%) and left eccentric (26\%). Neither stenosis nor insufficiency was observed in $11(22.44 \%)$ patients. Ascending aorta systolic $(2.29 \pm 0.36$ and $2.06 \pm 0.29 \mathrm{~cm}, \quad \mathrm{p}<0.05)$ and diastolic $(2.08 \pm 0.36$ and $1.90 \pm 0.27 \mathrm{~cm}, \mathrm{p}<0.05)$ measurements of the patient group were found significantly higher than the control group. Mitral valve early and late diastolic (MA) flow velocities of the patient group were significantly lower than the controls (E:0.97 \pm 0.13 $\mathrm{A}=0.59 \pm 0.13, \mathrm{E}: 0.89 \pm 0.13$ A: $0.50 \pm 0.07 \mathrm{~m} / \mathrm{sn})$. IVS early diastolic flow velocity of the patient group was significantly lower than the controls in TDI $(11.28 \pm 1.82$ and $12.9 \pm 1.73, \mathrm{p}<0.05)$. A positive correlation was found between BNP and ascending aorta stiffness, ascending aorta systolic, descending aorta systolic and diastolic diameters, also between AR length and aortic valve diastolic diameter measurements and between AR thickness and TDI MA.

Conclusion: The systolic functions were preserved whereas diastolic functions were disturbed in patients with isolated BAV. AR thickness and length, BNP measurement and TDI may be used to follow these patients.

\section{P-92 \\ Increases Atherosclerosis and Cardiovascular risk in in Prematurely Born School Children \\ Levent E. (1), Turksoylu M.(2), Koroglu O.A. (2), Doğan E. (1), Ozyurek A.R (1) \\ Ege University, Faculty of Medicine, Pediatric Cardiology (1); Neonatology (2) Department, izmir, Turkey}

Introduction: Bronchopulmonary dysplasia (BPD) is an important complication of prematurity and has long-term pulmonary consequences. Cardiovascular sequelae related to BPD have also been reported in severe BPD patients of the presurfactant era. The aim of this study is to investigate cardiovascular risk of BPD at school 
ages and to find out possible risk factors related to cardiovascular sequelae especially atherosclerosis

Methods: In our study totally 43 children (21F/22M: 23 aged $104 \pm 7.4$ months born preterm with BPD, 20 aged $112 \pm 8.2$ months born preterm without BPD and 21 healthy children aged $100 \pm 16$ months born at term (control group) were evaluated lipid profile and specifically echocardiographic methods (MPI, carotid artery intima media thickness - cIMT, brachial artery flow-mediated vasodilatation - FMD\%). And also Left (LV) and Right ventricular (RV) strain and strain rate were assessed by speckle-tracking echocardiography. Echocardiographic examination was performed using a Vivid 9 ultrasound machine, and LV and RV myocardial strain were analyzed offline using Echo PAC software.

Results: CIMT values were also significantly higher in the patients compared to the controls $(\mathrm{P}=0.001)$ and flow-mediated dilatation values were significantly lower $(\mathrm{P}=0.001)$ in $\mathrm{BPD}$ patients. These patients had a decreased pulmonary artery acceleration time and higher left and right ventricular myocardial performance indexes in school ages. Longitudinal and circumferential strains were abnormal in premature with BPD group even in the presence of normal RV and LVEF.

Conclusions: Subclinical cardiovascular dysfunction and risk in children with BPD persists at school age. Negative effects of BPD on global cardiac performances of ventricles, atherosclerosis and pulmonary arterial pressure persist up to school ages.

\section{P-93}

Assessment of right ventricular performance by right ventricular spekle-tracking strain in teenage patients with repaired Tetralogy of Fallot. Comparison with Magnetic Resonance Imaging

Mese T. (1), Yilmazer M.M.(1), Özdemir R.(2), Güven B.(3)

Izmir Dr.Behcet Uz Children's Hospital Department of Pediatric Cardiology, Izmir, Turkey (1); Dumlupinar University, Evliya Celebi Training and Research Hospital, Department of Pediatric Cardiology, Kütahya, Turkey (2); Tepecik Training and Research Hospital, Department of Pediatric Cardiology, Izmir, Turkey (3)

Background: Right ventricular performance is a major prognostic factor in patients with repaired tetralogy of Fallot (TOF). The objective of this study was to evaluate the utility of right ventricular speckle-tracking strain as an assessment tool for right ventricle $(\mathrm{RV})$ function in patients with repaired TOF compared with magnetic resonance imaging (MRI) parameters.

Methods: Twenty-six consecutive surgically corrected TOF patients aged $12-18$ years (mean $14.35 \pm 1.8$ ) were studied. Echocardiographic recordings of two-dimensional [2D] strain measurements from apical four chamber views focus on right ventricle were evaluated offline. Right ventricle free wall longitudinal speckle-tracking strain and RV septal wall longitudinal speckle-tracking strain were measured. Results were compared to RV indexed end-diastolic volume (EDV), indexed end-systolic volume (ESV), RV ejection fraction (EF) and pulmonary rejection fraction at MRI obtained in three to seven days after echocardiographic study.

Results: Correlations between global, lateral, septal longitudinal RV strain measurements and MRI derived parameters were assessed by stepwise linear regression analyses. RV apical longitudinal strain could be identified as an independent variable for MRI derived RVEF (B: -0.48 , p:0,02) and basal infero septal longitudinal strain was found to be an independent variable for RV EDV measured by MRI (B: -0.52, p:0,01) through a stepwise multivariate linear regression model.
Conclusions: Although MRI is the gold but not feasible tecnique for assesment of right ventricle in daily practice, echocardiographic measurement of $2 \mathrm{D}$ regional speckle tracking of $\mathrm{RV}$ is a promising method to estimate RV systolic function in patients operated on for TOF.

Table 1 Stepwise linear regression analysis of variables predicting right ventricular ejection fraction and end-diastolic volume measured by MRI.

\begin{tabular}{|c|c|c|c|c|}
\hline & $\mathbf{R}^{2}$ & $\boldsymbol{\beta}$ & $\begin{array}{l}95 \% \\
\text { Confidence } \\
\text { Interval }\end{array}$ & $\begin{array}{l}P \\
\text { value }\end{array}$ \\
\hline \multicolumn{5}{|c|}{ Right ventricular ejection fraction } \\
\hline $\begin{array}{l}\text { RV apical longitudinal } \\
\text { strain (APEX) }\end{array}$ & 0.23 & -0.48 & $(-2.38)-(-0.18)$ & 0.02 \\
\hline \multicolumn{5}{|c|}{ Right ventricular end-diastolic volüme } \\
\hline $\begin{array}{l}\text { Basal infero septal } \\
\text { longitudinal strain (BIS) }\end{array}$ & 0.27 & -0.52 & $(-5.99)-(-0.71)$ & 0.01 \\
\hline
\end{tabular}

\section{P-94 \\ Improved visualization of coronary arteries in patients with congenital heart disease using whole-heart image navigated $\mathrm{MR}$ angiography}

Velasco Forte M.N. (1, 2), Pushparajah K. $(1,2)$, Narayan S. $(1,2)$, Mathur S. (1, 2), Valverde I (1, 2, 3), Bell A. (2), Bellsham-Revell $H$. (1, 2), Razavi R. (1, 2), Henningson M. (1)

King's College London, UK (1), Department of Congenital Heart Disease, Evelina London Children's Hospital, Guy's and St Thomas NHS Foundation Trust, UK (2), Cardiovascular Pathology Unit, Institute of Biomedicine of Seville, IBIS, Virgen del Rocio University Hospital/CSIC/University of Seville, Spain (3)

Background: Whole-heart MR angiography (CMRA) is commonly used in patients with congenital heart disease (CHD) to assess morphology and structural disease. However, respiratory motion remains an impediment in a substantial amount of patients undergoing CMR. Studies in healthy subjects and cardiac patients have shown that image-based navigation (iNAV) improves respiratory motion compensation compared to conventional methods. Here, we investigated the use of iNAV with advanced respiratory gating in patients with $\mathrm{CHD}$.

Methods: iNAV allowed for direct tracking of the respiratory heart motion and was generated using the bSSFP startup echoes. Gating was achieved using the diminishing variance gating (DVG) algorithm with a $50 \%$ gating efficiency. Whole-heart CMRA was acquired with $1.3 \mathrm{~mm}$ isotropic resolution and $\mathrm{SENSE}=2$. For comparison, CMRA with identical imaging parameters were acquired using conventional diaphragmatic navigator with gating window of $3-5 \mathrm{~mm}$ and 0.6 tracking factor. Use of contrast agent before whole-heart imaging acquisition and need for general anaesthesia (GA) was recorded. Scan time, visualization of coronary artery origins and distal course and imaging quality was compared between the two sequences. Image quality was scored 1-5 for each dataset.

Results: A total of 27 patients (19 males; median weight: $49 \mathrm{~kg}$; range:6.5-70; mean age:13, 5months-18years) were recruited. Diagnosis are summarised in table $1.66 \%$ scans were performed in awake patients and 33\% under GA. A contrast agent was used in $51 \%$. Scan time was significantly shorter using iNAV (mean:6:59 $\pm 1: 23$ ) compared to conventional $(9: 17 \pm 2: 34$, $\mathrm{p}<0.05)$. Difference in visual score, using Wilcoxon sign-rank, 
was improved using iNAV, the difference was statistically significant $(p<0.001)$. The coronary arteries origin was depicted in all patients using iNav and $96 \%$ using conventional techniques. Distal course of all coronary arteries was more often visualized when using iNav (85\%vs57\%). In 3.5\% none of the coronary arteries could be visualized distally using respiratory navigation and in 35\% they were depicted partially.

Conclusion: iNav allows for a higher success-rate and clearer depiction of the distal course of the coronary arteries in patients with CHD. Its acquisition time is shorter and image quality score was found to be equal or superior to the conventional method in all cases.
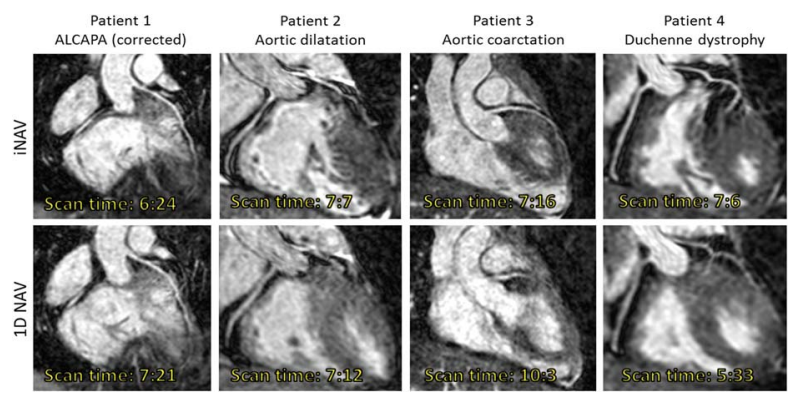

\section{P-95}

Experience in the management of abnormal origin of coronary artery from the opposite sinus at childhood age Courand PY.(1), Bozio A.(1), Veyrier M.(1), Bakloul M.(1), GaloinBertail C.(1), Ninet J.(1), Boussel L.(2), Di Filippo S.(1)

(1) Cardiovascular Hospital Louis Pradel, University Claude Bernard Lyon1, Hospices Civils de Lyon, Lyon, France, (2) Department of Radiology and Imaging, Cardiovascular Hospital Louis Pradel, University Claude Bernard Lyon1, Hospices Civils de Lyon, Lyon, France

The objective of this study was to assess the clinical presentation, management and outcomes of children diagnosed with abnormal course of coronary artery from the opposite aortic sinus (ACAAO).

Material and methods: All patients with ACAAO and less than 18y of age at diagnosis were included in this single-center retrospective study. Demographics, clinical presentation and symptoms, echocardiographic data treatment and outcomes were assessed.

Results: From 1993 to 2016, 34 patients aged 10.5 \pm 5.8 y, had ACAAO $(23$ males $=67.6 \%): 19$ right coronary artery $(\mathrm{RCA})$ from left sinus (56\%), 9 left coronary artery (LCA) from right sinus $(26 \%)$ and 6 others (single coronary artery and circumflex from right coronary artery). An Inter-arto-pulmonary course was present in $79.4 \%$ and intramural segment in $67.6 \%$. Mean age at diagnosis was $8 y$ in females and $11.7 y$ in males $(p=0.08)$ and did not differ with anatomy. Three patients had cardiac arrest (9\%), 7 chest pain at exercise (21\%), 8 syncope (23\%), 3 dyspnea (9\%), 2 acute pulmonary edema (6\%), 2 arrhythmias $(6 \%)$ and 9 had no symptom $(27 \%)$. All patients with severe symptoms (i.e. $59 \%$ of the cases) had an intramural segment, $57 \%$ of RCA and $89 \%$ of LCA had severe symptoms. Time from onset of symptoms to diagnosis was $1.9 \pm 2.6 y$ (median 9months), not different with anatomy and shorter in females $(0.9 y)$ than males $(2.5 y, p=0.27)$. Surgical reimplantation was performed in 20 cases (59\%): $63 \%$ of RCA and $89 \%$ of LCA from the opposite sinus, none of the single coronaries and abnormal circumflex. Surgery was more frequent in case of intramural segment $(80 \%$ versus $28 \%, p=0.0027)$ and severe symptoms ( $86 \%$ versus $27 \%$ in mild or no symptoms, $p=0.01$ ).
Age at surgery was $12.9 \pm 4.5 \mathrm{y}$ (median $13.6 \mathrm{y}$, min 5.3y). Time from diagnosis to surgery was $190 \pm 470$ days (median 50 days): shorter in LCA (50days) than RCA (253days, $\mathrm{p}=0.44$ ) and in severe symptoms (67 versus 723 days, $\mathrm{p}=0.02)$. Follow-up was $4.4 \pm 3.7 y$. No death occurred.

Conclusion: ACAAO in children can impair vital prognosis, even in RCA from opposite sinus. Intramural trajet is associated with worse outcome. Surgical option depends on clinical status and anatomy, and has been uneventful.

\section{P-96}

Abnormal regional myocardial deformation properties in essential pediatric hypertension patients despite successful treatment: an ABPM, standard echocardiography and strain rate imaging study

Celik S.F.(1), Karakurt C. (1), Tabel Y.(2), Elmas T. (2), Yoloğlu S. (3) Inonu University; Faculty of Medicine; Department of Pediatric Cardiology, Malatya, Turkey; 2 Inonu University; Faculty of Medicine; Department of Pediatric Nephrology, Malatya, Turkey; 3 Inonu University; Faculty of Medicine; Department of Biostatistics Malatya, Turkey

Introduction: Although there are few studies of subclinical myocardial dysfunctions in children and adolescents with essential hypertension (HTN), there is no study in successfully treated normotensive patients who had essential HTN by using twodimensional speckle tracking echocardiography (2DSTE).

Methods: This study consisted of 45 children (5-17 years) previously hypertensive nonobese patients, but currently on medication and normotensive and 45 sex, age, and body mass index-matched healthy subjects.

Results: Left ventricular wall thickness, dimensions, systolic functions, Tissue doppler echocardiography (TDI) of patients and controls were within normal limits and statistically similar. Global longitudinal strain (GS) $(-18,70 \pm 3,41$ and $-19,77 \pm 1,77)$, Global longitudinal strain rate $(\mathrm{SR})(-1,16 \pm 0,24$ and $-1,25 \pm 0,20)$, Global longitudinal four-chamber strain $(-17,88 \pm 2,67$ and $-19,77 \pm 1,77)$, and Global longitudinal four-chamber strain rate $(-1,04 \pm 0,18$ and $-1,19 \pm 0,14)$ values were lower in the patient group than in the control subjects. Left ventricular septal and lateral wall longitudinal and transverse strain values according to segmental analysis showed statistically significantly lower levels of Positive systolic peak circumferential strain and End systolic longitudinal displacement of the apical lateral segment in the patient group.

\begin{tabular}{lrrr}
\hline & Patients & Controls & P value \\
\hline GS & $-18,70 \pm 3,41$ & $-20,01 \pm 2,82$ & $\mathbf{0 , 0 3}$ \\
SR & $-1,16 \pm 0,24$ & $-1,25 \pm 0,20$ & $\mathbf{0 , 0 2}$ \\
GSRe & $1,77 \pm 0,47$ & $1,95 \pm 0,51$ & 0,09 \\
GSRa & $0,64 \pm 0,30$ & $0,72 \pm 0,41$ & 0,10 \\
VEbasL & $-12,03 \pm 2,54$ & $-12,24 \pm 2,11$ & 0,56 \\
VEbasR & $-10,46 \pm 3,39$ & $-10,96 \pm 2,73$ & 0,29 \\
VAbasL & $-5,85 \pm 2,11$ & $-6,46 \pm 2,02$ & 0,68 \\
VAbasR & $-5,26 \pm 2,80$ & $-4,99 \pm 2,14$ & 0,49 \\
\hline
\end{tabular}

GS: Global peak longitidunal strain (\%), SR: Global peak systolic longitudinal strain rate (1/sec), GSRe: Global peak 'E' longitudinal strain rate (1/sec), GSRa: Global peak 'A' longitudinal strain rate (1/sec), VEbasL: left basal Peak 'E' longitudinal velocity $(\mathrm{cm} / \mathrm{sn})$, VEbasR: Right basal Peak 'E' longitudinal velocity point $(\mathrm{cm} / \mathrm{sn})$, VAbasL: Left basal Peak 'A' longitudinal velocity $(\mathrm{cm} / \mathrm{sn})$, VAbasR: Right basal Peak 'A' longitudinal velocity $(\mathrm{cm} / \mathrm{sn})$ 
Conclusions: Despite normal diastolic function, LV longitudinal function was found to be impaired in children and adolescents in the treatment of HT who have normal LV ejection fraction by 2DSTE.

\section{P-97 \\ Are Microvolt T-Wave Alternans and Doppler Echocardiography Useful Methods for the Effect of Hypothermia and Rewarming on Myocardial Dysfunction in Neonates with Hypoxic-Ischemic Encephalopathy? Karpuz D.(1), Celik Y.(2), Giray D.(1), Tasdelen B.(3), Hallioglu O.(1) Department of Pediatrics, Division of Pediatric Cardiology, University of Mersin Faculty of Medicine, Mersin, Turkey (1), Department of Pediatrics, Division of Neonatology, University of Mersin Faculty of Medicine, Mersin, Turkey (2), Department of Biostatistics, University of Mersin Faculty of Medicine, Mersin, Turkey (3)}

Introduction: Hypoxic-ischemic encephalopathy (HIE) due to perinatal asphyxia is an important cause of newborn mortality and disability. Myocardial dysfunction is a crucial factor for the prognosis which is developed by the myocardial cell damage due to the hypoxia. This is the first study which aims to evaluate predictive value of myocardial performance on arrhythmia and mortality in hypoxic infants via tissue Doppler and the microvolt T-wave alternans (MTWA) with therapeutic hypothermia and rewarming. Methods: The study included 23 term newborns having the diagnostic criteria for HIE and scored using Sarnat staging as well as age and gender matched 12 healthy as control. To assess myocardial involvement, pulse and tissue Doppler echocardiography and MTWA were performed in the first six hours after birth, and after hypothermia and rewarming treatment on the fifth day of life.

Results: Comparing with the controls, the basal MTWA values were significantly higher in newborns with moderate and severe HIE in lead $\operatorname{aVF}(87.39 \pm 8.63,73.50 \pm 27.43, \mathrm{p}<0.001$, respectively). The values in lead aVF were correlated with the existing acidemia $(r=0517$ $\mathrm{p}=0$ 012). Moreover; when basal MTWA values compared with the post hypothermic and rewarming lead V1 (82.74 \pm 13.71 , $56.05 \pm 18.95, \quad p<0.001$, respectively) and aVF $(87.39 \pm 8.63$, $59.24 \pm 22.48, \mathrm{p}<0.001$, respectively) values, the former was found out to be decreased significantly. Right Ventricle Diastolic Diameter and estimated Systolic Pulmonary Artery Pressure in HIE newborns in the first 6 hours found out to be higher significantly compared with the controls ( $p=0,03, p<0001$, respectively). Although there was no degradation in ejection fraction of the patients, the basal values of left and right ventricular systolic and diastolic functions in tissue Doppler measurements were lower comparing with the controls initially which raised significantly after treatment.

Conclusion: The cardiac functions of the newborns with HIE are effected negatively related with the severity of the acidemia. However; it was observed that via MTWA and tissue Doppler measurements, the myocardial dysfunction can be cured with therapeutic hypothermia. Thus, MTWA and tissue Doppler measurements can be used as a marker of myocardial dysfunction for the prognosis of newborns with HIE for further studies.

\section{P-98}

Numerical simulation of blood flow across the fenestration in Total Cavopulmonary Connection

Muskawad S. D., Sharma S. D.

Indian Institute of Technology, Bombay, Mumbai

Introduction: To assess and predict the flow across the fenestration in total cavopulmonary connection using numerical simulation. The provision of fenestration or connecting IVC graft with the atrium is very important to reduce the systemic venous pressure. The reduction in systemic venous pressure is done at the cost of reduced pulmonary blood flow as some volume of the venous return is shunted out via fenestration to the atrium. The flow across the fenestration is function of fenestration area and configuration.

Method: An idealized CAD geometry of extra-cardiac total cavepulmonary connection with atrial chamber is designed. The IVC conduit of $18 \mathrm{~mm}$ diameter follows curvature of atrium, before joining the confluence. The fenestration is at midpoint of IVC graft. The outlet to atrium is placed diametrically opposite to the fenestration site. The inlet boundary conditions at IVC and SVC are in terms of steady volumetric flow. Outflow boundary conditions at LPA and RPA are steady pressures whereas outlet boundary condition at right atrium is pulsatile pressure. The simulation is carried out for the varying diameter of fenestration ( $4 \mathrm{~mm}, 8 \mathrm{~mm}, 12 \mathrm{~mm}, 16 \mathrm{~mm}$ and $20 \mathrm{~mm}$ diameter).

Results and Conclusions: The flow simulation for a given set of atrial, LPA and RPA outlet pressures helps in optimization of fenestration diameter in order to provide adequate pulmonary blood flow. This information is very important for determining appropriate fenestration diameter.

\section{P-99}

MRI lymphatic imaging in patients affected by recurrent chylothorax

Latus H., Mkrtchyan N., Shehu N., Ewert P., Stern H., Meierhofer C. German Heart Centre Munich, Technical University, Germany

Background: Recurrent chylothorax can be due to different underlying causes and is associated with significant morbidity. As the pathogenesis of chylothorax may involve leakage of thoracic duct and other lymphatic vessels, we planned to visualize lymphatic vessel abnormalities in patients with recurrent chylous pleural effusions using magnetic resonance imaging (MRI).

Methods: Eight patients (three with Fontan circulation, two after complex cardiac surgery, three with primary pulmonary disease and normal cardiac anatomy) at a median age of $4.3(0.2-18.7)$ years with recurrent chylothorax resistant to conventional therapies underwent MRI lymphatic imaging using a T2-weighted respirator-navigated and cardiac-gated 3D turbo spin echo sequence. A dynamic contrastenhanced MR lymphangiogram was performed after bilateral inguinal lymph nodes were assessed by conventional venous cannulas and MRI contrast media supplied.

Results: In all but one patient MR lymphatic imaging was possible and demonstrated abnormally dilated paravertebral lymphatic networks connected to the area of pleural effusion. A thoracic duct was identified in 7 of the 8 cases. Abnormal branching of the lymphatic vessels was seen in 6 patients. Contrast enhanced imaging of the lymphatic system was complicated by insufficient local contrast uptake probably due to difficult puncture of small inguinal lymph nodes.

Conclusions: T2-weighted MRI imaging may serve as a noninvasive tool to detect abnormal lymphatic connections to pleural effusions including potential leaks. Contrast enhanced MRlymphangiography may be limited by small subcutaneous lymphatic structures in young patients.

\section{P-100}

Early myocardial modifications in preterm infants

Bordin G., Castaldi B., Padrini M., Nardo D., Favero V., Baraldi E., Milanesi $O$

Department of Women's and Children's Health - University of Padova, Padua, Italy 
Background: Preterm newborns undergo hemodynamic challenges in the postnatal period. The cardiovascular disarray plays a role in the pathophysiology of brain injury in very preterm infants. Speckle tracking echocardiography is a novel and feasible technique to assess cardiac function.

Aims: To investigate the evolution of myocardial performance in the early postnatal period in preterm infants using speckle tracking echocardiography.

Material and Methods: We prospectively performed cardiac ultrasound evaluation in preterm infants $\leq 34$ weeks' gestation, during the first 96 hours of life. Echocardiographic assessment involved left ventricular ejection fraction, mitral $\mathrm{E} / \mathrm{A}$ ratio, $\mathrm{S}^{\prime}$ and $\mathrm{E}^{\prime}$ velocities, E/E' ratio, TAPSE, left atrium-to-aorta ratio, ductal diameter and ductal shunt pattern.

Left ventricular longitudinal, circumferential and radial strain, apex-basal rotation and twist were measured from the apical 4 chamber and short axis views and Calculated by speckle tracking echocardiography.

A second echocardiographic examination was conducted at about 3 weeks of postnatal age.

Results: Thirty-nine preterm infants were evaluated over a 4-month period. The mean gestational age was $30 \pm 2.7$ weeks with a mean birth weight of $1318 \pm 485$ gr.

Deformation parameters were higher in infants with a hemodynamically significant PDA. Apical segments demonstrated higher longitudinal strain than basal and mid ones. In all gestational ages, endocardial longitudinal strain was higher than the epicardial one. Epicardial longitudinal strain significantly increased during the first 3 weeks $(-15.3 \pm 3.6$ vs $-17.4 \pm 2.1 ; p=0.028)$, resulting in the change of basal rotation from counter-clockwise to clockwise and thus in the acquisition of twist $\left(4.9 \pm 4.4^{\circ}\right.$ vs $\left.8.4 \pm 4.8^{\circ}, \mathrm{p}<0.001\right)$. Conclusions: Echocardiographic assessment of myocardial deformation parameters is feasible in preterm infants. Our data suggest that the maturational process of the myocardium might develop from fetal to postnatal period, spreading from endocardial to epicardial layer.

\section{P-101}

How to clinically diagnose a fixed pulmonary hypertension in left-to-right shunts: a medical challenge for humanitarian organizations

Gouton M. (1,2), Lucet V. (1), Bical O. (1), Leca F. (1)

Mécénat-Chirurgie Cardiaque - Paris - France (1); Cardiologie

Congénitale Montsouris - Paris - France (2)

Background: Mécénat-Chirurgie Cardiaque (MCC) is a humanitarian organization whose purpose is to treat in France children from developing countries suffering from congenital heart diseases that cannot be treated in their home country. Their coming is decided solely on the analysis of medical records often without diagnostic certainty. It happens that children arrive in France with a wrong diagnosis or too late, and have to return without surgery. Aim: The aim of this work is to highlight simple diagnostic criteria to suspect fixed PAH in children from developing countries with left-to-right shunt heart disease to avoid an unnecessary coming in France.

Material and methods: we studied retrospectively the files of all children with left-to-right shunt heart disease sent to MCC that gone back without surgery because of a fixed PAH. We analysed only the data that can be available in all countries: skin saturation, clinical examination (heart murmur or not) and chest x-ray.

Results: Since 1996, 51 children with left-to-right shunt heart disease out of 2700 supported by MCC (1.9\%) are returned to their countries without surgery, because of a fixed PAH. In 20 children, the inoperability due to fixed PAH was diagnosed on clinical and echocardiographic data, without heart catheterization: 17 show no more any signs of high pulmonary blood flow, attesting to the fixed $\mathrm{PAH}$. The other 3 were recused because of the high complexity of their disease. Heart catheterization with pulmonary vascular resistance measurement was made in 31 children (15 without any shunt signs), and led to recuse the surgery in all.

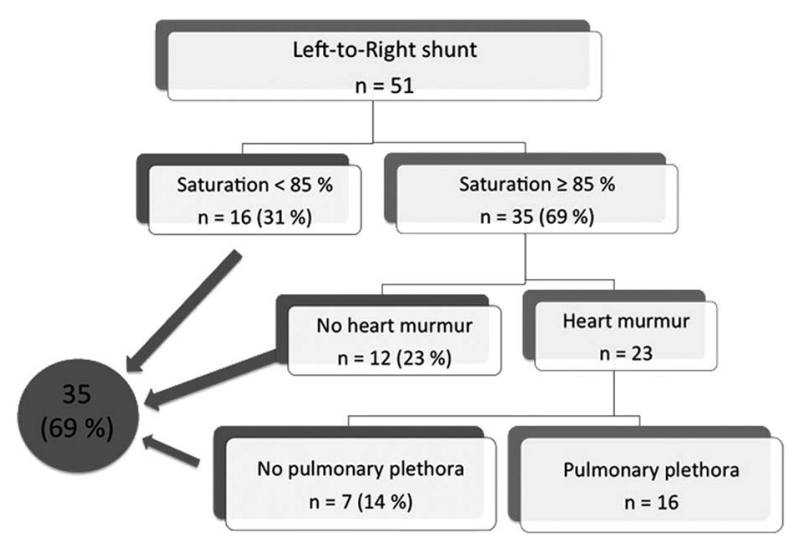

The proposed chart shows that with simple information (saturation $<85 \%$, no heart murmur, absence of pulmonary plethora on chest x-ray), 35 children out of 51 wouldn't have come: only patients with saturation $\geq 85 \%$, heart murmur and pulmonary plethora $(16 / 51)$ remain possibly able to be treated, warranting rapid catheterization.

Conclusion: With simple clinical and radiographic data, 70\% of children having a fixed PAH consecutive to a left-to-right shunt could be detected, allowing humanitarian teams to focus on the $30 \%$ remaining for a rapid and efficious coming to France to win the race against the fixed $\mathrm{PAH}$.

\section{P-102}

Evaluation of myocardial stiffness in healthy adult and hypertrophic cardiomyopathy with HFpEF populations using noninvasive ultrasound shear wave imaging Villemain O. (1), Correia M. (1), Mousseaux E. (2), Baranger J. (1), Zarka S. (2), Podetti I. (1), Soulat G. (2), Hagège A. (2), Tanter M. (1), Messas E. (2), Pernot M. (1)

Institut Langevin, ESPCI, CNRS, Inserm U979, PSL Research University (1); Hôpital Européen Georges Pompidou, Université Paris Descartes (2)

Objectives: Myocardial stiffness is an important prognostic and diagnostic parameter in heart failure. $U p$ to now, there is no noninvasive tools for quantitative evaluation of this parameter. We introduce recently a new ultrasound based technique called Shear wave imaging (SWI) to quantitatively assess the intrinsic tissue stiffness. The goal of our study was to investigate the potential of Myocardial SWI, to quantify noninvasively the diastolic myocardial stiffness (MS, $\mathrm{kPa}$ ) in healthy adult volunteers (HV) and in hypertrophic cardiomyopathy populations with heart failure with preserved ejection function (HCM-HFpEF).

Methods: We included prospectively 80 adults: $60 \mathrm{HV}$ (divided into three groups: $20-40$ yo $(n=20) ; 40-60$ yo $(n=20) ; 60-80$ yo $(\mathrm{n}=20))$ and $20 \mathrm{HCM}-\mathrm{HFpEF}$. An echocardiography, a cardiac magnetic resonance (CMR) and a biological exploration were achieved in all the study population. The MS estimation was 
performed using an ultrafast ultrasound scanner with cardiac phased array, on the basal antero-septal segment during the enddiastole, in long and short axis views. Fractional anisotropy (FA) of shear wave speed was also estimated.

Results: For 20-40, 40-60, and 60-80 yo group respectively, the mean MS was $2.59 \pm 0.58 \mathrm{kPa}, 4.70 \pm 0.88 \mathrm{kPa}, 6.08 \pm 1.06 \mathrm{kPa}$ ( $\mathrm{p}<0.01$ between each group). MS strongly correlated with age $(\mathrm{r}=0.88)$. For the HCM-HFpEF group (mean MS $=12.68 \pm 2.91$ $\mathrm{kPa}$ ), the MS was significantly higher than in the healthy volunteer $\left(\mathrm{p}<10^{-4}\right)$, with a cut-off identified at $8 \mathrm{kPa}(\mathrm{AUC}=0.993, \mathrm{Se}=$ $95 \%, \mathrm{Sp}=100 \%)$. The FA was lower in HCM-HFpEF (mean = $0.170 \pm 0.082)$ than in $\mathrm{HV}(0.289 \pm 0.073), \mathrm{p}<0.01$.

Positive correlations were found between the MS and parameters in echocardiography (E/e', r $=0.783 ; \mathrm{E} / \mathrm{Vp}, \mathrm{r}=0.616$; left atrial volume index, $r=0.623$ ) and CMR (late gadolinium enhancement, $r=0.804)$.

Conclusion: The MS estimation was found to increase with age in healthy population. A MS cut-off of $8 \mathrm{kPa}$ could differentiate healthy and HCM-HFpEF patients. We hope that this new noninvasive parameter will help to better diagnose the diastolic function and its prognosis in clinical practice. (Non-Invasive Evaluation of Myocardial Stiffness by Elastography: NCT02537041).

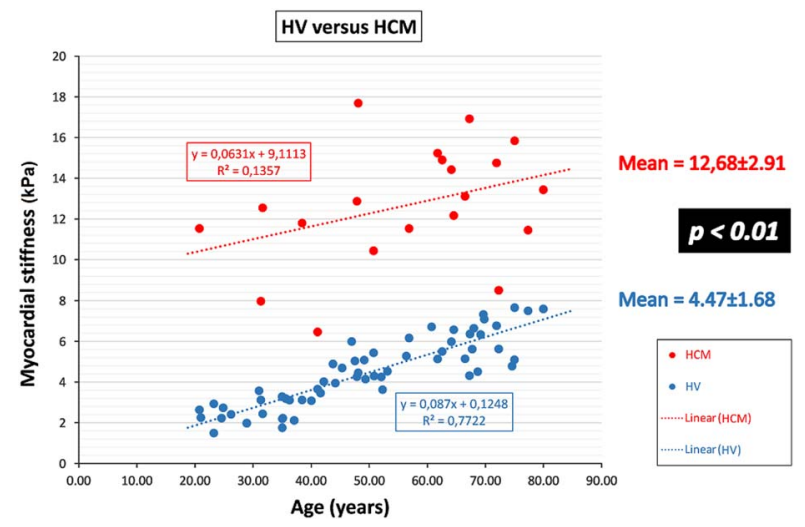

\section{P-103}

Myocardial stiffness assessment using shear wave imaging in healthy children and hypertrophic cardiomyopathy

Villemain O. (1), Correia M. (2), Khraiche D. (1), Pernot M. (2), Bonnet D. (1),

(1) M3C Institut Langevin U979, Paris, France, (2) Necker-Enfants Malades, Paris, France

Background: The evaluation of diastolic left ventricular function is critical for hypertrophic cardiomyopathy (HCM) evaluation. The pathophysiology of HCM is characterized by an increase of ventricular stiffness in most patients. However, myocardial stiffness cannot be measured non-invasively by the existing techniques.

Purpose: The goal of our study was to investigate the potential of Myocardial Shear Wave Imaging (SWI), to quantify noninvasively the passive diastolic myocardial stiffness in a pediatric population of HCM.

Methods: We included prospectively 20 children between five and eighteen years old, 10 healthy volunteer and 10 patients with HCM. A complete clinical echocardiography was as well achieved in all the study population. SWI was performed using an ultrafast ultrasound system (Aixplorer, Supersonic Imagine, France) and a phased-array probe $(2.75 \mathrm{MHz}$ central frequency, 96 elements, SuperSonic Imagine, France) to track and evaluate the shear wave speed (SWS). SWI acquisitions were performed on the basal antero-septal segment during the end of the diastole with ECG triggering, in a short axis (SA) and long axis (LA) views. SWS were compared between the two groups and analyzed with the clinical parameters of echocardiography.

Results: Shear wave imaging was performed successfully in $90 \%$ of healthy volunteer and in 95\% of HCM patients. The mean SA-SWS was $1.15 \pm 0.23 \mathrm{~m} / \mathrm{s}[\min =0.79 ; \max =1.58]$ in the healthy volunteer and $1.95 \pm 0.41 \mathrm{~m} / \mathrm{s}[\mathrm{min}=1.22 ; \max =3.21]$ in the HCM patients $(\mathrm{p}=0.01)$. The mean LA-SWS was $0.94 \pm 0.22 \mathrm{~m} / \mathrm{s}[\min =0.67 ; \max =1.51]$ in the healthy volunteer and $1.81 \pm 0.36 \mathrm{~m} / \mathrm{s}[\mathrm{min}=1.05 ; \max =2.94]$ in the HCM $(p=0.01)$. The averaged SWS is higher in SA than in LA $(p<0.05$ for both group), due essentially to the local elastic anisotropy of the myocardial muscle. Statistical analysis showed good repeatability of SWS measurements. Positive correlations were found between the evaluation of SWS and clinical parameters in echocardiography such as interventricular septum thickness, left ventricular mass, left atrium volume and doppler parameters (E/A, $\mathrm{Ea} / \mathrm{E}, \mathrm{Ea} / \mathrm{Aa})$.

Conclusion: Non-invasive SWI evaluation of diastolic myocardial stiffness can distinguish healthy children and HCM. SWI could be a new clinical parameter for the diagnosis of HCM.

\section{P-104}

Echocardiographic diagnosis of intramural anomalous origin of the left coronary artery from the pulmonary artery: a single center experience

D’ Anna C., Del Pasqua A., Chinali M., Esposito C., Iacomino M., Filippelli S., Carotti A., Iorio F., Drago F., Rinelli G.

Ospedale Pediatrico Bambino Gesù, Rome, Italy

Background: Anomalous origin of the left coronary artery arising from the pulmonary artery (ALCAPA) is a rare and underdiagnosed congenital cardiac anomaly with potentially severe outcome. Transthoracic Echocardiographic (TTE) diagnosis of ALCAPA is challenging, especially in case of intramural course, often requiring additional imaging techniques.

Aim of the study: This study sought to assess the echocardiographic ability in defining coronary anatomy and intramural course in patients with ALCAPA.

Methods: We retrospectively analyzed records of 13 patients that underwent surgical procedure for ALCAPA at our hosdpital from May 1999 to March 2015. We excluded from this study one patients due to the association of caorctation of aorta with perimembranous ventricular septal defect. Data on the remaining 12 patients (45\% males, mean age $66 \pm 65$ months, range 0-201 months) was collected and data were retrieved from the clinical records including TTE evaluation, CAT or MRI study, if performed.

Results: Among the 12 patients, eleven (91\%) were symptomatic, 1 patient was asymptomatic. At diagnosis, the mean left ventricle ejection fraction was 33.5\% (range 10-60\%). Mitral regurgitation was present in all patients (mild in $2(18 \%)$, moderate in $7 \%(64 \%)$, severe in $2(18 \%))$. Three patients $(27 \%)$ underwent further imaging technique including cardiac CAT ( 2 cases) and MRI scan (1 case) before surgery. Echocardiographic accuracy of ALCAPA diagnosis was $91,8 \%$ as it was surgically confirmed in 11 out of the 12 patients. In four patients ALCAPA was associated with intramural course (ALCAPA-IAR). In 2 of these patients ALCAPAIAR was suspected at echocardiogram and then confirmed by surgery. In contrast, in 2 patients diagnosis was made at the time of 
surgery (50\% sensitivity and $90 \%$ specificity). Among the 2 patients with echocardiographic diagnosis of ALCAPA-IAR, a CAT scan was also performed in one case before surgery. It is important to underline that correct echocardiographic diagnosis of intramural course were done in the two most recent patients, thus suggesting the existence of a learning curve needed to suspect this very challenging anatomic phenotype.

Conclusion: Transthoracic echocardiography is a reliable tool in detecting ALCAPA and, after a needed learning curve, also in the diagnosis of ALCAPA-IAR.

\section{P-105}

3D "modeling" and "printing" based on MR and CT data in neonate with complex twisted heart: new frontier for clinical decision and optimal surgical approach

Secinaro A., Muscogiuri G., Tozzi A., Carotti A., Ciliberti P. Bambino Gesu' Children's Hospital, Rome, Italy

Objectives: To produce a three dimensional (3D) anatomical prototype of the heart of a patient with complex congenital heart disease and evaluate clinical usefulness in determining the best therapeutic approach.

Methods: One neonate (male, $2.7 \mathrm{Kg}$ ) with Goldenhar syndrome, right lung hypoplasia, right sided heart, twisted superoinferior ventricles with hypoplastic LV, aortic coarctation and large ventricular septal defect underwent computed tomography angiography (CTA) and cardiovascular magnetic resonance (CMR) in order to better define cardiovascular anatomy and hemodynamic consequence related to the complex malformation. CTA was acquired using post-iodinate contrast ECG-gated highpitch acquisition modality (Somatom Definition Flash, Siemens). CMR sequences were focused on the evaluation of anatomical morphology, flows and function. In particular, CMR anatomy was assessed using a 3D T1 turbo spin echo with respiratory triggering. Both CTA and CMR volumetric sequence were acquired in systolic phase avoiding the artefact due to ventricular tachycardia.

Data achieved from CTA and CMR were combined using a postprocessing software (MIMICS, Materialise) in order to evaluate and isolate cardiac chambers, myocardium, great vessels and proximal coronary arteries.

Results: The left ventricle volumes (indexed LVEDV $21 \mathrm{ml} / \mathrm{m} 2$ ) and aortic forward flow $(>11 / \mathrm{min} / \mathrm{m} 2)$ were considered adequate to support biventricular circulation with no evidence of endomyocardial fibroelastosis at late gadolinium enhancement imaging. Stereolithography (STL) file was obtained from 3D data from CTA and CMR. Wall model was printed out with TuskT (rigid and trasparent) material and divided in three pieces in order to optimize intracardiac views and better define relationship between structures.

VSD septal defect, its margin and its relationship with surrounding structures such as outflow tracts, RV free wall and branching right coronary artery course were identified and three-dimensionally displayed. Surgical LV to aorta connectibility and VSD closure were considered feasible and biventricular repair was successfully performed.

Conclusions: Modern advanced integrated imaging approach supported by new 3D technologies provide additional insight into complex congenital heart disease in neonates, particularly in twisted or criss-cross heart. 3D print models can reduce uncertainties as regards to the patient's specific anatomy and concretely help in the clinical decision-making.

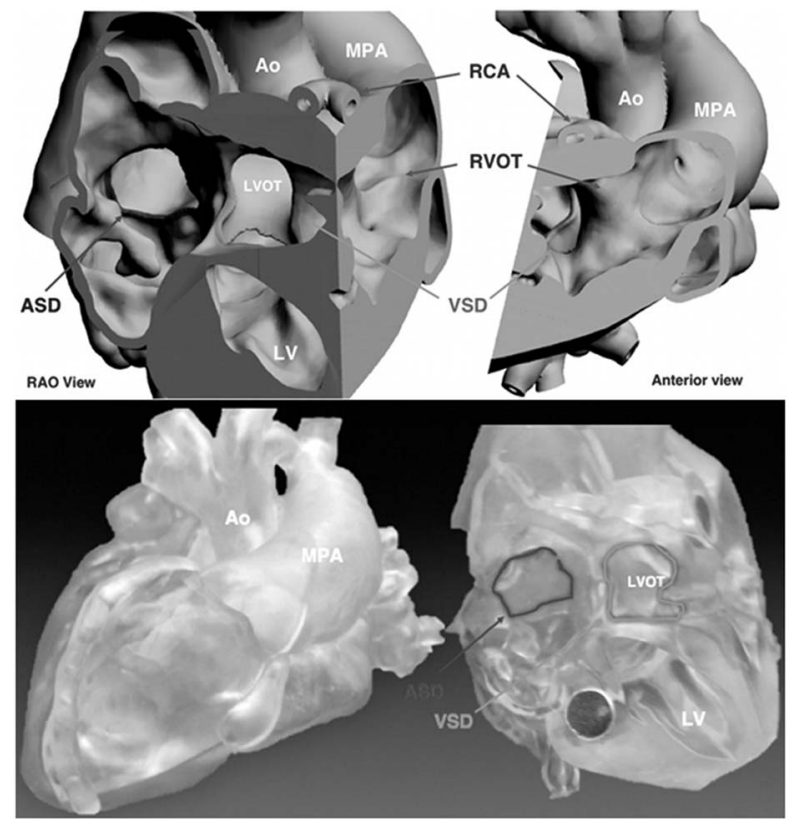

P-106

Cardiac function associated with home-ventilator care in Duchenne muscular dystrophy

Eun L., Kang S.W.

Yonsei University Severance Hospital, Seoul, Korea

Background: Duchenne muscular dystrophy (DMD) is prevalent neuromuscular disorder that affects one in 3600 live male births. Most leading cause of mortality in DMD patients is usually respiratory complications or cardiomyopathy. But nowadays, cardiomyopathy is becoming the leading cause of death in DMD patients because mechanically assisted lung ventilation and assisted coughing help resolving respiratory complication. We investigated to reveal the relation of cardio-pulmonary function in DMD patients.

Method: We retrospectively reviewed the DMD patients diagnosed from 2010 January to 2016 March in Gangnam Severance Hospital. Demographic characteristics, pulmonary function factor, Echocardiography data were assessed.

Results: We reviewed 54 case of DMD and divided into two groups (Home-ventilator assisted group vs Non-ventilator assisted group). In pulmonary function analysis, Home-ventilator group $(1038 \pm 620.41 \mathrm{ml})$ show lower sitting Functional vital capacity than Non-ventilator group $(1455 \pm 603.12 \mathrm{ml})$. In echocardiographic measurement, mean LV Ejection fraction and Fractional shortening were higher in Homeventilator group. Home-ventilator assisted group's E/A ratio $(1.7 \pm 0.44)$ was more stable than Non-ventilator assisted group (2.02 \pm 0.62$)$. With estimation by the multiple linear regression test, TDI S' was higher in Home-ventilator group (Estimated $\beta$ : 1.06, Standard error: 0.48).

Conclusion: DMD patients with ventilator care might have better systolic and diastolic cardiac function. Especially, patients without ventilator assistance may need more meticulous evaluation for cardio-pulmonary function, which may help to care the early cardiopulmonary dysfunction.

\section{P-107}

Pulmonary hypertension is mainly induced by high post-capillary pressure in patients with tetralogy of Fallot after definitive repair

Hamamichi Y., Horimoto Y., Takeguchi M., Mastui T., Saito M., Ishii T., Inage A., Ueda T., Yazaki S., Yoshikawa T.

Sakakibara Heart Institut, Tokyo, Japan 
Introduction: We find pulmonary hypertension $(\mathrm{PH})$ uncommonly in patients with tetralogy of Fallot (TOF) after repair. We predicted increased post-capillary pressure, such as pulmonary capillary-wedge pressure (PCWP) and end-diastolic pressure of left ventricle (LVEDP) would be main cause of $\mathrm{PH}$ which was induced by LV dysfunction. The purpose of this study is to investigate what factors triggered $\mathrm{PH}$ in repaired-TOF patients.

Methods: The medical records of 95 repaired-TOF patients aged from 7 to 53 years were reviewed. We performed cardiac catheterization to perceive hemodynamic state between 2010 and 2015. We defined $\mathrm{PH}(\mathrm{n}=11)$ as mean pulmonary-artery pressure $25 \mathrm{mmHg}$ or over. First, indexes were determined which would affect $\mathrm{PH}$. Second, we compared cardiac performances of right ventricle (RV) between repaired-TOF patients with and without $\mathrm{PH}$.

Results: In monovariate analysis $\mathrm{PH}$ was associated with 5 factors: PCWP ( $\geq 16 \mathrm{mmHg}, \mathrm{p}<0.01$ ); LVEDP $(\geq 18 \mathrm{mmHg}, \mathrm{p}<0.01$ ); 2 or more shunting from systemic artery to pulmonary artery $(p=0.011)$, age at definitive repair $(\geq 9.5$ years, $p=0.017)$; period after definitive repair ( $\geq 16.0$ years, $p=0.043$ ). Contrary to expectation, small pulmonary-artery size and systemic disease were not connected with $\mathrm{PH}$ after surgery. After multivariate analysis $\mathrm{PH}$ was independently related to only high PCWP (odds ratio 24.6). Explanatory coefficient was high (0.43). High PCWP had relation to high LVEDP ( $\mathrm{p}<0.0001)$. Of 11 patients with $\mathrm{PH}, 8$ patients $(73 \%)$ possessed whichever high PCWP or high LVEDP at least. Patients with $\mathrm{PH}$ possessed more compressed RV than non-PH patients: increased pressure-ratio of RV to LV on endsystole (0.74 vs. $0.54, \mathrm{p}=0.0043)$; increased EDP $(13 \mathrm{mmHg}$ vs. $10 \mathrm{mmHg}, \mathrm{p}=0.018)$; decreased ejection fraction ( $40 \% \mathrm{vs} .47 \%$, $\mathrm{p}=0.043)$.

Conclusion: Our study showed high post-capillary pressure, such as high PCWP or high LVEDP, mainly provoked $\mathrm{PH}$ in repairedTOF patients. Patients with $\mathrm{PH}$ possessed more RV overloads. Intensive RV overloads might lead to high post-capillary pressure through interaction between RV and LV. High post-capillary pressure owing to left heart disease is said to have a bad prognosis and needs shaped medical attention. We should search for PH in repaired-TOF patients with intensive RV overloads to set up therapy for it.

\section{P-108 \\ Upper limit of end-diastolic ventricular pressure is very low in Fontan patients which is set by decreased liver function \\ Hamamichi Y., Takeguchi M., Horimoto Y., Mastui T., Saito M., Ishii T., Inage A., Ueda T., Yazaki S., Yoshikawa T. \\ Sakakibara Heart Institut, Tokyo, Japan}

Background: The upper limit of end-diastolic pressure (EDP) of systemic ventricle is said to be approximately $12 \mathrm{mmHg}$ in normal heart. If EDP of systemic ventricle is held in this range, hepatic damage is never provoked by EDP in bi-ventricular heart. In Fontan patients without right-sided ventricle, however, they have the potential to suffer liver disturbance induced by this limit value. We attempt to estimate safety margin of systemic ventricular EDP set by decreased liver function in Fontan patients.

Methods: The medical records of 174 Fontan patients were reviewed from 1 year to 42 years. They underwent cardiac catheterization and routine blood test between 2010 and 2015. Four laboratory data, such as total protein (TP), albumin (Alb), total bilirubin (T-bil), and platelet (Plt), were employed as indexes for liver impairment. We adopted EDP as state variable and 4 hepatic-function data as assay variables. We examined whether the area under a receiving operating characteristics curve (AUROC) was calculated to determine the best discriminating each liverfunction factor for predicting abnormal EDP.

Results: We gained significant AUROC for predicting EDP $\geq$ $13 \mathrm{mmHg}(\mathrm{n}=16)$ by 3 hepatic-function indexes, such as Alb (0.336, $p=0.331)$, TP $(0.300, p=0.008)$, and Plt $(0.329$, $\mathrm{p}=0.025)$. Similarly, we gained significant AUROC for predicting $\mathrm{EDP} \geq 12 \mathrm{mmHg}(\mathrm{n}=26)$ by all indexes, and EDP $\geq$ $11 \mathrm{mmHg}$ by 3 indexes (Alb, T-bil, Plt). Only by Plt we obtained significant AUROC for predicting EDP $\geq 10 \mathrm{mmHg}(\mathrm{n}=60)$, $\mathrm{EDP} \geq 9 \mathrm{mmHg}(\mathrm{n}=85)$, and EDP $\geq 8 \mathrm{mmHg}(\mathrm{n}=98)$. Significant AUROC were not acquired for predicting EDP $7 \mathrm{mmHg}$ or below by any of 4 indexes.

Conclusion: We judged upper limit of EDP was $10 \mathrm{mmHg}$ at a maximum in Fontan patients which was estimated by hepatic disorder. If we adopted only Plt counts as index which indicated liver abnormality, safety margin of EDP went down to $7 \mathrm{mmHg}$ from $10 \mathrm{mmHg}$. If it was, more than half of Fontan patients in this study would have the potential to suffer liver damage by increased EDP. We might lower EDP drastically to avoid liver impairment in Fontan patients.

\section{P-109}

Ventricular interaction provokes diastolic dysfunction of left ventricle in patients with tetralogy of Fallot after repair Hamamichi Y., Horimot Y., Takeguchi M., Mastui T., Saito M., Ishii T., Inage A., Ueda T., Yazaki S., Yoshikawa T.

Sakakibara Heart Institut, Tokyo, Japan

Background: Intensive overload to right ventricle (RV) metamorphoses geometry of left ventricle (LV), which induces LV dysfunction (ventricular interaction). Patients with tetralogy of Fallot (TOF) after repair often possess intensive overloads on right heart. We sometimes encounter diastolic dysfunction of LV (DDLV) in repaired-TOF patients. We predicted DDLV would be provoked by RV overloads in repaired TOF patients.

Methods: The medical records of 117 repaired-TOF patients aged from 1.3 to 53.0 years were reviewed. We performed cardiac catheterizations to grasp hemodynamic status between 2010 and 2015. We defined DDLV $(\mathrm{n}=38)$ as end-diastolic pressure of LV (LVEDP) $13 \mathrm{mmHg}$ or over. First, indexes were determined which would affect DDSV. Second, we compared clinical features between repaired-TOF patients with and without DDLV.

Results: After multivariate analysis DDLV was independently associated with odds ratio of $35.7(\mathrm{p}<0.001)$ for RVEDP $(\geq$ $14 \mathrm{mmHg}), 3.6(\mathrm{p}=0.038)$ for ejection fraction of $\mathrm{RV}(\leq 52 \%)$, and $3.1(\mathrm{p}=0.044)$ for end-diastolic volume of RV $(\geq 163 \mathrm{ml} /$ $\mathrm{m} 2$ ). In monovariate analysis DDLV was additionally related to end-systolic volume of RV ( $\geq 79 \mathrm{ml} / \mathrm{m} 2$ ). Contrary to expectance, elevated end-systolic pressure of $\mathrm{RV}$ had no association with DDLV. Explanatory coefficient was high (0.49). In DDLV group, patients were operated at higher age ( $\geq 3.0$ years; $57 \%$ vs. $34 \%, p=0.014)$, and advanced in age ( $\geq 17.0$ years; $63 \%$ vs. $25 \%$, $\mathrm{p}<0.0001)$. They had lower cardiac output $(<2.6 \mathrm{~L} / \mathrm{min} / \mathrm{m} 2$; $57 \%$ vs. $16 \%, p<0.0001)$, and low ejection of LV ( $\leq 50 \% ; 28 \%$ vs $7 \%, \mathrm{p}=0.0052)$. The rate of patients with DDLV was only $16 \%$, if patients had risk factors 0 or 1 ; it was $53 \%$, if patients had risk factors 2 or more. Clinically, repaired-TOF patients with DDLV had some form of symptoms more than those without DDLV (39\% vs. $6 \%, \mathrm{p}<0.0001)$.

Discussion: Our study showed DDLV in repaired-TOF patients was independently associated with abnormal RV function: elevated EDP, decreased ejection fraction, and increased enddiastolic volume. Overloads of RV in repaired-TOF patients 
would be related to DDLV through ventricular interaction. Patients with DDLV fell into lower cardiac output. We should attention DDLV in repaired-TOF patients with intensive RV overloads.

\section{P-110 \\ $\mathrm{N}$-terminal pro-brain natriuretic peptide elevation in Kawasaki disease with clinically normal cardiac function: Relation with cytokines}

Kato M., Ayusawa M., Watanabe H., Komori A., Abe Y., Nakamura T., Kamiyama $H$.

Department of Pediatrics and Child Health, Nihon University, Tokyo, Japan

Background: N-terminal pro-brain natriuretic peptide (NTproBNP) is often elevated in patients with Kawasaki disease (KD) that shows clinically normal cardiac function in acute phase, and is reported as a marker for diagnosis, as well as an index of heart failure. Cardiac function in acute phase of KD has not been investigated 3D speckle tracking.

Objectives: We sought the cause of NTproBNP elevation by investing the correlation NTproBNP with cytokines and 3DGLS.

Methods: Forty-eight patients in our hospital in 2015 were studied. Age ranged from $3 \mathrm{~m}$ to $11 \mathrm{y}$. We measured NTproBNP, TNF $\alpha$, its receptors, IL-6, IL-10 and indices of cardiac functions simultaneously before and 1 week after immunoglobulin treatment (IVIg).

Results: The mean plasma NTproBNP before/after IVIg were $750 / 132 \mathrm{pg} / \mathrm{ml}$, TNF $\alpha$ were $4.6 / 2.9 \mathrm{pg} / \mathrm{ml}$, sTNFR 1 and 2 were $2827 / 1112 \mathrm{pg} / \mathrm{ml}$ and $6617 / 3143 \mathrm{pg} / \mathrm{ml}$, IL-6 were $101 / 9 \mathrm{pg} / \mathrm{ml}$, IL-10 were $32 / 8 \mathrm{pg} / \mathrm{ml}$. The cytokines after IVIg were significantly lower than before IVIg $(\mathrm{P}<0.01)$. 2DEF were 70.5 / $75.9 \%$, 3DEF were $66.8 / 69.2 \%$, GLS were $-19.8 /-21.3 \%$. GLS was not correlated with NTproBNP, but 3DEEF was weakly correlated. Correlation between sTNFR's and NTproBNP were sTNFR $(\mathrm{r}=0.57 / 0.48), \quad$ TTNFR2 $(\mathrm{r}=0.35 / 0.59)$, and IL-6 $(r=0.65 / 0.42)$, which showed significant correlations.

Conclusion: Although correlations between NTproBNP and 3D cardiac function were not apparent, correlation of NTproBNP and TNF-family was distinct. NTproBNP can be a marker reflecting inflammation of $\mathrm{KD}$ even in a case without clinically significant cardiac dysfunction.

\section{P-111}

3D transthoracic echocardiography to assess ventricular septal defect anatomy and severity

Hadeed K., Hascoet S., Amadieu R., Karsenty C., Dulac Y., Acar P., Pediatric cardiology unit, Children Hospital, Toulouse, France.

Background: Ventricular septal defect (VSD) is assessed classically by bi-dimensional transthoracic echocardiography (2D-TTE) which seems to underestimate defect size. Our aim was to compare VSD size and morphology obtained by 2D-TTE with those obtained by three-dimensional transthoracic echocardiography (3D-TTE), and to correlate morphological parameters with shunt severity. Methods: 48 patients with muscular (22.9\%) and membranous (77.1\%) VSDs were included (median age 21.4 months). Patients were classified according to shunt severity. Type 1 minor shunt, $2 \mathrm{a}$ moderate shunt, and $2 \mathrm{~b}$ severe shunt. Minimal (min-2D) and maximal $(\max -2 \mathrm{D})$ diameters were assessed using 2D-TTE. Minimal (min-3D), maximal (max-3D) diameters, systolic (sVSDA) and diastolic (dVSDA) areas of the VSD, and aortic valvular area (AVA) were obtained from 3D full volume dataset.
Results: Max-3D was higher than Max-2D diameter $(\mathrm{p}<0.0001)$. The difference was less between min-3D and min-2D diameters although it remained significant $(p=0.001)$. VSDs were asymmetric with a mean asymmetry ratio $2.3 \pm 1.4$ by $3 \mathrm{D}-\mathrm{TTE}$. Asymmetry ratio was higher in muscular than in membranous VSDs. Mean VSD surface area variation was $32 \%$. It was significantly higher in muscular than in membranous VSDs $(p=0.0001)$. VSD severity was better correlated with minimal rather than maximal diameter. Better correlation was found using the defect area (sVSDA/BSA ratio, sVSDA/AVA ratio; $r=0.60$, $p=0.0008 ; r=0.63, p=0.0002)$. Shunt severity was inversely correlated with the asymmetry ratio. The best parameter to predict shunt severity was sVSDA/AVA ratio.

Conclusion: 3D TTE allows a precise morphological and quantitative assessment of VSD. sVSDA/AVA ratio is an accurate diagnostic tool to predict shunt severity.

\section{P-112}

\section{CMR Feature Tracking shows Preserved Myocardial} Deformation Long-term after Coarctation Repair Dijkema E.J. (1), Slieker M.G. (2), Leiner T. (3), Breur J.M.P. (1), Grotenhuis H.G. (1)

Wilhelmina Children's Hospital, The University of Utrecht, Utrecht, The Netherlands (1), The Radboud University Medical Center, Nijmegen, The Netherlands (2), University Medical Center Utrecht, Utrecht, The Netherlands (3)

Background: Arterial vasculopathy and residual aortic obstruction can lead to left ventricular (LV) dysfunction in patients with coarctation of the aorta $(\mathrm{CoA})$ due to adverse ventriculo-arterial coupling. This study aimed to investigate potential differences in LV myocardial deformation indices between well-repaired CoApatients and healthy controls.

Methods: Twenty-two well-repaired CoA patients (age $30 \pm 10.6$ years) after surgical repair (12) or balloon angioplasty (10) (at 0.316 years) with $>10$ years of follow-up were compared to 22 healthy matched controls (age $30 \pm 3.8$ years). Five CoA patients had been treated for re-coarctation. CMR-FT was used for LV longitudinal-, circumferential- and rotational deformation indices. Results: Global systolic LV function was preserved in CoA patients (LV ejection fraction $58 \pm 4.8 \%$ vs. $60 \pm 6.8 \%, p=0.56$ ) when compared to controls, with normal indexed LV end-diastolic volume $(93 \pm 15.2 \mathrm{ml} / \mathrm{m} 2$ vs. $93 \pm 9.3 \mathrm{ml} / \mathrm{m} 2, \mathrm{p}=0.79)$, indexed $\mathrm{LV}$ end-systolic volume $(26 \pm 7.0 \mathrm{ml} / \mathrm{m} 2$ vs. $31 \pm 7.3 \mathrm{ml} / \mathrm{m} 2$, $\mathrm{p}=0.05)$ and indexed LV mass $(68 \pm 8.5 \mathrm{~g} / \mathrm{m} 2$ vs. $63 \pm 8.6 \mathrm{~g} / \mathrm{m} 2$, $\mathrm{p}=0.08$ ). Twelve CoA patients (55\%) were hypertensive (systolic and diastolic blood pressure of $144 \pm 9.3 \mathrm{mmHg}$ and $80 \pm 6.0 \mathrm{mmHg}$, respectively) of whom 4 were on antihypertensive medication.

LV global longitudinal strain was preserved in the horizontal $(-18 \pm 4.4 \%$ vs. $-16 \pm 4.7 \%, p=0.06)$ and vertical $(-22 \pm 5.1 \%$ vs. $-20 \pm 6.0 \%, \mathrm{p}=0.22)$ long axis in CoA patients. Global circumferential strain was preserved at basal $(-29 \pm 4.1 \%$ vs. $-28 \pm 4.8 \%$, $\mathrm{p}=0.43)$, mid-ventricular $(-27 \pm 4.2 \%$ vs. $-25 \pm 3.0 \%, \mathrm{p}=0.09)$ and apical levels $(-35 \pm 7.8 \%$ vs. $-32 \pm 34.9 \%, p=0.32)$. Segmental increase of circumferential strain was seen in the basal-anterior $(-28 \pm 10.1 \%$ vs. $-21 \pm 9.6 \%, \mathrm{p}=0.03)$ and the mid-posterior $(-23 \pm 6.3 \%$ vs. $-27 \pm 7.3 \%, p=0.04)$ segments. No differences were found in global torsion $\left(2.4 \pm 1.3^{\circ} / \mathrm{cm}\right.$ vs. $2.0 \pm 1.4^{\circ} / \mathrm{cm}$, $\mathrm{p}=0.28)$, twist $\left(14 \pm 5.8^{\circ}\right.$ vs. $\left.12 \pm 6.3^{\circ}, \mathrm{p}=0.34\right)$ and recoil rate $\left(-17 \pm 9.7^{\circ} / \mathrm{cm} / \mathrm{s}\right.$ vs. $\left.-17 \pm 7.1^{\circ} / \mathrm{cm} / \mathrm{s}, \mathrm{p}=0.97\right)$. Analysis of intra-observer variability demonstrated good reproducibility for all deformation indices.

Conclusion: Global and regional myocardial deformation indices are preserved in well-repaired CoA patients long-term after repair. 


\section{P-113}

Prognostic significance of myocardial ischemia detected by single-photon emission computed tomography in children with hypertrophic cardiomyopathy

Ziolkowska L., Boruc A., Sobielarska-Lysiak D., Paszkowska A., Brzezinska-Rajszys G.

Department of Pediatric Cardiology, The Children's Memorial Health Institute, Warsaw, Poland

Background: Myocardial ischemia caused by microvascular dysfunction is an important pathophysiologic component of hypertrophic cardiomyopathy (HCM), promoting myocardial fibrosis, adverse LV remodeling and impacting on clinical course and outcome in HCM patients. The aim of study was to assess the prevalence and clinical significance of myocardial ischemia in children with HCM using 99mTc-MIBI single-photon emission computed tomography (SPECT).

Methods: Seventy-nine children with HCM, median age 13.4yrs (IQR 9.9-15.5yrs), underwent SPECT evaluation from January 2006 to January 2015. Imaging were performed at rest and after maximal exercise.

Regional perfusion defects were identified in 59 of the 79 children (75\%; gI), median age 13.6yrs (IQR 10.5-15.9yrs). Fixed perfusion defects were evident in 21 patients. In 38 children perfusion defects were present only during exercise and they completely resolved at rest. In 20 of the 79 children $(25 \%$; gII), median age 11.8yrs (IQR 7.9-15.2yrs) myocardial perfusion defects were not detected. Patients demographics, cardiovascular events, ECG, 24-h Holter ECG and myocardial fibrosis in CMR were analyzed and compared between the groups. The clinical endpoints were defined as cardiovascular events: sudden cardiac death (SCD), HF-related death, appropriate ICD discharges (ICDdx), resuscitated cardiac arrest (rCA), heart transplant $(\mathrm{HTx})$, nonsustained ventricular tachycardia (NSVT), syncope, progression of heart failure to NYHA class III.

Results: During a median follow-up period of 6.1yrs (IQR $3.7-7.4 \mathrm{yrs})$, in children with myocardial ischemia significantly more often clinical endpoints occurred (39 vs $6 ; p=0.048$ ) and significantly more patients reached a clinical endpoint (28 [47\%] vs $4[20 \%] ; p=0.03$ ).

In children with myocardial ischemia, the following cardiovascular events occurred: SCD $(n=2), \operatorname{ICDdx}(n=2)$, rCA $(n=1)$, HFrelated death $(n=1), \operatorname{HTx}(n=1)$, NSVT $(n=12)$, syncope $(n=11)$, progression to NYHA class III $(n=9)$. In children with myocardial ischemia significantly more frequent myocardial fibrosis (68\% vs $25 \%$; $p=0.019)$ was detected.

Conclusions: (1) Myocardial perfusion defects may reflect an ischemic process which affects the clinical manifestations in children with HCM. (2) Myocardial ischemia is an important predictor of adverse clinical events and risk of death in children with HCM. (3) Myocardial ischemia in HCM patients frequently correlates with myocardial fibrosis.

\section{P-114}

The unnatural course of right ventricular dilatation in patients with severe pulmonary regurgitation after repair of Tetralogy of Fallot

Hoelscher M. (2,3), Bonassin F. (1,2,3), Oxenius A. (1,2,3), Greutmann M. (1,2,3) Leonardi B. (4), Kellenberger C.J. (3,5), Valsangiacomo Buechel ER. $(2,3)$

1 Clinic for Cardiology, Cardiovascular center, University Hospital Zurich, Switzerland, 2 Paediatric Heart Centre, University Children's Hospital, Zurich, Switzerland, 3 Children's Research Centre, University Children's Hospital, Zurich, Switzerland, 4 Department of Cardiology and Cardiac Surgery Bambino Gesù Children's Hospital, IRCCS Rome, Italy, 5 Department of Diagnostic Imaging, University Children's Hospital, Zurich, Switzerland

Background: Pulmonary valve regurgitation (PR) and related right ventricular (RV) dilatation are frequent sequelae after surgical repair for Tetralogy of Fallot (TOF). Timing of pulmonary valve replacement is still subject of debate and little is known about the rate of progression of $\mathrm{RV}$ dilatation. We sought to analyze the natural course of RV dilatation using serial magnetic resonance (CMR) data.

Methods: This retrospective multicentric case control study included all TOF patients with significant PR and RV dilatation, who underwent serial CMR scans between 2002 and 2016 without any intervention between CMR exams. RV volumes and degree of PR were quantified in both centers using the same CMR technique. Clinical and volumetric CMR data were analyzed regarding progression of ventricular dilation and related potential risk factors.

Results: 87 consecutive patients (82 TOF, 3 DORV, 2 PA/VSD) underwent at least 2 (range 2-6) CMR examinations. Median age at first CMR was $13.7 \mathrm{yrs}$ (range $1.4-49 \mathrm{yrs}$ ), time interval to last CMR was 3.3 yrs (6 months-10.5 yrs).

In the overall group no significant change was observed between first and last CMR for RV end-diastolic volume (RVEDV $151 \pm 32$ vs $155 \pm 34 \mathrm{ml} / \mathrm{m} 2$ ), RV end-systolic volumes (RVESV $79 \pm 28$ vs $80 \pm 28 \mathrm{ml} / \mathrm{m} 2$ ), RV function (EF $49 \pm 8 \%$ vs $49 \pm 8 \%$ ) Progression of dilatation, defined as an increase $>20 \mathrm{ml} / \mathrm{m} 2$, occurred in 20 patients $(22 \%)$. In this group RVEDV increased from $153 \pm 31 \mathrm{ml} / \mathrm{m} 2$ to $183 \pm 30 \mathrm{ml} / \mathrm{m} 2$ ( $\mathrm{p}<0.001$ ); RVESV changed from $89 \pm 33 \mathrm{ml} / \mathrm{m} 2$ to $105 \pm 30 \mathrm{ml} / \mathrm{m} 2 \quad(\mathrm{p}<0.001)$; ejection fraction did not deteriorate significantly $(46 \pm 9 \% \%$ vs $46 \pm 7 \%$ ). Presence of a transannular patch, initial RV volume and age at repair were not predictive for progressive RV dilatation. In contrast longer time interval between repair and first CMR ( $p$ 0.01 ) and to last CMR (p 0.03) were correlated to increasing RV dilatation.

Conclusion: During a follow-up of 3.3 yrs, only in $22 \%$ of all TOF patients with PR experience significant RV dilatation.

Time since surgical repair was the only factor predictive for progression of dilatation.

\section{P-115}

Endovascular Embolization In Children With Pulmonary Sequestration

Aypar E., Karagöz T., Emiroglu N., Gümüstas M., Ozcelik U., Yalcın E., Dogru D., Kiper N., Celiker A., Demircin M., Dogan R., Alehan D.

Hacettepe University Hospital, Ankara, Turkey

Introduction: Pulmonary sequestration (PS) is a very rare congenital pulmonary malformation, which is a cystic or solid mass composed of nonfunctioning primitive tissue that does not communicate with the tracheobronchial tree and has anomalous blood supply from systemic circulation rather than pulmonary circulation. The ideal treatment strategy for PS, whether surgery or embolization is not clearly identified. In literature, This study aimed to explore clinical features, diagnosis and outcomes of children who had PS and had endovascular embolization of the aberrant systemic arterial supply.

Methods: Clinical records of children who had PS and had embolization between 2000 and 2016 were analyzed.

Results: 13 patients had definite diagnosis of PS and had embolization. Median age: 12 months (15 days-15 years), weight: $8 \mathrm{kgs}$ 
(3-54), male/female: 8/5. Patients presented with recurrent pneumonias (6/13), persistent cough (3/13), murmur (2/13), dextroposition of heart $(1 / 13)$, cystic lesion in prenatal ultrasound $(1 / 13)$. Chest X-ray showed dextroposition of heart in $8 / 13$. First diagnosis was made by CT angiography in 5/13 and all confirmed by conventional angiography. Associated lesions were present in 11/13 patients; Scimitar syndrome (5 patients), hypoplasia of right PA and right lung (7 patients), secundum ASD (3 patients), aortic coarctation (2 patients), PDA (1 patient). Most of the PSs were located on the right side (10/13). Aberrant supply was mostly from abdominal aorta (11/13). 3/13 patients had more than one anomalous arterial supply. All patients had successful embolization without any catheteter related complications. Devices used were Amplatzer Vascular Plugs (5/13), different coils (6/13), Amplatzer Duct Occluder II (3/13), glue (cyanoacrylate) (1/13). Multiple devices were necessary in 2/13. Mean procedure time: $84 \pm 29 \mathrm{~min}$ (30-135), fluoroscopy time: $16 \pm 9 \mathrm{~min}(6-31) .5 / 13$ patients required surgery for associated lesions, (4/13 for Scimitar syndrome, 1/13 for PDA). 2/13 patients required segmentectomy and $1 / 13$ required lobectomy after embolization. Median followup period was 18 months ( 2 months- 8 years), 2/13 patients were lost to follow-up. There were three deaths unrelated to the procedure.

Conclusions: Clinicians must consider PS in patients with recurrent pneumonias, persistent cough, dextroposition of heart and Scimitar syndrome. CT angiography has high accuracy in the diagnosis of PS. Endovascular embolization is a safe and effective treatment in PS. Surgery related complications are avoided. If surgical resection is necessary, risk of vascular complications is greatly reduced with this procedure.

\section{P-116}

Transcatheter Embolization Of Coronary Artery Fistulas in Pediatric Patients:Clinical, Angiographic Findings And Follow-Up Results Of A Single Center

Aypar E., Karagoz T., Celiker A., Aykan H.H., Guvener M., Demircin $M$.

Hacettepe University Hospital, Ankara, Turkey

Introduction: Coronary artery fistulas (CAF) can be defined as a direct connection between a coronary artery and one of the cardiac chambers, large vessels or other vascular structures. CAFs have a $0.002 \%$ incidence among general population, $0.1 \%$ of coronary anomalies. Clinical presentation of CAF varies depending on size of fistula, age of patient, presence of myocardial ischemia. Most patients with CAF are asymptomatic during the first two decade, some children may present with murmur. Complications include 'steal' from the adjacent myocardium, thrombosis/embolism, heart failure, atrial fibrillation, rupture, endocarditis/endarteritis, arrhythmias. Therefore closure of CAFs are recommended even in asymptomatic patients.

Methods: We retrospectively analyzed clinical, angiographic findings, follow-up results of CAF patients diagnosed between 20002016.

Results: 26 patients had angiographic diagnosis of CAF, mean age: $6.5 \pm 5.8$ years ( 3 days-17 years), weight: $25 \pm 21 \mathrm{kgs}$ (3.5-67), male/female: $14 / 12$. 13/26 patients presented with murmur, 10/ 26 were incidentally diagnosed during echocardiography, 3/26 presented with dyspnea. Associated cardiac anomalies were present in $3 / 26$ patients. $24 / 26$ patients had single, $2 / 26$ had multiple CAF. Right and left coronary artery were involved in $8 / 26$ and $18 / 26$ patients, respectively.13/26 patients had coronary-cameral, $7 / 26$ had coronary artery-pulmonary artery, 4/26 had coronary artery-left atrium, 2/26 had coronary artery-right atrium fistula.
CAFs were embolized in 14/26 patients, successful in 12/14 (86\%) patients, partially successful in one, unsuccessful in one. Amplatzer Vascular Plugs were used in 6/14 patients, different coils used in 6/ 14 patients, Amplatzer Duct Occluder II Additional size device was used in one patient, glue (cyanoacrylate) was used in one. Mean procedure time: $112 \pm 64 \mathrm{~min}$ (45-220), fluoroscopy time: $27 \pm 16 \mathrm{~min}(8-60)$. No catheteter related complications were observed. Surgery were performed in $3 / 26$ patients (one for associated cardiac anomalies, two patients for unsuccessful embolization). $9 / 26$ patients had clinical follow-up without intervention due to small fistula size. Mean follow-up period was $3.9 \pm 3.7$ years (1 month-14 years), two patients were lost to follow-up, No patients with intervention had recurrence of fistula. Patients who had clinical follow-up had no complications asscociated with fistula during the follow-up. Control angiography were performed in 4 patients after successful embolization, all had remodeling, regression of the size of the involved coronary artery.

Conclusions: Transcatheter embolization of CAFs is an effective and safe procedure in pediatric patients. Follow-up is necessary for early diagnosis of recurrence of fistula.

\section{P-117}

Retrieval of Intravascular Foreign Bodies by Transcatheter Procedure

Duman D., Aykan H., Karagöz T., Behbudov V., Sel K., Gümüştaş M., Aypar E., Özkutlu S.

Hacettepe University Medical Faculty Pediatric Cardiology Department, Ankara- Turkey

Introduction: Nowadays as the common usage of venous port catheteters in the treatment of malignency and the invasive cardiac cateheterization procedures in pediatric pateints have been rising in quantity; the complications via these practices occurs widely. The embolisation of port catheteters, interventional devices, guide wires, stent types and/or other associated materials into the intracardiovascular structures is one of the important complications. We aimed to share our experiences about removal of these embolized intravscular foreign bodies (IVFB) by transcatheteter method.

Method: We evaluated 15 patients retrospectively in whom transcatheteter retrieval of intravascular foreign body had been performed between the years 2004-2016.

Results: We summarized the characteristic features of 15 patients in table 1 . The mean age of the patients were 9,8 years $(1,5-18)$ and the average weight was calculated as $37,3 \mathrm{~kg}(8-85)$. The mean process and scopy time was 67 minutes $(\mathrm{mn})(20-225)$ and $17,6 \mathrm{mn}$ $(2-69,5)$ respectively. The embolisation location of IVBF was toward to pulmonary artery in 4 patients, to right ventricle in one patient and to inferior venae cavae, superior venae cavae (SVC) and right atrium in the rest of the patients. The kind of embolised foreign bodies were venous port catheteter fragments in 12 patients, VSD and PDA occluder devices in 2 patients and proximal part of the coronary baloon catheter in one patient. 5 of 12 oncologic patients diagnosed as they were receiving a treatment and in the7 patients distal part of port catheter was broken away while taking off it. In 7 of those 12 patients, port catheter seemed to be mobile comparing with the rest of 5 patients whose cateheters estimated to be stuck on vessel. We used 6-11 french and long sheaths, snare, pigtail and cournand catheteters. The IVFB retrieval became unsuccessful in 2 patients due to cohesiveness of port catheteters and additonally obstruction of SVC by thrombosis in one patient. There were no complication up to the processes. Conclusion: Transcatheter retrieval of IVFB is an effective, realiable and alternative therapy to surgery. It must be hold in mind that 
especially removing the chronic adherent residual port catheteter pieces may lead to vascular injury up to traction maneuverings.

\begin{tabular}{|c|c|c|c|c|c|c|c|}
\hline No & $\begin{array}{l}\text { Age } \\
\text { (year) }\end{array}$ & $\begin{array}{l}\text { Weight } \\
\text { (kg) }\end{array}$ & Diagnosis & $\begin{array}{l}\text { Embolized } \\
\text { Substance }\end{array}$ & $\begin{array}{l}\text { Emboliza- } \\
\text { tion } \\
\text { Region }\end{array}$ & Success & $\begin{array}{l}\text { Complica- } \\
\text { tion }\end{array}$ \\
\hline 1 & 14 & 40 & NHL & PORT & $\mathrm{VCI}$ & YES & NO \\
\hline 2 & 1,5 & 13 & HBM & PORT & RV & YES & $\mathrm{NO}$ \\
\hline 3 & 6 & 17 & NBM & PORT & VCS & YES & NO \\
\hline 4 & 8 & 29 & ALL & PORT & VCS & YES & $\mathrm{NO}$ \\
\hline 5 & 7 & 30 & ALL & PORT & VCS & YES & $\mathrm{NO}$ \\
\hline 6 & 8 & 30 & MBL & PORT & PA & YES & $\mathrm{NO}$ \\
\hline 7 & 16 & 85 & PNET & PORT & VCS & NO & $\mathrm{NO}$ \\
\hline 8 & 4 & 20 & TOF & CTHTR & VCI & YES & NO \\
\hline 9 & 18 & 61 & VSD & PFM & PA & YES & $\mathrm{NO}$ \\
\hline 10 & 1,5 & 8 & PDA & COIL & $\mathrm{PA}$ & YES & $\mathrm{NO}$ \\
\hline 11 & 17 & 70 & AML & CTHTR & VCS & YES & $\mathrm{NO}$ \\
\hline 12 & 15 & 62 & NHL & CTHTR & VCS & NO & $\mathrm{NO}$ \\
\hline 13 & 6 & 15 & NBL & CTHTR & $\mathrm{PA}$ & YES & NO \\
\hline 14 & 7,5 & 28 & ALL & CTHTR & VCI & YES & $\mathrm{NO}$ \\
\hline 15 & 11 & 32 & ALL & CTHTR & $\mathrm{RA}$ & YES & NO \\
\hline
\end{tabular}

NHL, non-Hodgkin Lymphoma; HBM, hepatoblastom; ALL, acute lymphoblastic leukemia; AML, acute myeloblastic leukemia; PNET, primitiv neuroectodermal tumor; TOF, Fallot tetralogy; VSD, ventricular septal defect; PDA, patent ductus arteriosus; CTHTR, catheter; RA, right atrium; VCI, venae cava inferior; VCS, venae cavae superior; PA, pulmonary artery; RV, right ventricul.

\section{P-118}

Transcatheter closure of a Right Pulmonary Artery to Left Atrial Fistula using the Amplatzer Muscular VSD Occluder Device

Gümüştaş M., Karagöz T.

Hacettepe University, Pediatric Cardiology, Ankara, Turkey

A fistula formation between the right pulmonary artery (RPA) and left atrium (LA) is a very rare cyanotic congenital heart disease and the traditional treatment involves surgery. We presented the successful closure of the RPA to LA fistula using the Amplatzer Muscular VSD occluder in a child with cyanosis.

Case report: A 4-year-old girl was admitted with cyanosis and heart murmur. She had severe cyanosis, digital clubbing and effort intolerance (NYHA Class II-III). Cardiac MRI and MR Angiography showed a congenital fistula between the RPA and LA. Diagnostic cardiac catheterization was performed. The RPA showed dilatation and the inferior branch of the RPA, along with the right pulmonary veins via a large fistula, was draining to an aneurysmatic sac and then to the left atrium. A guide wire was advanced into the sac passing through the fistula via the RPA and the small ASD antegradely. An arteriovenous loop was created. The narrowest part of the fistula was $11 \mathrm{~mm}$ in balloon sizing. A 12-mm Amplatzer Muscular VSD occluder device was deployed at the narrowest site and the fistula was closed successfully (Figure 1) and control angiography demonstrated complete occlusion of the fistula. After the procedure, arterial oxygen saturation in room air increased from $63 \%$ to $97 \%$. The patient was asymptomatic on follow-up evaluations at 3, 6, 12, 18 and 30 months with a mean oxygen saturation of $97 \%$.

Discussion: A congenital RPA to LA fistula is a rare congenital heart disease that is typically associated with a normal cardiovascular system examination except for central cyanosis. There have been only a few cases reported in the literature where fistula closure using the Amplatzer Muscular VSD occluder device. In select cases, transcatheter closure appears to be a safe and effective alternative to surgical treatment.

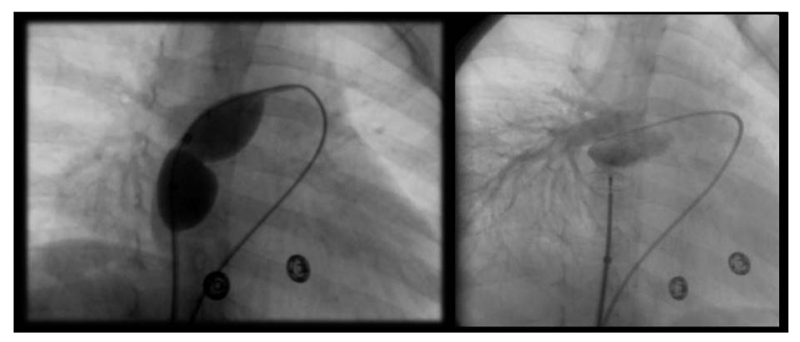

Figure1.

A. Balloon sizing of the fistula, B. Pulmonary arteriogram in the posteroanterior projection shows complete occlusion of the fistula by the Amplatzer Muscular VSD occluder device.

\section{P-119}

Endovascular Treatment of Aortic Coarctation: Early and Mid-term Results

Varan B (1), Unsal H (2), Erdogan I (1), Gokdemir M (1), Oktay A (1), Yakut K (1), Tokel K (1)

Baskent University Ankara Turkey (1); Zekai Tahir Burak Hospital Ankara Turkey (2)

Objective: To evaluate early and mid-term outcome of endovascular stent treatment of aortic coarctation in children and adolescents.

Patients and Methods: Twenty-five patients with aortic coarctation who underwent stent implantation between 2008-2015 were retrospectively evaluated (18 male, 7 female). Mean age was $13 \pm 3.8$ years (5-21), and weight was $46.6 \pm 16.2 \mathrm{~kg}(20-73)$. Eight patients had (32\%) native, 17 had $(68 \%)$ residual or recoarctation. Covered stents were implanted in 17 and bare in 8 patients. Computed tomography was made in 3-6 months.

Results: Catheter-measured gradient before and after the procedure were $36 \pm 16.3(15-83)$ and $7.0 \pm 6.9(0-25) \mathrm{mmHg}$, respectively. Early complications were observed in four patients: stent migration, pseudoaneurysm next to femoral artery, arteriovenous fistula and femoral artery injury requiring surgical intervention in one patient each. Mean follow-up time was $15.3 \pm 14$ months. Computed tomography showed no problems. Cardiac catheterization and angiography was performed in 9 patients. Stent fracture was detected in one patient after balloon dilatation of the stent. Two patients were shown to have aneuryms at the border of the covered stents. One underwent surgery for proximal residual coarctation and aneurysm repair, the other underwent second covered stent implantation. Four patients required balloon dilatation of their stent.

Conclusion: Stent implantaion is an effective treatment for native and recurrent aortic coarctation in children and adolescents. Early and late complications may occur thus cautious follow-up is necessary.

\section{P-120}

Catheter Closure of Patent Ductus Arteriosus in Adult Patents Using an Outpatient Protocol

Thanopoulos B. (1), Dardas P. (1), Ninios V. (1), Giannopoulos A.

(2), Giannakoulas G. (2), Deleanou D. (3), Lancovici S. (3)

"Agios” Lukas Klinic, Thessaloniki, Greece (1), 1 Ahepa”University Hospital,

Thessaloniki, Greece (2) Ares Medical Center, Bucharest, Romania (3)

Introduction: Transcatheter closure is the treatment of choice for the majority of patients with a patent ductus arteriosus (PDA). However, the standard technique of this procedure uses an arterial access and requires immobilization of the patients for 24 hours and may be associated with arterial complications. The aim of this study was to report experience with catheter closure of PDA in 68 consecutive adult patients the Cocoon PDA occluder on outpatient basis using an exclusive venous approach. 
Methods: The age of the patients ranged from 16-72 (median 36 years) and the weight from $52-74 \mathrm{Kg}$ (median $64 \mathrm{Kg}$ ). The anatomy and size of PDA were defined by transvenous retrograde aortography using a Pigtail or a Berman catheter. The PDA occluder was implanted through an 8-9 F delivery sheath (DS). The procedure was guided using hand injections of contrast media through the DS and 2D and color Doppler echocardiography from suprasternal and parasternal long and short axis, respectively.

Results: The PDA occluders were permanently implanted in 65/ 68 of the 68 patients. The mean PDA diameter (at the pulmonary end) was $3.8 \pm 0.9 \mathrm{~mm}$ (range, 1.2 to $9.8 \mathrm{~mm}$ ). The mean device diameter was $6 \pm 3 \mathrm{~mm}$ (range 4 to $12 \mathrm{~mm}$ ). Complete echocardiographic closure of the ductus at 1-month follow-up was observed in all 65 patients (100\%). Eight minor groin venous hematomas were the only complications of the procedure. The hospital stay of the patients ranged from 6-8 hours.

Conclusions: Exclusive transvenous PDA occlusion using combined angiographic and echocardiographic guidance is an effective and safe method that prevents the arterial complications of the standard approach. In addition, in adult patients, this technique, may be used on outpatient basis resulting in reduce hospital cost.

\section{P-121}

Comparison of the Lifetech Cera and Amplatzer ventricular septal occluders for ventricular septal defects closure in children

Baspinar O. (1), Sulu A. (1), Sahin D.A. (1), Yildiz H. (1), Ozer O.

(2), Kervancioglu M. (1), Kilinc M. (1), Gokaslan G. (3)

Gaziantep University, Dept of Pediatric Cardiology, Gaziantep, Turkey

(1); Gaziantep University, Dept of Adult Cardiology, Gaziantep,

Turkey (2); Gaziantep University, Dept of Cardiovascular Surgery,

Gaziantep, Turkey (3)

Objectives: The Amplatzer and modified double-disc Lifetech Cera ventricular septal defect (VSD) occluders allow the transcatheter closure of the VSDs. The Amplatzer membraneous devices are not used anymore because of increased complete atrioventricular block risk. Therefore, a comparison of these devices will show us the exact risk of the differences of the devices.

Methods: From May 2009 to July 2016, 147 consecutive patients (mean age $8.5 \pm 4.2$, range 1.4-26 year) underwent transcatheter closure of VSD. Used devices were Amplatzer membranous in 35 patients $(23.8 \%)$, Amplatzer muscular in $32(21.8 \%)$, Cera symmetric in $42(28.4 \%)$, Cera muscular in 23 (15.6\%), Cera asymetric in $4(2.7 \%)$ patients. And also 9 patients took Amplatzer ductal occluder I or II and 2 patients Occlutech muscular VSD occluders. Results: There were no differences in age, sex, defect type and shunt ratio between groups. Membranous defect ratio was $71 \%$. Amplatzer device sizes $(7.22 \pm 2.11$, range $4-16 \mathrm{~mm})$ were bigger than Cera devices $(6.22 \pm 1.83$, range $4-10 \mathrm{~mm})(p=0.009)$. Pacemaker implantation was performed temporary in 3 and permanently in 1 patient at Amplatzer group and none in Cera group. The follow-up period was statistically longer at the Amplatzer group $(22.15 \pm 16.56$ vs $4.76 \pm 5.52$ months, $\mathrm{p}<0.001)$. The other complication and residual flow and success rate were similar at both devices ( $p>0.05$ ). Membranous Amplatzer devices used in $72.9 \%$ of membranous defect but Cera symmetric and asymmetric devices used $94.5 \%$ of membranous defects ( $p<0.001)$, because of increased block risk of Amplatzer devices.

Conclusion: Although success rate was similar, Cera devices compare favorably with lower complete block risk. And they make more alternative with choosing symmetric, asymmetric and eccentric types.
P-122

How to size ASD's during percutaneous closure

De Wolf D. (1), Vertongen K. (1), Boon I. (1), Paelinck B. (2),

Demulier L. (1), Van Berendoncks A. (2), Panzer J. (1),

Vandekerckhove K. (1), De Groote K. (1), De Wilde H. (1)

Ghent University Hospital, Gent, Belgium (1); Antwerp University

Hospital, Antwerpen, Belgium (2)

Background: Percutaneous closure is the treatment of choice for secundum type ASD's. Adequate imaging of the ASD is essential. Incorrect sizing of the ASD may lead to complications. Oversizing may cause cardiac erosion and undersizing causes residual leak and device embolization. Balloon sizing (BS) was the method of choice for deciding on the appropriate device size. Improved 2Dand 3D-transesophageal echocardiographic (TEE) imaging raised questions about the necessity of BS.

Objective: For years, BS was our sizing method. We used 2 alternative techniques to measure the stretched diameter of the ASD. The 1st method uses a stiff guide which stretches the ASD. This stretched diameter is measured by $2 \mathrm{D}$ TEE in 2 planes at least. The second technique is the direct measurement of the ASD using 3D TEE. We compared the results of ASD closure using these 3 sizing methods.

Methods: 236 patients with follow-up of minimum one year were enrolled in this study. The occluders used were the AGA and Occlutech devices. The population was classified into 3 groups according to the sizing method: BS (group 1) $n=90,2 \mathrm{D}-\mathrm{TEE}$ (group 2) $n=87$ and 3D-TEE (group 3) $n=59$.

Results: All groups showed a distinct correlation between the baseline dimensions and the device size $(\mathrm{Rs}=0.821)$. In case of a floppy septum, the correlation was lower. The relative expansion rate (baseline dimension to device size ratio) did not differ significantly between BS (group 1: 32.50\%) and 3D-TEE (group 3: $32.80 \%$ ). Group 2 with 2D-TEE sizing showed a significantly lower expansion rate $(14.38 \%)$ compared to group $1(\mathrm{p}<0.000)$ and group $3(\mathrm{p}=0.003)$. The immediate success did not differ between the 3 groups $(98.9 \%, 96.6 \%$ and $96.6 \%)$. Sizing-related issues as oversizing, undersizing, arrhythmias, device embolization and cardiac erosion were comparable between the groups.

Conclusion: These data showed no difference in sizing-related issues for the three methods. Moreover, 2D TEE sizing is the simplest method and available for all age groups with comparable results and complications. The relative device size (compared to baseline) was lower for this sizing technique without causing more undersizing. Maybe this could be interesting for the prevention of erosions.

\section{P-123}

Transcatheter Interventions After Glenn Anastomosis and Fontan Operation in Patients with Univentricular Heart Celebi A., Yucel I.K., Bulut M.O., Balli S., Oner T., Basar E.Z., Kucuk $M$.

Dr. Siyami Ersek Hospital for Cardiology and Cardiovascular Surgery, Department of Pediatric Cardiology, Istanbul, Turkey

Introduction: We aimed to present transcatheter treatment of patients with a single ventricle physiology, experiencing low cardiac output (LCOS) or severe systemic desaturation (SSD).

Method: We retrospectively evaluated 33 patients between 2007 and 2016.

Results: The mean age was 7.6 years ( 6 months- 21 years) and the weight was $25.2 \mathrm{~kg}$ (6-54). The procedures were performed after a Kawashima, Glenn and Fontan surgery in 3, 12 and 18 patients, respectively. SSD was encountered in 17. Amongst these patients, 
closure of a Fontan fenestration was performed in 9. We occluded a decompressing vein in 5 and a pulmonary arteriovenous fistula closure in one. Closure of a residual right SVC-atrium connection was performed in one and stent implantation to reroute the hepatic blood flow to the right lung in one, after a Kawashima operation. The oxygen saturation of $79.3 \pm 8.1 \%$ increased to $92.2 \pm 5.6$ and the PA pressure increased from $11.9 \pm 2.2 \mathrm{mmHg}$ $(8-16)$ to $13.5 \pm 2.1 \mathrm{mmHg}(10-17)$. LCOS and / or increased PA pressure was detected in the remaining 16 . One was on an ECMO support. Amongst these 16 patients, antegrade pulmonary flow (APF) was occluded using a number of devices in 7 , APF was closed with the use of a covered stent, resolving an associated left PA stenosis at the same time in one. Among 5 patients suffering from branch PA stenosis, 4 received stent implantation while the remaining was treated via cutting balloon angioplasty. Two separate stents were needed to treat branch PA and extracardiac conduit stenosis in one. In the patient on ECMO, Fontan fenestration was dilated with a balloon to ensure cardiac output at the expense of systemic desaturation. Fenestration was created in one. In patients with LCOS, the PA pressure decreased from $20.6 \mathrm{mmHg}(15-27)$ to $14.9 \pm 1.8 \mathrm{mmHg}(11-18)$. There was no procedural mortality. Circulatory failure regressed in all except one.

Conclusion: To avoid reopening of the APF surgeons should not only ligate but divide the PAs from the ventricle. In the presence of LCOS or SSD, urgent catheterization should be considered. Significant PA stenosis should be treated even if there exists no pressure gradient throughout the circulation.

\section{P-124}

\section{The experience in percutaneous closure of patent ductus} arteriosus using various types of devices

Kusa J.(1,2), Czesniewicz P.(2), Skierska A.(1), Ras M.(2), Szydlowski L.(1), Mazurak M.(2)

Pediatric Cardiology Department, Medical University of Silesia, Katowice, Poland (1); Pediatric Cardiology Department, Regional Specialized Hospital-Research and Development Centre, Wroclaw, Poland (2)

Introduction: Percutaneous closure of patent ductus arteriosus (PDA) was applied for over 20 years, but in the initial period, the proportion of residual shunts was relatively large.

Objective: The aim of our study was to analyze the results of treatment using various devices, not always dedicated PDA closure. This study analyze our experience during last 5 years because in this period we have had available broad variety of implants.

Material and Methods: In this period 315 patients were catheterized with intention of PDA closure. Mean age was 2,8 $\pm 4,1$ years and mean weight was $13,4 \pm 6,6 \mathrm{~kg}$. Six pts had pulmonary hypertension but it was lower than $2 / 3$ of systemic pressure. According to Kirchenko classification 168 pts $(53,3 \%)$ had conical type, 3 pts $(0,95 \%)$ - window type, 21 pts $(6,7 \%)$ - tubular type, 77 pts $(24,4 \%)$ complex type and 46 pts $(14,6 \%)$ - elongated type PDA.

Results: PDA was closed in 309 pts $(98,1 \%)$. In 6 pts the ductus was very small and we decided not to close. Because of the wide anatomical variety of PDA the eight different types of implants were applied: coils- 160 (51,8\%), Amplatzer Duct Occluder- 124 $(40,1 \%)$, Amplatzer Duct Occluder II-2 (0,6\%), Amplatzer Duct Occluder II-AS- 11 (3,6\%), muscular Ventricular Septal Occluder- 3 (1\%), PFM coil- 4 (1,3\%), Amplatzer Vascular Plug II- 2 $(0,65 \%)$ and Amplatzer Vascular Plug4- 3 (1\%). All implantations were successful. Residual shunt at $24 \mathrm{~h}$ after procedure was in 5 pts
$(1,65 \%)$ but in all pts but one shunts disappeared during 3 months of follow up. In 7 month-old boy with pulmonary hypertension (PA-63/28/39, Ao-70/35/45) PDA was closed using ADO II but after 2 years the moderate residual shunt was still present. During repeated catheterization the normal pulmonary pressure and resistance was confirmed and coil 5/5 was implanted. The total occlusion was confirmed.

Conclusions: The introduction of new types of implants caused that the percentage of residual shunts after percutaneous closure is negligible. This is possible thanks to the diversity of shapes of these implants, because almost in even very complex anatomy we are able to choose the implant almost perfectly corresponding to the shape of PDA.

\section{P-125}

Experience of management secondary arterial hypertension during pregnancy due to of aortic coarctation

Yashchuk N. (1), Cherpak B.(1), Dytkivskyy I. (1), Davydova Ju. (2), Batsak B.(1), Siromakha S. (1), LazoryshynetsV. (1)

(1) Amosov National Institute of Cardiovascular Surgery, Kiev,

Ukraine, (2) Institute of Pediatrics, Obstetrics and Gynecology, Kiev, Ukraine

Introduction: Native severe coarctation is a condition in which pregnancy is at risk - WHO IV, which means pregnancy is contraindicated. Diagnosis of aortic coarctation is quite poor in developing countries. Management of hypertension during pregnancy is challenging due to the fetal toxic impact of some drugs. It is important to remember that strict blood pressure control in such patients may lead to fetal hypoperfusion.

Matherials and methods: We are presenting seven cases of secondary arterial hypertension management in pregnant women due to aortic coarctation. One of the women had mid-aortic syndrome and another one was diagnosed with hypoplastic transverse arch after coarctation repair. Mean age of patients was 25,71 $\pm 5,28$ years. Mean term of gestation at the time of diagnosis was $23,28 \pm 5,76$ weeks. Mean systolic blood pressure on admission was $175,71 \pm 32,58 \mathrm{mmHg}$.

Results: All patients received antihypertensive drugs. Mean SBP on medication was $147,86 \pm 29,70 \mathrm{mmHg}$. Four patients had percutaneous intervention for their coarctation. Three of them had coarctation stenting. The woman with transverse aortic arch hypoplasia had arch stenting when she was at the 15-th week of pregnancy. She experienced spontaneous rupture of the fetal membrane at the day of intervention which was managed conservatively. Spontaneous uneventful vaginal delivery occurred in three women who had intervention before labor. One patient with severe coarctation stenting had caesarian section. Three patients had coarctation stenting after childbirth. One woman experienced acute aortic dissection type A on the 4-th day after caesarian section. On the same day she had coarctation stenting than supracoronary ascending aorta replacement and patent ductus arteriosus ligation. All pregnancies were completed successfully with healthy babies born in term. Mean SBP after intervention was $126,42 \pm 10,69 \mathrm{mmHg}$. Mean pressure gradient decreased from $55,0 \pm 20,81$ to $13,71 \pm 8,79 \mathrm{mmHg}$.

Conclusion: Stenting of coarctation during pregnancy seems to be safe and effective option. There is no sufficient evidence still to draw definite conclusions about the optimal time of interventions. But, in our opinion it should be done before the labor due to high risk of cardiovascular complications despite strict blood pressure control. Interventions before 24-th week of gestation should be avoided in order to prevent miscarriages. 
P-126

Age-related humoral and cellular human immune response after pulmonary valve implantation using xenografts in patients with congenital heart defects

Wagner R. (1), Nieschke K. (1), Thorn M. (1), Vollroth M. (2), Kostelka M. (2), Lurz P. (3), Dähnert I.(1)

Department of Pediaetric Cardiology, Heart Centre Leipzig, Leipzig, Germany (1); Department of Cardiac Surgery, Heart Centre Leipzig, Leipzig, Germany (2); Department of Cardiology, Heart Centre Leipzig, Leipzig, Germany (3)

Objectives: Pulmonary valve implantation (PVI) led to a substantial increase in life expectancy for patients with congenital heart disease. Optimal valve function could be critically influenced by immune response related to the used xenograft material itself or related to the surgical or interventional transcatheter procedure (TPVI). Since immune response depends on immune maturity detailed age related analysis of this could help to understand clinical signs and advance the promising results of PVI.

Methods: At defined time points before and after preliminary stent implantation, TPVI or surgical valve replacement (SPVI) blood samples were analyzed for interleukins (12p70/1beta/2/6/8/10, TNF) $(n=33)$ and leukocyte analysis $(n=26)$. Patients were divided to five age groups (see table 1).

Table 1. Age related number of patients

\begin{tabular}{|c|c|c|c|c|c|}
\hline $\begin{array}{l}\text { age in } \\
\text { years }\end{array}$ & $\begin{array}{l}\text { group I } \\
0,5 \\
\text { to }<2\end{array}$ & $\begin{array}{l}\text { group } \\
\text { II } 2 \\
\text { to }<6\end{array}$ & $\begin{array}{l}\text { group } \\
\text { III } 6 \\
\text { to }<12\end{array}$ & $\begin{array}{l}\text { group IV } \\
12 \\
\text { to }<18\end{array}$ & $\begin{array}{l}\text { group } \\
\text { IV } 18 \text { to } \\
55\end{array}$ \\
\hline $\begin{array}{l}\text { cellular } \\
\text { pre- } \\
\text { stenting } \\
\text { \& TPVI }\end{array}$ & $\mathrm{n}=0$ & $\mathrm{n}=0$ & $n=3$ & $\mathrm{n}=2$ & $\mathrm{n}=3$ \\
\hline $\begin{array}{l}\text { SPVI } \\
\text { humoral }\end{array}$ & $\mathrm{n}=5$ & $n=6$ & $\mathrm{n}=7$ & $\mathrm{n}=0$ & $\mathrm{n}=0$ \\
\hline $\begin{array}{l}\text { pre- } \\
\text { stenting } \\
\text { \& TPVI }\end{array}$ & $\mathrm{n}=0$ & $\mathrm{n}=0$ & $n=3$ & $n=3$ & $\mathrm{n}=7$ \\
\hline SPVI & $\mathrm{n}=5$ & $\mathrm{n}=7$ & $\mathrm{n}=8$ & $\mathrm{n}=0$ & $\mathrm{n}=0$ \\
\hline
\end{tabular}

Results: Only IL 6 increased $72 \mathrm{~h}$ after TPVI in group V up to $28 \mathrm{pg} /$ $\mathrm{ml}$ without a significant difference compared to stent implantation. Leukocyte cell counts did not change after TPVI. Up to $4 \mathrm{~h}$ after SPVI IL 10 increased in groups I $(36 \mathrm{pg} / \mathrm{ml})$, II $(35 \mathrm{pg} / \mathrm{ml})$ and III (41 pg/ml). IL2R raised later (24h after SPVI) in groups I (262\%), II $(212 \%)$ and III (186\%). For 72h after SPVI, PCT and IL 8 increased in groups II (936 and $12 \mathrm{pg} / \mathrm{ml})$ and III (157 and $12 \mathrm{pg} / \mathrm{ml})$. Group I revealed IL 8 elevation even up to $72 \mathrm{~h}$ after SPVI $(27 \mathrm{pg} / \mathrm{ml})$. IL 6 was raised in all three groups for at least $72 \mathrm{~h}$. The older the patients the longer time was needed until normalization. Other IL did not differ from pre-values. Granulo-, Mono- and Lymphocytes reacted all after SPVI, granulocytes raised up to $7 \mathrm{~d}$ after SPVI in groups I (209\%), II (433\%) and III (275\%). Monocytes increased in group I $24 \mathrm{~h}$ after SPVI. Lymphocytes decreased in group II more and longer than in groups I and II (72h).

Conclusions: Immune response related to interventional or surgical pulmonary valve replacement differs to age and immune maturity. Explicit immunity patterns provoked by xenograft material were not apparent. Further Samples are needed to understand the different immune patterns in detail.
P-127

Transcatheter membranous VSD closure by Nit-Occlud Lê VSD Coil. A French multicentre study

Houeijeh A. (1), Khaled H. (2), Jalal Z. (3), Fraisse A. (2), Hascöet S.

(2), Heitz F. (2), Thambo J.B. (3), Godart F. (1)

Pediatric and Congenital Cardiology Department, Lille University

Hospital, Lille, France (1); Pediatric and Congenital Cardiology

Department, Toulouse University Hospital, Toulouse, France (2);

Pediatric and Congenital Cardiology Department, Bordeaux University

Hospital, Bordeaux, France (3)

Background: Interventionists are interested by nonsurgical closure of membranous VSD (mVSD). The Nit-Occlud Lê VSD Coil (PFM, Cologne, Germany) was used to close mVSD with the advantage of absence of atrioventricular block.

Methods: We describe here French multicentric experience in mVSD closure with Nit-Occlud Lê VSD Coil.

Results: We had 24 patients with mean age of 15 years 5 months (1.5-59 years), mean weight of $58 \mathrm{~kg}$ (9-79.6 kg). Mean VSD size was $8.5 \mathrm{~mm}$ on the left side and $4 \mathrm{~mm}$ on the right side. Mean procedures time was 112 minutes (45-200 min), with mean fluoroscopy time of $33.8 \mathrm{~min}$ (17-63 min). Closure failed in 5 patients: 4 because of insufficient aneurysmal tissue, and one because of retention of the coil into the aortic valve. Trivial residual shunt persisted in 6 patients. Major complications occurred in five patients including: hemolysis in two patients due to residual shunt requiring shunt closure by catheterization in one patient and by surgery in the other, tricuspid regurgitation due to cordage lesion, traumatic aortic valve regurgitation by cusp lesion, and finally severe transient hemodynamic collapses requiring short chest compression. Transient grade 1 atrioventricular block occurred in one patient. Mean left ventricle end diastolic diameter decreased significantly from 45.6 to $41.2 \mathrm{~mm}$ in patients with succeeded percutaneous closure.

Conclusion: mVSD closure by The Nit-Occlud Lê VSD Coil is effective with no permanent atrioventricular block. The procedure is complicated requiring high experience and learning curve which explains the high complications rate, but also this demonstrates the need of meticulous selection of patients.

\section{P-128}

PDA stenting in duct dependent pulmonary circulation- 5 year single centre experience

Kalantre A., Bowes M., Ramaraj R., Ladusans E., Peart I., Gladman G., Oswal N., Dhannapuneni R., Guerrero R., Shauq A.

Alder Hey Children's Hospital, Liverpool UK

Background: The Blalock-Taussig shunt (BTS) is considered gold standard initial palliative procedure for duct dependent pulmonary circulation in neonates but is fraught with morbidity and in recent times the mortality rate has risen throughout the world. PDA stenting is an alternative and its low morbidity and mortality makes it an attractive option. Our centre has employed ductal stenting as primary palliation for duct dependent circulation from 2011. We report our experience.

Methods: All patients were considered, excluding: - weight $<2.5 \mathrm{~kg}$, presence of pulmonary artery stenosis, or spiral-type tortuous PDA on echocardiography.

Results: 43 patients presented from April 2011 to August 2016. 6 patients were excluded after angiography. 4 cases were abandoned due to haemodynamic instability. 33 patients underwent successful PDA stenting. The basic demographics are illustrated in table 1. $23 / 33$ patients had "biventricular" repair while $10 / 33$ had "univentricular" future. Table 2 illustrates the spectrum of 
Table 1. Patient characteristics

\begin{tabular}{lcc}
\hline PARAMETER & MEAN & RANGE \\
\hline Age (days) & 36.4 & $1-175$ \\
Weight (kg) & 3.54 & $2.6-6.4$ \\
Flouroscopy time (min) & 18.4 & $4-41.6$ \\
Procedure time (min) & 87.4 & $40-153$ \\
\hline
\end{tabular}

Table 2. Ductal morphology

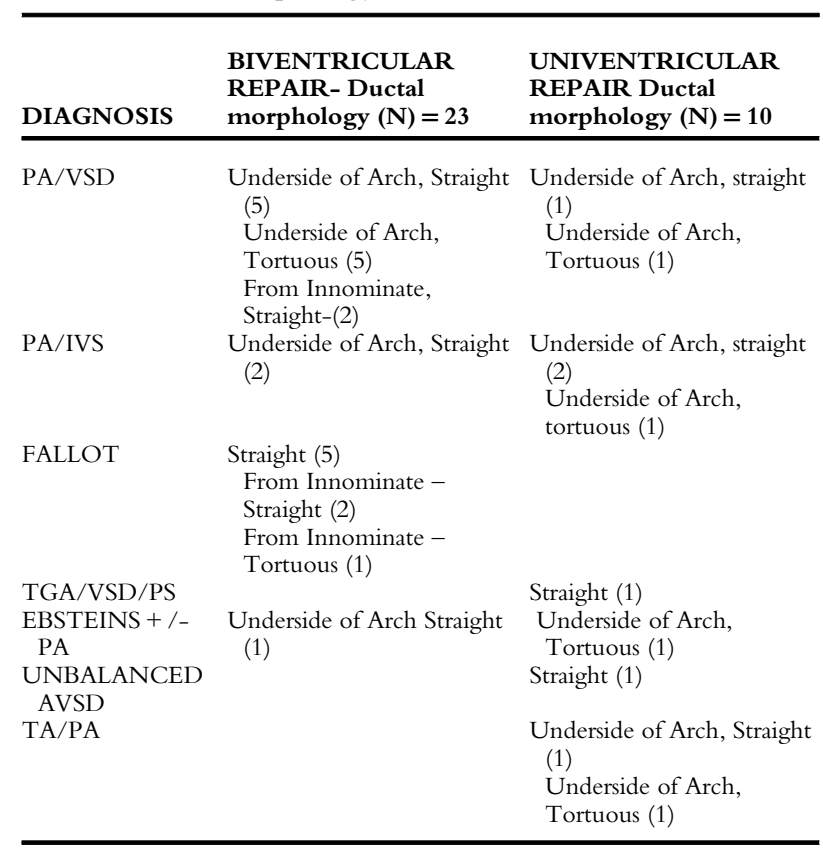

diagnoses and their ductal morphology based on "biventricular" or "univentricular" repair.

In all but 4 cases the stents were deployed from the arterial side through 4 F Mullins sheath. There were 2 early (within 30 days) and 1 late death. These were unrelated to the procedure. 2/33 patients developed femoral clots needing heparin. 2/33 needed re-intervention (1-stent, 1-balloon dilatation)

Conclusions: With careful case selection, PDA stenting is a safe, feasible and good alternative to BTS as an initial palliative procedure for duct dependent pulmonary circulation.

\section{P-129}

Percutaneous valvuloplasty versus surgical valvulotomy in neonates with critical aortic stenosis

Bakloul M., Metton O., Ducreux C., Veyrier M., Henaine R., Ninet J., Di Filippo $S$.

Cardiovascular Hospital Louis Pradel, University Claude Bernard

Lyon1, Hospices Civils de Lyon, Lyon, France

This study compared outcomes and prognosis of newborns with critical aortic valve stenosis (AVS) who underwent either percutaneous or surgical intervention as first therapeutic option.

Methods: Neonates with diagnosis of critical AVS (defined as aortic flow dependent on ductus arteriosus patency) were included in the study, and divided in group P (percutaneous valvuloplasty) and group S (surgical valvulotomy).
Results: 23 cases (19 males) were analyzed: 9 in group P and 14 in group S, aged 0 to 28 days at diagnosis ( $<7$ days of age: $78 \%$ of group $\mathrm{P}$ vs $50 \%$ in $\mathrm{S}, \mathrm{p}=\mathrm{NS}$ ). Weight was $>2.5 \mathrm{~kg}$ in $100 \%$ of group $\mathrm{S}$ and $78 \%$ of group P. Symptoms of heart failure were more frequent in group P. Echocardiography showed: LVSF $<28 \%$ in $67 \%$ of group P vs $43 \%$ group S (NS), LV fibroelastosis in $88 \%$ of group $\mathrm{P}$ and $21 \%$ group $\mathrm{S}(\mathrm{p}<0.05)$, mitral regurgitation in $77 \%$ of group $\mathrm{P}$ and $7 \%$ group $\mathrm{S}(\mathrm{p}<0.05)$. Aortic valve anatomy included bicuspidia in $52 \%$ of cases, aortic tricuspide valve dysplasia in $43.5 \%$. Events occurring after first intervention included restenosis in 6 cases $(26 \%$, i.e. $55.5 \%$ in group $\mathrm{P}$ and $7 \%$ in group $\mathrm{S})$, aortic valve regurgitation $>$ grade 2 in 11 cases $(47.8 \%=44 \%$ of group $\mathrm{P}$ and $50 \%$ of group $\mathrm{S}$ ), Ross procedure in 5 cases $(21.7 \%=22.2 \%$ of group $\mathrm{P}$ and $21.4 \%$ of group S). Prognosis factors for reintervention in group $\mathrm{P}$ were: aortic valve dysplasia, LV fibroelastosis and aortic annulus diameter $<6 \mathrm{~mm}$ (respectively $=100 \%, 75 \%$ and $83 \%$ ), and in group S were: bicuspidia, aortic valve annulus $<6 \mathrm{~mm}$ (respectively $=33 \%$ and $50 \%$ ).

Conclusion: This study showed a higher rate of reintervention in the percutaneous valvuloplasty group and more severe aortic regurgitation in the surgical group. Aortic annulus diameter $<6$ $\mathrm{mm}$ was related to reintervention risk.

\section{P-130}

Acute pulmonary oedema: An immediate complication after percutaneous pulmonary valve implantation

Alvarez-Fuente M., Fernandez-Ruiz A., Cuesta M., Arnalich A., Del Cerro M.J.

Hospital Ramón y Cajal. Madrid. Spain

Background: Percutaneous implantation of pulmonary valve is an alternative to surgery in right ventricular outflow tract (RVOT) residual lesions. Acute pulmonary oedema is a rare complication that appeared in 2 of our 25 valve implants.

Case 1: Twenty year old male $(56 \mathrm{Kg})$ with Tetralogy of Fallot operated with transannular patch as an infant. At age 13 a surgical bioprothesis pulmonary valve was implanted. Seven years after, the valve degenerated to stenosis and regurgitation. The patient was catheterized and a $39 \mathrm{~mm}$ CP stent was implanted over the bioprosthetic valve, in the same procedure a Melody valve was implanted. The procedure ended with good results and a right ventricle (RV) pressure at $40 \%$ of systemic pressure. No complications occurred. The patient received $98 \mathrm{ml}$ of contrast and under $1000 \mathrm{ml}$ of saline infusion. Minutes after extubation the patient suffered abrupt desaturation with radiologic image of acute pulmonary oedema.

Case 2: Twenty-three year old male $(60 \mathrm{Kg})$ with TGA. Arterial switch was performed in neonatal period. In the evolution, at 4 years of age, he required RVOT surgery with transannular patch due to pulmonary stenosis. The stenosis evolved to severe RVOT obstruction with systemic pressure in RV. Two stents were implanted in RVOT and 6 months after a Melody valve was implanted, with good results and RV pressure at $47 \%$ of systemic pressure. No complications occurred during the procedure. The patient received $269 \mathrm{ml}$ of contrast and $1000 \mathrm{ml}$ of saline. Minutes after extubation the patient presented serohematic secretions in the airway and had radiologic findings of acute pulmonary oedema.

Both cases resolved after 24 hours of mechanical ventilation and intravenous diuretic treatment.

Comment: A latent diastolic dysfunction of the left ventricle could explain the acute pulmonary oedema after the increase of flow in the pulmonary circulation due to the valve implantation. Larger series of patients could help identify risk factors for this complication. 


\section{P-131}

Double Edwards Sapien XT valve-in-valve implantation Alvarez-Fuente M. (1), Haas N.A. (2), Fernández Pineda L. (1), Tamariz R.(1), Del Cerro M.J. (1).

Hospital Ramón y Cajal, Madrid, Spain (1), Heart and Diabetes Center NRW, Bad Oeynhausen, Germany (2)

Background: Percutaneous implantation of pulmonary valves is now a common procedure in patients with congenital heart disease and pulmonary regurgitation and/or stenosis. The Melody ${ }^{\circledR}$ valve was the first pulmonary percutaneous valve to achieve this indication. In 2011 the first CE-marked transcatheter Sapien pulmonic valve was implanted in Europe and the first percutaneous valve-in-valve was implanted in a patient with a bioprosthetic pulmonary valve without a conduit or prestenting, being an off-label use. The implantation of the Sapien valve in tricuspid position is not yet approved by the EMA, although it is a good alternative to surgery for patients with high surgical risk. We present the case of a double valve-in-valve implantation of two Sapien XT valves in pulmonary and tricuspid bioprosthetic valves.

Case Report: A 20 year old male with severe pulmonary stenosis and hypoplastic right ventricle, palliated at neonatal age by percutaneous valvuloplasty. At age 14 two surgical prothesis were implanted: Edwards $25 \mathrm{~mm}$ in pulmonary and $29 \mathrm{~mm}$ in tricuspid position. Both valves degenerated to stenosis and regurgitation, developing supraventricular arrhythmia due to severely dilated right atrium. Angiographies show severe combined tricuspid and pulmonary lesion with great dilation of the pulmonary trunk. Under general anaesthesia, through right femoral venous access, initial balloon predilation was performed (Andralec $30 \times 40 \mathrm{~mm}$ ). Thereafter we implanted a $26 \mathrm{~mm}$ Sapien XT in pulmonary position and a $29 \mathrm{~mm}$ Sapien XT over the pulmonary and tricuspid bioprosthetic valves with the support of a super stiff wire (Lunderquist). Based on the anatomy, no prestenting was performed. There were no complications, although due to the severely dilated right atrium and hypertrabeculated right ventricle the implantation of the pulmonary valve required multiple manoeuvres and changing the wire position from the left pulmonary artery to the right artery. Both valves were implanted successfully and with good angiographic and echocardiographic results. Comments: This case shows the feasibility of implanting two percutaneous Sapien XT valves in the same procedure. It also reinforces the feasibility and security of the implantation of the Sapien $\mathrm{XT}$ valve over a degenerated tricuspid bioprosthetic valve, which is still an off-label procedure.

\section{P-132}

Successful treatment of tortuous abdominal aorta-right atrial fistula by vascular plug: a previously unreported cardiac malformation

Alkan F. (1), Pabuscu Y. (2), Cetin M. (3), Coskun S. (1)

Departments of Pediatrics, Division of Pediatric Cardiology, School of Medicine, Celal Bayar University, Manisa, Turkey (1); Departments of Radiology, School of Medicine, Celal Bayar University, Manisa, Turkey (2); Departments of Pediatrics, Division of Pediatric Cardiology, School of Medicine, Yüzüncü Yal University, Van, Turkey (3)

Objective: Aorta-right atrial fistula is a rare congenital anomaly characterized by a fistulous communication between the aorta and the right atrium. We report a child in whom a fistula connected the descending abdominal aorta to the right atrium.

Methods: A 2- year-old asymptomatic boy was referred to our institute for evaluation of a murmur.
Results: The physical examination was normal apart from a continuous murmur, which was best audible at the left upper sternal border. The electrocardiogram was normal with no evidence of volume overload or chamber hypertrophy. Chest X-ray showed no abnormalities. During echocardiographic examination, a small shunt from the proximal descending aorta to the main pulmonary artery was seen. The diagnosis of patent ductus arteriosus (PDA) was made. Left ventricle was slightly dilated with good function, intact ventricular septum, normal aortic and pulmonic valves, and normal aortic arch with no aortic coarctation but extra cardiac a structure as parallel to vc1 was opened into right atrium. Computed tomography angiography cofirmed the diagnosis and delineated the anatomy. Cardiac catheterization was done to confirm the diagnosis and possibly attempt closure of the PDA and aorto- right atrial fistula. Right and left heart catheterization were done and confirmed the diagnosis of the very small PDA, unusual communication and tortuous fistula, which originated from the descending abdominal aorta to the right atrium. The fistula ended in a sac beside the junction of the inferior vena cava with the RA, which opened into the RA with a large orifice. The anomaly was successfully treated by closing the proximal of fistula using an Amplatzer vascular plug IV.

Conclusions: This is a previously unreported cardiac malformation. We were successfully closed by trans-catheter embolisation using an Amplatzer vascular plug IV.

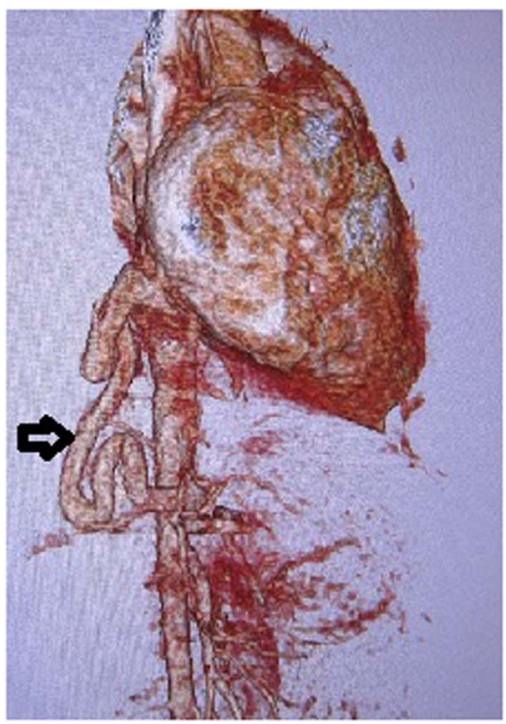

Figure 1.

Computed tomography angiography image showing a tortuous fistula (arrow), which originated from the descending abdominal aorta to the right atrium.

\section{P-133}

Large stents may infold during implantation - how to avoid and treat?

Georgiev S. (1), Krings G. (2), Tanase D. (1), Ewert P. (1), Eicken A. (1) (1) Department of Pediatric Cardiology and Congenital Heart Disease, German Heart Center Munich, Germany (2) Department of Pediatric Cardiology, University Medical Center, Utrecht, Netherlands

Introduction: Stent infolding or "Diabolo" configuration can occur during implantation of long stents on large low pressure balloons. Methods: We review 3 cases with stent infolding and discuss potential mechanisms as well as possible treatment options. 
Results: Two patients long after tetralogy of Fallot surgical correction were indicated for percutaneous pulmonary valve implantation. As both had "native" outflow tracts, the first step to create a landing zone. In the first patient a $57 \mathrm{~mm}$ Andra XXL stent was mounted on a VACS balloon $30 / 60 \mathrm{~mm}$, but during implantation into the left pulmonary artery the stent showed a "Diabolo" configuration and the balloon ruptured. It was possible to pass the infolded stent centrally and dilate it with high pressure balloons. A second $57 \mathrm{~mm}$ Andra XXL stent was implanted uneventfully over a $24 \times 50$ BiB balloon and Sapien valve $26 \mathrm{~mm}$ was implanted. In the second patient an Andra XXL $48 \mathrm{~mm}$ stent, which was mounted on a Crystal Balt CBV 30/60 mm balloon showed a "Diabolo" configuration during attempted implantation into the right ventricular outflow tract (figure 1). The Crystal Balt balloon could not be retrieved or passed centrally

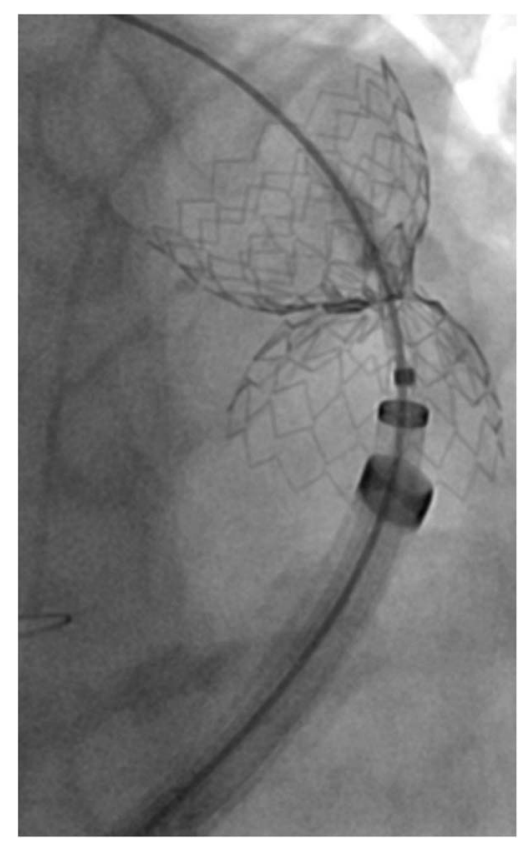

through the infolded stent for additional dilation. The patient was operated and recovered well. The third patient had a "failing" Fontan due to a highly stenotic extracardiac conduit. An Andra XXL $57 \mathrm{~mm}$ stent was mounted on a $22 \mathrm{~mm}$ BiB. During an implantation attempt into the conduit with the outer balloon only stent infolding occurred. With inflation of the inner balloon, followed by implantation of additional stenting and dilatations with high pressure balloons the stent could be expanded to the desired diameter. This patient recovered uneventfully.

Conclusions: Stent infolding is a possible during implantation of long stents on large low pressure balloon. Using a BiB for stent implantation could help to avoid this complication. After crossing the infolded region centrally, dilation with high pressure balloons can be a feasible treatment.

\section{P-134}

Fate of Duct-dependent, Discontinuous Pulmonary Arteries After Arterial Duct Stenting

Santoro G., Capozzi G., Gaio G., Palladino M.T., Giordano M., Fratta F., Mahmoud H.T., Di Nardo G., Carrozza M., Morelli C., Ricci C., Russo M.G.

Paediatric Cardiology, A.O.R.N. "Ospedali dei Colli", 2nd University of Naples, Naples, ITALY
Background: Significant and balanced PA growth following AD stenting has already been consistently reported in literature. However, no data are so far available about the role of this approach as palliation of congenital heart disease (CHD) with duct-dependent dPA. Aim of this study was to evaluate the fate of discontinuous pulmonary artery ( $\mathrm{PPA}$ ) following trans-catheter arterial duct (AD) stabilization.

Methods: Angiographic PA evaluation was performed in 7 patients submitted to neonatal AD stenting as palliative recruitment of dPA. Five patients showed discontinuity of one PA, while 2 patients had both PAs served by bilateral ducts. PA growth was evaluated as Nakata index, McGoon ratio as well as dPA $(n=9)$ vs. heart-dependent PA (hPA) $(\mathrm{n}=5)$ size and $\mathrm{z}$-score changes.

Results: AD stabilization was performed using coronary stents dilated to $3.2 \pm 0.3 \mathrm{~mm}$ (median 3.4), with significant increase of O2 saturation (from $83 \pm 11$ to $95 \pm 5 \%, p<0.02$ ). Control angiography was performed $5.1 \pm 2.8$ months (median 6) after duct stenting, showing significant growth of $\mathrm{dPA}$ (from $3.7 \pm 1.0$ to $7.6 \pm 2.7 \mathrm{~mm}, \mathrm{p}<0.001$; $\mathrm{z}$-score from $-0.7 \pm 1.4$ to $1.7 \pm 2.2$, $\mathrm{p}<0.01)$. A trend toward a better growth of $\mathrm{dPA}$ as compared to hPA was found $(117 \pm 87 \%$ vs. $54 \pm 34 \%, p=N S)$. The final vessel size was still significantly different between the groups (dPA $7.6 \pm 2.7$ vs. $\mathrm{hPA} 11.9 \pm 3.4 \mathrm{~mm}, \mathrm{p}=0.02$ ), although the final z-score value did not significantly differ (dPA $1.7 \pm 2.2$ vs. hPA $3.8 \pm 0.9 \mathrm{~mm}, \mathrm{p}=\mathrm{NS}$ ).

Conclusion: In conclusion, percutaneous AD stenting is effective in promoting a significant catch-up growth of duct-dependent dPA, being therefore advisable as a reliable alternative to surgical palliation.

\section{P-135 \\ Use of the IPCCC framework for reports of complications to pediatric heart catheterizations \\ Ravndal M., Christensen A.H., Døhlen G., Holmstrom H.}

Pediatric Department, Oslo University Hospital (OUS), Oslo, Norway

Introduction: Studies describing complications after cardiac catheterization differ in the way adverse events are reported, resulting in strikingly different occurrence rate of complications. In 2011 The International Paediatric and Congenital Cardiac Code (IPCCC) published a nomenclature system for complications of heart catheterization in children with the potential of making results more comparable. We reclassified all complications after cardiac catheterization in children at Oslo University Hospital (Norway) between 2010 and 2015 according to the new system. To our knowledge, no previous publications are based on the IPCCCnomenclature.

Methods: All procedures and adverse events were prospectively registered during a five-year period. Medical records for cases with complications were reviewed to confirm the event and to reclassify the type, severity and attributability of the complication according to the IPCCC nomenclature. Preventability and timing were difficult to re-classify retrospectively and were not included. Results: A total of 1318 catheterizations performed on 941 patients were included in the study. The total complication rate was 5.5\%. As for severity level 1 to 5 , level three was the modality (43.1\%). The low prevalence of severity levels 1 and 2 probably represents underreporting of minor complications. Trauma to vessels or myocardium, hemodynamic adverse events and arrhythmias were the most common types of complications. Some of the IPCCCcategories for complication types (renal, metabolic, infection, gastrointestinal) were not found in our material. We found complications in 12 of 13 categories of attributability. Complications related to catheter manipulation were clearly the most common (36.1\%). 
Conclusions: Diverse reporting systems remain an obstacle for precise cross-center comparisons. By using the IPCCC system we were able to reclassify most of our complications into predefined categories. In our opinion, the IPCCC classification system should be generally applied for both registration and reporting of complications after pediatric heart catheterizations.

\section{P-136 \\ Use of Micro Vascular Plug System Covidien in children: indications and results \\ Malekzadeh-Milani S., Sitefane F., Boudjemline Y. \\ Centre de Référence Malformations Cardiaques Congénitales Complexes - M3C, Necker Hospital for Sick Children, George Pompidou European Hospital, Assistance Publique des Hopitaux de Paris, Paris, France}

Introduction: In various conditions, devices are needed to occlude abnormal vascular connections. In children with congenital heart disease, access to vascular abnormalities might be hasardous: need of rigid delivery system to deploy the device, small or tortuous vessel. The micro vascular plug MVP Covidien is an alternative to existing device specially in small children or in complex anatomy. The MPV is available in 2 size currently: MPV-3 for occlusion of vessel size from 1,5 to $3.0 \mathrm{~mm}$ and MPV-5 for vessel from 3.0 to $5.0 \mathrm{~mm}$. We report our initial experience with MPV-3 and MPV-5 in children.

Methods: We reviewed all children who received Micro Vascular Plug Covidien from April 2015 until October 2016. Demographics, indication for plug implantation, number of plugs, size of plugs (3Q and 5Q) and immediate outcome are reported.

Results: 42 patients received 60 microplug (27 MPV-3 and 33 MPV-5). Plugs were inserted through $4 \mathrm{Fr}$ catheter or through Microcatheter (Progreat, Terumo). Mean age was 4,7y (14 d-18,46y). Mean weight was $17,4 \mathrm{~kg}(2,2 \mathrm{~kg}-66 \mathrm{~kg})$. Mean BSA was $0,75(0,18-1,8)$. Indications were PDA closure in 9 patients (4 new borns or premature patients), closure of venovenous collaterals in 8 patients and left SVC in 3 patients. In 15 cases occlusion of aorto-pulmonary collaterals were achieved. 4 patients had pulmonary sequestration occlusion and one pulmonary arterio venous malformation was occluded. Plugs were used to close 3 Blalock Taussig and 2 coronary artery fistula. Plug embolisation occured in 2 patients with successful lasso retrieval (1 PDA and 1 coronary artery fistula) and further occlusion with larger plugs was performed. In 3 patients, residual shunt was closed with additional vascular plugs or coils. In the remaining 37 patients, immediate complete occlusion of the shunt was obtained. Conclusion: The Micro Vascular Plug Covidien is a good device to close various abnormal vascular communications in children. Rate of closure is high with no serious adverse event.

\section{P-137}

Transcatheter closure of perimembranous ventricular septal defects using the second-generation AmplatzerTM vascular occlude device

Brunet L., Carretero J., Prada F., Perez A., Zambrano M.D., Arroyave J., Sanchez-de-Toledo J.

Hospital Sant Joan de Déu (Barcelona, Spain)

Objectives: Ventricular septal defect (VSD) is the most common congenital heart defect. Non-surgical closure of VSDs has become increasingly accepted. Several occluding devices are now available and some of them are being used off-label. The aim of this study was to review our institutional experience with VSD closure using the AmplatzerTM Duct Occluder II (ADO II) device.
Methods: Retrospective review of all patients who underwent percutaneous closure of perimembranous VSD using ADO-II device between Sep 2010 and Jul 2016 in our institution. VSD size and VSD-Aortic valve distance was measured by transesophageal echocardiography (TEE) and ventriculography. VSD size was measured at the largest diastolic phase in all patients. Appropriate device size was chosen to be between 0.5 to $1 \mathrm{~mm}$ larger than the VSD size. The procedure was performed with fluoroscopy and TEE guidance. Transthoracic echocardiography and ECG were performed at discharge and during follow-up. Data is presented as median [IQR].

Results: The procedure was successfully done in 11 consecutive patients with median age of 63 months (47-185) and weight of $17.9 \mathrm{~kg}(13.7-30.3)$. VSD diameter was $4.5 \mathrm{~mm}$ (4-5) with a VSDaortic valve distance of $5.5 \mathrm{~mm}$ (4.4-8.3). All patients were discharged within the first 24 hours. No major complications were observed. A trivial residual shunt was present in 5/11 (45\%) patients at discharge. During follow-up [31 months (15-61)], only $3 / 11(27 \%)$ remained with trivial residual shunt and 1/11 (9\%) developed mild aortic valve regurgitation.

Conclusions: In our experience, transcatheter perimembranous VSDs closure with ADO II device can be safely and effectively done in children despite its proximity to the aortic valve. Larger series, encompassing more centers and longer follow-ups, are needed to assess whether this technique should be more broadly available as an alternative to the surgical repair.

\section{P-138}

Implantation of bioabsorbable scaffolds in a baby with repeated stenoses of all pulmonary veins

Zartner P., Bierbach B., Asfour B., Schneider M.

German Paediatric Heart Centre, Sankt Augustin, Germany

History: At the age of 3 months a male patient developed severe stenosis of all pulmonary veins. In the cathlab all stenosis were dilated using Paclitaxel covered balloons (IN. PACT Falcon, Invatec, Germany) and conventional balloons (Sapphire, Orbus Neich, Hong Kong). Patient was dismissed home. After three months restenoses occurred. Surgical correction with the sutureless operation was performed and assisted by intraoperative balloon dilation with Paclitaxel covered balloons (Elutax, Aachen resonance, Germany). Again the patient was dismissed home. Three months later he reappeared with hypersystemic right ventricular pressures. Under compassionate care regulations and after having obtained informed consent by the parents, decision was made to implant bioabsorbable Novolimus covered scaffolds (DeSolve, Elixir, USA).

Angiography and/or other diagnostic tests: stenosed and stented left lower pulmonary vein.

Procedure: A hybrid approach was chosen to reduce procedure time and radiation. All but the right upper pulmonary veins were found severely obstructed and were consequently stented with $4 \star 18$ $\mathrm{mm}$ and $3.5 \star 14 \mathrm{~mm}$ DeSolve scaffolds under x-ray guidance. After proper placement the delivery material was completely removed and the chest closed. The patient could be extubated after 24 hours and was sent home twelve days later. Medication was diuretics, aspirine and clopidogrel.

Conclusion: Bioabsorbable scaffolds with cytostatic drugs may help to overcome the otherwise often fatal prognosis of intrinsic pulmonary vein stenosis. It rapidly improves the acute life-threatening situation and possibly reduces the chance of restenosis by its continuous cytostatic delivery to the tissue.

Comments: This is a single case with only short term follow-up, so more data and longer follow-up will be needed to allow a 
conclusion on the possible efficiency of scaffolds in congenital heart diseases. The use of bioresorbable material is still of great interest is all young patients with further growth of their vessels to expect.

\section{P-139}

Complications of trancatheter atrial septal defect closure Dimitrov L., Kuneva-Nencheva A., Levunlieva E., Nenova K., Velkovski I.

National Heart Hospital, Sofia, Bulgaria

Over the last three decades the transcatheter closure (TCC) of secundum type Atrial Septal Defect (ASD II) established itself as an alternative to surgical treatment.

Our objective was to assess the safety of the procedure by analyzing retrospectively the complications of the TCC of ASD over a period of 14 years. We divided the complications into 2 groups according to their severity: severe (death, embolization of the device with surgical removal, brain embolization, perforation, erosion, severe hemodynamic disorder, etc.), and mild (embolization with transcatheter retrieval of the device, transient disorders of cardiac rhythm, hematoma at the puncture site, etc.), and into early and late according to the time they originated (before and after the 24th hour).

164 patients with transcatheter closure of ASD II, treated at the National Heart Hospital were followed up retrospectively for a period of 14 years (from 2002 to 2016). We divided the patients into 2 groups according to age- children up to 18 years of age $(66 \%)$ and adults over 18 years of age $(34 \%)$.

The procedure has been done to 164 patients. In 3 cases, because the device could not be stabilized, the procedure was cancelled and the patients were referred for scheduled operation. These patients come under the group of mild complications alongside with the cases of pericardial effusion, gastrointestinal bleeding, transient disorders of rhythm (a total of 7 cases) and hematomas in the region of the puncture site- a total of 12 cases Percutaneous retrieval of the embolized device with subsequent TCC with a bigger device was performed in 3 of the cases ( 2 within the same procedure and 1 as a scheduled second procedure). A total of 25 mild complications have been observed.

In 6 of the cases $(3.5 \%)$ there were severe complications: fatal outcome in a 69-year-old woman with retroperitoneal hematoma; 4 embolizations necessitated surgical intervention (3 early and 1 late) and acute left ventricular failure with pulmonary oedema in a patient on hemodialysis.

TCC of ASD is a secure and safe method of treatment. The major complications which have occurred are mainly in adults and are related mainly to co-morbidity.

\section{P-140}

Immediate and long term results of balloon angioplasty of recurrent aortic coarctation in children less than one year

Lefort B. (1), Lachaud M.(2), El Arid J.M.(1), Neville P.(1), Soule N. (1), Guerin P.(2), Chantepie A.(1)

Pediatric Cardiology, Children Hospital Gatien de Clocheville, University Hospital of Tours, Tours, France (1), Cardiology, Institut du Thorax, University Hospital of Nantes, Nantes, France (2)

Background: Various publications have considered results of percutaneous angioplasty of aortic recoarctation, but none of them focused on procedures performed in children before one year of age.
Aims: We aimed to describe the immediate and long term results of balloon angioplasty of recoarctation before the age of one year, and to define factors that may influence the outcome.

Methods: We retrospectively reviewed data from 20 consecutives children undergoing percutaneous dilatation of aortic recoarctation before one year of age in University Hospitals of Tours and Nantes.

Results: In all patients except one, dilatation improved the median recoarctation diameter $\mathrm{Z}$ score (from -5.8 (range -1.8 to -8.3 ) to -3.2 (range -4.3 to 0.7 ); $\mathrm{p}<0.01$ ), and reduced the median peak systolic gradient (from $31 \mathrm{mmHg}$ (range 20 to 60 ) to $21 \mathrm{mmHg}$ (range 6 to 50$) ; \mathrm{p}<0.001)$. There was no procedure-induced mortality and no acute intimal flap or long term aneurysm. Three patients experienced femoral artery thrombosis and one had a transient ischemic stroke. Eight children (40\%) needed reintervention for further recoarctation (surgery $n=4$ or new dilatation $\mathrm{n}=4$ ). A smaller size of the balloon was significantly associated with the risk of reintervention (balloon to recoarctation diameter ratio: 1.66 (range 1.16-3.33) vs 2.72 (range 2.07-3.53); balloon to descending aorta ratio: 0.87 (range $0.69-1.20$ ) vs 1.00 (range $0.86-$ 1.43); $\mathrm{p}<0.05)$.

Conclusion: Percutaneous balloon angioplasty for recoarctation in young infant less than one year is safe and immediately effective as well as in older children and adults. Although most of children achieve a good result during follow-up, the need for further intervention is high.

\section{P-141}

Transcatheter closure of a giant right pulmonary artery-topulmonary vein fistula in two neonates - a lifesaving procedure

Zubrzycka M. (1), Brzezinska-Rajszys G. (1), Kolesnik A. (1), Rewers B. (1), Mirecka A. (2), Kowalczyk M. (2)

Heart Catheterization Laboratory (1); Cardiology Clinic (2), The

Children's Memorial Health Institute, Warsaw, Poland

Introduction: Direct fistulous connection between the right pulmonary artery and the right pulmonary vein (rPA-rPV fistula), reported also as pulmonary artery to left atrium fistula, is extremely rare congenital anomaly. In neonates can cause severe cyanosis and heart failure, and as a life-threatening anomaly requires urgent, most often surgical treatment. To our knowledge only two cases of transcatheter closure of $\mathrm{rPA}-\mathrm{rPV}$ fistulas in neonates were reported.

Material: Two neonates age 3 days - patient A, 1 day - patient B with echo diagnosis of rPA-rPV fistula (patient B diagnosed prenatally) were accepted for urgent percutaneous closure of fistulas using Amplatzer Duct Occluder (ADO). Both patients were in critical general condition, with severe cyanosis (patient A - 68\% $\mathrm{HbO} 2$, patient $\mathrm{B}-55 \% \mathrm{HbO} 2$ ) and heart failure.

Methods: In each case both femoral veins access was obtained. Two diagnostic catheters were introduced, one to the rPA, second to the $\mathrm{rPV}$ (through the right atrium, foramen ovale, left atrium). On the base of pulmonary artery angiography diameter of the rPArPV connection was $4.7 \mathrm{~mm}$ (patient A) and $6 \mathrm{~mm}$ (patient B). A guidewire was inserted through the catheter positioned in $\mathrm{rPV}$ and snared in the rPA, a veno-venous guidewire circuit was created. The long sheath was introduced over the guidewire from the $\mathrm{rPV}$ via fistula to the rPA and 6/4 mm ADO (patient A) and 12/10 mm (patient B) were deployed in fistulas.

Results: Complete occlusion of fistula was achieved in both patients with immediate oxygen saturation reached $99 \% \mathrm{HbO} 2$. No procedural related complications were observed. The result of 
the procedure has remained excellent during 8 years (patient A) and 6 months (patient B) follow-up.

Conclusions: Transcatheter closure of giant rPA-rPV fistula in neonates using $\mathrm{ADO}$ can be an effective method of treatment this life-threatening anomaly.

\section{P-142}

Difficult decisions. Percutaneous closure of Postinfarction Ventricular Septal Defect - peri-procedural and long-term observation

Galeczka M. (1,2), Knop M. (1,2), Litwin L. (1,2), Szulik D. (2), Fiszer R. (2), Szkutnik M. (1,2), Bialkowski J. $(1,2)$

Department of Congenital Heart Defects and Pediatric Cardiology, SMDZ in Zabrze, Medical University of Silesia, Katowice, Poland (1); Silesian Center for Heart Diseases, Zabrze, Poland (2)

Introduction: Postinfarction Ventricular Septal Defect (PIVSD) is a rare and severe complication after myocardial infarction (MI) with poor prognosis. Transcatheter closure (TC) of such defect can be a good alternative to surgery in selected patients.

Materials and methods: All data of 26 consecutive patients (pts) $(64,6 \pm 10 y$; 9 female) in whom TC of PIVSD was attempted in our center between 2000-2015 were retrospectively analyzed. Initially, all pts were in NYHA III or IV in whom 18 pts with cardiogenic shock; 4 pts with recanalization of previously operated PIVSD. Every pt had coronary arteries angiography performed before TC, subsequently: 12 pts PCI, 6 pts CABG. Mean time between PIVSD occurrence and its TC was $10 \pm 5$ weeks (2-56). Mean PIVSD diameter was $11,4 \pm 3,8 \mathrm{~mm}(5-21 \mathrm{~mm})$ in angiography. Implants used during TC: 16 Amplatzer Atrial Septal Occluders, 4 Amplatzer Postinfarction VSD Occluders, 2 Amplatzer Muscular Ventricular Septal Occluders, 1 Amplatzer Cribriform Septal Occluder, 2 Cardi-O-Fix ASD Occluder, 1 Cera ASD Occluder. All procedures were performed under fluoroscopic (10-87 min; mean time $39 \mathrm{~min}$ ) and echocardiographic guidance.

Results: Twenty (74\%) from 26 attempted PIVSD TCs were successful. In 3 pts procedure was abandoned because of unfavorable morphology, in 2 pts in acute phase because of occluder instability and in $1 \mathrm{pt}$ embolization occurred. No peri-procedural death was observed. Significant immediate improvement occured in $14 \mathrm{pts}$ and they were discharged from hospital but 6 pts died before discharge because of increasing multiorgan failure. Mean follow-up was $5,2 \pm 4,4 y, 4$ pts needed percutaneous or surgical reintervention.

Conclusions: Transcatheter PIVSD closure is feasible procedure and should be limited to properly selected pts.

\section{P-143}

Anomalies of the coronary arteries: a fatal under-diagnosed entity

Quarti A., Iezzi F., Colaneri M., Merlino E., Santoro G., Pozzi M. Pediatric and Congeital Cardiac Surgery and Cardiology. Ospedali

Riuniti, Ancona, Italy

Introduction: The anomalies of the coronary arteries are currently blamed to be the second major cause of sudden death among young athletes.

In the last years, we have increased our rate of surgical repair on coronary arteries, as we improved our capabilities of make a correct diagnosis. However, we strongly believe that this dangerous entity is still under-diagnosed.
Methods: Between 2012 and 2016, 13 patients presented to our Institution with a high risk coronary anomaly. Eleven showed an origin from the wrong aortic sinus (AOCA) associated to an interarterial course, and two presented with a myocardial bridge $(\mathrm{MB})$. At the beginning of our experience, the AOCA was an accidental finding and was diagnosed fortuitously during a CT scan or a MRI. Subsequently, in the last two years, we were able to diagnose the AOCA using the echocardiographic examination and the role of the MRI was just confirmation and acquisition of details. The MB was suspected in young people who presented symptoms of angina, evidence of myocardial ischemia, and normal origin of the coronary arteries.

Surprisingly one of the AOCA had had surgical correction for a VSD in his youth, nevertheless the AOCA had not been diagnosed!

Results: Among the AOCA group, 3 patients had a coronary reimplantation, 2 a coronary unroofing, one had a pulmonary translocation and, finally, 1 had a patch plasty of the ostium. One patient was not operated because of a single coronary ostium facing the pulmonary artery, one refused the operation and one arrived with cardiac arrest after a basketball game and suffered a brain death despite being rescued with an ECMO support. Finally one patient is 4 years old and is currently in follow-up.

The two patients with MB had a surgical bridge opening.

All surgical patients survived and presented complete normalization of their functional tests.

Conclusions: We believe that AOCA and $\mathrm{MB}$ are more common than generally suspected. Their potential fatal role is currently well known, thus it is worth making an effort to have a diagnosis.

The role of ultrasound is crucial as it is the major contributor for the general screening.

\section{P-144}

Different pulse pressure variations in predicting fluid responsiveness in children after cardiac surgery for ventricular septal defect or tetrology of Fallot

Li J.(1), Han D.(1), Luo Y.(1), Ou-Yang C.(1,2), Pan S.D.(1) Capital Institute of Pediatrics, Beijing, China (1); Capital Medical University affiliated Beijing Anzhen Hospital, Beijing, China (2)

Introduction: Pulse pressure variation (PPV) derived from pressure record analytical method (PRAM) is based on heart-lung interaction during mechanical ventilation. We have shown that PPV is predictive of fluid responsiveness in children after surgical repair of ventricular septal defect (VSD). The preoperative pulmonary vasculature in tetralogy of Fallot (TOF) is underdeveloped as compared to that in VSD. In some of the TOF patients with severe pulmonary arterial stenosis and even more poorly developed pulmonary vasculature, Blalock-Taussig shunt is performed instead of complete repair. Postoperative pulmonary blood flow relative to the development of pulmonary vasculature in the three groups of patients would affect heart-lung interaction and therefore PPV.

Methods: VSD (VSD group, $\mathrm{n}=38,1.1 \pm 0.8$ years) and TOF (TOF-repair group, $\mathrm{n}=36,1.2 \pm 0.7$ years) infants undergone complete repair, TOF infants undergone Blalock-Taussig shunt procedure (TOF-BT group, $\mathrm{n}=24,1.3 \pm 1.2$ years) clinically present low cardiac output were enrolled. $5 \%$ albumin or blood plasma was given over 15 minutes. PPV was recorded using PRAM along with cardiac index (CI) before and after volume replacement. Patients were considered as responders to fluid loading when CI increased $\geq 15 \%$.

Results: The overall PPV was the lowest in TOF-BT group, and less so in TOF-repair group as compared to that in VSD group 
$(10.7 \pm 2.7 \%, 15.2 \pm 4.4 \%, 19.3 \pm 4.4 \%$, respectively, $\mathrm{P}<0.01)$. In VSD group, 27 were responders and 11 non-responders. Area under the curve (AUC) was 0.89 (95\% confidence interval, $0.77 \sim 1.01, \mathrm{P}<0.01)$ and cutoff value $17.4 \%$ with a sensitivity of 0.89 and a specificity of 0.91 . In TOF-repair group, 26 were responders and 10 non-responders. AUC was 0.79 (95\% CI, $0.64 \sim 0.94, \mathrm{P}=0.01)$ and cutoff value $13.4 \%$ with a sensitivity of 0.81 and a specificity of 0.80 . In TOF-BT group, 15 were responders and 9 non-responders. AUC was 0.81 (95\% CI, $0.61 \sim 0.97, \mathrm{P}=0.01)$ and cutoff value $9.5 \%$ with a sensitivity of 0.80 and a specificity of 0.78 .

Conclusion: The degree of PPV and cutoff values are different among the three groups of patients. This should be paid attention to in clinical practice in order to avoid both under- and over-estimate fluid requirement in different congenital heart defects.

\section{P-145}

Can audit lead to improved outcome in critical congenital heart disease?

Drobni Z.D.(1,2), Parry A.J. (2), Tulloh R.M.R. (2)

Cardiovascular Imaging Research Group, Heart and Vascular Center, Semmelweis University, Budapest, Hungary (1), Department of Congenital Heart Disease, Bristol Royal Hospital for Children, Bristol, $U K(2)$

Introduction: Primary repair of congenital heart disease now has low mortality, but palliation remains sometimes appropriate. With increasing complexity of treated disease, the relative risk of palliation appears to be increasing. Routine audit of our management led us to change our approach in three different time periods. This study documents how altering our approach impacted on outcome.

Methods: Retrospective analysis was performed of first shunt (MBTS) for inadequate pulmonary blood flow. Three periods were audited 2002-2006, 2007-2011 and 2012-2015. After each audit, a change in approach aimed to improve outcome. Adverse outcomes, defined as shunt failure (blockage, redo or additional early MBTS) or death, are presented with median (interquartile range) and $\mathrm{p}$ values (Kruskal-Wallis).

Results: In total 137 children underwent first MBTS, 60 were performed at 18 (7-47) days age in 2002-2006 (period A) (78\% had thoracotomy), 51 at 9 (6-34) days from 2007-2011 (period B) (41\% thoracotomy) and 26 at 12 (8-19) days 2012-2015 (period C) (5\% thoracotomy, period A vs C \%thoracotomies $\mathrm{p}=0.0001)$. Median weight at time of MBTS fell from 3.4 to $3.1 \mathrm{Kg}$ from period A to $C, p=n s$. The number undergoing MBTS with functional single ventricle was stable during the study period (period $A=15, B=14, C=13$ ), but the number of patients with two ventricles decreased from 45 (period A) to 13 (period C) $\mathrm{p}=0.043$. Despite increased complexity of cases, adverse outcome rate did not change for MBTS (A 42\%, B 41\% and C 40\%). In period A we saw MBTS blockages, in period B there was more pulmonary over-circulation and in the last we performed alternative interventions (eg outflow tract stent, duct stent, or sano shunt, $n=48$ ) in increasing frequency (MBTS in $4.8 \%$ of all operations in period $\mathrm{A}$ and $1.8 \%$ in $\mathrm{C}, \mathrm{p}=0.0001)$ despite a rise in overall number of operations. Only 3 deaths occurred with alternative intervention, so that adverse events for all conditions fell significantly during the study period (period $A=42 \%$ vs $C=26 \%$ $\mathrm{p}=0.04)$.

Conclusion: With improved operative outcomes, the MBTS is now regarded as high risk. A program of repeated audit, planning allows adverse outcomes to be minimised with alternative interventional strategy.

\section{P-146}

A successful surgical correction for a rare aortic arch interruption

Sousa A.R., Marinho J., Rosa J., Anjos R., Neves J.P.

Hospital de Santa Cruz - CHLO, Lisbon - Portugal

Introduction: Aortic arch interruption (AAI) is a rare congenital heart defect. Blood flow to the descending aorta is exclusively dependent on the ductus arteriosus making survival without treatment rare after the neonatal period. In rare cases AAI is associated with right-sided descending aorta and in these cases it is usually associated with DiGeorge syndrome. Treatment is surgical with anastomosis of both aortic ends.

Clinical case: We present the case of a newborn whose parents were first cousins. Full-term pregnancy and childbirth without complications. The newborn was admitted to a Neonatal Intensive Care Unit on day one of life for cyanosis. A diagnose of ostium secundum atrial septal defect (ASD), perimembranous ventricular septal defect (VSD) and type A aortic arch interruption (distal to the origin of the left subclavian artery) with left-sided aortic arch and right-sided descending aorta was made. DiGeorge syndrome was suspected and later confirmed. An angio-TC was performed that confirmed the echocardiographic findings and showed that the distance between the left-sided distal end of the aortic arch and the right-sided proximal end of the descending aorta was $15 \mathrm{~mm}$, making an anatomic correction technically challenging. Corrective surgery was performed at 11 days of life. The descending aorta was anastomosed to the posterior wall of the ascending aorta, proximal to the emergence of the supra-aortic trunks, and the ASD and VSD were closed. Time of extra-corporeal circulation was 2 hours and 34 minutes. There were no significant post-operative complications.

Three months after surgery the infant is doing well. The aorta has laminar blood flow with $10 \mathrm{mmHg}$ of maximal pressure gradient in the neo-aortic isthmus.

Conclusion: In AAI with left-sided aortic arch and rightsided descending aorta anatomic correction is technically challenging. The anastomosis of the descending aorta to the ascending aorta overcame this problem with good surgical and clinical outcome.

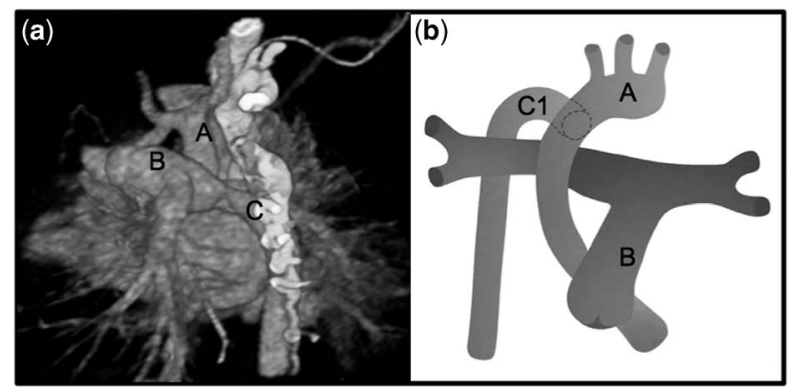

Figure 1.

Preoperative and post-operative anatomy - a) Preoperative thoracic angioTC image, b) Scheme of the postoperative anatomy.

$A$ : aortic arch, B: main pulmonary artery, $C$ : descending aorta, C1: descending aorta anastomosed to the posterior face of the ascending aorta. 


\section{P-147}

Surgical repair of the left AV valve in atrioventricular septal defect: incidence and modes of failure

Strubbe I., Bové T., Vandekerckhove K., De Groote K., Panzer J., De Wilde H., De Wolf D., François K.

University Hospital of Gent

Introduction: Late attrition of surgical correction of atrioventricular septal defect (AVSD) is strongly dependent on the result of left atrioventricular valve (LAVV) repair. The study aim is to investigate the long-term outcome of AVSD repair, based consistently on the use of a double-patch technique and primary closure of the LAVV cleft.

Methods: Retrospective investigation of 202 children with AVSD, operated on in our center between August 1992 and February 2016, was performed. Analysis focused on survival and freedom from reoperation for recurrent LAVV dysfunction, with specific attention to the mode of valve failure.

Results: The study population consisted of $129(64 \%)$ cAVSD and $73(36 \%)$ pAVSD patients, corrected at the mean age of $7.6(95 \% \mathrm{CI}$ 5.2-9.9) and 81 (95\%CI 53-109)months respectively. Survival at 10 year was $86 \pm 4 \%$ for $\mathrm{cAVSD}$ and $98 \pm 2 \%$ for $\mathrm{pAVSD}(\mathrm{p}=0.012)$ yielding a significantly better 8 -year survival during the second decade of the study $(91 \pm 4 \%$ versus $81 \pm 7 \%, \mathrm{p}=0.02)$. Within a mean follow-up of 7.6(95\%CI 6.5-8.7)years, $27(13 \%)$ patients required reoperation for LAVV dysfunction, respectively in 17 $(13 \%)$ cAVSD and 10(14\%) pAVSD patients. Freedom from LAVV reoperation at 10 year was $86 \pm 4 \%$ for cAVSD and $91 \pm 4 \%$ for $p A V S D(p=0.97)$. The mode of failure was predominantly technical in cAVSD (69\% versus $22 \%$ in pAVSD), while LAVV failure was preferentially due to residual anatomical abnormalities in pAVSD ( $78 \%$ versus $31 \%$ in cAVSD) $(p=0.04)$. Most frequent causes of technical failure were cleft suture dehiscence $(n=8)$, incomplete cleft closure $(n=5)$, whereas anatomical reasons were remnant distortion of the subvalvular apparatus $(\mathrm{n}=10)$, small asymmetric bridging leaflet $(\mathrm{n}=1)$ or double orifice $(n=2)$, and additional cleft $(n=1)$. Eight patients underwent second LAVV surgery, and 2 patients even a third procedure. Other reoperations were required for acquired LVOTO $(n=8)$ and pacemaker implantation $(\mathrm{n}=14)$.

Conclusion: Whereas advances in the peri-operative management have improved their survival, the long-term outcome of AVSD patients is still importantly affected by the faith of the LAVV. Despite the systematic closure of LAVV cleft, patients with cAVSD remain at risk of LAVV failure, mainly by technical inadequacy, while pAVSD patients need increased attention for additional anatomical features at the time of surgical repair.

\section{P-148}

Single-center experience with mechanical valve replacement in children and adolescents: a lifelong challenge

Verbeke J., Bové T., De Groote K., Vandekerckhove K., Panzer J., De Wilde H., De Wolf D., De Backer J., Demulier L., François K.

University Hospital of Gent - Gent - Belgium

Introduction: Although valve repair is the preferred treatment of valve diseases in children and adolescents, valve replacement is sometimes unavoidable. The purpose of this study is to evaluate the late clinical and valve-related outcome of a pediatric population with a mechanical valve prosthesis.

Methods: Retrospective investigation is performed of all patients, treated with a mechanical prosthesis before the age of 20 years, for a congenital or acquired heart valve disease during past 2 decades.
Analysis focused on late outcome in terms of survival and freedom from valve- and anticoagulation (AC)-related events.

Results: The study population comprised 41 patients, with a mean age of 11.6 y (95\% CI 7.6-15.5), presenting dysfunction of the mitral $(56 \%)$, aortic $(24 \%)$, tricuspid $(10 \%)$, pulmonary $(5 \%)$ or multiple valves (5\%). Etiology was primarily congenital in $80 \%$, rheumatic in $12 \%$, genetic in $5 \%$ and infectious in $3 \%$. The majority of children (83\%) underwent already previous valve surgery, of whom $39 \%$ more than 1 procedure. Survival at 20 years was $76 \pm 10 \%$. Within a mean follow-up time of $9(95 \%$ CI $5-14)$ years, $43 \%$ of the patients remained free from any valve-related event, which were predominantly thrombo-embolic (20\%), prosthetic dysfunction (17\%), endocarditis (14\%) and major bleeding $(6 \%)$. Late valve-related reoperation was required in $39 \%$ of the patients for prosthesis outgrowth $(33 \%)$, prosthesis dysfunction (3\%) and endocarditis (3\%). AC-related events were observed in $46 \%$, of which occurred in $73 \%$ of the patients showing an INR variability $\geq 30 \%$. Patients with INR-selfmonitoring $(n=5)$ showed lower INR variability compared to INR control by the local physician: INR variation was $29 \pm 21 \%$ versus $43 \pm 21 \%(p=0.05)$. Patients with selfmonitoring demonstrated no AC-related event: $0 \%$ versus $50 \%(\mathrm{p}=0.04)$. The rate of serious adverse events, including death, is $66 \%$ of all patients in follow-up.

Conclusion: This single-center study confirms that $2 / 3$ of the patients receiving a mechanical heart valve prosthesis during childhood experience at least one serious adverse event during later life. Half of these events are related to the need for anticoagulation, through showing a high INR-variability in this young population. Self-monitoring of INR might decrease this specific complication rate, yielding however the limitation of possibly including a selection bias.

\section{P-149}

Two Stage Arterial Switch in the Transposition of the Great Arteries with an Intact Ventricular Septum beyond Neonatal Period: Which is the best option?

Pulmonary Banding and Arterial BT Shunt or Pulmonary Banding and Bidirectional Cava-Pulmonary Shunt

Cevik A., Polat B., Zeybek C., Tuncel Z., Morkoç E.E., Yazicioglu V., Karaci A.R., Yalcin Y. Istanbul Bilim University Faculty of Medicine, Istanbul, Turkey.

Objective: Left ventricle retraining is necessary for the patients who have left ventricle dysfunction and transposition of great vessels with intact ventricular septum (TGA-IVS) before anatomical repair beyond the neonatal period.

Material and methods: Between July 2013 and August 2016, fortyseven patients who had left ventricle dysfunction and had applied twostage arterial switch operation with the diagnosis of TGA-IVS were assessed retrospectively. The criteria for the left ventricle (LV) retraining were defined as a combination of these conditions including being older than 2 months old, having LV mass index in echocardiography less than $35 \mathrm{gr} / \mathrm{m}^{2}$ and having an interventricular septum with "banana-shape" appearance. The patients were divided into two groups: one with patients receiving pulmonary artery banding and arterial BT shunt (n:19) and the other with patients receiving pulmonary artery banding and venous bidirectional cava-pulmonary shunt (n:28) in preparation to the arterial switch operation.

Results: The average age was found to be $122.3 \pm 45,6$ days in arterial shunt group and $145.9 \pm 37.2$ days in venous shunt group. No statistically significant difference $(p=0232 ; p=0373)$ was found between the LV mass index averages of the two groups which had been evaluated before the first stage and second stage (before the first stage $26,6 \pm 4,8 \mathrm{gr} / \mathrm{m}^{2} ; 25,0 \pm 4,9 \mathrm{gr} / \mathrm{m}^{2}$; before 
the second stage $\left.70,5 \pm 12 \mathrm{gr} / \mathrm{m}^{2}, 6 ; 73,8 \pm 12,0 \mathrm{gr} / \mathrm{m}^{2}\right)$. However, the average of time interval for the $\mathrm{LV}$ to retrain was $97,7 \pm 42,9$ days for the arterial shunt group and 117,3 \pm 40,3 days for the venous shunt group and the interval period was significantly low in the arterial shunt group $(p=0027)$. Time spent in intensive care unit, length of the period during which inotropic support was required and duration of hospital stay were significantly higher in arterial shunt group than the venous shunt group ( $\mathrm{p}<0001 \mathrm{p}<0001 \mathrm{p}<0001)$.

Conclusion: Bidirectional cava-pulmonary shunt can be performed as an alternative to arterial BT shunt for the cases of TGA-IVS in which two-stage arterial switch and LV retraining are indications beyond the neonatal period since venous bidirectional cavapulmonary shunt requires a shorter duration of hospital stay and has less complications. However, LV retraining period can be longer in venous shunt probably due to LV afterload.

\section{P-150}

Near infrared spectroscopy as supplementary hemodynamic monitoring after cardiac surgery

Hansen J.H. (1), Kissner L. (1), Logoteta J. (1), Jung O. (1), Dütschke P. (2), Scheewe J. (3), Kramer H.-H. (1,4)

Department of Congenital Heart Disease and Pediatric Cardiology, University Hospital of Schleswig-Holstein, Campus Kiel, Kiel, Germany (1); Department of Anesthesiology and Intensive Care Medicine, University Hospital of Schleswig-Holstein, Campus Kiel, Kiel, Germany (2); Department of Cardiovascular Surgery, University Hospital of Schleswig-Holstein, Campus Kiel, Kiel, Germany (3); DZHK (German Center for Cardiovascular Research), partner site Hamburg/ Kiel/Lübeck (4)

Background: Near infrared spectroscopy (NIRS) allows continuous and non-invasive monitoring of regional tissue oxygen saturation. We aimed to evaluate the value of NIRS as supplementary hemodynamic monitoring after cardiac surgery with cardiopulmonary bypass in neonates and infants.

Methods: Cerebral $(\mathrm{ScO} 2)$ and somatic $(\mathrm{SsO} 2)$ tissue oxygen saturations (INVOS, Covidien ${ }^{\circledR}$ ) were obtained after 83 cardiac surgeries (neonates: $\mathrm{n}=38$, infants: $\mathrm{n}=45$ ). NIRS data was recorded for 48 hours and matched with routine monitoring data. The relationship between regional tissue oxygen saturation and central venous saturation $(\mathrm{SvO} 2)$ was of special interest.

Results: In neonates, $\mathrm{ScO} 2$ declined in the first postoperative hours, reaching a nadir after approximately 4 hours. In infants, a rise of $\mathrm{ScO} 2$ was noted directly after surgery. In both groups, $\mathrm{ScO} 2$ reached a plateau after approximately 20 and 8 hours, respectively. Mean $\mathrm{ScO} 2$ of the entire postoperative course was not different between neonates and infants $(71 \pm 12 \%$ vs. $67 \pm 8 \%, p=0.071)$, while mean $\mathrm{SvO} 2$ was lower in infants $(73 \pm 8 \%$ vs. $63 \pm 5 \%, \mathrm{p}<0.001)$.

For the entire cohort, $\mathrm{ScO} 2$ and $\mathrm{SvO} 2$ were moderately correlated $(r=0.644, p<0.001)$, a weaker correlation existed between $\mathrm{SsO} 2$ and $\mathrm{SvO} 2(\mathrm{r}=0.472, \mathrm{p}<0.001)$. For estimation of $\mathrm{SvO} 2$ from $\mathrm{ScO} 2$, Bland Altman analysis showed a mean bias of $1.4 \%$, but wide limits of agreement (-19.2\% to $22.1 \%)$. Prediction of $\mathrm{SvO} 2$ slightly improved using a combination of $\mathrm{ScO} 2$ and $\mathrm{SsO} 2$ values. In neonates, correlation between $\mathrm{ScO} 2$ and $\mathrm{SvO} 2$ was weaker in the early compared to the later postoperative course $(0-8 \mathrm{~h}$ : $\mathrm{r}=0.383$ vs. $9-48 \mathrm{~h}: \mathrm{r}=0.662, \mathrm{p}=0.001)$, while no difference was found in infants $(0-8 \mathrm{~h}: \mathrm{r}=0.768$ vs. $9-48 \mathrm{~h}: \mathrm{r}=0.735, \mathrm{p}=0.211)$. In neonates, the mean bias in the early postoperative course was $-11 \%$ (limits of agreement: $-40.6 \%$ and $19.2 \%$ ) compared to $1,6 \%$ (limits of agreement: $-17.4 \%$ and $20.7 \%$ ) in the later course.

For the estimation of the difference between two consecutive SvO2 measurements from corresponding $\mathrm{ScO} 2$ values, Bland Altman analysis showed a bias of -0.5 (limits of agreement: $-18.1 \%$ and $17.1 \%$ )
Conclusion: Despite moderate correlation, absolute values and changes in $\mathrm{ScO} 2$ do not necessarily reflect $\mathrm{SvO} 2$ measurements. Especially in neonates, the relationship between $\mathrm{ScO} 2$ and $\mathrm{SvO} 2$ in the early postoperative course is weak.

\section{P-151}

Long-term outcomes after Ross procedure in different age groups: a single - institution experience

Klymyshyn Y., Lebid I., Khanenova V., Romanyuk O., Rudenko N., Artemenko $Y$.

Government institution "The Scientific-Practical Children's Cardiac

Center the Ministry of Health of Ukraine» Kyiv, Ukraine

Treatment of patients in different age groups with congenital pathology of aortic valve is still one of the important problem in cardiac surgery. The Ross procedure is an alternative to the use of mechanical, biologic, and homograft valves for aortic valve disease. Objective: To examined long-term outcomes after Ross procedure for twenty-year period.

Patients and Methods: During the period from 1996 to 2015 y 200 consecutive patients underwent Ross procedure at our institution. The mean age of patients was $145 \pm 101$ months, $24 \%(n=48)$ of them -in the age of 5 years, $18 \%(\mathrm{n}=36)$ - from 6 to 10 years, $30 \%$ $(n=59)-$ from 11 to 17 years and $28 \%(n=57)$ older then 18 years. Aortic stenosis was the lesion in $103(51,5 \%)$ patients, aortic insufficiency in 68 (34\%) and mixed lesion in 29(14,5\%) patients. Indications for reoperations after Ross procedure on autograft were severe autographt insufficiency and aortic root dilatation and on RV-PA conduit was severe conduit stenosis. Long-term follow up at median was $11,5 \pm 6,5$ years.

Results: There were 18 deaths $(9 \%)$. Mortality in early postoperative period was 13 deaths $(6,5 \%)$, in late postoperative period was $5(2.9 \%)$. Long-term results followed in 170 (93.4\%) patients. Ten $(5,9 \%)$ patients required reoperations on the pulmonary autograft (4 repairs and 6 replacements), $32(18,8 \%)$ patients in the RV-PA conduit (4 plasty and 28 replacement) and were performed $32(18,8 \%)$ transcatheter procedure.

Freedom from any reinterventions after Ross procedure was $66.0 \%, 61.4 \%, 58.5$ after 10,15 and 20 years. Freedom from reoperation on autograft was $-98.9 \%, 95.4 \%, 93.7 \%$ after 10,15 and 20 years. Freedom from reoperations on RV-PA conduit was $84.1 \%, 83.6 \%, 82.4$ after 10,15 and 20 years. Freedom from transcatheter procedure on RV-PA conduit was $83.7 \%, 82.5 \%$, $82.5 \%$ at 10,15 and 20 years.

Conclusions: The Scientific-Practical Children's Cardiac Center have the highest amount of patients after Ross procedure in Ukraine. The Ross procedure is a safe procedure with low mortality at early and long-term postoperative period. Reoperations are due to progressive autograft root dilation were rare in our patients. More frequent reoperations were on RV-PA conduit and require further study.

\section{P-152 \\ ChyloBEST - Chylothorax in Infants and Nutrition with Low Fat Breast Milk}

Springer T. (1), Nieschke K. (1), Abedini M. (2), Kostelka M. (3), Dähnert I. (1)

(1) HeartCenter Leipzig, Germany, Pediatric Cardiology, (2) Asklepios Hospital St. Augustin, Pediatric HeartCenter, Germany, (3)

HeartCenter Leipzig, Germany, Pediatric Heart Surgery

Objectives: The incidence of postoperative chylothorax (CT) is approximately $5-9,2 \%$ in neonatal heart surgery (10\% at Heart Center 
Leipzig). CT is treated by drainage and low fat diet using Formula ("Basic-F"). Low fat diet is usually mandatory for six weeks to prevent re-accumulation of chyle. Advantages of breast milk especially for operated infants are well known. The possibility of using breast milk for this patients would be a great development. The Trial ChyloBEST is focused on the treatment of CT with low fat breast milk (LFBM) as well as the cause of CT. Incidence of CT may be potentially related to increased complexity of neonatal heart surgery.

Methods: We report a prospective non-randomized multicenter (Leipzig, St. Augustin) pilot study. ChyloBEST includes neonates with congenital heart diseases and postoperative CT $(n=16)$. To receive LFBM milk will undergo kryo centrifugation for 15 minutes at $2^{\circ}$ Celsius. The fatty layer will separate on the top of the milk sampling. It can be easily removed mechanically. By adding high quality fat additives (MCT-Oil) and common human milk fortifier LFBM is prepared for feeding. The amount of fat, carbohydrates, proteins and energy were determined. To prove the efficacy of this diet following items were monitored: drained amount of pleural effusion, recurrence of CT, physical development within three months after diagnosis.

Results: By kryo centrifugation the fat content of breast milk could be reduced significantly. The content of other nutritional milk components of LFBM remained unaltered (meanFat: $0,36 \% \star$, meanProtein: 1,5\%, meanCarbohydrate: 7,1\%, meanEnergy: $39,3 \mathrm{kcal} / 100 \mathrm{ml})$. To date, 16 patients $(\mathrm{n}=5$ Norwood-stage 1 , $\mathrm{n}=6$ arterial switch, $\mathrm{n}=1$ TAPVD-redir., $\mathrm{n}=1$ Glenn, $\mathrm{n}=1$ TAC-correction, $\mathrm{n}=1$ CoA-resection, $\mathrm{n}=1$ complex VSD-closure) received LFBM diet. CT resolved in all cases. There was no CT relapse when returning to full fat breast milk even in cases diet was performed less then six weeks. After LFBM diet ten patients $(62,5 \%)$ achieved exclusive breastfeeding and well thriving. Conclusions: Producing LFBM is technically easily feasible. In case of postoperative CT LFBM diet seems to be a reliable nutrition form including the advantages of breast milk feeding.

\section{P-153}

Early results after cone reconstruction of the tricuspid valve in Ebstein's anomaly

SchrempfJ., Sames-Dolzer E., Kreuzer M., Benedikt P., Gitter R., Grohmann E., Tulzer G., Mair R.

Children's Heart Center Linz, Austria

Objectives: Patients with Ebstein's anomaly often suffer from severe tricuspid regurgitation, right ventricular dysfunction or rhythm disturbances. Our study shall evaluate early results after cone reconstruction of the tricuspid valve in pediatric and adult Ebstein patients. Methods: Our single center retrospective study reports 16 patients with Ebstein's anomaly corrected by cone reconstruction since 2012 . Four of them were infants including two neonates; median patient age was 5,79 years (ranging from 5 days until 56,34 years). Tricuspid reconstruction was done according to da Silva's method including the septal leaflet in the repair. A longitudinal ventricular plication and an annular plication were done as reported by Carpentier. A $4 \mathrm{~mm}$ patent foramen ovale was left open.

Three patients also had a pulmonary atresia, additionally treated with an RVPAC and a modified Blalock-Taussig shunt. The cone procedure was done primarily in one of these patients and at time of conduit change in the other 2 patients.

Results: Both neonates needed ECMO therapy postoperatively, but could be weaned successfully. Unfortunately one of them died despite good cardiac function on the fourteenth postoperative day because of massive cerebral hemorrhage. The other patients are doing well. Postoperative ultrasound showed none or minimal tricuspid insufficiency in 12 patients and moderate tricuspid valve insufficiency in 4 patients. There was no need for reoperation because of tricuspid valve insufficiency or stenosis during follow up. Postoperative rhythm disturbances occurred in 7 cases ( 4 patients showed episodes of atrial tachycardia, 3 had a temporary AV block grade III without need for pacemaker implantation).

Conclusions: The cone reconstruction can achieve excellent tricuspid valve reconstruction with good right ventricular function. Our study shows a promising option for symptomatic infants with severe $\mathrm{Mb}$. Ebstein. Rhythm disturbances occurred usually self-limiting, but should be treated thoroughly to avoid negative effects on cardiac output.

\section{P-154}

Neurological Complications after Surgery for Congenital Heart Defects in Children - A 15-Year Retrospective Survey Askfelt A. (1), Johansson Ramgren J. (2), Malm T. (2), Wingren P.(3), Liuba P.(1)

Pediatric Cardiology (1), Cardiac Surgery (2) and Radiology (3), Skåne University Hospital, Lund, Sweden

Background: Major advances in cardiac surgery and postoperative care for patients with congenital heart defects have resulted in significantly improved survival. Postoperative neurological complications remain an important concern in terms of morbidity. We sought to determine the incidence and onset of neurological complications in children after cardiac surgery.

Method: We performed a retrospective survey of all cases with suspected neurological complications after cardiac surgery between 2001 and 2015 at the Skåne University Hospital. Data included birth characteristics, cardiac diagnosis, pre-, intra- and postoperative variables. A postoperative neurological complication was defined as detected brain injury on either computer tomography (CT) or magnetic resonance imaging (MRI) within 30 days after cardiac surgery. Results: During the study period, a total of 4707 pediatric cardiac surgeries were performed at our center. Neurological complications based on clinical findings were suspected in $40(0.8 \%)$ patients and were eventually confirmed in 20 patients $(0.4 \%)$. Confirmed brain injury had no correlation with cyanotic CHD, age at surgery, preoperative oxygen saturation or cardiopulmonary bypass (CPB) time. Paresis was the initial symptom that was most often associated with positive CT or MRI findings $(51 \% ; p=0.02)$, whereas seizure was more often encountered among patients with negative CT or MRI findings $(55 \% ; p=0.01)$. In the majority of cases with confirmed neurological complication, the symptoms started within the first week after surgery (median: 2 days; 1-29).

Conclusion: The incidence of neurological complications in children operated for congenital heart defects at our center is very low. The majority of such complications occur during the first week after surgery. There was a significant correlation between the clinical presentation in form of paresis and verified brain injury.

\section{P-155}

Prognosis of children with Kawasaki Disease and compromised Haemodnamics

Rapin S.(1), Veyrier M.(2), Teyssier G.(1), Patural H.(1), Durrousset C.(2), Ducreux C.(2), Bakloul M.(2), Di Filippo S.(2)

(1) Pediatric Intensive care unit, Saint Etienne University Medical center, Saint-Etienne, France, (2) Cardiovascular Hospital Louis Pradel, University Claude Bernard Lyon1, Hospices Civils de Lyon, Lyon France

The aim of this study was to assess cardiac features and outcomes of children with severe hemodynamically-compromised Kawsaki Disease (KD). 
Material and Methods: Retrospective single-center study of children admitted from 1997 to 2014 in Intensive care unit for hemodynamics failure. Clinical features, cardiac lesions and short and longterm outcomes were analyzed.

Results: Six patients were included in the study, 3 males and 3 females. Median age at admission in ICU was 48 months. Cardiogenic shock was present in 4 cases $(66 \%)$ and hypovolemic shock in 2 (33\%), 5 (83\%) had typical KD clinical features and 5 had multivisceral failure (83\%) of which 3 neurological complications (encephalitis, meningitis). Left ventricle dysfunction (LV shortening fraction $=20 \%$ ) was present in the 4 cases with cardiogenic shock, 4 had pericardial effusion and 2 mitral valve regurgitation. Three cases $(50 \%)$ had transient coronary artery dilations an 2 had coronary arteries aneurisms (33\%), i.e. coronary arteries lesions were present in $83 \%$ of the cases. Median C-reactive protein was $278 \mathrm{mg} / 1$ (267 to 305$)$, albuminemia was $21 \mathrm{~g} / 1$ (15 to 33), hemoglobin level was $91 \mathrm{~g} / 1$ (70 to 101) and platelet count was $254.109 / 1$ (161 to 745). Mean time from onset of symptoms to IVIG infusion was 4 days ( 3 to 10 days). IVIG resistance occurred in 3 patients $(50 \%)$ who received second-line steroids therapy. No death occurred. Median hospital stay was 9.5 days (5 to 30 ). Coronary arteries aneurysms persisted in long-term follow-up in 2 patients (33\%).

Conclusion: Severe KD in children presents with hemodynamic and cardiogenic shock. Incidence of cardiac lesions is very high although IVIG infusion is not delayed, with higher IVIG resistance than in classic KD. One third of the cases suffer from long-term cardiac sequelae. These results might lead to recommend first-line treatment with IVIG resistant-type therapeutics.

\section{P-156}

Infective endocarditis in children with normal heart

Veyrier M., Duperril M., Ducreux C., Durousset C., Ninet J., Bakloul M., Walton C., Perouse de Monclos T., Di Filippo S.

Cardiovascular Hopital, Université Claude Bernard Lyon1, Hospices

Civils de Lyon, Lyon, France

The objective of this study was to assess the features of infective endocarditis (IE) and long-term prognosis of children with normal heart.

Material and methods: Data of all patients less than $18 \mathrm{y}$ diagnosed with IE and no previous cardiac lesion from 1972 to 2016 were retrospectively analyzed. Demographics, cause of IE, microbial agent, cardiac location, short and long term outcomes and survival were assessed. Diagnosis of IE relied on Duke criteria.

Results: 34 patients ( 20 females $=59 \%$ ) aged $8.8 \pm 5.4 \mathrm{y}$ at diagnosis were included. All presented with fever and a heart murmur, and had echographic evidence of vegetations left-heart sided in 27 cases $(80 \%=17$ mitral valve, 7 aortic valve, 3 mitral and aortic) or right-heart sided in 6 (tricuspid valve) (18\%); vegetations were present on aortic and mitral and tricuspid valves in 1 case. Microbial agents were identified in $88 \%$ of the cases (17 staphylococcus (57\%), 4 streptococcus (13\%), 4 kinghella kingae, 4 corynebacterium and 1 propionibacterium acnes), unknown in $12 \%$. Causes of IE were identified in 16 cases (47\%), of which $50 \%$ were cutaneous, $25 \%$ dental and 25\% miscellaneous. Cerebral embolic events occurred in 10 cases $(29.5 \%)$, other embolic localisations in $8(24.5 \%)$ (total of embolic events $=53 \%$ ), severe HF in 6 cases $(18 \%)$. Early surgery was performed in 22 of 34 patients (65\%) because of embolic events and/or severe HF, at $19 \pm 5$ days after diagnosis of IE (median 13 days, range 1 to 68 days): 15 valvular plasties (68\%) and 4 valvular replacements. Hospital stay ranged 19 to 95 days (mean 43days). Mean follow-up was 10 years (median $4.5 y)$. No patient died, 3 experienced a second episode of IE (9\%).
Sequellae (valvular regurgitation) were present in patients who did not require surgery.

Conclusion: IE can occur in children with normal heart is more frequently due to Staphylococcus agent, from a cutaneous cause. Early surgery is performed in most of the cases with favorable outcome, sequaella persisted in unoperated cases.

\section{P-157}

Does heart-type fatty acid-binding protein (H-FABP) predict clinical outcomes after pediatric cardiac surgery? Walavalkar V. (1), Evers E.S. (2), Pujar S. (1), Balasubramanian L. (1), Prinzen F.W. (2), Delhaas T. (2), Vanagt W.Y. (2), Maiya S. (1) Narayana Institute of Cardiac Sciences, Bengaluru, India (1), Cardiovascular Research Institute Maastricht, Maastricht University, The Netherlands (2).

Introduction: Children undergoing cardiac surgery can suffer considerable morbidity and mortality, in spite of recent advances in peri-operative cardio-protection. Early identification of ischemia / reperfusion injury could help clinicians provide timely support and prevent prolonged intensive care unit (ICU) stay. We evaluated Heart-type Fatty Acid Binding Protein (H-FABP), a cardiac injury marker that is released almost immediately following myocadial injury, as a predictor of clinical outcome, and compared it with the commonly used bio-markers creatine kinase-myocardial band (CK-MB) and Troponin-I (both peaking 3-6 hours after injury), as well as with bypass time.

Methods: This is a retrospective study of patient data from a blinded randomized, controlled trial (RCT) with sildenafil preconditioning aiming to reduce ischemia/reperfusion injury, aged 1- 16 years, undergoing open heart surgery for ventricular septal defect (VSD) closure. Patient data was pooled together for the current analysis as there was no difference between the sildenafil and placebo groups. We measured H-FABP, Troponin-I and CK-MB preoperatively and 1, 3 and 6 hours after aortic de-clamping in 32 patients. Area under the release curve was calculated for the injury markers. Spearman's Rho Correlations were calculated between laboratory and clinical parameters including inotropic support duration, aortic cross-clamp time, total bypass-time, ventilationweaning-time and total ICU-stay. We considered Spearman's rho correlations 0-0.19: very weak, 0.2-0.39: weak, 0.4-0.59: moderate, 0.6-0.79: strong, 0.8-1: very strong. Patients' data are expressed as median (interquartile range Q1-Q3).

Results: Age was 47 months (24-94), ACC time 30 minutes (26-36), bypass time 57 minutes (50-67), ventilation-weaning time 116 minutes (87-362), inotropy time 26 hours (20-38), ICU stay 38 hours (25-47), and pre-discharge LVEF 64\% (60-66).

The correlations of H-FABP, CK-MB, Troponin-I and bypass time with clinical outcome measures were only weak-moderate. There were strong correlations between all three injury markers. Conclusion: The predictive value of H-FABP for clinical outcome is not stronger than that of CK-MB, Troponin-I or bypass times. $\mathrm{H}-\mathrm{FABP}, \mathrm{CK}-\mathrm{MB}$ and Troponin-I are not clinically useful predictors of clinical outcomes in pediatric cardiac surgery.

\section{P-158}

Atrial switch operation: our experience in the last decade Polo L., Sánchez R., González A., Rey J., Monteagudo M., Bret M., Labrandero C., Deiros L., Durán P., Aroca Á.

La Paz University Hospital. Madrid. Spain

Introduction: Surgical results of D-Transposition of the great vessels $(\mathrm{TGV})$ are a good quality marker of a neonatal intensive care unit, 
because TGV is a prevalent pathology with reproducible surgery, and comparable between Hospitals. We present our surgical results and follow-up during the last 10-years.

Methods: Retrospective analysis of 94 infants with biventricular heart and TGV operated with arterial switch operation (ASO) during the period 2007-16. We excluded patients with pulmonary stenosis needing other techniques different than ASO. Statistical study was done with SPSS-15.0

Results: Mean age: 0,4 $\pm 0,4$ months, mean weight $3 \pm 0,6 \mathrm{~kg}, 69 \%$ were males. Prenatal diagnosis was made in $63 \%$, and $13 \%$ were premature. TGV was associated with ventricular septal defect (VSD) in 32\%, and coarctation in 6\%. Rashkind was performed in $77 \%$, and intravenous Prostaglandin was retired in $57 \%$ before surgery. Preoperative respiratory assistance was needed in $25 \%$, and neurological anomalies were detected in 11\%. Anomalous coronary pattern was present in $14 \%$ with high correlation with echocardiography findings (95\%). Three patients had previous surgeries: two received pulmonary band, and one a modified Blalock Taussig shunt. All patients were repaired with an ASO, $24 \%$ had their VSD closed, $6 \%$ had a coarctation resection with selective cerebral perfusion, and $79 \%$ had delayed sternal closure. Coronary buttons were transferred with trap-door in $81 \%$. Mean extracorporeal and aortic-clamp times were $208 \pm 66$ and $139 \pm 33$ minutes respectively. Hospital mortality was $8,6 \%$. Complicated postoperative stay (p:0,05), VSD closure (p:0,04) and coarctation (p: 0008 ) were risk factors of mortality. Hospital morbidity was (64\%), with arrhythmia, reoperation, pleural/peritoneal drainage, infection, phrenic nerve palsy and low cardiac output as the more frequent complications. Median intubation time was 120 hours (IQR 92-168), and postoperative stay was 21 days (IQR 15-35). Mean follow-up is $40 \pm 31$ months. Percutaneous reintervention was needed in 15 patients and reoperation in 10 patients during follow-up. Nowadays all patients are alive in good clinical condition and $91 \%$ have no cardiac medication.

Conclusions: Surgery and postoperative management of TGV are a challenge. Preoperative echocardiogram accurately defines the anomalous coronary patterns and helps the surgeon to plan the operation. Our results show acceptable mortality but moderated morbidity.

\section{P-159}

Do children who have been operated on for aortic arch during the first months of life need educational and psychotherapeutic support?

Sanchez R.(1), Polo M.L.(1), Tirado P. (2), Herrera C. (2), Rey J. (1), González A. (1), Deiros L. (3), Uceda A. (3), Climent F.J. (4). Garcia M. (4), Diez J. (5), Hernández L. (2), Moran P. (6). Aroca A. (1) Pediatric Cardiac Surgery, Hospital La Paz. Madrid. Spain (1); Neuropediatrics, Hospital La Paz. Madrid. Spain (2); Pediatric Cardiology. Hospital La Paz. Madrid. Spain (3); Unit Complex Pediatric Pathology, Hospital La Paz. Madrid. Spain (4); Unit Biostatistics Hospital La Paz. Madrid. Spain (5); Pediatric Anesthesia. Hospital La Paz. Madrid. Spain (6)

Objective: Study of the neuropsychological development of school-age children who have been operated on during the first weeks of life for hypoplasia of the aortic arch using selective anterograde cerebral perfusion.

Methods: Neuropyschological analysis of children who have been operated on for aortic arch during the period 2004-2012 in our center. We evaluated their intelligence, psycho-linguistic aptitudes, attention, behavior, memory, motor functions and autistic characteristics.

Primary outcome: Need for educational or psychotherapeutic support for school age children.
Criteria for inclusion: School age children who are currently alive, who were operated on through aortic arch surgery using selective anterograde cerebral perfusion.

Criteria for exclusion: Prematurity less than 34 weeks of gestational age and genetic alteration that affects neuropsychological development. Results: 52 patients were included. 57\% male and $43 \%$ female. Average age of intervention 1.5 months (range 10 days to 9 months). Average weight during surgery: $3.5 \mathrm{Kg}$. (range: $1.9 \mathrm{~kg}-9 \mathrm{~kg}$ ). Surgical technique: $36 \%$ aortic arch repair exclusively and $64 \%$ using aortic arch repair and intracardiac surgery. Average time of extracorporeal circulation: 150 minutes (range: 63-480 minutes). Average time of selective anterograde cerebral perfusion: 32 minutes (range: 18-63 minutes). Attention deficit: 35\%. Receiving Methylphenidate treatment: 15\%. Intelligence: $57 \%$ normal, $28 \%$ borderline. Severe intellectual disability: $8 \%$. Speech: $64 \%$ normal, $16.7 \%$ constitutional speech delay, 9.5\% mixed disorder, communication disorder with autistic characteristics: $4.8 \%$. Motor function: $26 \%$ normal, $65 \%$ fine motor alteration. $65 \%$ have required school support at some point in time.

Conclusions: The majority of children who have received aortic arch surgery early in life require educational support. It is important to study the long term evolution of children operated on due to congenital heart disease in order to improve surgical techniques, understand the long term prognosis and to be able to initiate preventative and therapeutic treatment that will help improve the quality of life of operated children and their families.

\section{P-160}

Surgical management of atrioventricular septal defects with double orifice left atrioventricular valve

Gran C., Ellouali F., Ovaert C., Fouilloux V., Mace L.

Department of pediatric and congenital heart disease, La Timone Hospital, Marseille, France

Objectives: To compare the post operative results of patients with atrioventricular septal defect (AVSD) and double orifice of the left atrioventricular valve (LAVV) to a matched control group.

Methods: A retrospective matched cohort method was used to compare group 1 (11 patients with AVSD and LAVV double orifice) to 11 control patients, matched on weight, age and AVSD type. LAVV double orifice could be native or after Alfieri plasty. All have been operated on between 2008 and 2016.

Results: The median lenght of stay in intensive care unit was significantly higher in the first group $(\mathrm{p}=0$ 038). There were two deaths in group 1 and no death in group $2(\mathrm{p}<00001)$. The subgroup analysis of double orifice shows that total cleft closure was not a risk factor for LAVV stenosis. There was more significant regurgitation when the cleft was left open or when it was partially closed. The main indication for reoperation was a significant LAVV regurgitation. Conclusion: For patients with AVSD, double orifice of LAVV is a risk factor for death and reoperation. The total cleft closure seems to limit regurgitation, without implicating stenosis.

P-161

$3 \mathrm{D}$ printing of heart with congenital heart disease-A case study Rao G.K (1,2), Muskawad S.D (2), Mohanty S.R (3) Imaginarium India Pvt.Ltd Mumbai, INDIA(1); Indian Institute of Technology Bombay, Mumbai, INDIA(2); Kokilaben Hospital \& Research Centre, Mumbai, INDIA (3)

Introduction: Objective of this study is to highlight the insight provided by patient specific 3D printed model of heart with 
congenital heart disease. The "3D printed physical model" provides detail morphological information for patient specific clinical management. The management of congenital heart disease includes invasive, non-invasive and catheter based intervention with accurate timing. "3D printed physical model" provides information for cardiologist and cardiac surgeons to manage patient from their perspective.

Method: A 5 month old boy with complex congenital heart disease with severe Pulmonary artery hypertension underwent assessment at our centre. Echocardiography revealed, heterotaxy, situs ambiguous, mesocardia, L-loop ventricles with L-posed aorta, common atrium, systemic venous anomaly in the form of interrupted IVC draining through azygous vein to the left sided SVC and hepatic veins draining into the right sided aspect of the common atrium (Fig.1) The pulmonary veins were lateralised. The baby had two good sized ventricles with large mid muscular VSD with severe right ventricular dilation.

CT scan was done to reconfirm the anatomy and subsequently $3 \mathrm{D}$ model was printed to aid in planning the surgical correction. The DICOM data set obtained from CT scan was processed to reconstruct a 3D digital model (Fig.2) using mimics software aided by skilful manual segmentation. The model was exported as STL file for 3D printing. The model was 3D printed by selective laser sintering of nylon powder (Fig.3). With Clinician's inputs, the model is pre-cut and built so as to maximise the visualisation of the

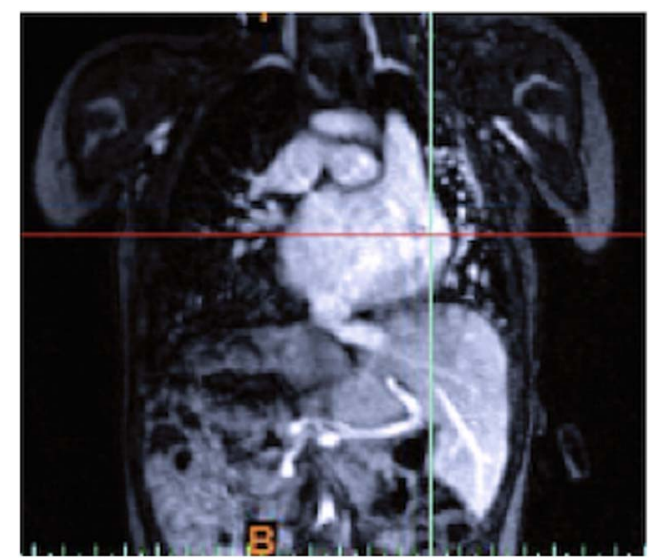

Figure 1.

$C T$ scan reveling situs ambiguous, $A S D$, VSD.

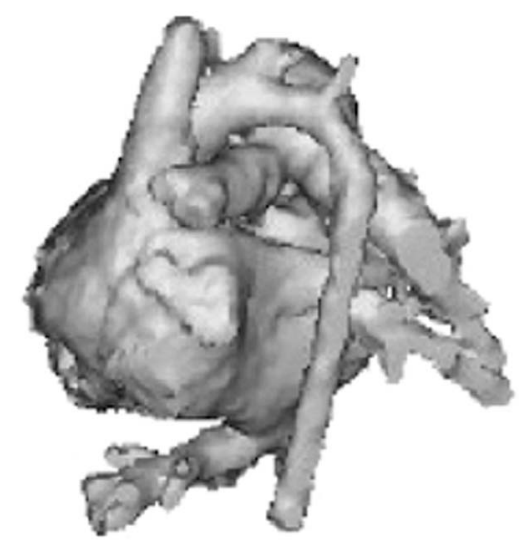

Figure 2.

Reconstructed Heart model.

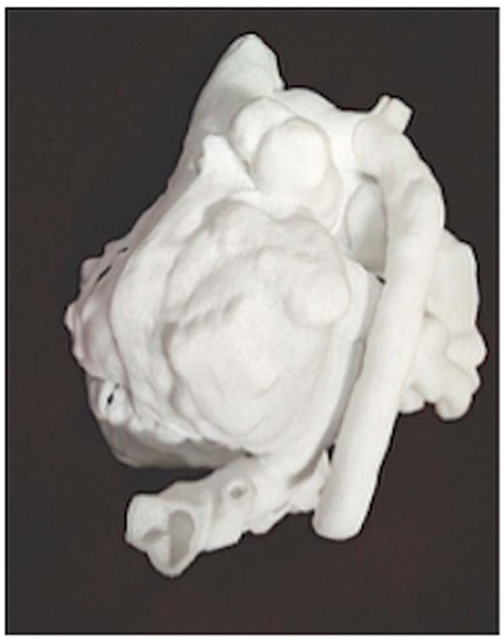

Figure 3.

SLS Nylon heart model.

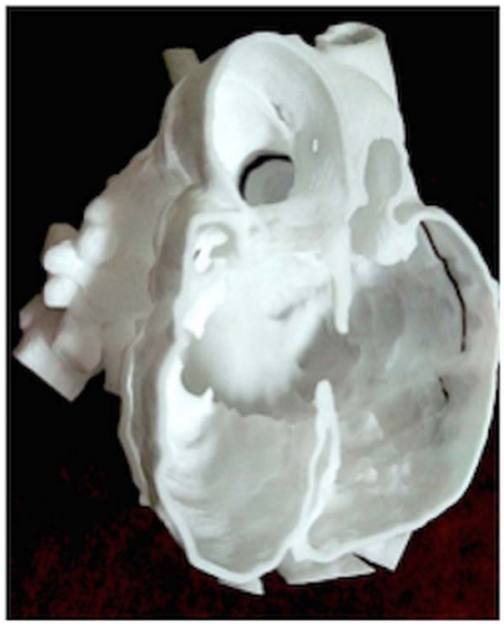

Figure 4.

Cut Sectional model showing details.

defects (Fig.4). A virtual planning with a CAD generated surgical patch is also exported as STL and printed and is evaluated for its suitability on physical model

Results and Conclusions: Though the anatomy was confirmed by both echocardiography and CT scan the 3D model enabled us to visualise and strategise the way to close the VSD and baffle the systemic veins into the left sided pulmonary ventricle and pulmonary veins to the right sided systemic ventricle. It was more evident planning the baffle after studying the $3 \mathrm{D}$ printed model in depth.

\section{P-162}

Postoperative chylothorax after congenital heart surgery Bernheim S., Mostefa Kara M., Bensemlali M., Meot M., Atallah V., Bonnet D., Vouhe P.

Paediatric Cardiology, Centre de Référence Malformations Cardiaques Congénitales Complexes - M3C, Necker Hospital for Sick Children, Assistance Publique des Hopitaux de Paris. Université Paris Descartes, Sorbonne Paris Cité, Paris, France. 
Background: Postoperative Chylothorax following cardiothoracic surgery is the most frequent etiology of Chylothorax in the pediatric population. Chylothorax is a severe complication of cardiothoracic surgery with an increased length, of hospital stay and mortality. Only few studies have described large cohorts of patients with Chylothorax. The aim of the study is to describe a French cohort of patients suffering from Chylothorax, their evolution and risk factors of severe Chylothorax.

Methods: We conducted a retrospective review of all cases of Chylothorax following congenital heart surgery in Necker Enfant Malades Hospital from October 1997 to December 2015. In order to evaluate the risk factors for severe Chylothorax we chose a composite endopoint that combines death, recurrence of Chylothorax, surgical treatment and drainage duration superior to 15 days.

Results: 195 patients were diagnosed with Chylothorax between october 1997 and December 2015. Prevalence of Chylothorax was $1,9 \%$. Almost half of the patients were treated for univentricular hearts $(40,5 \%)$. In the remaining half, the principal congenital heart diseases were tetralogy of Fallot and its variants $(17 \%)$, transposition of the great arteries $(10 \%)$ and coarctation of the aorta or interrupted aortic arch $(8 \%)$. Chylothorax occurred most after Bi directional Glenn surgery (22\%), Fontan surgery $(12 \%)$, Tetralogy of Fallot repair (12\%) and coarctation of the aorta repair (8\%). Risks factors for severe Chylothorax were Bi Directional Glenn procedure, OR $=2,9$ IC $95 \%=1,4-6,3, p=0004$, delayed sternal closure $0 \mathrm{R}=4,2$ IC95\% $=1,4-12, \quad \mathrm{p}=0007$. Concerning treatment, 195 patients were treated with fat free diet with addition of medium chain triglycerides (MCT diet). $120(61 \%)$ patients had a satisfying evolution following the MCT diet only and 75 patients needed additional treatment. 43 of those patients had surgery and 32 patients were treated with octreotid. In the 32 patients treated with octreotid, 13 had surgery, after failure of medical treatment. In total, $56(28 \%)$ patients needed revision surgery. Mortality rate was $8,7 \%$ (17 deaths).

Conclusion: Chylothorax is a severe complication after congenital cardiac surgery and the mortality is high enough to be of concern. Early treatment of this complication may lead to a decreased morbidity and mortality.

\section{P-163}

Diagnostic Accuracy and Prognostic Valued of Plasmatic Cystatin-C in Children Undergoing Cardiac Suregry for Congenital Heart Disease

Cantinotti M. (1, 2), Melo M. (1), Storti S. (1), Poli V. (1), Marotta M. (1), Crocetti M. (1), Pak. V (1), Arcieri L. (1), Giordano R. (1), Assanta N. (1), Murzi B. (1), Spadoni I. (1), Clerico A. (1,3).

1 Fondazione Regione Toscana G. Monasterio, Massa, Pisa, Italy 2. Institute of Clinical Physiology, Pisa, Italy3. Scuola Superiore Sant'Anna, Pisa, Italy

Background: Diagnosis and treatment of acute kidney injury (AKI) is often delayed in pediatric patients undergoing cardiac surgery due to the lack of an early biomarker of renal damage. Our aim was to evaluate the diagnostic and prognostic accuracy of plasma cystatin-C, as early biomarker of AKI in pediatric cardiac surgery. Methods: Cystatin-C and creatinine were measured preoperatively and at 2-6-12 hours post-surgery in 248 children undergoing cardiac surgery (median age 6.5 months; IQR: 1.7-40.1 months; range 0-17 years). The primary outcomes were: AKI and a composite marker of outcome (including major complications plus extubation time $>15$ days). Some risk models were taken in consideration for Cox proportional hazards regression analysis: a basal risk prediction model was fitted, including age, body surface area and Aristotle-score as continuous predictors, and then peak cystatin- $\mathrm{C}$ values were added to this model. Analysis to test discrimination, calibration, and reclassification were also performed.

Results: Cystatin-C post operatory values were accurate early diagnostic markers of AKI showing the best area under the ROC curve value (AUC) at 12 hours $(0.746$, CI 95\% 0.674-0.818). Peak cystatin- $\mathrm{C}$ value at peak showed a significant hazard ratio $(\mathrm{HR}=$ 2.665 , CI 95\% 1.750-4.059, $\mathrm{p}<0.001)$, add significant information to the basal regression model $(\mathrm{p}<0.001)$, and finally significantly improved the AUC $(\mathrm{p}=0.017)$, resulting a net gain in reclassification proportion $(\mathrm{NRI}=0.417, \mathrm{p}<0.001)$.

Conclusions: our data show that cystatin-C is not only an accurate early biomarker of AKI, but also may improve the risk prediction in pediatric cardiac surgery, supporting its routine use in this setting.

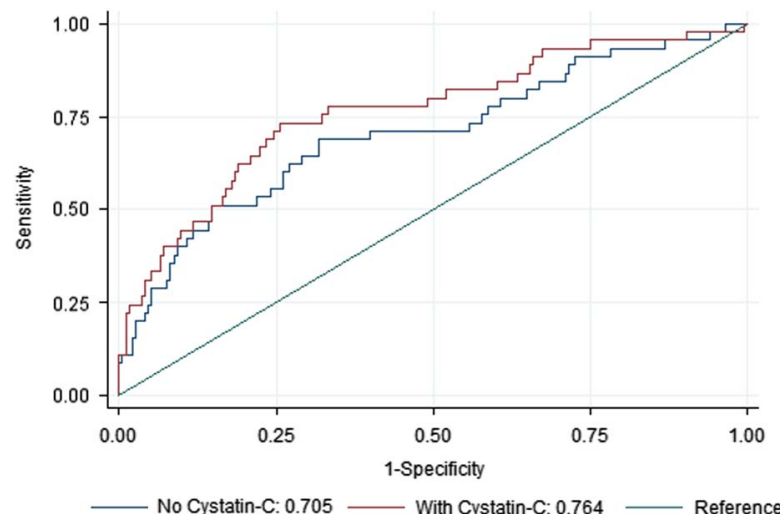

Figure 1.

The ROC curve showing diagnostic accuracy of costati-C in the diagnosis of $A K I$.

\section{P-164}

Endothelin-1 plays an important role in the Fontan circulation

Aoki M.(1), Hirono K. (2), Higuma, T. (1), Okano T. (3), Suzuki Y. (3), Nakayama K. (3), Ichida F. (2), Nishida N. (4), Imura J. (5), Emoto N. (3), Yoshimura N. (1)

Department of Cardiovascular Surgery, University of Toyama, Japan (1); Department of Pediatrics, University of Toyama, Japan (2); Department of Clinical Pharmacy, Kobe Pharmacentical University, Kobe, Japan (3); Department of Legal Medicine, University of Toyama, Japan (4); Department of Diagnostic Pathology, University of Toyama, Japan (5)

Objectives: To evaluate whether endothlin-1 (ET-1) plays an important role in the Fontan circulation.

Methods: First, we measured the serum ET-1 levels taken from the pulmonary artery in 32 patients with single ventricle (SV) physiology and compared this group with 28 patients with biventricular physiology (BV). Second, 13 patients who underwent right heart bypass were evaluated using lung histopathological and immunohistochemical studies. For 10 of these patients, quantitative real-time PCR analyses of ET-1, endothelin receptor type A and type $\mathrm{B}$ were performed.

Results: First, The serum ET-1 levels in the patients in the Fontan, Glenn and BV groups were $1.7 \pm 1.2,1.1 \pm 0.6$, and $0.9 \pm 0.5 \mathrm{pg} /$ $\mathrm{mL}$, respectively. The serum ET-1 level positively correlated with the mean pulmonary artery pressure in Fontan circulation $(p<0.01)$. Second, in patients with failed Fontan circulation, the pulmonary arteries exhibited severe medial hypertrophy and the 
overexpression of ET-1 in the endothelium and media. Quantitative real-time PCR analyses also confirmed that the mRNA expression of ET-1 was increased in patients with failed Fontan circulation,

Conclusions: Medial hypertrophy and the overexpression of ET-1 in the pulmonary arteries may induce failure of the Fontan circulation. There is an association between ET-1 and SV physiology in maintaining vasoconstriction of the pulmonary artery and promoting vascular remodeling.

\section{P-165}

Surgical management of muscular ventricular septal defects: Selection of appropriate technique according to each location and size of ventricular septal defect

Yoshimura N. (1), Aoki M. (1), Ikeno Y. (1), Nakaoka H. (2), Saito K. (2), Ozawa S. (2), Hirono K. (2), Ichida F. (2)

Department of Cardiothoracic Surgery, University of Toyama, Graduate School of Medicine, Toyama, Japan (1); Department of Pediatric Cardiology, University of Toyama, Graduate School of Medicine, Toyama, Japan (2)

Background: Although surgical results of multiple muscular ventricular septal defects (mVSDs) have been improved by new surgical techniques, standardized approach to these lesions has not been established. We have developed a treatment strategy that used direct closure, patch closure, and felt sandwich technique according to each location and size of mVSD. The present study evaluated the early and midterm results of this strategy.

Methods: One-hundred and eleven consecutive patients surgery for mVSDs and associated cardiac malformations from February 2005 to October 2016. They included 51 boys and 60 girls, with a median age of 9.0 months. Fifty patients had four or more VSDs (Swiss cheese septum).

Results: There were two early (pulmonary apoplexy, left ventricular dysfunction) and 1 late death (non cardiac death). There were no heart block. Two patients with Swiss cheese septum had postoperative congestive heart failure. Three mVSDs were closed by the sandwich technique in these 2 patients, whereas 1 or less VSD was closed by the sandwich technique in the other 109 patients. Two-hundred-and-sixty-eight mVSDs were closed in 111 patients. These included 35 high-trabecular (direct: 25, patch: 2, sandwich: 8), 141 mid-trabecular (direct: 130, patch: 3, sandwich: 8), 82 low-trabecular (direct: 66, patch: 1 , sandwich: 15), and 10 inlet muscular (direct: 10) septal lesions. Two patients required reoperation for residual VSD $(10 \mathrm{~mm}$ and $7 \mathrm{~mm}$ in high-trabecular lesion). The in-hospital echocardiograms demonstrated residual leakage in 39 lesions (high-trabecular: 6, mid-trabecular: 8, low-trabecular: 25). At the latest echocardiographic study, the residual leakage had disappeared in 24 lesions.

Conclusions: The outcome of the surgical repair of mVSDs was satisfactory. To avoid postoperative cardiac dysfunction, the surgeon should try to close the muscular VSD directly. Hightrabecular VSD especially that located at the junction between the ventricular septum and the right ventricular free wall was difficult to identify. Large residual VSD in this area caused congestive heart failure. This type of VSD was considered suitable for sandwich technique. Most of the mid-trabecular VSDs could be closed directly. Low-trabecular VSDs were difficult to close directly. However, small residual shunt disappeared spontaneously. Large low-trabecular VSDs were considered suitable for sandwich technique.

\section{P-166}

Results of delayed surgical treatment in children with tetralogy of Fallot from a devloping country

Hofbeck M. (1), Affangla A. (2), Hanser A. (1), Sieverding L. (1), Wiegand G. (1), Kaulitz R. (1), Kumpf M. (1), Haller C. (3), Neunhoeffer F. (1), Schlensak C. (3)

Department of Pediatric Cardiology, University of Tuebingen, Germany (1), Hôpital Saint Jean de Dieu, Cardiologie, Thiés, Senegal (2), Department of Cardiothoracic Surgery, University of Tuebingen, Germany (3)

Introduction: Corrective surgery of tetralogy of Fallot (TOF) is usually performed in the first year of life. Treatment options in developing countries are frequently restricted due to limited availability of diagnostic and therapeutic facilities. This results in a significant number of children and adolescents with native TOF and severe obstruction of the RVOT. We report the results in a cohort of children from Senegal who were referred to our center for surgical treatment.

Patients: From 05/2004 - 10/2016 16 children with TOF were referred with a mean age of 7.0 years (range $0.9-14.8$ years). The primary diagnosis established in Senegal by echocardiography was confirmed in all patients. Cardiac catheterization was required in 3/16 patients. Almost all patients were dystrophic (mean BMI 13.7, range $9.8-16.7$ ). All patients were significantly cyanosed and presented in NYHA class III-IV.

Results: Primary corrective surgery was performed in 13/16 patients. A valve sparing transatrial approach was possible in 10 patients, one of these patients required secondary transanular patch enlargement 2 days later. One patient, who presented with active endocarditis and vegetations on the aortic and pulmonary valve, underwent corrective surgery including aortic and pulmonary valve replacement. Corrective surgery with RVOT patchenlargement was performed in further 2 . A primary shunt procedure was required due to persistent hypoxemic spells necessitating treatment with noradrenalin in 2 (13.8 and 3.3 years) and excessive hypertrophy of both ventricles in 1 case (6.8 years). Two of these patients underwent secondary corrective surgery requiring RVOT patch enlargement in one, the third patient awaits corrective surgery. All patients were discharged in good condition (median 12 postoperative days).

Conclusion: Despite of late referral and severe obstruction of the RVOT corrective surgery was possible with low mortality. Preservation of the pulmonary valve was possible despite of severe obstruction of the RVOT in the majority of our patients avoiding the long term sequelae of significant pulmonary regurgitation. Palliative procedures remained reserved for patients with persistent hypoxemic spells and excessive RV hypertrophy. The unfavorable natural history and the disabling sequelae of chronic hypoxemia justify all efforts to attempt late corrective surgery in children with TOF.

\section{P-167}

Surgical outcome of isolated aortic coarctation with or without tubular aortic arch hypoplasia

Thölking T.W. (1), Slieker M.G. (2), Schoof P.H. (1), Grotenhuis H. B. (1)

Wilhelmina Children's Hospital/UMC Utrecht, Utrecht, The

Netherlands (1); Radboudumc, Nijmegen, The Netherlands (2)

Introduction: Coarctation of the aorta (CoA) can appear isolated or associated with tubular hypoplasia of the aortic arch (THAA). This study aimed to investigate potential differences in outcome between surgically repaired CoA patients with or without THAA. Method: 44 patients with surgically repaired CoA (age $10 \pm 5.5$ years) were retrospectively evaluated at $7.1 \pm 2.4$ years of follow up, of 
whom 26 patients had isolated CoA and 18 CoA/THAA. 29 patients were treated through a lateral thoracotomy and 14 through median sternotomy. All CoA patients were compared by type of coarctation for aortic and left ventricular (LV) dimensions by echocardiography, blood pressure and reinterventions at latest follow up. Results: Patients with CoA/THAA demonstrated a significantly higher corrected gradient across the aortic arch when compared to patients with isolated $\mathrm{CoA}(14.2$ vs. $7.7 \mathrm{mmHg}, \mathrm{p}=0.03)$ at latest follow up. Aortic root and aortic arch dimensions were similar for patients after CoA and CoA/THAA repair, except for CoA/ THAA patients having a larger sinus of Valsalva diameter ( $Z$ score 0.5 vs. $-0.1, p=0.02$ ). No significant differences were found in blood pressure and LV function for both groups. Three patients had aortic insufficiency in the isolated CoA group (2 mild, 1 moderate) compared to none in the CoA/THAA group.

Two patients in the isolated CoA group required reintervention (stenting and surgical aortic arch repair, respectively) vs. 2 patients in the CoA/THAA group (balloon angioplasty and stenting, respectively). Seven isolated CoA patients were hypertensive according to the AHA guidelines of the Task Force Report 2005, compared to 4 patients in the CoA/THAA group. Two isolated CoA patients used antihypertensive drugs compared to 3 in the CoA/THAA group. Conclusions: Despite similar aortic and LV dimensions at late follow up, patients after surgical repair of CoA/THAA demonstrate an increased corrected gradient across the aortic arch when compared to patients after isolated CoA repair, posing an increased risk for future reinterventions.

\section{P-168}

Hybrid Atrioventricular Valve Replacement in Patients with Hypoplastic Left Heart Syndrome

Michel-Behnke I.(1), Kitzmueller E.(1), Herbst C.(2), Greil S.(1), Laufer G. (2), Zimpfer D.(2)

Medical University, Dpt. of Pediatric Cardiology, Pediatric Heart Center, Vienna, Austria(1), Medical University, Dpt. of Heart Surgery, Pediatric Heart Center, Vienna, Austria (2)

Objectives: Severe atrioventricular (AV) valve regurgitation in patients with hypoplastic left heart syndrome is challenging given the lack of dedicated valve prosthesis for small infants. The Melody valve is a transcathether pulmonic valve that can be modified and implanted in AV position in a hybrid fashion. We report our experience with hybrid AV valve replacement in patients with hypoplastic left heart syndrome (HLHS).

Methods: The Melody valve was used for hybrid AV valve replacement in 3 infants (mean age 10.2 months, range 7-14; mean weight $6,8 \mathrm{~kg}$, range 6,6-6,9) with HLHS and severe AV (tricuspid) valve regurgitation. All patients were in refractory heart failure and unable to wean from the respirator. In one patient concomitant Berlin Heart implantation was performed. For hybrid AV valve replacement the following modifications of the Melody valve were performed. The Melody valves were shortened in order to suit the small ventricles and avoid pulmonary vein obstruction by inverting and crimping the last row of struts on both sides of the valve. Additionally, a skirt of pericardium or Cormatrix was sewn to the middle of the stent to anchor the valve in the native annulus and to prevent paravalvular leakage. The modified valves were then crimped on a balloon-in-ballooncatheter. After proper positioning, valves were balloon-inflated to appropriate diameters (mean $21 \mathrm{~mm}$, range $18-22 \mathrm{~mm}$ ) and secured to the valve annulus with several interrupted sutures.

Results: Implantation of the Melody valve in AV-position was successful in all patients with no procedural complications. The Melody valves functioned properly in the high-pressure environment without any relevant valve regurgitation, with unrestrictive antegrade ventricular inflow and no paravalvular leaks during the study period. Thirty-day mortality in this complex patient population was $0 \%$. One patient later on died from multi organ failure, one patient is awaiting cardiac transplantation and one patient is stable at home.

Conclusion: The Melody transcatheter pulmonic valve can be modified for hybrid AV-valve replacement in patients with HLHS. Percutaneous redilation is possible to adapt the valve to patient's growth.

\section{P-169}

Decellularized fresh pulmonary homografts for reconstruction of the right ventricular outflow tract: Intial experience with the ESPOIR valve

Dave H., Schmiady M., Valsangiacomo E., Greutmann M., Stambach D., Kretschmar O., Huebler $M$.

Children's Heart Centre and Children's Research Centre, University Children's Hospital, Zurich, Switzerland

Objectives: Reconstruction of the right ventricular outflow tract (RVOT) constitutes an essential part of the correction of a large group of congenital cardiac anomalies.

Herein we report the initial experience with the use of decellularized fresh pulmonary homografts used for reconstruction of the RVOT in the scope of a multi-centric European trial (ESPOIR).

Methods: 17 patients with a median age and weight of 11(2-59) years and 36(11-100) kg undergoing a Ross Procedure (10) or a pulmonary valve replacement (7) received the ESPOIR valve. Two patients with truncal and pulmonary valve disease underwent replacement of both these valves. Patients underwent a preoperative MRI and tests to document haemolysis. Most of the patients had 1 to 3 prior cardiac operations. Median size of ESPOIR valve was $24(14-30) \mathrm{mm}$ and the median duration from harvest to implantation was 1.9(1.3-4.4)months. The ESPOIR valved conduit was sutured distally and proximally using continuous suture technique. Postoperatively, acetyl salicylic acid was given for 3 months after removal of the drainages.

Results: One 60 year old patient undergoing triple valve replacement along with maze procedure died postoperatively; the death was unrelated to the ESPOIR valve. All the remaining patients are alive with well-functioning ESPOIR valve at a median follow-up of 6(0-19)months. Mean gradient across the valves was a median of 2.5(1 -20) $\mathrm{mm} \mathrm{Hg}$ and 9 patients had mild regurgitation. The handling of the ESPOIR valves was very good and the conduit was well accepted by the body without clinical signs of haemolysis. Two patients had mild temperature in the first postoperative days which resolved spontaneously.

Conclusion: Initial experience with the use of the ESPOIR valve is satisfying. Longer follow-up is necessary to determine whether decellarization leads to better long term outcome compared to its existing Peers.

\section{P-170}

Short term experience with surgical Melody valve implantation in mitral position in children

Schmiady M.(1), Dave H.(1), Sigler M.(3), Hofmann M.(1), Kretschmar O.(2), Hübler M.(1)

1.) University Children's Hospital Zurich, Department of Congenital Cardiovascular Surgery, University Zurich, Switzerland, 2.) University Children's Hospital Zurich, Division of Pediatric Cardiology, University Zurich, Switzerland, 3.) Department of Pediatric Cardiology and Intensive Care Medicine, Georg-August University, Goettingen, Germany 
Introduction: The options for mitral valve replacement in children with irreparable native valves have been limited to stented mechanical and bio-prosthetic valves. For very small infants, because of the small size of the annulus, even these options are not easily practicable. Additionally, these prostheses do not allow for expansion with somatic growth, necessitating frequent reoperations for valve replacement. We present our single center experience with a modified externally stented bovine jugular vein graft (Melody ${ }^{\circledR}$ Valve) for mitral valve replacement in children.

Methods: We retrospectively reviewed medical records of patients who have undergone surgical Melody ${ }^{\circledR}$ Valve implantation in left atrioventricular valve (LAVV) position from 2013 to 2016 at our center.

Results: Four patients have undergone Melody ${ }^{\circledR}$ valve implantation in LAVV position from December 2013 to March 2016 at our center. Indication for valve replacement was mitral stenosis or insufficiency in the course of complete atrioventricular defect $(n=2)$, congenital mitral valve dysplasia $(n=1)$ and combined mitral valve disease with aortic valve stenosis in a child with borderline left ventricle $(n=1)$. Median age of the patients at implant was 17.5 months (range 1-30). There was one early death in the cohort due to ventricular rupture. One child died 13 months after valve implantation due to an unrelated malignancy. Histopathological workup demonstrated good biocompatibility of the Melody valve with well-preserved valve function, complete endothelialization, and only little neointima formation. The remaining two valves are functioning well at 7 and 18 months follow-up.

Conclusion: LAVV replacement with the Melody® valve in small children with irreparable mitral valve disease shows acceptable short term results and allows trans-catheteral trans-septal dilatation to accommodate for somatic growth of the child. The demonstrated short-term performance of the Melody ${ }^{\circledR}$ valve, which was primary designed for the RVOT, in high pressure environment is encouraging with good valve function and biocompatibility. Special care must be taken in children with small mitral annulus and short ventricle to avoid LVOT obstruction, obstruction of the pulmonary vein, AV-block or compression of the circumflex artery.

\section{P-171}

\section{Left Ventricular Torsion in Clinically Stable Heart Transplant Recipients in Children}

Pac F.A.(1), Koca S.(1), Kavurt V. (1), Ece I.(1), Eris D.(1), Kervan U. (2), Sabitoglu S. (2), Sert D. (2), Pac M.(2)

Pediatric Cardiology, Yuksek Ihtisas Heart-Education and Research Hospital, Ankara, Turkey (1), Department of Cardiovascular Surgery, Yuksek Ihtisas Hospital, Ankara, Turkey (2)

Background: Left venctricular (LV) rotation is the dominant deformation during relaxation and links systole with early diastolic recoil. In adult heart transplant recipients, LV twist dynamics are significantly impaired. This study was aimed to explore LV twist in clinically stable heart transplant (HT) recipients in children.

Methods: Thirteen children underwent ortotopic heart transplantation (HT) in our hospital in last four years. In all HT recipients underwent scheduled cardiac catheterization and endomyocardial biopsy. Two dimentional and colour M-mode echocardiography with speckle-tracking analysis were performed to evaluate the changes of left ventricular systolic strain in heart transplant recipients at 1st, 3rd, 6th, 12th, 18th, 24th and 30th month after heart transplantation, and 10 healthy subjects served as controls. The strain curves and peak systolic strain values for each segment and overall left ventricular wall were obtained. Left ventricular global longitudinal strain (GLS), global radial strain (GRS), global circumferential strain (GCS) and left ventricular torsion (LV TOR) were measured and then statistically analyzed

Results and Conclusion: There were no significant differences in left ventricular ejection fraction (LVEF), GLS, GRS and GCS between heart transplant recipients and controls. LV twist dynamics were in normal range in all thirteen HT recipients after one month later from transplantation. However, significant impairment in LV TOR had begun to be identifed four months after transplantation. There was no rejection in endomyocardial biopsies except only one of the patients (Grade 2). It is suggested that left ventricular torsion can be used for monitoring changes of left ventricular functions in cardiac allograft.

\section{P-172}

Experience with restrictive cardiomyopathy in childhood age

Veyrier M., Ducreux C., Henaine R., Bakloul M., Ninet J., GaloinBertail C., Metton O., Durousset C., Walton C., Perouse de Monclos T., Mitchell J., Di Filippo S.

Cardiovascular Hospital Louis Pradel, University Claude Bernard

Lyon1, Hospices Civils de Lyon, Lyon, France

The objective of this study was to assess the clinical outcomes of children diagnosed with restrictive cardiomyopathy (RCM).

Material and methods: Retrospective data analysis of patients diagnosed with RCM at age less than 18y. Age at diagnosis, clinical oucome, therapeutics and survival were collected. Prognosis factors were assessed.

Results: From 1991 to 2016, 24 patients (15 males $=62.5 \%$ ) were diagnosed with RCM, at the age of $5.5 \pm 4.8 \mathrm{y}$ (median $4.2 \mathrm{y}$, range 1week to 14.8y). All patients had normal LV and RV systolic function. Events occurring over time included: supraventricular tachycardia in 5 cases (20\%), atrioventricular block in 1 (4\%), right heart failure in $10(40 \%)$, stroke in $1(4 \%)$ and syncope in $3(12 \%)$. Pulmonary hypertension occurred in 4 cases (16\%). Follow-up was uneventful in 4 patients at the time of analysis (16\%). Treatment included: diuretics in 13 cases $(52 \%)$, beta-receptor antagonists in $11(44 \%)$, amiodarone in $4(16 \%)$, and 2 patients had no treatment $(8 \%)$. NYHA class I, II, III and IV range was respectively: 2 cases (8\%), 11 (44\%), 4 (16\%) and 7 (28\%). Death occurred in 5 cases $(20 \%)$, at the age of $2 \pm 1.7 \mathrm{y}$ (median $1.5 \mathrm{y}$, range 0.5 to $4.8 \mathrm{y}$ ) and $0.6 \pm 0.7 \mathrm{y}$ after diagnosis (median $0.5 \mathrm{y}, 1$ week to $2 \mathrm{y}$ ), from HF in 1 and sudden death in 4 . Ten patients (40\%) underwent heart transplantation, at the age of $12.5 \pm 6.7 \mathrm{y}$ (median $14.8 \mathrm{y}$, range 3.7 to $22.1 \mathrm{y}$ ) and $5 \pm 7 \mathrm{y}$ after diagnosis (median $2 \mathrm{y}, 0.6$ to $19.4 \mathrm{y}$ ). Overall death or transplantation occurred in $60 \%$ of the cases. Age at diagnosis was a predictive factor for death $(1.4 \mathrm{y}$ in deceased patients versus $6.6 y$ in alive cases, $\mathrm{p}=0.03$ ).

Conclusion: RCM prognosis is poor in children with high rates of death or transplantation. Infant age at diagnosis is a predictive factor of bad outcome.

\section{P-173}

Paediatric Acute Myocarditis in University Hospital within Years 2010-2015

Bergmane I.(1), Ligere E.(1,2), Matsate-Matsone B.(1), Lubaua I. (1,2), Lace I.(1,2), Ozolins V.(1), Smits L.(1), Sikora N.(1) Department for Pediatric Cardiology and Cardiac Surgery, University Hospital for Children, Riga, Latvia(1); Department of Paediatrics, Riga Stradins University, Riga, Latvia(2)

Acute myocarditis (AM) in children is a rare disease and most often has viral ethiology but can also arise from bacterial, parasitic, 
autoimmune, fungal, toxic, drug-related causes. Myocarditis is connected with high mortality in small children and the diagnosis can be missed.

The objectives of the study were to analyse all cases of AM treated or discovered during autopsy in our hospital during years 20102015.

Methods All the cases with AM were selected from hospitals database and/or autopsy register. We analyzed case histories to explore the course of disease, causative agents, echocardiographic findings and the outcome.

Results: There were 11 cases of AM diagnosed during hospitalization: 4 girls and 7 boys (63\%), age $8.9 \pm 4$ years. $63,6 \%$ complained about chest pain, but $27 \%$ were too young to complain, $72,7 \%$ had heart rhythm disorders, all 11 patients had elevated troponin I $(3,6 \pm 2,7 \mathrm{ng} / \mathrm{L}), 45,5 \%$ of patients were in need of inotropic support, 3 - ventilation. Causative agents found in 5 cases (1- Coxacie B, 1- rheumatic fever, 1-EBV, 1-Dyphteria, 1-Rotavirus). There were 3 cases of death (27,3\%): 7 months old girl with large VSD, pneumonia (cause unrecognized, moderate myocardial intersticial lymphoid cell infiltration on autopsy),10 year old girl with severe heart rhythm disorders (atrial fibrillation, undulation), cause of myocarditis unclear, autopsy revealed large regions of myolysis, severe lymphocytic and moderate leucocytar interstitial infiltrations, 4 months old girl with atypical haemolyticuremic syndrome and myocarditis with poor cardiac function (EF $30 \%$ ) (only Rotavirus positive found).

There were 10 cases where myocarditis as a part of diagnosis was revealed only during autopsy, 6 boys (60\%), 4 girls, mean age $1,17 \pm 2,5$ years ( 5 days to 8 years), causative agent recognized in $90 \%$ (8 patients with severe septicaemia (2-Acinetobacter Baumani, 1-Micrococcus lutheus, 1- Pseudomona aeruginosa, 1-Serratia marcescens, 1- Salmonella enteritidis, 1- Clebsiella oxytoca, 1-Staphylococcus epidermidis (two cases in combination with RSV), 1 -Influenza AH1N1. 4 of the patients were newborn (3 premature).

Conclusions: AM remains serious disease with high mortality rates and diagnosis is still often recognized only post mortem. Miocardial damage should be evaluated in patients with severe disease with a rapid, progressive, downhill course.

\section{P-174}

A case of Barth syndrome of early-onset and severe cardiomyopathy associated with triple compound heterozygosity

Tonegawa H.(1), Tsujii N.(1), Hayashi T.(1), Nishikubo T.(1), Kimura A.(2), Yoshizawa H.(1), Takahashi Y.(1),

Shima M.(1)

Department of Pediatrics, Nara Medical University, Kashihara, Nara, Japan (1); Department of Molecular Pathogenesis, Medical Research Institute, Tokyo Medical and Dental University, Tokyo, Japan(2)

Background: Barth syndrome is an X-linked recessive mitochondrial disorder characterized by cardiomyopathy, neutropenia, growth retardation, 3-methylglutaconic aciduria. It is caused by mutations in the TAZ gene, which is known to encode for the protein tafazin. We report a neonatal case of the Barth syndrome sufferd from an early-onset and severe cardiomyopathy that may be associated with triple compound heterozygous mutations in TAZ, SDHA, and DTNA genes.

Case: A term male infant was born to a 33-year-old 5G4P mother. Acute heart failure from dilated cardiomyopathy with the left ventricular noncompaction developed to him immediately after birth. He was intubated and started on inotoropic support. After stabilization of cardiac function, carvedilol was added under continuous infusion of dobtamine. Though he somehow discharged at 5 months old, he have sufferd from acute aggravation of the chronic heart failure. The family history was reviewed and the second-born boy to the parents died suddenly several hours after birth and the maternal grandmother had spontaneous abortion twice. The whole exome sequencing of genes in the cardiomyopathy panel available at Laboratory for Molecular Pathogenesis at the Tokyo Medical and Dental University confirmed that the patient has novel mutations in TAZ, SDHA and DTNA genes. The novel mutations are identified in exon 6 , c.469C > T (p.Leu157Phe) of the TAZ gene, exon 9, c.1351_1355delCGCCT, (p. Arg451fs) of the SDHA gene, and exon 19 , c. $1763 \mathrm{G}>$ A, p.Ser588Asn) of the DTNA gene. In silico tools suggest these mutations might be disease causing. Further sequencing to the parents revealed his mother had the mutation in the TAZ gene with heterozygosis, and his father had mutations in the SDHA and DTNA genes with heterozygosis.

Discussion: This is the first case of Barth syndrome associated with triple compound heterozygous mutations. Hypertrophic cardiomyopathy with multiple mutations is known to be aggravated than that with single mutation. In this case, a similar mechanism might be involved in the early onset and severity of heart failure.

\section{P-175}

Heart failure clinic in pediatric popluation: a comparative analysis of outcome

Adorisio R., Cantarutti N., Baban A., Giannico S., Grutter G. Iorio F. S., F. Drago

Bambino Gesu' Hospital, Rome, Italy

Objectives: It has been reported that heart failure clinic (HFC) can improve the outcome of adult patients affected by heart failure, in terms of reduction of rehospitalisation, adherence to therapy and improvement of quality of life. No data are currently available on paediatric population. The aim of current study was to determine whether a specific HFC program is effective also in paediatric field Methods: Since 2013, a specific HFC program has been developed. Children are referred to HFC in case of: 1) left ventricular dysfunction with $\mathrm{EF}<45 \%$ due to dilated cardiomyopathy or after surgery, 2) restrictive and 3) hypertrophic cardiomyopathy 4) Fontan failure 5) pulmonary hypertension. To evaluate the impact on mortality, rehospitalisation, analysis of comorbidities and compliance to therapy, we made a comparative analysis on 291 children followed between 2011 and 2015, before and after the specific program. A multidisciplinary approach and specific disease protocols have been applied. HFC included two cardiologists and 1 nurse dedicated.

Results: The characteristic of our population $(11.2+5$ yrs old; $52 \%$ male) are: $45 \%$ affected by cardiomyopathies, $50 \%$ CHD with ventricular dysfunction or Fontan failure, 12\% pulmonary hypertension. After the introduction of this program, a significant reduction of rehospitalization was observed in particular for emergency reasons (2012 vs $201475 \%$ vs 33\%, p < 0.001; 2012 vs 201575 vs $12 \%, p<0.001$ ) and for acute HF (2012 vs $201452 \%$ vs $13 \%, \mathrm{p}<0.001)$. No differences in terms of mortality has been observed. Major reasons for rehospitalisation were: respiratory infection (52\%), gastroenterological infection (33\%), arrhythmias $(12 \%)$. Major comorbidities included: nutritional aspects, respiratory disease and orthopaedic problems. $45 \%$ of patients had more than two comorbidities.

Conclusions: In our center, HFC program allowed to reduce rehospitalisation but not mortality. Multidisciplinary approach is required to manage this complex group of patients. 


\section{P-176}

Long term results of heart rate reduction by beta blockers in children affected by left ventricular dysfunction

Adorisio R., Cantarutti N., Baban A., Lisignoli V., Iorio F.S., Drago F.

Bambino Gesu Hospital

Objectives: Heart rate is strongly related to prognosis in heart failure (HF). Although beta blockade showed significant benefit in adult population, these drugs did not showed the same benefit in children with HF. No data are currently available on baseline heart rate and treatment reduction in HF children. Aim of the study was to assess the long term effect of heart rate reduction (HRR) on cardiac function and remodelling in children.

Methods: We evaluated retrospectively the effect of long term HRR by beta blockade in children affected by left ventricular dysfunction. NYHA/Ross class, weight, and height, HR, blood pressure, echocardiographic parameters (LVEDD, LVESD, SF, $\mathrm{EF})$ were recorded before treatment, after 1 month of maximal dose tolerated, at 1 year, at 3 year.

Results: 90 patient (48\% male) are included in this analysis. Median age at the beginning was 6.5 yrs (range $0-18$ yrs). $66 \%$ of children had idiopathic cardiomyopathy, $15 \%$ after surgery. At the beginning $80 \%$ were in Ross/NYHA classe $>$ II and growth failure was present in $60 \%$. Beta blockade significantly reduced HR $>20 \%$ in the 3 months after beginning of treatment without significant reduction of blood pressure. LVEDD and LVESD significantly reduced at 1 year after treatment with significant increase of EF $(34.3 \%+8.2 \%$ vs $45.2+7.6 \%, \mathrm{p}<0.001)$ and after 3 year $(34.3 \%+8.2 \%$ vs $48.3+6.9 \%, \mathrm{p}<0.001)$.

Conclusions: In children with left ventricular dysfunction, long term HRR induced by beta blockade improves LV function. Further studies are required to evaluate heart rate in children with $\mathrm{HF}$ and its prognostic value.

\section{P-177 \\ Electrocardiographic Changes in the Pediatric Patients with Symptomatic Rejection after Heart Transplantation \\ Ko J.K, Jun H.O, Kim Y, H \\ Department of Pediatric Cardiology, Ulsan University College of \\ Medicine, Seoul, Korea}

Introduction: Many studies have defined rejection as a clinical event in which temporary augmentation of immunosuppression is initiated to treat presumed acute rejection. Endomyocardial biopsy remains the gold standard of diagnosis of acute cellular rejection after heart transplantation. However, biopsy is not always diagnostic and is also risky to critical patients. In this study we considered possibility of non-invasive diagnosis of acute symptomatic rejection using electrocardiography in pediatric patients after heart transplantation.

Methods: Records of patients having undergone heart transplantation were reviewed focusing specifically on surface ECGs performed in temporal proximity to symptomatic acute rejection episodes.

Results: 65 pediatric patients after heart transplantation were evaluated and 20 patients had episodes of acute symptomatic rejection. Surface ECGs within one or two days of events were analysed. Most prevalent finding was low QRS voltage in 16 patients and there were no recorded low voltage in patients without episode of significant rejection. Right bundle branch block was more frequent when comparing those with and without significant rejection. The mean QTc at the rejection was significantly increased compared with that of before episode of rejection $(525 \pm 40$ vs $469 \pm 18 \mathrm{~ms}, \mathrm{p}<0.001)$. The mean QTc before episode of rejection in patients with clinical rejection was also significantly increased compared with that of patients without rejection $(469 \pm 18$ vs $453 \pm 32 \mathrm{~ms}, p=0.01)$. The QTc difference between before and at the rejection was larger when comparing those between last follow-up and at one year after transplantation in patients without rejection $(60 \pm 32$ vs $10 \pm 27 \mathrm{~ms}, \mathrm{p}<0.001)$. However, the QTc difference between before rejection and at one year after transplantation was not significantly different from those between last follow-up and at one year after transplantation in patients without rejection $(23 \pm 24$ vs $10 \pm 27 \mathrm{~ms}, \mathrm{p}>0.05)$.

Conclusions: In this study, low QRS voltage and increasd QTc were important surface ECG findings in the patients who have experienced clinically significant rejection.

\section{P-178}

Systolic dysfunction after Fontan is provoked by pulmonary high flow before procedure

Hamamichi Y., Takeguchi M., Horimot Y., Mastui T., Saito M., Ishii T., Inage A., Ueda T., Yazaki S., Yoshikawa T.

Sakakibara Heart Institut, Tokyo, Japan

Background: For causes of systolic dysfunction of systemic ventricle (SDSV) innate factors were reported in Fontan patients, such as right ventricle and regurgitation of atrio-ventricular valve. However, we predicted pulmonary high flow before Fontan was much related to SDSV in Fontan patients. We investigated what factors were connected with SDSV in Fontan patients.

Methods: The medical records of 162 Fontan patients were reviewed aged from 1.7 to 42.9 years. They underwent cardiac catheterization between 2010 and 2015. We defined SDSV as ejection fraction of SV 0.40 or below $(n=21)$. Clinical features were determined which would affect SDSV.

Results: In monovariate analysis SDSV was associated with following 7 factors: study ages (> $6.0 \mathrm{yrs})$, Fontan ages ( $>2.9 \mathrm{yrs}$ ), ventricular type (single right ventricle), 1st strategy (native pulmonary stenosis before Glenn), catheter intervention (No history of coil embolization), pulmonary artery index $(\geq 350 \mathrm{~mm} / \mathrm{m} 2)$, and Fontan procedure (Non-fenestrated Fontan). After multivariate analysis SDSV was independently associated with odds ratio of 11.7 for larger pulmonary-artery index, 6.1 for single right ventricle, and 5.5 for elder ages at study. Explanatory coefficient was 0.42. Contrary to our expectation, the frequencies of regurgitation of atrio-ventricular valve or regurgitation of aortic valve, long sustentation of which would lead to SDSV finally, were not different between Fontan patients with SDSV and without SDSV. Afterloads, much increasing of which would cause SDSV particularly in Fontan circulation, were not different between two groups, such as systolic pressure of major ventricle and systolic pressure of aorta. The rate of isomerism heart was not different between two groups. Clinically, Fontan patients with SDSV had low cardiac output. Unexpectedly, indexes for liver function on blood test were inferior in patients with SDSV, such as gammaglutamyl transpeptidase and total bilirubin.

Conclusion: In Fontan patients SDSV was related to strategies before Fontan that suggested pulmonary high flow. Pulmonary high flow augments loads to ventricle. We might not raise pulmonary artery too large before Fontan in consideration of loading to ventricle. Additionally, liver congestion subsisted in SDSV patients. We should pay much attention to venous system failure in Fontan patients with SDSV. 
P-179

Monitoring of Cardiotoxic Effects of Anthracycline Chemotherapy in Childhood Malignancies

Vugrinec Mamic M.(1), Matijasic N.(2), Tokic Pivac V.(1), Stepan

Giljevic J.(1), Jakovljevic G.(1,2), Herceg Cavrak V.(1,2)

University Children's Hospital Zagreb, Zagreb, Croatia, Faculty of

Medicine, University of Osijek, Osijek, Croatia

Introduction: Cardiotoxicity is a known complication of anthracyclines' therapy in children treated for malignancies. Regular monitoring of heart function enables the identification of heart damage at a subclinical level.

Methods: This is a retrospective study based on the analysis of data obtained by echocardiographic monitoring of patients treated for malignant diseases in University Children's Hospital Zagreb from 2003 to 2016. The systolic function of the left ventricle was observed through shortening fraction (FS), while the diastolic function was studied through early (E) and late (A) diastolic filling velocities and their ratio $(\mathrm{E} / \mathrm{A})$. We also measured the dimensions of the left ventricle correlated with age, gender and the anthracyclines dose.

Results: The study included 378 children, 234 of which were boys $(62 \%)$, with an average age of 8.8 years $( \pm 7.36)$. The most common diagnosis was sarcoma, followed by hematologic malignancies. Total cumulative dose of anthracyclines was $<100 \mathrm{mg} /$ $\mathrm{m} 2$ in $67(20 \%)$ patients, $207(61 \%)$ patients received a dose of $101-300 \mathrm{mg} / \mathrm{m} 2$, and $66(19 \%)$ patients received a dose higher than $301 \mathrm{mg} / \mathrm{m} 2$. None of the patients had clinical signs of heart failure. Subclinical damage of the systolic function was found in $18 \%$ of children, mainly $(13.8 \%)$ during the chemotherapy and within 6 months of its completion. In $4.2 \%$ of patients the damage was still present and /or observed 6 months after the end of the chemotherapy. There was a clear correlation between reduced systolic function and the anthracyclines cumulative dose $(p<0.01)$. Diastolic dysfunction was registered in $5 \%$ of patients, but no statistically significant correlation was found between reduced dyastolic function and cumulative dose $(\mathrm{p}=0.213)$. A total of 55 $(14.5 \%)$ children received cardiac medication, ACE inhibitors and /or beta blockers.

Conclusion: Subclinical damage of the systolic cardiac function in children treated for malignancies was present in a significant percentage and correlated with the cumulative dose of anthracyclines, despite the normal cardiac status, which highlights the importance of regular cardiac monitoring of these patients.

\section{P-180}

Can a Structured Intervention Programme Improve the Biophysical and Psychosocial Wellbeing in Children with Congenital Heart Disease?

Callaghan S. (1,2), Morrison M.L. (1), McCusker C.(1,2), McKeown P. (1,2), Casey F.A (1)

Royal Belfast Hospital for Sick Children, Northern Ireland (1); Queen's University Belfast, Northern Ireland (2)

Introduction: Improved survival among children with congenital heart disease (CHD) has shifted focus to the long-term physical and psychological outcomes for these patients. There is evidence that children with CHD have lower levels of daily physical activity and a higher prevalence of obesity compared to their normal peers. The benefits of an active lifestyle within the general population have been well described. This study aims to determine if a structured intervention programme can improve both physical and psychological functioning in children with CHD.

Methods: This study is a prospective randomised control trial. Patients aged between 5-10 years with CHD were identified and invited to participate. Each patient underwent baseline assessment as detailed below:

Biophysical assessments:

- Weight, height, waist measurements

- Baseline heart rate, blood pressure, oxygen saturation

- Exercise stress test - Graded cycle ergometer protocol

- Actigraph accelerometer worn for 1 week

Psychosocial assessments:

- Kidscreen27

- Strengths and Difficulties Questionnaire

- Butler Self-image Profile

Following baseline assessment patients were randomised into intervention and control groups. The intervention group were invited to attend a one day education session during which motivational interviewing techniques were used to deliver advice on diet, exercise and positive lifestyle choices. They also received an individual written exercise plan to take home and implement. The control group continued with their usual level of care. After 4 months all participants were reassessed.

\section{Baseline Results}

- 163 patients were recruited, 100 were male $(61.3 \%)$ with a mean age of 8.4 years (range $5.3-11.5$ )

- Patient subgroups: $18.4 \%$ acyanotic no intervention, $37.4 \%$ acyanotic repaired, $27.6 \%$ cyanotic corrected, $16.6 \%$ cyanotic palliated

- EST duration mean 5.89mins (SD 2.02),

- Actigraph: Average time spent in MVPA (Moderate-Vigorous Physical Activity) 45 mins (SD -27.2)

- The 'cyanotic palliated' subgroup had significantly shorter EST duration and lower levels of daily MVPA

- The 'cyanotic palliated' group also scored significantly lower on HrQOL subscale, physical wellbeing

\section{Review Results}

- There was a significant improvement in performance at peak exercise in the intervention group following the exercise programme

Conclusions: The baseline assessments suggest that overall physical and psychological wellbeing is well preserved in the majority of children aged 5-11 years with CHD. A structured intervention programme significantly improved peak exercise capacity in young children with CHD.

\section{P-181}

Health-Related Quality of Life After Repaired Tetralogy of Fallot is Related to Prematurity and Systemic to Pulmonary Artery Shunt Palliation Svensson B. (1), Rosenkvist C.J. (2), Hanseus K. (1), Liuba P.(1) Cardiology, Children's Heart Center, Skåne University Hospital, Lund (1) and Dpt of Paediatrics, Kalmar Hospital, Kalmar (2), Sweden

Objective: We sought to assess the relationship between healthrelated quality of life (HRQoL) and early life variables in children with previous surgical repair for tetralogy of Fallot (ToF). To this aim, we used the Pediatric Quality of Life Inventory (PedsQL) 4.0 Generic Core Scales which encompass physical functioning, emotional functioning, social functioning and school functioning. Methods: Of a total of 224 patients with surgical repair for ToF between 1996 and 2013 at the Skåne University Hospital in Lund who were included in an earlier retrospective survey, we identified 
48 patients ( 31 male) aged 9 to 18 (median 13) years with available self-reported PedsQL 4.0 data. The total score (TS, 100 highest) was expressed as median and 10-90th percentiles.

Results: The overall TS was 84 (54-99). There was no significant association between TS and the weight, age or bypass time at the time of repair $(p>0.2)$. Also there was no association between TS and the number of reoperations $(\mathrm{p}>0.3)$. In the whole cohort, 11 patients were born preterm ( $<37$ weeks of gestation) and 13 patients were palliated with Blalock-Taussig shunt (BTs) prior to repair. The TS of patients with both prematurity and BTs $(n=6)$ was significantly lower $(69(33-76) ; \mathrm{p}<0.05)$ compared to the other 3 groups (fullterm \& no-BTs: 82(60-99), fullterm \& BTs: 93 (54-100), and preterm \& no-BTs: 86(70-93); Figure).

Conclusion: Systemic to pulmonary artery shunting prior to ToF repair in children born preterm appears to adversely influence HRQoL. Further larger studies are needed to confirm this association.

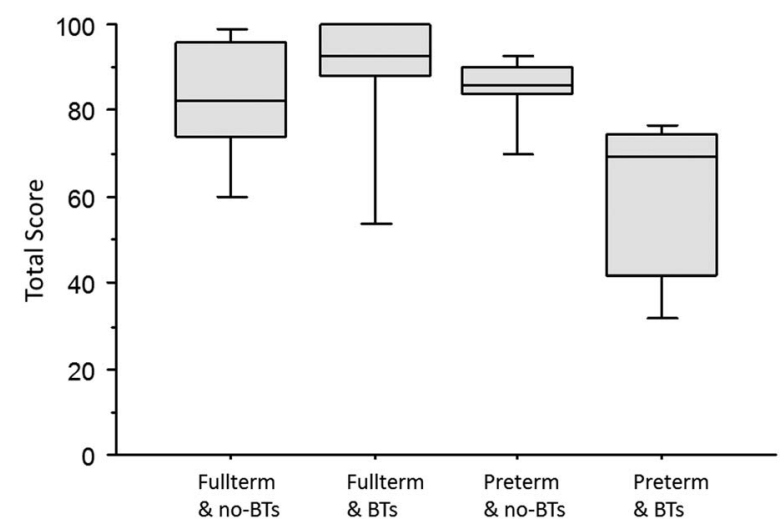

\section{P-182}

Bicuspid Aortic valve in children with Marfan Syndrome: high appearance without correlation to severity of clinical symptoms

Stark V.C., Mir T.S., Olfe J., Seggewies F.S., Arndt F., Mueller G. C., Kozlik-Feldmann R.

Clinic for Pediatric Cardiology, University Heart Center, Hamburg, Germany

Introduction: Due to age dependent development of most pathologies in Marfan syndrome (MFS) estimation of clinical outcome in childhood is difficult. Bicuspid aortic valve (BAV) is correlated with MFS and prevalence is up to $4.7 \%$ in adult Marfan patients. Data in childhood about appearance and correlation with severity of phenotype is missing. Subsequently it may be a predictor for onset of pathologies and helpful to determine individual patient care. We hereby evaluated correlation of present BAV with other Marfan pathologies in childhood.

Methods: Since 2008 we investigated 395 patients (11.4 \pm 5.5 y) with suspected MFS. In 145 patients MFS was diagnosed, thereby 16 patients showed BAV. We retrospectively analyzed correlation of prevalence and age of onset of cardiovascular pathologies (Dilatation sinus of valsalvae (SV), Mitral valve prolaps (MVP)), systemic manifestation of Ghent Criteria and FBN1 mutation with appearance of BAV.

Results: Prevalence of BAV in pediatric Marfan patients was $11.0 \%$ whereas age of patients with and without BAV did not differ significantly. There is no correlation of BAV with prevalence or age of onset of pathologies of MFS. (Table1).
Table 1. Correlation of Marfan pathologies with BAV appearance.

\begin{tabular}{|c|c|c|c|c|c|c|}
\hline \multirow[b]{2}{*}{ Pathology } & \multicolumn{2}{|c|}{ Prevalence } & \multicolumn{2}{|c|}{ Age of onset[years] } & \multirow{2}{*}{$\begin{array}{l}\text { P } \\
\text { (Prevalence) }\end{array}$} & \multirow{2}{*}{$\begin{array}{l}\text { P (Age of } \\
\text { onset) }\end{array}$} \\
\hline & BAV & noBAV & BAV & noBAV & & \\
\hline SV & $\begin{array}{l}12 / 16 \\
(75.0 \%)\end{array}$ & $\begin{array}{l}71 / 129 \\
(67.8 \%)\end{array}$ & $11.8 \pm 1.5$ & $9.5 \pm 0.7$ & ns & ns \\
\hline MVP & $\begin{array}{l}11 / 16 \\
(68.8 \%)\end{array}$ & $\begin{array}{l}76 / 129 \\
(58.9 \%)\end{array}$ & $11.1 \pm 1.7$ & $9.8 \pm 0.7$ & ns & ns \\
\hline $\begin{array}{l}\text { Systemic } \\
\text { manifestation }\end{array}$ & $\begin{array}{c}8 / 16 \\
(50.0 \%)\end{array}$ & $\begin{array}{l}62 / 129 \\
(48.1 \%)\end{array}$ & $12.4 \pm 1.8$ & $12.9 \pm 0.6$ & ns & ns \\
\hline Ectopia lentis & $\begin{array}{c}3 / 16 \\
(18.8 \%)\end{array}$ & $\begin{array}{l}25 / 129 \\
(19.4 \%)\end{array}$ & $7.2 \pm 1.8$ & $9.1 \pm 1.1$ & ns & ns \\
\hline FBN1 & $\begin{array}{l}12 / 16 \\
(75.0 \%)\end{array}$ & $\begin{array}{l}88 / 129 \\
(68.2 \%)\end{array}$ & - & - & ns & - \\
\hline
\end{tabular}

Conclusions: In our large collective prevalence of BAV in MFS was surprisingly higher compared to adult. The reason is unknown, but can possibly be an effect of selection. Indeed, occurrence of BAV did not correlate with severity of clinical phenotype. Especially SV dilatation did not appear earlier or more often in BAV patients. Thus BAV is present frequently in MFS but is no predictor for clinical outcome and estimation of need for therapy. Development of pathologies in those patients in adulthood is unknown but may be relevant for course of disease. Good predictors for severity of phenotype in childhood are unfortunately still missing.

\section{P-183}

Virtual Dissection by Polarised Light Imaging of The Deep Ventriculo-Indibular Fold in Normal and Malformed

Hearts

Truong B.L (1,3), Michalowicz G. (1,2), Usson Y. (1), Jouk P.S (1,2) (1) Univ. Grenoble Alpes, TIMC-IMAG, F-38000 Grenoble, France CNRS, Grenoble, France(2) CHU Grenoble-Alpes, Département de Génétique, F-38000 Grenoble, France(3) Cardiovascular Unit, Children's Hospital 2, Ho Chi Minh City, Vietnam

Introduction: The anatomic structure located between the inflow tract and the semi-lunar valve of the right ventricle "l'eperon de Wolf' or ventriculo-infundibular fold (VIF) has received much attention. Until now description of this specific landmark of the right ventricle relies essentially on visualization after right ventricular opening or sequential sectioning, that are disruptive methods making difficult to establish the connexity of the ventricular fold with the other parietal and septal components of the supraventricular crest. New developments of Polarized light imaging (PLI) with half sphere definition of the orientation of myocardial cells make possible to study the deep anatomy of VIF in three dimensions in normal and malformed hearts.

Methods: 8 normal hearts and 9 malformed hearts (atrio-ventricular septal defect (AVSD); tetralogy of Fallot (TOF)) were studied by PLI. In each voxel of the ventricular mass $\left(90 \times 90 \times 500 \mathrm{~mm}^{3}\right)$, the principal orientation segment was automatically and unambiguously extracted. The Line Integral Convolution (LIC) is used to help streamline visualisation, then the virtual dissection in threedimensional dimension (3D) were exploited with Image J Software.

Result: The Figure 1 showed the principal orientation of the VIF viewed in axial plan section.

A: Normal heart of 12 weeks of age; B: Zoom of selected square in A to show the VIF. myocardial cells are located transversely from parietal band to inter-ventricular septum.

C: Fetal heart of 22 week of gestational age with AVSD. D: Zoom of selected square in C. Myocardial cells of the VIF are placed obliquely in axial sections from parietal band to conal septum. 
(a)

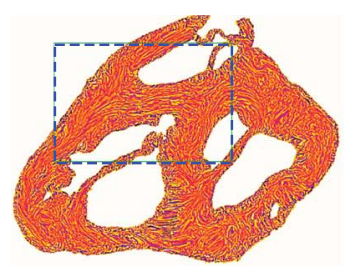

(c)

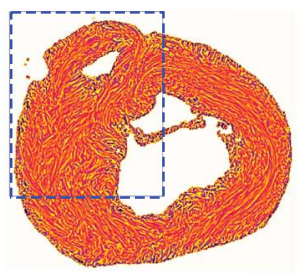

(e)

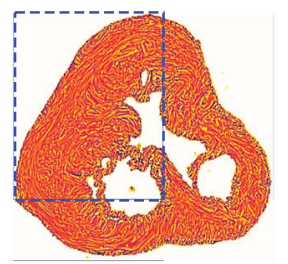

(b)

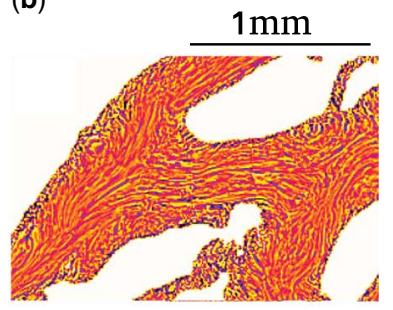

(d)

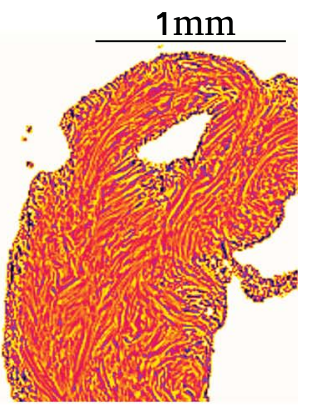

(f)

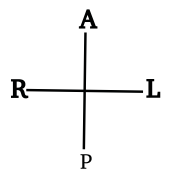

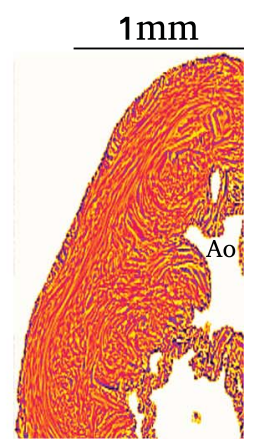

E. Neonatal heart of TOF, F: Zoom of selected square in E. Due to the overriding aorta, the VIF is hypoplasic and maintains the continuity with the outlet septum.

Conclusion: In the first time, we used PLI to describe the myocardial pattern in the deep of VIF. The organisation of myocardial cells in the VIF changes with different malformations. Complete AVSD and cono-truncal malformations disrupt the deep VIF in specific manner. This description completes description relying classically on superficial analysis of the relationships between the VIF and the septomarginal trabeculation.

\section{P-184}

Dissection of ductus arterious as a cause of sudden intrauterine death

Fedrigo M. (1), Visentin S. (2), Frascura C. (1), Cosmi E. (2), Thiene G. (1), Angelini A. (1)

Department of Cardiac, Thoracic and Vascular Sciences, University of

Padua, Italy (1), Department of Woman and Children Health,

University of Padua, Italy. (2)

Background: Sudden intrauterine death is a socio- epidemiological problem. In fact it accounts for 4-5/1000 births.

The causes of unexpected death were mostly in the setting of placenta injury in terms of placenta insufficiency, maternal malperfusion vascularization, fetal inadequate vascularization or ombelical cord causes, such as nodal cord, rupture, marginal insertion, etc. Most of the series in the literature showed the high percentage of cases with unexplained causes ("sine materia")

ranging from $20 \%$ to $50 \%$. Premature closure of ductus arteriosus or fossa ovalis are rear conditions for cardiac sudden death.

Methods: We report two cases of sudden intra-uterine deaths due to premature closure of ductus arteriosus caused by dissection of the ductus with ematoma of the parietal wall and ab-extrinseco compression of the lumen. In both cases the pregnancy was phisiological without maternal or fetal pathology, no administration of drugs during pregnancy was done. Time of stillbirth was 32 gestation weeks for both cases. We performed TUNEL analysis for apoptosis quantification and immunohistochemistry for MIB1 for scoring proliferation.

Results: The dissecting features showed hematoma of ductus arteriosus extending to the aortic arch and descending aorta and with involvement of carotid and succlavian artery. TUNEL analysis showed a high nuclear apoptosis of smooth muscle cells and absence of intimal MIB1 smooth muscle cell proliferation.

Conclusions: Dissecting aneurism of ductus arteriosus is a rare cause of stillbirth; the pathological marker is hematoma of adventitia that involves aortic arch, brachiocephalic vessels, descending aorta. The evidence of a high rate of apoptosis in the parietal wall of the ductus suggests a possible pathophysiological mechanism with an imbalance between apoptosis and proliferation of smooth muscle cells.

\section{P-185}

Fontan patients with right isomerism possess inferior cardiac performances irrespective of strong valve regurgitation

Hamamichi Y., Horimoto Y., Takeguchi M., Mastui T., Saito M., Ishii T., Inage A., Ueda T., Yazaki S., Yoshikawa T.

Sakakibara Heart Institut, Tokyo, Japan, Background.

When patients with right isomerism heart (Riso) possess intensive regurgitation of atrio-ventricle (AVVR), they suffer depressed cardiac functions. However, we often find Riso-patients to fall into cardiac impairment, although they have no strong AVVR. We investigated whether Riso-patients owned cardiac depression irrespective of intensive AVVR.

Methods: The medical records of 36 Fontan patients with Riso were reviewed who underwent cardiac catheterization between 2010 and 2015. Control was 181 Fontan patients without Riso who had examinations likewise during the same period. First, we compared cardiac performances and clinical features between Fontan patients with and without Riso. Second, we excluded patients with AVVR mild-to-moderate or over (Riso 10, nonRiso 20) from 217 patients, using rest of whom we compared cardiac performances in the same way.

Results: Riso-patients were operated for AVVR more (41\% vs. $16 \%, \mathrm{p}<0.001)$; they had residual AVVR mild-to-moderate or over more $(27 \%$ vs. $11 \%, p=0.0079)$. As for cardiac performances, Riso-patients possessed larger ventricular volume on end-systole $(64 \%$ vs. $51 \%, p=0.013)$ and on end-diastole $(125 \%$ vs. $106 \%$, $\mathrm{p}=0.023)$. They had higher capillary wedge pressure of pulmonary artery $(9.1$ vs. $6.9 \mathrm{mmHg}, \mathrm{p}=0.00082)$. Driving pressure of pulmonary artery was lower in Riso-patients (3.7 vs. $5.3 \mathrm{mmHg}, \mathrm{p}=0.0016)$. Between new Riso group $(\mathrm{n}=26)$ and new non-Riso group $(\mathrm{n}=167)$, where 30 patients with mild-tomoderate or over AVVR were excluded from origin groups, new Riso-patients also owned inferior cardiac performances: larger ventricular volume on end-systole $(60 \%$ vs. $49 \%, \mathrm{p}=0.042)$ and end-diastole ( $120 \%$ vs. $103 \%, \mathrm{p}=0.048)$; more elevated capillary wedge pressure (8.5 vs. $6.7 \mathrm{mmHg}, \mathrm{p}=0.0034)$; smaller driving pressure $(3.7$ vs. $5.6 \mathrm{mmHg}, \mathrm{p}=0.019)$. In the background for new groups almost all Riso-patients had no left ventricle as major 
systemic pomp ( $4 \%$ vs. $27 \%: p=0.00026$ ); they undertook shunting to pulmonary artery as 1 st strategy which forcedly dilated ventricle. In addition, Riso-patients more undertook repair of atrio-ventricular valve regurgitation which had stretched ventricle before surgery.

Conclusion: Our study showed Fontan patients with Riso possessed more dilated ventricle and higher capillary wedge pressure without reference to regurgitation of atrio-ventricular valve. Riso-patients potentially might have deteriorating cardiac function owing to inherent predisposition and pre-Fontan intervention.

\section{P-186}

\section{Cardiac tumors in newborn: Report of five cases}

Ayari F.

Neonatology Intensive Care Unit at Maternity and Neonatology Center of Tunis, Tunisia

Introduction: The primary cardiac tumor is a rare disease in children, with a frequency between $0.03-0.32 \%$. Eighty percent are benign tumors. Usually they are asymptomatic and discovered fortuitously. Methods: Recall the clinical and sonographic features of primitive cardiac tumors through 5 neonatal observations.

Results: The Five neonates: Leith, Taher, Youssef, Khaled and Eya are from a non consanguineous marriage. The pregnancy was well attended in all cases. Prenatal ultrasound showed cardiac tumors suggeging a left intraventricular rhabdomyoma in the first case, biventricular in 2nd case, right ventricular fibroma in the case of Youssef, two hyperechoic spots $2 \mathrm{~mm}$ each depends on the valvular pillars in the left ventricle in the case of Khaled and a tumor taking all the right ventricle in the case of Eya. The five newborns were eutrophic for the term and showed good adaptation to extrauterine life. The examination at birth was without anomalies. The post natal ultrasound pinpoint the tumor localization which was respectively: landlocked in the aorta which partially obstructed in the first case, multiple biventricular whose largest was opposite to the large mitral valve in the second case, in the trabecular room of the right ventricle in the third case, not individualized right ventricle with aspect of trabeculation in the latter case. Hemodynamically, these cardiac tumors were well tolerated in the 1st and 3rd case. In the case of Taher, this tumor generate a supraventricular tachycardia quick to $300 \mathrm{bpm}$ having been reduced by vagal maneuvers and in the case of Eya, the tumor was responsible for the death by refractory hypoxemia. The ultrasound transfontanellaire was without defects in the first two cases. Youssef showed convulsions type bending spasms evoking a tuberous sclerosis and Eya had a total agenesis of the corpus callosum.

Conclusion: Children's cardiac tumors are usually benign. The severity is related to functional consequences to which depends the support. Surgery is the best treatment with an excellent prognosis.

\section{P-187}

Subclinical Left Ventricular Systolic and Diastolic Dysfunction in Type 1 Diabetic Children and Adolescents with Good Metabolic Control

Ozgur S. (1), Yoldas T. (1), Orun U.A (1), Sagsak E. (1,2), Aycan Z. (1,2), Kaya O. (1), Ozgur S. (1), Karademir S. (1)

Dr. Sami Ulus Maternity and Children Research and Training Hospital, Pediatric Cardiology, Ankara - Turkey (1)Dr. Sami Ulus Maternity and Children Research and Training Hospital, Pediatric Endocrinology, Ankara - Turkey (2)

Objective: Cardiac dysfunction is a well-known consequence of diabetes and subclinical left ventricular diastolic and systolic dysfunction followed by overt left ventricular dysfunction and finally symptomatic heart failure. This study was designed to assess whether type 1 diabetic children and adolescents with good metabolic control have early echocardiographic signs of subclinical left ventricular dysfunction and whether diabetes duration is of influence, using conventional and non-conventional echocardiographic tools.

Methods: A total of 100 patients with type I diabetes mellitus and 80 gender and age-matched healthy controls were included. The cases underwent standard conventional transthoracic echocardiography, tissue Doppler imaging and two dimensional speckle tracking echocardiography. None of the diabetic patients had signs of renal, retinal or neurological complications of the disease and all were good metabolic control (mean $\mathrm{HbA}_{1 \mathrm{c}}<7.5 \%$ ).

Results: There was no difference among groups in relation to age, sex, body mass index and blood pressure. Conventional echocardiographic parameters were similar between diabetic and nondiabetic subjects except increased mitral valve peak A wave and significantly lower mitral E/A ration in diabetics. Diabetic patients had more advanced diastolic dysfunction in TDI analysis. In diabetic group, left ventricular global longitudinal strain and strain rate, left ventricular global circumferential strain and strain rate and left ventricular radial strain and strain rate were significantly lower compared with the controls. There was a positive correlation between diabetes duration and cardiac dysfunction.

Conclusion: The results of this study showed that the diabetic children and adolescents with good metabolic control had diastolic dysfunction when assessed with either conventional or tissue Doppler echocardiograph. In addition to diastolic dysfunction diabetic patients had subclinical LV systolic dysfunction in the longitudinal and short axis direction with a normal LVEF which can be detected with 2D speckle tracking echocardiography. Diabetic duration was the only independent predictor for the diastolic and systolic dysfunction.

\section{P-188}

Timing of diagnosis in readily treatable congenital heart diseases does not affect mortality

Khor K. (1), Gentles T. (1), Finucane K. (1), Sadler L. (2), Harpham M. (3), Parry E. (3)

Greenlane Paediatric and Congenital Cardiac Service, Starship Children's Hospital, Auckland, New Zealand (1); Department of Obstetrics \& Gynaecology, National Women's Hospital, Auckland, New Zealand (2); New Zealand Maternal Fetal Medicine Network, National Women's Hospital, Auckland, New Zealand (3)

Introduction: Timing of diagnosis in readily treatable congenital heart diseases does not affect mortality.

Methods: From a retrospective, population-based review of all major congenital heart disease (CHD) cases in New Zealand from 2006 to 2015 the outcome of two groups of readily treatable CHD (D-transposition of the great arteries (D-TGA) and critical left ventricular outflow tract obstruction including coarctation and interrupted aortic arch with two ventricles (LVOTO) were examined. Outcome was analysed based on the timing of diagnosis and survival to 6 months of age over two timeframes: 2006-2010 and 2011-2014.

Results: There was a slight increase in the prenatal diagnosis of D-TGA over the study period and a decrease in the number with LVOTO diagnosed after hospital discharge (Figure 1).

The rate decreased substantially between 2006-10 and 2011-14 (D-TGA $9.7 \%$ to $1.3 \%(\mathrm{p}=0.021)$ and LVOTO $20.7 \%$ to $2.2 \%$ $(p=0.005))$.

In both D-TGA and LVOTO mortality occurred prior to cardiac surgery in almost all cases. Within the time frame for outcome, in 

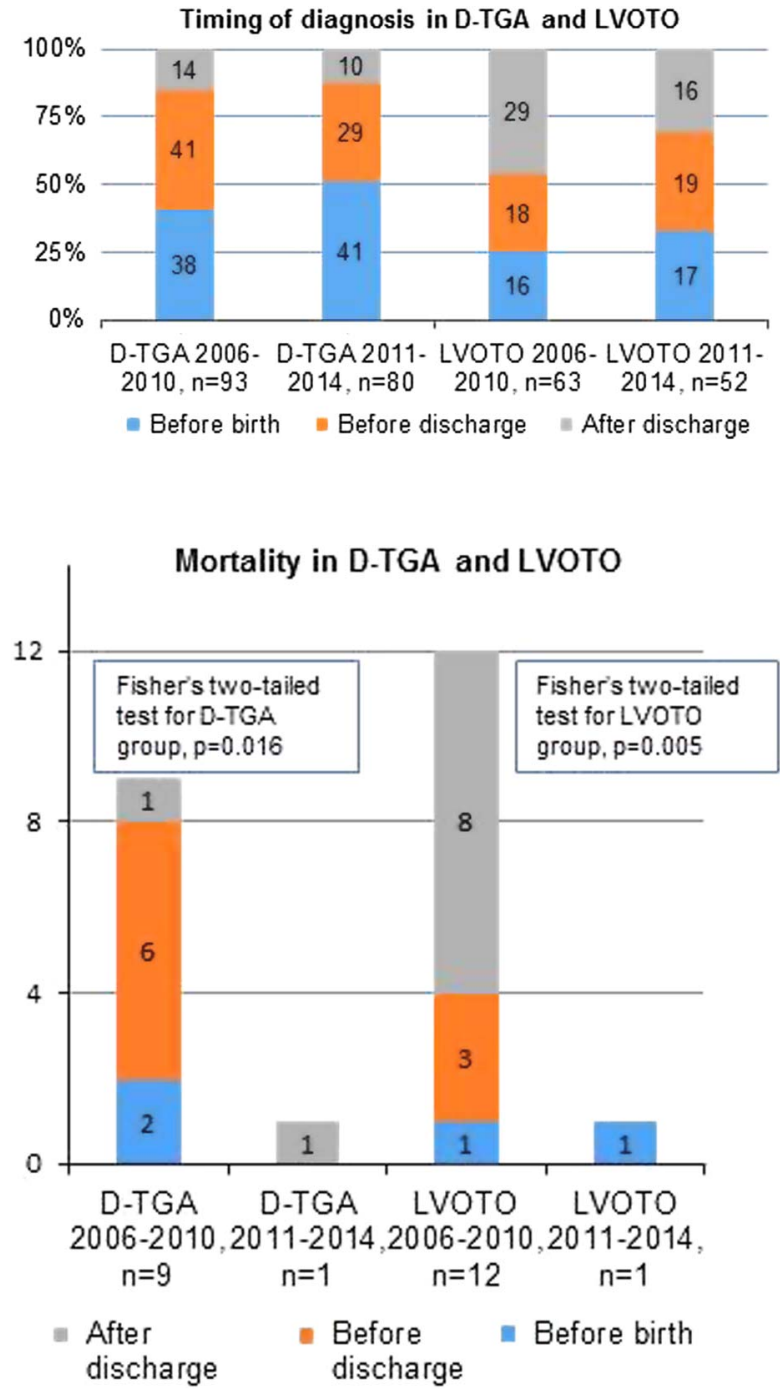

2006-2010, there was one post-operative death within 6 months of surgery in the D-TGA group and two in the LVOTO group. In 2010-2014, there were no deaths in the D-TGA group and one in the LVOTO group. All of these had significant non-cardiac comorbid issues contributing to death.

Conclusions: There has been a dramatic reduction in mortality for D-TGA and LVOTO. This has been accompanied by a modest increase in the antenatal detection of D-TGA and a reduction in the number with LVOTO identified after hospital discharge. In D-TGA, lower mortality is likely related to an emphasis on delivery at a cardiac centre and rapid identification, stabilisation and transfer of those born elsewhere, while in LVOTO it is most probably the result of a reduction in the number of cases diagnosed after hospital discharge.

\section{P-189}

Prevention of Infective Endocarditis is Necessary for all Risk Groups: Case Series

Kosger P. (1), Ayvaci B. (2), Karabel D.(2), Yildirim A. (2), Keskin T. (2), Ucar B. (2), Kilic Z. (2)

Clinic of Pediatric Cardiology, Eskisehir State Hospital, Turkey (1), Department of Pediatric Cardiology, Faculty of Medicine, Eskisehir Osmangazi University, Turkey (2)
Infective Endocardite (IE) is a serious disease with mortality rate of $16-25 \%$ in children. Incidence of IE increases with congenital heart diseases. Cyanotic congenital heart disease, history of previous IE, prosthetic heart valve, heart transplant patients with a valvulopathy in the implanted heart, repaired heart disease with residual significant lesion, or 6 months after repaired heart disesase with prosthetic material are defined high risk group for IE and current guidelines are recommend antibiotic prophylaxis for only the high risk group just before dental procedures to prevention of IE. Since the publications of these recommendations, at least one study has documented the lack of any demonstrable increase in IE as a cause of hospitalization for children. However, no data has been reported about whether it has changed frequency of IE in developing countries.

In our clinic, 4 patients aged between 3.5 and 11 years were diagnosed with IE in the last year. All of them had a congenital heart disease and 2 of them were in high risk group (vsd, bicuspid aortic valve, c-TGA with PS, opere d-TGA with peripheral pulmonary stenosis). Only one patient diagnosed with vsd had a history of dental prosedure who applied antibiotic prophylaxis. Methicillin-sensitive staphylococcus aeureus was identified in two patients and streptococcus viridans was identified in other patients in blood cultures. Moving vegetations $(>10 \mathrm{~mm}$ ) were detected over the aortic valve in the patient diagnoised with bicuspid aortic valve who died within the first 24 hours due to multisystemic septic embolism and heart failure despite broad spectrum antibiotics and intensive care support. Two cases underwent anticoagulant therapy due to pulmonary embolism in second week of follow-up due to the vegetations over the tricuspid valve and in the pulmonary arteries. Recovery was achieved In 3 patients after parenteral antibiotherapy given 4 to 8 weeks thus surgical treatment was not necessary.

We want to emphasize that limiting the use of antibiotic prophylaxis with high-risk patients in the prevention of infective endocarditis, which is known to have a high mortality and complication rate, may have negative consequences especially for developing countries.

\section{P-190}

EUROlinkCAT - a Horizon2020 study 2017-2021

Garne E. (1), Morris J.K. (2), Loane M.(3), Rankin J. (4), Barisic I.(5), Densem J.(6), Neville A. (7), Pierini A.(8), de Walle H. (9) [on behalf of the EUROlinkCAT Steering Committee]

Hospital Lillebaelt, Kolding, Denmark (1); Queen Mary University of London, London, UK (2); Ulster University, Belfast, N Ireland (3); Newcastle University, Newcastle upon Tyne, UK (4); Medical School University of Zagreb, Zagreb, Croatia (5); BioMedical Computing Limited, St Leonards on Sea, UK (6); University of Ferrara, Ferrara, Italy (7); CNR Institute of Clinical Physiology, Pisa, Italy (8); Department of Genetics, Groningen, The Netherlands (9);

Introduction: Congenital anomalies (CA or birth defects ) whilst often rare diseases, are a major cause of infant mortality, childhood morbidity and long-term disability. Over 130,000 children born in Europe every year have a congenital anomaly of whom a third will have a congenital heart defect (CHD) and a fourth of these children will have an isolated CHD.

Methods: EUROCAT is an established European network of population-based registries for the epidemiologic surveillance of CAs. The Horizon2020 funded EUROlinkCAT project will use the EUROCAT infrastructure to support 21 EUROCAT registries in 13 European countries to link their data on children with CA to mortality, hospital discharge, prescription and educational databases. Each registry will send standard aggregate tables and analysis results to a Central Results Repository (CRR) thus 
respecting data security issues surrounding sensitive data. The CRR will contain standardised summary data and analyses on an estimated 200,000 children with a CA born 1995-2014 up to age 10, enabling hypotheses on their treatment, health and education to be investigated at the EU level. Information on prognosis and outcome at the European level will be published and geographical differences in morbidity and mortality will be investigated. This enhanced information will allow optimisation of personalised care and treatment decisions for children with rare CAs including all types of CHD.

Registries will be supported in using social media platforms to connect with parents of childen with CAs in their regions including parents of children with severe CHDs. A novel sustainable e-forum, "ConnectEpeople", will link these families with local/national and international registries and information resources. ConnectEpeople will involve these families ensuring a meaningful dissemination of results.

Results: Findings will provide evidence to develop national treatment guidelines, such as concerning screening programs, to optimise diagnosis, prevention and treatment for these children and reduce health inequalities in Europe. An economic evaluation of the hospitalisation costs associated with CA will be provided. Conclusion: The CRR and associated documentation, including linkage and standardisation procedures and "ConnectEpeople" forum will be available post-EUROlinkCAT facilitating future local and EU level analyses to improve healthcare for children with $\mathrm{CA}$ and in particular with CHD.

\section{P-191}

Risk and Prediction of Clinically Relevant Pericardial Effusion after Pediatric Heart Surgery

Adrichem R. (1), Le Cessie S. (1), Hazekamp M.G. (1), Van Dam N. A.M. (1), Blom N.A. (1), Rammeloo L.A.J. (2), Filippini L.H.P.M. (3), Kuipers I.M. (4), Ten Harkel A.D.J. (1), Roest A.A.W. (1)

Leiden University Medical Center, Leiden, The Netherlands (1); Free University Medical Center, Amsterdam, The Netherlands (2); Juliana Children's Hospital, The Hague, The Netherlands (3); Academic

Medical Center, Amsterdam, The Netherlands (4)

Introduction: Pericardial effusion (PE) after pediatric cardiac surgery is common. Research on PE is hampered by the lack of a uniform classification of the presence and severity of PE. Therefore, we focused on PE altering clinical management: ie requiring additional echocardiograms, start or continuation of medication or pericardiocentesis: clinically relevant $\mathrm{PE}$ (crPE). The aim was to investigate risk factors for $\mathrm{crPE}$ and to create a prediction model. Methods: Pediatric patients who underwent cardiac surgery at the center for congenital heart disease Amsterdam-Leiden between January 2010 and December 2014 were selected and followed until 1 month after surgery. Preoperative variables, such as age, gender and type of heart defect were studied in the complete cohort using independent t-tests, chi-square tests, and logistic regression. Perioperative and postoperative variables were studied in a case-control manner. Each patient with crPE was matched to one without crPE. Matching was based on age, gender and diagnosis severity. Matched logistic regression was used to determine the effect sizes. To create the prediction model, the important preoperative variables were determined in the complete cohort and then the additional effect of perioperative and postoperative variables was determined in the case-control data. Inverse probability weighting was used for data of the controls.

Results: 1214 surgical episodes of 1039 patients were included. CrPE developed in 134 (11.0\%) of these episodes and 31 (2.6\%) required pericardiocentesis. No deaths were caused by PE. Older age at surgery, use of cardiopulmonary bypass (CPB) and longer duration of CPAP increased the risk of crPE, whereas a previous operation decreased the risk of crPE. The final model consisted of age, history of previous operation, $\mathrm{CPB}$ usage, duration of CPAP postoperatively and the amount of thoracic drain output. The area under the curve of the model was 0.71 .

Discussion: PE incidence corresponds with those in previous studies. Most of the observed risk factors were known from adult literature and are demonstrated to also apply to crPE in children, whereas $\mathrm{CPB}$ use was newly identified as a risk factor for crPE after pediatric cardiac surgery. The model can identify high-risk patients for crPE and may improve patient care.

\section{P-192}

Carotic intima media thickness in children with white coat and essential hypertension

Jurko A. Jr (1), Jurko T. (2), Jurko A.(1), Mestanik M.(3), Mestanikova A. (3), Visnovcova Z. (4), Tonhajzerova I. $(3,4)$ Paediatric Cardiology, Martin, Slovak Republic (1); Clinic of Neonatology, Jessenius Faculty of Medicine in Martin, Comenius University in Bratislava, Slovak Republic (2); Comenius University in Bratislava, Jessenius Faculty of Medicine in Martin (JFM CU), Department of Physiology JFM CU, Martin, Slovak Republic (3); Comenius University in Bratislava, Jessenius Faculty of Medicine in Martin (JFM CU), Biomedical Center Martin JFM CU, Martin, Slovak Republic (4)

Introduction: Sonographic intima media thickness measurement of the carotic artery (cIMT) is considered a valid surrogate marker for cardiovascular risk allowing assessment of atherosclerotic changes at very early stage. Elevated blood pressure in adults is associated with increased cIMT. However, its effect on arterial health is less understood in a paediatric population. Thus, the aim of this study was to evaluate cIMT in adolescents suffering from essential and white coat hypertension.

Methods: One hundred thirty eight children - 46 (23 boys) children suffering from WCH, 46 (23 boys) patients with essential hypertension and 46 age/gender-matched healthy controls (ageperiod from 14 to 18 years) - were examined under standard conditions. The mean IMT of the carotid arteries was measured by high-resolution B-mode ultrasound (Prosound F75 Aloka).

Results: Statistical analysis revealed significant differences in the mean cIMT between the both hypertensive groups (WCH, EH) and control group $(0,45 \pm 0,05 \mathrm{~mm}$ for $\mathrm{WCH} ; 0,47 \pm 0,06 \mathrm{~mm}$ for $\mathrm{EH}$ vs $0,40 \pm 0,04 \mathrm{~mm} ; \mathrm{p}<0.001)$. No significant differences were found between $\mathrm{WCH}$ and essential hypertensive groups.

Conclusions: Our results revealed significantly higher mean cIMT in children suffering from white coat and essential hypertension indicating early atherosclerotic changes associated with increased cardiovascular risk already in adolescent hypertension. Importantly, WCH should not be considered a harmless trait and has common features with essential hypertension.

Support: VEGA 1/0087/14, project "Biomedical Center Martin" ITMS code [26220220187] co-financed from EU sources.

\section{P-193}

Effect of blood pressure on carotid stiffness in children with white coat and essential hypertension

Jurko A. Jr. (1), Jurko T. (2), Jurko A.(1), Mestanik M.(3), Mestanikova A. (3), Visnovcova Z. (4), Tonhajzerova I. $(3,4)$ Paediatric Cardiology, Martin, Slovak Republic (1); Clinic of Neonatology, Jessenius Faculty of Medicine in Martin, Comenius University in Bratislava, Slovak Republic (2); Comenius University in Bratislava, Jessenius Faculty of Medicine in Martin (JFM CU), Department of Physiology JFM CU, Martin, Slovak Republic (3); Comenius University 
in Bratislava, Jessenius Faculty of Medicine in Martin (JFM CU), Biomedical Center Martin JFM CU, Martin, Slovak Republic (4)

Introduction: The term "arterial stiffness" denotes alterations in the mechanical properties of arteries, which may represent higher cardiovascular risk in middle-aged and older adults. Recently, the question regarding the arterial stiffness in patients with risk factors for the early progression of systemic atherosclerosis in vulnerable adolescent age-period are rare. Thus, the aim of this study was to evaluate the carotid stiffness and early atherosclerotic changes in adolescents suffering from essential and white coat hypertension using noninvasive markers.

Methods: One hundred thirty eight children - 46 (23 boys) children suffering from WCH, 46 (23 boys) patients with essential hypertension $(\mathrm{EH})$ and 46 age/gender-matched healthy controls were examined under standard conditions. The age-period of all children ranged from 14 to 18 years. Ultrasonography combined with echo-tracking system (Prosound F75 Aloka) on common carotid artery (CCA) was used to analyse of local arterial stiffness. In the study five physiological parameters of the CCA were analysed - mean stiffness index ( $\beta$ ), elastic modulus (Ep), arterial compliance (AC), augmentation index (AI) and pulse wave velocity (PWV $\beta$ ).

Results: Statistical analysis revealed significant differences in parameters $\beta, \mathrm{Ep}, \mathrm{AC}$ and PWV $\beta$ between the both hypertensive groups (WCH, EH) and control group (Tab 1). Significant differences were not found in the index AI between $\mathrm{EH}, \mathrm{WCH}$ and control groups. No significant differences were found between WCH and essential hypertensive groups.

Conclusions: Study revealed significantly increased carotid stiffness in children suffering from white coat and essential hypertension. This finding could help to understand hypertension atherosclerosis interaction. We suggest that further research regarding the potential atherosclerotic changes using the sensitive noninvasive parameters in pediatric hypertension is important. Support: VEGA 1/0087/14, project "Biomedical Center Martin" ITMS code [26220220187] co-financed from EU sources

Table 1. Parameters of carotid stiffness in children with white coat hypertension (WCH), essential hypertension (EH) and control group (C group)

\begin{tabular}{|c|c|c|c|}
\hline & $\mathrm{WCH} n=46$ & $\mathrm{EH} n=46$ & $C$ group $n=46$ \\
\hline$\beta$ & $6,14 \pm 0,9^{\star \star \star}$ & $6,01 \pm 1,01^{\star \star \star}$ & $4,8 \pm 0,9$ \\
\hline $\mathrm{Ep}(\mathrm{kPa})$ & $85,4 \pm 14,4^{\star \star \star}$ & $85,2 \pm 16,4^{\star \star \star}$ & $56,1 \pm 9,7$ \\
\hline $\mathrm{AC}\left(\mathrm{mm}^{2} / \mathrm{kPa}\right)$ & $0,84 \pm 0,2^{\star * *}$ & $0,84 \pm 0,18^{\star \star \star}$ & $1,10 \pm 0,24$ \\
\hline AI & $5,06 \pm 37^{\mathrm{NS}}$ & $6,7 \pm 32^{\mathrm{NS}}$ & $-6,1 \pm 6,7$ \\
\hline $\mathrm{PWV} \beta(\mathrm{m} / \mathrm{s})$ & $5,3 \pm 0,5^{\star \star \star}$ & $5,3 \pm 0,6^{\star \star *}$ & $4,4 \pm 0,4$ \\
\hline
\end{tabular}

$\beta$, mean stiffness index; Ep, elastic modulus; AC, arterial compliance;

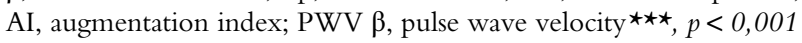
compared to control group

\author{
P-194 \\ Blunted reactivity to stress - a possible pathomechanism of \\ cardiovascular complications in adolescent hypertension? \\ Mestanik M.(1), Jurko A. (3), Jurko T. (4), Mestanikova A. (1, 2), \\ Visnovcova Z. (2), Langer P. (5, 6), Tonhajzerova I. $(1,2)$ \\ Comenius University in Bratislava, JesseniusFaculty of Medicine in \\ Martin (JFM CU), Department of Physiology JFM CU, Slovak \\ Republic (1); Comenius University in Bratislava, JesseniusFaculty of \\ Medicine in Martin (JFM CU), Biomedical Center Martin JFM CU, \\ Slovak Republic (2); Pediatric Cardiology, Martin, Slovak Republic (3); \\ Clinic of Neonatology JFM CU, University Hospital Martin, Slovak
}

Republic (4); Institute of Scientific Instruments of the ASCR, v.v.i., Brno, Czech Republic (5); International Clinical Research Center, St. Anne's University Hospital Brno, Czech Republic (6)

Introduction: Altered physiological reactivity to stress is a wellknown indicator of an increased risk for cardiovascular diseases. One of possible consequences of impaired cardiovascular regulation is elevated blood pressure. Hypertension is commonly associated with excessive sympathetic activity, however, the methods of the non-invasive assessment of sympathetic regulatory outputs are limited. Therefore, we aimed to study complex cardiac sympathetic control using novel promising method of nonlinear analysis of heart rate variability (HRV) - symbolic dynamics - at rest and in response to physiological stress in adolescents with newly diagnosed essential and white-coat hypertension.

Methods: The studied groups consisted of thirty adolescent boys with newly diagnosed essential hypertension $(16.9 \pm 0.2$ years, body mass index (BMI) $22.9 \pm 0.4 \mathrm{~kg} / \mathrm{m} 2$ ), thirty adolescents with newly diagnosed white-coat hypertension $(16.8 \pm 0.3$ years, BMI $22.6 \pm 0.5 \mathrm{~kg} / \mathrm{m} 2)$ and thirty healthy adolescent boys $(16.2 \pm 0.4$ years, BMI $22.1 \pm 0.4 \mathrm{~kg} / \mathrm{m} 2$ ). Continuous recording of RRintervals using DiANS PF8 (Dimea, Czech Republic) was performed in supine position and during active orthostatic test (duration of each phase: 6 minutes). Sympathetic modulation of cardiac autonomic control was assessed using nonlinear analysis of HRV complexity - symbolic dynamics - where index 0V\% (zero variation of $\mathrm{HRV}$ ) is considered to reflect beta-adrenergic sympathetic regulation.

Results: The cardiac beta-adrenergic sympathetic index $0 \mathrm{~V} \%$ was significantly increased during supine position in both white-coat and essential hypertension groups compared to control group ( $p<0.05$ for both). The reactivity (percentual increase) of $0 \mathrm{~V} \%$ to active orthostatic test was significantly lower in white-coat and essential hypertension groups compared to control group $\left(\mathrm{p}^{<} 0.05\right.$ for both). No significant between-groups differences were found in absolute values of $0 \mathrm{~V} \%$ during orthostatic test.

Conclusions: The novel method of nonlinear HRV analysis symbolic dynamics was sensitive to detect increased cardiac sympathetic stimulation in both white-coat and essential hypertension during rest. The sympathetic activation in response to orthostatic test was lower in hypertensive groups indicating abnormalities in the cardiovascular stress reactivity. Importantly, both exaggerated and diminished physiological reactivity to stress have been discussed in terms of negative health outcomes. Our findings could help to better understand the changes of sympathetic cardiac regulation in the adolescent hypertension.

Support: VEGA 1/0087/14.

\section{P-195 \\ Impairment of cardiovascular function in childhood cancer survivors \\ Reiner B. (1,2), Müller J. (1,2), Hager A. (1), Kühn A. (1), Schmid I. (3), Oberhoffer R. (1,2), Ewert P. (1), Weil J. (1) \\ Department of Pediatric Cardiology and Congenital Heart Disease, German Heart Centre Munich, Technical University Munich, Munich, Germany (1); Institute of Preventive Pediatrics, Technical University Munich, Munich, Germany (2); Dr. von Hauner Children's Hospital, Department of Pediatric Hematology and Oncology, Ludwig- \\ Maximilians-University, Munich, Germany (3)}

Introduction: Childhood cancer survivors (CCS) are at an increased risk of developing cardiovascular disease. It is the most common non-cancer cause of death in this patient group. Cardiovascular diseases are caused by cardiotoxic side-effects of anthracycline 
therapy. The main purpose of this study is to detect any deterioration of cancer therapy on vascular structure, function and exercise capacity.

Methods: 49 patients (25 female) aged 16.5 to 32.0 years $(21.5 \pm 3.5$ years) were examined in our institute between March 2015 and October 2016. All patients were asymptomatic and in NYHA class 1. In average cancer was diagnosed $11.2 \pm 4.5$ years ago. Ejection fraction (EF) and fractional shortening (FS) were measured by echocardiography to quantify changes of the left ventricle. $\mathrm{N}$-terminal pro-brain natriuretic protein (NT-proBNP) levels were determined as well-known biomarker of left ventricular dysfunction. Additionally, all patients underwent a cardiopulmonary exercise test to detect any limitation in exercise capacity. Patients were categorized in two groups by their anthracycline dosage: All patients with more than $250 \mathrm{mg} / \mathrm{m}^{2}$ anthracyclines were in the high dose group and compared to patients with moderate dose $\left(100-250 \mathrm{mg} / \mathrm{m}^{2}\right)$ anthracyclines.

Results: EF was reduced in $12 \%(\mathrm{EF}<54 \%)$ of female CCS and in $8 \%(\mathrm{EF}<52 \%)$ of male CCS. $59 \%$ of the patients showed EF values under $60 \%$. There was no sig. difference between the groups with high and moderate anthracyclines. NT-proBNP was increased in $36 \%$ of the female CCS (NT-proBNP $>116 \mathrm{ng} / \mathrm{l}$ ) and $21 \%$ of the male patients (NT-proBNP $>63 n g / l)$. There was no association between NT-proBNP and EF. Reduced cardiopulmonary exercise capacity was sign. associated with higher NTpro BNP levels $(\mathrm{r}=-.342 ; \mathrm{p}=.023)$ and higher anthracycline dosage $(r=-.293 ; p=.048)$.

Conclusion: Determination of EF, NT-proBNP levels and cardiopulmonary exercise test are useful parameters to detect beginning impairment of cardiovascular function in asymptomatic CCS after treatment with cardiotoxic drugs.

\section{P-196}

KidsTUMove: Background and outlook for healthy exercise in children and adolescents with congenital heart diseases

Stoecker N.(1), Oberhoffer R. (1,2), Ewert P. (2)

Chair for Preventive Pediatrics, TU Munich (1), Clinic for pediatric cardiology and congenital heart disease, German Heart Center Munich (2), Germany

The main focus of children with congenital heart defects (CHD) nowadays is no longer on survival, but on their quality of life and daily life. This is why their sport activities are playing an important role. Data of the project kidsTUMove is presented.

108 children with CHD as well as a control group (CHD $9,14 \pm 2,24$ years, 183 control $8,78 \pm 1,67$ years) absolved a motoric test battery to check force, coordination, reaction, balance, flexibility and speed. 'Physical activity (KA) was measured by accelerometry (Actigraph, gt $3 \mathrm{x}+$ ) (88 CHD $9.57 \pm 2.58$ years, 66 control $9.86 \pm 1.90$ years). Health-related quality of life (hLQ) (263 CHD 9,55 $\pm 2,93$ years, 73 control 9,67 $\pm 3,26$ years) was collected using KINDL questionnaire. The data was divided into CHD, CHD ambulant and children with CHD who participated in a kidsTUMove project.

Results: Motoric: CHD achieved lower values than heart-healthy children in the motor characteristics of strength and coordination. On the other hand, heart-healthy children achieved significantly worse values in reaction. No differences: speed, flexibility and balance. CHD who had participated in a kidsTUMove summer camp achieved higher scores than CHD ambulant.

KA: There was no difference in the daily KA. According to Freedson algorithm, children who had participated in a
kidsTUMove project reached higher KA than CHD ambulant (275.24 $\pm 67.40 \mathrm{~min}$.).

hLQ: CHD achieved higher values in hLQ (total) than heart-healthy children (CHD: $77.66 \pm 12.66$, heart disease: $75.1 \pm 10.62$ ).

Since the KA is an important health care provider and motor skills have an impact on many areas of life, they should be regularly examined. And patients should be motivated and advised to integrate KA as a central and permanent component in their daily life. Activities such as the kidsTUmove Sommercamp or kidsTUMove sports groups help children with CHD (also when having a reduced physical performance) to perform similar like healthy children. And since the development of overweight and further illnesses is already beginning in school age, it is urgently necessary to screen CHD already in childhood and provide interdisciplinary prevention offers (for example kidsTUMove).

\section{P-197}

First Evidence of Impaired Cardiac Geometry and Function in Children with Autosomic Recessive Polycystic Kidney Disease

Chinali M., Massella L., Esposito C., Di Zazzo G., Del Pasqua A., Iacomino M., Ricotta A., Emma F., Rinelli G., Drago F.

Bambino Gesù Children Hospital, Rome, Italy

Background: Autosomic recessive polycystic kidney disease (ARPKD) is a rare clinical condition associated with renal failure and neonatal death. In patients surviving the neonatal age, progressive renal dysfunction and liver fibrosis develop. No previous study has yet defined the prevalence of cardiac geometric and mechanical function abnormalities in children with ARPKD.

Methods: Standard echocardiograms were obtained in 22 children with ARPKD aged $0-18$ yr (50\% boys) and in 88 healthy children of similar age, gender distribution, and body build. Left ventricular (LV) mass was calculated by the Devereux formula and LV hypertrophy was defined according to our recently proposed criteria (J Ped 2016). Cardiac geometry was defined by age-adjusted relative wall thickness (RWT) while systolic function was assessed by both measurement of ejection fraction and fractional shortening at the midwall (MFS), representing cardiac mechanical function.

Results: Patients with ARPKD exhibited higher LV mass index as compared to normal individuals $(40.4 \pm 8.2$ versus $28.9 \pm 4.3 \mathrm{~g} /$ $\left.\left(\mathrm{m}^{2.16}+0.09\right) ; \mathrm{P}<0.001\right)$, mainly due to a more concentric LV geometry $(\mathrm{RWT}=0.33 \pm 0.07$ versus $0.27 \pm 0.03: \% ; \mathrm{P}<0.001$ ). Accordingly, prevalence of LV hypertrophy was significantly higher in ARPKD (18.2vs0\%; P < 0.005). No differences could be observed among patients and controls in ejection fraction $(66.2 \pm 4.6$ versus $68.4 \pm 5.9 \% ; \mathrm{P}=\mathrm{NS})$, while a significantly lower MFS $(19.3 \pm 3.6$ versus $21.6 \pm 2.1 \%$; $\mathrm{P}<0.05)$ could be observed. In analysis of covariance, adjusting for differences in age, systolic blood pressure and gender, both LV mass index and RWT remained significantly higher in the ARPKD group, while MFS remained significantly reduced (all $\mathrm{p}<0.05$ ). The prevalence of subclinical systolic dysfunction (defined by $\mathrm{MFS}<16 \%$ ), was significantly higher in patients with ARPKD as compared with control subjects $(22.7$ versus $0 \% ; \mathrm{P}<0.001)$, demonstrating significantly impaired cardiac function despite normal ejection fraction (figure).

Conclusions: This is the first study reporting on cardiac geometry and mechanical function in children with ARPKD. Children with ARPKD show significantly impaired cardiac phenotype, characterized by high rates of LV hypertrophy paired with significant systolic mechanical dysfunction, possibly contributing to the high cardiovascular risk of children affected by ARPKD. 


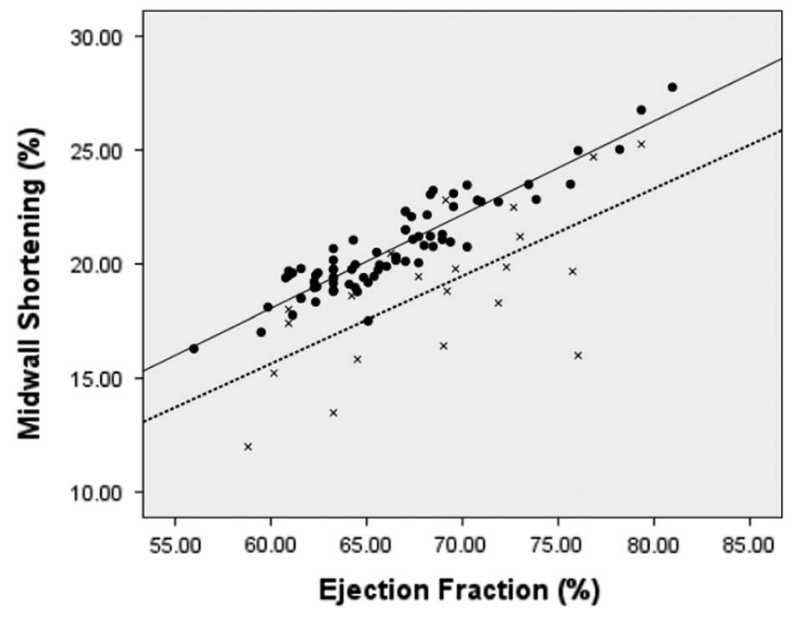

Figure 1.

filled circles (and bold line) represent normal controls; crosses (and dashed line) represent $A R P K D$ patients (see text for explanations).

\section{P-198}

Very rare association of hypertrophic cardiomiopathy with myocardial crypts, noncompaction of the left ventricle and myocardial bridge in a girl from a family with sudden cardiac deaths

Doros G. (1), Popoiu A.(1), Russu G.(2), Olariu C.(1), Ardelean A. (1), Gafencu M.(1), Miclaus G.(3)

University of Medicine and Pharmacy "Victor Babes", IIIrd Pediatric

Clinic, Timisoara, Romania (1); University of Medicine and Pharmacy

"Grigore T Popa", Cardiology Pediatric Clinic, Iasi, Romania (2);

Neuromed Clinic Timisoara, Romania (3)

Objective: To report a particular case of a 14 yo girl admitted in our clinic after her father's sudden cardiac death at the age of 53, a few days after he was diagnosed with hypertrophic cardiomiopathy. In the father's family there were several sudden cardiac deaths (SCD) at a very young age. The patient's cousin was recently diagnosed with hypertrophic cardiomiopathy.

Material and methods: The young girl performed clinical investigations: cardiac examination, ECG, Echocardiography, Holter ECG, angio CT and angio MRI, laboratory tests and genetic examination.

Results: The patient was asymptomatic, with grade II cardiac murmur, normal blood pressure, femoral pulse present. Impressive deep Q waves in limb leads and left ventricular hypertrophy was registered on ECG. Echocardiography revealed concentric hypertrophic cardiomiopathy with deep myocardial crypts in the interventricular septum, well seen after contrast examination, but also noncompaction of the left ventricle. Angio MRI confirmed the lesions, well describing the noncompaction and the crypts and angio CT discovered a tiny myocardial bridge on anterior descending coronary artery at this moment with no obstruction. The familial pedigree demonstrated sudden cardiac deaths in a very young age in father's sister, brother, father and hypertrophic cardiomiopathy diagnosed in father's sister son. Despite that on the girl's Holter ECG there were no PVC, the decision after guidelines was to implant this girl with an ICD (implantable cardioverter defibrillator), to prevent SCD.

Conclusions: Hypertrophic familial cardiomiopathy represent a risk factor for SCD. Myocardial crypts are new features of the disease, described in the literature, but with unknown importance at this moment. Noncompaction cardiomiopathy may induce sudden cardiac deaths due to the complications. Tiny myocardial bridge is irrelevant now. Hypertrophic familial cardiomiopathy with myocardial crypts, associated with noncompaction of the left ventricle and myocardial bridge are very rare associated and till now they were not reported in the literature in this association and when familial SCD exist in the family, increase the risk of SCD of the patient. ICD is the only option to prevent sudden death. Genetic tests were performed and the girl was implanted with a cardiacdefibrillator.

\section{P-199}

Prognosis after cardiac events due to hypertrophic cardiomyopathy under school supervision in AED era Abe Y., Ayusawa M., Kato M., Komori A., Nakamura T. Department of Pediatrics and Child Health, Niohn University, Tokyo, Japan

Background: The most common cause of sudden death under school management is cardiac accident in japan. Among them, the ratio of cardiomyopathy in recent years is somewhat increasing trend, and most cardiomyopathy is hypertrophic cardiomyopathy (HCM). The aim of this study is to examine the recent occurrence of cardiac accident due to HCM and effect of automated external defibrillator (AED) under school management.

Methods: We retrospectively studied cases with cardiac accident under school management between 2004 and 2011.

Results: The total number of cardiac accident in this study period was 218 cases. Among them, 29 cases had suddenly died for HCM. 15 cases had been successfully survived resuscitation. Survivor did not exist until 2006. All survival cases except a case implanted implantable cardioverter defibrillator (ICD) were used AED. Both death cases and survival cases ware often in boys than girls. Although death cases observed not only junior high and high school students but also kindergarten students and elementary school students, survival cases were only junior high and high school students. Percentage of cases who had been diagnosed was $48 \%$ (death cases) and 20\% (survival cases). Occurrence of cardiac accidents during exercise was most in both death cases and survival cases.

Conclusion: Because the general public has been able to use AED, cardiac arrest cases due to HCM under school management could be survival since 2007 in Japan. AED and ICD were important to prevent cardiac sudden death.

\section{P-200}

Internationalized normal ratio reflects not only warfarin dose but also liver function in Fontan patients

Hamamichi Y., Horimot Y., Takeguchi M., Mastui T., Saito M., Ishii T., Inage A., Ueda T., Yazaki S., Yoshikawa T.

Sakakibara Heart Institut, Tokyo, Japan

Background: Dosage amount of warfarin (Wf) is adjusted by internationalized normal ratio (INR) of prothrombin time in patients with bi-ventricle. Values of INR reflect not only drop of prothrombin time but also deterioration of other coagulation factors. Fontan patients are easy to suffer liver disturbance, which influences production of coagulation factors. We predicted high INR was influenced by liver disorder as well as warfarin dose in Fontan patients.

Methods: The medical records of 250 Fontan patients were reviewed who were administered Wf. They underwent cardiac catheterization and blood tests between 2010 and 2015. First, we divided 250 patients into two group: 22 patients with INR2.0 or over (INR2.0-up), and 228 patients without INR2.0-up. 
We employed clinical data as assay variable, and INR2.0-up as state variable. We examined whether the area under a receiving operating characteristics curve (AUROC) was calculated to determine the best discriminating each assay variable for predicting INR2.0-up. Second, we performed same analysis for INR1.5-up patients.

Results: There were significant differences in cardiac performances and hepatic indexes between Fontan patients with INR2.0-up and without INR2.0-up: end-diastolic ventricular pressure (10.5 vs. $8.0 \mathrm{mmHg}$ ), albumin ( 4.2 vs. $4.5 \mathrm{~g} / \mathrm{dl}$ ), total billirubin (1.1 vs. $0.9 \mathrm{mg} / \mathrm{dl}$ ), activated partial thromboplastin time (42 vs. $37 \mathrm{sec}$ ), and platelet counts $(17.2$ vs. $23.5 \times 104 / \mu \mathrm{l})$. However, doses of Wf were not different between two groups. We gained significant AUROC for predicting INR2.0-up by platelet counts $(0.284$, $95 \%$ C.I. $=0.163-0.405)$, by albumin $(0.339,95 \%$ C.I. $=0.219$ $0.458)$, and by activated partial thromboplastin time (0.692, 95\% C.I. $=0.541-0.843)$. Similarly, we gained significant AUROC for predicting INR1.5-up by doses of Wf $(0.598$, 95\% C.I. $0.525-$ $0.672)$, by platelet counts $(0.411,95 \%$ C.I.0.337-0.485), and by activated partial thromboplastin time $(0.714$, 95\% C.I. 0.620$0.809)$. Study ages were significantly higher both in INR2.0-up group (17.9 vs. $10.6 \mathrm{yrs}$ ) and in INR1.5-up group (14.3 vs. $9.7 \mathrm{yrs})$

Conclusion: Dose of Wf was associated with value of INR in Fontan patients with INR1.5-up. In INR2.0-up patients, however, INR value was not related to Wf dose but hepatic disorder or diminished production of coagulation factors. We should carefully interpret high INR values in Fontan patients.

\section{P-201}

\section{High post-capillary pressure reduces the efficacy of} pulmonary vasodilator in Fontan patients

Hamamichi Y., Mastui T., Takeguchi M., Horimot Y., Saito M., Ishii T., Inage A., Ueda T., Yazaki S., Yoshikawa T.

Sakakibara Heart Institut, Tokyo, Japan

Background: Efficacy of pulmonary vasodilator is limited in Fontan circulation. We predicted that elevation of post-capillary pressure, namely increased pressure of pulmonary-capillary wedge (PCWP) or increased end-diastolic pressure of ventricle (EDP), subsists mostly in Fontan patients with high central venous-pressure (HCVP).

Methods: The medical records of 139 Fontan patients were reviewed. They underwent cardiac catheterizations and blood tests between 2010 and 2015. First, we investigated clinical characters in patients with pulmonary vasodilator $(n=55)$. Second, we divided the whole into patients with CVP $16 \mathrm{mmHg}$ or over (HCVP: $n=21)$ and those without HCVP $(n=118)$. Related factors to HCVP were set up by square test. Third, we compared retention rate of these factors between vasodilator patients with $\operatorname{HCVP}(n=9)$ and without HCVP $(n=46)$.

Results: Fontan patients with Vasodilator possessed following medical histories more than those without Vasodilator: coil embolizations $(80 \%$ vs. $61 \%, \mathrm{p}=0.024)$, percutaneous transluminal pulmonary angioplasty $(50 \%$ vs. $27 \%, \mathrm{p}=0.0048)$, and repair of atrio-ventricular valve ( $30 \%$ vs. $14 \%, \mathrm{p}=0.018)$. They had small pulmonary artery index $(\mathcal{E} 180 \mathrm{~mm} 2 / \mathrm{m} 2)$ more $(41 \%$ vs. $16 \%$, $\mathrm{p}=0.0010)$. In monovariate analysis HCVP in all 139 patients was associated with 5 factors: PCWP $\geq 11 \mathrm{mmHg}(52 \%$ vs. $2 \%$, $\mathrm{p}<0.00001), \mathrm{EDP} \geq 12 \mathrm{mmHg}(42 \%$ vs. $10 \%, \mathrm{p}=0.00042)$, pulmonary artery index $\geq 370 \mathrm{~mm} 2 / \mathrm{m} 2(19 \%$ vs. $3 \%, \mathrm{p}=0.019)$, ventricular volume on end-diastole $\geq 117 \% \quad(57 \%$ vs. $29 \%$, $\mathrm{p}=0.027)$, ventricular volume on end-systole $>46 \%(71 \%$ vs. $44 \%, p=0.037)$. In Vasodilator group, patients with HCVP owned either of PCWP $\geq 11 \mathrm{mmHg}$ or EDP $\geq 12 \mathrm{mmHg}$ (high post-capillary pressure) much more than those without HCVP ( $77 \%$ vs. $10 \%$ : p < 0.0001). However, the ratio of patients with extended ventricular volume (On end-diastole $\geq 117 \%$ or On end-systole $>46 \%$ ) was not significantly different between vasodilator patients with HCVP and without HCVP (77\% vs. $54 \%$ ). Similarly, the ratio of Vasodilator patients with large pulmonary artery index was not different.

Conclusion: Most of Fontan patients with Vasodilator who had still HCVP possessed increased PCWP or increased EDP. Subsisting of high post-capillary pressure might be one of the reasons why vasodilator does not respond to high CVP so much in Fontan patients.

\section{P-202}

Fontan children with enarapril have decline of estimated glomerular filtration-rate owing to low blood pressure or combination use of drugs

Hamamichi Y., Takeguchi M., Horimoto Y., Mastui T., Saito M., Ishii T., Inage A., Ueda T., Yazaki S., Yoshikawa T.

Sakakibara Heart Institut, Tokyo, Japan

Background: In childhood criterion values of creatinine $(\mathrm{Cr})$ are very different according to ages, which makes difficult for us to evaluate children's renal function by raw $\mathrm{Cr}$ values. Fontan patients are often administered angiotensin converting-enzyme inhibitor (ACEI), who sometimes show reduced renal functions. Japan society of pediatric renal failure provides formula of estimated glomerular filtration-rate (eGFR). We assessed reduction of kidney functions by eGFR in Fontan children with ACEI and sought backgrounds of reduced eGFR.

Methods: The medical records of 139 Fontan children with or without ACEI were reviewed aged from 2 to 18 years. They underwent cardiac catheterization and routine blood tests between 2010 and 2015. We calculated eGFR by pediatric formula which was composed of quintic equation for body height. Patients with eGFR $80 \mathrm{~mL} / \mathrm{min} / \mathrm{m} 2$ under $(\mathrm{n}=27)$ were defined as decreased eGFR. We compared clinical characteristics between patients with decreased eGFR and non-decreased eGFR.

Results: There were significant differences in cardiac performances between Fontan patients with decreased eGFR and without decreased eGFR, such as ventricular volume on end-diastole and end-systole, and ventricular pressure on end-systole. However, significant area under a receiving operating characteristics curve for predicting decreased eGFR was gained only by end-systolic ventricular pressure $(0.328,95 \% \mathrm{CI}=0.212-0.443 ; \mathrm{p}=0.005)$. Contrary to expectance, following performances were not different between two groups; ventricular pressure on end-diastole, ventricular ejection fraction, central venous pressure, and cardiac output. Clinical backgrounds were also not different between two groups. As for medicine, the rate of decreased eGFR was significantly higher in ACEI group with high dose, in ACEI group with angiotensin receptor blocker, and in ACEI group with spironolactone. After multivariate analysis, decreased eGFR was independently associated with combined usage of ACEI or angiotensin receptor blocker (odds ratio 6.4, $\mathrm{p}=0.009$ ), and low ventricular pressure on end-systole $(\leq 71 \mathrm{mmHg}$ : odds ratio 3.6 , $\mathrm{p}=0.018)$.

Conclusion: We could estimate renal function in Fontan children by eGFR formula for Japanese. Fontan children with ACEI should take care of reduced renal function, if they took angiotensin receptor blocker or spironolactone. We also should mind low blood pressure in Fontan patients with ACEI to check up kidney function. 


\section{P-203}

High-dose administration of beta-adrenergic blocking agent does not cause reverse modeling for systemic ventricle in Fontan children

Hamamichi Y., Takeguchi M., Horimoto Y., Mastui T., Saito M., Ishii T., Inage A., Ueda T., Yazaki S., Yoshikawa T.

Sakakibara Heart Institut, Tokyo, Japan

Background: Concerning heart failure therapy against bi-ventricle beta-adrenergic blocking agent (beta-blocker) produces reverse modeling, such as a dose-related decrease in systemic ventricular size and a dose-related increase in ejection fraction. In betablocker therapy we should raise amount of drugs carefully. There are no reports about high-dose administration of beta-blocker to systemic ventricle in Fontan children. We estimated cardiac function in Fontan children who were administered high-dose beta-blocker.

Methods: The medical records of 176 Fontan children were reviewed aged from 2 to 14 years. They underwent cardiac catheterization between 2010 and 2016. We divided the whole into 4 groups: 25 with beta-blocker $0.6 \mathrm{mg} / \mathrm{kg}$ or over (High betablocker), 39 with beta-blocker between 0.30 and $0.59 \mathrm{mg} / \mathrm{kg}$ (Middle beta-blocker), 29 with beta-blocker $0.29 \mathrm{mg} / \mathrm{kg}$ or under (Low beta-blocker), and 83 without beta-blocker (No betablocker). We compared cardiac performances among 4 groups.

Results: End-diastolic ventricular volume was significantly larger in High beta-blocker than that in each other group, such as Middle beta-blocker, Low beta-blocker, and No beta-blocker $(174 \%$, $117 \%, 111 \%, 100 \%)$. However, it was dot significantly different among these 3 groups other than High beta-blocker. Similarly, end-systolic volume was significantly larger in High beta-blocker than in each other group $(86 \%, 55 \%, 43 \%, 45 \%)$, which was not significantly different among 3 groups. Ejection fraction of ventricle was also lower in High beta-blocker $(47 \%, 55 \%, 57 \%, 55 \%)$, which was not different among 3 groups; value of NT-pro BNP $(\mathrm{pg} / \mathrm{ml})$ was significantly higher in a like manner $(1711,280,293$, 155). The rate of patients was not significantly different among 3 beta-blocker groups (High, Middle, Low) who underwent catheterization for the purpose of other than periodic examination, such as suspicion of cardiac dysfunction and catheter intervention ( $48 \%, 46 \%, 44 \%, 15 \%)$.

Conclusion: For these Fontan children we could not obtain the results that high-dose beta-blocker provoked reverse modeling, such as reducing ventricular volume and improving ventricular ejection fraction. We gained the results that cardiac functions in High beta-blocker were inferior to those in other lower betablocker or no beta-blocker groups. We should support Fontan children with high-dose beta-blocker carefully to make sure whether cardiac functions improve.

\section{PN-1}

\section{Transition program in pediatric cardiology}

Geiser Mages V., Gendre C., Sekarski N., Di Bernardo S., Mivelaz Y., Boulos Ksontini T., Prsa M.

University Hospital, Lausanne, Switzerland

Introduction: Thanks to advances in diagnosis, medical treatments and surgery, children born with congenital heart disease (CHD) have a good chance to reach adulthood with an excellent quality of life. Several studies have shown the importance of a transition program for adolescents with a chronic disease. Our center covers an area of 2 Mio inhabitants and all CHD patients are followed at our clinic. Objective: Creation of a transition program targeting the specific needs of adolescents with CHD in our outpatient clinic.
Method: Patients with CHD from our clinic between 14 and 18 years old and their parents received each a questionnaire targeting their educational needs and the actual satisfaction of them.

At the same time we began a program of thematic workshops for these patients and organized two sessions on the theme "A Healthy Heart".

Results: We sent 187 questionnaires to adolescents with CHD aged14 to 18 years and their parents (374 questionnaires in total). We received 136 answers (61 adolescents and 75 parents).

The main topics where adolescents had insufficient knowledge were: signs of gravity $(50.8 \%)$, physical restrictions (46.1\%), adulthood follow-up (71.7\%), future risks (45.9\%). According to their parents they lack information about their CHD (39.2\%), the effect of alcohol and tobacco $(61.2 \%)$, contraception and pregnancy (67.4\%) and adulthood follow-up (72.3\%).

$77 \%$ of the adolescents and $90.7 \%$ of their parents found important that the nurses in pediatric cardiology see the patients to give them additional explanations and answer their questions.

The two sessions of thematic workshops on the theme "A Healthy Heart" were followed by 11 adolescents. Nine of them reported to have learned something new and were ready to participate in further workshops.

Conclusion: The answers to the questionnaires show the need for a personalized educational program for adolescents with CHD including their present condition and their future.

The thematic workshops answer to some needs of the adolescents and will be extended and completed with the following themes: "My Cardiopathy", "Daily life with a Congenital Heart Disease" and "My future".

\section{PN-2}

Transition Care Models in adolescents with Congenital Heart Disease: an overview of literature reviews

Flocco S.F.(1), Magon A.(1), Dellafiore F.(1), Pittella F.(2), Chessa M. (3), Caruso R.(1)

Health Professions Research and Development Unit, IRCCS Policlinico San Donato, Italy (1); Nursing Degree Course, University of Milan, section of IRCCS Policlinico San Donato, Italy (2); Pediatric and Adult Congenital Heart Disease Center, IRCCS Policlinico San Donato University Hospital, Italy (3)

Introduction: The number of adolescents with Congenital Heart Disease (CHD) continues to grow, considering the improving of surgical and medical techniques. Indeed, CHD are the most common congenital anomaly with a worldwide prevalence of 9 per 1000 live births. CHD represents a new challenge for the health care systems, also considering that these patients are relatively a new population with specific peculiarities, such as the need of a specific care planning and a life-time clinical follow-up. The literature shows how transition care models from adolescence to adulthood are strategic to ensure the continuity of the care, and to improve adolescents' knowledge and self-managing skills, even considering the psychological issues related to their condition. However, the literature shows how the number of follow-up is reduces with the increasing of the patients' age, and the literature appears diverse considering the growing of papers related to this topic in the last ten years. Hence, the aim of this overview of systematic reviews is to summarize and critically assess the main results of the included papers to define the state of art of the transition care models, highlighting the current limits.

Methods: The authors performed this overview searching on PubMed, CINAHL, Web of Science, Cochrane, and using keywords, queries, inclusion criteria and critical appraisal to assess the eligible papers. The 'Preferred Reporting Items for Systematic 
Reviews and Meta-Analyses' (PRISMA) flow-diagram guided the inclusion process, and a narrative analysis of papers' content was performed to analyze the included papers.

Results: The included papers were five. Our findings show that there are not available (a) specific and standardized paths of transition care models, (b) effectiveness studies, and (c) the competencies' required for the transition multidisciplinary team are not well defined.

Conclusion: In conclusion, our findings problematize the current state of art of transition care models for adolescents with CHD, highlighting the current limits related to research and practice. More methodological and empirical research is needed to best implement an evidencebased approach for the transition period of these patients.

\section{PN-3}

How could the clinicians assess the CHD adolescents' and their parents' needs? A preliminary theoretical basis underpinning a tool development

Flocco S.F.(1), Magon A. (1), Dellafiore F. (1), Pittella F. (2), Chessa M. (3), Caruso R (1)

Research and Development Unit, IRCCS Policlinico San Donato, Milan, Italy (1); Nursing degree Course, IRCCS Policlinico San Donato, Milan, Italy (2); Pediatric and Adult Congenital Heart Disease Center, IRCCS Policlinico San Donato, Milan, Italy (3)

Introduction: Literature currently shows a growing interest in understanding the peculiarities of both the patients with congenital heart disease (CHD) and their parents, whom are living the transition period from childhood to the adulthood, known as adolescents. However, considering the parents' crucial role and some risky behaviors related to their duties (e.g., hyper- protection, fear), there are not yet available tools to specifically assess the needs of both the adolescent patients and their parents. Therefore, it could be strategic the identification of a clear theoretical framework that could guide a CHD-specific tool development to assess their needs. Thus, the aim of this study is to identify a theoretical framework, which will guide a future tool development. Methods: The theoretical framework will be defined by a literature review (phase I) and a phenomenological study (phase II). The phase I was performed by a search on PubMed, Scopus, CINHAL and Web of Science, with the sub-aim to identify papers related to the lived experience of adolescents with CHD and their parents. The findings of the phase I represents the preliminary theoretical framework. The phase II (phenomenological study) will be conducted to validate the preliminary theoretical framework (phase I findings), customizing the same phase I findings to shape the semistructured interviews for patients and parents, and using a purposive sampling.

Results: Seven papers were included (phase I), coming from a selection and assessment of 405 potentially relevant studies. These papers were analyzed, discussed and a qualitative meta-synthesis was performed. The meta-synthesis findings were 4 main domains, exploring 4 main contradictions that characterize the CHD adolescents' parents experiences: 'fear and uncertainty of the future versus positive coping strategies'; 'parents hyper-responsibility and overprotection versus adolescents' independence desire'; 'desire to give support, but not to be supported'; 'normality desire versus awareness to live with particular conditions'. Those domains represents the preliminary theoretical framework to test with phase II (not yet implemented).

Conclusions: Our strategy to develop a CHD-specific scale for adolescents patients with CHD and their parents should help the delivery of a tailored care, where the needs assessment is helped by the use of a disease-specific tool.
PN-4

Intensive Care Nursing - Analysis of Current Practice and Implications for an ECMO Training Program

Kröger Y., Stoffel G.

University Children's Hospital Zurich, Zurich, Switzerland

Introduction: Extracorporeal Membrane Oxygenation (ECMO) is a mechanical circulatory support system used in treating patients in respiratory or cardiac failure. ECMO has been provided in the Intensive Care Unit (ICU) of the Children's Heart Center (CHC) since 2004. Ten nurses ensure 24-hour care for 6-7 patients treated per year.

Numbers of patients requiring ECMO have increased 3-fold. Therefore, all ICU nurses with at least $1 / 2$ year's experience are trained in ECMO.

Method: The CHC ECMO-training program was evaluated using an IST-Analysis. Two questionnaires were developed and available online (Survey Monkey). Questionnaire-I, for newer nurses, focused on a theoretical and practical introduction to ECMO (Group 1). Questionnaire-II was for nurses experienced with ECMO, and focused on practical training (Group 2).

Results: 10 from 12 (83\%) nurses in Group 1 answered Questionnaire-I (mean age: 33.9 years; mean years of ICU experience: 5.5). The theoretical content of the ECMO training course was rated as good, but the practical aspect such as ,trouble shooting' or case studies, was rated as insufficient. Nurses working part-time said that they initially needed more practical training.

All 12 nurses from Group 2 answered Questionnaire-II (mean age: 41.25 years; mean years of ICU experience: 12.75 ; mean years of ECMO experience: 5.2). These nurses had positive attitudes toward the practical training and assessed the differing levels of professional experience and capabilities of the co-workers (SkillMix) as helpful in reducing the burden. Staff-planning to provide a balance in experienced vs less-experienced nurses was important. Conclusion: It is recommended that all ICU nurses are trained in caring for patients on ECMO. Staff-planning should allow time for practical training and consider the needs of the part-time nurses.

Refresher courses need to be periodically offered. Troubleshooting and hands-on sessions should be integrated in the program. Online tools such as Easy Learning allow individuals to test their knowledge.

A balanced skill-mix within the nursing teams has been achieved through the specialized training of all ICU nurses. By improving nurses' abilities in working with ECMO, the burden for the ECMO-experienced nurses is lessened, and patient safety and the quality of nursing care are optimized.

\section{PN-5}

Like being in another world Parents' experiences of their child being admitted to a pediatric intensive care unit: a qualitative study

Dahav P., Sjöström-Strand A.

Department of Health Sciences, Lund, Sweden

Background: There is very little research on the parent's experiences of having a child admitted to a pediatric intensive care unit (PICU). Identifying and describing "such experiences could facilitate better parental support from the PICU team and help the parents manage a stressful situation.

Aims: This study aims to describe parents' experiences of having their child admitted to a pediatric intensive care unit.

Methods: Interviews were conducted with 12 parents whose children had been admitted to a PICU. A qualitative design was used and the interviews were analyzed based on content analysis. 
Findings: The analysis revealed two categories: "being involved" and "being informed" with seven sub-categories: "caring for the parents", "security and trust", "altering the parental role" "stress and fear" ,"the importance of knowing", "interaction in the care process" and "being prepared"). An overarching theme emerged: the experience was that of "being in another world". The study concludes that a child's PICU admission is a stressful situation, and for the parents to be able to handle the anxiety and stress they needed to be informed of and involved in their child's care.

Conclusions: The parents' experience when their child is admitted to a PICU is fraught with a range of emotion and fear. There are indications that things such as good information, involvement and a positive experience of the transfer to the pediatric ward reduce the stress and anxiety associated with PICU admission. The result of this study could be used as basis for a post-PICU follow-up service for the children and their families.

\section{PN-6}

Implantation of ICDs in children and teenagers development of a nurse-led integrated care pathway Walsh H., Hudson M.E., Lord E., English J., Kaski J.P., Mangat J., Field E.

Great Ormond Street Hospital, London

Introduction: Implantation of cardioverter defibrillators (ICDs) may be indicated in children with an underlying heart rhythm disorder or cardiomyopathy. ICDs may also be implanted for the secondary prevention of ventricular arrhythmias in children who have presented with an out of hospital cardiac arrest.

Our centre has seen increasing numbers of patients requiring ICD implantation in recent years. The complex needs of this patient group and the need to minimise the length of admissions while simultaneously optimising quality of care has resulted in development of an integrated care pathway and a new specialist nurse role to support and educate families and to coordinate these admissions.

Objective: To improve the care of children and families undergoing ICD implantation in our centre by developing a new nurse-led care pathway. The aim is to standardise patient care using safe, evidence-based practice, to minimise delayed discharges and to improve the preparation of children and families prior to surgery and discharge.

Method: Inpatient admissions for ICD implantation at our centre were monitored over a twelve-month period. Any complications or delays were noted and the causes analysed. In order to develop a comprehensive care pathway, information and advice was sought from cardiologists, electrophysiologists, clinical psychologists, theatre staff, ward nurses and other relevant specialists.

Results: Seventeen children required ICD implantation during this period, with four devices implanted as secondary prevention following a cardiac arrest and thirteen implanted electively for primary prevention. Inconsistencies and delays identified during these admissions included those relating to education, psychology input, pain relief, wound dressings and rehabilitation. By drawing on these experiences and seeking input from relevant specialists, an integrated care pathway was constructed which included a comprehensive plan for the education and psychological preparation of children and families.

Conclusions: The process of ICD implantation is a challenging time for children and their families. An integrated care pathway enables a consistent, safe and evidence-based approach. Specialist nurses are ideally placed to lead and coordinate the holistic care required by these patients and the development of a role specifically dedicated to ICD patients is warranted in centres carrying out significant numbers of implantations.
PN-7

Computer assisted Learning to understand hemodynamics in congenital heart defect

Hjorth-Johansen Elin., Kynø N.M

Oslo University Hospital, Norway (1); Lovisenberg Diakonale

Hoegskole (2)

Background: Computer assisted learning has shown to be an approved and cost effective strategy to improve knowledge and skills in various nursing objectives. So far, no studies have published results regarding e-learning as a tool to understand congenital heart defect and well-judged nursing decisions.

We developed an e-learning course with six modules regarding; normal circulation, transition from fetal circulation, hemodynamics in congenital heart defect and nursing actions. We tested whether students and nurses could learn and understand the theory of hemodynamics and nursing, related to congenital heart defect, when taught using computer-assisted learning and compared with conventional teaching in a classroom.

Method: A group of 30 nurses and neonatal nurse specialist students was random distributed into two groups. The intervention group used the interactive, self-directed computer-assisted learning modules. An experienced lecturer in an auditorium taught the control group. Both groups received the same content and illustrations within the framework of the modules. All participants answered a knowledge test in advance of their lecture. Straight after the lecture, all participants answered a questionnaire about their perception of the learning method they participated in. After one week a second knowledge test was completed.

Results: Preliminary results shows that knowledge scores increased significantly from baseline in both groups and no significant differences were detected between the scores of the two groups. There was a significant difference in former experience with caring for infants with congenital heart disease and this reflected slightly higher knowledge in the e-learning group in both pre and posttests. The e-learning group used significantly less time to study than the lecture group. The participants in the lecture group perceived the lecture method to be significant more positive than the e-learning group.

Conclusion: The six modules of computer-assisted learning was an effective strategy for teaching both theory of hemodynamics and nursing to infants with congenital heart defects. Further analyzes may reveal complementary results of interests but are not yet completed.

YIA-1

3D volume analysis by transesophagial echocardiography of right and left ventricle just after transcatheter device closure of atrial septal defect

Saito M.(1), Mahara K.(2), Terada M.(2), Ishii T(1), Hamamichi Y. (1), Inage A.(1), Ueda T.(1), Yazaki S.(1), Yoshikawa.T(1) Sakakibara Heart Institute, Tokyo, Japan, Pediatric Cardiology(1); Cardiology(2)

Introduction: Little is known about quantitative change of ventricles just after transacatheter device closure of ASD. The objective of this study was to clarify the effects of ASD closure on ventricular functions and volume by three-dimentional transeshophageal echocardiography (3DTEE).

Methods: We investigated consecutive 29 patients who underwent ASD device closure in our institute guided by TEE from January to October 2016. Eight patients were excluded due to their moderate and severe tricuspid valve regurgitation or their poor image quality. Of the total, 18 patients (ranged 6 to 71 , median 27 
year-old) were enrolled in this study. 3D ventricular volume data were derived before and after closure during operation to assess right ventricular end-diastolic volume (RVEDV), right ventricular end-systolic volume (RVESV), left ventricular end-diastolic volume (LVEDV) and left ventricular end-systolic volume (LVESV). The pulmonary to systolic flow ratio (Qp/Qs) was calculated by using estimated systolic volume from the difference between EDV and ESV by 3D TEE: (RVEDV -RVESV) / (LVEDV -LVEDV), comparing with $\mathrm{Qp} / \mathrm{Qs}$ calculated by catheter.

Results: The Bland-Altman plots revealed Qp/Qs derived from 3D volume data (mean $\pm \mathrm{SD}$ : $1.72 \pm 0.41)$ showed good agreement with that from catheter (mean $\pm \mathrm{SD}$ : $1.87 \pm 0.43$ ) (mean difference \pm SD: $-0.16 \pm 0.15)$. While the mean EDV was changed dynamically from 110.6 to $98.6 \mathrm{ml}$ (RV), 60.2 to $70.2 \mathrm{ml}$ (LV), the mean ESV showed only slight changes: 57.7 to $57.0 \mathrm{ml}$ (RV), $29.4 \mathrm{ml}$ to $29.3 \mathrm{ml}(\mathrm{LV})$, respectively. Thus, the changes of EDV were likely to correlate with the decrease of Qp/Qs.

All of the Qp/Qs derived from 3D TEE after ASD closure showed around 1.0 (mean $\pm \mathrm{SD}: 1.04 \pm 0.07$ ), which reflected the ventricle-adjusts after shunt closure.

Conclusions: Volume data by 3D TEE can assess the effects of preload changes on bilateral ventricles correctly.

\section{YIA-2}

Long-term outcome following percutaneous closure of isolated secundum atrial septal defects in children: a french nationwide series of $\mathbf{1 0 0 0}$ consecutive patients

Jalal Z. (1), Mauri L. (2), Dauphin C. (3), Gronier C. (4), Hascoet S. (2), Lefort B. (5), Lachaud M. (6), Petit J. (3), Ovaert C. (7), Fraisse A. (8), Pillois X. (1), PHD, Thambo JB. (1), Baruteau A-E. (8) University Hospital of Bordeaux, Bordeaux, France (1), Centre Chirurgical MarieLannelongue, Le Plessis Robinson, France (2), University Hospital of Clermont Ferrand, Clermont Ferrand, France (3), University Hospital of Strasbourg, Strasbourg, France (4), University Hospital of Tours, Tours, France (5), Institut du Thorax, Nantes, France (6), Hôpital la Timone, Marseille, France (7), Division of Cardiology, Department of Pediatrics, Royal Brompton Hospital, London, UK (8)

Introduction: Transcatheter closure has become the preferred treatment strategy in most cases of isolated, secundum atrial septal defect (ASD). Although widely used, data on long-term outcomes in the pediatric population are scarce. We aimed to assess procedural characteristics, early clinical outcome, long-term devicerelated complications and the electrical remodeling after transcatheter closure of isolated ASD in children.

Methods: A 1998-2014 retrospective multicentre study was performed at 8 French tertiary institutions, including all patients $<18$ yo who attempted a percutaneous ASD closure with an Amplatzer Septal Occluder.

Results: 1000 children [38\% males, median age: 9 yrs (0.7-18.0), median weight: $27 \mathrm{~kg}(6-92)]$ were referred for transcatheter ASD closure. They all had a significant left-to-right shunting assessed by right ventricular dilation and/or a 1.5:1 Qp/Qs ratio; Median ASD size was $15 \mathrm{~mm}$ in transthoracic echography (TTE).

ASD closure was guided by fluoroscopy and transoesophageal echocardiography in 627 cases $(62.7 \%)$ or TTE in 373 cases (37.3\%). Procedural success rate was $94 \%$ with a median occluder size of $19-\mathrm{mm}(4-40)$.

Device placement was unsuccessful in 60 patients $(6 \%)$ due to unfavourable anatomy in 38, early device embolization in 12 and other causes in 10 patients.

Follow-up (FU) data were available for 829 patients. After a mean FU of $53 \pm 31$ months (range, 5-204), all patients were alive and $96 \%$ were asymptomatic. Long-term complications included supraventricular arrhythmias $(n=6)$ and pulmonary hypertension $(n=2)$. No cardiac erosion, late ASO dislodgement or stroke occurred.

Seventy-one women had pregnancy during FU without any associated complication. From an electrical standpoint, supraventricular arrhythmias occurred in 6 patients, no late atrioventricular block was observed and there was no significant difference between preprocedural and last follow-up ECG.

Conclusions: Our large-scale pediatric cohort confirms that transcatheter closure of isolated secundum ASDs is a safe procedure in children, with a favourable long-term outcome and no lifethreatening delayed complication.

\section{YIA-3}

Clinical Evaluation of Regional Myocardial Viability and Segmental Strain in Adults with Repaired Tetralogy of Fallot

Kitagawa A. (1), Kimura S. (1), Ando H. (1), Sugimoto K. (2), Miyaji K. (2), Ishii M. (1)

Department of Pediatrics, Kitasato University School of Medicine, Kanagawa, Japan (1); Department of Cardiovascular Surgery, Kitasato University School of Medicine, Kanagawa, Japan (2)

Background: Myocardial fibrosis detected by late gadolinium enhancement (LGE) using cardiovascular magnetic resonance imaging (CMR) was often present in adults with repaired tetralogy of Fallot (TOF). The purpose of this study was to evaluate regional myocardial viability and segmental strain in repaired TOF patients. Method: Thirty-one repaired TOF patients $(20$ males, 11 females, $17.0 \pm 6.3$ years of age) were examined. Ventricular volumes were measured by cine MRI, and blood flow were quantified by phasecontrast cine MRI. Myocardial viability was evaluated using LGE. Regional myocardial performances in left ventricle were measured by peak systolic radial and circumferential strains using tagged MRI. All patients examined blood samplings including plasma brain natriuretic peptide (BNP).

Results: LGE lesions were detected in 11 patients. All LGE lesions were in medial inferior septal wall. Patients with LGE were significantly older than without $(20.3 \pm 9.3$ vs. $15.1 \pm 2.8$ years of age, $p=0.006)$. Patients with LGE had lower left ventricular peak radial strain at inferior septal wall than without $(1.3 \pm 0.7$ vs. $3.5 \pm 2.6 \%, \mathrm{P}=0.026)$. Patients with LGE had significantly higher plasma BNP levels than without $(35.8 \pm 23.2$ vs. $19.6 \pm 14.9 \mathrm{pg} / \mathrm{mL}, \mathrm{P}=0.012)$. Left and right ventricular volumes, ejection fraction, pulmonary regurgitant fraction and left ventricular peak circumferential strain, there were no significant differences between patients with LGE and without.

Conclusions: LGE lesions detected in adult patients with repaired TOF could be associated with lower peak radial strain and higher plasma BNP level. There would be good relationships between regional myocardial viability and segmental strain.

\section{YIA-4}

Children after Fontan surgery have liver hypoperfusion Shiraga K. (1,2), Ono K. (2), Okubo T. (2), Nagamine H. (2), Shirayama A. (2), Nakajima H. (2)

The University of Tokyo Hospital, Tokyo, Japan (1); Chiba Children's Hopsital, Chiba, Japan (2)

Introduction: Liver cirrhosis is one of the critical complications in patients after Fontan surgery. It is well accepted that liver cirrhosis is associated with reduced liver perfusion. Previous reports showed that MRI-based Intravoxel Incoherent Motion-diffusion imaging (IVIM) which evaluates the change in liver parenchymal perfusion 
can monitor disease progression of liver fibrosis and liver cirrhosis. IVIM parameters ( $D^{\star}$ and $f$ values) in the Liver were reported to correlate with the blood perfusion.

Hypothesis: We hypothesized that children after Fontan surgery have liver hypoperfusion.

Materials and Methods: Five consecutive patients after Fontan surgery who visited our cardiology clinic and 4 age-matched controls were enrolled in this study. IVIM parameters ( $D^{\star}, D$ and $f$ values) were compared between the Fontan and normal children. We calculated the cardiac output from the IVC and SVC flow volume using phase contrast method. MRI scans were performed with a 1.5-T MR imaging system (Signa HDxt, General Electric, U.S.A). IVIM parameters were calculated using a non-linear least square fit to the bi-exponential model curve, and four circular ROIs were placed manually within the right hepatic lobe.

Results: Age at MRI scan was $12.8 \pm 1.5$ years old. Body weight was $40.0 \pm 11.1 \mathrm{~kg}$. Age at Fontan surgery was $2.6 \pm 0.5$ years old and post-operative time was $10.5 \pm 1.5$ years, and cardiac output was $2.61 \pm 0.23 \mathrm{~L} / \mathrm{min} / \mathrm{m} 2$. $\mathrm{D}^{\star}$ and $\mathrm{f}$ values of IVIM in children after Fontan surgery were significantly lower than controls (see Table). There was no significant difference in D value (see Table). Limitations: The number of patients is small. This trial is the nonrandomized controlled one.

Conclusions: Liver hypoperfusion exists in children after Fontan surgery. IVIM imaging may be a noninvasive and quantitative technique for monitoring disease progression of liver fibrosis and liver cirrhosis in patients after Fontan surgery.

\begin{tabular}{llcr}
\hline & Fontan & Control & $\boldsymbol{p}$ \\
\hline $\mathrm{D}^{\star}\left(10^{-3} \mathrm{~mm}^{2} / \mathrm{s}\right)$ & $30.8 \pm 24.8$ & $113.2 \pm 25.6$ & $\underline{0.002}$ \\
$\mathrm{D}\left(10^{-3} \mathrm{~mm}^{2} / \mathrm{s}\right)$ & $1.07 \pm 0.04$ & $1.25 \pm 0.16$ & 0.11 \\
$\mathrm{f}(\%)$ & $13.2 \pm 3.1$ & $22.4 \pm 2.4$ & $\underline{0.001}$ \\
\hline
\end{tabular}

\section{YIA-5}

Ascending aortic haemodynamic flow changes are stable in bicuspid aortic valve disease

Bissell M.M., Hess A.T., Loudon M., Neubauer S., Myerson S.G. University of Oxford, Oxford, United Kingdom

Introduction: Aortic dilation in bicuspid aortic valve disease is thought to be at least in part caused by helical flow changes in the ascending aorta. We sought to examine whether these haemodynamic changes develop over time.

Methods: All participants from our initial cohort were invited to undergo a 4D flow MRI assessment 3 years after the initial visit. The datasets were analysed using well described CMR parameters. Aortic valve function and ascending aortic diameters were assessed using 2D flow and anatomical MRI sequences.

Results: 64/100 participants returned for follow-up at a mean of 3.4 years $(2.8$ to 4.4$)$. Of the remaining 36 participants 16 had undergone aortic valve ( \pm ascending aortic) replacement (AVR \pm AA), 3 had undergone valve repair, 1 patient had died of endocarditis, 2 had MRI contraindications at follow-up, 12 declined follow-up and 2 were lost to follow-up. Age range at initial study visit was 8-69 years. Mean ascending aortic growth was $0.3 \mathrm{~mm}$ (range $0-1.7 \mathrm{~mm} /$ year). There was no difference in growth rate between the flow groups normal flow, right-handed helical flow and complex flow $(p=0.78)$. There was also no statistically significant progression of flow angle, flow displacement, rotational flow and wall shear stress. Interestingly, 4/6 (67\%) patients with a left-handed flow underwent AVR +-AA, compared to $19 \%$ in the right-handed flow group.
$15 / 64(23 \%)$ participants had an aortic growth rate $>0.5 \mathrm{~mm} /$ year. Even in this group progression of haemodynamic flow abnormalities was minimal: Peak velocity $2.4 \pm 0.6 \mathrm{~m} / \mathrm{s}$ to $2.4 \pm 0.6 \mathrm{~m} / \mathrm{s}$; flow angle $20 \pm 8$ degree to $20 \pm 10$ degree; normalised flow displacement $0.139 \pm 0.063$ to $0.144 \pm 0.51$; wall shear stress $0.88 \pm 0.28$ to $0.96 \pm 0.40$.

Conclusion: This is the first large prospective longitudinal follow-up study examining haemodynamic flow changes using 4D flow MRI. We have shown that both ascending aortic growth and haemodynamic flow changes are largely stable over a 3 year follow-up period suggesting slow disease progression in the majority of patients. Previous work has shown left handed helical flow is not seen in health and a high proportion of these participants underwent aortic valve or ascending aortic surgery, suggesting that left handed helical flow may be a predictor of likelihood of intervention.

YIA-6

Risk stratification of sudden cardiac death in pediatric patient with accessory pathway: is there a difference between symptomatic and asymptomatic ventricular preexcitation?

Atallah V. (1), Lacotte J. (2), Salerno F. (2), Ait Said M.(2), Maltret A. (1,2) Centre de référence "Malformations cardiaques Congénitales ComplexesM3C ", Université Paris-V, Necker-Enfants Malades, AP-HP, Paris, FRANCE (1); Institut Cadiovasculaire Paris Sud, Hopital Jacques Cartier, Massy, FRANCE (2)

Introduction: Incidence of atrial fibrillation is known to increase at teen age. Its rapid conduction by an accessory pathway with a short anterograde refractory period can be dramatic. If symptomatic accessory pathway are usually followed and managed either by medication at younger age or by catheter ablation, many asymptomatic ventricular pre-excitation are under the radar and first symptom can be life-threatening. Hopefully, with the EKG generalization many accessory pathway are now diagnosed at teen age. The aim of this study was to determine the electrophysiological characteristics of young patients with ventricular pre-excitation to assess and compare their potential risk of sudden cardiac death among symptomatic and asymptomatic patients.

Methods: We retrospectively investigate data of every patients with ventricular pre-excitation with or without symptom who underwent endocardial electrophysiology testing. Procedure was done under general anesthesia for every patient under 12 years old. Accessory pathway anterograde effective refractory period was determined with an extrastimuli protocol at baseline and after isoproterenol infusion. Accessory pathway was said to be malignant if anterograde effective refractory period was equal or under $240 \mathrm{~ms}$ at baseline and $200 \mathrm{~ms}$ after Isoproterenol infusion or if the shorted pre excited RR interval was less than $250 \mathrm{~ms}$.

Results: From october 2009 to october 2016, 265 consecutive pediatric patients under the age of 18 had an electrophysiology testing for ventricular pre-excitation on baseline electrocardiogram. Mean age at procedure was 12.4 years old (range 5 weeks to 18 years). If the great majority of patients with accessory pathway were symptomatic $(208-78 \%)$, for 57 (22\%) ventricular pre-excitation was discovered on an electrocardiogram done incidentally. Among asymptomatic ventricular preexcitation patients $24 / 56(43 \%)$ had malignancy criteria on electrophysiology testing ( 1 missing data), while there were only 58/ 189 (31\%) on the symptomatic group (19 missing data).

Conclusion: Children and adolescents with ventricular pre-excitation seems to have greater risk if asymptomatic. Endocardial electrophysiological study is mandatory for every patients with ventricular pre-excitation before teen age. 